\title{
THE UNIVERSITY OF SHEFFIELD
}

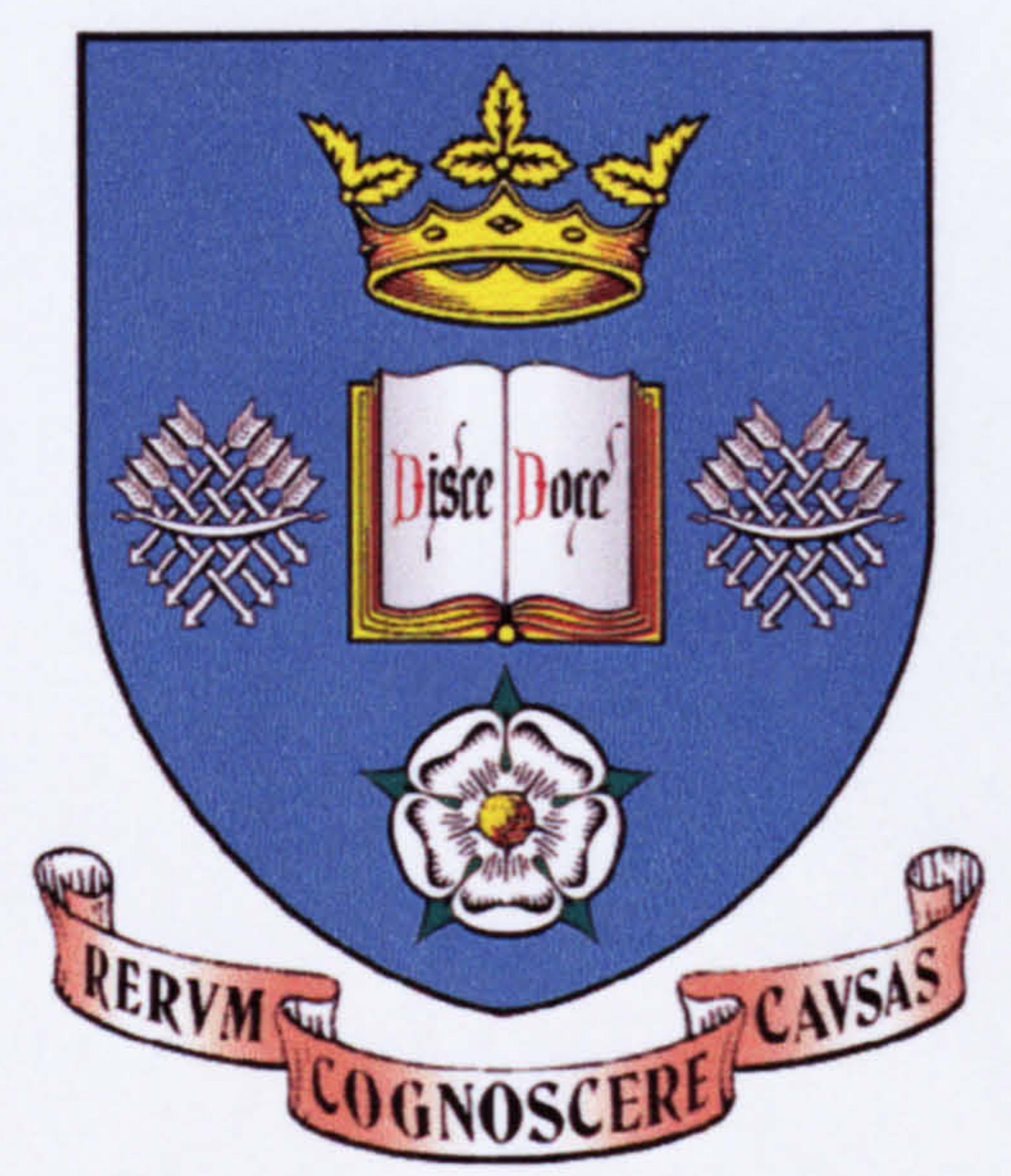

\section{DESIGN OF A HIGH SPEED SWITCHED \\ RELUCTANCE MACHINE FOR AUTOMOTIVE TURBO-GENERATOR APPLICATIONS}

\author{
By
}

Stuart David Calverley

\begin{abstract}
A Thesis submitted for the degree of Doctor of Philosophy in the Department of Electronic and Electrical Engineering, The University of Sheffield.
\end{abstract}

OCTOBER 2001 


\section{SUMMARY}

The efficient generation of relatively large quantities of electrical energy in vehicles is becoming an increasingly important issue, as a result of increasing demands of ancillary equipment and the emergence of hybrid power-train vehicles. An attractive solution to meeting these demands is to extract the electrical energy by means of a generator driven by a high-speed exhaust mounted turbine, a technology that is beginning to emerge commercially. This thesis is concerned with the design of a system, which extends this concept to enable both electrical generation and highly flexible air-management. The heart of the system is a high-speed switched reluctance machine, the rotor of which is located on a common shaft with the turbine and compressor wheels of a standard commercial turbocharger.

The design synthesis of a -switched reluctance machine capable of meeting the required performance specification is particularly onerous given the harsh environment in which it operates, specifically in terms of the restricted space envelope, the high ambient temperature and the very high rotational speeds. This thesis describes the design of a switched reluctance machine for a prototype system, a procedure that encompasses the detailed analysis of centrifugal stress in the rotor, aerodynamic losses, iron loss, rotordynamic performance and dynamic performance. The design and analysis of the system is supported by experimental evaluation at both component and system level. 


\section{ACKNOWLEDGEMENTS}

I would like to express my sincere thanks to Geraint Jewell and Robin Saunders for their invaluable guidance, encouragement and support during the course of the research and the writing of this thesis

I would also like to thank the EPSRC for the award of a research studentship and Holset Engineering for the construction of prototype equipment, and in particular Henry Tennant and David Green for their valuable advice and technical support.

Thanks are also due to members of the Electrical Machines and Drives Group, past and present, for both their technical advice and for providing a lively and friendly atmosphere. Thanks are also extended to all the technical staff in both the Group, IC engines lab and the Departmental Mechanical Workshop, for their skill and technical assistance in the manufacture of prototypes and test rigs.

Finally, many thanks to Helen for her continual support during the writing of this thesis. 


\section{CONTENTS}

SUMMARY ii

ACKNOWLEDGEMENTS iii

CONTENTS iv

NOMENCLATURE $\quad$ ix

\section{CHAPTER 1: INTRODUCTION}

1.1 DIESEL ENGINES 1

1.2 DIESEL ENGINE POLLUTION, FORMATION, CONTROL AND 3 REGULATION

1.3 TURBOCHARGING 6

1.4 TURBOCHARGER ASSISTING SYSTEMS 10

1.5 TURBOCHARGER RETARDING SYSTEMS AND EXCESS 12 ENERGY RECOVERY

1.6 ELECTRICAL MACHINE TECHNOLOGIES 16

1.7 SWITCHED RELUCTANCE MACHINES 20

1.7.1 Selection of pole number 23

1.7.2 Selection of stator and rotor pole arc 24

$\begin{array}{lll}1.8 & \text { SUMMARY } & 24\end{array}$

CHAPTER 2: ROTOR MECHANICAL DESIGN

2.1 INTRODUCTION 40

2.2 FINITE ELEMENTS ANALYSIS 41

2.3 FINITE ELEMENT ANALYSIS OF CYLINDRICAL ROTORS 42

2.4 FINITE ANALYSIS OF SR ROTORS 48

2.4.1 Introduction 48

2.4.2 Mesh discretisation 49 
2.4.3 Analysis of stress distribution 53

2.4.4 Rotor cross sectional design 54

2.4.5 Selection of rotor diameter 58

2.4.6 Shaft bore diameter 59

$\begin{array}{ll}2.4 .7 & \text { Rotor moment of inertia }\end{array}$

2.4.8 Radial growth of the rotor and airgap selection 62

2.5 PROTOTYPE ROTOR DESIGN AND MANUFACTURE 63

$\begin{array}{ll}\text { APPENDIX 2.1 } & 90\end{array}$

CHAPTER 3: MACHINE DESIGN SYNTHESIS AND DYNAMIC SIMULATION

3.1 INTRODUCTION 92

3.2 DESIGN SYNTHESIS

$\begin{array}{lll}3.3 & \text { DYNAMIC SIMULATION }\end{array}$

$\begin{array}{lll}\text { 3.3.1 Dynamic SR operation } & 97\end{array}$

3.4 NUMERICAL MODELLING OF SR MACHINES 101

3.4.1 Simulation stage 1: Characterisation or Electromagnetic Performance

101

$\begin{array}{ll}\text { 3.4.2 Simulation stage 2: Solution } & 108\end{array}$

3.5 LINEAR SIMULATION MODEL 109

$\begin{array}{lll}\text { 3.5.1 Current calculation } & 111\end{array}$

$\begin{array}{ll}\text { 3.5.2 Torque calculation } & 112\end{array}$

3.6 NON-LINEAR SIMULATION MODEL 113

$\begin{array}{lll}\text { 3.6.1 } & \text { Phase winding resistance } & 113\end{array}$

$\begin{array}{lll}3.7 & \text { POST-PROCESSOR STAGE } & 115\end{array}$

$\begin{array}{lll}\text { 3.7.1 Winding design } & 115\end{array}$

3.7.2 Current waveforms and results from non-linear model 124

$\begin{array}{lll}\text { 3.7.3 Optimal commutation angles } & 127\end{array}$

APPENDIX 3.1 Post processing finite element solutions to calculate winding flux linkage and inductance 
APPENDIX 3.2 Analytical calculations of inductance in aligned and unaligned positions

APPENDIX 3.3 Approximating the gauge curves using established curve fitting techniques

\section{CHAPTER 4: CALCULATION OF IRON LOSS}

4.1 INTRODUCTION

4.2 PREDICTION OF IRON LOSS

4.3 WINDING CONFIGURATION

4.4 CALCULATION OF FLUX DENSITY WAVEFORMS IN THE 170 MACHINE

4.4.1 Analytical

4.4.2 De-coupled finite-element method

4.4.3 Rotational components of flux density

4.5 LOSS MODEL 185

4.5.1 Introduction 185

4.5.2 Hysteresis 186

4.5.2.1 Major loop hysteresis loss component

4.5.2.2 Existing techniques for the estimation of minor hysteresis loop loss

4.5.2.3 Alternative method for estimating minor loop losses

4.5.2.4 Comparison between measured and predicted minor loop losses

4.5.3 Classical eddy current loss 196

4.5.4 Excess loss - Bertotti model 197

4.5.5 Calculation of dynamic component of iron loss based on the analytical method 200

4.6 MACHINE LEVEL PREDICTIONS OF IRON LOSS 203

4.7 DYNAMIC LOSS MEASUREMENTS AND SEPARATION 207

$\begin{array}{lll}\text { 4.7.1 Material samples } & 208\end{array}$

4.7.2 Measuring the loss induced by arbitrary waveforms 209 
4.8 EXPERIMENTAL VALIDATION OF LOSS MODEL

APPENDIX 4.1 Hysteresis loss measurement equipment

APPENDIX 4.2a Evaluation of the integrals in the excess loss equation for winding connection type $\mathrm{A}$

APPENDIX 4.2b Evaluation of the integral $s$ in the eddy current loss equation for winding connection type $A$

APPENDIX 4.2c Evaluation of the integrals in the excess loss equation for 263 winding connection type $B$

APPENDIX 4.2d Evaluation of the integrals in the eddy current for the 264 winding connection type B

APPENDIX 4.3 Components for iron loss for the 5kW, 100,000rpm 265 operating conditions

\section{CHAPTER 5: AERODYNAMIC LOSS}

\subsection{INTRODUCTION}

5.2 SIMULATION TECHNIQUES

5.3 SIMULATION RESULTS

5.3.1 Two-dimensional analysis

5.3.2 Three-dimensional analysis

5.4 EXPERIMENTAL VALIDATION

\section{ANALYSIS}

\subsection{INTRODUCTION}

6.1.1 Jeffcott analysis

6.2 BEARING CONFIGURATION

6.3 'FREE-FREE' MODAL ANALYSIS

\subsection{BEARING DESIGN}


6.4.1.1 Self-excited instability 303

$\begin{array}{lll}\text { 6.4.1.2 Parametrically excited instability } 304 & 308\end{array}$

$\begin{array}{ll}\text { 6.4.2 Rolling element bearings } & 308\end{array}$

$\begin{array}{lll}\text { 6.4.2.1 Rolling element bearing stiffness } & 311\end{array}$

$\begin{array}{lll}\text { 6.5 CRITICAL SPEED ANALYSIS } & 314\end{array}$

CHAPTER 7: PROTOTYPE EATG CONSTRUCTION AND EXPERIMENTAL VALIDATION OF FINDINGS

7.1 PROTOTYPE MACHINE CONSTRUCTION 339

7.1.1 Introduction 339

7.1.2 Design and manufacture of the rotor assembly 339

7.1.3 Stator laminations 341

7.1.4 Turbine end-cap $\quad 344$

7.1.5 Compressor end-cap 345

7.2 PROTOTYPE CONVERTER DESIGN AND CONSTRUCTION 346

7.2.1 Control system implementation 348

7.3 TEST RESULTS 349

7.3.1 Measurement of phase self-inductance and resistance 349

7.3.2 Low-speed, low-power bench testing 350

$\begin{array}{lll}\text { 7.3.3 Measured dynamic performance } & 351\end{array}$

7.3.4 Simulated performance at test-points 353

7.4 ENGINE TESTING 357

7.4.1 Experimental apparatus 357

7.4.2 Base testing results 358

7.5 TESTING ENGINE WITH EATG FITTED 362

CHAPTER 8: DISCUSSION AND CONCLUSIONS

8.1 CONCLUSIONS 


\section{NOMENCLATURE}

\section{CHAPTER 1 - INTRODUCTION}

$\begin{array}{ll}N_{\text {phase }} & \text { Number of turns per phase } \\ \mathrm{N}_{\mathrm{r}} & \text { Number of rotor poles } \\ \mathrm{N}_{s} & \text { Number of stator poles } \\ \mathrm{T} & \text { Torque } \\ W^{\prime} & \text { Co-energy } \\ \varepsilon_{0} & \text { Stroke angle } \\ \psi & \text { Flux linkage }\end{array}$

\section{CHAPTER 2 - ROTOR MECHANICAL DESIGN}

\begin{tabular}{ll}
$D_{b o r e}$ & Rotor bore diameter \\
$D_{r}$ & Rotor diameter \\
$D_{r, \max }$ & Maximum rotor diameter \\
$E$ & Young's modulus \\
$I_{p}$ & Polar moment of inertia \\
$k_{\alpha \gamma}$ & Inertia constant \\
$k_{\delta}$ & Radial growth constant \\
$k_{\sigma}$ & Rotor stress constant \\
$L_{\alpha x}$ & Axial length of lamination stack \\
$m_{v}$ & Mass per unit volume \\
$R_{l}$ & Cylinder inner radius \\
$R_{2}$ & Cylinder outer radius \\
$r_{f i l l e t}$ & Fillet radius \\
$S r$ & Element size ratio \\
$u_{r \max }$ & Maximum radial growth \\
$\alpha_{r}$ & Rotor pole arc \\
$\beta$ & Bore diameter ratio \\
$\gamma$ & Fillet radius ratio \\
$\gamma_{\max }$ & Maximum allowable fillet radius ratio \\
$\xi$ & Convergence parameter \\
$\sigma_{1}$ & First principal stress \\
& \\
\hline &
\end{tabular}




$\begin{array}{ll}\sigma_{2} & \text { Second principal stress } \\ \sigma_{e} & \text { Equivalent (Von-Mises) stress } \\ \sigma_{\max } & \text { Maximum calculated stress } \\ \sigma_{\max , F E} & \begin{array}{l}\text { Maximum calculated stress using finite element } \\ \text { technique }\end{array} \\ \sigma_{\max , i} & \text { Maximum stress of } i^{\text {th }} \text { level of discretisation } \\ \sigma_{\max , i-1} & \text { Maximum stress of } i^{\text {th }}-1 \text { level of discretisation } \\ \sigma_{r} & \text { Radial stress } \\ \sigma_{\theta} & \text { Circumferential (hoop) stress } \\ \omega & \text { Rotational speed } \\ \omega_{\max } & \text { Maximum rotational speed }\end{array}$

\section{CHAPTER 3 - MACHINE DESIGN SYNTHESIS AND DYNAMIC SIMULATION}

$\begin{array}{ll}a & \text { Parabola constant } \\ A_{s l o t} & \text { Area of winding slot } \\ B_{p} & \text { Peak flux density in stator tooth } \\ d_{b} & \text { Depth of stator back-iron } \\ D_{b} & \text { Rotor bore diameter } \\ D_{r} & \text { Rotor diameter } \\ d s & \text { Depth of the winding slot } \\ D_{s} & \text { Stator inner diameter } \\ i_{0} & \text { Current at beginning of mechanical overlap } \\ \hat{i}_{l} & \text { Peak current calculated with linear model } \\ i_{m} & \text { Maximum phase current } \\ \hat{i}_{n l} & \text { Peak current calculated with non-linear model } \\ i_{n o r m} & \text { Normalised current } \\ i_{r m s} & \text { RMS current } \\ i_{s} & \text { Phase current at onset of saturation } \\ i_{s 0} & \text { Current at apex of parabolic curve-fit } \\ i_{u} & \text { Phase current in unaligned position } \\ k_{p} & \text { Winding packing factor }\end{array}$




\begin{tabular}{|c|c|}
\hline$k_{T R V}$ & Torque per unit rotor volume constant \\
\hline$L$ & Phase inductance \\
\hline$L_{a}$ & Aligned phase inductance \\
\hline$L_{a x}$ & Axial length of lamination stack \\
\hline$l_{c u}$ & Mean length of a single winding turn \\
\hline$L_{\text {end_wdg }}$ & Length of end winding conductors \\
\hline$L_{u}$ & Unaligned phase inductance \\
\hline$N_{p h}$ & Number of winding turns per phase \\
\hline$P_{l}$ & Power calculated with linear model \\
\hline$P_{n l}$ & Power calculated with non-linear model \\
\hline$q_{r}$ & Number of rotor poles \\
\hline$q_{s}$ & Number of stator poles \\
\hline$r_{\text {fillet }}$ & Fillet radius \\
\hline$R_{p h}$ & \\
\hline$R_{p h, 2}$ & Resistance of 2-turn phase winding \\
\hline $\begin{array}{c}T \\
T\end{array}$ & $\begin{array}{l}\text { Torque } \\
\text { Average toraue }\end{array}$ \\
\hline$T_{\text {ave }}$ & \\
\hline$T_{\text {norm }}$ & Normalised torque \\
\hline$V_{d c}$ & DC link voltage \\
\hline$W_{s}$ & Stator tooth width \\
\hline$W^{\prime}$ & Co-energy \\
\hline$\alpha_{r}$ & Rotor pole arc \\
\hline$\alpha_{s}$ & Stator pole arc \\
\hline$\delta$ & Skin depth of a conductor \\
\hline$\Delta_{\text {ovl }}$ & Degree of rotor/stator mechanical overlap \\
\hline$\xi_{1}$ & Beginning of mechanical overlap \\
\hline$\xi_{2}$ & Beginning of complete mechanical overlap \\
\hline$\xi_{a}$ & Aligned position \\
\hline$\xi_{h r}$ & Midpoint of $\xi_{1}$ and $\xi_{2}$ \\
\hline$\xi_{0}$ & Offset angle \\
\hline$\xi_{u}$ & Unaligned position \\
\hline$\theta_{\text {dwell }}$ & Switch-dwell angle \\
\hline$\theta_{o f f}$ & Switch off angle \\
\hline$\theta_{o n}$ & Switch-on angle \\
\hline$\mu$ & Permeability \\
\hline$\rho_{c u}$ & Resistivity of \\
\hline$\sigma$ & Conductivity \\
\hline
\end{tabular}




$\begin{array}{ll}\psi_{a} & \text { Flux linkage } \\ \psi_{a} & \text { Aligned flux linkage } \\ \psi_{m} & \text { Maximum flux linkage } \\ \psi_{s} & \text { Flux linkage at onset of saturation } \\ \psi_{s 0} & \text { Flux linkage at apex of parabolic curve-fit } \\ \psi_{u} & \text { Flux linkage in unaligned position } \\ \omega & \text { Rotational speed }\end{array}$

\section{CHAPTER 4 - CALCULATION OF IRON LOSS}

$\begin{array}{ll}A & \text { Cross sectional area of sample material } \\ A_{1,2} \text { (section 4.5.2.3) } & \text { Area of sections of hysteresis curve } \\ A_{i} & \text { Cross sectional area of } i^{\text {th }} \text { region } \\ a \text { (section 4.4.3) } & \text { Eccentricity of orthogonal waveforms } \\ a \text { (section 4.5.2.1) } & \text { Hysteresis constant } \\ b & \text { Hysteresis constant } \\ B_{i} & \text { Flux density in } i^{t h} \text { region } \\ B_{m} & \text { Peak flux density } \\ B_{\text {meas }} & \text { Measured flux density waveform } \\ B_{r e f} & \text { Reference flux density waveform } \\ B_{\text {sat }} & \text { Saturation flux density } \\ d & \text { Lamination thickness } \\ e & \text { Induced emf } \\ E_{\text {emf }} & \text { Error between measured and reference emf } \\ e_{\text {meas }} & \text { Measured emf waveform } \\ e_{\text {ref }} & \text { Reference emf waveform } \\ G_{i} & \text { Effective cross sectional area of } i^{t h} \text { region } \\ G V_{0} S & \text { Excess loss constant } \\ H & \text { Magnetic field strength } \\ H_{0} & \text { Magnetic field strength when B=0 } \\ H_{c} & \text { Coercive magnetic field strength } \\ \hat{i} & \text { Peak demagnetising current } \\ k & \text { Loop correction coefficient } \\ K_{1,2} & \text { Gains in error correction algorithm } \\ k_{c} & \text { Coercivity constant } \\ k_{h} & \text { Hysteresis constant } \\ k_{i A} & \text { Element of k-matrix } \\ & \end{array}$


$k_{\tau}$

$l$

$m_{v}$

$M H$

$N$

$N_{p}$

$N_{s}$

$P_{C L A}$

$P_{D Y N}$

$P_{E X C}$

$P_{H Y S}$

$P_{\text {TOT }}$

$Q_{\text {minor }}$

$T$

$W_{c l}$

$W_{c l}^{s}$

$W_{\text {exc }}$

$W_{e x c}^{s}$

$W_{h}$

$W_{\text {tot }}^{s}$

$\alpha$

$\Delta B$

$\rho$

$\tau$

$\phi_{m}$

$\psi_{A, B, C}$

$\omega_{\text {demag }}$
Rotor angle modifier to k-matrix

Mean length of flux path of sample material

Mass per unit volume

Value of moving horizon integration filter

Number of turns per coil

Number of turns on primary winding

Number of turns on search coil

Classical eddy current compoent of iron loss

Dynamic components of iron loss

Excess component of iron loss

Hysteresis power loss

Total iron loss

Loop correction function

Period of fundamental flux density variation

Work done by classical eddy currents

Work done by classical eddy currents with sinusoidal induction

Work done by excess loss mechanism

Work done by excess loss with sinusoidal induction

Hysteresis work done

Total iron loss work done under sinusoidal induction

Alignment of principal axes

Flux density excursion

Resistivity

Pulse width

Maximum induced flux

Flux linking phases $\mathrm{A}, \mathrm{B}$ or $\mathrm{C}$.

Angular frequency of demagnetisation waveform

\section{CHAPTER 5 - AERODYNAMIC LOSSES}

$\begin{array}{ll}\text { A } & \text { Curve fitting exponent } \\ B & \text { Curve fitting exponent } \\ l_{g} & \text { Length of airgap } \\ P & \text { Aerodynamic power loss } \\ R e & \text { Reynolds number } \\ u_{w} & \text { Tangential Wall velocity }\end{array}$




$\begin{array}{ll}v & \text { Kinematic viscosity } \\ \omega & \text { Rotational speed }\end{array}$

\section{CHAPTER 6 - BEARING SELECTION AND ROTORDYNAMIC}

\section{ANALYSIS}

$\begin{array}{ll}A & \text { Location of accelerometer } \\ B & \text { Width of bearing } \\ B_{n} & \text { Normal component of flux density } \\ B_{t} & \text { Tangential force } \\ C & \text { Rotor/journal clearance } \\ d & \text { Bearing inner diameter } \\ D & \text { Bearing outer diameter } \\ D_{0} & \text { Diameter of shaft } \\ D_{b} & \text { Diameter of rolling elements } \\ D_{i} & \text { Diameter of } i^{\text {th }} \text { cylinder } \\ D_{m} & \text { Pitch diameter of bearing } \\ d_{t} & \text { Axial distance between turbine and turbine bearing } \\ e & \text { Rotor eccentricity } \\ f_{i, o} & \text { Groove radius coefficient for the inner/outer race } \\ F_{n} & \text { Normal component of force } \\ F_{t} & \text { Tangential component of force } \\ F\left(\rho_{i l o}\right) & \text { Curvature function for the inner/outer race } \\ G_{x y} & \text { Shear modulus } \\ H & \text { Location of modal hammer } \\ I_{p} & \text { Polar moment of inertia } \\ I_{p i} & \text { Polar moment of inertia of } i^{t h} \text { cylinder } \\ I_{t} & \text { Transverse moment of inertia } \\ I_{t i} & \text { Transverse moment of inertia of } i^{t h} \text { cylinder } \\ K_{n} & \text { Point contact Hertzian stiffness } \\ K_{r r} & \text { Incremental direct bearing stiffness } \\ K_{t r} & \text { Incremental cross coupled bearing stiffness } \\ k_{m} & \text { Modal stiffness of the } m^{\text {th }} \text { mode } \\ L & \text { Axial length of bearing } \\ L_{a x} & \text { Axial length of lamination stack } \\ l_{t} & \text { Axial length of } i^{t h} \text { cylinder } \\ & \end{array}$




$\begin{array}{ll}l_{t o t} & \text { Axial length of compressor/turbine } \\ m_{i} & \text { Mass of } i^{t h} \text { cylinder } \\ m_{m} & \text { Modal mass of the } m^{\text {th }} \text { mode } \\ m_{\text {tot }} & \text { Mass of compressor/turbine } \\ p & \text { Fluid film pressure } \\ p_{0} & \text { Oil supply pressure } \\ p_{a} & \text { Ambient pressure } \\ Q & \text { Radial force on bearing } \\ R & \text { Radius of journal } \\ r & \text { Radius of curvature of the raceway } \\ r_{c} & \text { Location of mass centre } \\ Z & \text { Number of rolling elements } \\ \alpha & \text { Contact angle of bearing } \\ \delta_{i} & \text { Deflection of inner race } \\ \delta_{n} & \text { Total radial deflection of the shaft centre } \\ \delta_{o} & \text { Deflection of outer race } \\ \varepsilon & \text { Dimensionless rotor eccentricity } \\ \mu & \text { Viscosity of oil } \\ \mu_{0} & \text { Permeability of free space } \\ \varphi & \text { Angular position of the line of centres } \\ \sum \rho_{i, o} & \text { Bearing 'curvature sum' for the inner/outer race } \\ \omega & \text { Rotational speed } \\ \omega_{m} & m^{t h} \text { critical speed } \\ & \end{array}$

\section{CHAPTER 7 - PROTOTYPE EATG CONSTRUCTION AND EXPERIMENTAL VALIDATION}

$\begin{array}{ll}c_{p} & \text { Specific heat capacity at constant pressure } \\ c_{p e} & \text { Specific heat capacity of exhaust gas at constant pressure } \\ L_{p h} & \text { Phase inductance } \\ Q & \text { Reactive power } \\ \dot{W}_{c} & \text { Compressor power } \\ \dot{W}_{t} & \text { Turbine power } \\ \gamma & \text { Ratio of specific heats } \\ \eta_{c} & \text { Compressor efficiency }\end{array}$




\section{CHAPTER 1}

\section{INTRODUCTION}

This thesis is concerned with the design of a prototype electrical machine for use as a high-speed electrically assisted turbocharger for automotive applications. The research presented encompasses the mechanical and electromagnetic issues associated with the design of a high-speed electrically assisted automotive turbocharger, the techniques developed and many of the conclusions drawn are generally applicable to the design of any high-speed electrical machine.

The principal motivations for the development of an electrically assisted turbocharger are to enhance the efficiency and drive-ability of the automotive compression ignition diesel engine and to facilitate a reduction in the emission of exhaust pollutants. The potential merits of this type of device and the legislative obligations placed upon engine manufactures to reduce harmful engine exhaust emissions and hence adopt such exhaust after-treatment technologies are discussed in this introduction. The specification of the electrical machine, i.e. the maximum speed and power ratings, the thermal endurance and the available space envelope, are derived from consideration of the specific features of automotive turbochargers and the environment in which they operate.

\subsection{Diesel Engines}

Compression-ignition diesel engines are very widely employed in applications where the demand for robustness, high reliability and longevity is paramount. Indeed the heavy-duty-commercial transport, agricultural, military, marine and locomotive sectors are dominated by the diesel engine, particularly in the United Kingdom and Western Europe. The power rating of compression ignition engines varies between 5 $\mathrm{kW}$ to $22 \mathrm{MW}$ with swept volumes between 0.3 and 1600 litres per cylinder. 
Combustion in the compression ignition diesel engine is initiated by injecting liquid fuel into a combustion chamber containing compression-heated air. The liquid fuel evaporates, entraining air at temperatures exceeding the ignition point of the fuel, until the localised air/fuel ratio is in the correct proportion to initiate combustion. After the initial combustion delay, the fuel spray exhibits multi-point ignition and a rapid rate of heat release is observed as the pre-mixed fuel burns in a few crank angle degrees, figures 1.1 and 1.2. Combustion slows after the pre-mixed fuel has been consumed and the rate of heat release is dictated by the rate at which fuel and air become available for combustion. As oxygen is consumed and the pressure and temperature in the combustion chamber are reduced in the late phases of combustion the heat release slows allowing the formation of sustainable soot within the combustion chamber, figure 1.2 [1.1].

Much greater expansion ratios may be adopted in diesel engines without the characteristic pre-ignition of fuel observed in spark ignition engines. The higher compression ratios give the diesel engine much higher thermal efficiency than a similar spark ignition petrol engine. Output power in C.I. engines is controlled by varying the amount of fuel injected into the cylinder, while the air inducted per cycle remains relatively constant due to the absence of any throttling system. The overall air/fuel ratio within the combustion chamber is therefore variable. At low load the combustion occurs under very lean conditions whereas at high load the air/fuel ratio approaches the stoichiometric value. The combustion at air/fuel ratios approaching or below the stoichiometric condition is characterised by excessive particulate matter formation, which due to the low oxygen content in the combustion chamber cannot be burned prior to exhaust. Ultimately the power output of the diesel engine, particularly at low speed, is governed by the minimum air/fuel ratio that will allow effective and complete combustion of the fuel with tolerable levels of particulate matter in the exhaust gas. 


\subsection{Diesel Engine Pollution, Formation, Control and Regulation}

The combustion efficiency (as distinct from the overall thermal efficiency) of the diesel engine typically exceeds $98 \%$ [1.1], however diesel engine emissions produce a significant level of air pollution particularly in urban environments [1.2].

The primary pollutant associated with diesel engines is the particulate emission, which consists primarily of combustion generated carbonaceous material (soot) with absorbed hydrocarbon and organic compounds. Most particulate matter arises from incomplete combustion particularly in the late stages of combustion (figure 1.2). However engine oil from poorly maintained vehicles may also be a significant contributor [1.1, 1.3]. Soot formation occurs at temperatures between 1000 and $2800 \mathrm{~K}$, at pressures in the range of 5-10MPa and occurs in two stages [1.1]:

I. Particle formation: Where the first solid matter arises due to the oxidation and pyrolysis of the fuel in the form of a laminar structure of bonded carbon atoms. These first particles are typically in the range of $2 \mathrm{~nm}$ [1.1].

II. Particle growth. This either occurs by coagulation of the individual particles or by surface growth where gaseous phase substances are deposited on the surface of the solid particulate. The mean equivalent diameter of the particles emitted after growth is $28 \mathrm{~nm}$ [1.1]

At either stage of the particulate formation and growth oxidation can occur where typically, in an oxygen abundant environment, the product will be $\mathrm{CO}_{2}$. The resulting emission of particulate matter is a summation of the factors contributing to the formulation and growth of the particles and the subsequent oxidation.

The UK government, addressing the apparent health risks associated with airborne particulate matter have prepared a long term air quality standard [1.4]. Particles with equivalent diameters of less than $10 \mu \mathrm{m}$, referred to as $\mathrm{PM}_{10}$, are limited to mean concentrations of $50 \mu \mathrm{g}$ per cubic metre over a 24 hour period, with a target of 
achieving $99 \%$ compliance in the UK by the year 2005. In parallel with this UK based regulation the European Union has agreed a two stage air-quality standard, from which a summary of the requirements regarding the limits of $\mathrm{PM}_{10}$ mean concentrations is shown in table 1.1 [1.4]

\begin{tabular}{|c|c|c|c|c|}
\cline { 2 - 5 } \multicolumn{1}{c|}{} & Year & $\begin{array}{c}\text { Maximum daily mean } \\
\text { concentration }\left(\mu \mathrm{g} / \mathrm{m}^{3}\right)\end{array}$ & $\begin{array}{c}\text { Number of permissible } \\
\text { daily exceptions annually }\end{array}$ & $\begin{array}{c}\text { Mean concentration } \\
\left(\mu \mathrm{g} / \mathrm{m}^{3}\right) \text { (annual) }\end{array}$ \\
\hline STAGE I & 2005 & 50 & 35 & 40 \\
\hline STAGE 11 & 2010 & 50 & 7 & 20 \\
\hline
\end{tabular}

Table 1.1 Summary of the EU two stage air-quality standard regarding $\mathrm{PM}_{10}$ concentrations.

Nationally road transport contributes $25 \%$ of the airborne particulate matter in the UK (1996 figures), and the contribution from diesel engines is considerably higher than that from spark ignition engines [1.4]. However in regions such as London where there is no appreciable industrial contribution road traffic accounts for $77 \%$ of the airborne particulate matter [1.4]. Figure 1.3 shows the spatial distribution of $\mathrm{PM}_{10}$ emissions in 1996, indicating higher concentrations in urban areas, which is particularly prevalent in the South East and the North of England. However this problem is not solely due to the effect of traffic emissions of $\mathrm{PM}_{10}$, as other urbanised concentrations such as domestic combustion, construction and aerosol usage contribute. Table 1.2 shows estimates of exhaust emission factors for diesel vehicles $(\mathrm{g} / \mathrm{km})$ with varying road conditions, weighted for engine capacity and vehicle load. The figures quoted refer to diesel vehicles compliant with European stage II regulation regarding emission of particulate matter and other pollutants implemented in 1996/1997. 


\begin{tabular}{|l|c|c|c|c|}
\cline { 2 - 5 } \multicolumn{1}{l|}{} & Urban & $\begin{array}{c}\text { Rural single } \\
\text { carriageway }\end{array}$ & $\begin{array}{c}\text { Rural dual } \\
\text { carriageway }\end{array}$ & Motorway \\
\hline Diesel cars & 0.02 & 0.012 & 0.011 & 0.028 \\
\hline Diesel LGV & 0.061 & 0.043 & 0.042 & 0.083 \\
\hline Rigid HGV & 0.310 & 0.189 & 0.169 & 0.116 \\
\hline Articulated HGV & 0.343 & 0.212 & 0.189 & 0.131 \\
\hline Buses and coaches & 0.347 & 0.153 & 0.136 & 0.092 \\
\hline
\end{tabular}

Table 1.2. $\mathrm{PM}_{10}$ exhaust emission factors $(\mathrm{g} / \mathrm{km})$ with varying load conditions [1.4]

Table 1.2 highlights that the emissions of $\mathrm{PM}_{10}$ for most categories of vehicle are considerably higher for urban driving cycles where typically a higher degree of transient engine operation is observed, whereas the lowest emission factors are observed under conditions where the engine is operated for long periods in a steady state condition, such as motorway travel.

\begin{tabular}{|l|c|c|c|c|c|c|c|c|c|c|c|}
\cline { 2 - 12 } & 1970 & 1975 & 1980 & 1985 & 1990 & 1991 & 1992 & 1993 & 1994 & 1995 & 1996 \\
\hline $\begin{array}{l}\text { Combustion in energy } \\
\text { production and transformation. }\end{array}$ & 78 & 61 & 60 & 53 & 60 & 59 & 59 & 57 & 49 & 40 & 38 \\
\hline $\begin{array}{l}\text { Commercial, institutional and } \\
\text { residential combustion. }\end{array}$ & 235 & 139 & 109 & 99 & 55 & 59 & 53 & 54 & 44 & 34 & 36 \\
\hline \begin{tabular}{l} 
Industrial combustion. \\
\hline $\begin{array}{l}\text { Non-combustion processes, viz. } \\
\text { mining and construction. }\end{array}$
\end{tabular} & 54 & 43 & 33 & 25 & 26 & 26 & 27 & 25 & 23 & 20 & 17 \\
\hline $\begin{array}{l}\text { Road transport, including petrol } \\
\text { and diesel exhaust emissions } \\
\text { and brake and tyre wear. }\end{array}$ & 46 & 50 & 55 & 58 & 67 & 66 & 64 & 61 & 60 & 56 & 52 \\
\hline \begin{tabular}{l} 
Diesel exhaust sub-category \\
\hline Other Transport.
\end{tabular} & $(33)$ & $(35)$ & $(37)$ & $(37)$ & $(45)$ & $(46)$ & $(44)$ & $(43)$ & $(43)$ & $(40)$ & $(37)$ \\
\hline Waste treatment and disposal. & 44 & 44 & 46 & 45 & 38 & 37 & 34 & 33 & 26 & 7 & 6 \\
\hline TOTAL & 538 & 400 & 362 & 341 & 314 & 313 & 299 & 293 & 267 & 220 & 213 \\
\hline
\end{tabular}

Table 1.3 Emissions of $\mathrm{PM}_{10}$ by sector per year for the period 1970-1996, in Kilotonnes [1.4] 
Table 1.3 shows the emissions of $\mathrm{PM}_{10}$ by sector in the UK, and for comparison the output of $\mathrm{PM}_{10}$ from diesel exhaust is also shown as a subset of the road transport category.

It is apparent from table 1.3 that the greatest reduction in particulate matter emission has arisen in the commercial, institutional and residential combustion sector, where the reduced use of coal as a residential fuel has had the most pronounced effect [1.4]. Indeed, emission of $\mathrm{PM}_{10}$ from diesel engines accounted for $17 \%$ of the total UK $\mathrm{PM}_{10}$ emission in 1996 and hence diesel engines are the subject of increasingly stringent regulations regarding particulate matter emission. Table 1.4 shows the European Community limits for exhaust emissions from new diesel cars. Reductions by a factor of 5.6 are required over the period 1993-2005

\begin{tabular}{|c|c|c|c|c|}
\hline Stage & Date & $\mathrm{PM}(\mathrm{g} / \mathrm{km})$ & $\mathrm{NO}_{\mathrm{x}}+\mathrm{HC}(\mathrm{g} / \mathrm{km})$ & $\mathrm{NO}_{\mathrm{x}}(\mathrm{g} / \mathrm{km})$ \\
\hline I & 1993 & 0.14 & 0.97 & - \\
\hline II & 1997 & 0.08 & 0.7 & - \\
\hline III & 2001 & 0.05 & 0.56 & 0.5 \\
\hline IV & 2005 & 0.025 & - & 0.25 \\
\hline
\end{tabular}

Table 1.4. European Community limits for particulate matter and $\mathrm{NO}_{\mathrm{x}}$ emission for new diesel cars [1.3].

\subsection{Turbocharging}

The power output of an internal combustion engine is limited by the amount of fuel that can be efficiently burned in the combustion chamber which in turn is governed by the mass of air present in the cylinder. Supercharging is a method of raising the inlet air density and hence providing a greater mass of air into the cylinder. 
The turbocharger, an example of which is shown in Figure 1.4, is a device used to elevate the air pressure at the inlet manifold. Power is developed as the exhaust gas passes through the turbine, and this power (minus shaft losses) is delivered to the compressor where the density of the inlet gas is elevated above atmospheric and the mass of air entering the combustion chamber is greater than that of a naturally aspirated engine. Figure 1.5 shows a typical turbocharger-engine arrangement for a four cylinder four stroke engine utilising a twin entry turbine to exploit the pulsating nature of the exhaust gas. The specific power of a turbocharged diesel engine is generally higher than that of an equivalent naturally aspirated engine, and furthermore, turbocharged engines exhibit lower specific particulate matter emissions and slightly lower specific fuel consumption.

The turbocharger environment is particularly arduous, typical automotive units experiencing exhaust gas temperatures at entry to the turbine in the region of $1000 \mathrm{~K}$, shaft speeds in excess of 100 000rpm and pressure ratios across a stage as high as 3:1. A typical maximum shaft power rating for a medium sized automotive type turbocharger is in the region of $35 \mathrm{~kW}$.

The first attempts to use turbochargers were made by Buchi in 1909, however, measurable success was only achieved in 1925 [1.5]. The widespread use of turbochargers since 1945 is mainly attributed to the development of materials able to withstand the stress and temperature levels associated with high-speed turbomachinery. Legislation regarding engine emissions and specific power, advanced modelling and improved manufacturing techniques have also played a significant role in their development. Turbochargers are currently widely utilised in medium and large engine applications, most notably in locomotive, marine, road haulage and static electrical generation applications, however their use in the high speed automotive diesel engines is becoming increasingly prevalent.

For automotive applications where the engine operates over a wide load and speed envelope the centrifugal compressor is almost exclusively used. Axial compressors exhibit higher efficiencies, however these devices are typically more expensive to 
manufacture, less robust and exhibit a smaller operating envelope. [1.6]. The choice of turbine for the automotive application is mainly dictated by the size of the turbocharger. The radial flow turbine is mainly utilised for small turbochargers, whereas at diameters over $250 \mathrm{~mm}$ the axial flow turbine may be advantageous [1.6]; again the discriminators are cost, manufacturing complexity and reliability.

The energy availability of the exhaust gas is dictated, in terms of engine parameters, by the load and speed. The turbine and compressor are matched to provide an acceptable energy balance over a wide range of operating conditions. However, when utilising a fixed geometry turbine, a good match over the entire engine operating range is difficult to achieve. The internal combustion piston engine, an example of a positive displacement device, exhibits a mass flow characteristic that is almost linear with engine speed and inlet density. The turbocharger however, shows an increase in pressure differential across the respective flow device with increased mass flow rate. Hence only a single point match between the engine and turbocharger may be achieved and any deviation from this point is subject to degradation of the performance of the overall system. The components are typically matched to provide an acceptable minimum amount of boost at low speeds. At high speeds and loads the boost often becomes too high in terms of pre-ignition limits in a spark ignition engine or metallurgical/cooling limits in a compression ignition engine. At high engine speeds and loads the turbocharger speed may reach limits based upon the centrifugal stress and rotordynamic performance of the rotor/bearing system. Figure 1.6 shows a typical torque-limiting curve for a diesel engine (where in this case the torque is presented as brake mean effective pressure, BMEP), detailing the individual mechanisms that limit the performance of the engine. The smoke limit at high load, low speed and the turbocharger over-speed limit are attributable to the turbocharger design and subsequent matching to the engine.

Further to these matching considerations, the transient response of the turbocharger must be considered. The mass flow rate of fuel injected into the combustion chamber can be increased rapidly, however the turbocharger has a much higher time constant and the time taken for the air flow rate to increase to appropriate levels may be 
considerable. This provides fuel rich combustion conditions where a large amount of soot is formed in the diffusion flame and little subsequent oxidation occurs, leading to the characteristic plume of black smoke and poor drive-ability of the diesel engine.

It is apparent that, over the full range of engine operating conditions, the general requirements of any system designed to improve the turbocharger/engine match varies according to the engine operating condition. Consequently any turbocharger ancillary system designed to improve the match may be broadly categorised as either:

1. Turbocharger assist. Systems/devices designed to improve the turbocharger performance under low load engine operating conditions, including the Hyperbar system of turbocharging [1.7,1.8], compressed air injection into the compressor [1.9], the wastegate control method, variable geometry turbine $[1.10,1.11,1.12]$, three wheel turbochargers [1.13,1.14], electrically driven compressors [1.15], and the use of low inertia ceramic turbo-machinery components [1.16].

2. Turbocharger retard. Systems/devices used to slow the turbocharger down at high engine operating conditions in order to prevent over-boost and shaft over-speed. The use of such systems allows a considerable degree of power to be extracted from the exhaust gases, which may be converted to mechanical drive shaft power using turbocompounding, useful mechanical power for ancillary vehicle functions or into electrical power where the requirements of the conventional alternator may be eliminated.

In order to gain insight into the requirements, specifications, complexity and performance of the wide variety of conventional systems described, and hence to define a tentative specification which may be used in the design of such systems, it is useful to discuss each system, both assisting and retarding, individually. 


\subsection{Turbocharger Assisting Systems}

The wastegate and variable geometry turbine systems may be classified, in some applications, as a device which retards the turbocharger system. However, since these devices provide a variable match between the turbocharger and engine by altering the characteristics of the turbine and hence produce no useful output power, it is appropriate in this case to classify them as assisting.

1. The Hyperbar system essentially de-couples the compressor flow from the (low compression) engine by introducing a bypass valve, controlling the flow rate, in the inlet manifold so the compressor can be used in a region of higher yield, closer to the surge line of the device. Figure 1.7 shows a schematic representation of the Hyperbar system. The excess air is recombined with the exhaust gases from the engine in a secondary combustion chamber. Under low load operating conditions, typically around $20 \%$ of maximum, fuel is introduced into the auxiliary combustion chamber to increase the supercharging pressure to compensate for the low engine compression ratio. At low engine speed and high load the auxiliary chamber is again utilised to increase the supercharging pressure to increase the air/fuel ratio and hence reduce specific engine emissions and thermal loading. To exploit the maximum advantage of the Hyperbar system the engine is designed with lower compression ratios, however the compression ratio is typically too low to achieve self ignition without the boost pressure being above ambient and such systems require an electrically actuated turbocharger to allow starting. A further disadvantage of the system is the increased fuel consumption penalty of the secondary combustion chamber, the specific fuel consumption is increased by $2-8 \%$ at rated speed and load [1.7] and by as much as $30 \%$ at low engine operating conditions [1.17]

2. Compressed air injection into the compressor is a method primarily utilised to improve the transient response of the turbocharger, however the system aids the scavenging process in two-stroke petrol engines and enables starting 
of large, typically marine, diesel engines. The system comprises generally several angled nozzles (although a single nozzle system is in utilisation [1.9]) located at the rotor tips around the centre of rotation, figure 1.8. Compressed air is introduced into the compressor to aid the acceleration of the turbocharger under the application of a suddenly increased engine load. The system has the added advantage of increasing the air/fuel ratio since the injected air enters the combustion chamber and the transient emissions, most notably particulate matter, can be significantly reduced. However, considerable air storage and management systems must be available for the system to operate.

3. The wastegate control method is widely commercially utilised. The wastegate allows a proportion of the exhaust gas to bypass the turbine under conditions of high engine load and speed, preventing turbocharger shaft overspeed and over-boost of the inlet air. This ability to alleviate excess energy at high end operating conditions allows a turbocharger-engine match to be achieved whereby the boost at low end conditions is significantly increased via a smaller capacity turbine.

4. Variable geometry turbochargers are currently commercially available. The system utilises either a moving turbine scroll wall [1.18], or more typically [1.6], a series of adjustable guide vanes [1.19], shown in figure 1.9. Adjusting the angle of the vanes (or indeed narrowing the sidewall entry distance) allows the inlet area of the turbine to be optimised given the engine operating conditions. Reducing the nozzle area at low end operating conditions accelerates the turbocharger shaft and allows significant improvement to be made to the low-end transient operation of the engine.

5. Three wheel turbochargers consist of a third turbine hydraulically powered via an external supply of high-pressure oil. The third wheel is either a hydraulic turbine or a pelton wheel. Again the power addition occurs at lowend engine operating conditions and hence the third wheel is often optimised for lower speeds than the turbocharger. Encouraging results are reported for this system, indeed the low speed maximum torque characteristic of the 
engine is greatly enhanced with no increase in particulate matter emissions [1.13].

6. Electrically assisted compressors, such as the so-called 'turbo-pac' device, provide significant reductions in the development of exhaust particulate matter under transient engine loading [1.15]. The device comprises a radial compressor driven with a brushless permanent magnet (PM) motor and may be operated in parallel with a standard automotive turbocharger.

7. Ceramic turbo-machinery components, most commonly the utilisation of Silicon Nitride turbine wheels significantly reduces the inertia of the turbocharger rotor. The steady state low speed performance of the engine shows no improvement as there is no power addition to the turbocharger shaft, however the transient response of the engine is improved by the shortened turbocharger response time. The commercial development of such devices is poorer than predicted [1.16], mainly due to the mechanical interfacing between the steel and ceramic components, the high cost of manufacture and high rejection rate of the components.

\subsection{Turbocharger Retarding Systems and Excess Energy Recovery}

Many of the assisting devices described sufficiently enhance the low end performance of the turbocharger and allow the turbine, in particular, to be downsized and hence re-matched to the engine at higher end operating conditions. In the majority of cases this re-matching eliminates the need for a retarding system on the turbocharger, indeed the use of assisting systems is much more prevalent than retarding systems. However several systems have been developed to retard the turbocharger by extracting the excess exhaust energy, providing power to perform other functions.

1. Turbo-compounding by combining the excess power available to the turbine under high engine operating conditions with that from the engine shaft has 
been utilised in aircraft, ground transport and marine applications [1.20]. Although many combinations of arrangement and number of turbines exist, each system comprises a turbine transmitting power via a gear train to the engine crankshaft (figure 1.10 shows a schematic of the most widely used two-turbine, series system [1.20]). Research on turbocompound engines widely indicates increased transient response [1.21, 1.22], lower particulate emissions with no increase in $\mathrm{NO}_{\mathrm{x}}$ output [1.22] and lower specific fuel consumption [1.23, 1.21]. Figure 1.11 shows typical fuel consumption characteristics of a standard engine and a series turbocompound engine [1.20]. Turbocompound systems are relatively complex and the largereduction gearboxes needed between the turbine and the crankshaft require, typically, fluid couplings to reduce the torsional oscillations on the turbine shaft generated by the crankshaft [1.23].

2. Ancillary power turbines. Woollenweber [1.24] argues that commercial acceptance of the turbocompound engine is hampered by the cost and mechanical complexity and suggests that the power provided by the second turbine is best utilised in the engine cooling system. The second turbine is coupled to an axial compressor, in an arrangement similar to a conventional turbocharger, but to provide cooling air for the engine and heat exchanger, it is proposed to mount the device and appropriate heat exchangers at the rear of the vehicle, eliminating the need for a conventional radiator. The system reduces the parasitic load on the engine, allows the vehicle body to be redesigned reducing aerodynamic drag and ultimately reduces the fuel consumption of the vehicle [1.24].

3. Turbogenerators are emerging as a technology capable of supplying useful quantities of electrical power primarily for utilisation in hybrid electric vehicles. Atkinson et al. [1.25] have reported the design of a 3kW, 100000 rpm three-phase brushless permanent magnet turbo-alternator. Figure 1.12 shows a schematic layout of the system in a hybrid vehicle drive train, while figure 1.13 shows the detail of the prototype turbo-alternator. The system operates on a naturally aspirated engine, and as a consequence, the increase in exhaust back-pressure reduces engine performance and ultimately leads to a 
degradation of system fuel efficiency over some parts of the operating envelope. However the operating range of the engine in hybrid vehicles is very constrained, in some cases even to a single operating point. This provides scope to optimise the turbo-alternator/engine characteristics so as to minimise the adverse effects of the increased exhaust back pressure [1.25]. The system was tested both on a constant pressure flow bench and an internal combustion engine, from which results indicate electrical power generation of $3 \mathrm{~kW}$ at a system efficiency of $65 \%$ with a corresponding reduction in specific fuel consumption of $4-5 \%$ [1.25].

The objective of the research presented here is to extend this concept to a system that both exploits the excess exhaust gas energy for electrical generation, and also provides motoring torque to the compressor to enhance the controllability of inlet boost. The proposed system is referred to throughout this thesis as an EATG system (Electrically assisted turbo charger/generator). The basic layout of the EATG is shown schematically in figure 1.14. Both the exhaust turbine and the inlet compressor are mounted on a common shaft either side of an electrical machine rotor.

Under low engine speed and high load conditions where the air/fuel ratio in the cylinder is very low, the compressor speed can be increased by the motoring torque produced by the electrical machine, thus facilitating more efficient combustion than a standard turbocharger. Further, the ability to accelerate the compressor by the addition of torque from the electrical machine will also reduce exhaust emissions under transient engine loading. Further, at high engine speed and low load, the shaft speed can be retarded by operating the electrical machine as a generator, with little or no detriment in engine performance. In the absence of any specification of the power levels attainable with such devices, the initial design was based on delivering a net electrical power of $3 \mathrm{~kW}$ over a typical driving cycle and a peak power of $\pm 5 \mathrm{~kW}$. These figures are comparable with the power levels reported by Atkinson et al. [1.25]. 
A similar integrated turbine, compressor and electrical machine design is currently being developed by Reuter for cryogenic application [1.26, 1.27]. The outline design is shown in figure 1.15. The system is much larger than is typically encountered in automotive turbocharger applications and allows the inclusion of a magnetic bearing to reduce the friction loss in the bearing system. The power modulation by the electrical machine is specifically to constrain the speed of the shaft to $60000 \mathrm{rpm}$. The power rating of the prototype brushless 6 phase AC machine is $\pm 50 \mathrm{~kW}$ (research is continuing into a $450 \mathrm{~kW}$ machine), operating over a temperature range of $-180^{\circ} \mathrm{C}$ to $120^{\circ} \mathrm{C}$.

During the course of the research reported in this thesis, Turbodyne Systems Inc. (USA) released limited details of a turbo-generator system known as the 'Dynacharger' $[1.28,1.29]$. The concept is similar to that proposed in this thesis, a motor/generator being placed on a common shaft with the turbine and compressor of a standard turbocharger. The unit is inter-changeable with a standard turbocharger installation with the machine windings connected to a power converter as shown in figure 1.16. However, few specific details were provided as regards measured performance or detailed design data. A revised design was also reported, albeit again with limited technical data [1.28], where the motor/generator is mounted at the compressor end of the shaft to assist with cooling. The claimed benefits of the dynacharger system are:

- Improved specific fuel consumption. Reductions as high as 10-20\% are quoted with a redesign of the gearbox to accommodate the increased torque envelope of the engine [1.30].

- Non-zero turbocharger speed at engine start up. This allows the compression ratio of the engine to be optimised for fuel consumption, where currently the design is compromised in order to facilitate cold start of the engine [1.29].

- Effective elimination of turbo-lag. Motive power can be added to overcome turbo-lag, and hence the turbine and compressor can be re-matched to allow 
elevated turbocharger speeds at low engine operating conditions without the risk of shaft over-speed [1.30].

- Reduced emissions. Under urban drive cycle conditions the characteristic plume of black smoke from a diesel engine under sudden load application is greatly reduced due to the elevated boost levels and increased transient response of the turbocharger. Further, $\mathrm{NO}_{\mathrm{x}}$ may be reduced by the high rates of exhaust gas recirculation that may be afforded by the device [1.29].

It should be noted that there are very limited published results that rigorously validate these findings at present.

\subsection{Electrical Machine Technologies}

The recent practical realisation and preliminary low-volume commercialisation of high-speed automotive turbo-generators has been facilitated, to a large extent, by advances in brushless electrical machine technologies. The three turbo-generators described in section 1.5, i.e. the turbo-generator described by Atkinson, the motor/generator for cryogenic pump applications developed by Reuters and the turbocharger motor/generator commercially manufactured by Turbodyne systems, have in each case been based around brushless DC permanent magnet machines (BLDC).

However switched reluctance (SR) machines are also likely to be well suited to turbo-generators, and may in the long term offer a more cost effective solution for high volume, mass market applications. SR machines continue to attract significant interest in the aerospace [1.31, 1.32], domestic appliance [1.33] and automotive sectors [1.34]. The principles of SR machines are very well established, and indeed as far back as 1838 their basic principles were identified [1.35]. However, they have only recently emerged as a competitive machine format as power electronic converter technology has become established. The absence of magnets and brushes make SR machines highly competitive in terms of robustness and cost as compared 
to brushless PM machines [1.36,1.31,1.32]. Further, it has been claimed [1.31] that they inherently afford a higher degree of fault tolerance as compared to permanent magnet machines.

In common with PM machines, SR machines are capable of very high levels of performance in terms of specific power and high-speed capability, and can be controlled in a highly flexible manner, albeit that the control strategies may be more complex than an equivalent PM machine. Although many comparisons between the relative merits of SR and PM machines have been published [1.37,1.31], these draw few if any definitive conclusions that can be supported by quantitative data. Indeed, it is apparent that many of the conclusions drawn are inevitably subjective in nature, application specific and highly influenced by the constraints imposed in the comparison. For the purposes of this study, it is therefore useful to identify and qualitatively compare the key features of each of these topologies within the context of the likely requirements of the EATG system.

1. Power density. As will be demonstrated in chapters 2 and 6 , the rotor diameter is highly constrained by the centrifugal stress induced at high speeds and the axial length by rotor-dynamic considerations. This constraint on the rotor volume is likely to require that the machine has a very high power density if useful levels of power are to be derived. Although the power densities which can be achieved in electrical machines are highly dependent on specific design features, particularly the cooling mechanism employed, it is generally recognised that PM and SR machines are capable of comparable power densities [1.34].

2. Fault tolerance. The ability of the electrical machine to provide generating torque (hence limiting the shaft speed) allows the turbine and compressor to be re-matched to provide higher power levels without the risk of over-speed at high engine speed and load conditions. However failure of the electrical machine may allow the turbocharger shaft speed to reach levels where catastrophic failure may occur. Thus, the degree to which the machine is fault 
tolerant, i.e. it can remain operational (albeit at some reduced rating) with faults such as short/open circuit phase windings, is likely to be a key factor. SR machines are generally regarded as exhibiting a higher degree of fault tolerance than conventional PM machines [1.31], which generally require the addition of specific design features to achieve comparable levels of faulttolerance with consequent reductions in specific power capability [1.31].

3. No-load iron loss. Although the duty cycle of the turbo-generator will be a complex function of both engine operating conditions and turbine/compressor matching, it is likely that its principal use will be at high engine speeds and/or during engine transients. As such, it is likely to be essentially idling for a considerable proportion of the time, during which it is potentially a parasitic loss. In this regard, SR machines offer a considerable advantage since the noload (i.e. un-excited) iron losses are essentially zero, while for a conventional PM machine, the iron losses under no-load are still significant. This is likely to be a particularly important factor in this case, since the iron loss will comprise a very significant proportion of the system losses given the high operating speeds of the machines.

4. Rotor high-speed capability. The rotors of both SR and brushless PM machines are well suited to high-speed operation. The single piece laminations and the absence of any magnets and rotor windings in SR machines, make them ideally suited to high speeds in small to medium machines. However the maximum peripheral speed is ultimately limited by the intrinsic material strength since there is little, if any, scope to contain the rotor within a high-tensile sleeve since they cannot readily accommodate large airgaps without a marked reduction in performance. Below this limit they offer a low-cost and robust rotor structure. High-speed PM machines on the other hand, require some form of magnet containment (e.g. a carbon fibre sleeve), even in the case of small machines. Ultimately, their ability to accommodate large airgaps and hence thick-walled containment may enable them to exceed the peripheral speed capabilities of SR machines. However, 
small to medium high-speed PM machines will inevitably require a more complex, multi-component rotor structure.

5. Elevated Temperature Capability. The high temperatures encountered within the turbocharger environment place particular demands on the materials in the electrical machine. The critical materials in terms of temperature capability are the winding insulation and the permanent magnets used in the BLDC design (most conventional lamination materials being well capable of operating up to $500^{\circ} \mathrm{C}$ or so). The maximum temperature that the winding insulation can be exposed to without reducing lifetime dramatically is typically some $200-220^{\circ} \mathrm{C}$. For the permanent magnet materials, the maximum operating temperature varies between different materials, e.g. high performance Hard ferrite $-150^{\circ} \mathrm{C} ; \mathrm{NdFeB}-220^{\circ} \mathrm{C} ; \mathrm{Sm}_{2} \mathrm{Co}_{17}-250-350^{\circ} \mathrm{C}$ [1.38]. In many cases where either the machine is located in a high ambient temperature or a high temperature is generated due to high dissipation levels within the machine, the temperature capability of high-performance PM materials is usually adequate since the winding insulation is equally susceptible to over-temperatures. Consequently Jack et al. [1.31] concluded that the penalties for using a PM machine in terms of maximum thermal endurance is minimal. However, in the case of a turbo-generator, there is likely to be a considerable temperature differential between the rotor and the stator, due to a heat-soak effect from the turbine wheel (which itself may be at temperatures as high as $800^{\circ} \mathrm{C}$ ) and the difficulty in cooling a high-speed rotor. Thus the ability of an SR rotor to operate at considerably higher temperatures is likely to be of considerable benefit. Further, the performance of an SR rotor improves with increasing temperature due to the increasing resistivity of the laminations and the consequent reduction in the eddy current component of iron loss. By contrast, in a PM machine, the negative temperature coefficient of remanence, which is typically some $-0.1 \%$ per ${ }^{\circ} \mathrm{C}$ for $\mathrm{NdFeB}$, will give rise to a marked reduction in performance with temperature. 
6. Aerodynamic drag. Aerodynamic losses in high speed rotating machinery are likely to be a highly significant loss mechanism. Intuitively, one would expect higher aerodynamic losses with an SR rotor (which in many respects resembles a fan like structure) than with the smooth cylindrical rotors of BLDC, behaviour that is borne out by the fluid dynamic studies presented in chapter 5 . Further, the much smaller airgaps that are typically required by SR machines to ensure comparable power densities and power factors, are also disadvantageous in terms of aerodynamic loss as compared to the larger airgaps which can be accommodated in PM machines.

Although much of the above discussion is qualitative, an SR machine was adopted as the preferred topology for this application. The principal justifications were the absence of any no-load iron loss, the simple and robust rotor structure and the absence of any permanent magnet material in the high temperature environment. Further, since PM machines have already been employed in the target application, this study will provide a useful comparison in terms of relative machine merits

\subsection{Switched Reluctance Machines}

SR machines are doubly salient machines, i.e. the rotor and stator both exhibit magnetic saliency. Although there are many variants, in terms of stator pole, rotor pole and phase number, the basic underlying torque producing mechanism is common, and is a result of the tendency of the rotor to align itself in a position of minimum magnetic reluctance. In terms of the torque producing mechanism, it is useful to consider the particular case of a 3 phase SR machine equipped with 6 stator teeth and 4 rotor teeth (a so-called ' $6 / 4$ ' machine). A cross-section of a typical $6 / 4$ SR machine is shown in figure 1.17. Each stator pole carries a concentrated winding connected in series or parallel with the winding on the diametrically opposing tooth to form one phase of the machine. The coils are connected such that the flux produced by currents in the windings is additive. 
The position of minimum reluctance (i.e. maximum inductance) with respect to a given phase is referred to as the aligned position, figure 1.17a. The position of maximum reluctance (i.e. minimum inductance) is known as the un-aligned position, figure $1.17 \mathrm{~b}$. By virtue of being a singly excited machine, it is desirable to have a small airgap in the aligned position in order to minimise the stator mmf requirements. As a consequence, SR machines are often manufactured with very small airgaps, typically $0.1-0.2 \mathrm{~mm}$ in medium power machines.

Continuous torque can be generated by sequencing the excitation in the phases with respect to rotor position, such that the rotor is continually drawn towards a position of minimum reluctance with respect to the excited phases. By way of example, if current were flowing in phase $\mathrm{C}$ in figure 1.17a, the rotor would move in a clockwise direction, until it reached alignment, at which point phase B would be excited to continue the rotational motion (the other pair of rotor poles would then be drawn into alignment).

It is important to note that the direction of torque is independent of the polarity of current in a given phase. Hence, in principle an SR machine can be driven from a simple uni-polar supply without any loss of winding utilisation, a feature which is often cited as a major advantage as compared to PM machines. In the case of a 6/4, 3 phase machine, bi-directional operation can be achieved by employing the appropriate phase sequence of uni-polar currents, i.e. in the machine shown in figure 1.17, A-C-B produces clockwise rotation while A-B-C produces anticlockwise rotation.

An established and widely employed approach for modelling SR machines is the use of so-called ' $\psi-i$ characteristics'. These characteristics represent the relationship between the flux-linkage, $\psi$, and the current, $i$, at a particular rotor angle. Of particular interest are the limiting characteristics in the aligned and un-aligned positions, typical examples of which are shown in figure 1.18. The area under the $\psi-i$ 
curve at a particular value of current is referred to as the co-energy, which is defined as:

$$
W^{\prime}(\theta, i)=\left.\int_{0}^{i} \psi(\theta, i) d i\right|_{\theta \approx \mathrm{const}}
$$

The change in co-energy during rotation from the un-aligned to the aligned position can be employed to calculate the torque produced for a given current, i.e.:

$$
T(\theta, i)=\frac{\partial W^{\prime}(\theta, i)}{\partial \theta}
$$

The static torque produced for a given constant current can be readily calculated from the change in co-energy between the aligned and un-aligned positions, i.e. from area $W^{\prime}$ in figure 1.18 , and the angle of the stroke ( $30^{\circ}$ mechanical in a $6 / 4$ machine). However, it should be noted that SR machines rarely operate with constant current under static conditions, and as will be demonstrated in chapter 3, dynamic operation reduces the torque capability markedly from that of static operation. Another important feature of SR machines is the relatively high proportion of energy that is drawn from the supply which is stored as magnetic energy and subsequently returned to the supply. This can often be of the order of $50 \%$ of the energy drawn from the supply, and hence gives rise to a relatively poor power factor.

The direction of net energy flow and hence the mode of operation, i.e. generating or motoring, is determined by the commutation angles of the machine. If the machine is to be operated as a motor, current must flow in the phase winding during the period of rising inductance of that winding, i.e. the period when the rotor is approaching the aligned position. Generation is achieved with current flowing in the winding when the rotor is drawn away from the aligned position by the prime mover, i.e. during a period of falling inductance. 


\subsubsection{SELECTION OF POLE NUMBER}

A wide variety of pole/phase numbers can be employed, although feasible combinations require an appropriate superposition of the torque from each phase. In general machines with lower pole/phase numbers tend to exhibit high torque ripple, and indeed in the case of single and two-phase machines, the torque nulls prevent self-starting from all rotor positions unless specific additional design features are incorporated [1.39,1.40]. Machines with higher pole/phase numbers have less torque ripple and can self-start from all rotor positions. Table 1.5 shows a published summary of the merits of the most commonly employed pole number and phase number combinations [1.35].

\begin{tabular}{|l|l|l|l|l|l|}
\hline$N_{\text {phases }}$ & $\mathrm{N}_{\mathrm{s}}$ & $\mathrm{N}_{\mathrm{r}}$ & $\varepsilon_{0}$ & Strokes/rev & Comments \\
\hline 1 & 2 & 2 & 180 & 2 & Needs assistance starting \\
\hline 3 & 3 & 2 & 60 & 6 & Unbalanced magnetic pull makes impractical \\
\hline 2 & 4 & 2 & 90 & 4 & $\begin{array}{l}\text { Non-uniform rotor/parking magnet required to } \\
\text { assist starting }\end{array}$ \\
\hline 3 & 6 & 2 & 60 & 6 & May need assistance starting \\
\hline 3 & 6 & 4 & 30 & 12 & Lowest strokes/rev for self starting \\
\hline 3 & 6 & 8 & 15 & 24 & \\
\hline 3 & 12 & 8 & 15 & 24 & \\
\hline 3 & 18 & 12 & 10 & 36 & Low inductance ratio \\
\hline 3 & 24 & 16 & 7.5 & 48 & Low inductance ratio \\
\hline 4 & 8 & 6 & 15 & 24 & \\
\hline 4 & 16 & 12 & 7.5 & 48 & Low inductance ratio \\
\hline
\end{tabular}

Table 1.5. Summary of SR pole/phase combinations [1.35]

As was demonstrated by the discussion of the Hyper-bar system, compressed air injection and turbo-pac systems in section 1.4, there is considerable merit in terms of engine performance if boost pressure is available at start-up. Thus, reliable self- 
starting capability is highly desirable. As is evident from table 1.5 , the selection of a phase number is restricted to 3 and above to meet this criteria. Given that any increase in phase and/or tooth number is accompanied by increases in electrical frequency (a particular concern given the high-speed nature of the machine), the simplest 3 phase topology, viz. 6 stator teeth and 4 rotor teeth (6/4), is the leading candidate, and as a consequence, was adopted for the prototype.

\subsubsection{SELECTION OF STATOR AND ROTOR POLE ARC}

The selection of the stator and rotor pole arcs, i.e. the angles subtended by the stator and rotor poles respectively, is a critical design parameter. Lawrenson et al. [1.41] provides useful guidance to determine a range of feasible pole arcs. In order to maximise the specific power of the prototype SR machine the following guidelines are adopted:

- The mutual inductance between phases must be minimised

- The rotor and stator must exhibit cyclic symmetry

- The switching frequency must be minimised, given that the machine must be self starting.

These conditions give rise to a series of constraints regarding the selection of appropriate pole arcs that are given in detail by Lawrenson et al. [1.41]. For the prototype application a $30^{\circ}$ stator and a $35^{\circ}$ rotor pole arc were selected. This leaves the largest possible area for the windings in order to minimise the copper loss, while the $5^{\circ}$ overlap will assist the fluxing of the machine prior to the generating period of falling inductance.

\subsection{Summary}

This chapter has described the magnitude and scope of diesel engine application, the inherent problems with turbo-charged diesel engines and a series of measures that have been adopted in an attempt to correct the mismatch between the positive 
displacement engine and the aerodynamic compressor. The EATG system that forms the basis of this thesis has been introduced and its basic operating mode described. Switched reluctance machines have been identified as the preferred electrical machine topology for this application and some design features have been established. 


\section{REFERENCES}

[1.1] Heywood, J. B., 'Internal Combustion Engine Fundamentals.' McGraw-Hill, 1988.

[1.2] Environment Agency, 'Emissions to the Atmosphere.' Public Document. 1999. (URL: http://www.environment-agency.gov.uk/s-enviro/stresses/4userel-dis/2emissions)

[1.3] Parliamentary Office of Science and Technology, 'A Clean License? Graduating Vehicle Excise Duty.' POST technical report N N 116. June 1998.

[1.4] Report of the Airborne Particles Expert Group, 'Source Apportionment of Airborne Particulate Matter in the United Kingdom.' Department of the Environment, Transport and the Regions. January 1999.

[1.5] Watson, N. And Janota, M. S., 'Turbocharging the Internal Combustion Engine.' MacMillan Press Ltd. 1982.

[1.6] Flaxington, D. And Swain, E., 'Turbocharger Aerodynamic Design.' Proc. Institution of Mechanical Engineers, Vol 213, Part C pp43-57. 1999.

[1.7] Melchior, J. And Andre-Talamon, Th. 'Hyperbar System of High Supercharging.' SAE 740723, 1974

[1.8] Andre-Talaman, Th., 'A New Hyperbar Engine Propulsion System for Ship Propulsion.' SAE 830506, 1983.

[1.9] Ledger, J. D., Benson, R. S. And Furukawa, H., 'Improvement in Transient Performance of a Turbocharged Diesel Engine by Air Injection into the Compressor.' SAE 730665, 1973.

[1.10] Harp, J. L. And Oatway, T, P.'Centrifugal Compressor Development for a Variable Area Turbocharger.' SAE 790066, 1979.

[1.11] Matsumo, K., Jinnai, Y. and Suzuki, H., 'Development of variable geometry turbocharger for diesel passenger car.' Proceedings of the $6^{\text {th }}$ ImechE international conference on turbocharging and air management systems, London UK, 3-5 November 1998, p329-346.

[1.12] Hawley, J. G., Cox, A., Pease, A. C., Bird, G. L. and Horrocks, R. W. 'Use of a VGT to improve the limiting torque characteristics of a D1 automotive 
diesel engine.' Proceedings of the $6^{\text {th }}$ ImechE international conference on turbocharging and air management systems, London UK, 3-5 November 1998, p347-357.

[1.13] Maulice, S. K. Lee et. Al, 'Improvement of a Turbocharged and Intercooled Diesel Engine Powered Vehicle's Startability by Means of a Three Wheel Turbocharger (TWT).' SAE 945018, 1994.

[1.14] Winterbone, D. E. Et. Al, 'Transient Response of Turbocharged Diesel Engines.' SAE Preprints 7701221977.

[1.15] Page, D.L., 'Optimization of the air/fuel ratio for improved engine performance and reduced emissions.' SAE 961714, 1996.

[1.16] Larson, R. P. 'The Future of Ceramic Turbochargers - Promises and Pitfalls.' Proceedings of the $22^{\text {nd }}$ Automotive Technology Development Contractors' Co-ordination Meeting. Pp541-547, SAE, 1985.

[1.17] F. J. Wallace and Winkler, G., 'Very High Output Diesel Engines - a Critical Comparison of Two-Stage Turbocharged, Hyperbar and Differential Compound Engines' SAE 770756. 1977.

[1.18] Rogo, C., Hajek, T. And Roelke, R. 'Aerdynamic Effects of Moveable Sidewall Nozzle Geometry for Rotor Exit Restriction on the Performance of a Radial Turbine.' SAE 831517. 1983.

[1.19] Roessler, M. And Swenson, K. R., 'Variable Nozzle turbo-chargers for medium speed diesel engines.', SAE 880119. 1988.

[1.20] Tennant, D. W. H. And Walsham, B. E., 'The Turbocompound Diesel Engine.' SAE 890647, 1989. Presented at the International Congress and Exposition, Detroit. 1989.

[1.21] Brands, M. C. Et al., 'Vehicle Testing of a Cummins Turbocompound Diesel Engine.' SAE 810073, Presented at the International Congress and Exposition, Detroit. 1981.

[1.22] Holtman, R. H., 'Testing of a Low Specific Fuel Consumption Turbocompound Engine.' SAE 870300, Presented at the International Congress and Exposition, Detroit. 1987. 
[1.23] Wilson, D. E., 'The Design of a Low Specific Fuel Consumption Turbocompound Engine.' SAE 860072, Presented at the International Congress and Exposition, Detroit. 1986.

[1.24] Woollenweber, W. E., 'Turbo-Cooling of Heavy Duty Diesel Engines.' Diesel Progress, Engines and Drives, pp 20, 22-23. June 1995

[1.25] Atkinson, M.J., Kono, S. And Pride, A. A., 'A Turbo-Alternator for Hybrid Electric Vehicles.' 96EL059.

[1.26] Reuter, K. 'Integrated Machinery Systems for Cryogenic Processes Consisting of Turboexpander, Compressor, High Frequency Motor and Generator with Magnetic Bearings.' Presented IEE colloquium on high speed bearings for electrical machines, pp1-12. 1997.

[1.27] Reuter, K. And Cancers, W. R., 'Integrated System of Turboexpander with Magnetic Bearing and High Frequency Generator.' Presented at the Third International Symposium on Magnetic Bearings, Alexandria, USA. 1992.

[1.28] Woollenweber, W. E. 'New System Looks At Dealing With Turbo Lag.' Diesel Progress, August 1998.

(Reprinted at URL: http://www.turbodyne.con/dieselprogress.html)

[1.29] Anon., 'Dynacharger' Turbodyne systems Inc Sales literature, URL: http://www.turbodyne.com/product2.html.

[1.30] Hofbauer, P., 'Improvement of Automobile Fuel Consumption Using Turbodyne Technology.' URL: http://www.turbodyne.com/improvefuel.html.

[1.31] Jack, A. G., Mecrow, B. C. and Haylock, J. A., 'A comparative study of permanent magnet and switched reluctance motors for high-performance fault-tolerant applications.' IEEE Trans. on Ind. Appl. Vol. 32, No 4, July 1996 p889-895.

[1.32] Radun A. V., 'High-power density switched reluctance motor drive for aerospace applications.' IEEE Trans. on Ind. Appl. Vol. 28, No 1, Jan 1992 p113-119.

[1.33] Harmer. K., 'An energy efficient brushless drive system for a domestic washing machine.' Ph.D. Thesis. University of Sheffield. 1997. 
[1.34] Fulton, N. N. and Lawrenson, P. J., 'Switched reluctance drives for electric vehicles: a comparative assessment.' Proceedings of $23^{\text {rd }}$ conference on Power conversion/Intelligent motion, Nuremberg, Germany, 1993.

[1.35] Miller T. J. E., 'Switched reluctance machines and their control.' Magna Physics, 1993.

[1.36] MacMinn, S. R., Rzesos, W. J., Szczesny, P. M. and Jahns, T. M., 'Application of sensor integration techniques to switched reluctance motor drives.' Proc. Industry Applications Society Annual Meeting, Vol. 1, 1988, pp584-588.

[1.37] West, J. G. W., 'DC, Induction, Reluctance and PM motors for electric vehicles.' Power engineering journal, Vol. 8, No 2, April 1994, p77-88.

[1.38] Group Arnold, Swift Levick Magnets Ltd, 'Sales literature'. Group Arnold, Marengo, Illinois, USA.

[1.39] Jenkinson, G. C. and Stephenson, J. M., 'Starting of a single-phase switched reluctance motor' Proceedings of the $9^{\text {th }}$ international conference on electrical machines and drives, p391-395, 1999.

[1.40] Stephenson, J. M. And Jenkinson G. C., 'Single-phase switched reluctance motor design.' IEE Proceedings - Electric power applications, Vol 147, No 2, p131-139, March 2000.

[1.41] Lawrenson, P. J., Stephenson. J. M., Fulton, N. N., Blenkinsop, P. T. and Corda, J., 'Variable speed switched reluctance motors.' IEE Proceedings B Electric power applications, Vol. 127, No 4, July 1980, p253-265. 


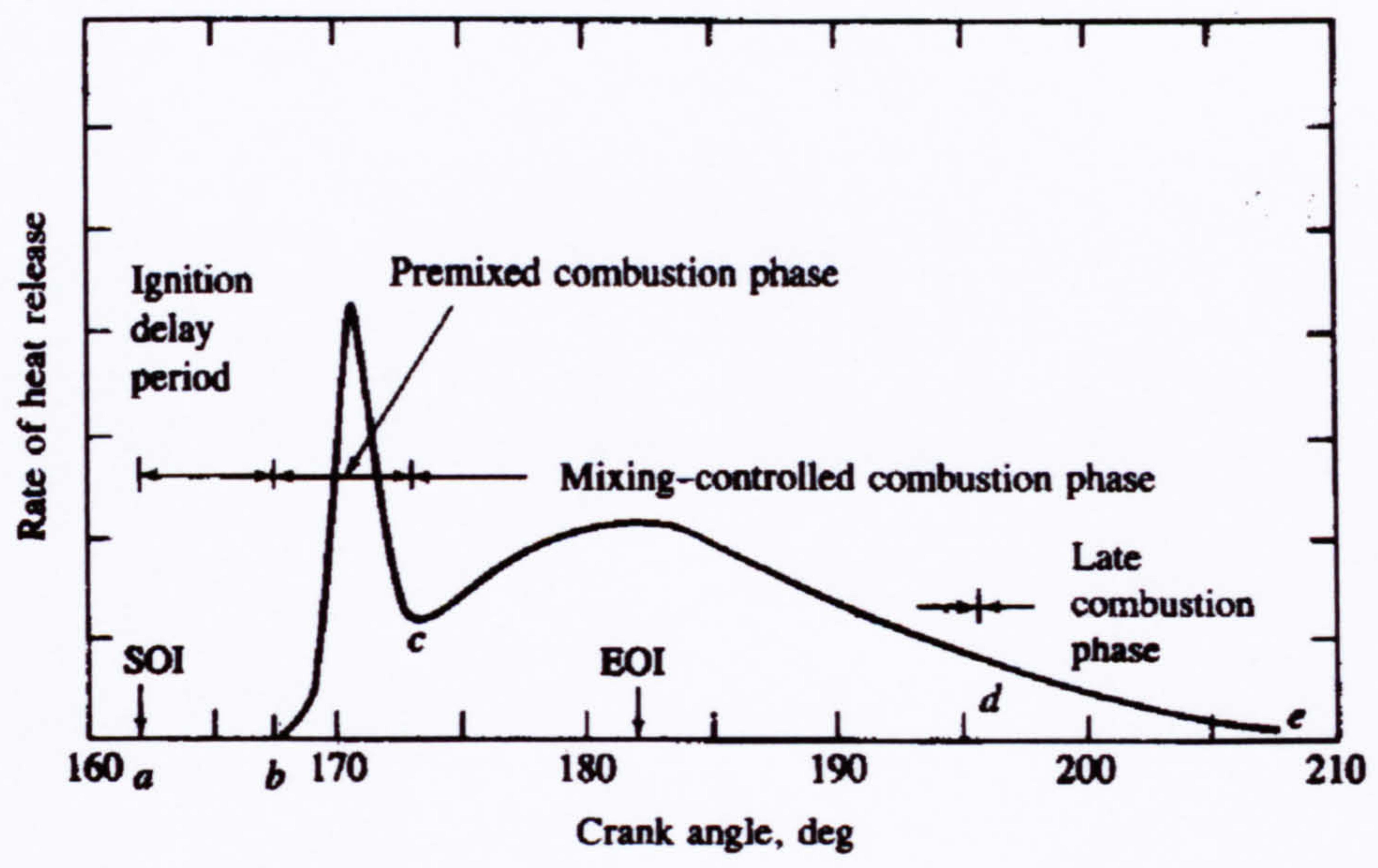

Figure 1.1. Typical rate of heat release diagram for a direct injection diesel engine. [1.1].

Start of injection (SOI)

Start of combustion

Pre-mixed combustion

Mixing controlled combustion

Late combustion and soot formation

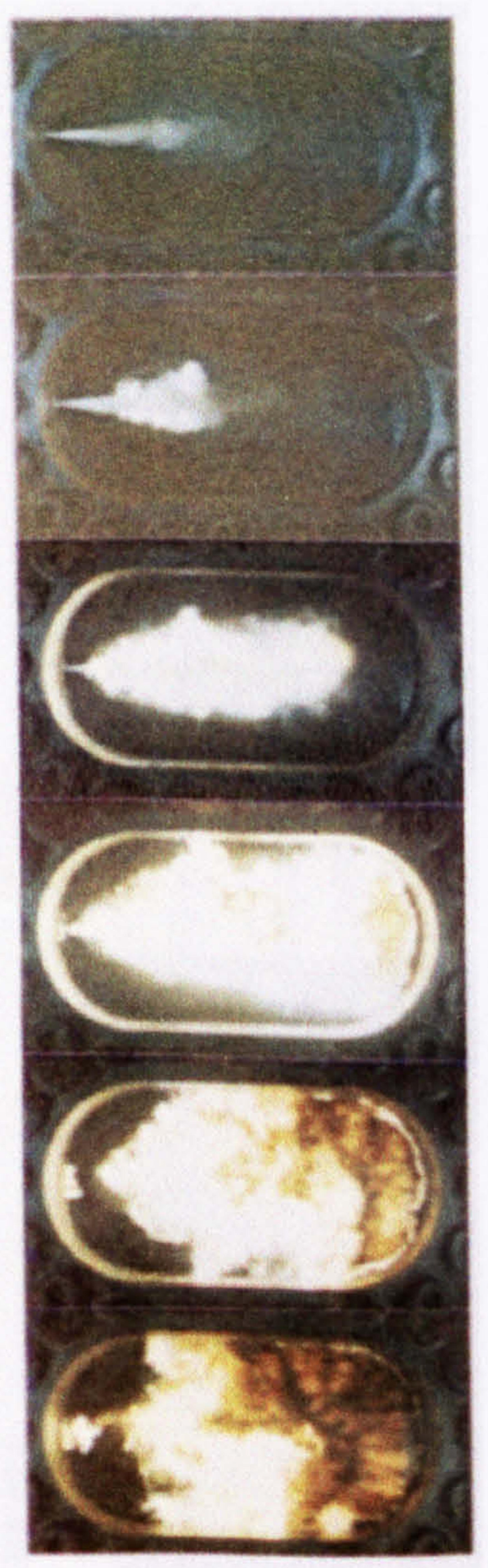

Figure 1.2 Stages of combustion in a single spray direct injection diesel engine. [1.1]. 




Figure 1.3. Spatial distribution of $\mathrm{PM}_{10}$ emissions (tonnes $/ \mathrm{km}^{2}$ ) in 1996 [1.4]. 


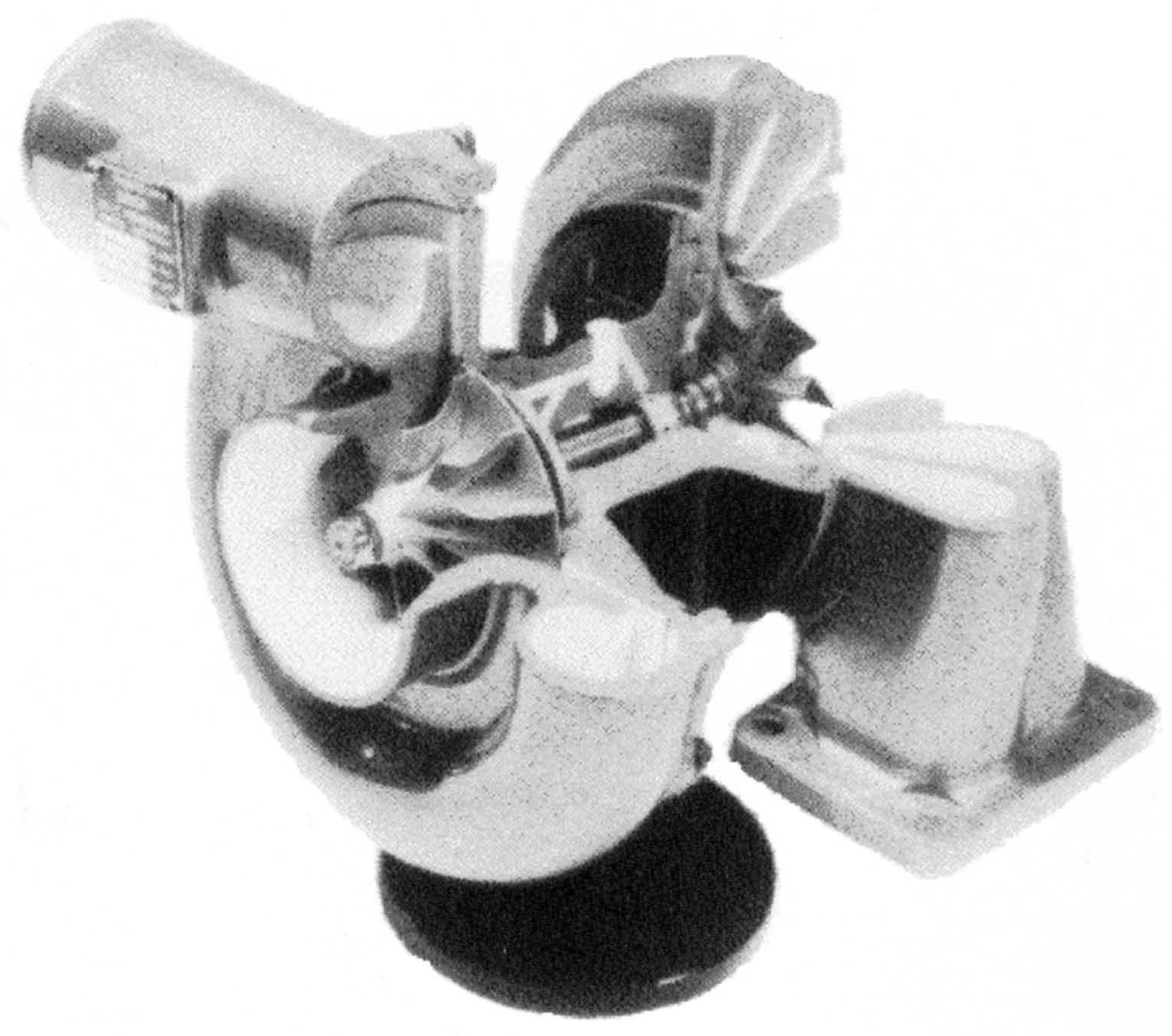

Figure 1.4. Typical automotive turbocharger [1.5]

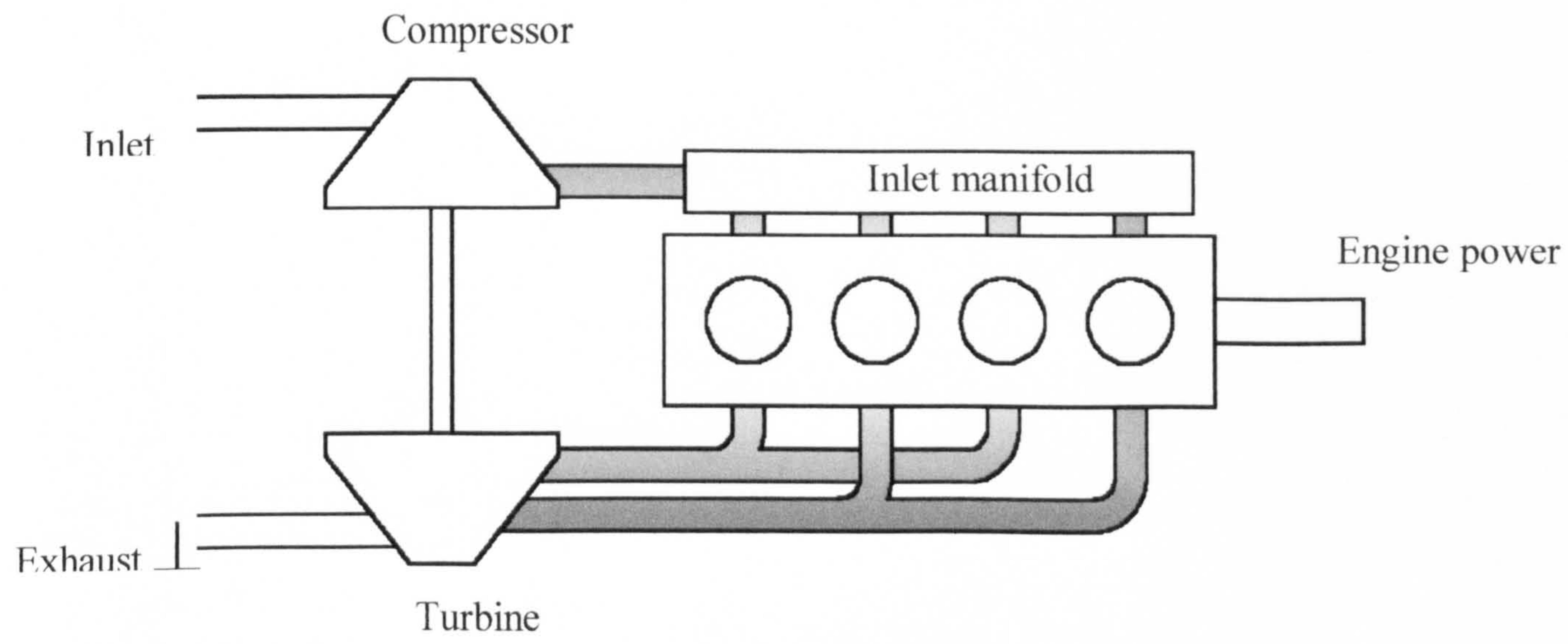

Figure 1.5 Typical turbocharger arrangement on a four stroke, four cylinder engine. 


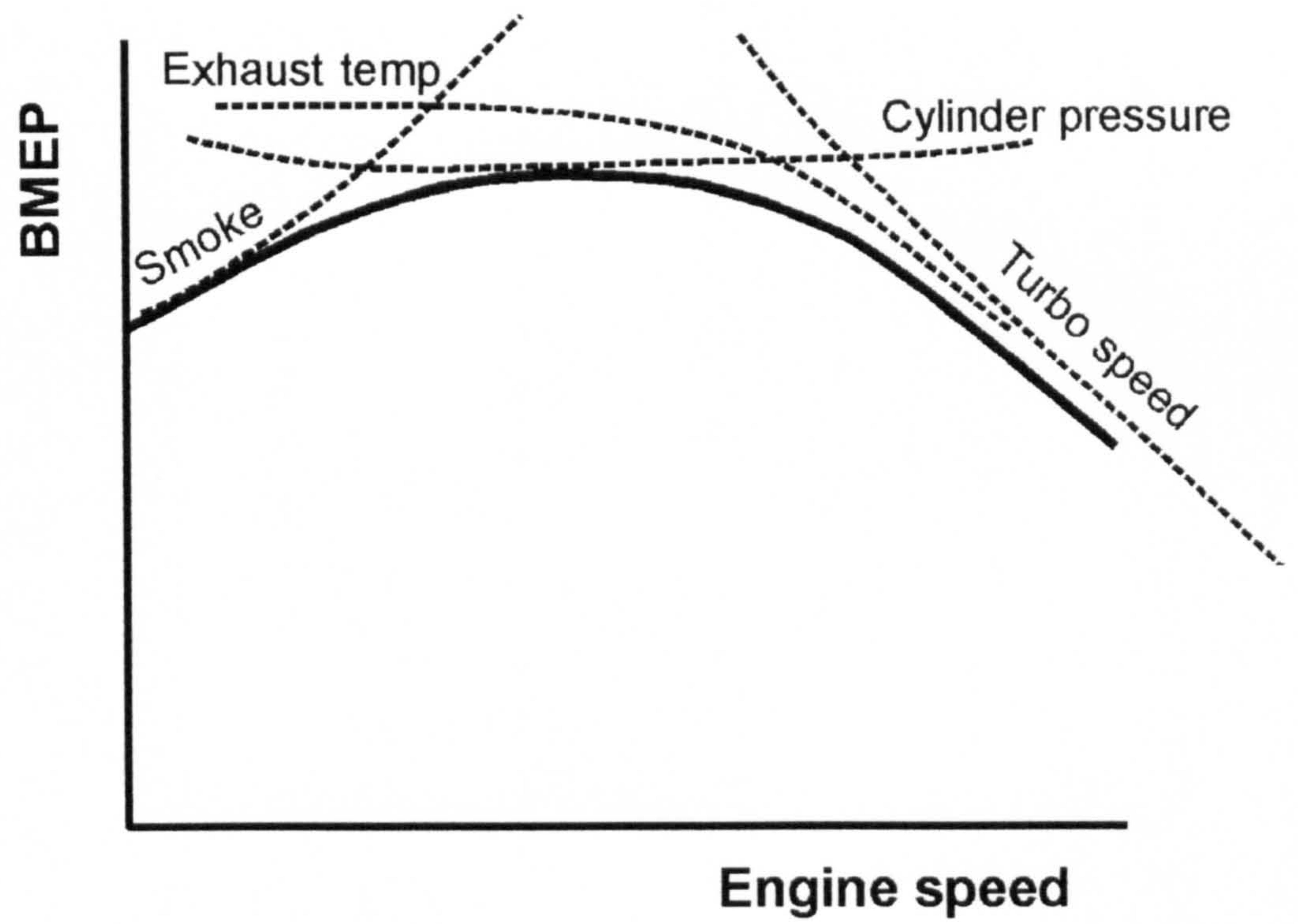

Figure 1.6. Typical characteristics of a torque-limiting curve in a diesel engine. [1.5]

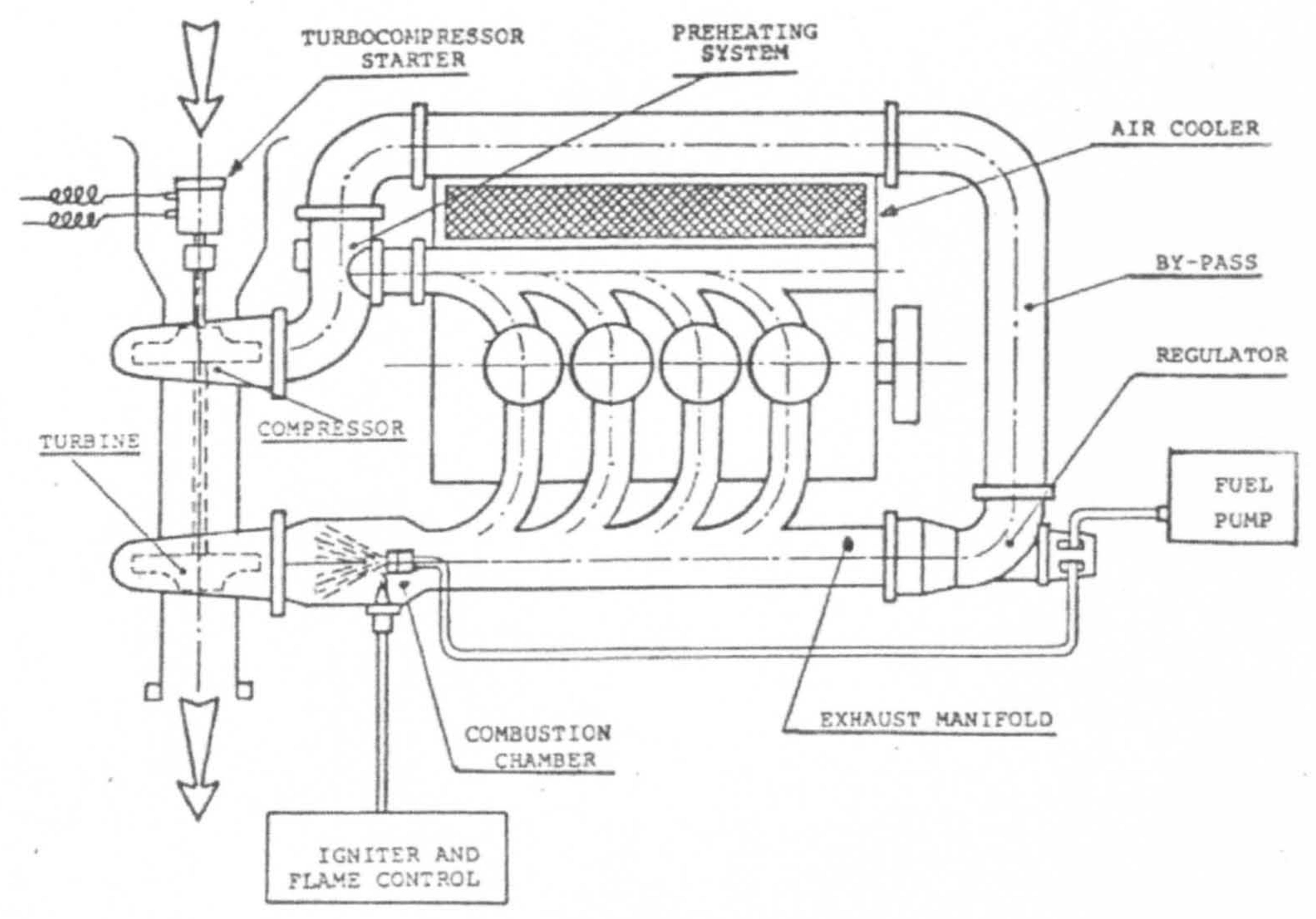

Figure 1.7 Schematic of the Hyperbar system [1.7] 


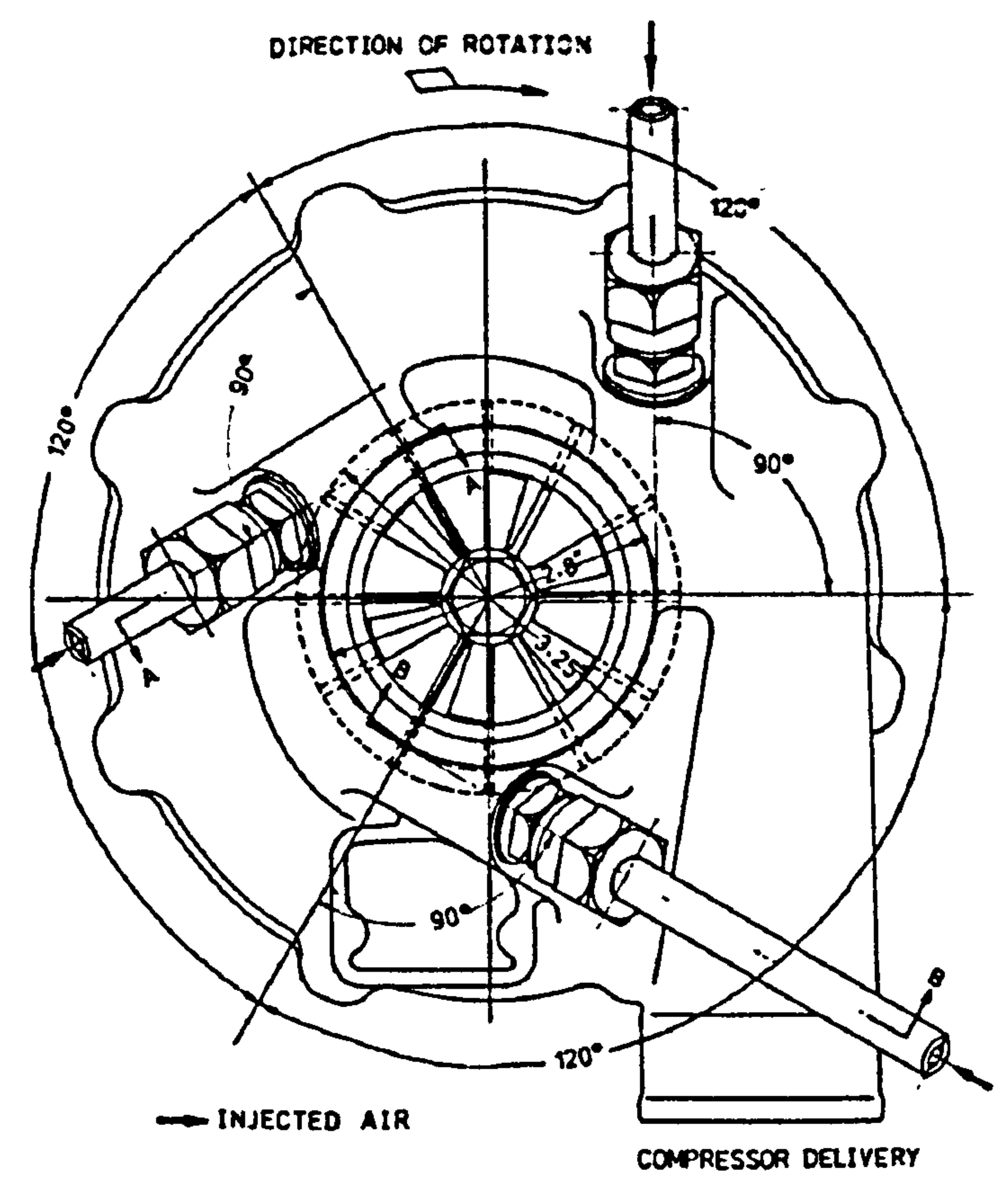

Figure 1.8 3-point compressed air injection into the compressor housing. [1.9]

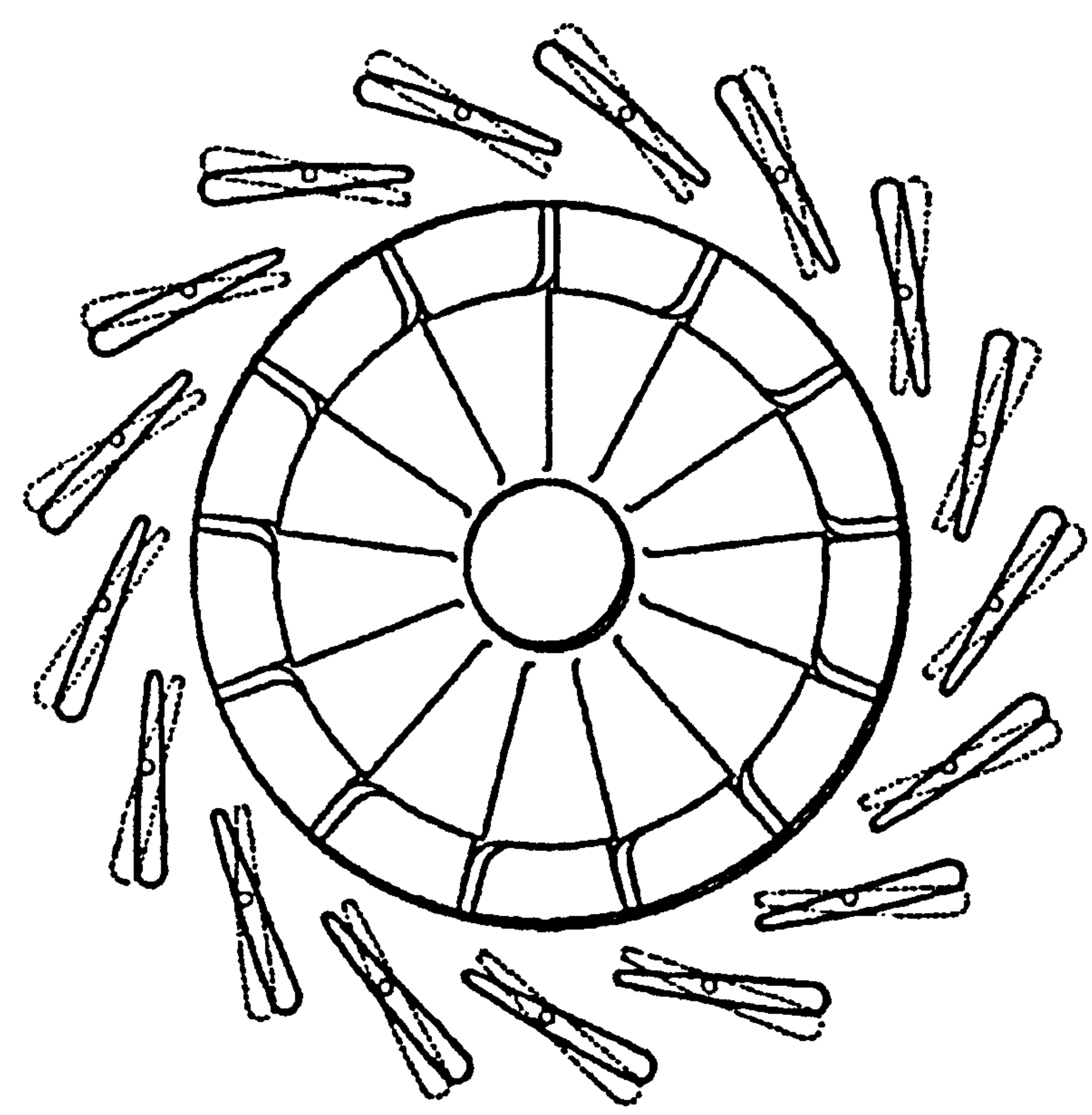

Figure 1.9. Swinging vane variable geometry turbine [1.6] 


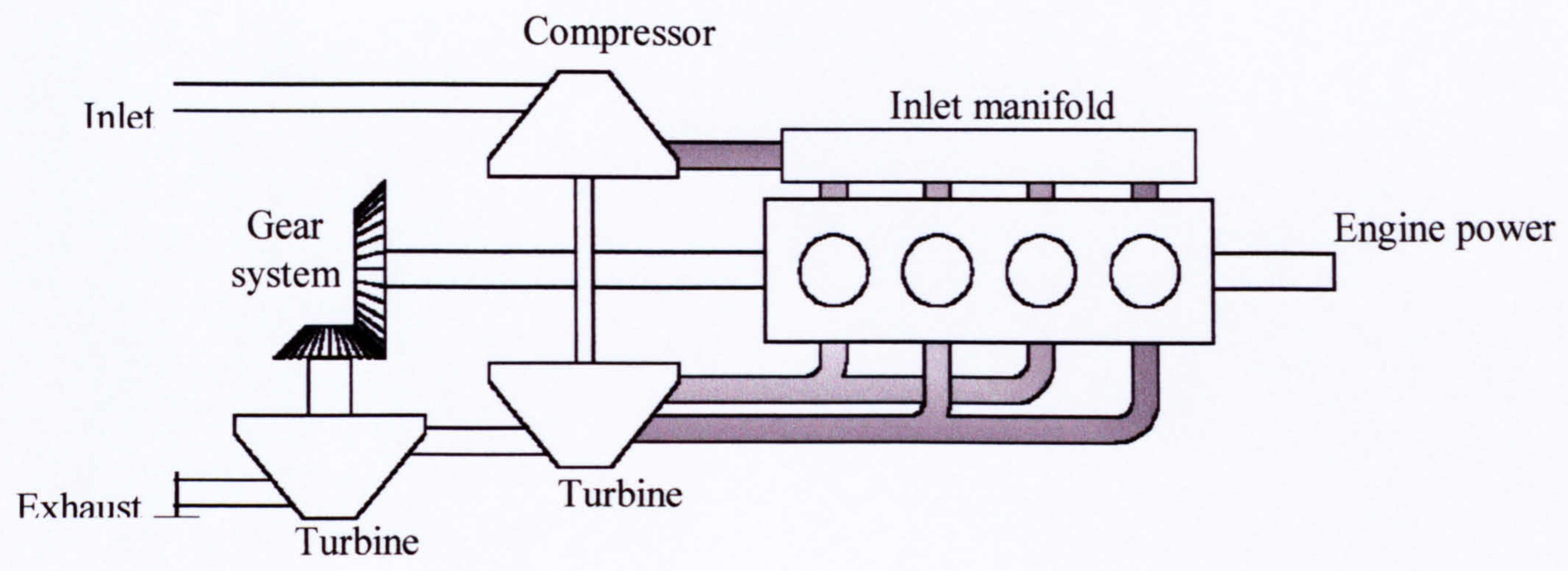

Figure 1.10. Two turbine turbocompund engine with the turbines connected in series with the free power turbine mounted downstream.

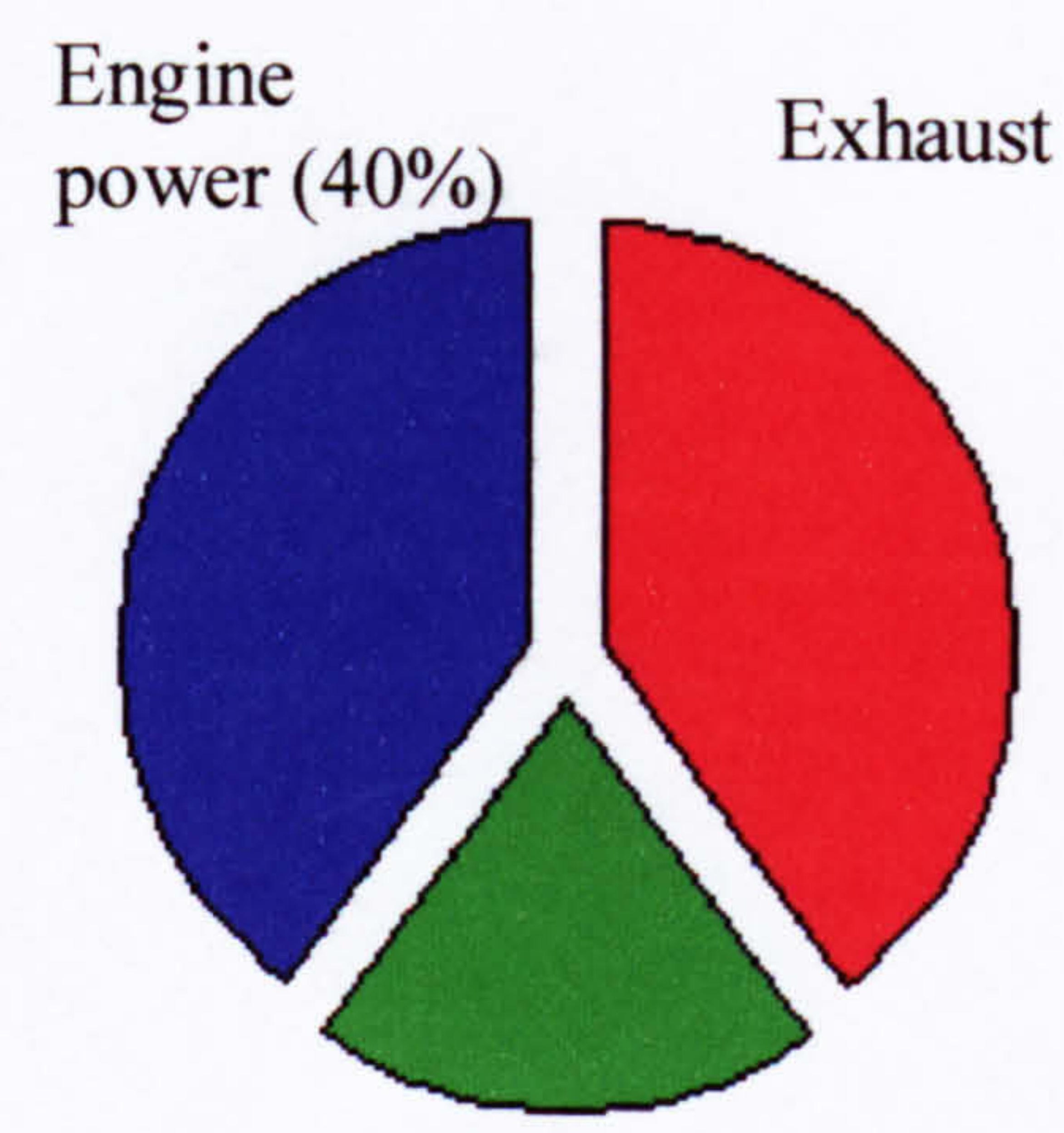

Coolant $(20 \%)$
Engine

power $(36 \%)$

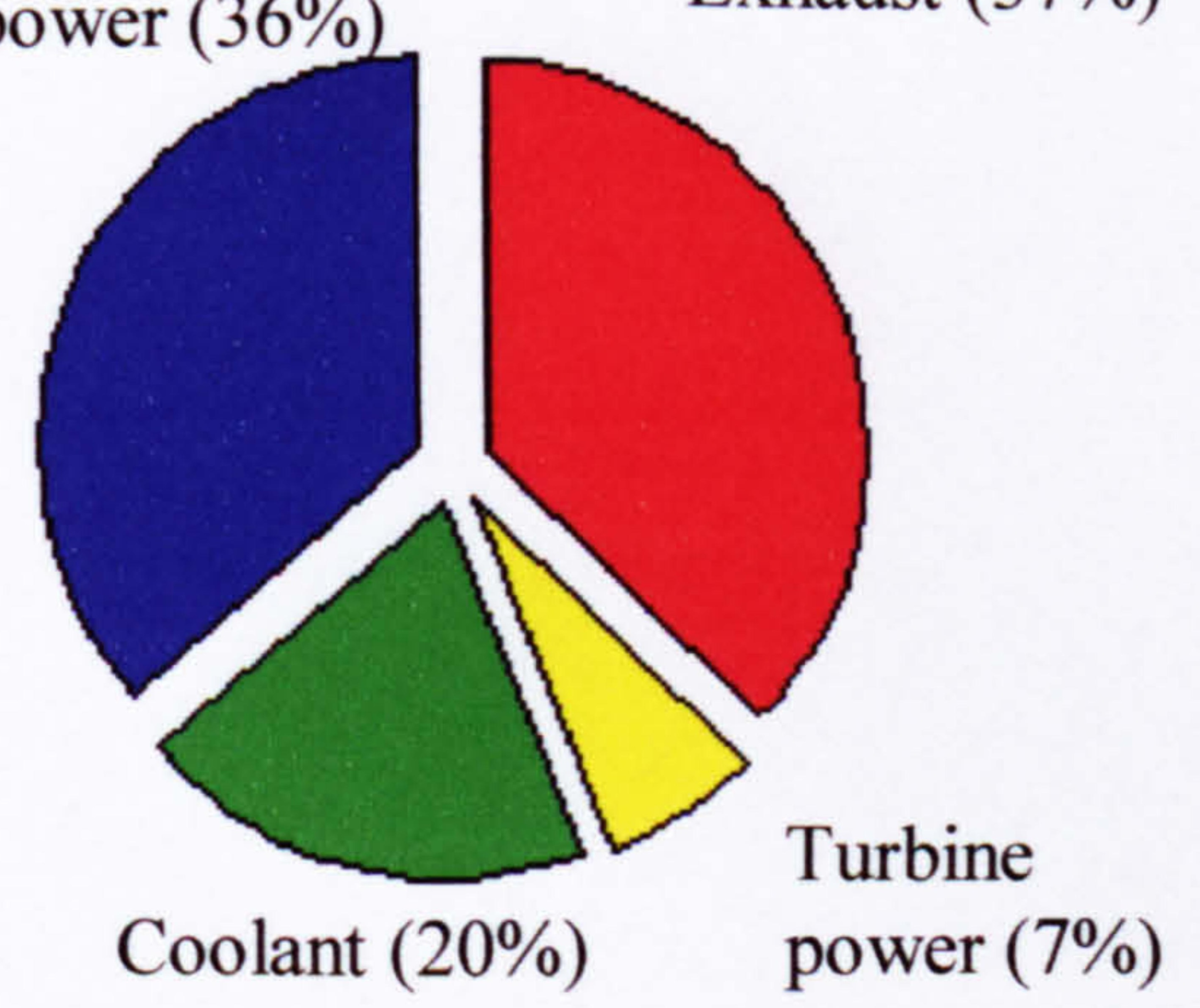

Turbocompound Engine

Figure 1.11. Typical fuel utilisation characteristics for standard and turbocompound engines [1.20]. 


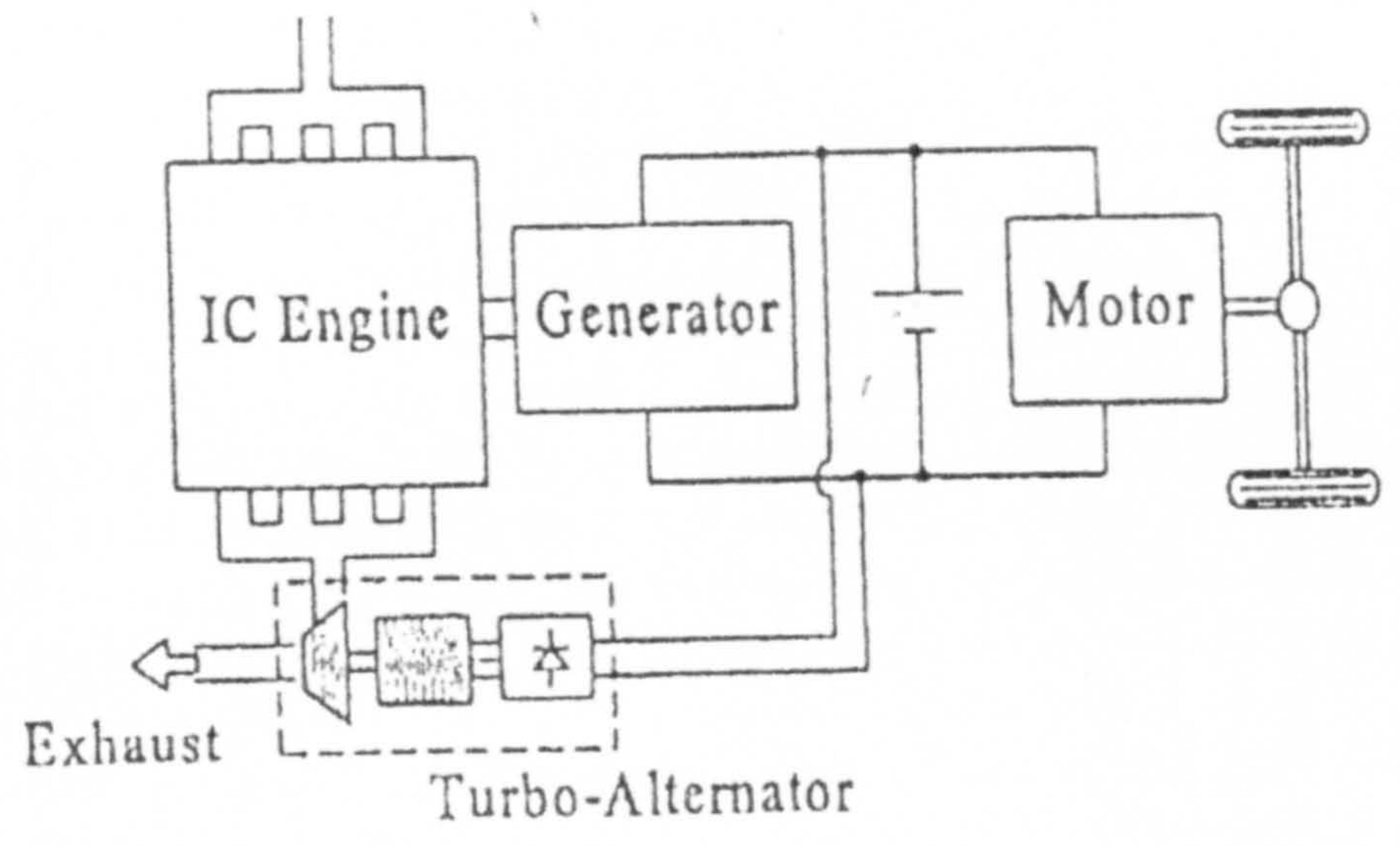

Figure 1.12. Schematic representation of the turbo-alternator and engine drive train system. [1.25]

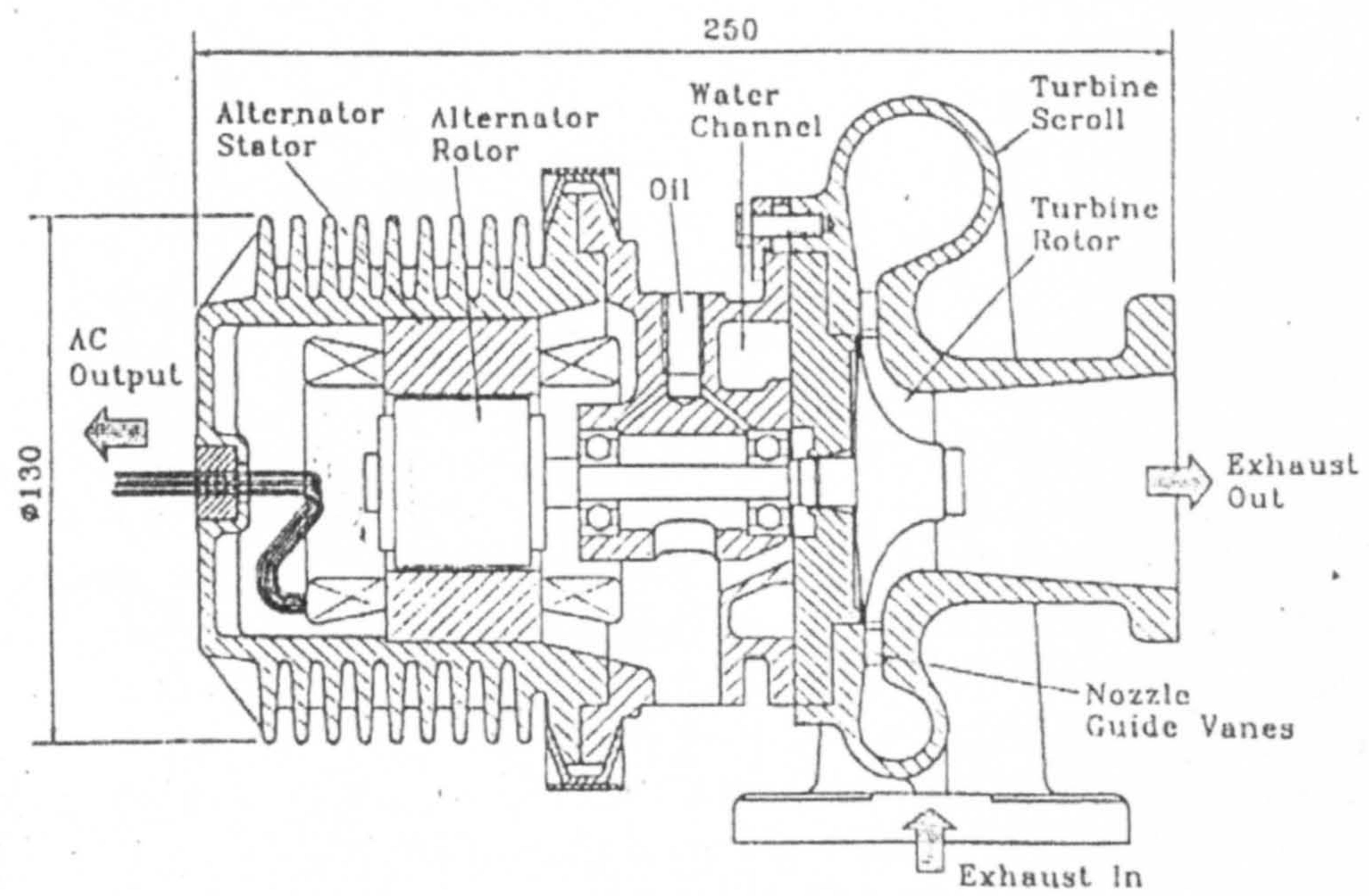

Figure 1.13. Prototype brushless permanent magnet turbo-alternator [1.25] 


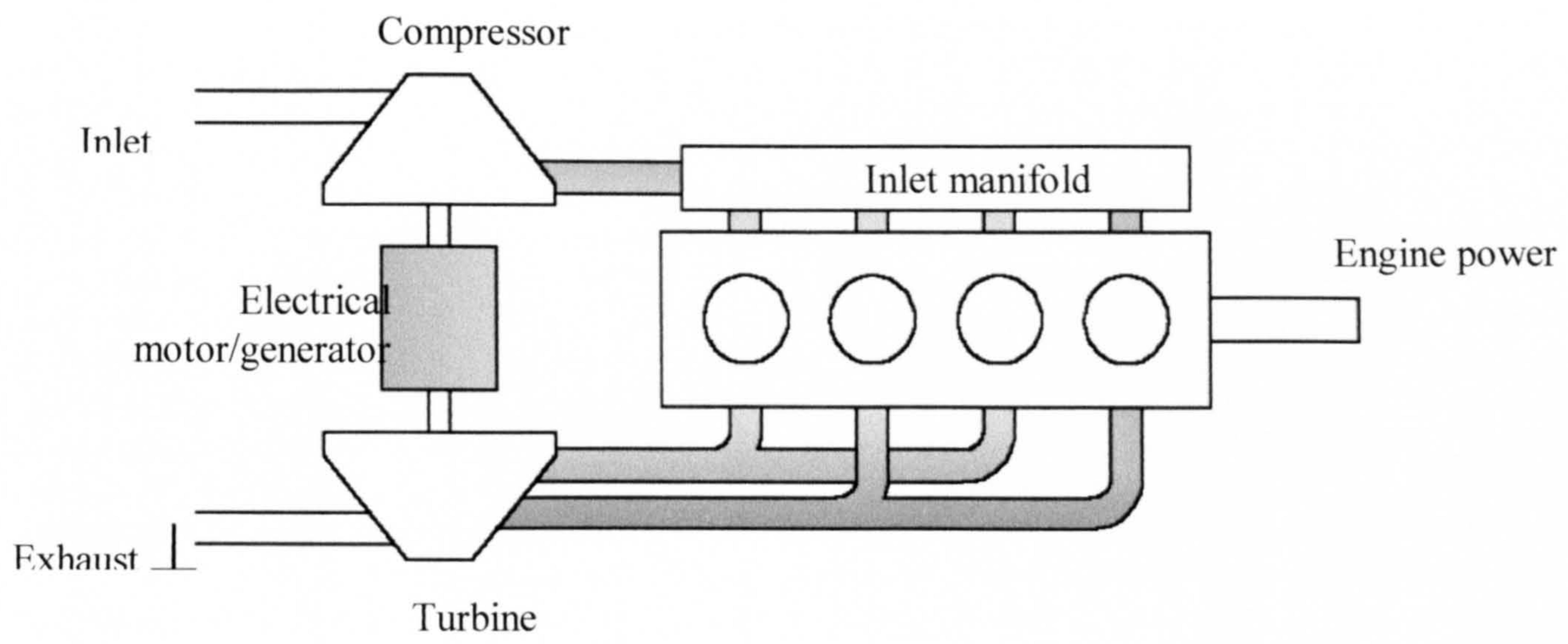

Figure 1.14. Schematic of turbo-generator system proposed.

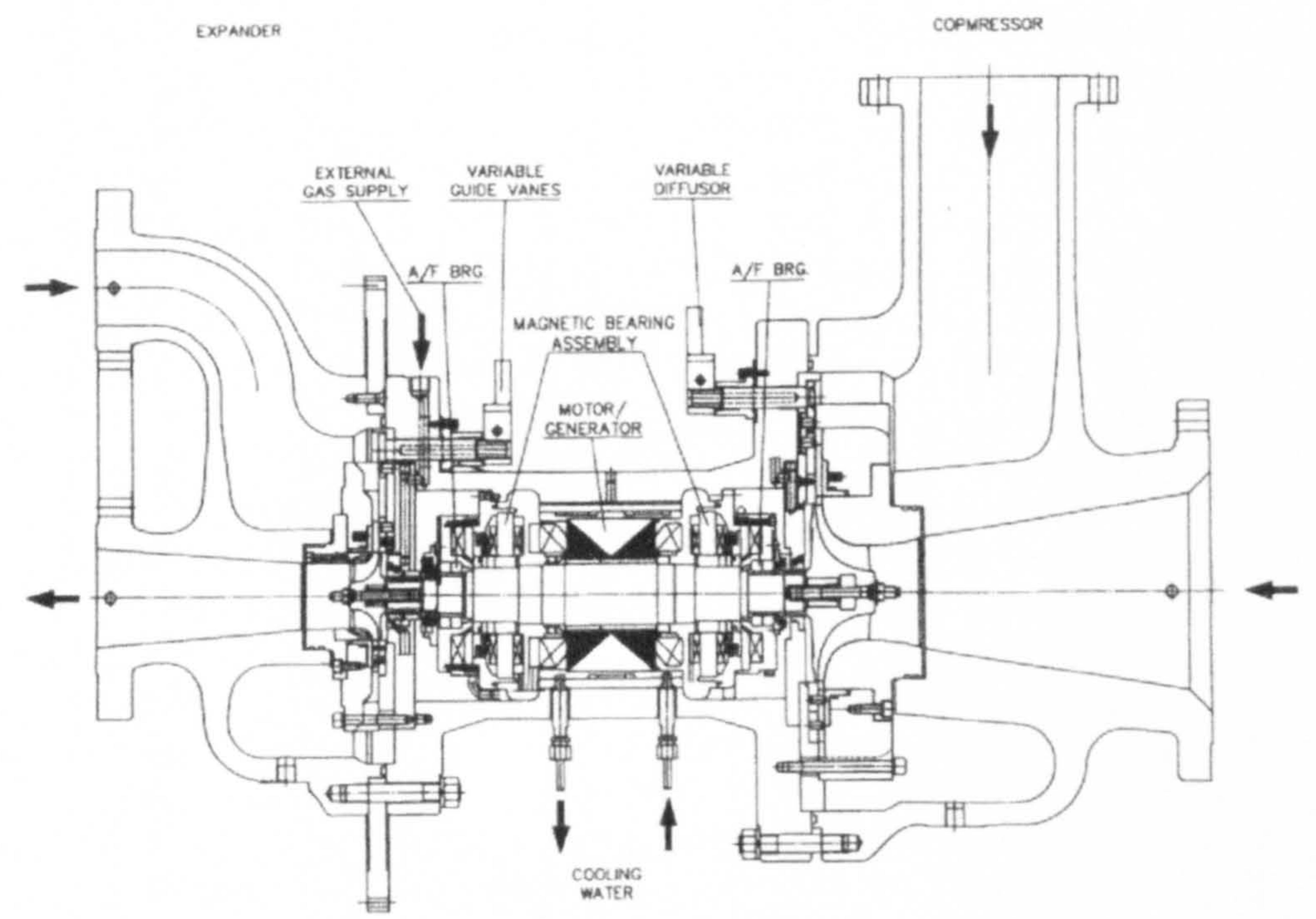

Figure 1.15. Turbine, compressor, motor/generator and magnetic bearing assembly for cryogenic applications [1.26]. 

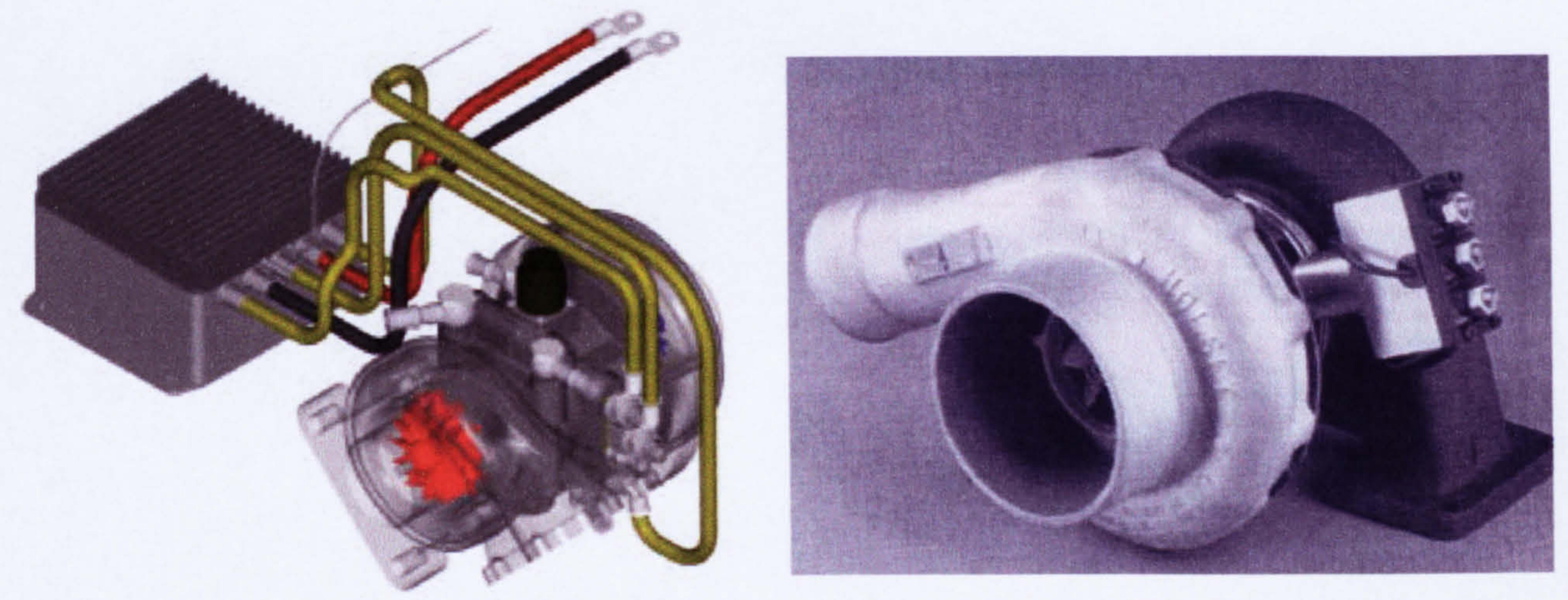

Figure 1.16. 'Dynacharger' combined turbocharger and motor/generator manufactured by Turbodyne systems Inc [1.28].

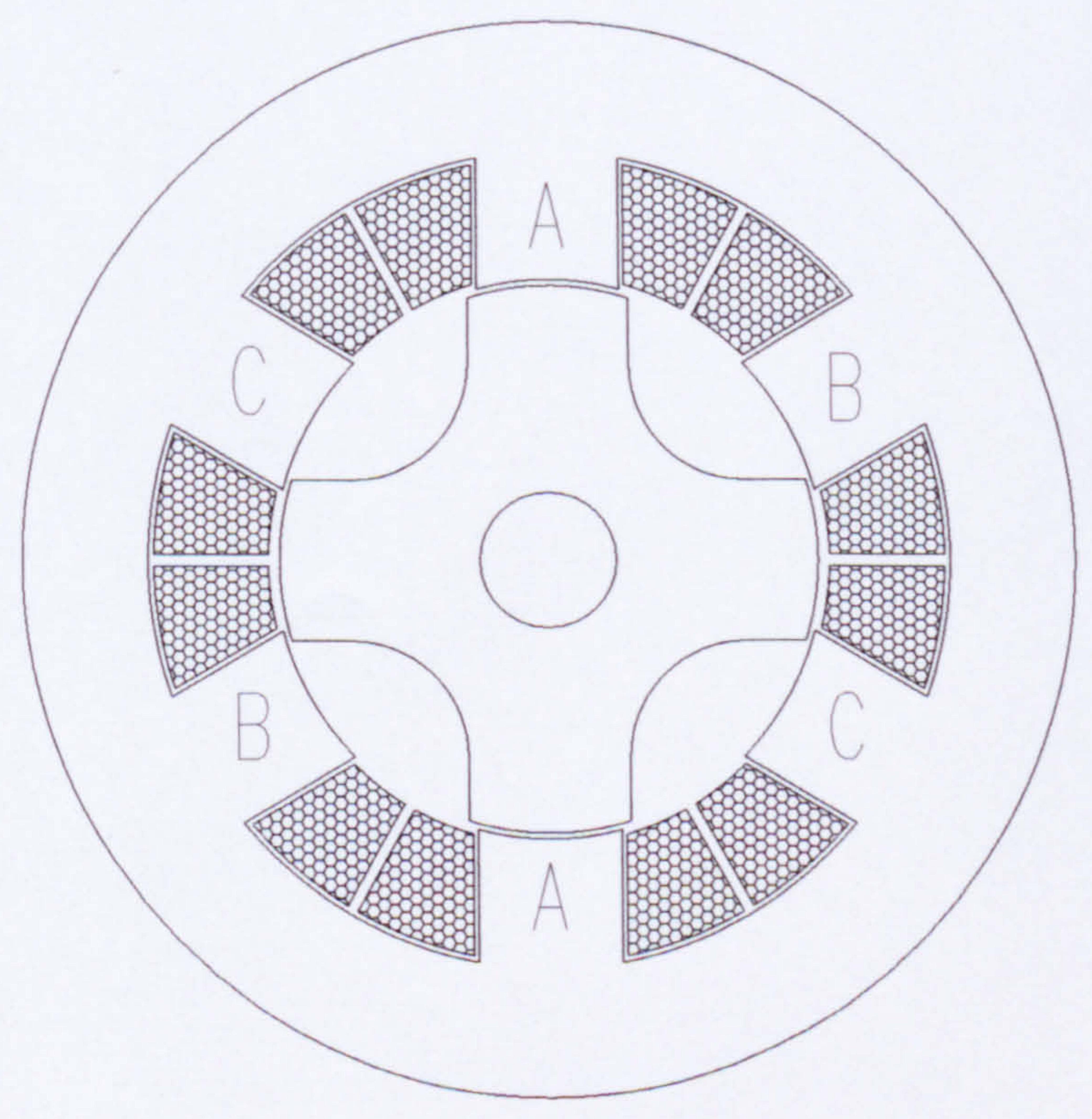

Figure 1.17a. Cross section of a typical $6 / 4$ SR machine with the rotor in the aligned position with respect to phase A. 


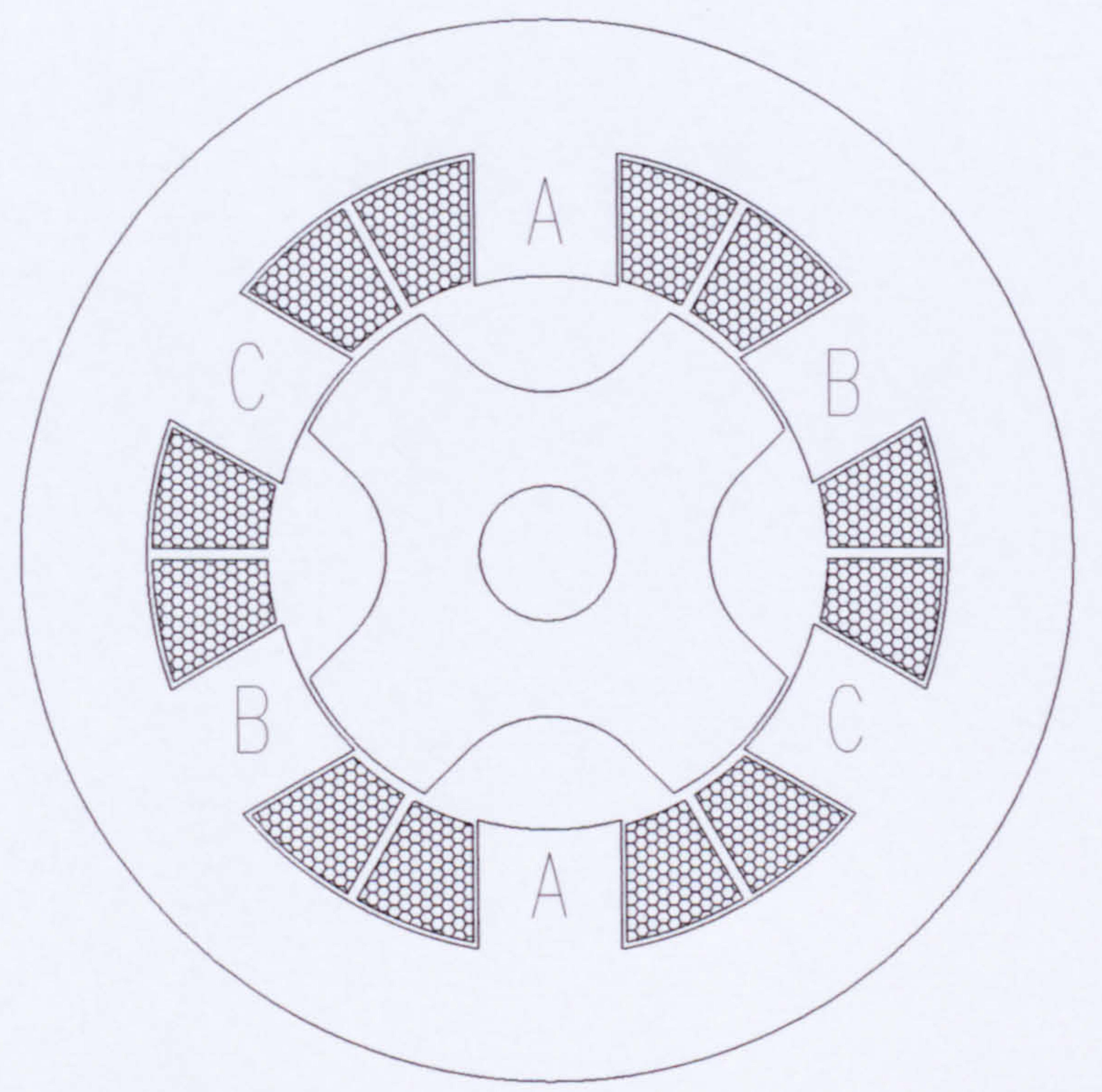

Figure $1.17 \mathrm{~b}$. Cross section of a typical $6 / 4 \mathrm{SR}$ machine with the rotor in the unaligned position with respect to phase A.

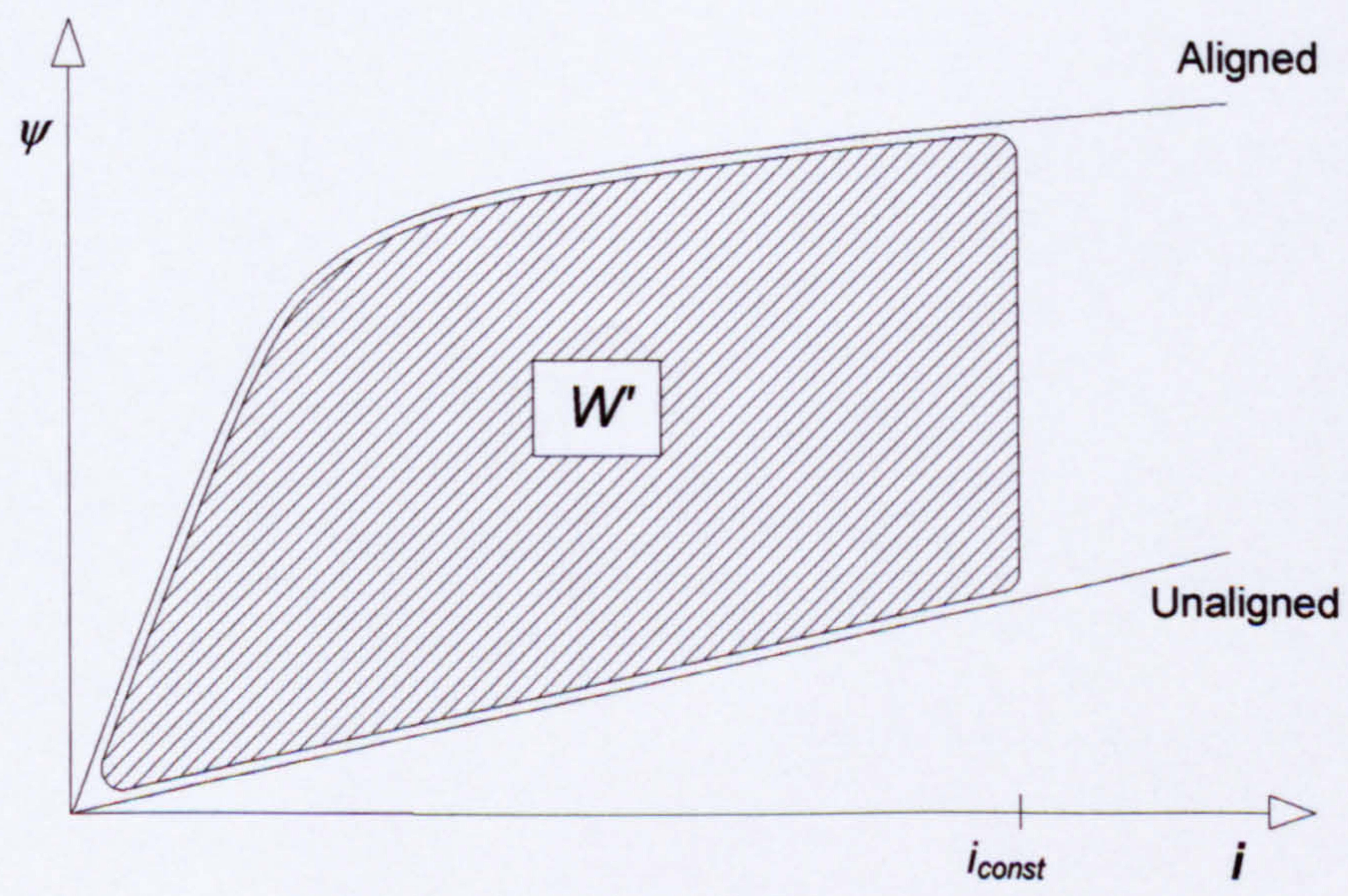

Figure 1.18. Typical aligned and unaligned $\psi$-i characteristic with a constant current operating tradjectory. 


\section{CHAPTER 2}

\section{ROTOR MECHANICAL DESIGN}

\subsection{Introduction}

The design of switched reluctance (SR) rotors for many conventional low to medium speed applications is dictated predominantly by electromagnetic considerations. However, in high-speed applications due consideration must also given to the mechanical design, specifically in terms of:

- Centrifugal stresses in the rotor - Medium sized automotive turbochargers typically operate at speeds of the order of $100,000 \mathrm{rpm}$ or so. The resulting centrifugal stresses induced in the rotor at such high rotational speeds impose severe constraints on the practical rotor diameter that can be employed while remaining within the safe operating stress limits of the rotor materials.

- Radial growth of the rotor - In SR machines the radial airgap between the rotor and stator is typically $0.1-0.25 \mathrm{~mm}$ or so in order to minimise the stator $\mathrm{mmf}$ requirement. Care must therefore be taken to ensure that the radial growth of the rotor under high levels of centrifugal stress does not become comparable with the nominal air-gap and associated tolerances in order to prevent potentially destructive rotor/stator contact.

- Moment of inertia of the rotor. The transient response of any turbocharger system is strongly influenced by the total moment of inertia. Given that the SR rotor is likely to contribute a significant proportion of the total moment of inertia of the complete shaft assembly, its minimisation in the mechanical design is desirable.

This chapter is concerned with a detailed finite element study of the stress distribution within SR rotors, with particular reference to establishing design 
guidelines for the prototype machine with due account of the consequent implications on the rotor moment of inertia.

\subsection{Finite Element Analysis}

Well-established and validated analytical techniques exist for the calculation of stress in simple rotating structures such as discs and cylinders. However the particular geometrical features of SR rotors preclude the use of such techniques, and recourse to numerical techniques such as finite-element analysis is required to calculate the stress distribution in the rotor to a level of accuracy sufficient to provide the necessary design guidelines.

The finite element method has been widely adopted for the calculation of centrifugal stresses and radial growth in rotating machinery [2.1,2.2]. The method is highly developed and has been shown to provide accurate results where appropriately applied. Given this well validated track-record, experimental validation of the technique was not deemed necessary, particularly given the significant practical difficulties involved in performing localised stress measurement and/or highly precise dimensional measurements at rotational high speeds [2.2].

One notable feature of the study presented in this chapter is that a linear stress-strain relationship is assumed throughout the study. While this simplification may give rise to the calculation of erroneous stress levels, specifically under load conditions where plastic deformation within the material will occur, the operation of the rotor under these loads will inevitably result in failure (albeit potentially under fatigue conditions after a number of cycles). Hence for all practical loading conditions, where yield of the electrical steel is avoided (with a considerable degree of safety) the linear material stress-strain curve remains valid.

Care must be taken when using the finite element method, since its ability to predict stress levels in a particular rotor geometry under given conditions is reliant on the 
imposition of the correct boundary conditions and loads. Further, the absolute accuracy of the solution is sensitive to the discretisation employed in the finite element mesh. Hence, a systematic investigation of the influence of these factors was undertaken so as to provide the necessary confidence in the findings derived for SR rotors.

\subsection{Finite Element Analysis of Cylindrical Rotors}

Cylindrical rotors provide an extremely useful starting point in terms of ensuring appropriate and accurate use of $\mathrm{FE}$ analysis, since exact analytical solutions of the stress distributions can be employed as a benchmark. The centrifugal stress distribution in a cylindrical rotor can be calculated using exact analytical solutions for the radial and circumferential stresses [2.3]:

$$
\begin{gathered}
\sigma_{r}=\frac{(3+v)}{8} m_{v} \omega^{2}\left(R_{1}^{2}+R_{2}^{2}-\frac{R_{1}^{2} R_{2}^{2}}{r^{2}}-r^{2}\right) \\
\sigma_{\theta}=\frac{(3+v)}{8} m_{v} \omega^{2}\left(R_{1}^{2}+R_{2}^{2}+\frac{R_{1}^{2} R_{2}^{2}}{r^{2}}-\frac{1+3 v}{3+v} r^{2}\right)
\end{gathered}
$$

As is evident from equations 2.1 and 2.2, the stress in both the radial and circumferential directions increase with the square of both the diameter and rotational speed, and proportionately with the material density.

Three rotors were selected as the basis for the comparison between FE analysis and the analytical solutions. The dimensions selected and the operating speeds for all three cases are shown in table 2.1. Rotors 1 and 2 have the same outer radius but different inner radii, while to confirm the scalability of the discretisation requirements relative to the overall dimensions, the third rotor was included with the same aspect ratio as rotor 1 , but with much smaller overall dimensions. 


\begin{tabular}{|c|c|c|c|}
\hline Rotor & Outer radius & Inner radius & Rotational speed $(\mathrm{rad} / \mathrm{s})$ \\
\hline 1 & $1 \mathrm{~m}$ & $0.5 \mathrm{~m}$ & 1000 \\
\hline 2 & $1 \mathrm{~m}$ & $0.25 \mathrm{~m}$ & 1000 \\
\hline 3 & $25 \mathrm{~mm}$ & $12.5 \mathrm{~mm}$ & 1000 \\
\hline
\end{tabular}

Table 2.1 Dimensions of the cylindrical rotors analysed in the convergence study.

Figure 2.1 shows the stress variation with radius in rotor 1 calculated using equations 2.1 and 2.2. The radial stress is normal to the surface at the boundaries of the rotor and is therefore zero, while the circumferential, or hoop, stress is a maximum at the bore.

Finite-element models of the three rotors were constructed at various levels of discretisation. A useful means of defining the level of discretisation is the element size ratio, which is defined as the ratio of the global element edge length to the overall diameter. In each of the rotor models this was reduced from $25 \%$ down to $1 \%$, the step size in each case being reduced as the reciprocal of the initial step size, i.e. $1 / 2,1 / 3,1 / 4, \ldots .1 / 25$. The rotational symmetry of the rotor was exploited to minimise the computational effort, and whereas an arc segment subtending any angle may be used for a simple cylinder, a $45^{\circ}$ segment was used in order to provide consistency with the rotational periodicity of a 4-pole SR rotor. To fully constrain the model, symmetry boundary conditions were applied to the nodes lying on the lines describing the plane of symmetry of the model.

For many electrical machine applications the lamination stack is fitted to the shaft using a radial interference fit or so called "shrink-fit" at the intersection. One consequence of this shrink-fit is to leave considerable stress in the rotor at zero speed, which in turn would greatly reduce the maximum operating speed of the rotor. For the current application, in common with the manufacturing techniques adopted in the production of standard turbochargers, the lamination stack is retained by axial 
compression and the initial radial displacement of the inner-bore nodes in the finite element model is assumed to be zero.

Figure 2.2 shows a typical mesh of rotor 1 which has 28 elements with a global element edge length equal to $6.25 \%$ of the rotor diameter, and includes a representation of the segmentation and corresponding symmetry boundary conditions applied to the rotor. The radial and circumferential stress contours obtained using the mesh shown in figure 2.2 are shown in figures $2.3 \mathrm{a}$ and $2.3 \mathrm{~b}$ respectively.

The radial and circumferential stress distribution across the radius of the machine was calculated at each node lying along a radial path, and a linear interpolation was performed by Ansys to obtain discrete stress values at 20 equal intervals along the radius. Figure 2.4 shows the stress distribution across the radius of rotor 1 obtained using Ansys. The correlation between the finite element analysis and the analytical solution can be considered using two measures:

- The root mean squared error of the interpolated stress values, normalised to the mean stress level in both the radial and circumferential direction respectively. Although providing a global indication of the performance of the finite element technique, individual nodal stresses may still have a marked discrepancy despite an apparently low mean error. Given that from a design point of view, regions of localised high stress may be critical, reliance on this error criterion alone may be somewhat questionable.

- Since failure of the rotor will occur at the location of the maximum effective stress, accurate prediction of this peak stress level is a requirement. It is useful therefore to investigate the error associated with the maximum stress in the cylindrical rotors, and hence the error in circumferential stress at the inner bore is defined using equation 2.3. It is evident from the definition in equation 2.3 that a positive error indicates that the finite-element solution underestimates the peak stress. 


$$
\text { nodal stress error at max stress }(\%)=\frac{\sigma_{\max }-\sigma_{\max , F E}}{\sigma_{\max }} \times 100
$$

Whereas the accuracy of the finite-element solution will inevitably increase as the mesh discretisation is increased, the computational requirements of models with very high levels of discretisation may become overwhelming and in the limit numerical truncation errors may occur. Further, the benefits of very high accuracy solutions are rather questionable if large and somewhat empirical safety factors are subsequently used in the rotor design.

An indication of the penalty in terms of additional computational time associated with increasing levels of mesh refinement is given by the so-called 'wavefront' of the solution. This is defined as the number of unsolved equations in the solution phase and is calculated by Ansys. The square of the mean wavefront is proportional to the computational time required to achieve a solution. The computational requirement figures quoted in this study are normalised to the requirement for the solution to rotor 1 with the minimum mesh discretisation.

Figures 2.5 and 2.6 show the variation in the root mean square error in both the radial and circumferential directions respectively, and the nodal stress error at the location of the maximum stress for the solution of rotor 1 as a function of the computational requirement as mesh discretisation is increased.

Table A2.1 lists the respective error values for rotor 1 where the element size is detailed as the ratio of the global element edge length to the outer diameter of the rotor. It is apparent from table A2.1 that the largest errors occur when the radial stress is calculated, because this is highest near the midpoint of the radius. The radial stress is much lower than the circumferential stress and, at the location of maximum stress, where ultimately rotor failure will occur, the radial component is zero. Therefore the larger errors observed in the radial stress are permissible, as these do not significantly affect the performance of the finite-element method in predicting rotor failure. 
In order to assess the convergence of the finite element solution, a convergence parameter expressing the incremental change of maximum nodal stress with increased discretisation is defined by:

$$
\xi=\frac{\left|\sigma_{\max , i}-\sigma_{\max , i-1}\right|}{\sigma_{\max , i}} \times 100
$$

The rate of change of discretisation is omitted from equation 2.4. As the solution converges $\xi$ will tend towards zero, whereas if the rate of change of discretisation is included in the denominator of equation 2.4 the convergence parameter would exhibit a minimum and rise again as convergence is approached, suggesting apparent divergence. However, upon inspection of equation 2.4 it is apparent that a small increase in discretisation may yield a small change in the stress maximum detected and hence convergence will be detected prematurely. Given the omission of the rate of change of discretisation, it is necessary therefore to ensure that its value is much greater than the convergence criterion applied to $\xi$. For the range of discretisation modelled, the smallest increase in discretisation is $4 \%$, where the convergence parameter threshold is chosen as $0.2 \%$ as discussed below. The convergence of rotor 1 based upon the parameter is plotted in figure 2.7.

Table 2.2 shows a summary of the minimum number of elements where the convergence parameter falls below and remains below $0.2 \%$ for increasing discretisation. The criterion is met in rotors 1 and 3 with an element size ratio of $4.2 \%$, whereas in rotor 2 with the smaller bore radius with respect to the outer radius the criteria is met with an element size ratio of $3.1 \%$. The need for higher discretisation in rotor 2 is due to the higher stress gradient at the inner bore due to the stress concentration effect. 


\begin{tabular}{|c|l|c|c|c|c|c|c|}
\hline Rotor & Sr & $\begin{array}{c}\text { Number of } \\
\text { elements }\end{array}$ & $\begin{array}{c}\text { Normalised } \\
\text { computational } \\
\text { requirement }\end{array}$ & $\begin{array}{c}\text { RMS error } \\
(\text { radial) } \\
(\%)\end{array}$ & $\begin{array}{c}\text { RMS error } \\
\text { (hoop) } \\
(\%)\end{array}$ & $\begin{array}{c}\xi \\
(\%)\end{array}$ & $\begin{array}{c}\text { Nodal error at } \\
\text { maximum stress } \\
(\%)\end{array}$ \\
\hline 1 & 4.2 & 60 & 2.85 & 2.25 & 0.205 & 0.127 & 0.39 \\
\hline 2 & 3.1 & 156 & 5.06 & 1.56 & 0.488 & 0.146 & 1.02 \\
\hline 3 & 4.2 & 60 & 2.85 & 2.25 & 0.205 & 0.126 & 0.39 \\
\hline
\end{tabular}

Table 2.2. Element size ratio and computational effort required with a $0.5 \%$ error criteria applied to each rotor.

It is apparent from consideration of the geometrically similar rotors 1 and 3 in table 2.2 that the solution error is attributable to the element size ratio and not the absolute size of the element, and hence the element size should be specified in this way.

The use of the structural finite element method to analyse the centrifugal stress in the cylindrical rotors and comparison of the results to the analytical formulae demonstrates the validity of the boundary conditions and loads applied, and the mesh discretisation of the finite element model. Specifically the use of symmetrical boundary conditions and the imposition of the loads as an angular velocity in Ansys is validated. Furthermore, methods have been established to identify appropriate mesh discretisation to ensure that the error associated with under-discretised mesh is minimised. 


\subsection{Finite Element Analysis of SR Rotors}

\subsubsection{INTRODUCTION}

Having established the influence of mesh discretisation in the modelling of cylindrical rotors, the study was extended to accommodate the more complex SR rotor geometry. The leading geometrical parameters of a typical 6-4 SR rotor are shown in figure. 2.8. The stress distribution within the rotor is strongly influenced by the inter-polar fillet radius and the central bore which accommodated the shaft. These two features can be conveniently represented by two dimensionless ratios viz. $\beta$, the ratio of bore diameter to the overall diameter, and $\gamma$, the ratio of the fillet radius to the overall diameter.

$$
\begin{aligned}
& \beta=\frac{D_{\text {bore }}}{D_{\text {rotor }}} \\
& \gamma=\frac{r_{\text {fillet }}}{D_{\text {rotor }}}
\end{aligned}
$$

The angle subtended by the rotor pole also affects the stress distribution, but for the current application this was maintained at $35^{\circ}$, a value selected to achieve high power density and to minimise torque pulsation as will be discussed in chapter 3 . The rotational periodicity of the machine was exploited to minimise the computational requirement and hence only 1/8 of the machine was modelled, as shown in figure 2.9, symmetry conditions being imposed at the relevant boundaries. The rotor material is a commercial grade of Silicon Steel (European Electrical Steels CK-26), the mechanical properties of which are listed below in table 2.3: 


\begin{tabular}{|c|c|}
\hline Physical Property & Value \\
\hline Elastic modulus & $196 \mathrm{GPa}$ \\
\hline Density & $7610 \mathrm{kgm}^{-3}$ \\
\hline Poisson's ratio & 0.3 \\
\hline
\end{tabular}

Table 2.3 Material properties of CK-26 (Manufacturer's specified data)

\subsubsection{MESH DISCRETISATION}

On the basis of the analysis presented in section 2.3 for the level of discretisation required for the cylindrical rotor, the following criteria were selected as forming the initial basis for the modelling of SR rotors:

1. Element size ratios should be less than $5 \%$, since the SR rotor is likely to exhibit more pronounced stress concentrations due to its shape.

2. The convergence parameter $\xi$ should not exceed $0.2 \%$ (equivalent to $1 \%$ error in maximum stress for cylindrical rotors)

A similar convergence study to that described for the cylindrical rotor was conducted on two SR rotors, details of which are given in table 2.4. Although the analysis of the cylindrical rotors demonstrated that the overall diameter of the finite-element model does not affect the solution accuracy, the SR rotors were modelled with a diameter of $50 \mathrm{~mm}$, this value being representative of the likely order of magnitude considered for the turbo-generator. Rotor 1 has a fillet radius ratio $\gamma$ of 0.325 , which corresponds to the maximum value which can be accommodated as this represents a full quadrant fillet for a rotor with a $35^{\circ}$ pole arc, while rotor 2 has a minimum fillet radius ratio of 0.025 in order to provide an example of extreme stress concentration. For both rotors, the bore diameter ratio was 0.24 , which corresponds to a representative practical bore diameter of $12 \mathrm{~mm}$. 


\begin{tabular}{|c|c|c|c|c|}
\hline Rotor & Diameter & Bore diameter ratio, $\beta$. & Fillet radius ratio, $\gamma$ & Rotational speed \\
\hline 1 & $50 \mathrm{~mm}$ & 0.24 & 0.325 & $10000 \mathrm{rad} / \mathrm{s}$ \\
\hline 2 & $50 \mathrm{~mm}$ & 0.24 & 0.025 & $10000 \mathrm{rad} / \mathrm{s}$ \\
\hline
\end{tabular}

Table 2.4. Geometrical parameters of the SR rotors used for a convergence study.

The global element edge size for each rotor was initially varied between $5 \%$ and $1 \%$ of the overall rotor diameter. Figure 2.10 shows the meshes for rotors 1 and 2, each with a global element edge length of $2.5 \%$ of the rotor diameter, and which consists of 145 and 123 elements respectively. Also shown are the symmetry boundaries. Convergence of the solution as the discretisation is increased was considered solely by reference to the convergence parameter $\xi$ defined in equation 2.4 .

The geometry of the rotor will inevitably give rise to regions of localised high stress concentration. As a consequence, more efficient use of a given overall number of elements may be achieved by specifying locally higher discretisation in regions where the stress gradient is anticipated to be highest. Hence for each mesh $\xi$ was calculated individually at the point of highest stress observed along the two paths A$\mathrm{A}^{\prime}$ and $\mathrm{B}-\mathrm{B}^{\prime}$ defined in figure 2.9.

It is apparent from table A2.2 and figure 2.11 that the convergence of the solution is not as straightforward as for the cylindrical rotor case shown in table A2.1 and figure 2.7. Although there is a general convergent trend, in some cases an increased number of elements and hence computational requirement does not necessarily lead to a decrease in the convergence parameter $\xi$. This behaviour is largely a consequence of the difficulty in discretising the complex geometry of the SR rotor, a procedure which is undertaken automatically by Ansys. This is compounded by high localised stress gradients, and the inability of the mesh pre-processor to correlate the degree of refinement with the stress distribution. Such limitations could be overcome to a large extent by employing so-called adaptive meshing [2.4], a facility which was however 
not available. The results in table A2.2 and figure 2.11 serve to illustrate that the distribution of elements is of equal importance to the overall number.

Criterion 2 above $(\xi<0.2 \%)$ is achieved along the path A-A' with a global element size ratio of $2.5 \%$. However along path $\mathrm{B}^{-\mathrm{B}^{\prime}}$ an element size ratio of $1.47 \%$ is required, as a result of the high stress concentrations within the fillet.

Similar analysis of rotor 2 yields a convergence in the region $A \rightarrow A^{\prime}$ when the element size ratio is again $2.5 \%$. However over the element size range modelled convergence is not achieved in the region $\mathrm{B} \rightarrow \mathrm{B}^{\prime}$, since the smaller fillet radius gives rise to a considerably higher localised stress concentration. In order to establish the element size ratio required to achieve convergence in rotor 2 , the element size ratio along path $\mathrm{A} \rightarrow \mathrm{A}^{\prime}$ was maintained at $2.5 \%$, while along path $\mathrm{B} \rightarrow \mathrm{B}^{\prime}$ it was varied from $1 \%$ down to $0.2 \%$, where again the element size in each subsequent mesh was reduced as the reciprocal of the initial element size, i.e. $1 / 2,1 / 3,1 / 4 \ldots 1 / 50$. Under these conditions a suitable convergence was achieved in both regions with an element size ratio of $0.38 \%$ along path $B \rightarrow B^{\prime}$.

Table 2.5 summarises the mesh density requirements determined from this analysis. The element size ratio in region $A \rightarrow A^{\prime}$ is essentially insensitive to fillet radius, and a fixed element size ratio of $2.5 \%$ appears appropriate for the entire range of interpolar fillet radii under consideration. In contrast, the discretisation required in the fillet region depends strongly on the fillet radius. Therefore in the generation of meshes for intermediate fillet ratios between 0.025 and 0.325 , the size ratio along path $\mathrm{B} \rightarrow \mathrm{B}^{\prime}$ was established by a linear interpolation between the two values in table 2.5. Figures 2.12 and 2.13 show the resultant final mesh for rotors 1 and 2 respectively. 


\begin{tabular}{|c|c|c|c|c|c|}
\hline Rotor & Region & $\begin{array}{c}\text { Element } \\
\text { Size } \\
\text { ratio } \\
(\%)\end{array}$ & $\begin{array}{c}\xi \\
(\%)\end{array}$ & $\begin{array}{l}\text { Number of } \\
\text { elements }\end{array}$ & $\begin{array}{l}\text { Normalised } \\
\text { computational } \\
\text { requirement }\end{array}$ \\
\hline \multirow{2}{*}{1} & $A-A^{\prime}$ & $2.5 \%$ & 0.0091 & \multirow{2}{*}{208} & \multirow{2}{*}{5.76} \\
\hline & ${\mathrm{B}-\mathrm{B}^{\prime}}^{\prime}$ & $1.5 \%$ & 0.053 & & \\
\hline \multirow{2}{*}{2} & $A-A^{\prime}$ & $2.5 \%$ & 0.11 & \multirow{2}{*}{596} & \multirow{2}{*}{10.45} \\
\hline & B-B' & $0.38 \%$ & 0.06 & & \\
\hline
\end{tabular}

Table 2.5. Required mesh density in the pole and fillet regions of the two SR rotors modelled. Again the computational requirement reported is normalised to cylindrical rotor 1 modelled with minimum mesh discretisation. 


\subsubsection{ANALYSIS OF STRESS DISTRIBUTION}

Several methods may be used to assess the stress levels in a given SR rotor design, including the following:

i) Global parameters such as the maximum or average element stress throughout the rotor. By means of example, table 2.6 contains a summary of useful global parameters for rotors 1 and 2 derived using the mesh in figures 2.12 and 2.13 .

\begin{tabular}{|c|c|c|}
\cline { 2 - 3 } \multicolumn{1}{c|}{} & Figure of merit & Stress, MPa. \\
\hline \multirow{4}{*}{ Rotor 1 } & Average radial stress & 66 \\
\cline { 2 - 3 } & Average hoop stress & 164 \\
\cline { 2 - 3 } & Maximum radial stress & 110 \\
\cline { 2 - 3 } & Maximum hoop stress & 340 \\
\hline \multirow{3}{*}{ Rotor 2 } & Average radial stress & 100 \\
\cline { 2 - 3 } & Average hoop stress & 129 \\
\cline { 2 - 3 } & Maximum radial stress & 175 \\
\cline { 2 - 3 } & Maximum hoop stress & 588 \\
\hline
\end{tabular}

Table 2.6. Summary of element stress values in rotors 1 and 2 operating at 10,000 $\mathrm{rad} / \mathrm{s}$.

ii) Graphical representations of the rotor cross-section, which indicate the location, magnitude and concentration of the stress within the rotor. In terms of assessing the merits of a given rotor in terms of the likelihood of yield and/or fracture, a useful guideline is provided by calculating the effective or so-called 'Von-Mises' stress distribution [2.3]. This is a well-established measure of the critical stress at a given point in a two-dimensional stress field. The Von-Mises stress at a particular location is given by [2.3]: 


$$
\sigma_{e}=\sqrt{\left(\sigma_{1}^{2}+\sigma_{2}^{2}-\sigma_{1} \sigma_{2}\right)}
$$

Contours of Von-Mises equivalent stress for rotors 1 and 2 calculated using the mesh of figures. 2.12 and 2.13 are shown in figures.2.14 and 2.15 respectively. Significant stress concentration effects are evident in the fillet and bore region of the rotor.

iii) Profiles of the variation of radial and/or hoop stress along specified paths. For the $1 / 8$ sector of an SR rotor, two convenient paths $A \rightarrow A^{\prime}$ and $B \rightarrow B^{\prime}$ are shown in figure 2.9. The radial and hoop stress variations along these planes for each rotor are shown in figures 2.16 and 2.17 respectively. It is evident that the finite element model predicts zero radial stress at the rotor surfaces, which is consistent with the necessary physical condition in a direction normal to a circumferential surface.

\subsubsection{ROTOR CROSS SECTIONAL DESIGN}

Having established the appropriate boundary conditions and degrees of discretisation required for representative SR rotors, a comprehensive study of the influence of various geometrical parameters was undertaken, with a view to calculating a rotor diameter suitable for use in the prototype and to establish general design guidelines. There are two conflicting requirements for the most appropriate rotor design:

i) To maximise the overall diameter within the material limits so as to maximise the torque and hence power capability.

ii) To minimise the rotor moment of inertia to improve the transient response of the system.

For the best use of the rotor material in terms of its mechanical strength, it would be desirable to avoid regions of stress concentration and hence establish a rotor design where a large proportion of the rotor material is subjected to similar maximum stress level. As discussed previously, the normalised stress for a combination of fillet radius ratio and bore diameter ratio is scalable by the material density and the square 
of the overall diameter and rotor speed. Thus, for a predetermined range of fillet radius ratio, the entire feasible rotor design space can be explored by reference to these two parameters and the rotor pole width.

There are a number of methods for incorporating an inter-polar fillet that alleviates regions of stress concentration. This study encompasses rotors in which the angle subtended by a circular fillet is $90^{\circ}$, e.g. figure. $2.18 \mathrm{a}$ and $2.18 \mathrm{~b}$, and rotors that employ fillet angles of less than $90^{\circ}$ such as that in figure. $2.18 \mathrm{c}$. The same definition of the size of the fillet i.e. the ratio of the radius of the fillet to the diameter of the rotor, is used for both cases. The maximum fillet radius ratio for fillets subtending an angle of $90^{\circ}$ which can be employed for a given rotor pole arc $\alpha_{p}$, is given by:

$$
\gamma_{\max }=\frac{1}{2}\left[\cos \left(\alpha_{p} / 2\right)-\sin \left(\alpha_{p} / 2\right)\right]
$$

The diameter of the shaft is often determined by the requirements of the specific application. In some cases, particularly when machines are integrated into compact, high-performance systems, such considerations may require a large shaft diameter, which may in turn impose limits on the geometry of the rotor. In the general case, the relationship between the shaft diameter and fillet radius ratio is constrained by an ultimate geometrical limit when the bore reaches the minimum point of the fillet. Thus the fillet radius ratio, $\gamma$, must satisfy the following constraint:

$$
\gamma>\frac{\beta-\sqrt{2} \sin \left(\alpha_{p} / 2\right)}{2(\sqrt{2}-1)}
$$

A second more arduous constraint may be defined where the need to maintain a thickness between the bore and the minimum point of the fillet (which is at least half the width of the rotor pole in order to avoid localised magnetic saturation in this region) gives rise to the constraint: 


$$
\gamma \geq \frac{\beta+(1-\sqrt{2}) \sin \left(\alpha_{p} / 2\right)}{2(\sqrt{2}-1)}
$$

The feasible range of fillet radius ratios based on this second constraint is shown in figure 2.19 as a function of bore ratio for a pole arc of $35^{\circ}$.

A series of 360 finite-element calculations were performed for rotor combinations within the entire feasible design space detailed in figure 2.19. The bore diameter ratio was modelled in increments of 0.04 (to a maximum of 0.6 ) and the fillet radius ratio in increments of 0.025 (to a maximum of 0.6 ). The minimum fillet radius ratio is taken to be 0.025 since zero is impractical in terms of stress concentration because the absence of a fillet creates a singularity and crack tip elements would be required in the finite element solution.

Figures 2.20 and 2.21 show the effect of the fillet radius on the maximum stress in the bore and the fillet of a $50 \mathrm{~mm}$ diameter rotor at $10,000 \mathrm{rad} / \mathrm{s}$, for bore diameter ratios of 0.20 and 0.40 respectively. The maximum stress in the fillet decays rapidly in both cases as the fillet radius is increased. At low values of $\gamma$ the maximum VonMises stress in the rotor occurs at the fillet surface. Increasing the fillet radius yields high returns in terms of reducing the stress concentration in this region. However, as the fillet radius is further increased, eventually the stress in the fillet is reduced to a level such that the maximum stress is present at the bore surface. A larger fillet radius slightly reduces the stress at the bore due to the stiffening of the central region, but in turn adds significantly to the rotor inertia. Ultimately the increased mass of the fillet region increases the centrifugal stress at the bore, however this occurs beyond the range of fillet radii considered.

For high-speed operation, the fillet radius should be sufficiently large to minimise the stress concentration in this region. Arguably the most efficient utilisation of the rotor material, particularly when rotor inertia is considered, is achieved when the stress in 
the fillet is equal to the stress in the bore. It is possible using the results obtained from the finite element analysis to determine the fillet radius ratio with a given bore diameter ratio that will provide equal stress in both regions as shown in figure 2.22. This relationship between the bore diameter and fillet radius ratio remains valid at any rotor speed, diameter and material density and is solely a function of the rotor pole arc. Figure 2.23 shows the calculated maximum stress levels for the combinations of fillet radius and bore diameter ratios shown in figure 2.22 as a function of the bore diameter ratio, where each rotor modelled has a $50 \mathrm{~mm}$ diameter operating at $10,000 \mathrm{rad} / \mathrm{s}$. The stress increases with bore diameter due to the thinning of the region between the fillet and the bore. At bore diameter ratios above 0.44 the stress in the fillet remains larger than that in the bore for all the values of fillet radius ratio considered, and hence the 'optimal' criteria in which the stress is equal in the bore and fillet cannot be satisfied.

Contours of peak stress calculated for rotors over the entire feasible design space can be calculated. Figure 2.24 shows the maximum rotor stress as a function of both bore diameter and fillet radius ratios. Also shown on figure 2.24 are the limits for the two criteria described, i.e.

1. The criteria defined by equation 2.10 required to ensure that localised magnetic saturation is avoided in the region between the bore and the centre of the fillet.

2. The criteria established where the stress in the bore region is equal to the stress in the fillet region, a condition that corresponds to maximum utilisation of the rotor material. The combinations of the fillet radius and bore diameter ratios at which this occurs are also shown in figure 2.22.

Preferred designs are those in the region above and to the left of both the limiting contours defined by the two dashed lines in figure 2.24. Outside this region, the peak stress in the rotor rises rapidly and localised magnetic saturation will occur in the rotor behind the fillet. Arguably the most appropriate rotor design is that at the intersection of the two criteria (with the minimum rotor inertia), i.e. at a fillet radius ratio of 0.16 and a bore diameter ratio of 0.26 . This combination of fillet radius and 
bore diameter ratio represents the most appropriate design in terms of the mechanical and electromagnetic utilisation of the material for a 4 pole rotor with a $35^{\circ}$ pole arc. However, the preferred design for a particular application may well be influenced by factors such as the relative importance of the rotor moment of inertia and any requirements to integrate the machine with a given shaft assembly. The Von-Mises stress contours for this combination of fillet radius and bore in a rotor with an outer diameter of $50 \mathrm{~mm}$ at $10,000 \mathrm{rad} / \mathrm{s}$ are shown in figure 2.25 .

\subsubsection{SELECTION OF ROTOR DIAMETER}

By inspection of equations 2.1 and 2.2 it is apparent that the stress in the rotor increases in direct proportion to the material density and with the squares of the rotor diameter and rotational speed. Hence the maximum stress for any given SR rotor can be calculated using an equation of the form:

$$
\sigma_{\max }=k_{\sigma} m_{\nu} \omega^{2} D^{2}
$$

Where $k_{\sigma}$ is a dimensionless constant which can be determined from finite element analysis for a given combination of fillet and bore radius ratios. For the rotor with a fillet radius ratio of 0.16 and a bore diameter ratio of 0.26 , i.e. the combination identified in section 2.4 .4 as having the best material utilisation, the value of $k_{\sigma}$ is 0.21 . Hence for a $50 \mathrm{~mm}$ diameter rotor operating at $10,000 \mathrm{rad} / \mathrm{s}$, with a density of $7610 \mathrm{~kg} / \mathrm{m}^{3}$ the maximum stress in this particular rotor is $400 \mathrm{MPa}$. Contours of $k_{\sigma}$ for the full range of fillet and bore ratios considered are shown in figure 2.26 . Thus, by reference to figure 2.26 and equation 2.11 , the maximum stress in any rotor design at any speed can be straightforwardly calculated.

By re-arranging equation 2.11, the maximum rotor diameter that can be used for a given maximum speed can be calculated by specifying the maximum stress that can be tolerated 


$$
D_{\max }=\sqrt{\frac{\sigma_{\max }}{k_{\sigma} m_{\nu} \omega_{\max }^{2}}}
$$

Hence for a given operating speed, rotor material and specified stress limit, the maximum rotor diameter may be established using the contours of figure 2.26 Since the rotor of a turbocharger has a large speed range and is frequently required to accelerate from zero to very high speeds, the maximum equivalent stress must incorporate some safety factor to avoid fatigue within the material. A standard criterion [2.5] corresponding to a fatigue limit for this type of cyclical loading is that the maximum equivalent stress in the rotor should be no higher than half the yield stress of the material, i.e. a safety factor of 2 is employed. The yield strength of the CK26 lamination iron is 400MPa and hence a limit of 200MPa was specified for this application.

Figure 2.27 shows the contours of maximum rotor diameter at an operating speed of $100000 \mathrm{rpm}$ with a stress limit of 200MPa determined using equation 2.12. Figure 2.28 shows the maximum rotor diameter for the rotor designs of figure 2.22 for the same 200MPa limit, in designs where the stress in the bore and the fillet are equal.

\subsubsection{SHAFT BORE DIAMETER}

The preceding analysis indicates that the basic geometry of a 4-pole SR rotor of a given overall diameter can be fully defined by the pole arc, the fillet radius ratio and the bore diameter ratio. If the rotor outer diameter is selected from consideration of the centrifugal stress constraints, the resulting bore diameter is then given by the bore diameter ratio, $\beta$. However for many applications the bore diameter may be predetermined by a number of factors, including:

1. The shaft/bore dimensions of existing system components.

2. Minimum lateral stiffness requirements of the shaft required ensuring the critical speeds of the rotor do not occur within the operating speed range. 
3. Torsional stress limits based on the material properties and the maximum torque transmission of the electrical machine.

If the rotor design must include a specified bore diameter, it is more convenient to present the data of figures 2.27 and 2.28 as a function of the absolute magnitude of the bore diameter, rather than as a normalised bore diameter ratio. This can achieved by multiplying the values of bore diameter ratio in figure 2.27 by the corresponding maximum rotor diameter magnitude for a particular operating speed. Hence, the generality of figures 2.27 and 2.28 is compromised somewhat by the imposition of a specific operating speed. For the particular case of an operating speed of 100,000 rpm and a safety factor of 2.0, the normalised characteristics of figures 2.27 and 2.28 can be converted into the speed specific characteristics of figures 2.29 and 2.30, which are expressed in terms of the actual rotor dimensions.

\subsubsection{ROTOR MOMENT OF INERTIA}

The preceding analysis was concerned with the design of a rotor cross section from the point of view the elimination of any significant stress concentration and localised magnetic saturation are the primary considerations. In establishing design guidelines, it is also useful to consider the polar moment of inertia of the rotor, which has particular relevance to the transient response of the turbo-generator system

Assuming that the rotor polar moment of inertia is independent of the bore diameter (i.e. the shaft and the rotor materials have similar densities), then it can be calculated by the appropriate superposition of the contributions of the individual geometric elements shown in figure 2.31. Using this approach, the moment of inertia, $I_{p}$, of the SR rotor with a fillet radius ratio of $\gamma$ which subtends a rotor pole arc of $\alpha_{r}\left(35^{\circ}\right.$ in the case of the particular $6 / 4$ rotor considered in this study) is given by:

$$
I_{p}=L_{\alpha x} m_{v} D_{r}^{4} k_{\alpha \gamma}
$$


Where, for quarter-circle fillets such as those shown in figures $2.18 \mathrm{a}$ and $\mathrm{b}$, the inertia constant is:

$$
\begin{aligned}
& k_{\alpha \gamma}=\left\{-\frac{1}{6} \cos ^{4}\left(\frac{\alpha_{r}}{2}\right)-\frac{1}{12} \sin \left(\frac{\alpha_{r}}{2}\right) \cos ^{3}\left(\frac{\alpha_{r}}{2}\right)+\left(\left(\frac{\pi}{2}-2\right) \gamma^{2}+\frac{1}{3}\right) \cos ^{2}\left(\frac{\alpha_{r}}{2}\right)+\right. \\
& \left.\frac{1}{8} \cos \left(\frac{\alpha_{r}}{2}\right) \sin \left(\frac{\alpha_{r}}{2}\right)+\left(\frac{20}{3}-2 \pi\right) \gamma^{3} \sin \left(\frac{\alpha_{r}}{2}\right)+\left(8-\frac{5 \pi}{2}\right) \gamma^{4}+\left(2-\frac{\pi}{2}\right) \gamma^{2}+\frac{\alpha_{r}}{16}-\frac{1}{6}\right\}
\end{aligned}
$$

And for fillets subtending an angle of less than $90^{\circ}$ (figure 2.18c), the inertia constant is:

$$
\begin{aligned}
& k_{\alpha r}=\left\{\frac{\alpha_{r}}{16}+\left(6 \sin \theta \cos \theta-2 \theta-4 \theta \cos ^{2} \theta\right) \gamma^{4}+\left(\frac{8}{3} \sin \left(\frac{\pi}{4}+\frac{\alpha_{r}}{2}\right) \sin \theta-4 \theta \cos \theta \sin \left(\frac{\pi}{4}+\frac{\alpha_{r}}{2}\right)+\right.\right. \\
& \left.\frac{4}{3} \cos ^{2} \theta \sin \left(\frac{\pi}{4}+\frac{\alpha_{r}}{2}\right) \sin \theta\right) \gamma^{3}+\left((\theta-\sin \theta \cos \theta) \cos ^{2}\left(\frac{\pi}{4}+\frac{\alpha_{r}}{2}\right)-\theta+\sin \theta \cos \theta\right) \gamma^{2}+ \\
& \left.\frac{1}{12} \cos ^{2}\left(\frac{\alpha_{r}}{2}\right)-\frac{1}{24}\left(\cos \left(\frac{\alpha_{r}}{2}\right) \sin \left(\frac{\alpha_{r}}{2}\right)+1\right)+\frac{1}{12} \cos ^{3}\left(\frac{\alpha_{r}}{2}\right) \sin \left(\frac{\alpha_{r}}{2}\right)\right\}
\end{aligned}
$$

Where in this case the fillet half-angle, $\theta$, is given by

$$
\theta=\sin ^{-1}\left(\frac{\cos \left(\frac{\pi}{4}+\frac{\alpha_{r}}{2}\right)}{2 \gamma}\right)
$$

It is useful to plot the variation of $k_{\alpha \gamma}$ as a function of the fillet radius ratio $\gamma$ for a fixed rotor pole arc of $35^{\circ}$, as shown in figure 2.32. As would be expected and is indeed evident from figure 2.32, the most appropriate designs in terms of reducing the polar moment of inertia are those with small fillet radii. However, the requirement to avoid significant stress concentration and localised magnetic saturation imposes a lower limit on the fillet radius. 


\subsubsection{RADIAL GROWTH OF THE ROTOR AND AIRGAP SELECTION}

The high stress levels induced in the rotor at high speed will inevitably give rise to a significant degree of strain, which will manifest itself as a radial expansion of the rotor. Although the magnitude of the radial expansion is not critical in many rotating machines, it may be of importance in SR machines since the airgap length is typically very small (of order of a few tenths of a mm). Hence the calculation of radial expansion is useful in order to ensure that the airgap length selected during the electromagnetic design is sufficient to avoid the possibility of rotor/stator contact, although rotor dynamic issues will also have an influence.

The radial growth of the rotor calculated from the finite element solutions described in section 2.4.4, can be used to establish a concise relationship between radial growth and the leading rotor dimensions using an approach analogous to that used to represent the maximum stress level. Hence the radial growth for a given combination of $\gamma$ and $\beta$ is given by:

$$
u_{r_{\max }}=\frac{k_{\delta} m_{v} \omega^{2} D^{3}}{E}
$$

The variation of the radial growth constant $k_{\delta}$ over the entire range of $\beta$ and $\gamma$ considered is shown in figure 2.33. Again for the rotor with a fillet radius ratio of 0.16 and a bore diameter ratio of 0.26 , the value of $k_{\delta}$ is 0.036 and for the case of a $50 \mathrm{~mm}$ diameter rotor operating at $10,000 \mathrm{rad} / \mathrm{s}$, with a density of $7610 \mathrm{~kg} / \mathrm{m}^{3}$ and elastic modulus of $196 \mathrm{Gpa}$ the maximum radial growth is $7.7 \mu \mathrm{m}$. 


\subsection{Prototype Rotor Design and Manufacture}

In order to realise a reliable prototype system within realistic cost constraints, the mechanical design of the rotor was based, where possible, on employing standard Holset turbo-machinery components and manufacturing techniques. In the volume manufacture of conventional turbochargers, the Inconel turbine wheel is frictionwelded onto a steel shaft, thus ensuring a high-integrity sub-assembly with excellent elevated temperature capability. One consequence of this manufacturing process is that the various components which are to be added to the rotor sub-assembly, viz. the bearings, the SR rotor laminations and the compressor wheel must be sequentially fitted from the compressor end of the shaft, as illustrated in figure. 2.34 (a detailed discussion of the rotor/bearing design is presented in section 6.2 of chapter 6).

The compressor wheel is retained by generating a high level of axial compression, which is achieved by tightening a nut against shoulder on the shaft (a typical torque setting for the nut being $200 \mathrm{Nm} / 150 \mathrm{ft} . \mathrm{lb})$. This in turn imposes a very significant axial tensile stress in the shaft. In order to accommodate this stress level, a minimum shaft diameter of $7 \mathrm{~mm}$ is employed in production by Holset, and even at this shaft diameter the stress approaches the yield stress of the material. Hence, there is little, if any scope to reduce the inner bore of the SR lamination below $7 \mathrm{~mm}$. Using the characteristic in figure 2.30 this gives a maximum rotor diameter of $34.5 \mathrm{~mm}$ for operation at $100,000 \mathrm{rpm}$ at a safety factor 2.0 .

It should be borne in mind that this limiting figure was based on a somewhat arbitrary and conservative safety factor of 2.0. In the detailed optimisation of highperformance machinery subjected to cyclical loading such as automotive-engine connecting-rods [2.6], considerably lower safety factors have been employed, once a very detailed understanding of long-term material behaviour has been proven. This ability to reliably operate with a reduced safety factor yields considerable dividends in terms of specific power capability and/or maximum operating speed. As will be demonstrated in chapter 3 , a diameter of $34.5 \mathrm{~mm}$ is relatively small for the potential 
power rating of the machine, with a consequent requirement for a relatively long lamination stack. As a consequence, for the prototype rotor, the safety factor was reduced by $20 \%$ to 1.6 , a figure which is still well within the range of values encountered in various high specific power cyclically loaded machine elements [2.6]. The viability of this safety factor in long-term production would necessitate a detailed study of the fatigue behaviour of the electrical steel (there being no published data in the current literature). The metallurgical data produced in such a study must be coupled to a detailed specification of the likely duty cycle of the machine in order to determine the residual, mean and peak-cyclical stresses for construction of a so-called "Goodman line"[2.7,2.8], to determine the "equivalent" stress.

Using this safety factor, a maximum rotor diameter of $38 \mathrm{~mm}$ can be employed. The bore diameter ratio, $\beta$, becomes 0.184 and the fillet radius ratio, $\gamma$, required to provide equal stress in the bore and fillet regions is 0.13 . Figure 2.35 shows the stress contours in the $38 \mathrm{~mm}$ diameter rotor operating at $100,000 \mathrm{rpm}$. The absolute maximum speed at which failure due to shaft burst will occur for this rotor is approximately $130000 \mathrm{rpm}$.

A prototype rotor was manufactured using a lamination stack with an outer diameter of $38 \mathrm{~mm}$. The rotor is held in place using the retaining nut to provide axial compression against a pre-machined shoulder in the shaft in a manner similar to that adopted for the compressor wheel in standard turbocharger design. This eliminates both the stress concentration effects (and unbalance) associated with machining keyways into the shaft/rotor, and the high levels of residual stress associated with shrinkfitting the laminations onto the shaft, which would (due to superposition with the centrifugal stresses) considerably reduce the maximum operating speed of a given rotor. However upon assembly, the action of compressing the laminations with the retaining nut against the shoulder on the shaft via a collar behind the compressor, caused the shaft to bend to an extent beyond the limits of the tolerances required for balancing. This bending occurred as the two faces of the rotor laminations tended to deviate from parallel as they compressed in a non-uniform manner forcing the shaft 
to bend, as shown in figure 2.36. Consequently the shaft was redesigned to incorporate a precision ground steel sleeve which was inserted into a redesigned rotor with a larger bore. The compressor collar and shaft shoulder act against the sleeve and the non-uniform compression of the lamination material was avoided. The diameter of the sleeve was selected as $13 \mathrm{~mm}$ in order to provide sufficient sectional area upon which the shaft shoulder and compressor collar can act, and hence the bore of the rotor was modified to accommodate this. For the purpose of producing a prototype machine the rotor diameter of $38 \mathrm{~mm}$ was maintained and hence the fillet radius ratio in this case was tailored to achieve the desired speed without exceeding the stress limits of the rotor. In this case the fillet radius ratio that, when combined with the bore diameter ratio of 0.34 , would give the same magnitude of stress in the two regions was beyond the limits of the saturation criterion and a fillet radius ratio of 0.275 was used. The stress distribution in this rotor at $10000 \mathrm{rad} / \mathrm{s}$ is shown in figure 2.37 and figure 2.38 shows the corresponding radial displacement where the poles can be seen to extend into the airgap by $8 \mu \mathrm{m}$.

The final rotor specifications, which were arrived at with due consideration of the practical issues associated with integrating the rotor onto the turbo-machinery components, are summarised in table 2.7. 


\begin{tabular}{|l|c|c|}
\hline Diameter & 38 & $\mathrm{~mm}$ \\
\hline Bore diameter ratio & 0.34 & - \\
\hline Bore diameter & 13 & $\mathrm{~mm}$ \\
\hline Fillet radius ratio & 0.275 & - \\
\hline Fillet radius & 10.5 & $\mathrm{~mm}$ \\
\hline Stress constant $k_{\sigma}$ (equation 2.11) & 0.206 & - \\
\hline Maximum stress level@100,000rpm & 248 & $\mathrm{MPa}$ \\
\hline Inertia constant $k_{\alpha \gamma}$ (equation 2.14) & 0.875 & - \\
\hline Additional inertia (50mm axial length - section 3.2) & $43 \times 10^{-6}$ & $\mathrm{kgm}$ \\
\hline Percentage increase of inertia w.r.t. to standard turbocharger & 43 & $\%$ \\
\hline Radial growth constant $k_{\delta}$ (equation 2.15) & 0.037 & - \\
\hline Maximum radial growth of rotor@100,000rpm & 8 & $\mu \mathrm{m}$ \\
\hline Maximum rotational speed to yield stress & 127,000 & $\mathrm{rpm}$ \\
\hline Safety factor for yield & 1.61 & - \\
\hline
\end{tabular}

Table 2.7 Summary of rotor design. 


\section{REFERENCES}

[2.1] Jun, C-H. and Nicolas, A. 'Analysis of the mechanical stresses on a squirrel cage induction motor by the finite element method.' IEE Trans. on Magn. Proc. of the 1998 18th Biennial IEEE Conference on Electromagnetic Field Computation (IEEE CEFC '98), pp1282-1285, May 1999.

[2.2] Subramani, D. A., Ramamurti, V. and Sridhara, K., 'Numerical analysis and experimental verification of the radial growth of a turbocharger centrifugal compressor impeller.' Jnl. of Strain Analysis for Eng. Design, Vol. 32 No 2, pp119-128, March 1997.

[2.3] Fenner, R. T., 'Mechanics of Solids' Blackwell Scientific Publications, 1989.

[2.4] Lee, C. K. and Lo, S. H., "An automatic adaptive refinement procedure using triangular and quadrilateral meshes." Engineering Fracture Mechanics, Vol. 50. No5, 1996. pp 671-686.

[2.5] Gere, J. M. and Timoshenko, S. P., "Mechanics of materials - $3^{\text {rd }}$ Edition." Chapman and Hall, 1992.

[2.6] Graham, J. A., Millan, J. F. and Franklin, J., "Fatigue design handbook." Publication of the Society of Automotive Engineers, USA. 1968.

[2.7] Chyn, C., Wu, R. C. and Tsao, T. P., "Torsional fatigue of turbine-generator shafts owing to network faults." IEE Proc. Generation, Transmission and Distribution, Vol. 143, No 5, 1996. pp479-486.

[2.8] Heath, A. R. and McNamara, P. M., "Crankshaft stress analysis-combination of finite element and classical analysis techniques." Trans. of the ASME, Jnl of Engineering for Gas Turbines and Power, Vol. 112. No 3. 1990. pp268-

275. 


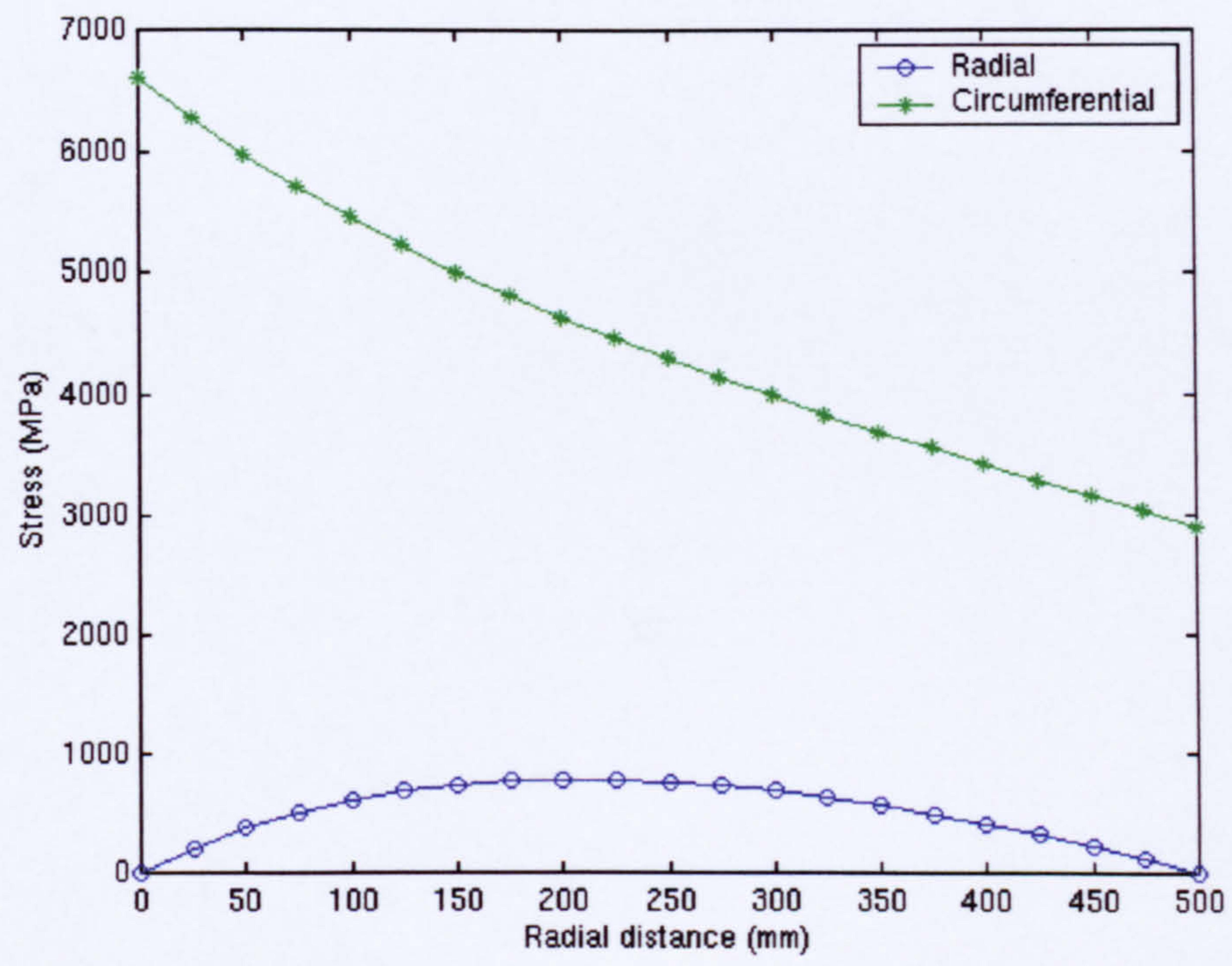

Figure 2.1. Radial and circumferential stress calculated using equations 2.1 and 2.2 respectively for rotor 1 .

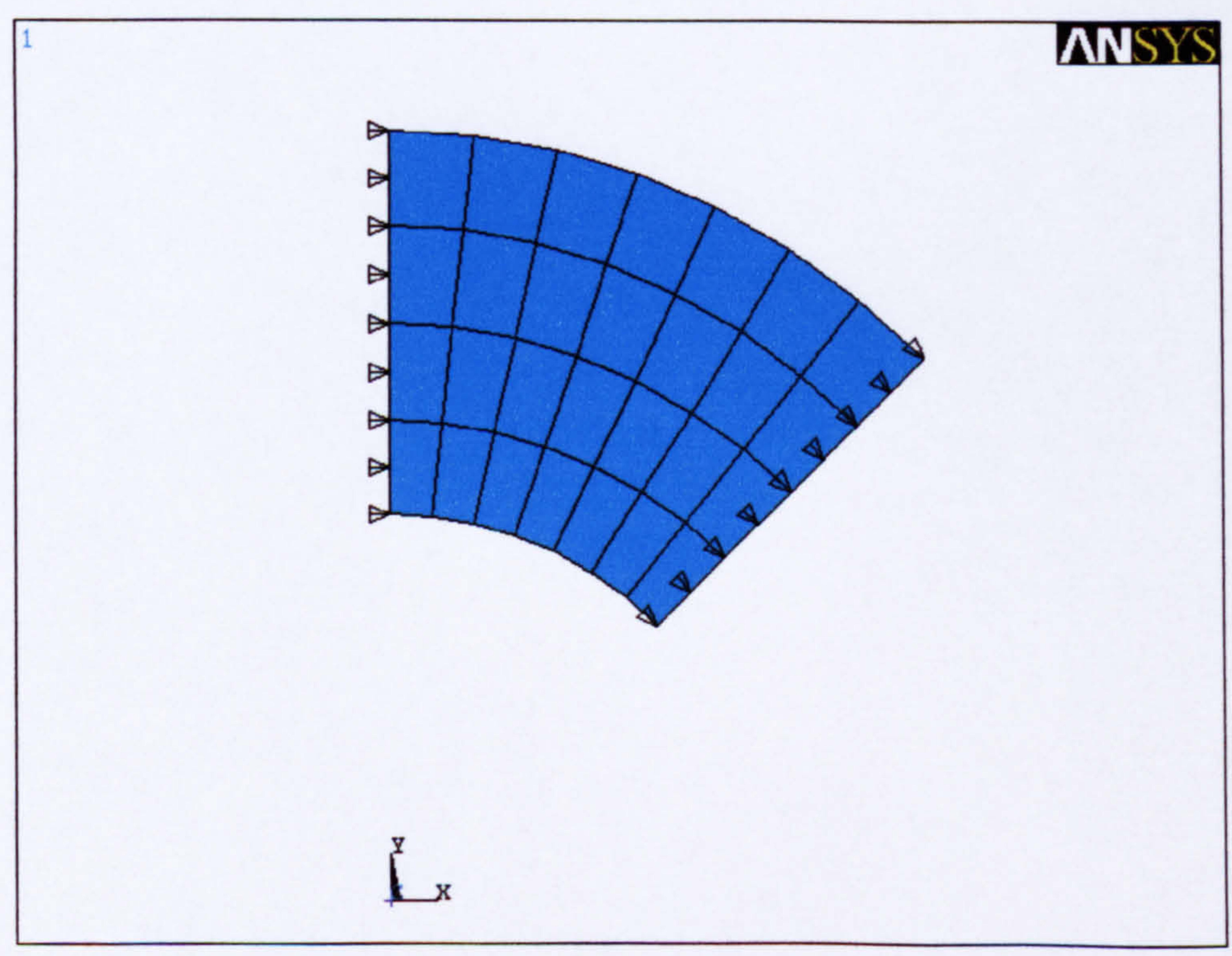

Figure 2.2. Typical finite element mesh of rotor 1 consisting of 28 second order structural elements. 


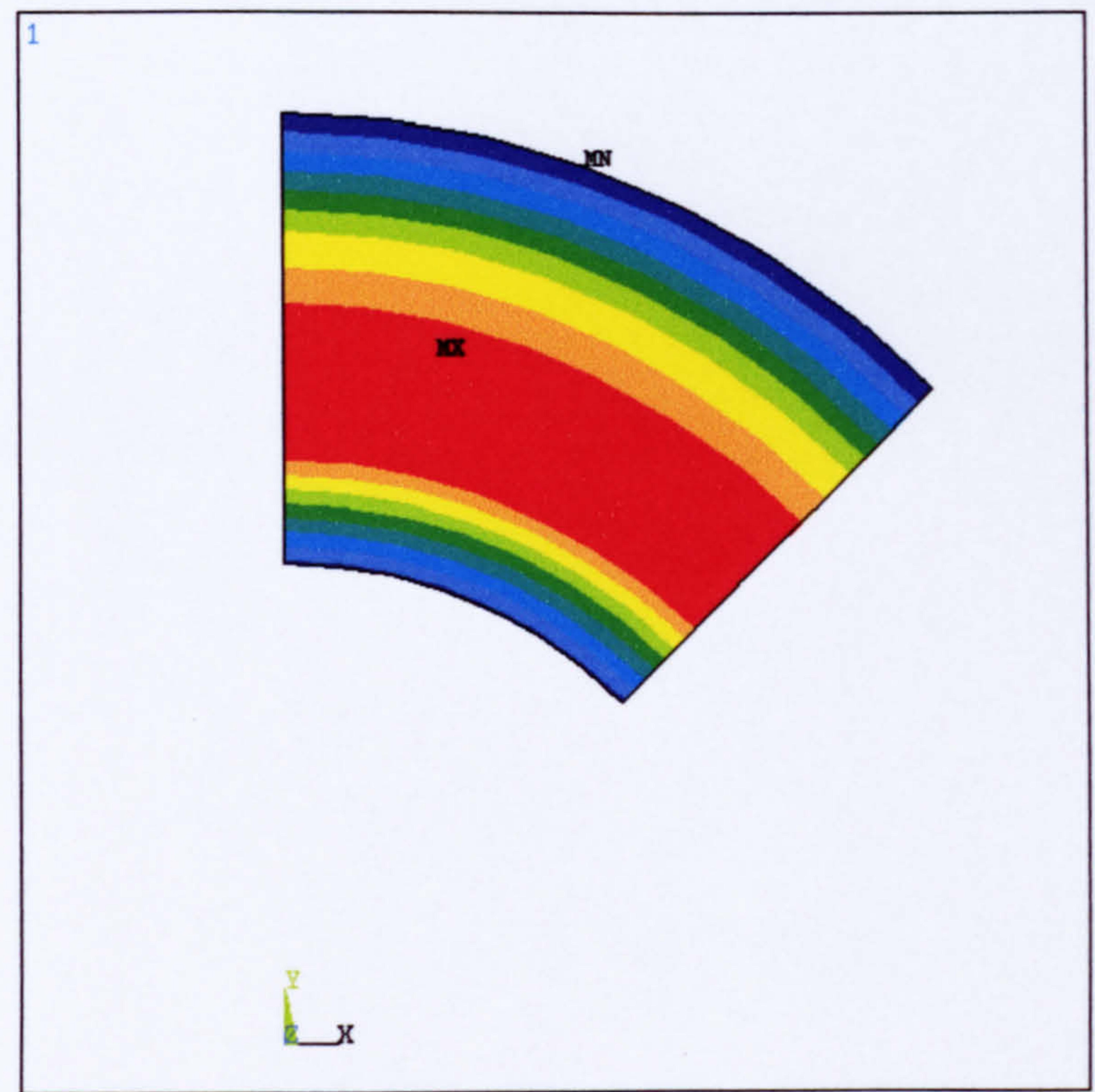

ANSYS 5.4

FEB 192000

$16: 30: 08$

NODAL SOLUTION

$\mathrm{STEP}=1$

RSYS $=1$

(AVG)

PowerGraphics

BFACET $=1$

AVRES $=$ Mat

$\mathrm{DMX}=.016528$

$S M N=.910 E+07$

$S M X=.784 \mathrm{E}+09$

$910 \mathrm{E}+07$

$952 \mathrm{E}+08$

$181 \mathrm{E}+09$

$267 \mathrm{E}+09$

$354 \mathrm{E}+09$

.

$526 \mathrm{E}+09$

$612 \mathrm{E}+09$

$698 \mathrm{E}+09$

$.784 \mathrm{E}+09$

Figure 2.3a. Radial stress contours in cylindrical obtained using the mesh detailed in figure 2.2.

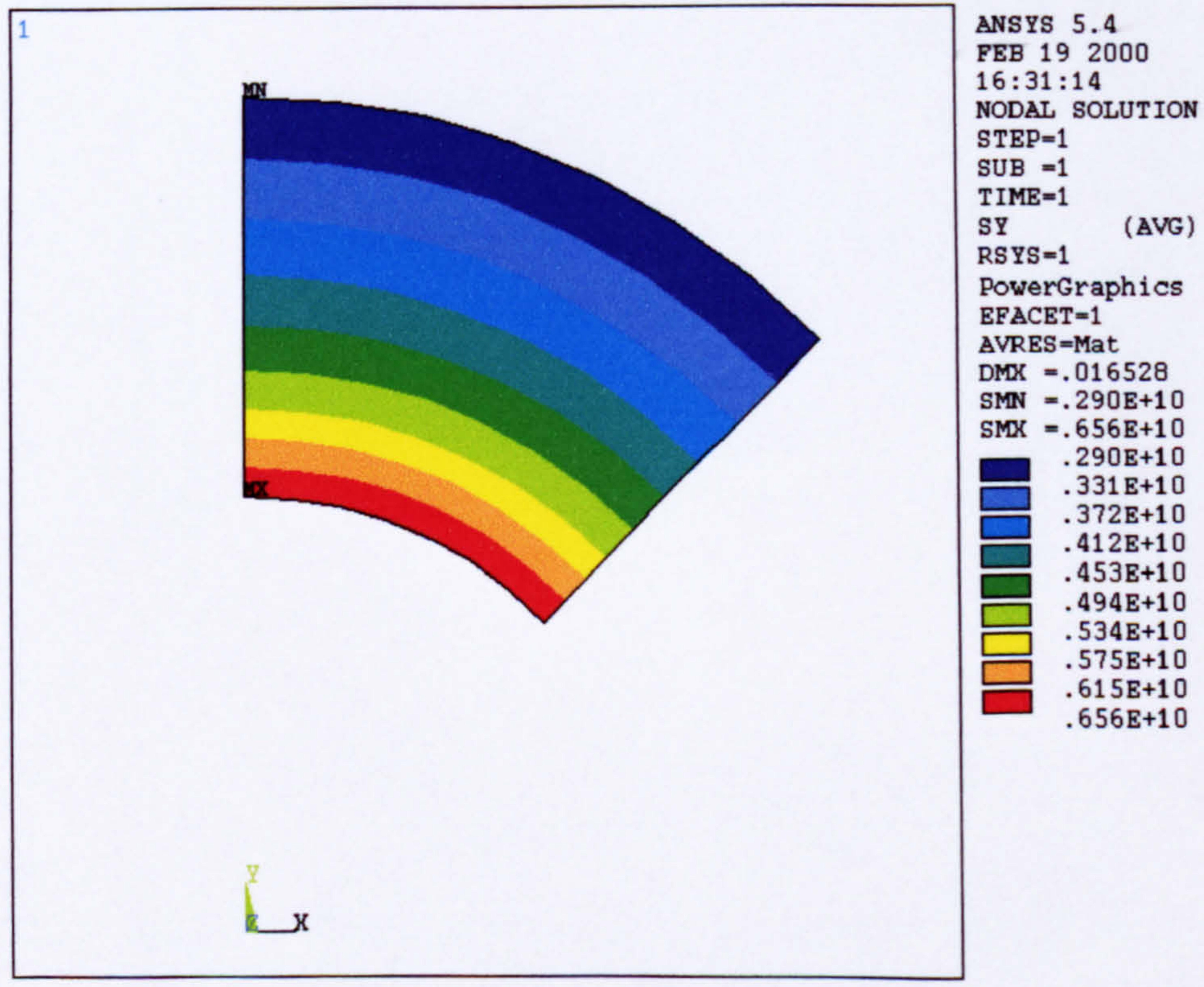

Figure 2.3b. Circumferential stress contours in cylindrical obtained using the mesh detailed in figure 2.2 . 


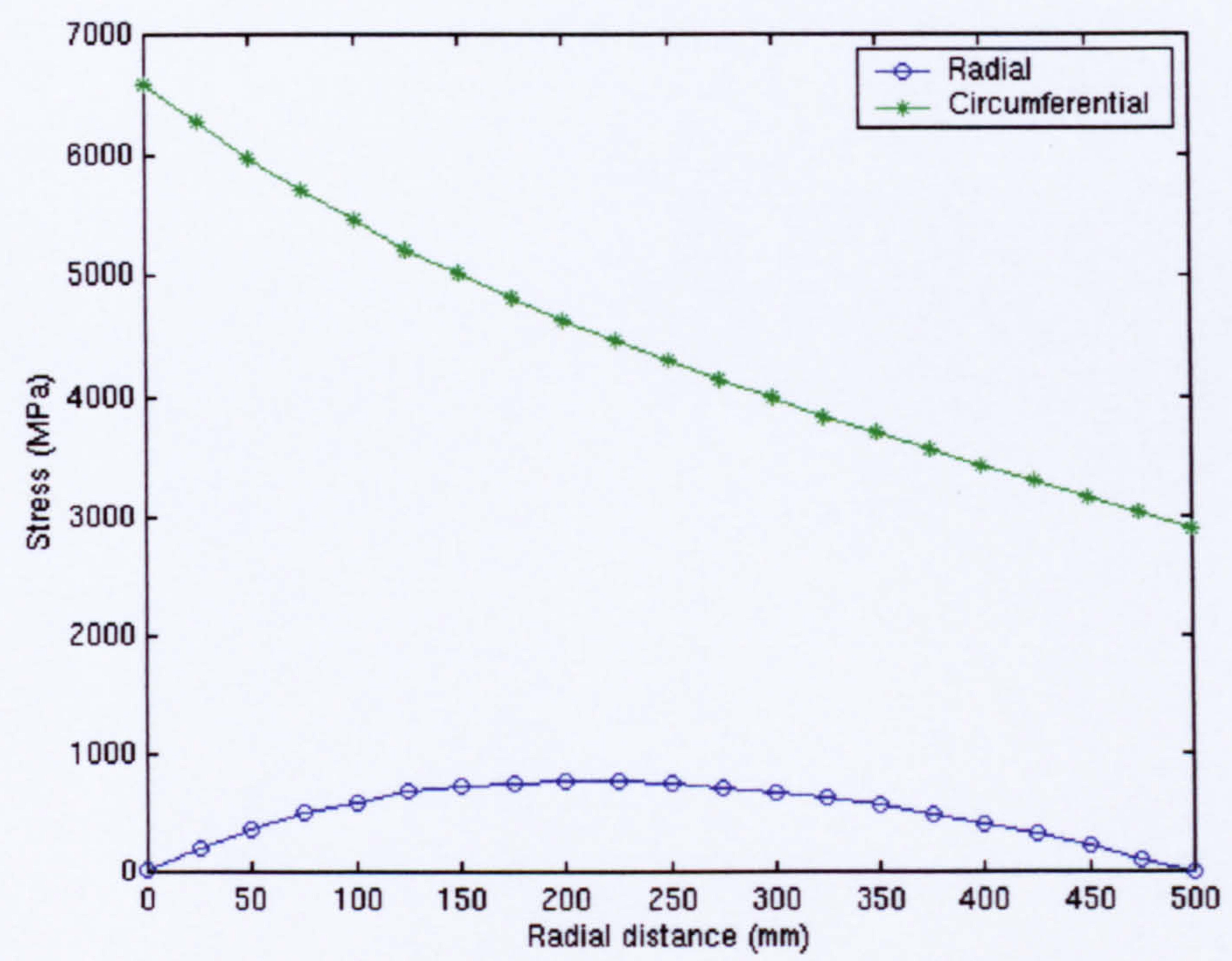

Figure 2.4. Radial and circumferential stress distribution calculated using Ansys for mesh consisting of 28 elements.

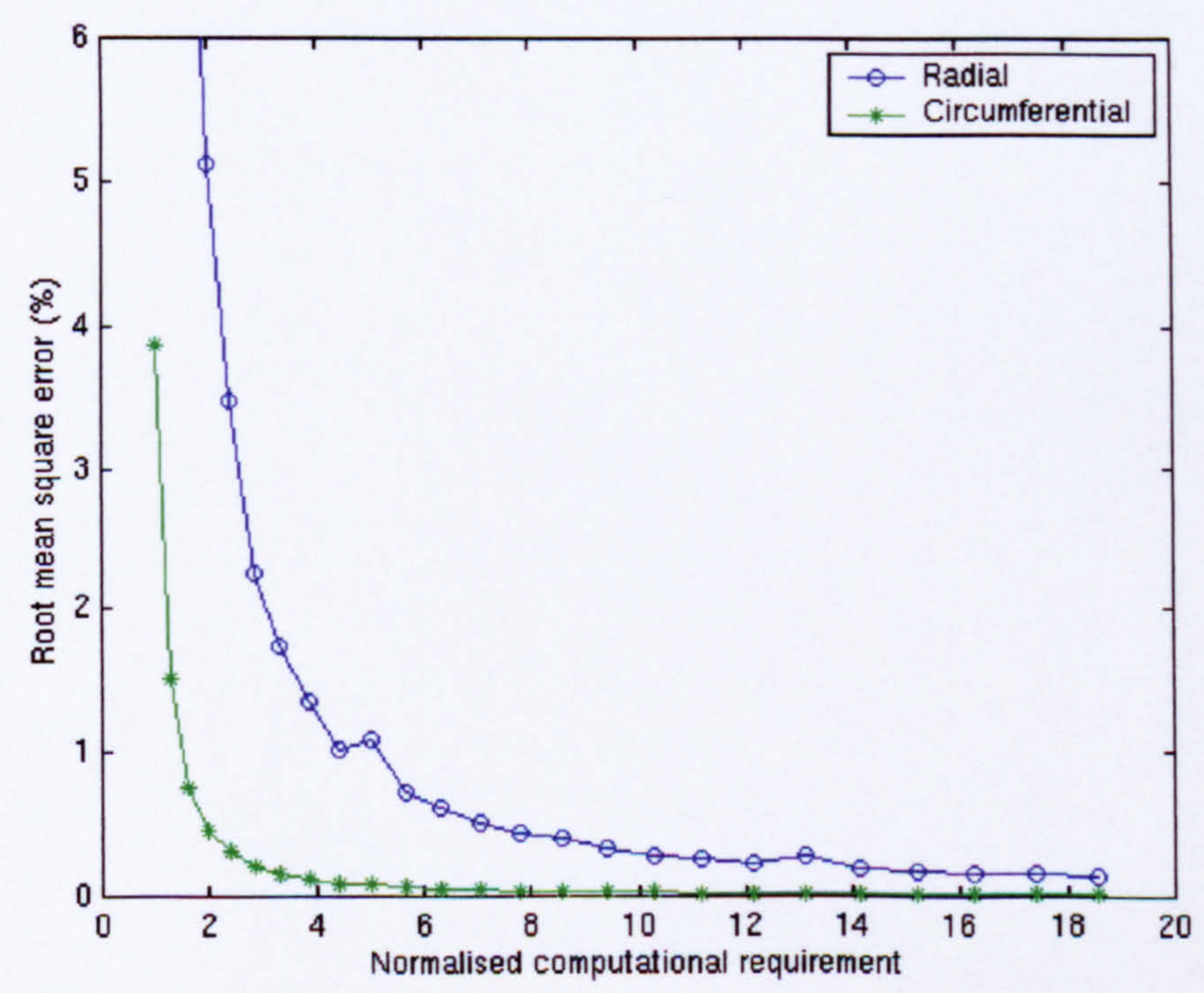

Figure 2.5. RMS error for cylindrical rotor 1 as the mesh discretisation is increased. 


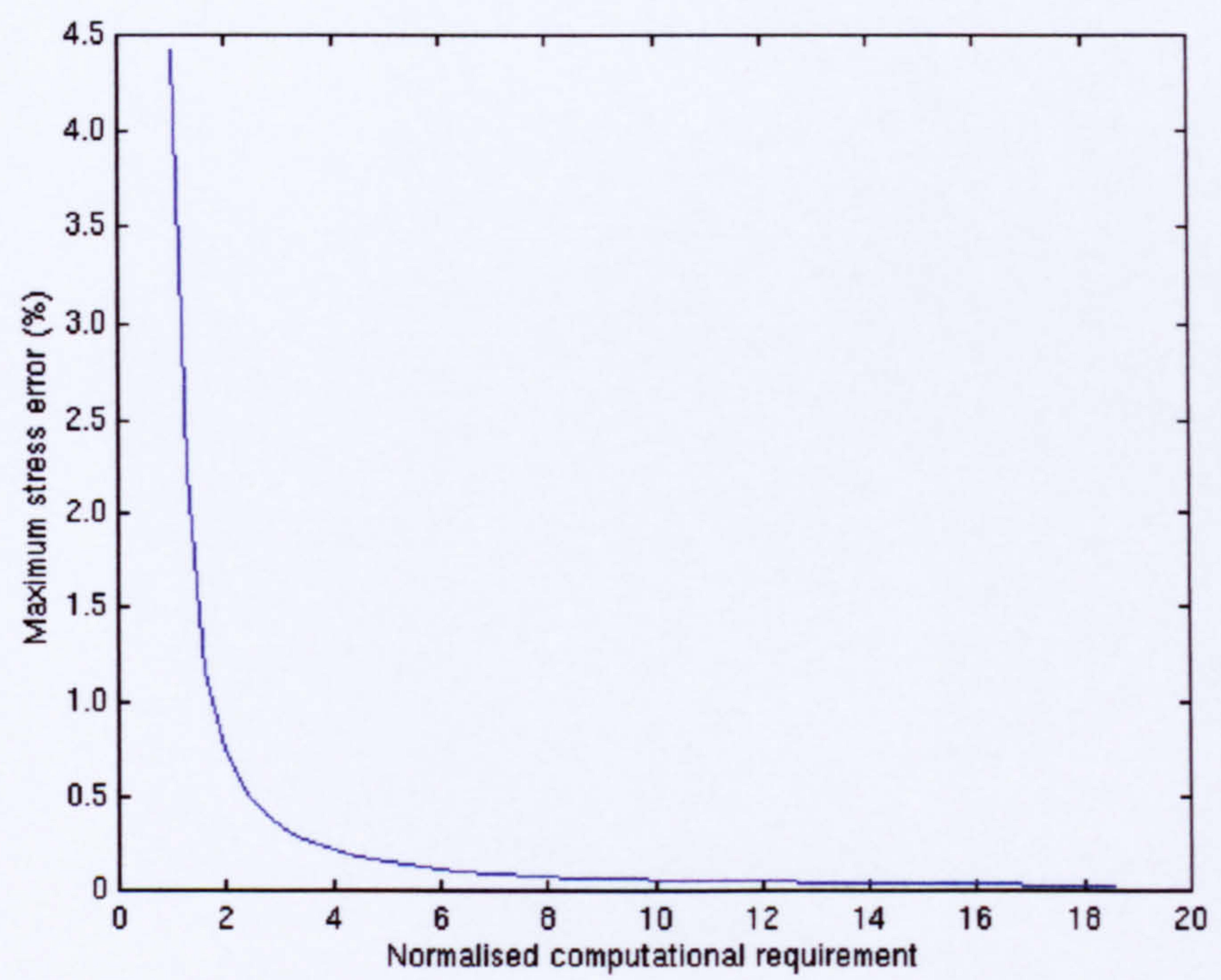

Figure 2.6. Variation in the peak stress error as a function of computational requirement as the element discretisation is increased.

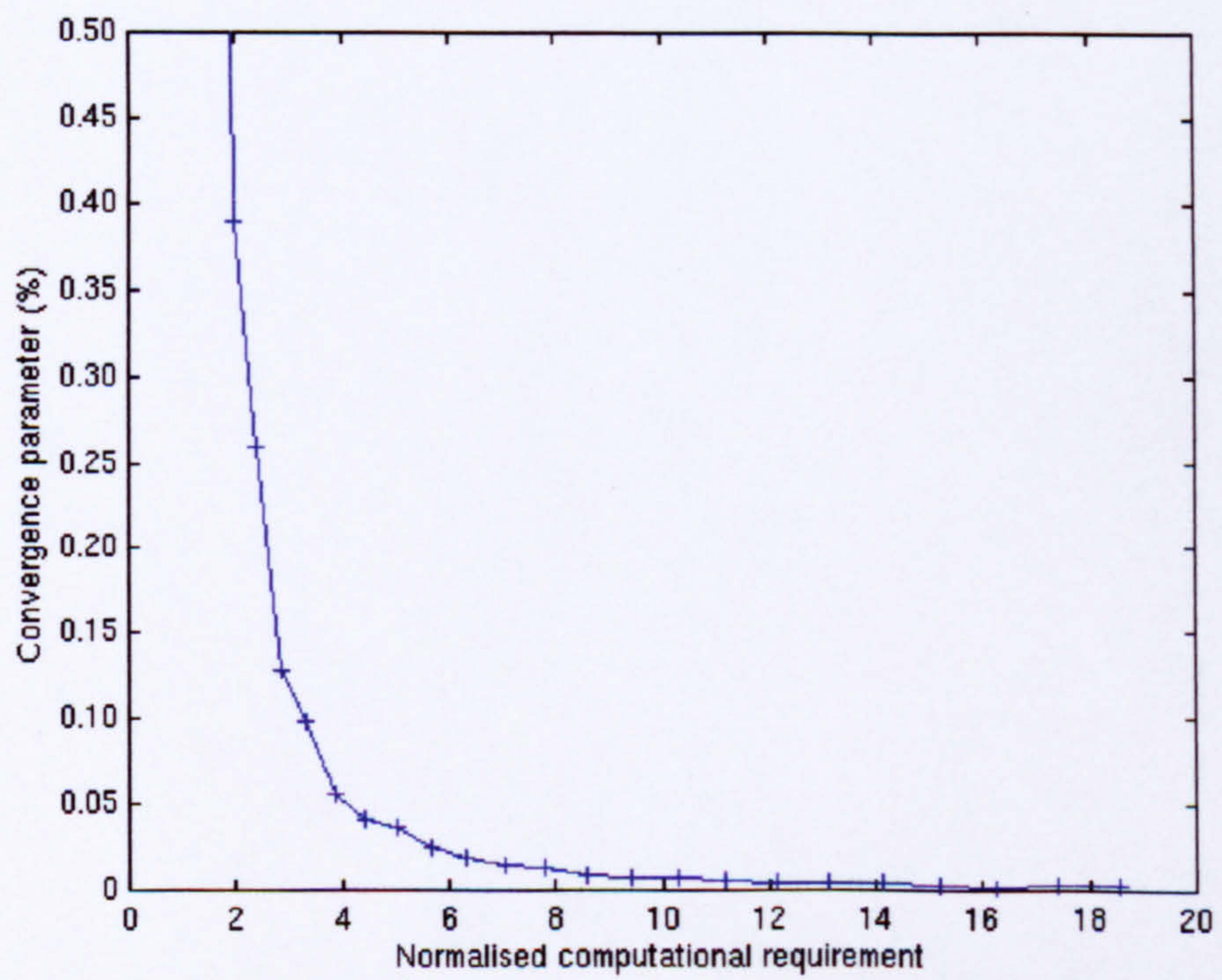

Figure 2.7. Variation in the convergence parameter, $\xi$ as the mesh discretisation is increased 


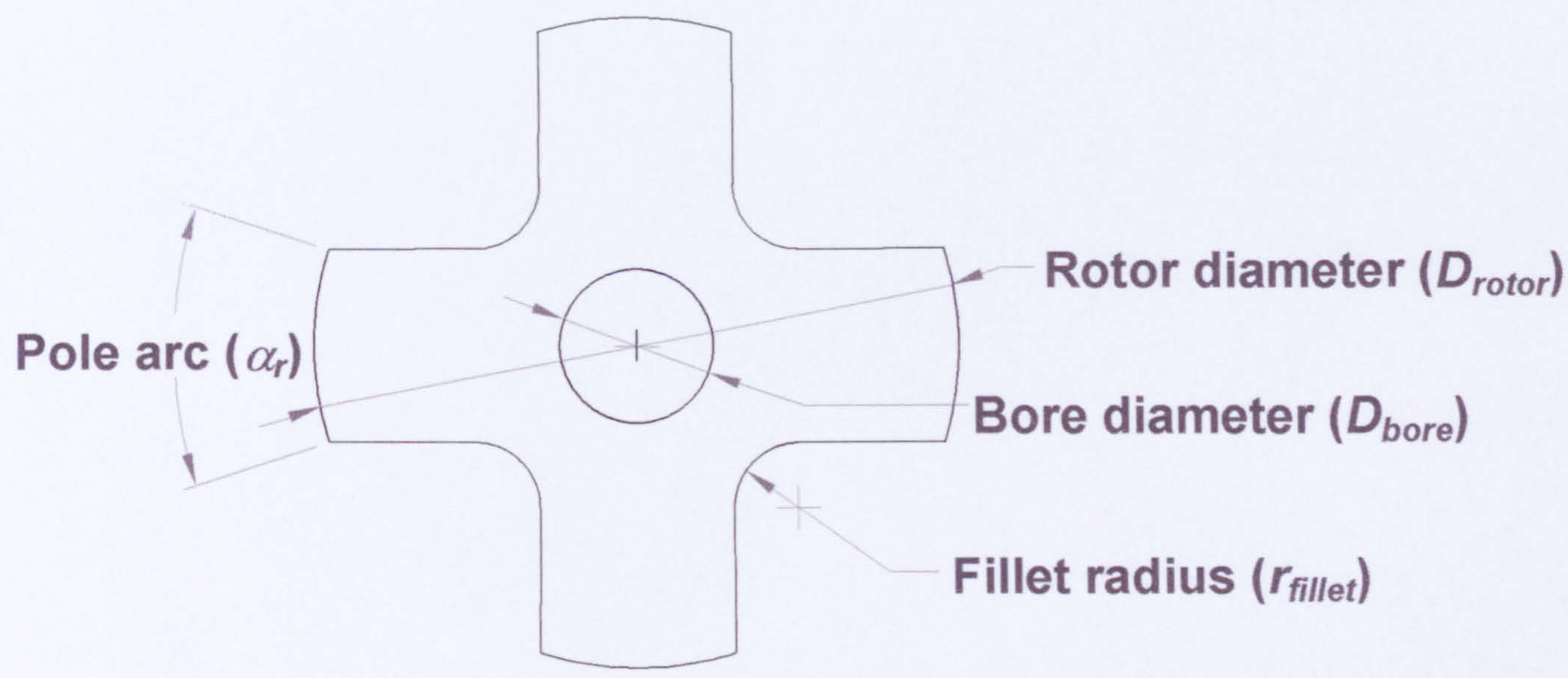

Figure 2.8. Switched reluctance rotor structure highlighting leading dimensions.

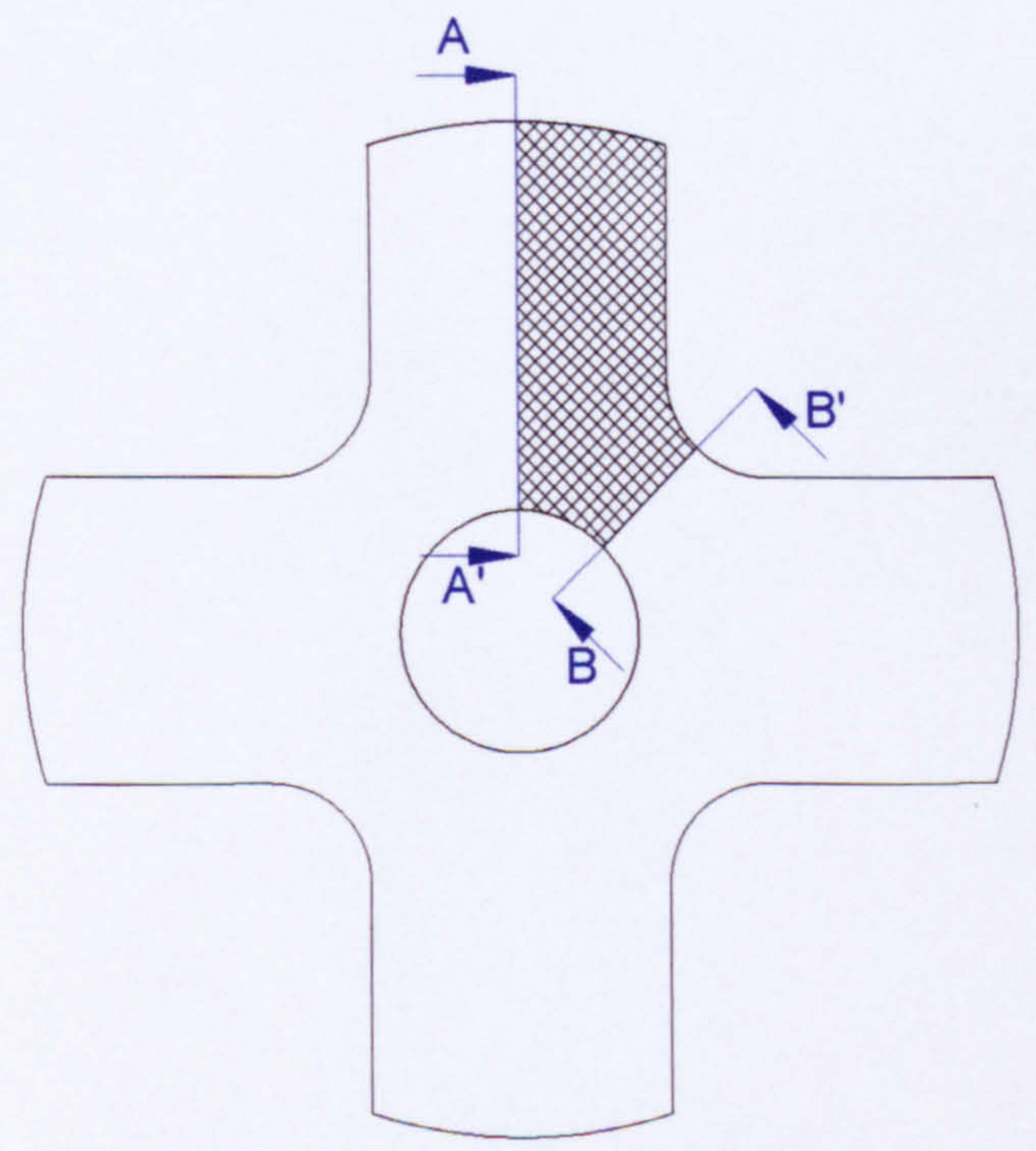

Figure 2.9 SR rotor schematic indicating region modelled and the paths A-A' and BB' defined on the lines of symmetry. 


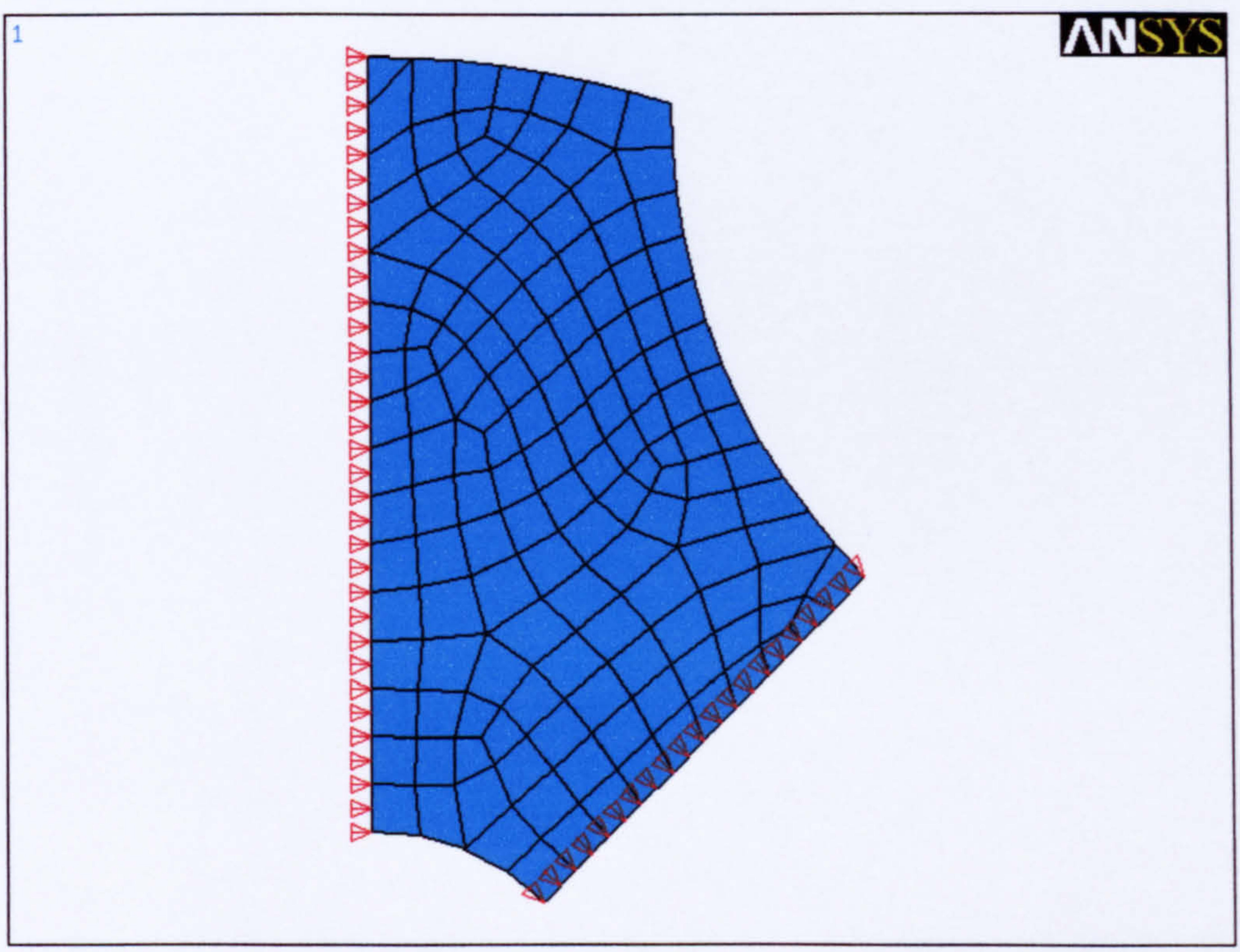

Figure 2.10a. Mesh of rotor 1 with a global element edge length of $2.5 \%$ of the rotor diameter consisting of 145 elements.

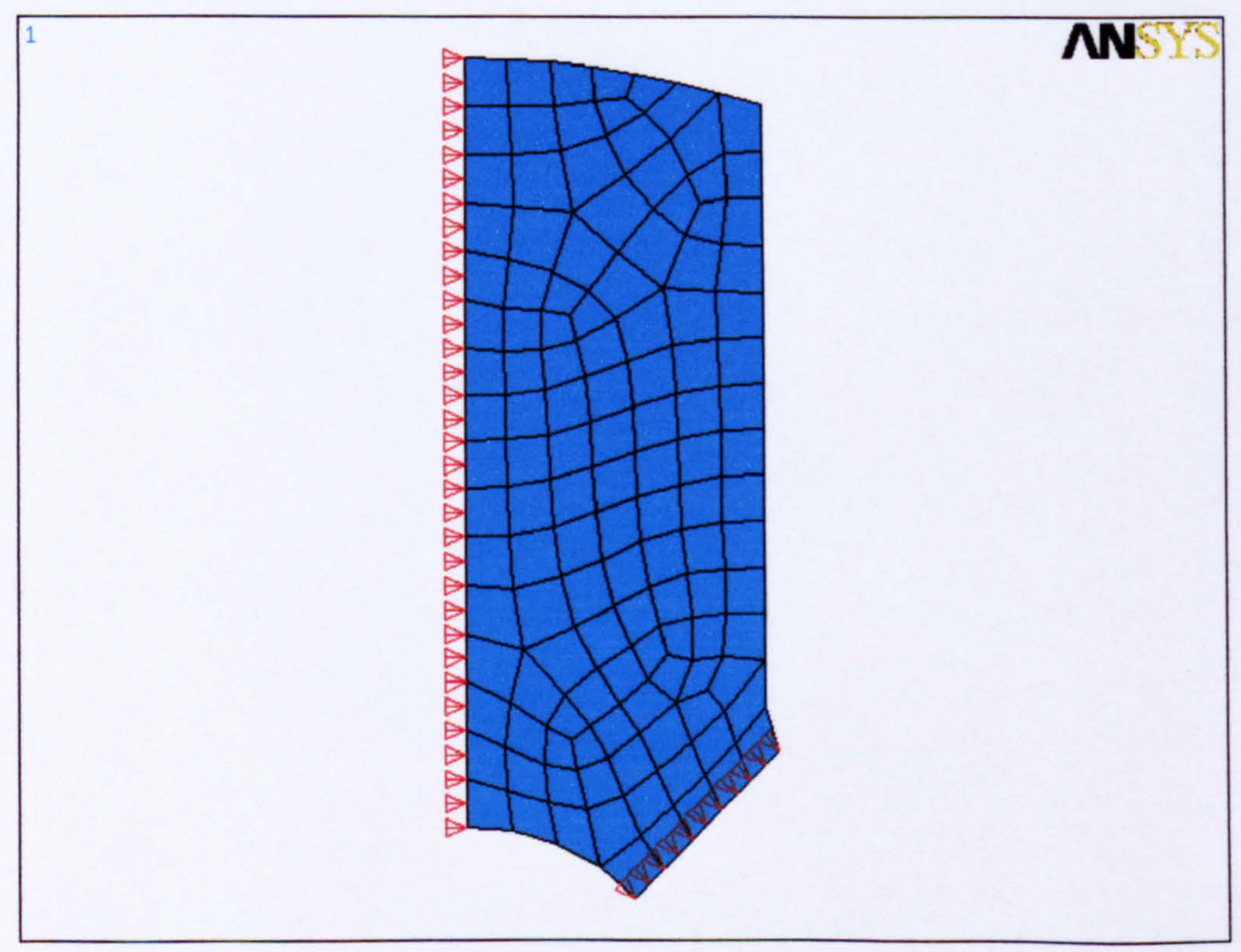

Figure $2.10 \mathrm{~b}$. Mesh of rotor 2 with a global element edge length of $2.5 \%$ of the rotor diameter consisting of 123 elements. 


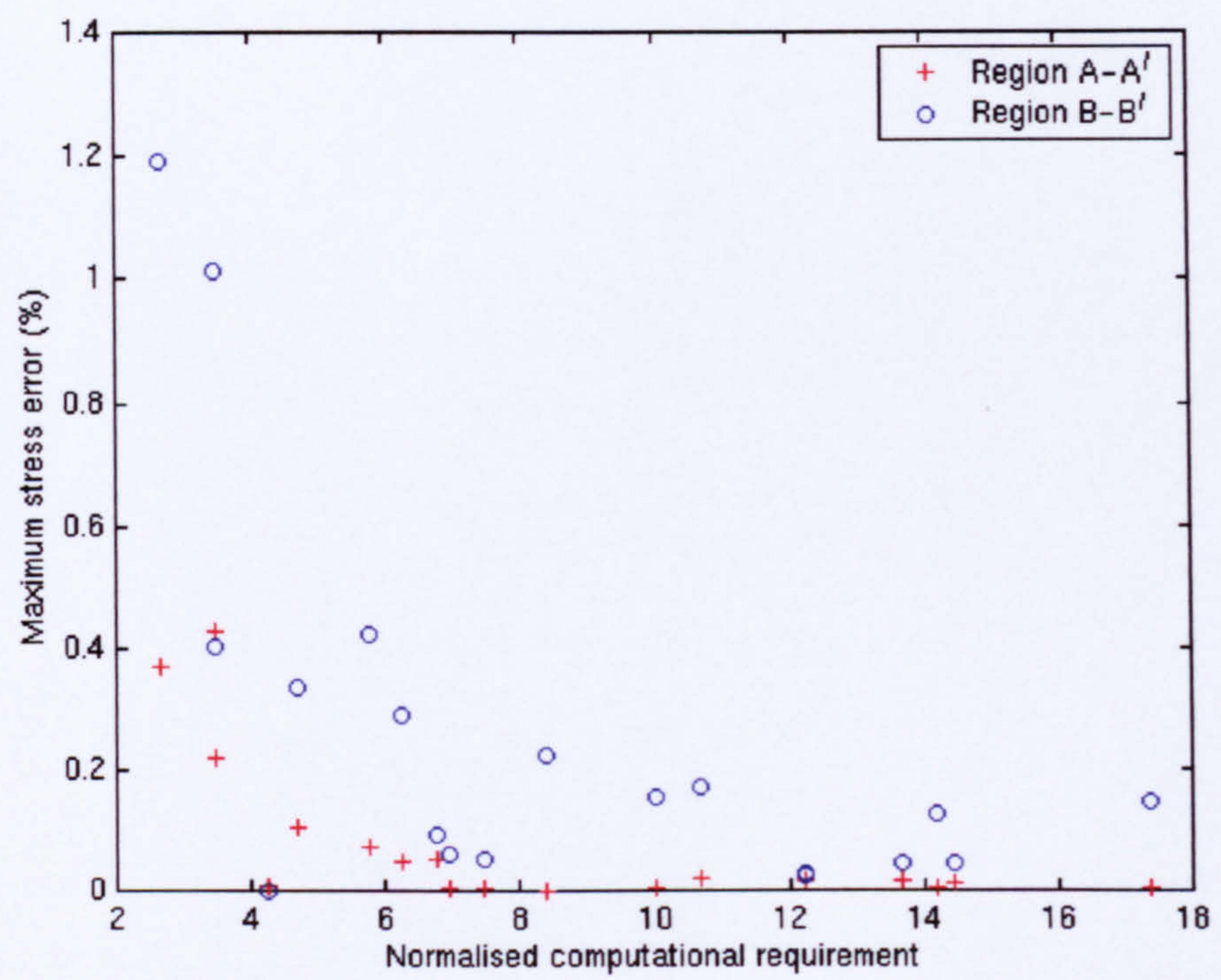

Figure 2.11. Variation of the convergence parameter, $\xi$, in the regions A-A' and B-B' of SR rotor 1 as the element discretisation is increased.

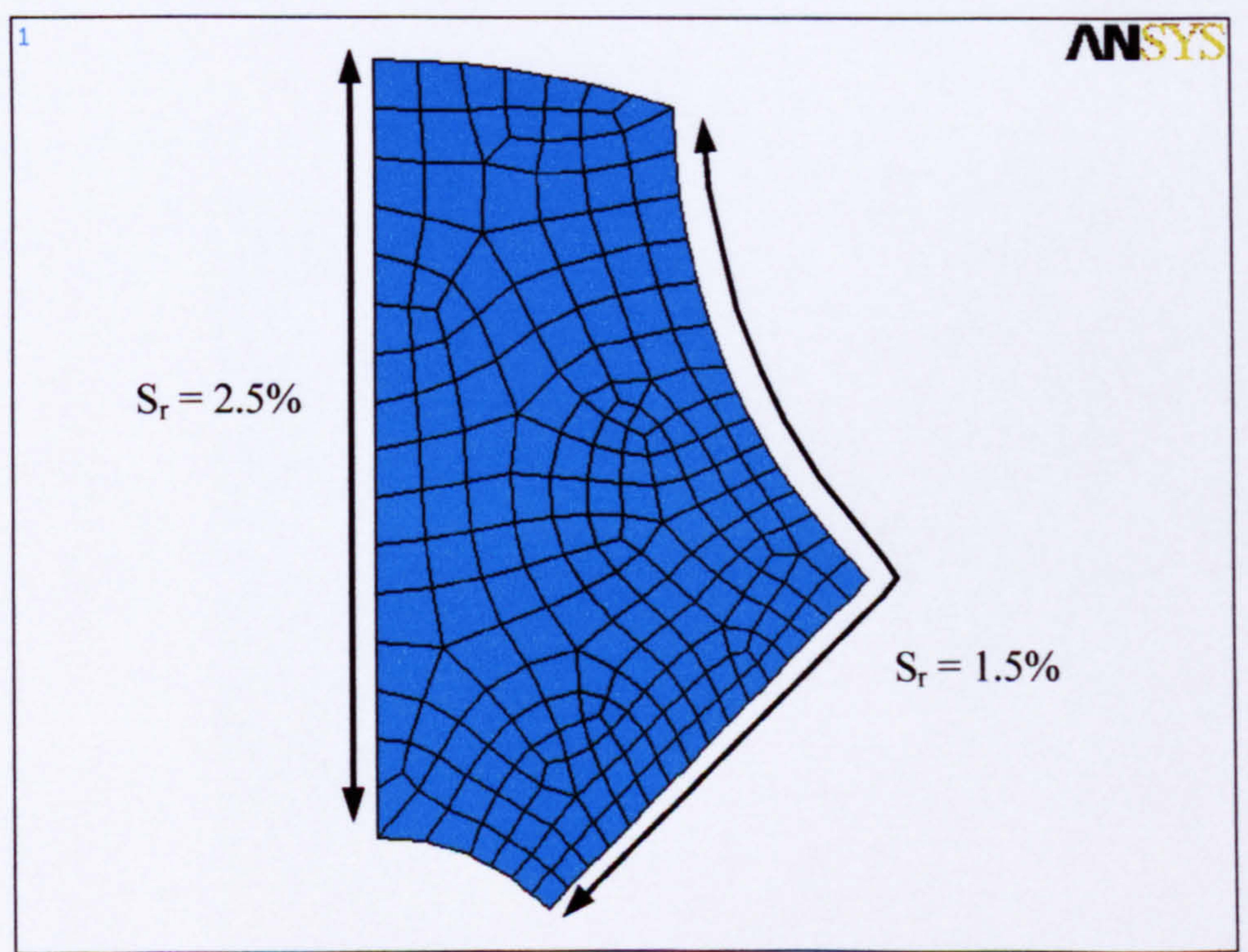

Figure 2.12. Revised mesh of rotor 1 with a global element edge length of $2.5 \%$ of the rotor diameter and a localised edge length of $1.5 \%$ in region B-B', consisting of 208 elements 


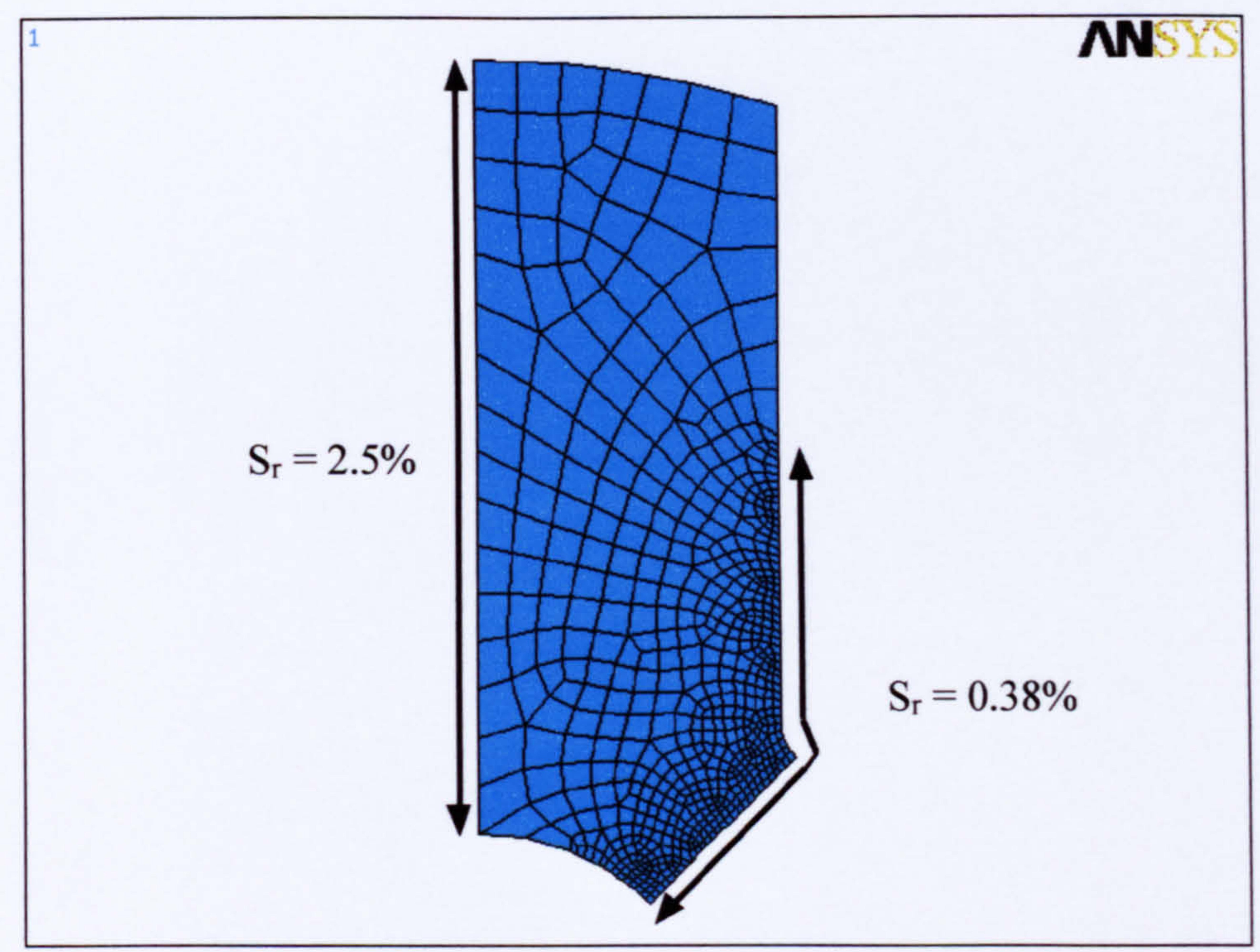

Figure 2.13. Revised mesh of rotor 2 with a global element edge length of $2.5 \%$ of the rotor diameter and a localised edge length of $0.38 \%$ in region B-B', consisting of 596 elements
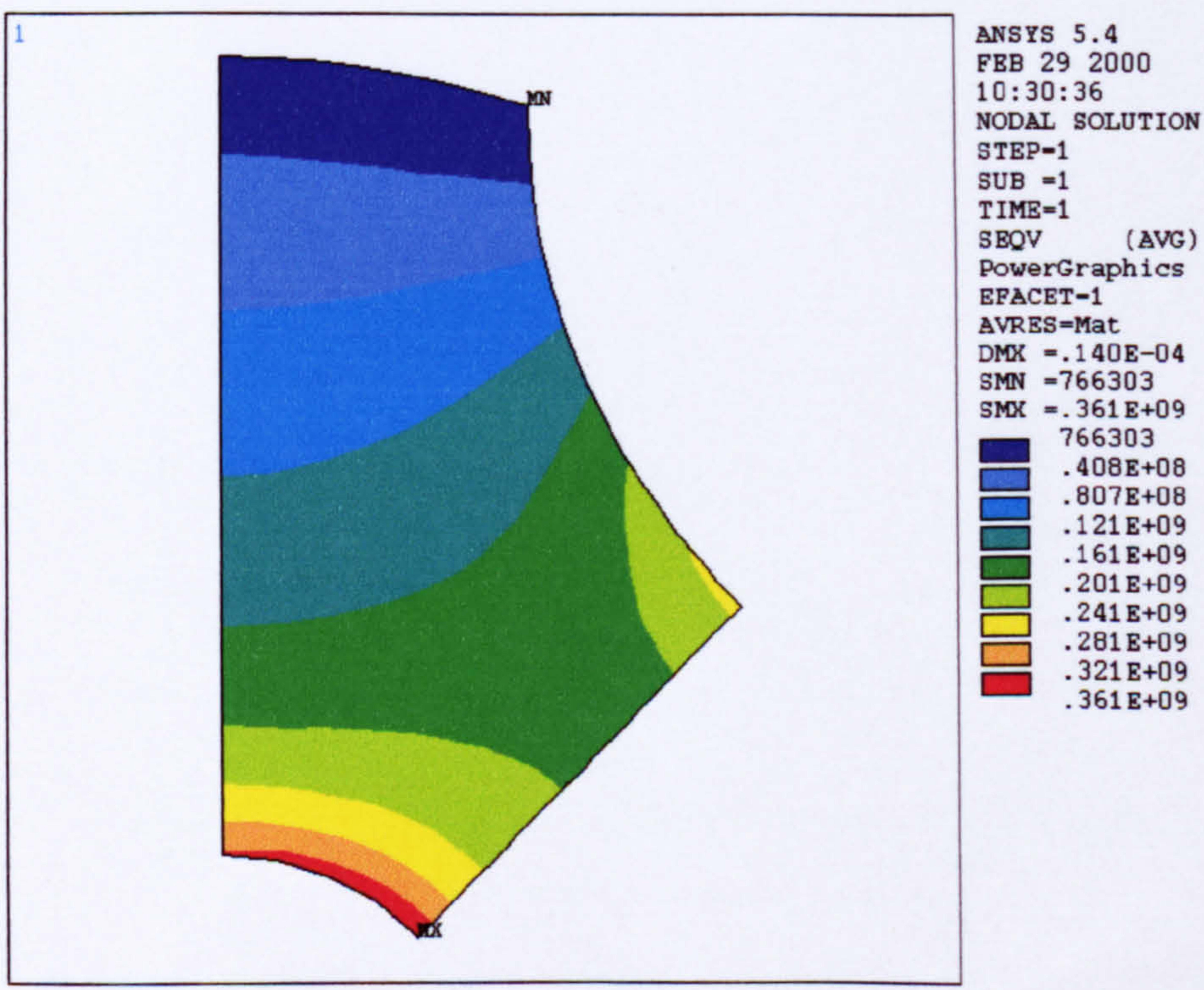

Figure 2.14. Von-Mises stress distribution in rotor 1 obtained using the mesh shown in figure 2.12 at $10000 \mathrm{rad} / \mathrm{s}$. 


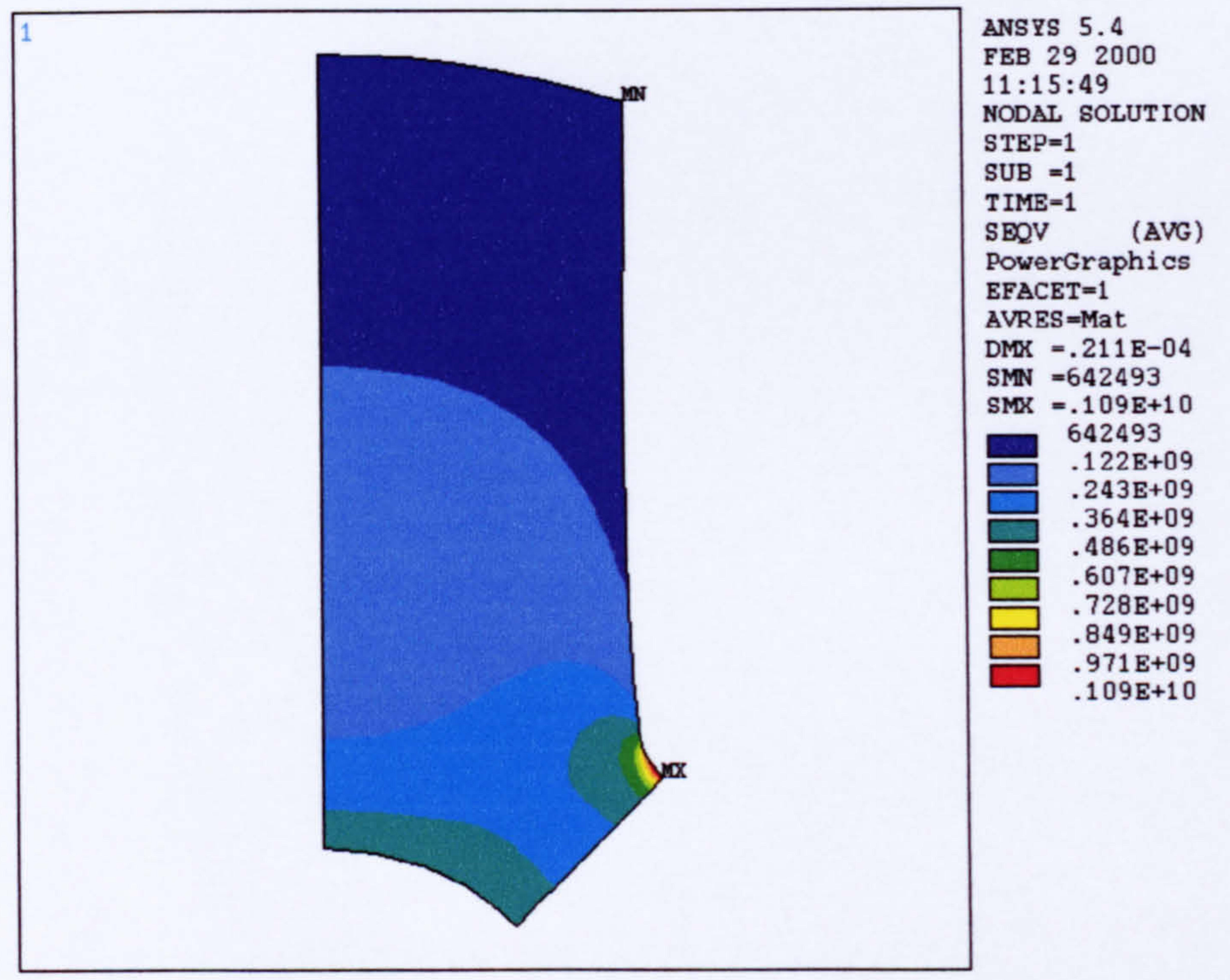

Figure 2.15. Von-Mises stress distribution in rotor 2 obtained using the mesh shown in figure 2.13 at $10000 \mathrm{rad} / \mathrm{s}$.

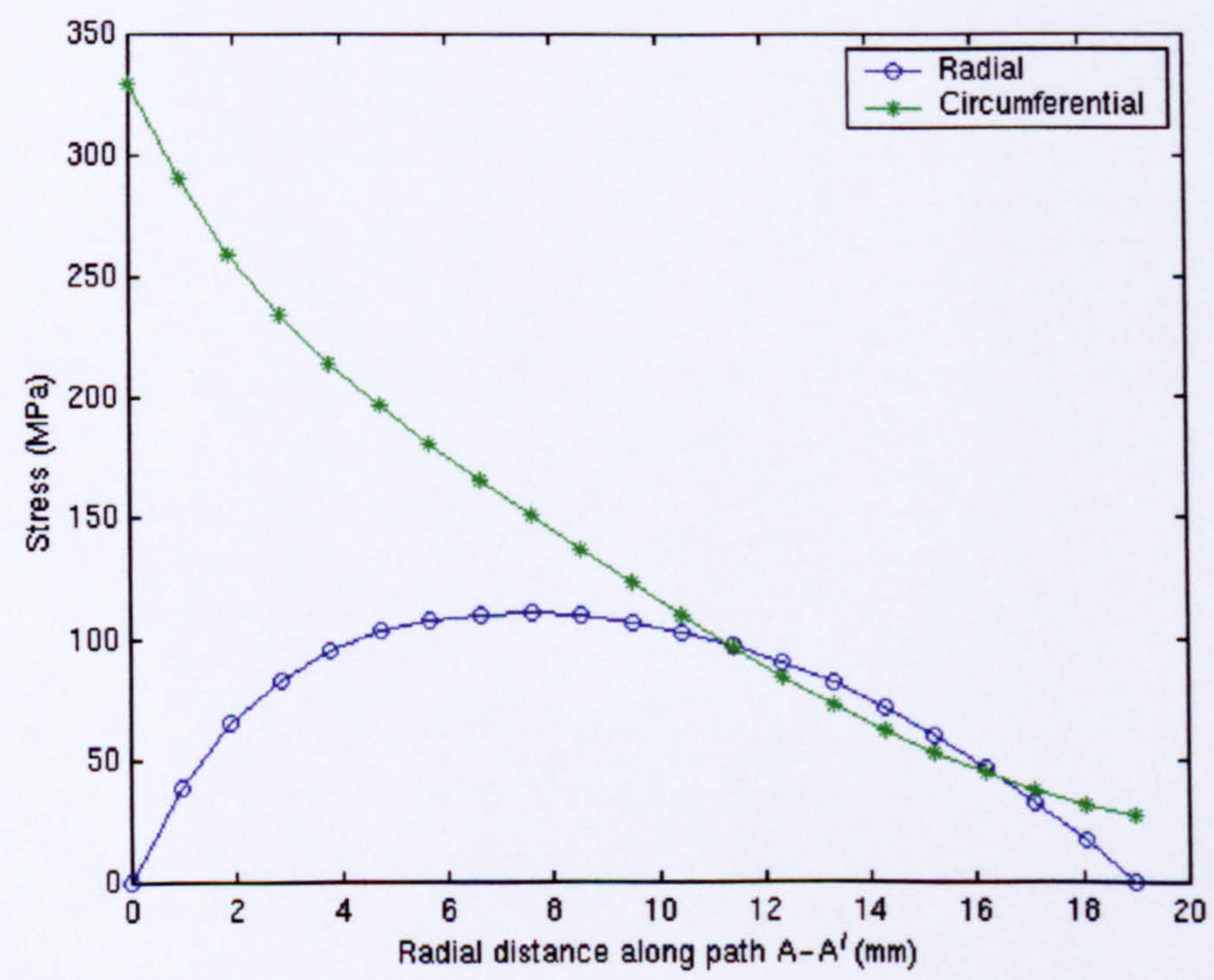

Figure 2.16a. Radial and circumferential stress along the profile A-A' in rotor 1 


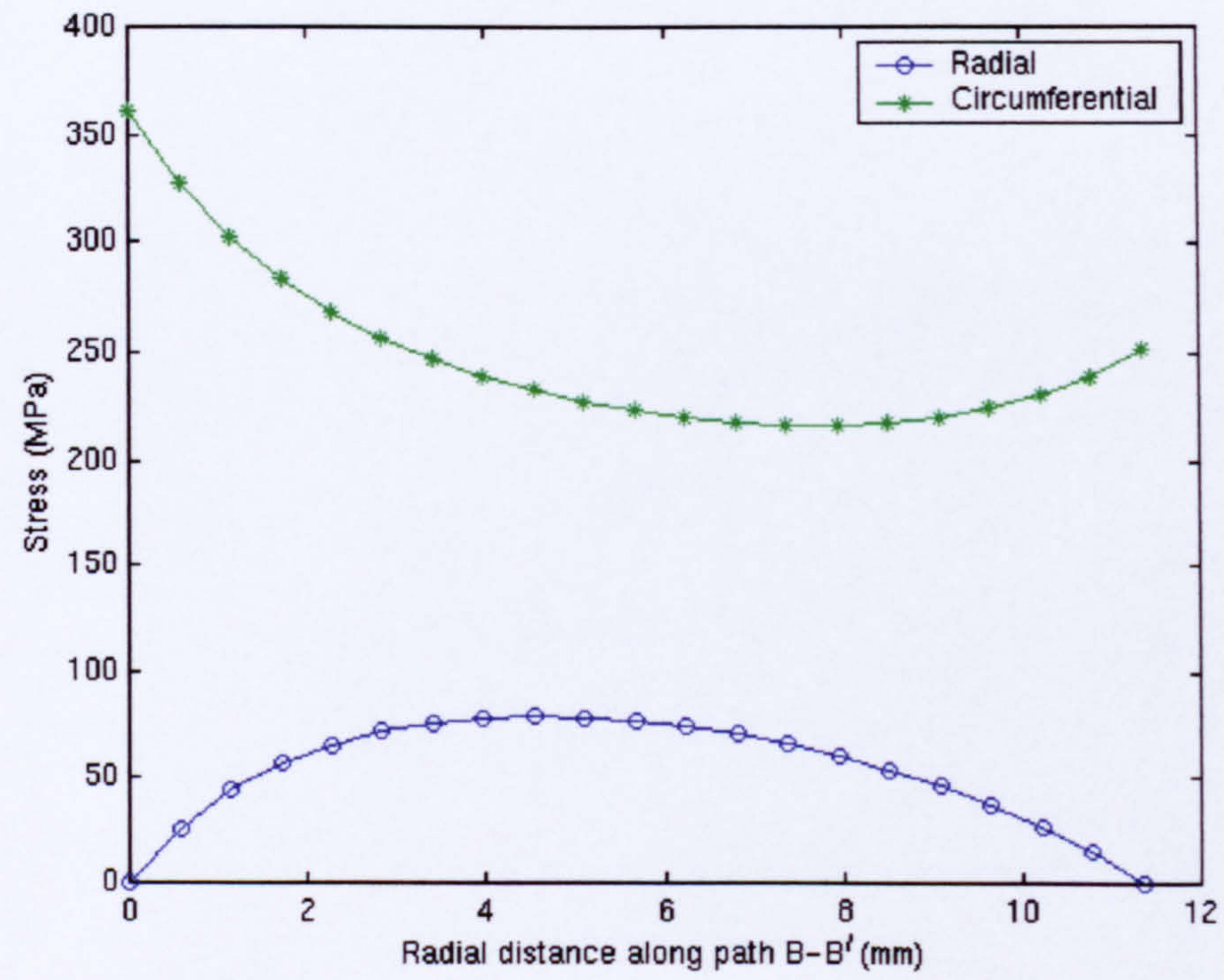

Figure 2.16b. Radial and circumferential stress along the profile B-B' in rotor 1

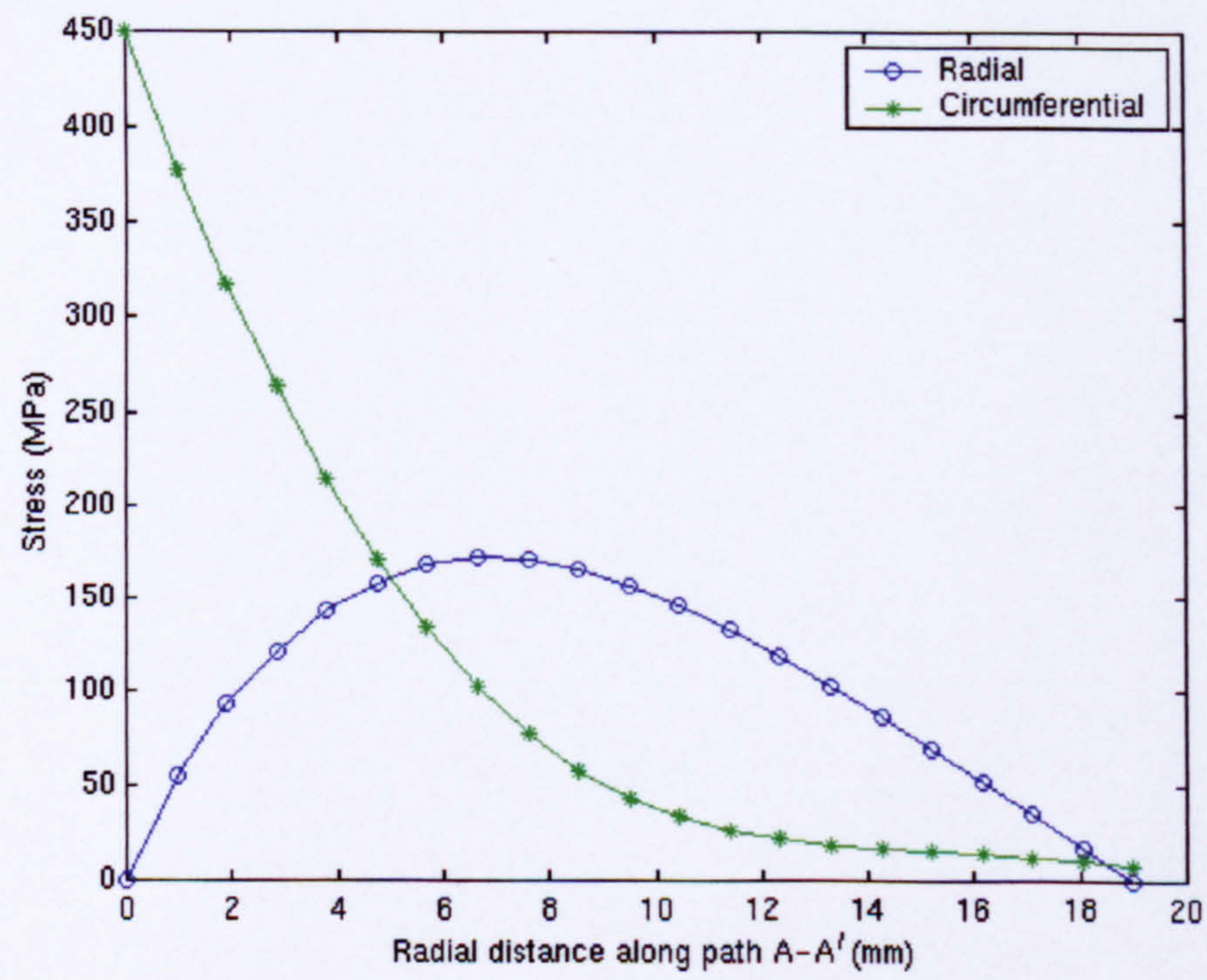

Figure 2.17a. Radial and circumferential stress along the profile A-A' in rotor 2 


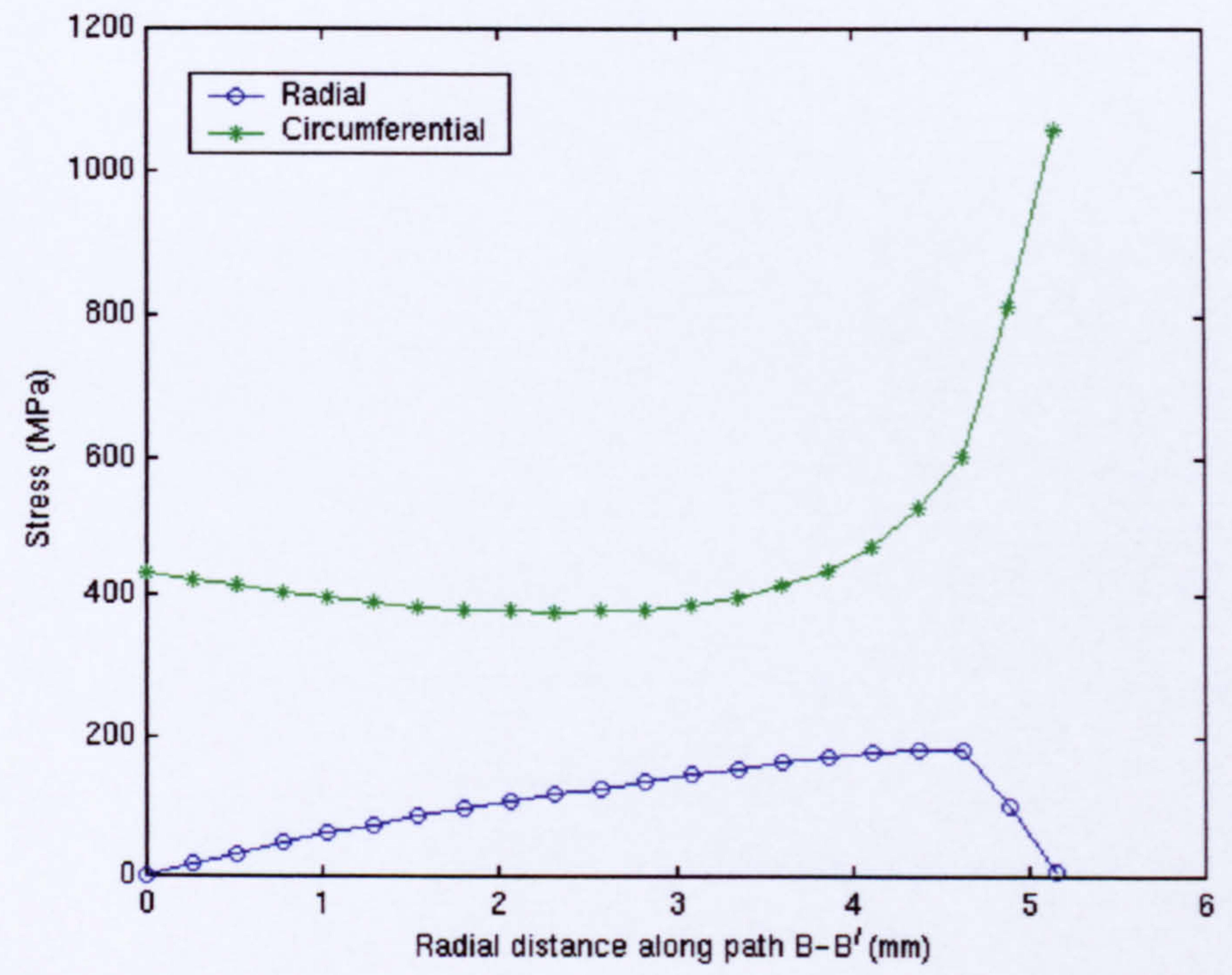

Figure 2.17b. Radial and circumferential stress along the profile B-B' in rotor 2 


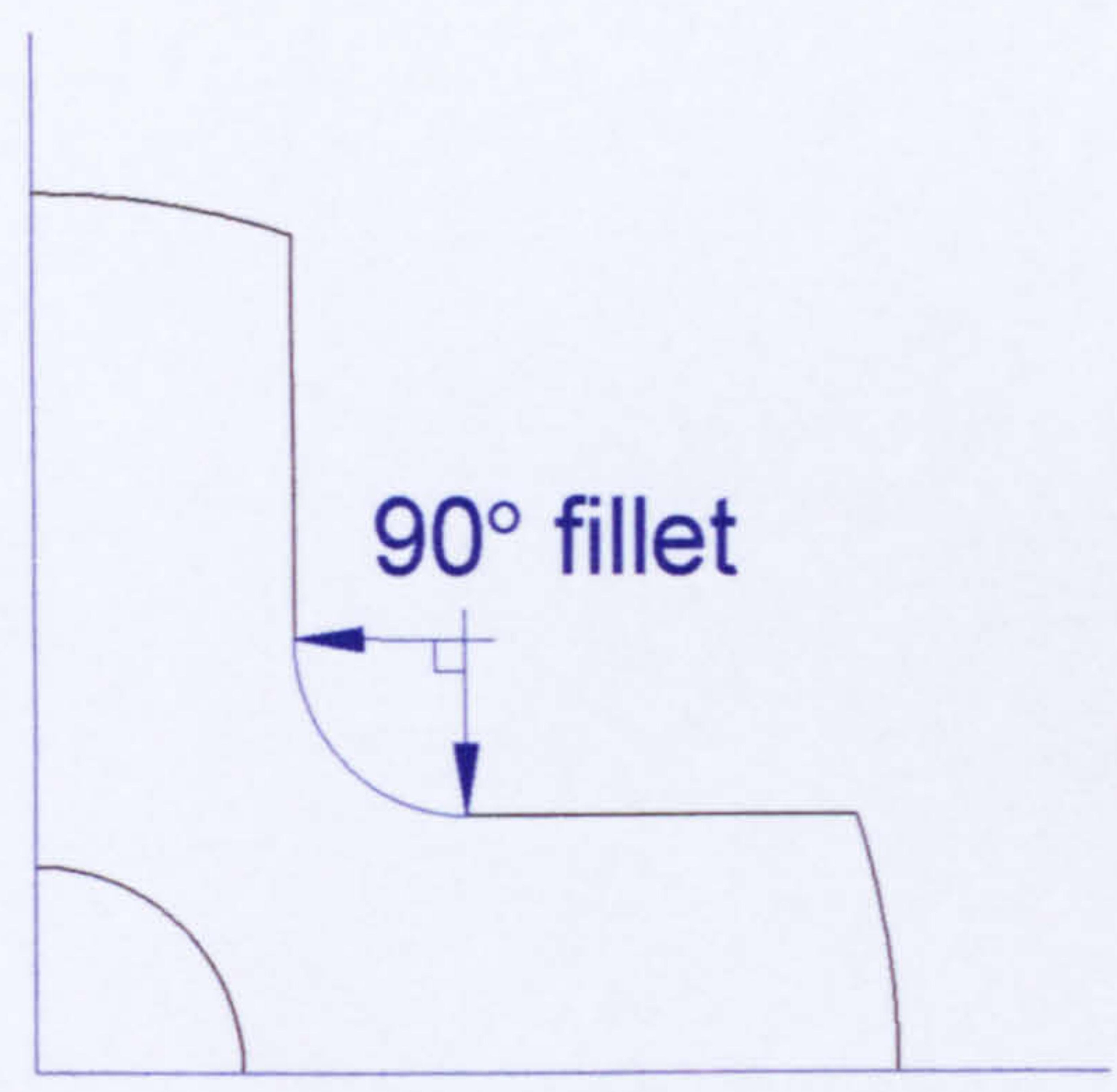

Figure 2.18a Segment of SR rotor exhibiting a quarter circle fillet.

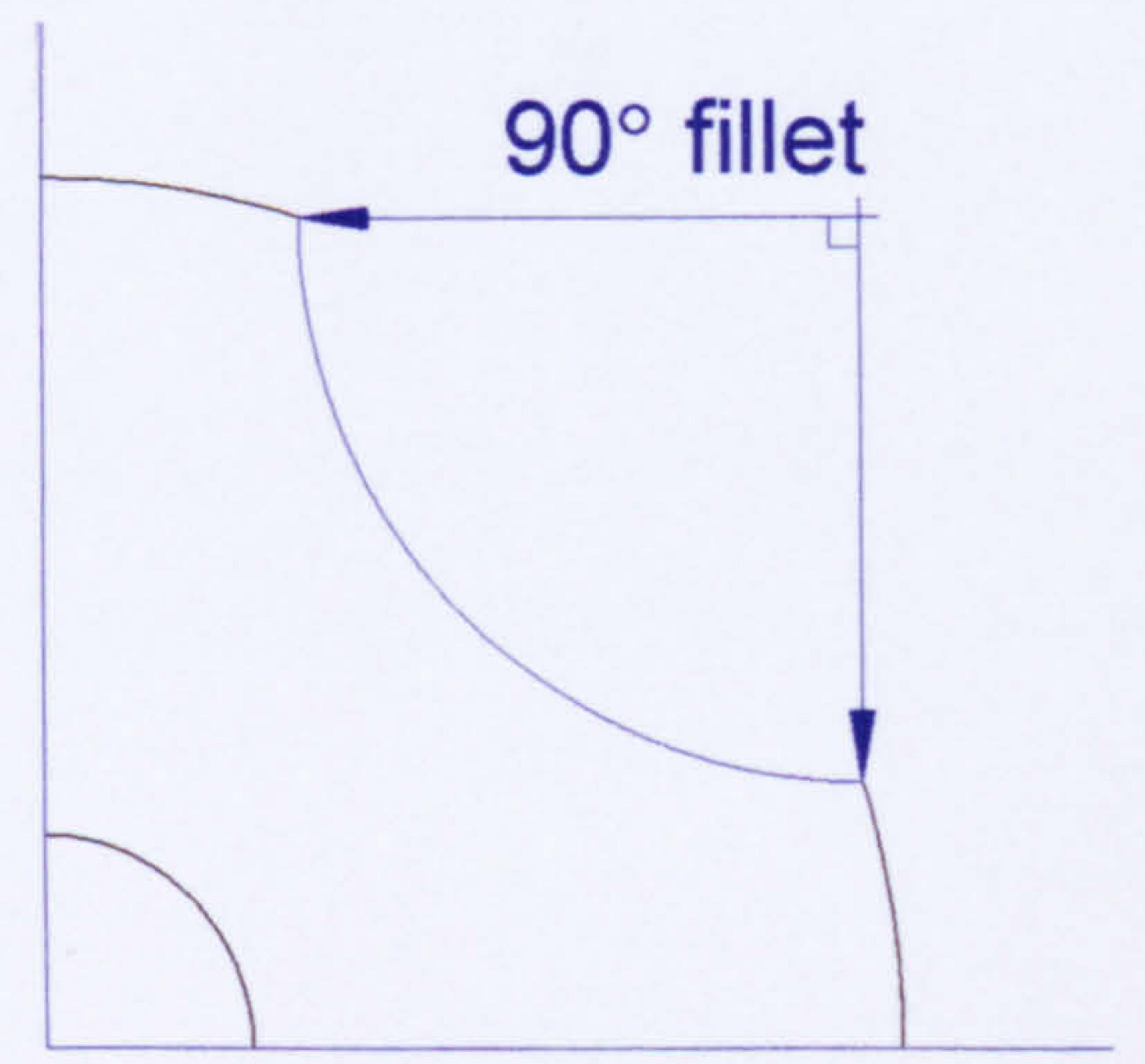

Figure 2.18b Segment of SR rotor exhibiting a quarter circle fillet of the maximum value.

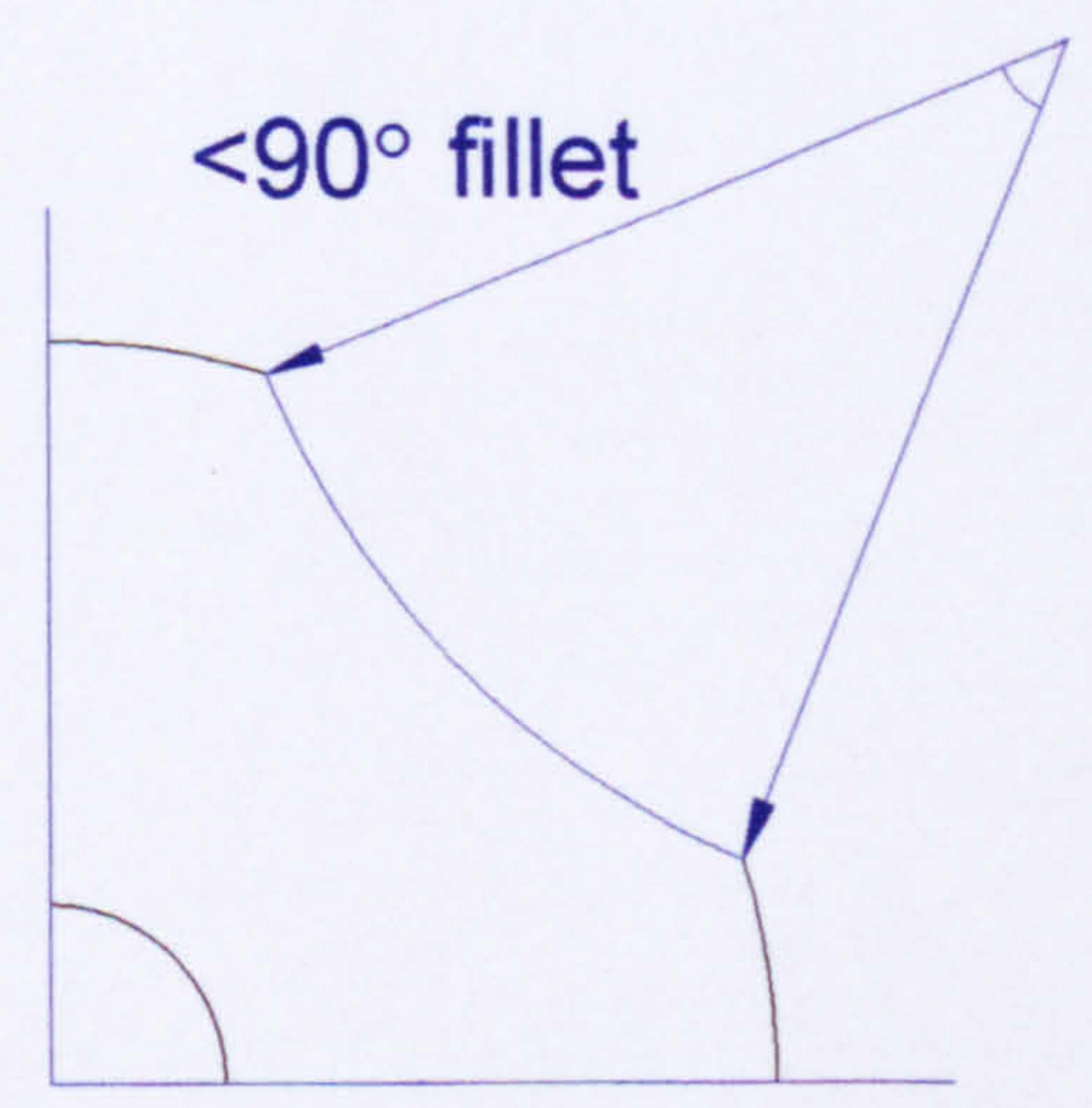

Figure 2.18c Segment of SR rotor exhibiting a fillet subtending an angle less than $90 .^{\circ}$ 


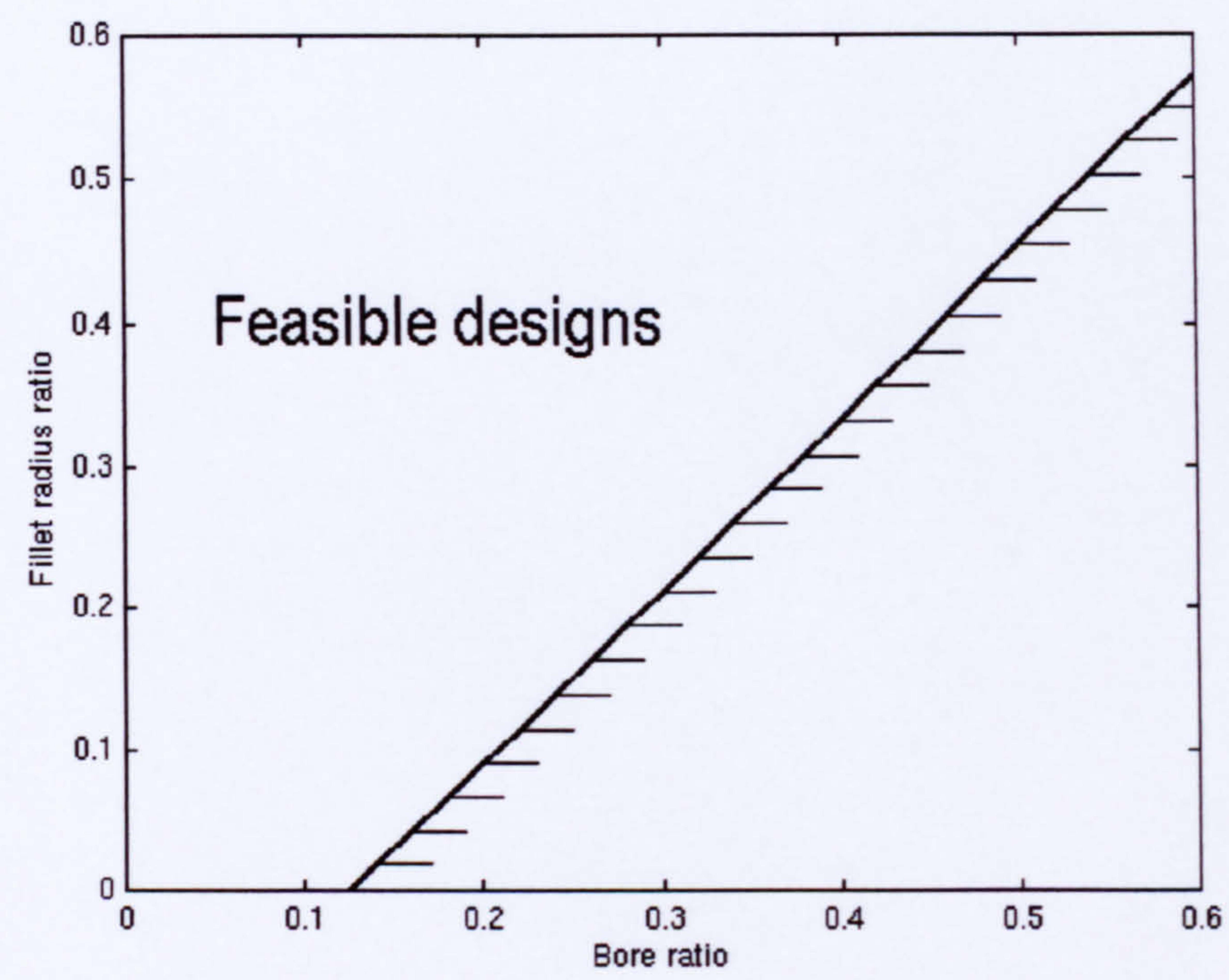

Figure 2.19. Locus of fillet radius ratio as a function of bore diameter ratio where localised saturation between the shaft bore and the minimum point of the fillet is avoided.

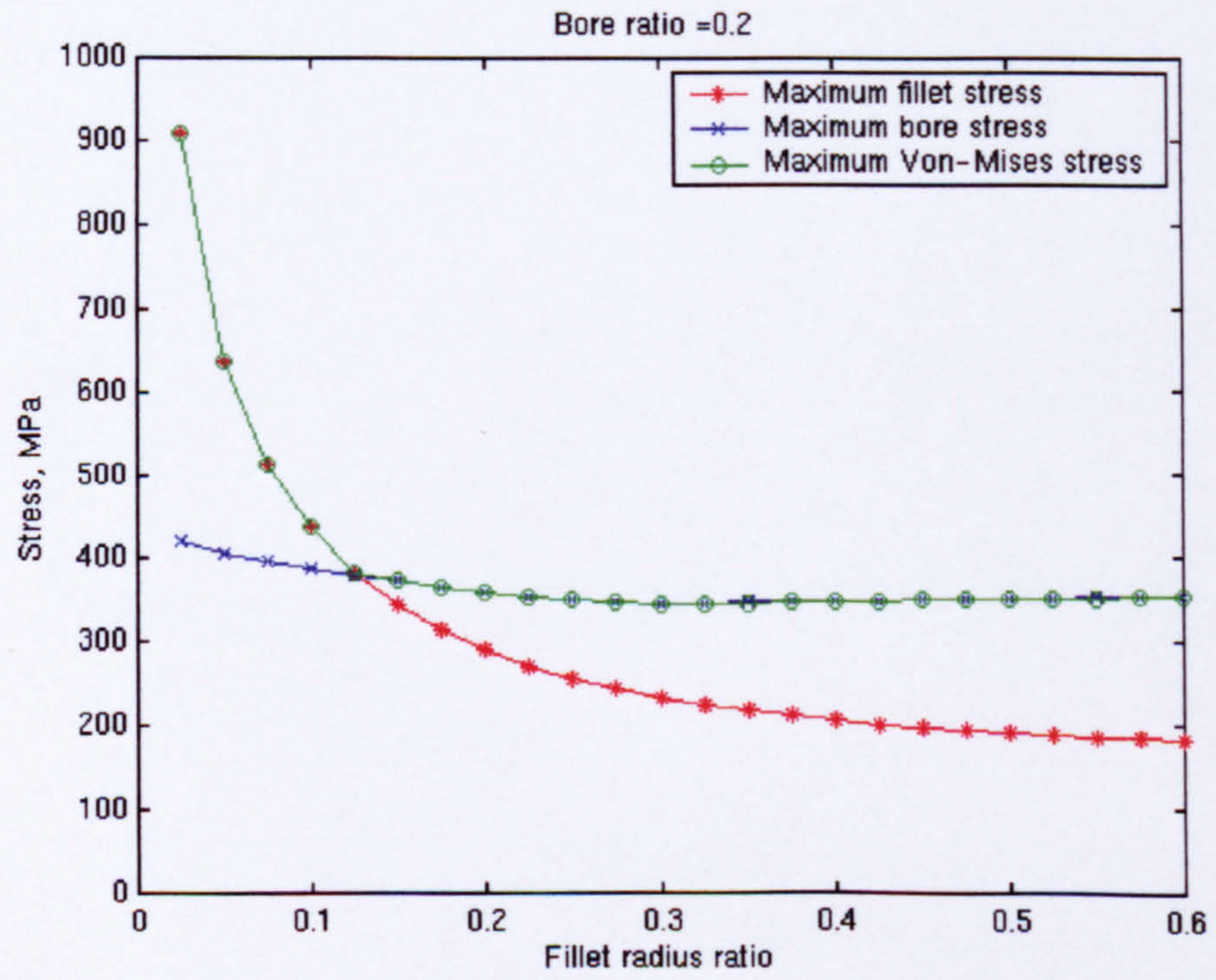

Figure 2.20 Maximum stress in the fillet and the bore of the SR rotor with a bore ratio of 0.2 as the fillet radius ratio is increased. 


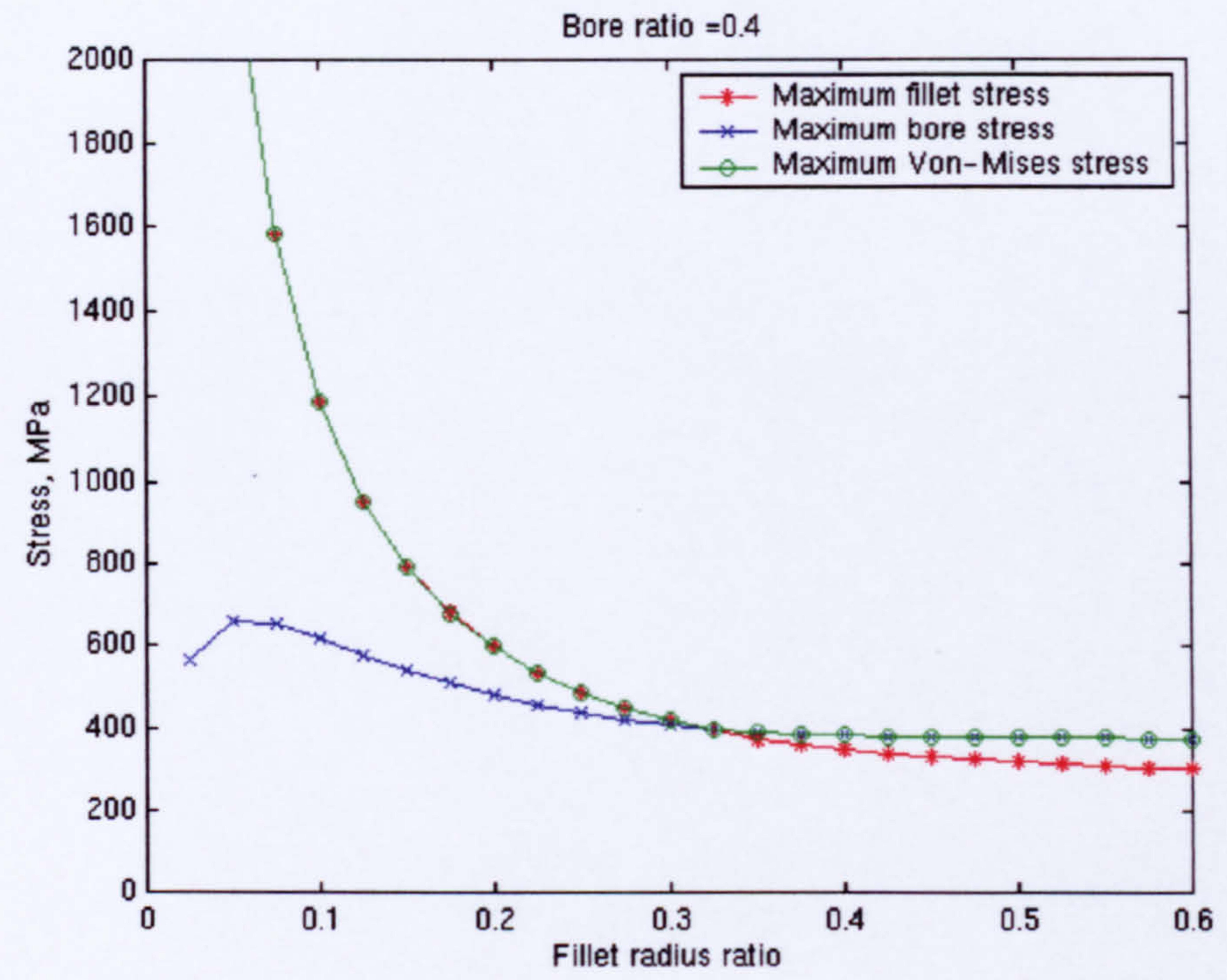

Figure 2.21 Maximum stress in the fillet and the bore of the SR rotor with a bore ratio of 0.4 as the fillet radius ratio is increased.

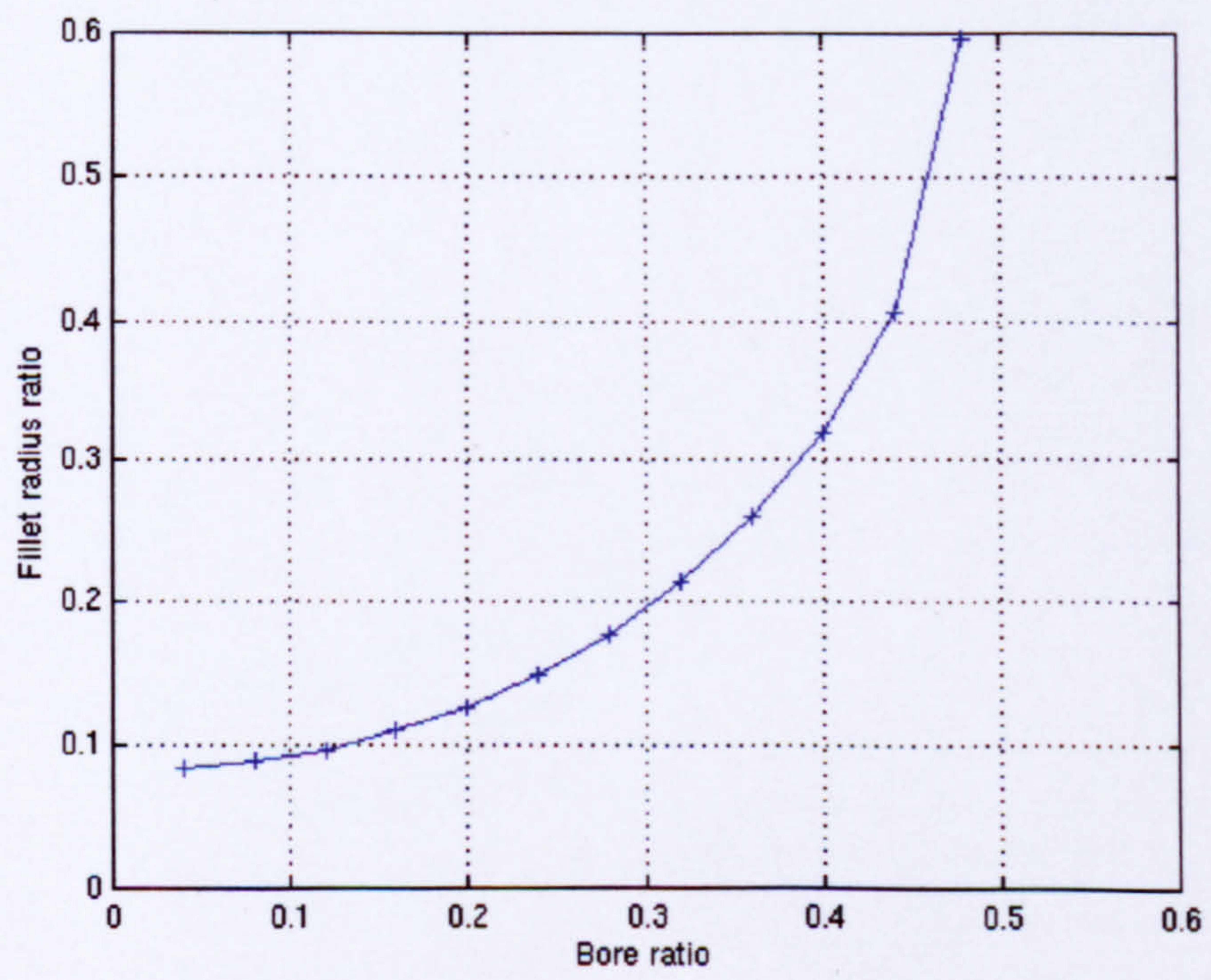

Figure 2.22 Locus of fillet radius ratio as a function of bore diameter ratio where the fillet stress is equal to the bore stress. 


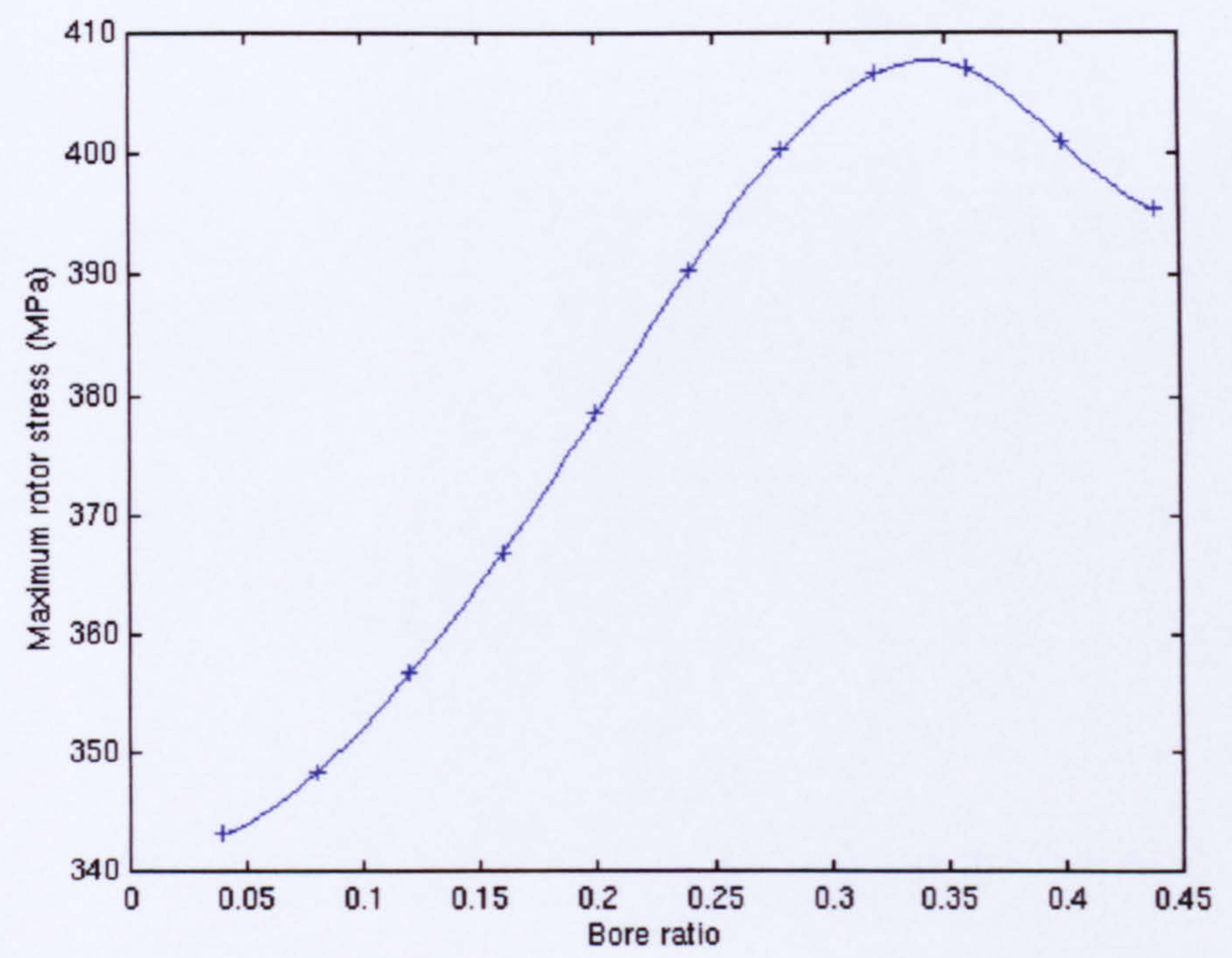

Figure 2.23. Maximum rotor stress as the bore diameter ratio is increased

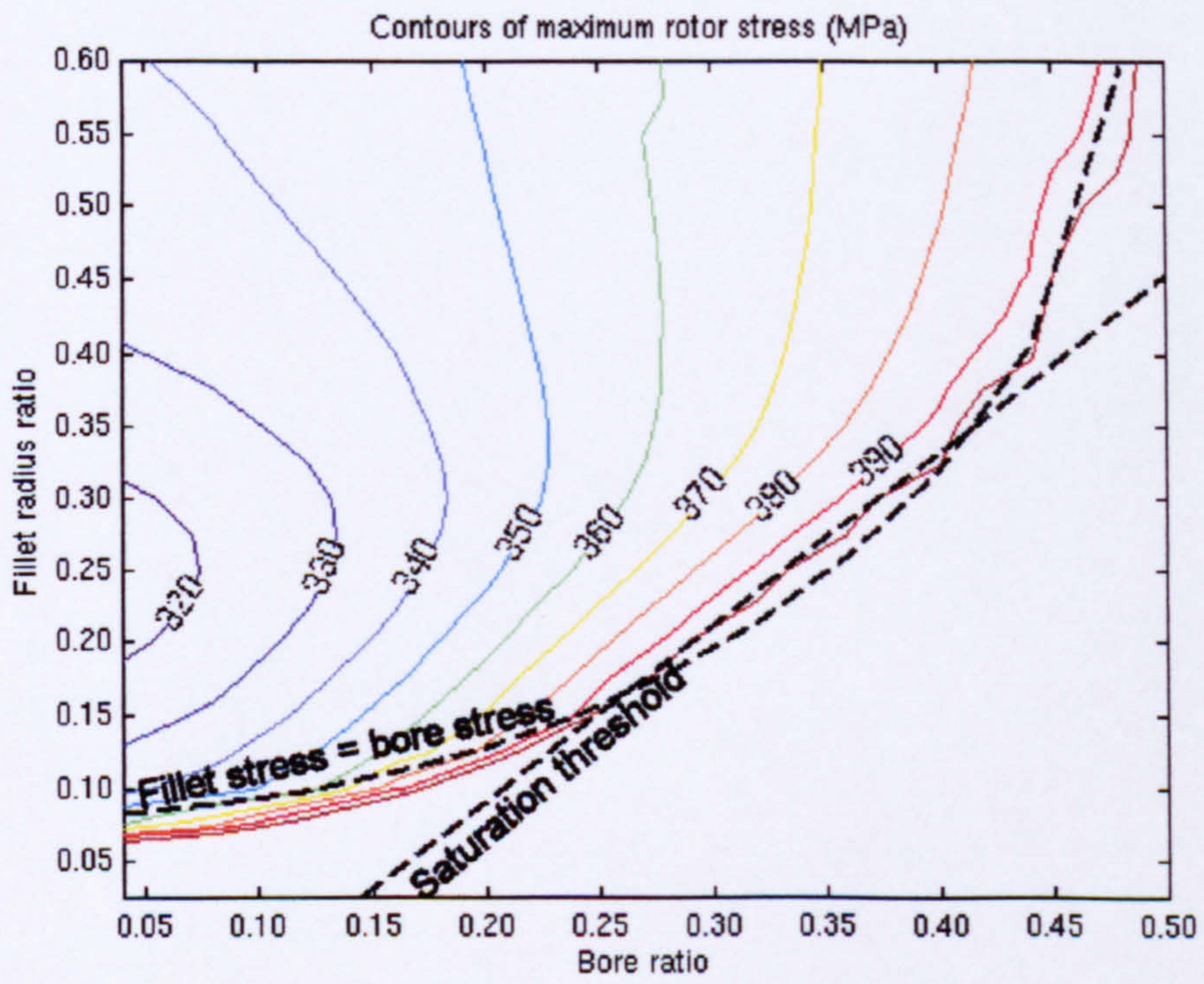

Figure 2.24. Contours of maximum stress in the $50 \mathrm{~mm}$ diameter rotor at a speed of $10000 \mathrm{rad} / \mathrm{s}$ as a function of bore diameter and fillet radius ratios. 


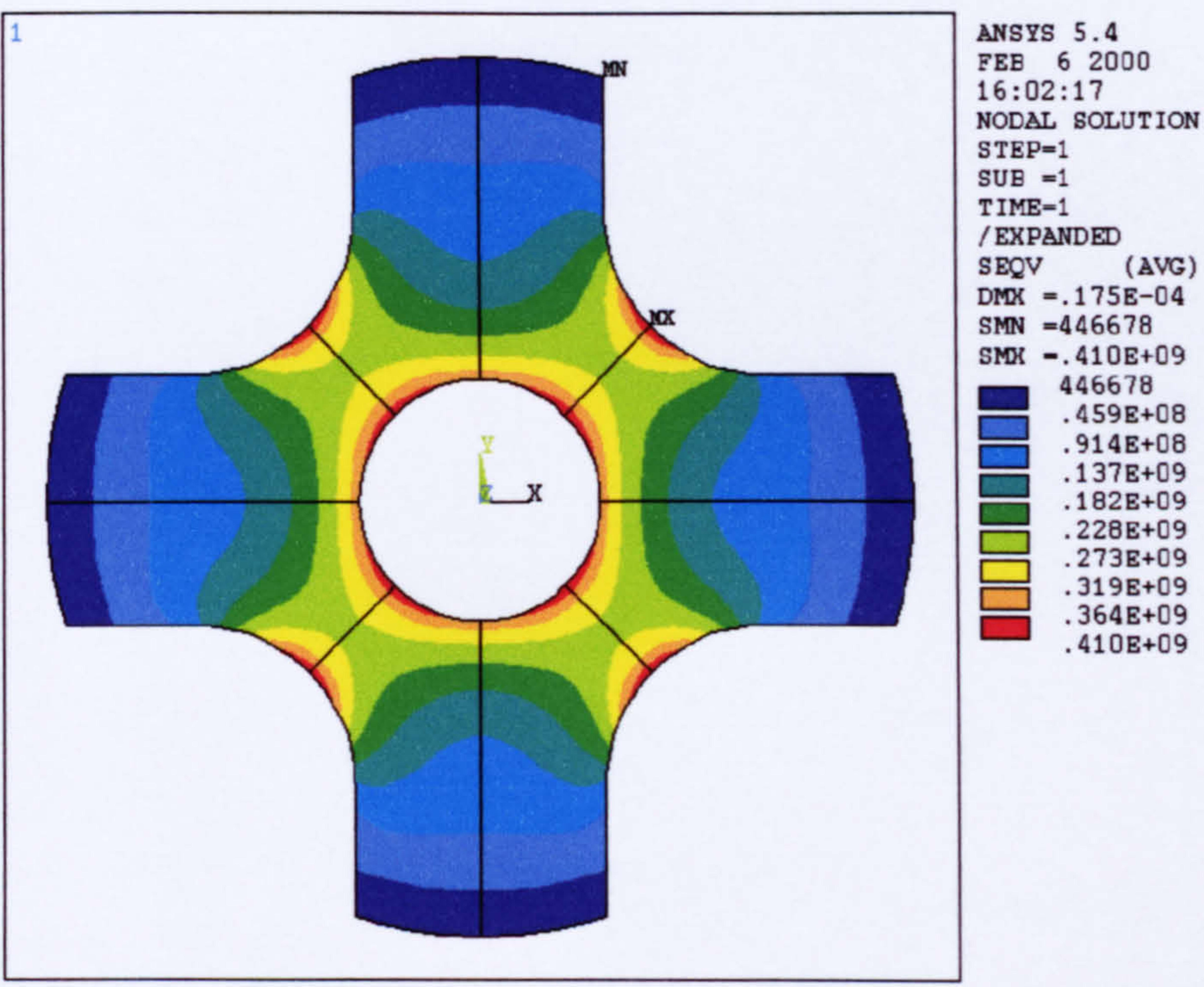

Figure 2.25. Von-Mises stress contours in the rotor design where the mechanical and electromagnetic utilisation of the material is a maximum.

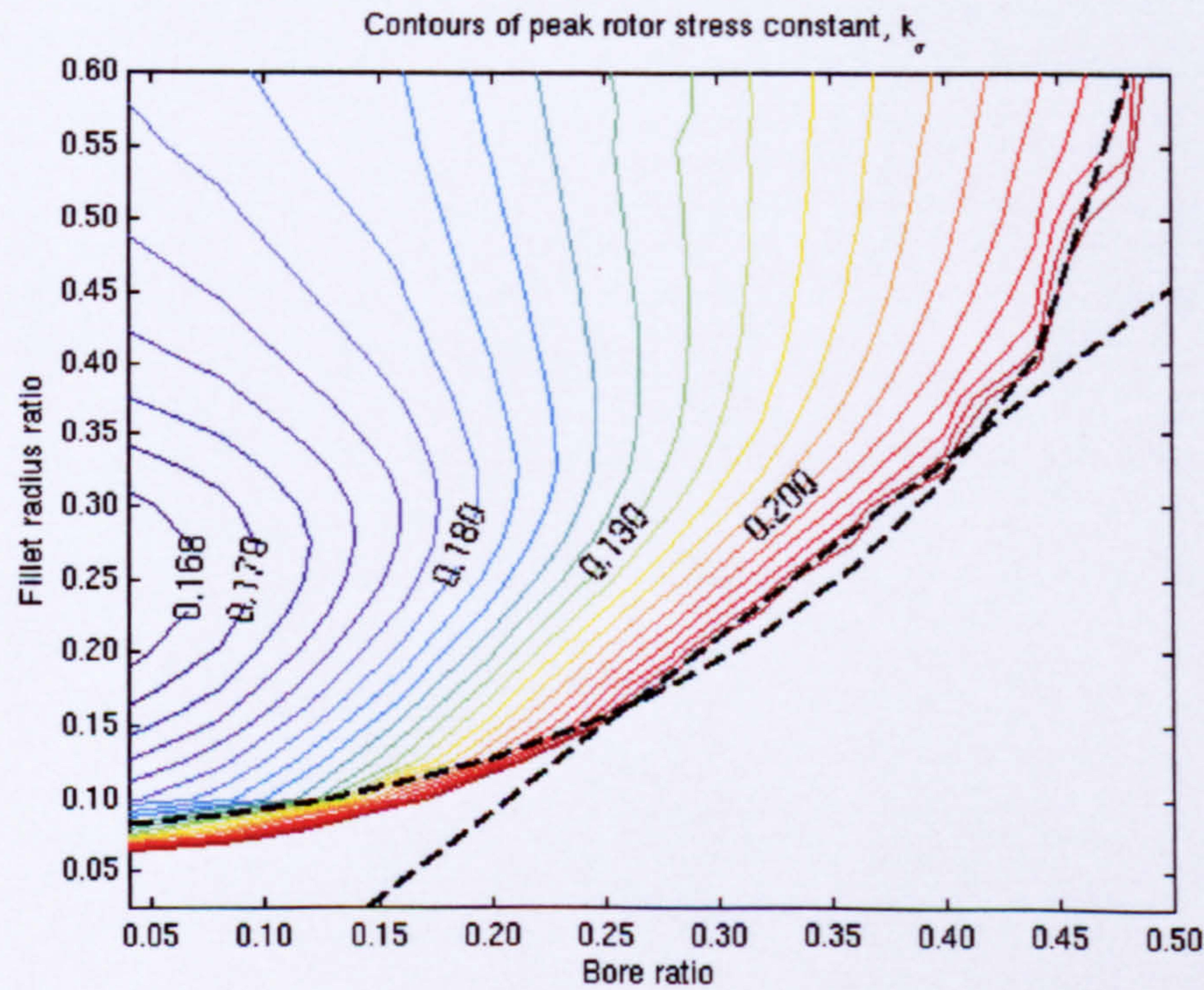

Figure 2.26 Contours of peak stress constant defined in equation 2.11. 


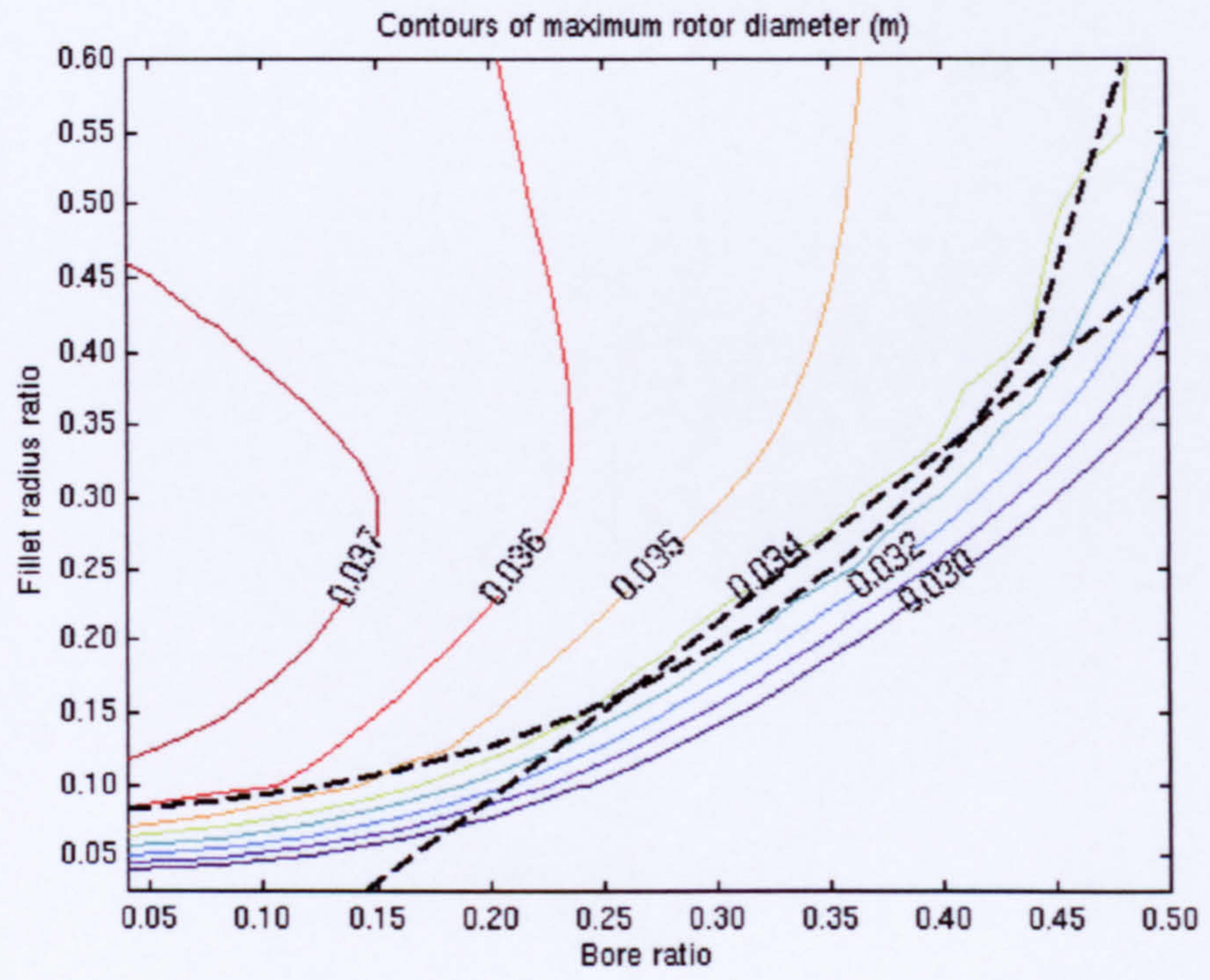

Figure 2.27 Contours of maximum rotor diameter over the feasible design range at $100000 \mathrm{rpm}$.

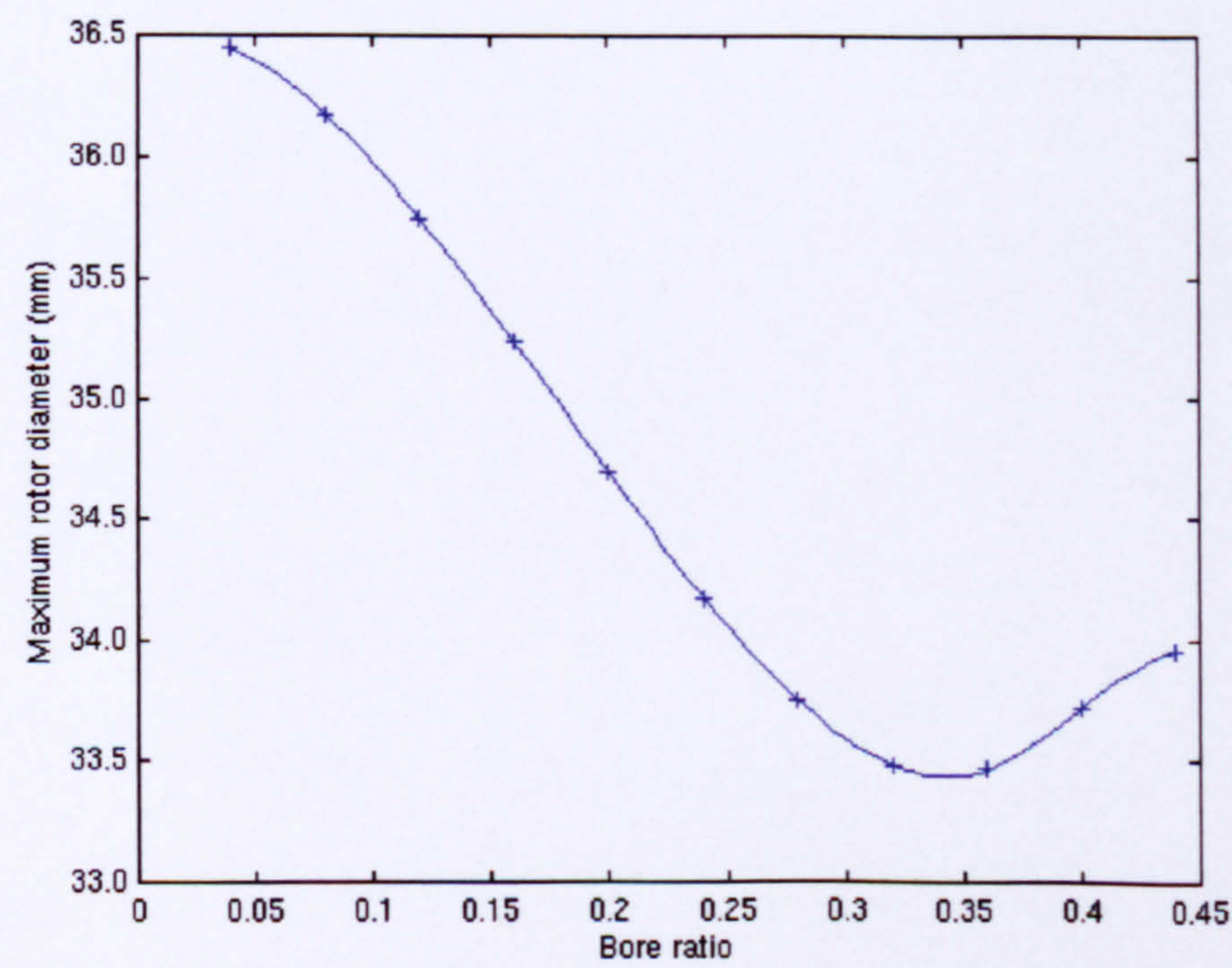

Figure 2.28. Maximum rotor diameter at the design speed of $100000 \mathrm{rpm}$ where the fillet stress is equal to the rotor stress. 


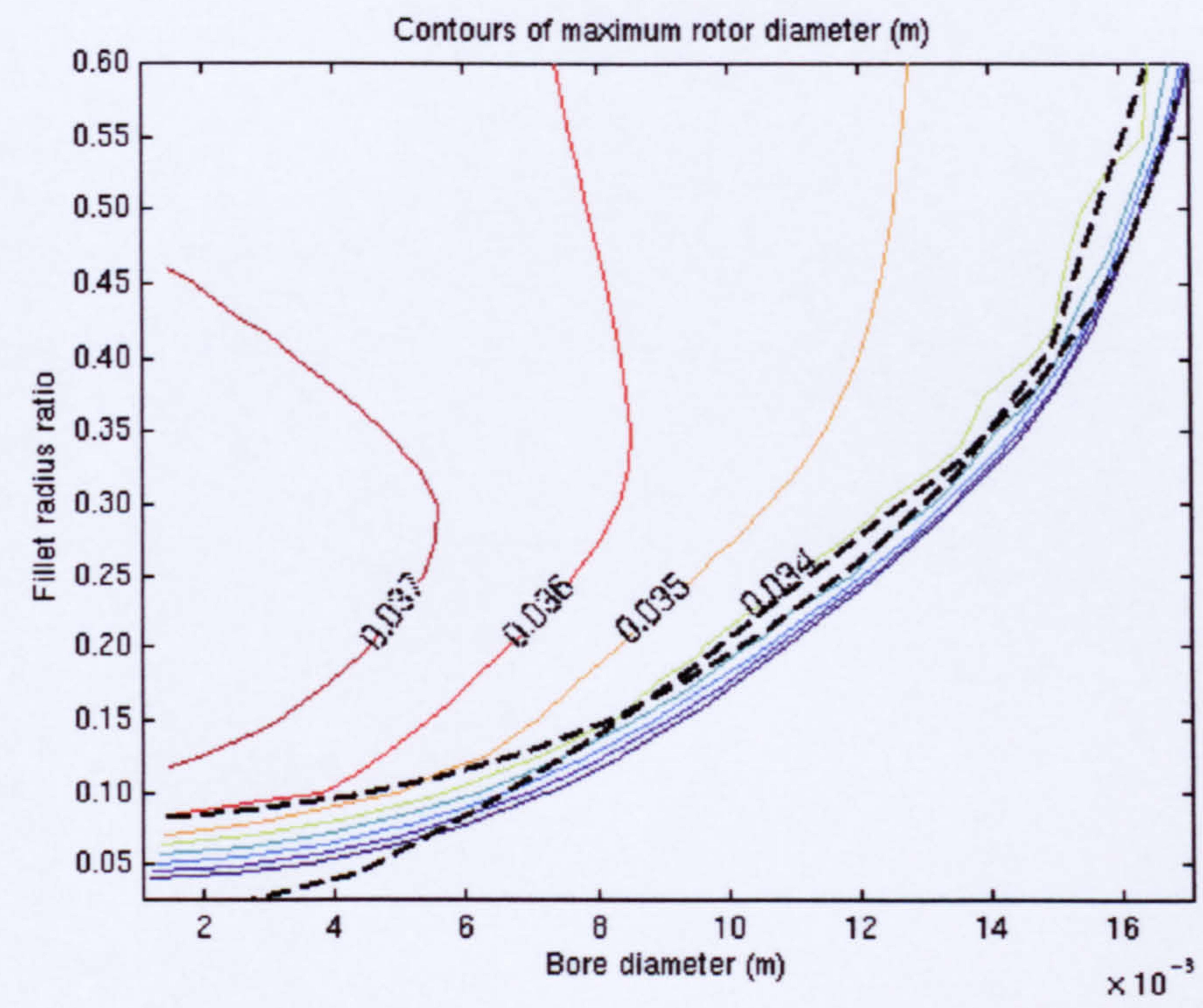

Figure 2.29 Contours of maximum rotor diameter as a function of the bore diameter at $100000 \mathrm{rpm}$

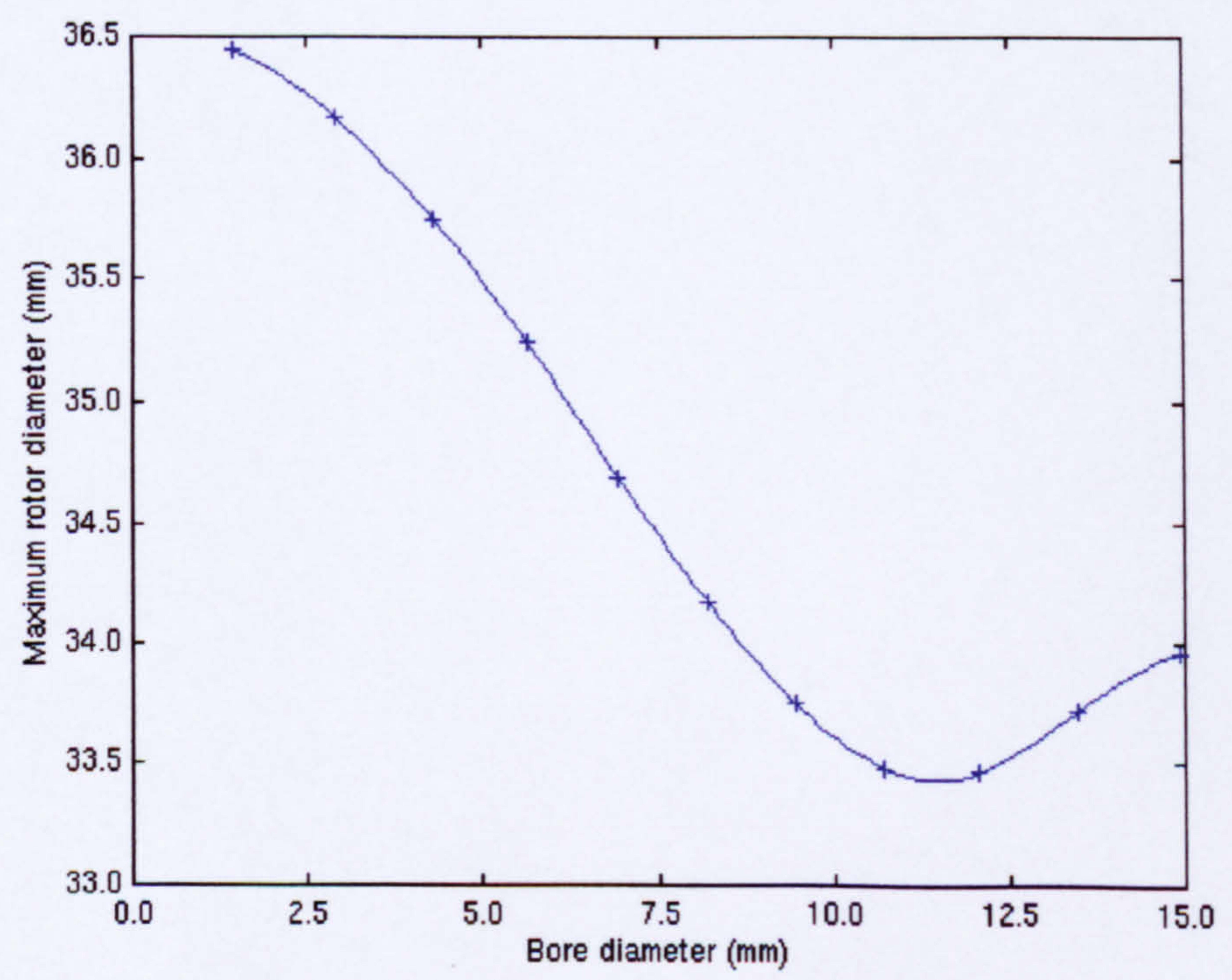

Figure 2.30. Maximum rotor diameter as a function of bore diameter at $100000 \mathrm{rpm}$ where the fillet stress is equal to the rotor stress. 


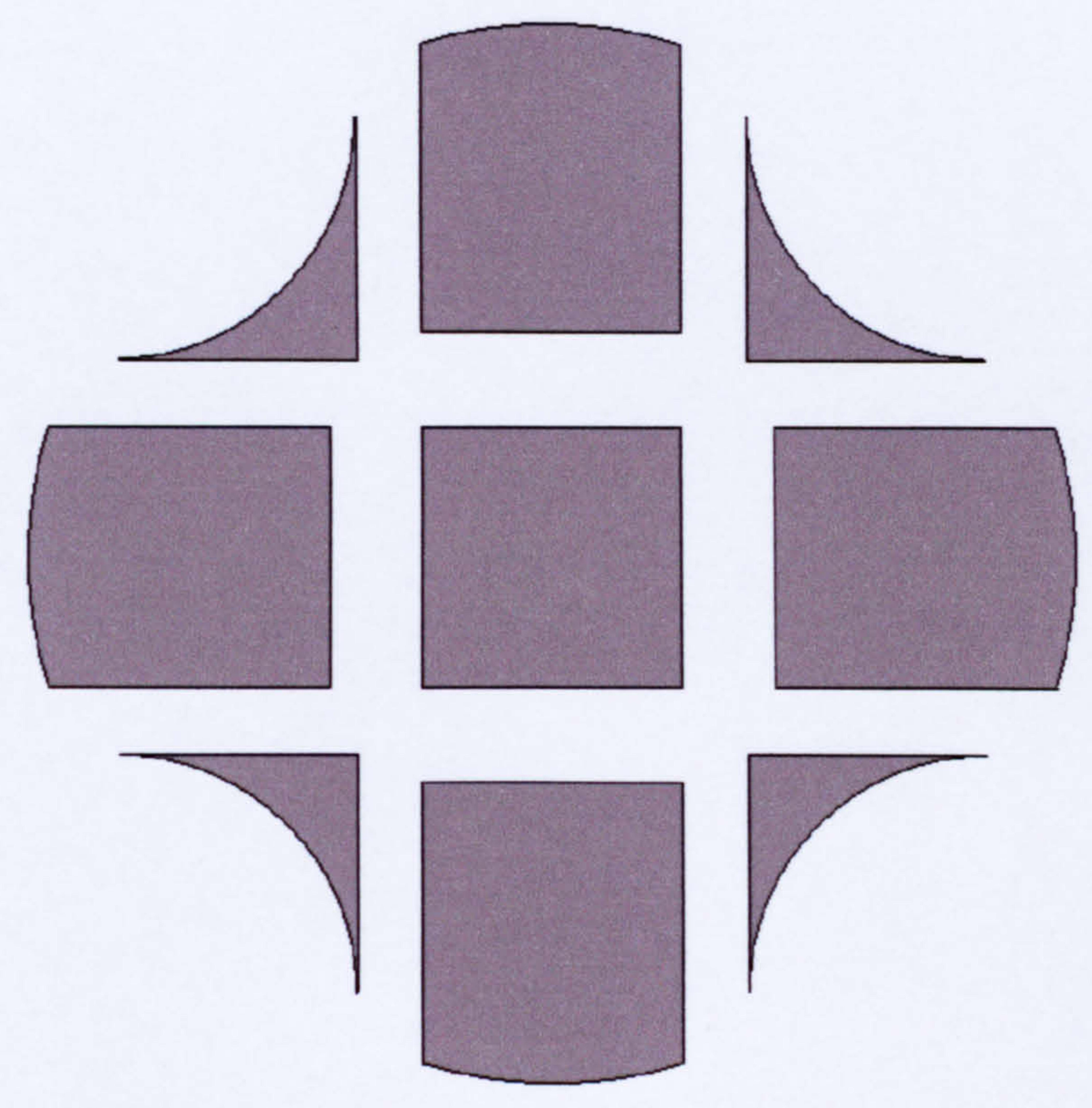

Figure 2.31 Segregation of rotor into simple geometric structures to determine rotor inertia.

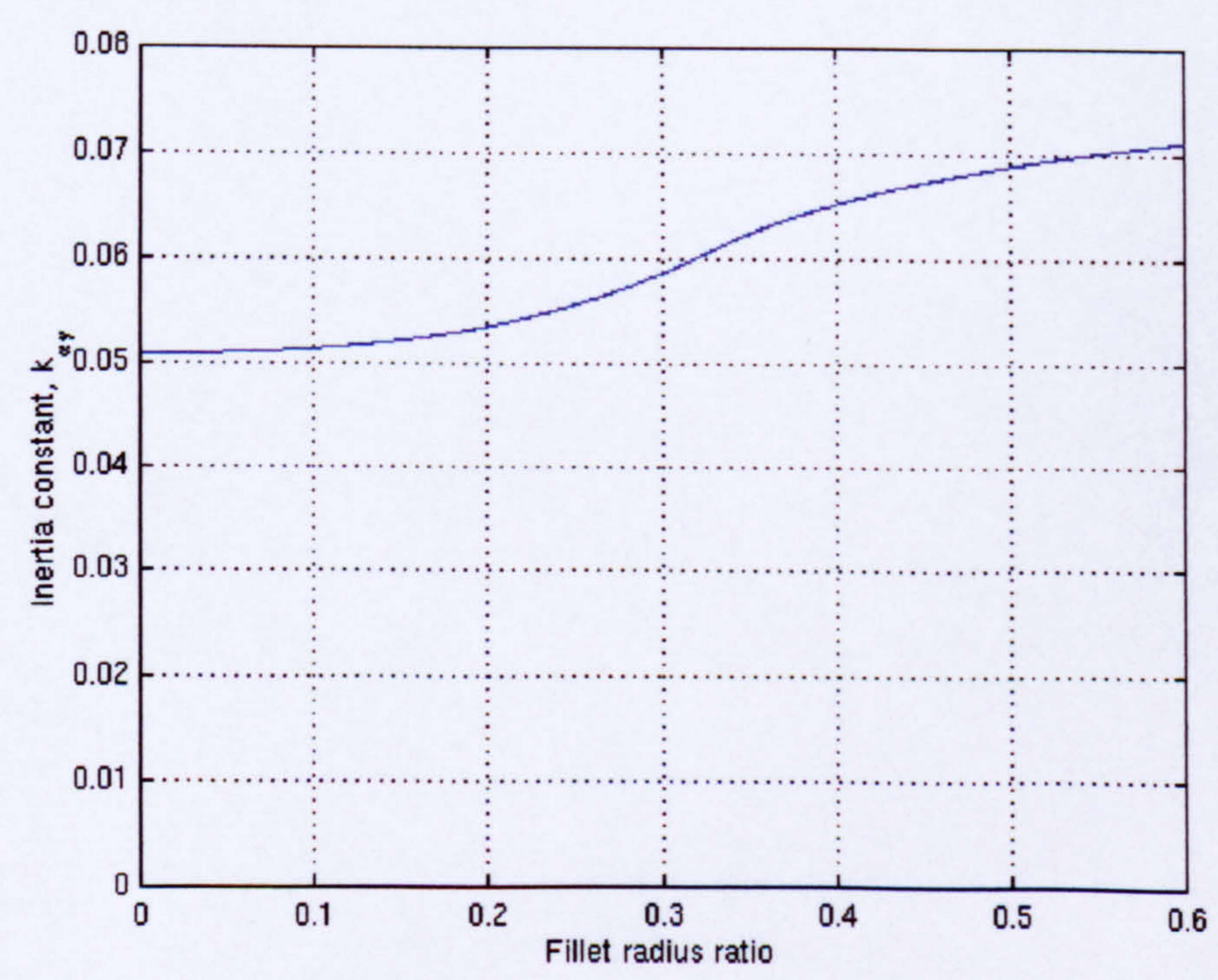

Figure 2.32 Rotor inertia constant as a function of the fillet radius ratio as defined in equation 2.14 . 


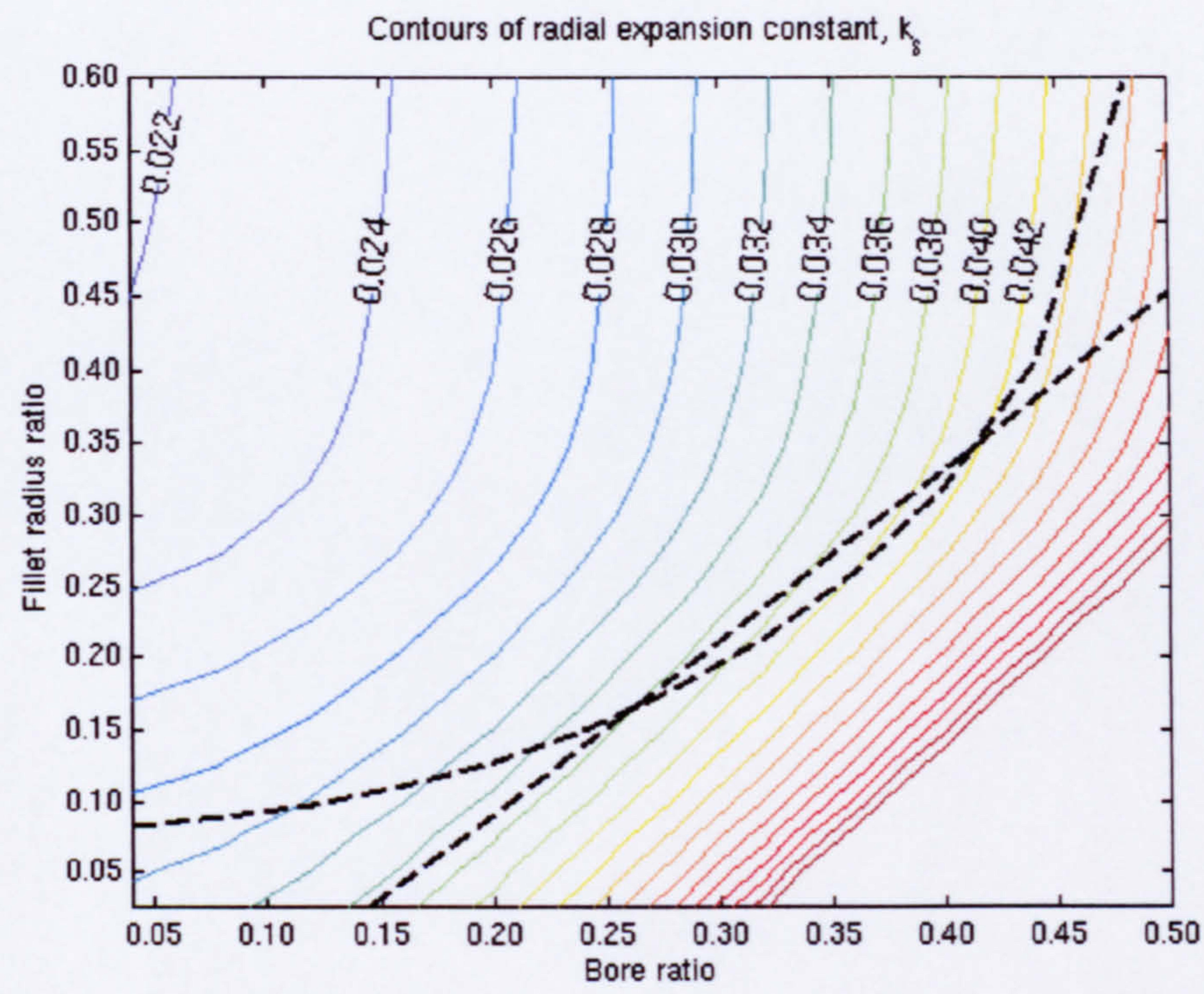

Figure 2.33. Contours of rotor radial growth constant defined in equation 2.15 .

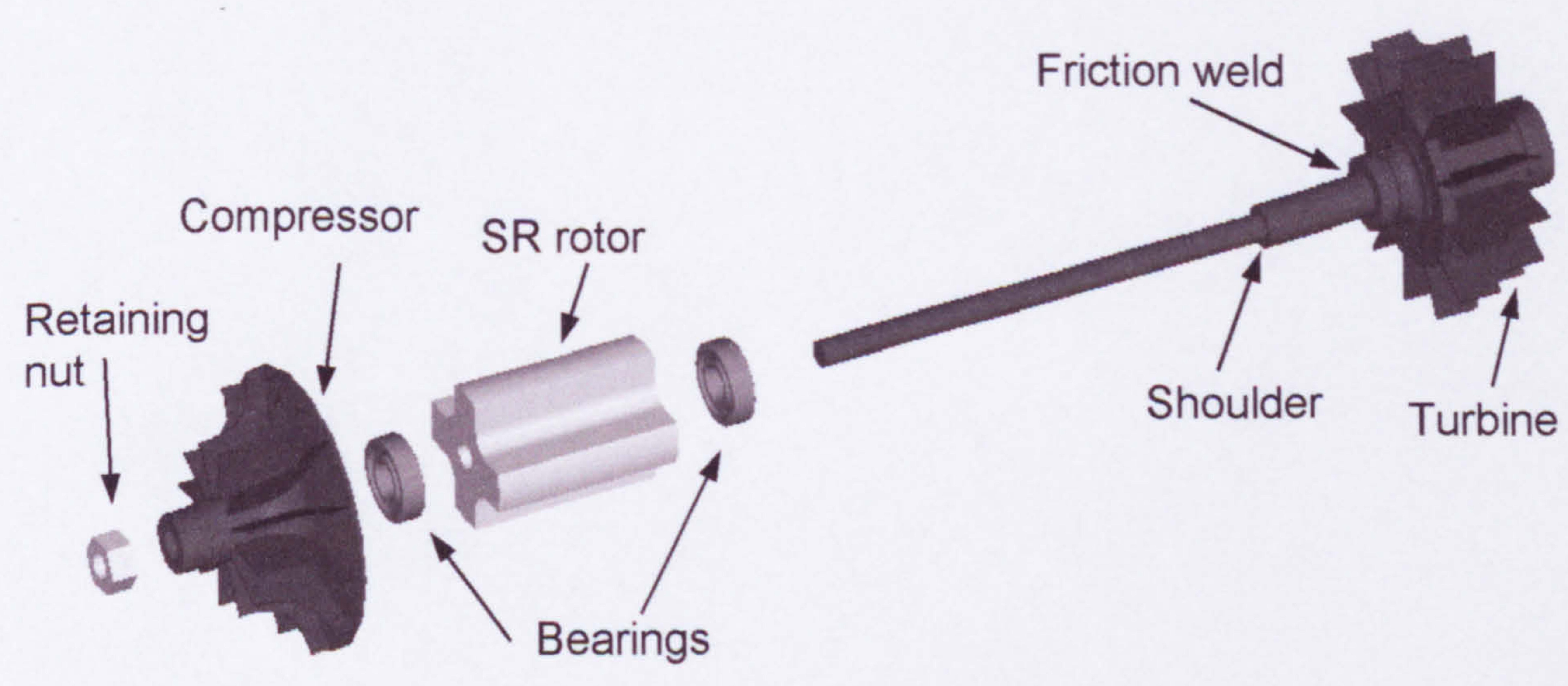

Figure 2.34. Rotor assembly from the compressor end of the shaft. 


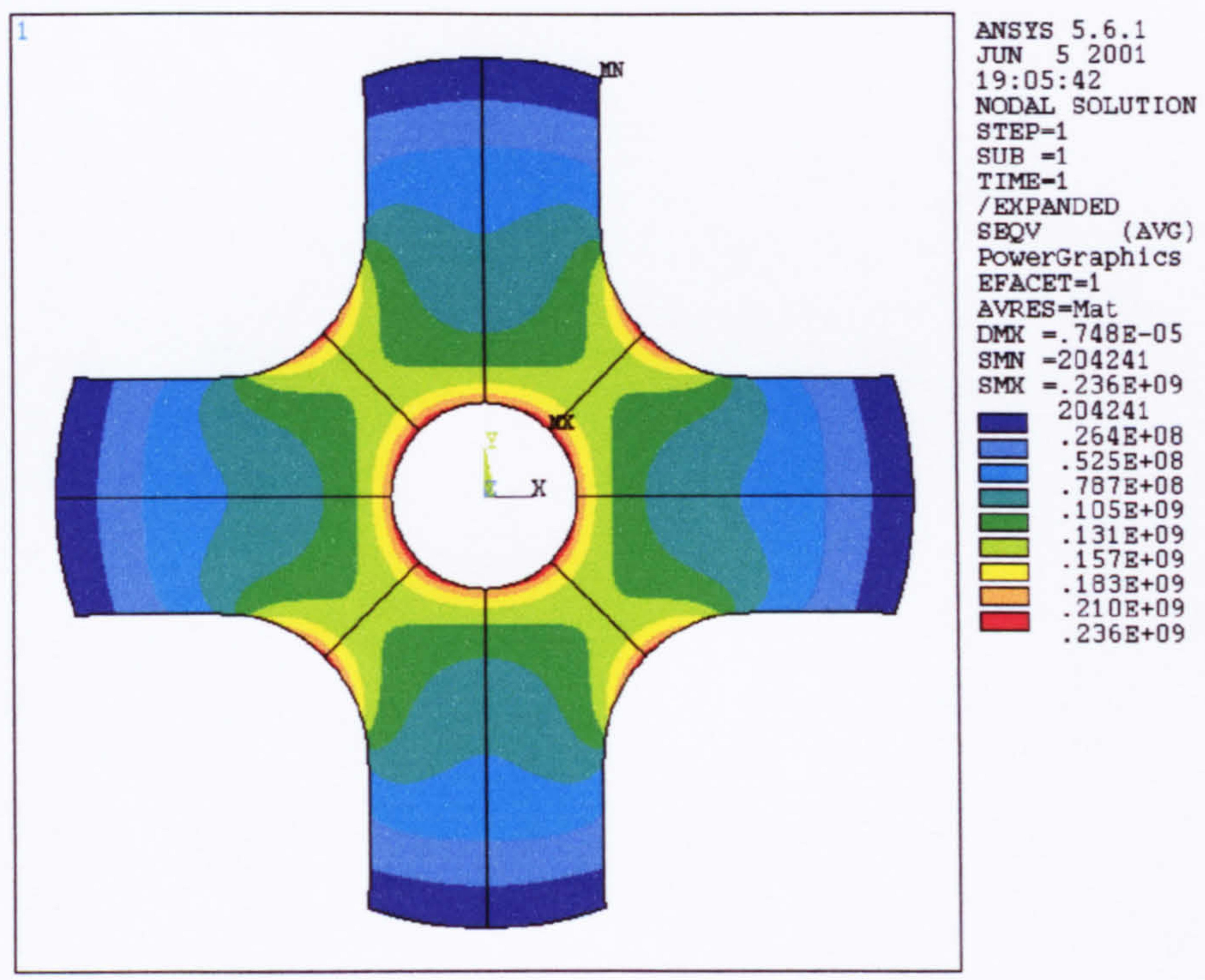

Figure 2.35 Stress contours for the rotor design with a $7 \mathrm{~mm}$ diameter shaft at $100000 \mathrm{rpm}$, where the fillet radius is selected such that the stress in the rotor bore and fillet are equal, i.e. $\beta=0.184$ and $\gamma=0.13$.

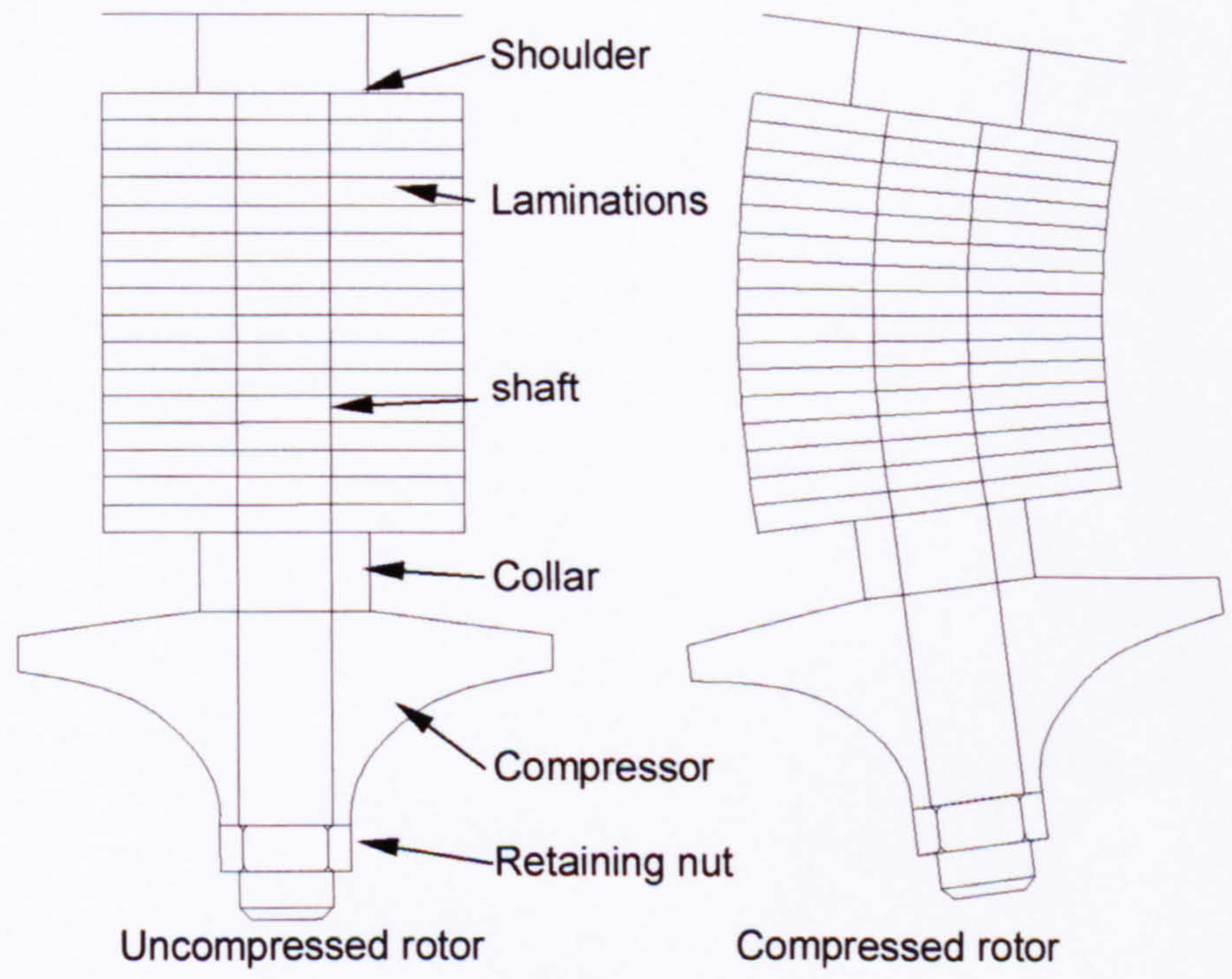

Figure 2.36. Uneven axial compression of rotor laminations causing shaft to bend. 


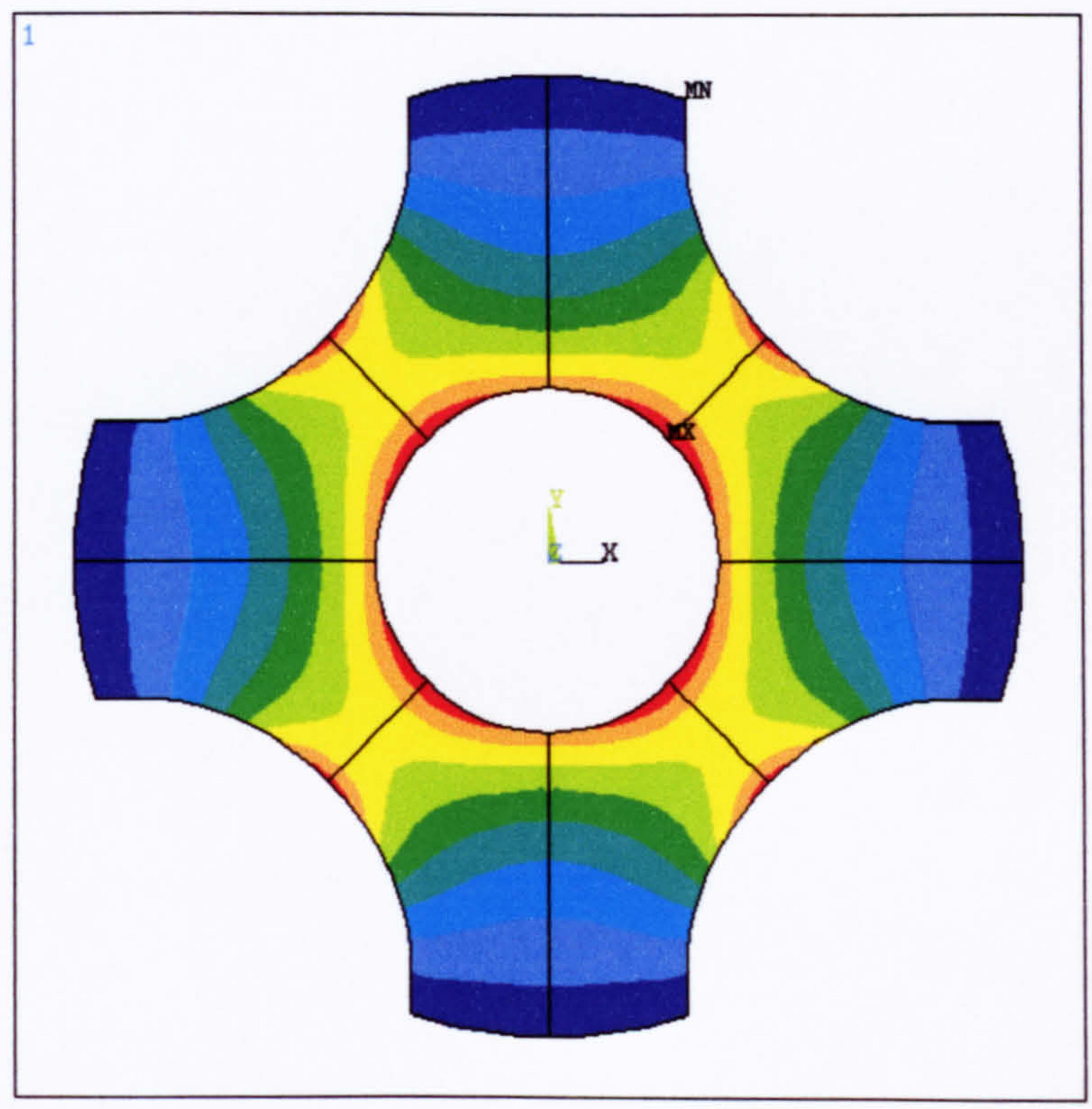

ANSYS 5.4

AUG 292000

$16: 24: 42$

NODAL SOLUTION

$\mathrm{STEP}=1$

SUB $=1$

TIME $=1$

/ EXPANDED

SEQV

(AVG)

$\mathrm{DMK}=.800 \mathrm{E}-05$

SMN $=141034$

$\mathrm{SMX}=.226 \mathrm{E}+09$

141034

$.252 \mathrm{E}+08$
$.503 \mathrm{E}+08$

$754 \mathrm{E}+08$

$.100 \mathrm{E}+09$

$125 \mathrm{E}+09$

$151 \mathrm{E}+09$

$.176 \mathrm{E}+09$

$.201 \mathrm{E}+09$

$.226 \mathrm{E}+09$

Figure 2.37 Stress contours for the final rotor design with a shaft bore of $13 \mathrm{~mm}$ and a fillet radius of 0.275 at $10000 \mathrm{rad} / \mathrm{s}$.

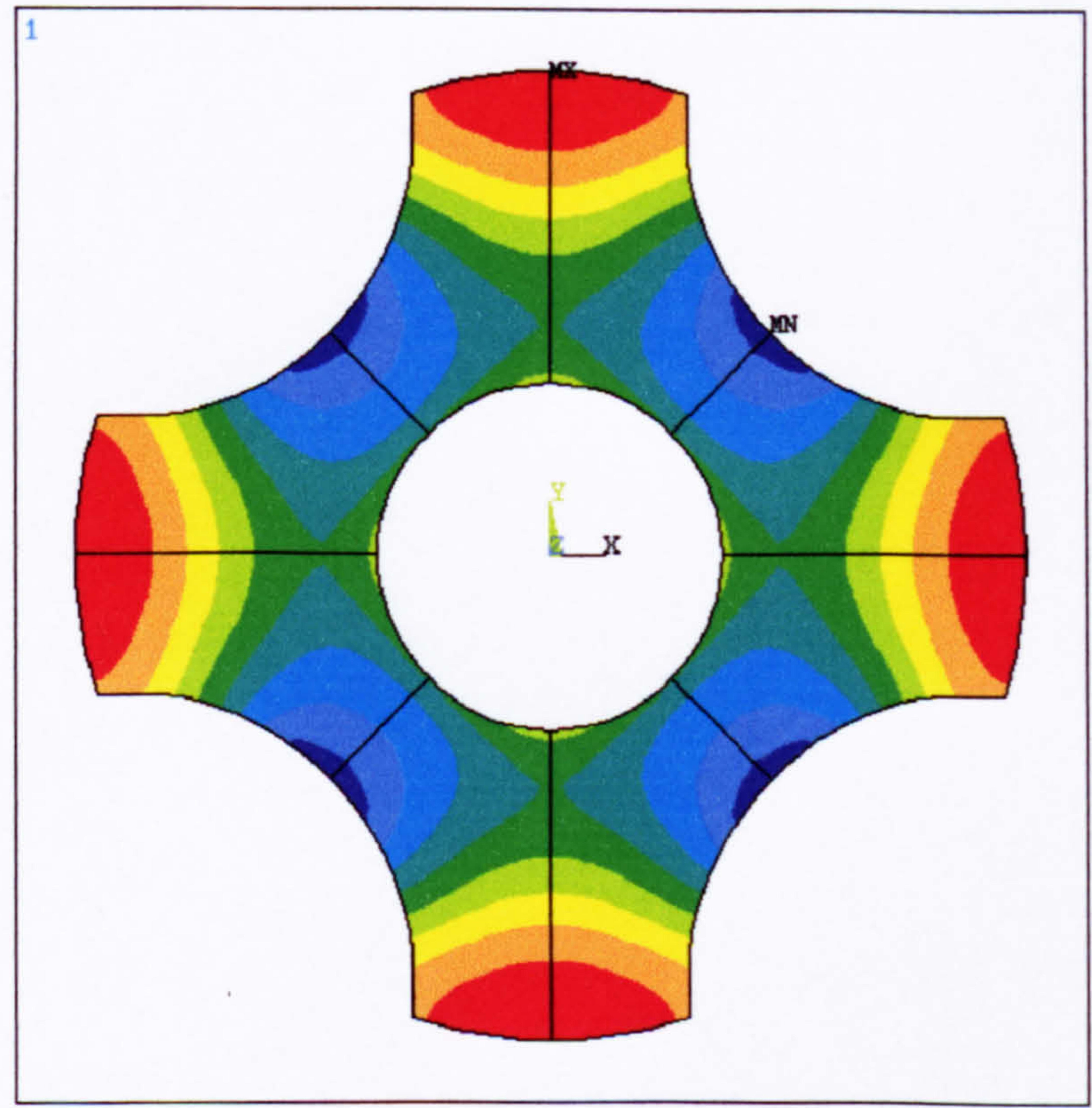

ANSYS 5.4

AUG 292000

$16: 28: 29$

NODAL SOLUTION

STEP $=1$

T IME $=1$

EXPANDED

UX

RSYS $=1$

$\mathrm{DMX}=.800 \mathrm{E}-05$

SMN $=.583 \mathrm{E}-05$

$\mathrm{SMX}=.797 \mathrm{E}-05$

$.583 \mathrm{E}-05$

$607 \mathrm{E}-05$

$.630 \mathrm{E}-05$

$.654 \mathrm{E}-05$

$.678 \mathrm{E}-05$

$.702 \mathrm{E}-05$

$.726 \mathrm{E}-05$

$.749 \mathrm{E}-05$

$.773 \mathrm{E}-05$

$.797 \mathrm{E}-05$

Figure 2.38 Radial displacement of the final rotor design at $10000 \mathrm{rad} / \mathrm{s}$. 
Appendix 2.1.

\begin{tabular}{|c|c|c|c|c|c|c|}
\hline $\begin{array}{c}S r \\
(\%)\end{array}$ & $\begin{array}{c}\text { Number } \\
\text { of } \\
\text { elements }\end{array}$ & $\begin{array}{c}\text { Normalised } \\
\text { computational } \\
\text { requirement }\end{array}$ & $\begin{array}{c}\text { RMS error } \\
\text { (radial) } \\
(\%)\end{array}$ & $\begin{array}{c}\text { RMS error } \\
\text { (hoop) } \\
(\%)\end{array}$ & $\begin{array}{c}\xi \\
(\%)\end{array}$ & $\begin{array}{c}\text { Nodal error at } \\
\text { maximum stress } \\
(\%)\end{array}$ \\
\hline 25 & 2 & 1.00 & 52 & 3.9 & - & 4.4 \\
\hline 12.5 & 8 & 1.29 & 17 & 1.6 & 2.23 & 2.2 \\
\hline 8.33 & 15 & 1.62 & 8.4 & 0.76 & 1.09 & 1.2 \\
\hline 6.25 & 28 & 1.99 & 5.1 & 0.45 & 0.390 & 0.77 \\
\hline 5.00 & 40 & 2.41 & 3.5 & 0.31 & 0.260 & 0.51 \\
\hline 4.17 & 60 & 2.85 & 2.2 & 0.20 & 0.127 & 0.39 \\
\hline 3.57 & 77 & 3.34 & 1.7 & 0.16 & 0.0986 & 0.29 \\
\hline 3.12 & 104 & 3.86 & 1.3 & 0.12 & 0.0561 & 0.23 \\
\hline 2.78 & 135 & 4.42 & 1.0 & 0.095 & 0.0409 & 0.19 \\
\hline 2.50 & 160 & 5.02 & 1.1 & 0.096 & 0.0364 & 0.15 \\
\hline 2.27 & 198 & 5.66 & 0.72 & 0.067 & 0.0242 & 0.13 \\
\hline 2.08 & 228 & 6.33 & 0.62 & 0.056 & 0.0197 & 0.11 \\
\hline 1.92 & 273 & 7.05 & 0.51 & 0.047 & 0.0151 & 0.095 \\
\hline 1.79 & 308 & 7.80 & 0.43 & 0.040 & 0.0136 & 0.082 \\
\hline 1.67 & 360 & 8.59 & 0.40 & 0.036 & 0.00908 & 0.072 \\
\hline 1.56 & 416 & 9.41 & 0.34 & 0.031 & 0.00757 & 0.065 \\
\hline 1.47 & 459 & 10.3 & 0.29 & 0.027 & 0.00757 & 0.057 \\
\hline 1.39 & 522 & 11.2 & 0.26 & 0.023 & 0.00605 & 0.051 \\
\hline 1.32 & 570 & 12.1 & 0.23 & 0.021 & 0.00454 & 0.047 \\
\hline 1.25 & 640 & 13.1 & 0.28 & 0.024 & 0.00454 & 0.042 \\
\hline 1.19 & 693 & 14.1 & 0.19 & 0.017 & 0.00454 & 0.038 \\
\hline 1.14 & 770 & 15.2 & 0.17 & 0.016 & 0.00302 & 0.035 \\
\hline 1.09 & 851 & 16.3 & 0.16 & 0.015 & 0.00151 & 0.033 \\
\hline 1.04 & 912 & 17.4 & 0.15 & 0.014 & 0.00302 & 0.030 \\
\hline 1.00 & 1000 & 18.6 & 0.15 & 0.013 & 0.00303 & 0.027 \\
\hline
\end{tabular}

Table A2.1. Effect of element size and computational requirement on the solution error for cylindrical rotor 1. 


\begin{tabular}{|c|c|c|c|c|c|c|}
\hline $\begin{array}{c}S r \\
(\%)\end{array}$ & $\begin{array}{c}\text { Number } \\
\text { of } \\
\text { elements }\end{array}$ & $\begin{array}{c}\text { Normalised } \\
\text { computational } \\
\text { requirement }\end{array}$ & $\begin{array}{c}\text { Number of } \\
\text { elements along } \\
\text { path A-A' }\end{array}$ & $\begin{array}{c}\xi_{\mathrm{A}-\mathrm{A}^{\prime}} \\
(\%)\end{array}$ & $\begin{array}{c}\text { Number of } \\
\text { elements along } \\
\text { path B-B' }\end{array}$ & $\begin{array}{c}\xi_{\mathrm{B}-\mathrm{B}^{\prime}} \\
(\%)\end{array}$ \\
\hline 4.17 & 51 & 2.45 & 9 & - & 5 & - \\
\hline 3.57 & 68 & 2.67 & 11 & 0.37 & 6 & 1.19 \\
\hline 3.12 & 92 & 3.48 & 12 & 0.43 & 7 & 1.01 \\
\hline 2.78 & 102 & 3.48 & 14 & 0.22 & 8 & 0.402 \\
\hline 2.50 & 143 & 4.27 & 15 & 0.00910 & 9 & 0 \\
\hline 2.27 & 161 & 4.69 & 17 & 0.106 & 10 & 0.337 \\
\hline 2.08 & 200 & 5.76 & 18 & 0.0729 & 11 & 0.424 \\
\hline 1.92 & 228 & 6.76 & 20 & 0.516 & 12 & 0.094 \\
\hline 1.79 & 262 & 6.25 & 21 & 0.0486 & 13 & 0.293 \\
\hline 1.67 & 286 & 6.93 & 23 & 0.00611 & 14 & 0.0608 \\
\hline 1.56 & 316 & 8.41 & 24 & 0 & 15 & 0.224 \\
\hline 1.47 & 374 & 7.47 & 26 & 0.00302 & 15 & 0.0526 \\
\hline 1.39 & 423 & 10.67 & 27 & 0.0213 & 16 & 0.174 \\
\hline 1.32 & 456 & 10.03 & 29 & 0.00612 & 17 & 0.158 \\
\hline 1.25 & 530 & 12.25 & 30 & 0.0182 & 18 & 0.0249 \\
\hline 1.19 & 562 & 12.25 & 32 & 0.0182 & 19 & 0.0277 \\
\hline 1.14 & 618 & 13.69 & 33 & 0.0182 & 20 & 0.0471 \\
\hline 1.09 & 691 & 14.44 & 35 & 0.0121 & 21 & 0.0470 \\
\hline 1.04 & 748 & 14.18 & 36 & 0.00300 & 22 & 0.127 \\
\hline 1.00 & 810 & 17.36 & 38 & 0.00300 & 23 & 0.0147 \\
\hline
\end{tabular}

Table A2.2 Convergence of the finite-element solution as the element discretisation is increased for switched reluctance rotor 1. 


\section{CHAPTER 3}

\section{MACHINE DESIGN SYNTHESIS AND DYNAMIC SIMULATION}

\subsection{Introduction}

In chapter 1, switched reluctance (SR) machines were selected as the preferred machine topology, with the 6/4 variant specifically identified in terms of its reasonably low torque ripple and self-starting ability. The detailed mechanical study presented in chapter 2 confirmed that the rotor construction is well suited to highspeed applications, although at the high speeds that are likely to be encountered, severe constraints are imposed on the rotor dimensions. The findings of the previous chapters, which serve to restrict the machine design at this stage, can be summarised as:

Pole and Phase numbers (Section 1.7.1). The previously published findings of Miller [3.1] and Lawrenson et al. [3.2] were used as the basis for selecting a 3 phase machine with 6 stator teeth and 4 rotor teeth.

Rotor and stator pole arc (Section 1.7.2). Again the guidelines presented by Lawrenson et al. [3.2] were used to establish stator and rotor pole arcs of $30^{\circ}$ and $35^{\circ}$ respectively. This combination maximises the area for stator windings while allowing a small overlap of $5^{\circ}$ to produce a region of constant inductance to assist with the 'fluxing' period for electrical generation.

Rotor cross-section (Section 2.5). The rotor cross-section design established on the basis of centrifugal stress has an outer diameter of $38 \mathrm{~mm}$, a bore diameter of $13 \mathrm{~mm}$ and a fillet radius of $10.5 \mathrm{~mm}$.

Airgap height (Section 1.7). An airgap of $0.2 \mathrm{~mm}$ was selected on the basis of balanced judgement between manufacturing tolerances and electromagnetic performance. This value also provides the necessary capability to accommodate the 
predicted radial growth of the rotor at high speed with sufficient remaining overhead to accommodate an additional narrowing of the gap due to any differential thermal expansion of the rotor and stator.

Preliminary performance specification (Section 1.5, 1.7). As discussed in chapter 1 , the target peak power is $\pm 5 \mathrm{~kW}$, with a maximum rotational speed of $100000 \mathrm{rpm}$, a specification which is predominantly determined by the engine, turbine and compressor characteristics.

\begin{tabular}{|c|l|c|}
\hline Symbol & Description & Value \\
\hline$\alpha_{s}$ & Stator pole arc & $30^{\circ}$ \\
\hline$\alpha_{r}$ & Rotor pole arc & $35^{\circ}$ \\
\hline$D_{s}$ & Diameter of stator bore & $38.4 \mathrm{~mm}$ \\
\hline$D_{r}$ & Diameter of rotor & $38.0 \mathrm{~mm}$ \\
\hline$W_{s}$ & Width of the stator tooth $\left[D_{s} \sin \left(\alpha_{s} / 2\right)\right]$ & $9.8 \mathrm{~mm}$ \\
\hline$r_{f}$ & Interpolar fillet radius & \\
\hline$D_{b}$ & Bore diameter & $10.5 \mathrm{~mm}$ \\
\hline
\end{tabular}

Table 3.1 Established machine dimensions.

Many of the principal geometrical parameters (which are defined in figure 3.1) are either specified from the above design guidelines, or can be derived directly, e.g. the width of the stator tooth can be determined from the stator bore diameter and stator pole arc. The numerical values of all the dimensions that are fixed at this stage of the design synthesis are listed in table 3.1 .

The remaining principal dimensions, viz. the depth of the stator back iron $\left(d_{b}\right)$, the depth of the stator slots $(d s)$ and the axial length of the rotor $\left(L_{a x}\right)$ must be selected with due regard to many factors, paramount amongst which are efficiency and temperature rise limitations. 


\subsection{Design Synthesis}

Depth of the stator back iron $\left(d_{b}\right)$. The stator back iron provides the return path for the machine flux. A useful design guideline is to select the back iron thickness such that it is sufficient to prevent saturation at excitation levels below those required to saturate the stator teeth. The selection of any thickness greater than this guideline value is likely to yield little return in terms of torque per amp capability, but would nevertheless reduce the iron loss, albeit at the expense of an increased stator outer diameter. Using this design guideline as a basis, with due recognition of the fact that multiphase operation gives rise to a degree of flux superposition in the back iron, the back iron thickness is given by:

$$
d_{b}=\frac{2}{3} W_{s} \approx 7.0 \mathrm{~mm}
$$

Depth of the stator slot. The depth of the stator slot is a critical design consideration in all electrical machines and its selection involves establishing the appropriate balance between copper losses, iron losses, temperature rise, and overall machine size:

The winding cross-sectional area increases with increasing slot depth thus allowing a given level of excitation to be achieved with a lower current density and hence copper loss, albeit at the expense of a larger stator. There is a minimum slot depth that can be practicably employed, since the maximum of copper loss that can be sustained is limited by temperature rise considerations. However, increasing slot depth has a detrimental influence on the iron losses, since the overall iron mass increases without affording any scope to reduce the flux density and hence the iron loss density. In selecting an appropriate slot depth which provides a compromise between the conflicting requirements of copper and iron losses, it should be borne in mind that iron losses can be more readily dissipated than the equivalent copper 
loss, by virtue of the considerably reduced thermal resistance to the ambient/cooling fluid. One important feature of high-speed machines is that they tend to have relatively few conductors per slot. In such cases, the dimensions of the individual conductors dictates that the slot depth can only be varied in discrete steps, which can in some cases limit the scope for precise optimisation.

In the absence of reliable estimates of the relative contribution of the iron and copper loss mechanisms, which in turn are reliant on detailed simulations of the machine performance, the selection of an appropriate slot depth at this stage of the design synthesis would involve a significant degree of empiricism. Fortunately however, the phase inductance of an SR machine (in both the un-aligned and more particularly the aligned position) is not particularly sensitive to the slot depth, providing it is of a reasonable depth. It is possible to infer a minimum slot depth using the guidelines established by Radun [3.3] where the ratio of aligned to unaligned inductance is maximised be ensuring that the length of the rotor tooth is greater than twice the mean circumferential spacing between the rotor and stator teeth. If this result is applied to the stator teeth, a minimum tooth height of $8.3 \mathrm{~mm}$ is established.

Hence, as a starting point it can be set with reference to typical proportions of previously published machines [3.1,3.4] and in this case was selected as $10 \mathrm{~mm}$. Although this is an apparently somewhat arbitrary approach, it enables detailed simulations to be performed, in order to generate reliable performance data (which itself is relatively insensitive to the actual value of slot depth providing the resistive drop is relatively small as compared to the supply voltage). This can then be used as the basis for a more rigorous selection of an appropriate slot depth,

Axial length of machine. The torque capability of electrical machines can be reasonably assumed to be proportional to the rotor volume. In this machine design, in which the diameter of the rotor is constrained by centrifugal stress 
considerations to a value of $38 \mathrm{~mm}$, the axial length is the only degree of freedom available in the rotor design, which can be tailored to meet the torque specification. An initial estimate of an appropriate axial length can be derived from equation 3.2, which is based on employing torque per unit volume figures, such as those shown in table 3.2:

$$
L_{a x}=\frac{T}{\pi / 4 D_{r}^{2} k_{T R V}}
$$

\begin{tabular}{|l|c|}
\hline Machine type/application & $k_{T R V}\left(\mathrm{kNm} / \mathrm{m}^{3}\right)$ \\
\hline Small totally enclosed motors & $2.5-7$ \\
\hline Industrial motors & $7-30$ \\
\hline High-performance servomotors & $15-50$ \\
\hline Aerospace machines & $30-75$ \\
\hline Large liquid-cooled machines & $100-250$ \\
\hline
\end{tabular}

Table 3.2 Typical performance coefficients of SR machines. Source [3.1]

As is evident from table 3.2, the values of $k_{T R V}$ cover a relatively wide range and are influenced by the cooling strategy employed and the size of the machine. For the particular case of the machine considered in this study, the oil cooling of the stator is likely to result in a relatively high value of $k_{T R V}$, although the scope for achieving very high values is limited by the relatively small size of the machine. As a consequence, initial estimates of axial length were derived using a value of $k_{T R V}=$ $40 \mathrm{Nm} / \mathrm{m}^{3}$ i.e. towards the lower end of the range which is likely to be achievable in aerospace machines. In terms of a torque rating, a worst case operating point of $5 \mathrm{~kW}$ at $20,000 \mathrm{rpm}$ was used, which corresponds to a torque of $2.4 \mathrm{Nm}$. Substituting these values into equation 3.2, yields an axial length of $53 \mathrm{~mm}$ for the case of a rotor diameter of $38 \mathrm{~mm}$, which formed the basis for selecting a final length of $50 \mathrm{~mm}$ 


\subsection{Dynamic Simulation}

The design synthesis described in sections 3.1 and 3.2, largely employs established design guidelines and empirical factors to determine many of the dimensions, whereas others are a result of mechanical analysis and space restrictions. As a consequence, the dimensions have hitherto been selected with little reference to the output power specification, with the exception of the axial length, $L_{a x}$ which was estimated from published specific torque figures. Further, the dynamic nature of SR machine operation dictates that the performance of a given machine/drive combination can often deviate markedly from idealised static considerations. This factor is a particular concern in this case given the high-speed nature of the machine, and the consequent high electrical frequencies that are likely to be encountered. Hence, recourse to dynamic simulation techniques that are capable of accurately predicting machine performance is essential. This in turn will allow detailed predictions to be made of the maximum power capability, the current waveforms (and hence copper and iron losses), thus providing a robust basis on which further refinement of the machine design and/or optimisation of the control strategy can be undertaken.

\subsubsection{DYNAMIC SR OPERATION}

The basic operating principles of SR machines, in both motoring and generating modes, were described previously in section 1.7, albeit for the somewhat idealised case of essentially static, constant current operation. In practice, SR machines tend to be operated in conjunction with voltage source inverters rather than being operated with a strictly constant current as shown previously in figure 1.18 (section 1.7). As such, each winding is excited by a nominally square wave voltage pulse, during which, the phase flux linkage increases in accordance with the governing electrical circuit equation:

$$
\psi=\int\left(V_{\mathrm{dc}}-i R\right) d t
$$


This process is often referred to as 'fluxing' the machine. At the end of the initial voltage pulse, conduction through the free-wheeling diodes 'de-fluxes' the machine, hence returning the vast majority of the stored magnetic energy to the supply. A typical drive circuit for one phase (comprising two series-connected concentrated windings) is shown in figure 3.2. Figure 3.2a shows the conduction path through transistors $\mathrm{Q}_{1}$ and $\mathrm{Q}_{2}$ during the fluxing period, while figure 3.2b shows that for the de-fluxing period in which current freewheels through diodes $D_{1}$ and $D_{2}$, and back into the supply. The relative phasing of the voltage pulses with respect to the rotor position determines the net energy transfer between the machine and the supply, with both motoring and generating modes being realisable.

In a practical machine operating over a wide speed range, some degree of closed loop control of the current is essential. Current control strategies for medium to highpower machines tend to achieve this by applying a series of pulses of the full DC link voltage across the winding with a duty cycle appropriate for achieving the desired current. The differences between the various techniques are, in essence, the means by which the duty cycle is determined, and the relationship between the machine electrical frequency and the switching frequency.

The most common strategy is to have a switching frequency that is considerably higher than the fundamental electrical frequency of the machine, such that many switching cycles with appropriate duty occur during one machine electrical cycle, e.g. pulse-width-modulation (PWM) techniques. Such techniques are well suited to very accurate control of the shape of the current waveform, and are extensively used for example in many SR drives for low to medium speed applications [3.5]. However, in the case of very high-speed machines, in which the electrical frequency of the machine tends towards the $\mathrm{kHz}$ range, such techniques become increasingly problematic. The switching frequency of medium to high-power inverters is generally restricted to some $10-20 \mathrm{kHz}$ in order to limit the switching losses and maintain efficiency. Hence, for a high fundamental electrical frequency, the number 
of switching intervals per cycle reduces to such an extent that the degree of control that can be exercised with a fixed frequency PWM is severely compromised.

An alternative control strategy is to limit the number of voltage pulses applied during one machine electrical cycle to a single pulse, with control being exercised by varying the instant at which the pulse is applied and its subsequent duration [3.5, 3.6]. These two events can be considered in terms of so-called turn-on and dwell angles, which are labelled as $\theta_{\text {on }}$ and $\theta_{\text {dwell }}$ respectively in figure 3.3. However, whereas close control of average current and power can be achieved, little direct control is exercised over the shape of the current pulse during conduction. This will tend to result in some degree of performance degradation as compared to a closely controlled waveform in terms of an increased copper loss (as a consequence of the higher rms to average current ratio) and an increased torque ripple. In terms of iron loss, it is more difficult to generalise as regards the merits of the two techniques, since this will depend on the relative contributions of the higher flux density from a single pulse as compared to the eddy-current losses generated by current ripple caused by high-frequency switching.

Given the apparent suitability of this latter technique to controlling a machine with a high electrical frequency, its merits were analysed in detail with reference to the proposed application. In terms of practical implementation, one drawback of this control strategy is that it is difficult to implement as a truly closed-loop technique for a number of reasons:

1) Since the current is not directly controlled, and can continue to increase even following the turning off of a switch (due to the falling inductance), the closedloop control of the turn-off instant to achieve a given power is difficult to reliably implement.

2) In the absence of any a-priori knowledge, establishing the turn-on angle required to obtain a given power demand could only be achieved iteratively on a cycle by cycle basis. 
3) A given power can often be achieved with more than one combination of turn-on and dwell angle. In principle, the merits of the particular combinations require detailed consideration of the resultant losses (which may involve temperature rise having an influence on the balance of the losses) as well as factors such as torque ripple etc.

As is evident from the above, the successful and robust implementation of such a control strategy is inevitably reliant on a significant degree of a-priori knowledge. A possible exception is the use of self-tuning/neural networks or genetic algorithms. However these have limited applicability in this case since the objective functions (i.e. minimisation of copper loss, iron loss etc) are difficult to measure, and the potential for catastrophic failure due to inappropriate commutation conditions at such high power level restricts the scope for searching/self-tuning. As a consequence, dynamic performance simulation allied to detailed loss estimates plays a key role in both assessing whether such a control strategy is well suited to the particular combination of machine electrical frequency/time constant, and generating reliable performance maps which form the basis of look-up tables in the implementation of the control system

It should be noted that whereas the proposed control strategy may well prove to be suited to high-speed operation, it is likely that recourse to a more conventional current control strategy will be required at low rotor speeds and during starting (the ability to self-start and provide engine inlet boost for the engine start procedure having been identified as a key performance feature in section 1.5 of chapter 1). This chapter however is restricted to the development of control strategy for application at high-speed, since many well-established and proven control techniques are available for low speed operation. 


\subsection{Numerical Modelling of SR Machines}

SR machines tend to be much more onerous to model than other topologies, because of their singly excited nature, the doubly salient geometry, and the tendency for machines to be operated with high levels of magnetic saturation in order to realise competitive power densities and power factors. The simulation of SR machines and subsequent analysis of the results can be considered in three distinct stages i.e.:

1. Characterisation of the electromagnetic performance in terms of the relationships between flux-linkage, current and rotor position.

2. Calculation of current, flux-linkage and torque under given commutation conditions when driven using a constant voltage supply.

3. Post-processing functions that provide key design and analysis data that facilitate, for example, winding design, specification of the drive VA and selection of optimal control parameters.

\subsubsection{SIMULATION STAGE 1 - Characterisation of Electromagnetic Performance}

The characterisation of a particular SR machine involves two stages:

1. The calculation of the $\psi-i-\theta$ relationships using methods such as magnetostatic finite element analysis or analytical magnetic field calculation techniques.

2. The representation of the $\psi-i-\theta$ relationships in the simulation code using methods such as curve fitting, interpolation or look-up tables.

The resulting three-degree of freedom characteristic, a typical form of which is shown in figure 3.4 is often considered in terms of two linked two-dimensional relationships, viz. $\psi-i$ and $\psi-\theta$ planes as shown in figures $3.5 \mathrm{a}$ and $3.5 \mathrm{~b}$. A wide variety of strategies, both linear and non-linear, have been reported for representing typical $\psi-i$ and $\psi-\theta$ relationships $[3.2,3.7 \& 3.8]$. Table 3.3 summarises the main 
features of 5 possible strategies (designated as FL1 to FL5), with particular emphasis on the number of data points required in each case.

\begin{tabular}{|c|c|c|c|c|c|c|}
\hline & & \multicolumn{2}{|c|}{ Variation with Current } & \multicolumn{2}{|c|}{ Variation with rotor angle } & \\
\hline & $\begin{array}{c}\text { Flux- } \\
\text { Linkage } \\
\text { Calculation } \\
\text { Technique }\end{array}$ & Representation & $\begin{array}{l}\text { Minimum } \\
\text { data } \\
\text { points } \\
\text { req'd } \\
\end{array}$ & Representation & $\begin{array}{l}\text { Minimum } \\
\text { data } \\
\text { points } \\
\text { req'd }\end{array}$ & $\begin{array}{l}\text { Total number of } \\
\text { data points } \\
\text { req'd for model }\end{array}$ \\
\hline FL1 & $\begin{array}{c}\text { Linear } \\
\text { analytical }\end{array}$ & $\begin{array}{l}\text { Assumed } \\
\text { linear }\end{array}$ & $\begin{array}{l}1-A \\
1-U\end{array}$ & Linear & 2 & 2 \\
\hline$\overline{\text { FL2 }}$ & Linear FE & $\begin{array}{l}\text { Assumed } \\
\text { linear }\end{array}$ & $\begin{array}{l}1-A \\
1-U\end{array}$ & $\overline{\text { Linear }}$ & $\overline{2}$ & $\overline{2}$ \\
\hline FL3 & $\begin{array}{l}\text { Non-linear } \\
\text { FE }\end{array}$ & $\begin{array}{l}\text { Non-linear } \\
\text { parabolic } \\
\text { curve fit }\end{array}$ & $\begin{array}{l}2-A \\
1-U\end{array}$ & Linear & 2 & 3 \\
\hline$\overline{\text { FL4 }}$ & $\begin{array}{c}\text { Non-linear } \\
\text { FE }\end{array}$ & $\begin{array}{l}\text { Non-linear } \\
\text { parabolic } \\
\text { curve fit } \\
\end{array}$ & $\begin{array}{l}2-A \\
1-U\end{array}$ & $\begin{array}{l}\text { Curve fit - } \\
\text { Frolich }\end{array}$ & 2 & 3 \\
\hline FL5 & $\begin{array}{c}\text { Non-linear } \\
\text { FE }\end{array}$ & Look up table & $10+$ & Look up table & $\overline{10 t}$ & $100+$ \\
\hline
\end{tabular}

Table 3.3. Alternative techniques for the calculation and subsequent representation of the flux linkage variation with current and rotor position (A-aligned; U-unaligned).

On the basis of table 3.3, two strategies were selected for use in this study, viz. FL4 and FL2. The non-linear method FL4 was selected as it is a computationally efficient method, at least in terms of the number of data points which must be generated, while at the same time representing the major non-linearities of the machine. The linear method FL2 was also selected to gauge the suitability of linear analysis to this particular high-speed application.

Method FL2 - Since the flux linkage is assumed to vary linearly with both phase current and rotor position, the full machine characteristic can be simply derived from flux-linkage calculations for only two cases, viz. the aligned and unaligned positions with an essentially arbitrary level of current. The two values of flux-linkage required are calculated using two-dimensional, linear finite element analysis in which the rotor and stator soft magnetic components are represented with a fixed relative permeability of 10,000 , a value which is typical of an unsaturated $2 \%$ Silicon steel 
[3.9]. The two-dimensional nature of the finite element model inherently neglects end-effects within the machine and the contribution of the end-windings to the overall flux-linkage. Figure 3.6 shows the two-dimensional mesh of the complete machine and a close-up of the mesh in the region around one stator pole, for the case of a rotor in the aligned position. The mesh of figure 3.6 comprises 17468 second order elements and 52659 nodes, and was constructed and solved using Ansys v5.4.

Figures 3.7 and 3.8 show calculated equi-potential and flux density contours respectively, for the case of a current density of $1 \mathrm{Amm}^{-2}$ in the entire slot area of phase $A$ with phases $B$ and $C$ remaining unexcited (the phase designation being that shown previously in figure. 1.17 chapter 1). The phase flux-linkages and hence selfinductances were calculated from the two finite element solutions using the techniques described in appendix 3.1.

The finite element predicted self-inductance for the aligned and unaligned positions are $6.58 \mu \mathrm{H}$ and $0.48 \mu \mathrm{H}$ respectively for the case of a single turn around each of the two teeth that constitute a phase. The merits of established analytical techniques for the calculation of flux-linkage and inductance were assessed by employing the simplified model proposed by Radun [3.3], details of which are described in Appendix 3.2. Using this model, values of $6.25 \mu \mathrm{H}$ and $0.41 \mu \mathrm{H}$ are obtained for the aligned and unaligned inductance respectively. The agreement between the analytical model and the finite element method is reasonable in this particular case, the lower values predicted by the analytical technique being consistent with the fact that it necessarily neglects some components of leakage flux, whose contribution is particularly significant in the unaligned position.

Having established the flux-linkage per unit current for both the aligned and unaligned positions, a representation of their variation with respect to rotor angle must be established. Rather than a simple linear variation between the two values corresponding to aligned and unaligned rotors, a trapezoidal variation is more representative of the variation observed in SR machines [3.2]. The form of the 
trapezoidal variation can be calculated from a simple consideration of the overlap of the rotor and stator teeth, as shown in figure 3.9. Using the definitions in figure 3.9, the flux-linkage per unit current, and hence the self-inductance at any current and rotor position is given by:

$$
L(\theta)=L_{u}+\Delta_{\text {ovl }}\left(L_{a}-L_{u}\right)
$$

Method FL4 - Whereas method FL2 gives rise to a single equation which enables flux-linkage to be calculated at any rotor position and current, the assumption that the inductance does not vary with current limits its applicability due to saturation. As is evident from the $\psi-i$ characteristics shown previously in figure $3.5 \mathrm{a}$ the variation of flux-linkage with current in the aligned positions is significantly more influenced by saturation than is the case for the unaligned position. Indeed, for all reasonable levels of excitation, the unaligned $\psi-i$ characteristic will be essentially linear [3.7], and as such can be fully represented by the single value of unaligned inductance calculated previously. By contrast, for the aligned position, some form of non-linear curve-fit is required, and a number of methods have been proposed. The data on which any curve-fit is based, necessarily requires the calculation of flux-linkage values within the saturated region of the $\psi-i$ characteristic. Although a non-linear lumped reluctance modelling approach [3.4] could be used to establish the necessary data, in this study non-linear, magnetostatic, two-dimensional finite element analysis was employed.

Although the use of a single curve-fit based on a limited number of coefficients would offer a convenient approach, the form of a typical aligned $\psi$-i characteristic is not well suited to the use of such techniques within dynamic simulations. By means of example, although inverse tangent and inverse hyperbolic tangent functions have been employed to model the non-linear magnetisation curves of soft magnetic materials which have a similar form to a typical $\psi$-i characteristic [3.10], such functions cannot be readily inverted, and hence are cumbersome in simulations in which the flux linkage and current are solved sequentially. 
An alternative approach is to model the $\psi$-i characteristic as consisting of two distinct regions, corresponding to unsaturated and saturated conditions. The most straightforward techniques are based on a two-stage piecewise linear approximation, such as the method implemented by Krishnan et al. [3.8]. Although simple in its formulation, such a two stage linear approach is limited in terms of accuracy, particularly near the onset of saturation. The approach adopted in this study is based on a combination of parabolic and linear functions as proposed by Miller and McGilp [3.7] and shown schematically in figure 3.10. The $\psi-i$ characteristic is represented by the linear function at low levels of excitation below saturation, and by the parabolic function near the onset of saturation and beyond. Using the coefficients defined in figure 3.10, the equation of the parabola is [3.7]:

$$
\left(\psi-\psi_{s 0}\right)^{2}=4 a\left(i-i_{s 0}\right)
$$

In principle, only two finite element calculations are required to establish the coefficients for the curve-fit, viz. one at the intersection of the parabolic and linear curves and the other at high levels of saturation. However, in the absence of a-priori knowledge, the intersection is difficult to directly establish, and a number of solutions are usually required to generate the appropriate data. Table 3.4 contains the curve-fit parameters for both the linear and parabolic regions which were calculated using finite element mesh for the SR machine design described above with the application of non-linear B-H characteristics for the rotor and stator iron. The resulting $\psi-i$ characteristic derived using these parameters is shown in figure 3.11, together with that established from a series of 60 non-linear finite element calculations. As is evident, the curve-fit provides a reasonable representation of the $\psi$-i characteristic, the accuracy of this particular curve-fit being limited by the extent of saturation the machine is likely to encounter [3.7]. 


\begin{tabular}{|c|c|c|}
\hline$i_{s}$ & 297.4 & $A$ \\
\hline$i_{m}$ & 2230 & $\mathrm{~A}$ \\
\hline$I_{u}$ & 2230 & $\mathrm{~A}$ \\
\hline$\psi_{s}$ & 0.0163 & Wb-turns \\
\hline$\psi_{m}$ & 0.0223 & Wb-turns \\
\hline$\psi_{u}$ & 0.0107 & Wb-turns \\
\hline$a$ & $4.94 \times 10^{-11}$ & \multicolumn{1}{|c}{} \\
\cline { 1 - 2 } & &
\end{tabular}

Table 3.4 Curve-fit coefficients for parabolic curve-fitting function for machine design of table 3.1 (with two turns/ phase and $1 \mathrm{~m}$ axial length.)

The variation of flux-linkage with respect to the rotor position can also be represented using a number of alternative approaches, ranging from a simple trapezoidal variation such as that employed in method FL2 through to a complete series of finite element solutions. One approach that has been demonstrated to provide an improved degree of accuracy as compared to a trapezoidal variation, while at the same time remaining computationally efficient, is the use of mathematical functions to represent the variation of flux linkage with respect to rotor position, with simple correction factors to account for the influence of current at intermediate rotor positions. A convenient formulation is that proposed by McGilp et al.[3.7], which is based on separating the variation with respect to rotor position into three discrete regions, as shown by the schematic $\psi-\theta$ curve of figure 3.12. Regions I and III of the $\psi-\theta$ curve are represented by two independent Frohlich curves, while region II is assumed to be linear. The key reference angular positions which form the basis of the three curve-fits, labelled as $\xi_{u}, \xi_{l}, \xi_{h r}, \xi_{2}$ and $\xi_{a}$ in figure 3.12, are determined predominantly from simple geometrical considerations, the exception being $\xi_{0}$ which is related to leakage flux and is a function of the level of excitation. Table 3.5 contains a summary of the means by which these various angles are determined. 


\begin{tabular}{|c|l|c|}
\hline & \multicolumn{1}{|c|}{ Description } & Rotor angle (degrees) \\
\hline$\xi_{u}$ & Unaligned position & -45 \\
\hline$\xi_{0}$ & $\begin{array}{l}\text { Offset angle. The intersection of the } \\
\text { linear variation of region II and the } \\
\text { unaligned inductance. }\end{array}$ & $\left.\begin{array}{c}\text { Determined such that: } \\
\xi_{1} \leq \xi_{0} \leq\left[\xi_{1}-\frac{\left(\xi_{a}-\xi_{u}\right)}{12}\right]\end{array}\right]$ \\
\hline$\xi_{1}$ & Beginning of mechanical overlap & -32.5 \\
\hline$\xi_{h r}$ & Midpoint of $\xi_{1}$ and $\xi_{2}$. & -17.5 \\
\hline$\xi_{2}$ & $\begin{array}{l}\text { Beginning of complete mechanical } \\
\text { overlap }\end{array}$ & -2.5 \\
\hline$\xi_{a}$ & Rotor aligned position & \\
\hline
\end{tabular}

Table 3.5 List of parameters for the determination of the inductance variation.

It is important to note that the form of the $\psi-\theta$ curve is itself a function of the level of excitation. Thus there is a unique set of $\psi-\theta$ curve curve-fit coefficients for each level of excitation, which are determined using the appropriate limiting flux-linkage values, i.e. $\psi_{a}(i)$ and $\psi_{u}(i)$ derived from the representation of the $\psi$-i characteristics. A detailed description of the curve fitting functions is presented by McGilp and Miller [3.7], and the particular coefficients described in this study are presented in appendix 3.3.

Figure. 3.13 shows a comparison between the $\psi-\theta$ curve calculated using this curvefitting approach, and that derived from a comprehensive series of finite element solutions for 3 levels of excitation, viz. 10,20 and $30 \mathrm{~A} / \mathrm{mm}^{2}$, which correspond to operation within the non-linear region of the $\psi-i$ curve at various levels of saturation. As is evident, this approach provides a reasonable representation of the flux-linkage variation at intermediate positions between the unaligned and aligned position, despite the fact that the curve fitting technique employed is based solely on data for the aligned and unaligned positions. 


\subsubsection{SIMULATION STAGE 2 - Solution}

Having established appropriate representations for the $\psi-i-\theta$ characteristics of the SR machine, the dynamic performance in terms of the current waveform and hence power, can be determined for a given combination of winding design, supply voltage and rotor speed by solving the governing electrical circuit equation:

$$
V=i R+\frac{d \psi}{d t}
$$

For the case of a non-linear $\psi$-i- $\theta$ relationship, an iterative time-stepped approach is required if the resistive voltage drop across the winding is to be included. However in many machines, efficiency considerations during the design process dictate that the resistive voltage drop is very low compared to the DC link voltage [3.11]. As a consequence, adequate levels of accuracy in terms of predicting the current waveform may well be achievable by neglecting the resistance, in which case the calculation of current and flux linkage can be de-coupled. One further simplification that can be introduced to reduce the computational requirement is to neglect the influence of any mutual coupling that exists between the individual phases. It has been demonstrated that in the case of well designed SR machines, the mutual coupling between phases is very low [3.2] and the error associated with adopting a de-coupled phase model is generally acceptable [3.7]. On the basis of these potential simplifications, it is possible to identify a range of techniques of increasing sophistication for solving the governing electrical circuit equation. Table 3.6 contains a summary of 3 potential techniques: 


\begin{tabular}{|c|c|c|l|}
\hline & $\begin{array}{c}\text { Resistive } \\
\text { Voltage Drop }\end{array}$ & $\begin{array}{c}\text { Mutual } \\
\text { Coupling }\end{array}$ & \multicolumn{1}{|c|}{ Basis of Technique } \\
\hline EC1 & $\boldsymbol{x}$ & $\boldsymbol{x}$ & $\begin{array}{l}\text { De-coupled phase based sequential, } \\
\text { time stepped solution }\end{array}$ \\
\hline EC2 & $\checkmark$ & $\boldsymbol{x}$ & $\begin{array}{l}\text { De-coupled phase based iterative, time } \\
\text { stepped solution }\end{array}$ \\
\hline EC3 & $\checkmark$ & $\checkmark$ & $\begin{array}{l}\text { Circuit coupled solution (finite } \\
\text { element based) }\end{array}$ \\
\hline
\end{tabular}

Table 3.6. Summary of electrical circuit simulation techniques.

Solution methods EC1 and EC2 can in principle be used in conjunction with any of the techniques shown previously in table 3.3 for calculating and representing the $\psi-i$ $\theta$ characteristic, while EC3 is based on a series of discrete finite element magnetostatic solutions for each time step within an electric circuit model. In this study only two combinations have been investigated in detail and employed during the machine and control system design synthesis, viz. EC1 in conjunction with FL2 and EC2 in conjunction with FL4. These two particular combinations were selected on the basis that $\mathrm{EC} 1$ allows a large number of operating conditions to be simulated very rapidly, albeit with some simplifications, while EC2 allows consideration of the major nonlinearities without an excessive computational overhead. It is convenient to classify the FL2/EC1 and FL5/EC2 combinations as linear and non-linear models respectively.

\subsection{Linear Simulation Model}

The use of a linear model allows the three principal machine operating variables, viz. phase current, flux linkage and rotor position to be fully represented as a single, two dimensional, relationship. The linearity of the $\psi-i$ relationship is exploited and the machine is fully characterised by the phase inductance and rotor position. Hence the 
calculation of phase current and flux linkage can be de-coupled. However, the calculation of the instantaneous value of flux-linkage is useful in order to provide the means by which the co-energy, and hence torque can be determined, and to provide an estimation of the flux density waveforms within the stator and rotor. This latter feature is useful both for iron loss calculations and to assess the likely degree of magnetic saturation, and thus the validity of the linear model. The calculation of the flux linkage is relatively straightforward and is achieved using the governing circuit equation, which if the resistive voltage drop is neglected reduces to:

$$
\psi=\int V_{\mathrm{dc}} d t=\int \frac{V_{\mathrm{dc}}}{\omega} . d \theta
$$

For the case of a single voltage pulse, the flux linkage variation has the form shown in figure 3.14. A key feature of this flux linkage waveform is that it is solely defined by the switch dwell angle $\theta_{\text {dwell }}$, the DC link voltage and the rotor speed, i.e. it is independent of the angular position of the rotor and the relative phasing of the commutation interval. The linear analytical model therefore provides a convenient method by which the flux density waveforms in various regions of a machine can be calculated for the purpose of estimating the iron loss and its dependency on $\theta_{\text {dwell }}$. The determination of the flux density variation within the rotor and stator by superposition of the components from each phase is discussed in detail in chapter 4.

It is interesting to note that as the excitation is increased to a level at which significant magnetic saturation occurs, the largest errors are likely to be apparent in the phase current, rather than the flux linkage. Thus, providing the resistive voltage drop is small compared to the supply voltage, the linear model, despite its drawbacks, is likely to prove useful for iron loss prediction even under conditions of magnetic saturation. 


\subsubsection{CURRENT CALCULATION}

The current waveform can be calculated analytically by considering the variation of inductance with angular position in terms of three distinct regions, as shown in figure. 3.15. By representing the flux-linkage in terms of the product of the phase current and the appropriate value of inductance, and neglecting the resistive voltage, the governing electrical circuit equation can be written as:

$$
V_{\mathrm{dc}}=\omega \frac{d[i L(\theta)]}{d \theta}
$$

When the rotor is located within regions 1 and 3 of figure 3.15, i.e. when the rate of change of inductance is zero, the currents at a given rotor angle are given straightforwardly by equations 3.9 and 3.10 respectively:

$$
\begin{aligned}
& i(\theta)=\int \frac{V_{\mathrm{dc}}}{\omega L_{u}} d \theta+i_{0} \\
& i(\theta)=\int \frac{V_{\mathrm{dc}}}{\omega L_{a}} d \theta+i_{0}
\end{aligned}
$$

where $i_{0}$ is the initial current in the winding at the beginning of the respective region. The calculation of current in region 2 requires the full solution of equation 3.8 in which the rate of change of inductance is assumed to be linear, i.e.:

$$
\frac{d L(\theta)}{d \theta}=\frac{L_{a}-L_{u}}{\xi_{2}-\xi_{1}}
$$

which gives rise to the non-homogeneous first order differential circuit equation:

$$
\frac{d i}{d \theta}+\frac{L_{a}-L_{u}}{L_{u}\left(\xi_{2}-\xi_{1}\right)+\left(L_{a}-L_{u}\right)\left(\theta-\xi_{1}\right)} i=\frac{V_{\mathrm{dc}}\left(\xi_{2}-\xi_{1}\right)}{\omega\left[L_{u}\left(\xi_{2}-\xi_{1}\right)+\left(L_{a}-L_{u}\right)\left(\theta-\xi_{1}\right)\right]}
$$


When the phase winding is excited prior to the rotor passing angular position $\xi_{l}$ and the current at this instant, $i_{\xi_{1}}$ is non-zero, the variation of current with rotor angle within region 2 is given by:

$$
i(\theta)=\frac{V_{\mathrm{dc}}\left(\xi_{2}-\xi_{1}\right)\left(\theta-\xi_{1}\right)}{\omega\left[L_{u}\left(\xi_{2}-\xi_{1}\right)+\left(L_{a}-L_{u}\right)\left(\theta-\xi_{1}\right)\right]}+\frac{i_{\xi_{1}} L_{u}\left(\xi_{2}-\xi_{1}\right)}{L_{u}\left(\xi_{2}-\xi_{1}\right)+\left(L_{a}-L_{u}\right)\left(\theta-\xi_{1}\right)}
$$

Under some operating conditions, the excitation can be applied when the rotor is already within region 2 , in which case equation 3.13 can be simplified to:

$$
i(\theta)=\frac{V_{\mathrm{dd}}\left(\xi_{2}-\xi_{1}\right)\left(\theta-\theta_{\mathrm{on}}\right)}{\omega\left[L_{u}\left(\xi_{2}-\xi_{1}\right)+\left(L_{a}-L_{u}\right)\left(\theta-\xi_{1}\right)\right]}
$$

\subsubsection{TORQUE CALCULATION}

In terms of establishing the output power capability, the key quantity of interest is the average torque generated over one cycle, which is given by [3.7]:

$$
T_{\mathrm{ave}}=\frac{q_{r} q_{s}}{2} \cdot \frac{\Delta W^{\prime}}{2 \pi}
$$

Where $q_{r}$ and $q_{s}$ are the number of rotor and stator teeth respectively and the coenergy $W^{\prime}$ is calculated using equation 1.1 in section 1.7 


\subsection{Non-Linear Simulation Model}

In this case, the calculation of flux linkage and phase current cannot be de-coupled, and hence a time-stepped approach is required, in which the following procedure is undertaken at each time step.

1. Calculation of the flux linkage from the governing circuit equation using the phase current obtained from the previous time step.

2. For a given rotor position (which is assumed to have a linear relationship with respect to time) the appropriate region of the $\psi-\theta$ variation of figure 3.12 is identified.

3. Calculation of the phase current by inverting the electrical circuit appropriate to the rotor position. The method for calculating current differs in each case; regions I and III requiring the flux linkages from the previous time step, while for region II the calculation is independent of the excitation from the previous time step

4. Calculation of the aligned and unaligned flux linkages for the given phase current, which act as boundary conditions for performing step 3 in the subsequent time step.

5. Calculation of performance related quantities such as instantaneous copper loss and stored magnetic energy.

The exact implementation and calculation techniques for each of the rotor positions for the saturated and unsaturated cases are discussed in detail by Miller and McGilp[3.7] and are not included in this thesis for brevity.

\subsubsection{PHASE WINDING RESISTANCE}

The phase resistance for a given machine design is calculated assuming that the stator teeth are parallel-sided and that the end-windings take a semicircular path around the end of each tooth as shown in figure 3.16. The total conductor area for 
one half-slot, $A_{s l o t}$ and the mean length of a single turn, $l_{c u}$, are given by equations 3.16 and 3.17 respectively.

$$
\begin{gathered}
A_{s l o t}=\frac{\left(2 \pi-\alpha_{s} q_{s}\right) d_{s} D_{s}}{q_{r} q_{s}} \\
l_{c u}=2 L_{a x}+\frac{\pi D_{s}}{2}\left[\sin \left(\frac{2 \pi-q_{s} \alpha_{s}}{2 q_{s}}\right)+\sin \left(\frac{\alpha_{s}}{2}\right)\right]
\end{gathered}
$$

The resistance of a phase comprising two individual series connected windings each with $N_{p h} / 2$ turns is given by:

$$
R_{\mathrm{ph}}=\frac{N_{p h}^{2}}{2} \frac{\rho_{c u} l_{c u}}{k_{p} A_{s l o t}}
$$

where $k_{p}$ is the packing factor of the winding, which in this study was assumed to be 0.35 , a value which is typical of many medium power electrical machines. Table 3.7 summarises the calculated parameters and the resistance of the coil.

\begin{tabular}{|l|l|}
\hline Slot area, $A_{\text {slot. }}$ & $50.2 \mathrm{~mm}^{2}$ \\
\hline Mean turn length, $l_{c u}$. & $14.6 \mathrm{~mm}$ \\
\hline Resistance of basic (2 turn) winding, $R_{p h, 2 \cdot} \cdot$ & $0.28 \mathrm{~m} \Omega$ \\
\hline
\end{tabular}

Table 3.7. Summary of the winding geometry and resistance.

The calculation of the phase winding resistance is further complicated by reduced depth of penetration of the winding at high frequencies. The skin depth of a conductor, $\delta$, is given by [3.12]: 


$$
\delta=\frac{1}{\sqrt{\pi f \mu \sigma}}
$$

Where $\mu$ is the permeability and $\sigma$ the conductivity of the conductor. For the rotor at $100000 \mathrm{rpm}$, and assuming a sinusoidal current (at a frequency of $6.66 \mathrm{kHz}$ ) the skin depth is $0.81 \mathrm{~mm}$ Wire diameters much greater than twice this skin depth will yield little benefit in terms of current density reduction in the conductor cross section, and under these conditions the value of resistance used in the simulation must reflect this.

\subsection{Post-Processor Stage}

Having established the dynamic current waveforms, either from linear or non-linear electric circuit simulations, the final stage is to employ these waveforms to calculate performance figures (e.g. average torque, copper loss) and/or to finalise design features such as the number of winding turns per phase required to achieve the desired torque capability with a given supply.

\subsubsection{WINDING DESIGN}

The design of a winding for an SR machine, i.e. selection of an appropriate number of turns and their gauge, is arguably more difficult than is the case for machines with separate excitation. Although SR machines can accommodate a very wide range of winding parameters (there being an inherent degree of self-compensation in such self-excited machines), the performance achieved in terms of inverter VA, copper loss and maximum power capability will vary significantly for different windings. Further, the commutation angles provide an additional and useful degree-of-freedom with which control of the machine can be exercised. To cater for these factors, and to provide a realistic estimate of the likely number of turns, recourse to at least linear dynamic simulation is required. In contrast, for machines with permanent magnet and/or wound field excitation the requirement to match the induced emf to the terminal phase voltage severely constrains the winding design. In these machines, a 
reasonably good estimate of the number of turns can be made using essentially first order analysis, although recourse to more detailed analysis to fine-tune the winding design is often necessary.

In terms of initial winding design, the linear simulation is particularly useful in that the average torque figures for a given operating speed and combination of commutation angles can be readily scaled with respect to the DC link voltage and the number of turns. Thus, the linear simulation is useful in generating normalised performance data, which can be used to rapidly and efficiently identify the likely optimal number of turns to meet a given criterion, e.g. minimum copper loss and/or inverter VA. Having established an estimate of the most appropriate winding design, this can be refined using a non-linear simulation for a particular winding design.

The first stage in analysing the performance of the machine, with a view to designing the winding and identifying preferred commutation angles, is to develop a normalised torque characteristic for the machine by performing simulations for all feasible combinations of $\theta_{\text {on }}\left(-45^{\circ}\right.$ to $\left.+45^{\circ}\right)$ and $\theta_{\text {dwell }}\left(0\right.$ to $\left.+45^{\circ}\right)$ in $2.5^{\circ}$ increments, for the particular case of 1 turn per tooth (i.e. $\mathrm{N}_{\mathrm{ph}}=2$ ) and a DC link voltage of $1 \mathrm{~V}$. The resulting normalised output torque characteristic, $T_{\text {norm}}$, (with units of $\mathrm{Nm} \mathrm{rad}^{2} \cdot \operatorname{turn}^{2} / \mathrm{V}^{2} \mathrm{~s}^{2}$, where $\omega=1$ ) as a function of both $\theta_{\text {on }}$ and $\theta_{\text {dwell }}$ is shown as a three-dimensional plot in figure 3.17, and a series of constant torque contours (in increments of approximately $4 \times 10^{5} \mathrm{Nm} \mathrm{rad}^{2} \cdot \mathrm{turn}^{2} / \mathrm{V}^{2} \mathrm{~s}^{2}$ ) in figure 3.18 .

$$
T_{\text {norm }}=\frac{\omega^{2} N_{p h}^{2} T_{a v e}}{V_{d c}^{2}}
$$

One interesting feature which is highlighted in figure 3.18 are the commutation conditions which yield zero net torque, a condition that occurs when the switch-off angle $\theta_{o f f}$, falls exactly on the aligned or unaligned position and hence the motoring power is equal and opposite to the generating power over one stroke. As is evident 
these zero torque conditions form lines of symmetry within the torque map of figure 3.18 .

As is evident from figure 3.17, there are a number of possible combinations of $\theta_{\text {on }}$ and $\theta_{d w e l l}$ which would give rise to a given desired output power. However different combinations result in varying degrees of copper loss as evidenced by figures 3.19 and 3.20 which show a three dimensional mesh and a corresponding series of contours of normalised $\mathrm{rms}$ current, $i_{n o r m}$, (in increments of approximately $1.8 \times 10^{5}$ Ampere.rad.turn ${ }^{2} / \mathrm{Vs}$ ) as a function of $\theta_{o n}$ and $\theta_{d w e l l}$, where $i_{n o r m}$ is given by:

$$
i_{n o r m}=\frac{\omega N_{p h}^{2} i_{r m s}}{V_{d c}}
$$

As a consequence, for a given output power there will one preferred combination in terms of minimum copper loss, and hence one means of discriminating between candidate combinations is to establish that which gives rise to the lowest value of rms current. This can be illustrated by considering the superposition of a particular constant power contours onto the rms current contours, as shown in figure.3.21 For the cases shown, the preferred combination of $\theta_{o n}$ and $\theta_{d w e l l}$ can be established from the intersection of the constant power contour with the lowest rms current contour that it crosses. Determining preferred values of $\theta_{o n}$ and $\theta_{d w e l l}$ is a procedure that can be readily implemented as part of the post processing of the simulation results. Figure 3.22 shows the preferred values of $\theta_{o n}$ and $\theta_{d w e l l}$ calculated for operation up to a maximum attainable normalised torque for this design of approximately $4 \times 10^{6} \mathrm{Nm}$. It should be noted that these values of $\theta_{\text {on }}$ and $\theta_{\text {dwell }}$ are derived solely with regard to minimising copper losses, whereas other factors such as iron losses may modify these preferred values somewhat.

Having established the preferred commutation conditions using normalised per turn data, the design of the winding can be undertaken. As shown previously by equation 3.7 , the rate of change of flux-linkage is determined solely by the magnitude of the 
supply voltage, and hence is independent of the number of turns providing the resistance is assumed to be negligible. However, under dynamic operating conditions at a fixed rotational speed, the rate of rise of current, and hence the magnitude of the resulting torque is limited by the inductance of the winding, which itself is proportional to the square of the number of turns. Hence, for a fixed DC link voltage and in the absence of a converter current limit, the maximum power capability at a particular speed decreases in proportion to the square of the number of turns. Therefore, in order to realise a particular maximum power capability at a given rotational speed, there is an upper limit on the number of turns which can be employed for a given combination of machine parameters, DC link voltage and commutation conditions.

The normalised power data can be employed to directly establish the maximum normalised torque output of the machine and hence place an upper limit on the number of turns for this particular machine design with a specified power of $5 \mathrm{~kW}$ and a DC link voltage of $96 \mathrm{~V}$ (both of which form part of the target specification described in chapter 1). From figure 3.17, the maximum normalised torque is approximately $4 \times 10^{6}$, which for a $5 \mathrm{~kW}$ capability with a DC link voltage of $96 \mathrm{~V}$ leads to a maximum number of turns per phase of 28 (i.e. 14 turns on each of the two coils which constitute a phase). One interesting feature of the normalisation procedure discussed and the data presented in figure 3.22 is that the ideal commutation angles for any given number of turns on the winding can be readily obtained for a given operating condition. By means of example figure 3.23 shows the commutation angles required to obtain $5 \mathrm{~kW}$ motoring power at $100,000 \mathrm{rpm}$ as a function of the number of turns on the winding, up to the limit of 28 turns.

Below this upper limit of 28 turns, there are a number of factors that must be considered in selecting an appropriate number of turns, viz. the magnitudes of the peak currents and resulting flux densities, the average copper loss and practical issues such as the ease with which the winding can be formed. 
Peak current - The variation in the magnitude of the peak current as a function of $N_{p h}$ at $96 \mathrm{~V}, 5 \mathrm{~kW}, 100000 \mathrm{rpm}$ is shown in figure 3.24 . As is evident a higher value of $N_{p h}$ is beneficial in terms of reducing the peak current, hence minimising the converter VA rating, and the peak rate of change of current (which has direct implications on electromagnetic emissions).

Copper Loss -As shown previously in figure 3.15, the inductance variation with respect to rotor angle comprises three distinct regions, of which only the central region (denoted as region 2) gives rise to a significant rate of change of inductance. For the particular machine design considered, the inductance increases between rotor angles of -32.5 and -2.5 and decreases between 2.5 and 32.5. In terms of making best utilisation of a given current, it is desirable to operate the machine such that current only flows during the period of changing inductance, since any current flow which overlaps into regions 1 and 3, essentially produces no torque. In the case of a linear model, conduction is initiated at a rotor angle $\theta_{o n}$, the switch is turned off at $\theta_{o n}+\theta_{d w e l l}$, and the current is fully extinguished at an angle $\theta_{o n}+2 \theta_{d w e l l}$.

Figure 3.25 shows the limits of the conduction period (i.e. variation of $\theta_{o n}$ and $\left.\left(\theta_{o n}+2 \theta_{d w e l l}\right)\right)$ as a function of $N_{p h}$ for an operating point of $5 \mathrm{~kW}$ motoring at $100,000 \mathrm{rpm}$ (similar behaviour is observed in the case of generating). Also shown on figure 3.25 are the regions of rising and falling inductance (labelled as motoring and generating) for the particular design. As is evident, for values of $N_{p h}$ greater than 2, current overlap into regions 1 and/or 3 occur, the extent of which increases as $N_{p h}$ is increased. Indeed, for values of $N_{p h}$ greater than 2, the current continues to flow at rotor positions which correspond to a falling inductance, which gives rise to a period of electrical generation, which detracts from the net motoring power (although a net $5 \mathrm{~kW}$ is still achieved). Hence, from the point of view of current utilisation, a value for $N_{p h}$ of 2 appears the most suitable.

The implications of this varying degree of current utilisation in terms of copper loss can be calculated. At a given operating point (i.e. output power level and speed), the 
magnitude of the copper loss for a particular value of $N_{p h}$ can be calculated from the normalised rms current values in figure 3.19 and the resistance of given winding design which is given by:

$$
P_{\mathrm{cu}}=I_{\mathrm{rms}}^{2} N_{\mathrm{ph}}^{2} \frac{\rho_{\mathrm{cu}}\left(L_{a x}+L_{\text {end_wdg }}\right)}{k_{p} A_{\text {slot }}}
$$

Figure. 3.26 shows the calculated variation of copper loss as a function of output power at $100,000 \mathrm{rpm}$ as the value $N_{p h}$ is progressively reduced from the upper limit of 28 (the characteristics being equally applicable to motoring or generating power) down to a value of 6 . The values of $\theta_{\text {on }}$ and $\theta_{\text {dwell }}$ required to minimise copper loss at a given power vary as a function of $N_{p h}$ as illustrated in figure 3.22. As is evident from figure 3.26, benefits can be achieved in terms of reducing copper loss by using a lower number of turns, by virtue of the better current utilisation. For values of $N_{p h}$ down to 6 , the improved utilisation more than compensates for the fact that the form factor of the current waveform is increasing (i.e. ratio of rms to average). However, for values of $N_{p h}$ of 6 or less, a lower $N_{p h}$ value gives rise to a higher copper loss due to the higher form factor of the waveform. This is evident in figure 3.27 which shows the variation of copper loss for values of $N_{p h}$ of 2,4 and 6 as a function of power at $100,000 \mathrm{rpm}$.Thus, on the basis of copper loss consideration a clear optimum value of 6 is obtained for $N_{p h}$.

It is interesting to note that the above analysis that gives rise to an optimal value of $N_{p h}$, is specific to the conditions of $5 \mathrm{~kW}$ at $100,000 \mathrm{rpm}$. As the power and/or speed are reduced, the ability to restrict the current flow to regions of changing inductance can be realised with higher values of $N_{p h}$, and at these operating points, a value of $N_{p h}$ equal to 6 will inevitably give a higher copper loss. This can be illustrated by using the principles discussed to calculate the optimal values of $N_{p h}$ as a function of power, as shown in figure 3.28. 
Peak flux density/iron loss. The variation of iron loss (which is discussed in detail in chapter 4) is somewhat more complex to evaluate for the various winding designs and increases as a complex and non-linear function of both the magnitude of the peak flux density and its rate of change with respect to time. For a linear simulation, the rate of flux density waveform can be simplified to that of figure 3.29 , in which the change of flux density within a region of the machine and its peak magnitude are given by equations 3.23 and 3.24 respectively:

$$
\begin{gathered}
\frac{d B}{d t}=\frac{V_{\mathrm{dc}}}{N_{\mathrm{ph}} A \omega} \\
B_{p}=\int_{\theta_{\mathrm{on}}}^{\theta_{\mathrm{m}}+\theta_{\text {omen }}} \frac{d B}{d t} d t=\frac{V_{\mathrm{dc}} \theta_{\mathrm{dwcll}}}{N_{\mathrm{ph}} A \omega}
\end{gathered}
$$

Whereas the rate of change of flux density is inversely proportional to $N_{p h}$, the relationship between $B_{p}$ and $N_{p h}$ for a given output power is somewhat more complicated since the value of $\theta_{d w e l l}$ required is a non-linear function of $N_{p h}$. As was demonstrated for the calculation of the copper loss, the switch dwell angle, $\theta_{\text {dwell, }}$, decreases rapidly as the number of phase turns is decreased, offsetting the apparent increase in iron loss. Figure 3.30 shows the variation of $B_{p}$ as a function of $N_{p h}$ based on the optimised values of $\theta_{d w e l l}$ (defined in figure 3.23) and a value of $A=502 \mathrm{~mm}^{2}$ (which corresponds to the stator teeth). Thus, as is evident from equations 3.23 and 3.24 and figure 3.30, considerable benefits are likely to be accrued in terms of reducing iron loss by employing a high value of $N_{p h}$. The absolute magnitude of the iron loss is a trade off between these generally conflicting non-linear trends and the variation of iron loss with the number of turns over the entire operating envelope remains beyond the scope of the current discussion.

Further, given that the magnitude of the peak flux density that can be practicably realised is limited by magnetic saturation within the stator and/or rotor, this consideration places a limit on the lower number of turns that can be employed. 
However, at $5 \mathrm{~kW}$ and $100,000 \mathrm{rpm}$, even a value of $N_{p h}=2$ only gives rise to a peak flux density of $1.35 \mathrm{~T}$, which is well below the saturation level of the Silicon Steel employed in the prototype. Despite not causing saturation at 100,000 rpm low values of $N_{p h}$ are likely to give rise to problems if useful levels of power are to be developed over a wide speed range. This can be illustrated by figure 3.31 which shows the variation of the lower speed at which a $B_{p}$ of $1.8 \mathrm{~T}$ is achieved as a function of $N_{p h}$ using the commutation conditions discussed while maintaining an output power of $5 \mathrm{~kW}$.

Current control and drive switching loss. Consideration of the drive circuit provides further insight into the design of the phase winding. The efficiency of the switching devices in the drive circuit will decrease as the RMS phase current is increased. Furthermore for a machine operated in current control mode the decreased time constant of a winding with fewer turns will require higher switching frequencies, and the efficiency of the drive operating under these conditions will be compromised further. The drive efficiency and dynamics require that a higher number of turns is used and may ultimately provide a lower limit on the number of turns. It is beyond the scope of the present research to provide a model of the drive circuit whereby system efficiency may be optimised by the number of turns throughout the operating range of the machine, and the discussion is limited to these qualitative issues regarding the drive circuit.

Slot area utilisation and manufacturing considerations. For machine designs where the space envelope is restricted (in this case by the existing geometry of the turbomachinery and engine) with a low number of turns, the efficient utilisation of the slot area becomes a key design issue. For the current application the windings are to be pre-formed and inserted into the machine individually. This removes the requirement for laborious in-situ winding, but the area available for the winding is greatly reduced by the need to insert each of the adjacent windings as shown in figure 3.32. Furthermore the machine design requires that the shaft and end-caps are gas sealed in order to maintain the efficiency of the compressor and turbine. The winding terminations must therefore emerge radially from the stator back iron, rather 
than axially from the end-caps as in conventional machine design. This onerous constraint requires that:

i. The number of winding layers around the stator teeth must be an integer multiple of two to allow both ends of the winding to emerge through the stator back iron, i.e. for a two layer winding the central layer is made with an external termination emerging from the stator, the second layer can then begin at the tooth tip and upon completion the connection can again be made through the stator.

ii. The series phase connections must be made externally.

It is apparent from the preceding discussion that the selection of the number of turns for SR machines, although much less constrained by the requirement to match the induced emf to the supply voltage as in brushless dc machines, is a critical parameter in terms of overall system optimisation. The upper and lower limits of $N_{p h}$ for the current application have been established as 28 and 6 respectively, figures based on the maximum power attainment and the minimisation of the copper loss at the highest speed and power. It is possible to determine the optimum number of turns based upon the criteria defined above with weightings applied to each parameter (e.g. converter VA ratings and cost and overall system efficiency etc.). This apparent optimum will however inevitably be specific to a given duty cycle. Given that the operating duty cycle is difficult to establish at this stage, for the current application $N_{p h}=16$ was selected, on the basis that it is a reasonable compromise between the conflicting requirements identified above. For an $N_{p h}$ of 16 , the resulting power 'map' for the machine at 100 000rpm calculated using the linear simulation is shown in figure.3.33. Also shown in figure 3.33 are the commutation angles derived using the techniques discussed in section 3.7.1.

The use of 16 turns per phase (eight turns per pole) allows the winding to be conveniently arranged as two layers of four conductors in the slot, as shown in figure 3.34. For the current slot aspect ratio, i.e. depth initially defined as $10 \mathrm{~mm}$ (discussed in section 3.2) and a width of $4.6 \mathrm{~mm}$, this winding arrangement allows efficient utilisation of the available slot area with conductor diameters within the 
range $1.5 \mathrm{~mm}$ to $2 \mathrm{~mm}$. Although a diameter of $2 \mathrm{~mm}$ would be preferable in terms of minimising the copper loss, practical winding trials established that a maximum diameter of $1.7 \mathrm{~mm}$ was feasible. This reduction of diameter to $1.7 \mathrm{~mm}$ was largely required to produce sufficiently compact end-windings such that they could be accommodated within the recesses in the machine end-caps (as discussed in chapter 7).

\subsubsection{CURRENT WAVEFORMS AND RESULTS FROM NON-LINEAR MODEL.}

Having established the number of turns using the linear model, the phase resistance can be calculated, hence allowing the non-linear simulation to be employed. In order to generate current waveforms for direct comparison with a linear simulation, a series of linear and non-linear simulations were performed for a machine with 16 turns at a fixed speed of $100,000 \mathrm{rpm}$, using 11 values of $\theta_{\text {on }}\left(-44.6^{\circ}\right.$ to $-24.6^{\circ}$ in $2.0^{\circ}$ increments) and a fixed value of $\theta_{d w e l l}$ of $20^{\circ}$. The current waveforms calculated by both methods are compared in figure. 3.35, while figure 3.36 shows the variation of the average calculated power as a function of $\theta_{o n}$. One interesting feature of figure 3.35 is the particular waveform in the linear case where the phase current remains constant during the fluxing period when the inductance is rising. This condition is achieved when the applied voltage and back emf are equal. The switch-on angle, $\theta_{o n}$, required to achieve this condition, remains fixed for a given machine design $\left(-34.6^{\circ}\right.$ for this particular case) and for the general case is given by:

$$
\theta_{\mathrm{on}}=\frac{L_{a} \xi_{1}-L_{u} \xi_{2}}{L_{a}-L_{u}}
$$

Under these operating conditions, the agreement between the linear and non-linear simulation methods is satisfactory in terms of both average torque and current, with a maximum error in the calculated torque for example of some $-17 \%$ in the case of $\alpha=$ -44.6. The general agreement between the two simulation methods is a consequence 
of the relatively low peak flux densities in the machine and hence the absence of any significant magnetic saturation. These low flux densities in turn result from the short time intervals involved at this high speed. The peak flux density in the stator tooth (the region most susceptible to saturation) can be estimated using the specific form of equation 3.24 , i.e.:

$$
B_{p}=\frac{V_{\mathrm{dc}} \theta_{\mathrm{dwell}}}{\omega N_{\mathrm{ph}} L_{a x} D_{s} \sin \left(\alpha_{s} / 2\right)}
$$

By means of example, for the conditions shown in figure 3.35 for the linear simulation, i.e. $\theta_{\text {dwell }}=20.0$ and $\omega=10472$, the peak stator tooth flux density is only some $0.4 \mathrm{~T}$. Figure 3.37 shows a performance map at $100,000 \mathrm{rpm}$ for the 16-turn machine with a dc link voltage of $96 \mathrm{~V}$ calculated using the non-linear simulation. Again the commutation conditions corresponding to the maximum power output per unit RMS current squared are superimposed upon the power contours. The commutation conditions are in good general agreement with those calculated using the linear model (presented in figure 3.33) under these conditions. The only discrepancy is that for values obtained with very small dwell angles, where the error is attributable to the initial (and final) conditions imposed on the non-linear simulation and the ill conditioning of the ratio of output power and the square of the RMS current in the non-linear model.

If the speed is reduced to $20,000 \mathrm{rpm}$, the peak flux density derived from equation 3.26 increases to approximately $2.0 \mathrm{~T}$ for the same commutation angles. With this flux density level in the linear simulation, there is a considerable discrepancy between the current waveforms and the average torque values predicted by the two simulation methods, as evidenced by figure 3.38 and figure 3.39 respectively. The phase current rises to much higher levels in the non-linear model, due to the reduction in the effective inductance caused by the saturation of the magnetic circuit, further the non-linear model generally predicts higher power levels for the same commutation conditions. By means of example, the average torque calculated using 
the linear method shows a maximum discrepancy of $158 \%$ when compared to the value calculated using the non-linear model when $\theta_{o n}=-32.6\left(\theta_{\text {dwell }}=20.0\right)$.

On the basis of the predicted performance at $100,000 \mathrm{rpm}$ and $20,000 \mathrm{rpm}$, it can be anticipated that the linear model is likely to be adequate for operating conditions in which the peak flux density is less than some $1.6 \mathrm{~T}$, which is satisfied if the current rises for a period of less than $0.13 \mathrm{~ms}$. (The duration of the period during which current rises is given by $\theta_{d w e l l} / \omega$.)

The range of speeds over which the linear model is likely to be valid can be investigated by calculating for example, the peak current and output power using each of the models for a range of operating conditions at constant commutation angles. A useful means of comparing the techniques is to calculate the percentage discrepancies in peak current and output power, which are defined as:

$$
\begin{gathered}
\text { Discrepancy } i(\%)=100 \frac{\hat{i}_{n l}-\hat{i}_{l}}{\hat{i}_{l}} \\
\text { Discrepancy } P(\%)=100 \frac{P_{n l}-P_{l}}{P_{l}}
\end{gathered}
$$

Where the subscripts $n l$ and $l$ denote the values calculated using the non-linear and linear models respectively.

Figure 3.40 shows the calculated percentage discrepancies as a function of rotor speed in which the commutation conditions correspond to those discussed above, i.e. $\theta_{o n}=-34.6^{\circ}$ and $\theta_{\text {dwell }}=20^{\circ}$. It is apparent that the correlation of the two models is reasonably good at high speeds for this dwell angle. However at low speeds (i.e. $<35,000 \mathrm{rpm}$ or so) even the $20^{\circ}$ dwell angle yields a high degree of saturation, and the linear model underestimates both the peak current and output power of the machine. Indeed at the lowest operating speed the peak current predicted by the nonlinear model is approximately five times the value calculated using the linear model. 
Given that this degree of discrepancy will be present at even higher speeds as the dwell angle is increased, it is apparent that at least some of the performance mapping of the device required to establish the best commutation angle combinations will require the use of the non-linear simulation.

\subsubsection{OPTIMAL COMMUTATION ANGLES}

Having demonstrated the need for non-linear simulation over part of the operating range of the machine, a comprehensive and systematic series of non-linear simulations were performed in order to establish optimal commutation angles. To this end, the process was automated so that the penalty in employing non-linear simulations even for cases where a linear simulation would arguably have been sufficient, was incurred purely in processing time rather than user interaction.

Clearly, given the three input variables involved, viz. speed, $\theta_{o n}$ and $\theta_{d w e l l}$, a vast array of data was generated. In the vast majority of cases, the best combination of commutation angles can be directly established by the simple application of two criteria, highest efficiency (in terms of copper loss only) in conjunction with minimum peak current. However, as will be discussed in chapter 7, it is highly desirable in terms of real-time computational overhead in the control system and the sensitivity of the performance to commutation angle, if a series of simple curve fits at various speeds is employed to map the commutation angles as a function of power rather than a 2D look up table with interpolation. Thus, a graphical user interface, an example of which is shown in figure 3.41, was developed in order to allow manual refinement of the commutation angles automatically selected, such that a smooth curve fit could be employed. In a few cases, this necessarily gave rise to some small compromises in efficiency on order to simplify the implementation of the control system The resulting optimal commutation angle characteristics, which form the basis of the control algorithm described in chapter 7 are shown in figures $3.42 \mathrm{a}$ and $3.42 \mathrm{~b}$. 


\section{REFERENCES}

[3.1] Miller T. J. E., 'Switched reluctance machines and their control.' Magna Physics, 1993.

[3.2] Lawrenson, P. J., Stephenson, J. M., Blenkinsop, P. T., Corda, J., and Fulton, N. N., 'Variable-speed switched reluctance motors.' Proc. IEE Vol 127, Pt. B, July 1980. pp253-265.

[3.3] Radun, A. V., 'Design considerations for the switched reluctance motor.' IEEE Trans. on Ind. Appl. Vol. 31, No. 5, Sept. 1995.

[3.4] Faiz, J. and Finch, J. W., "Aspects of design optimisation for switched reluctance motors." IEEE Trans. on Energy Conversion, Vol. 8, No 4, Dec 1993, pp 243-258.

[3.5] Radun A. V., "High power density switched reluctance motor drive for aerospace applications." IEEE Trans. on Ind. Appl. Vol. 28, No 1, Jan 1992, pp113-119.

[3.6] Mese, E., Sozer, Y., Kokernak, J. M. and Torrey, D. A., "Optimal excitation of a high speed switched reluctance generator." Proceedings of the Fifteenth Annual IEEE Applied Power Electronics Conference and Exposition. Vol. 1, 2000, pp362-368.

[3.7] Miller, T. J. E., and McGilp, M., 'Nonlinear theory of the switched reluctance motor for rapid computer-aided design.' Proc IEE Vol. 137 Pt. B, Nov. 1990, pp337-347.

[3.8] Krishnan, R., Arumugam, R. and Lindsay, J. F., 'Design procedure for Switched-Reluctance motors.' IEEE Trans. on Ind. Appl. Vol 24, No 3, June 1998, pp456-461.

[3.9] "Non-oriented electrical steels". Publication of European Electrical Steels, 1992.

[3.10] Low, W. F., "The computation of magnetostatic fields in permanent magnet devices." Ph. D., University of Sheffield, 1985.

[3.11] Ray, W. F. and Davis, R. M., 'Inverter drive for doubly salient reluctance motor: its fundamental behaviour, linear analysis and cost implications.' IEE Jnl. Electric Power Applications, Vol. 2, No 6, Dec. 1979 pp185-193. 
[3.12] Hayt, W. H. Jnr. "Engineering electromagnetics." 5th edition, McGraw-Hill, 1989.

[3.13] Gyorki, J. R. 'Design secrets of switched-reluctance motors.' Machine Design, Sept. 1996. pp87-91. 


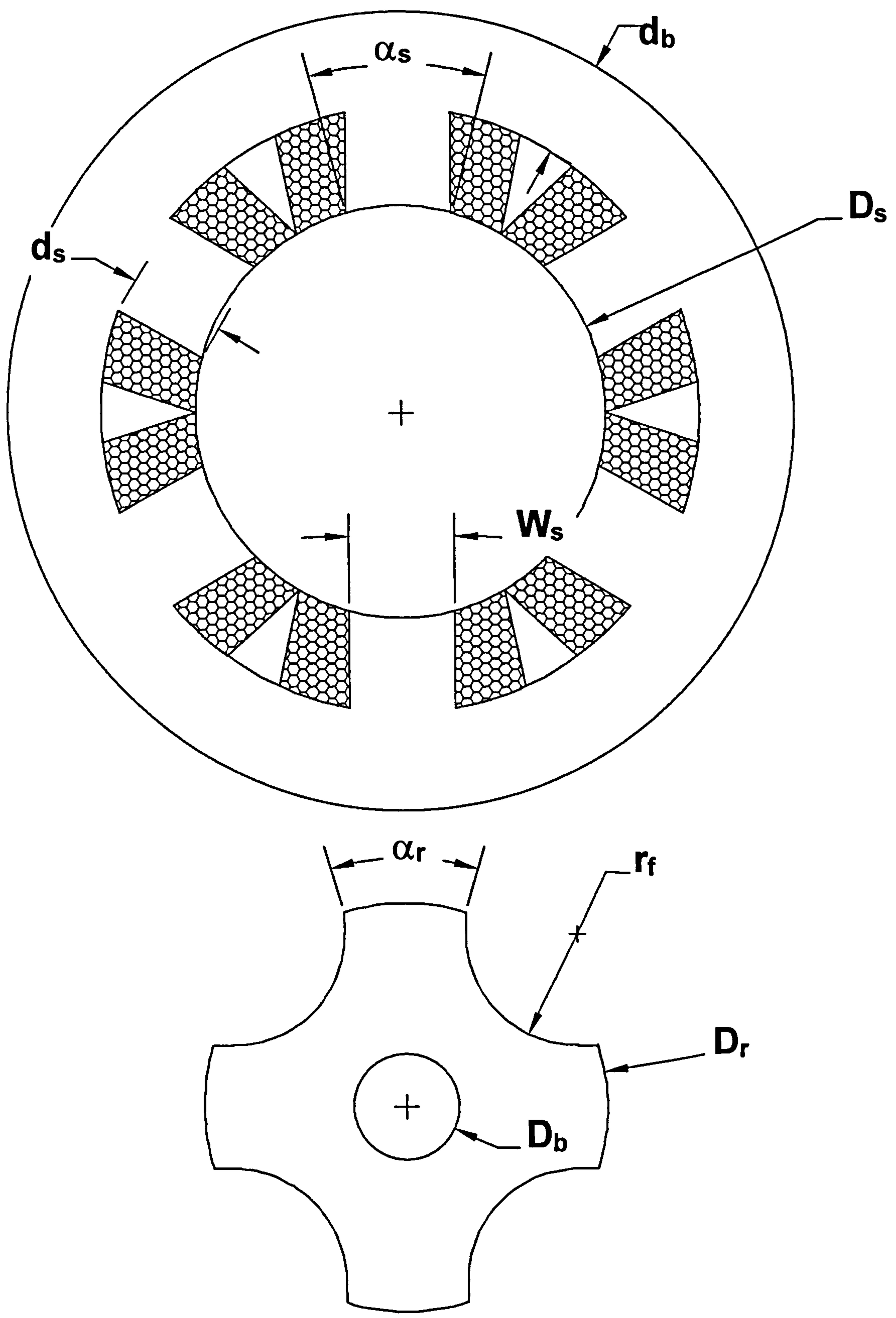

Figure 3.1 Principal dimensions of the 6/4 SR machine stator and rotor. 


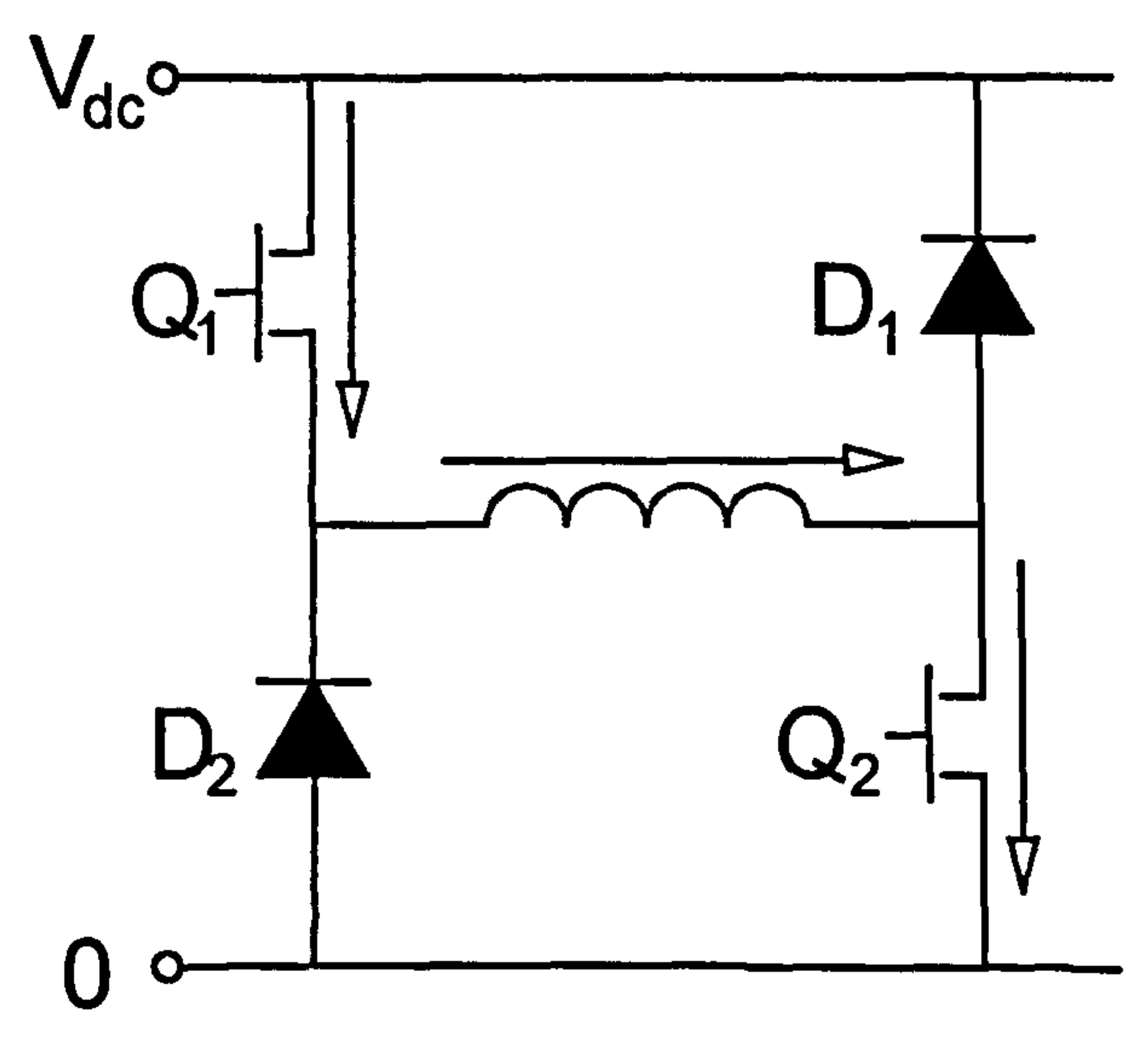

Figure 3.2a. $Q_{1}$ and $Q_{2}$ on (fluxing)

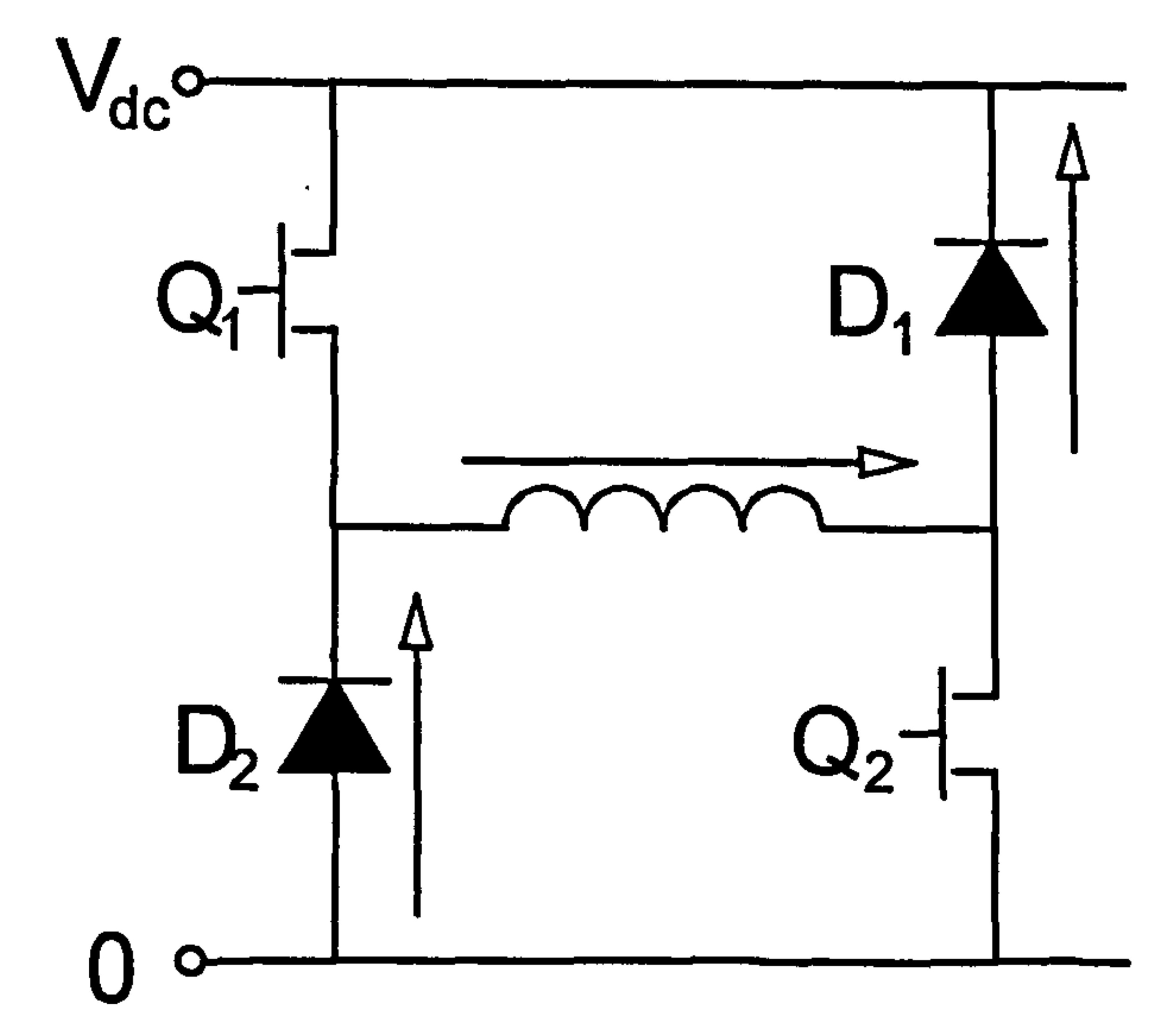

Figure 3.2b. $D_{1}$ and $D_{2}$ on (freewheeling/de-fluxing)

Figure 3.2. SR drive circuit operation. Reference [3.13] 


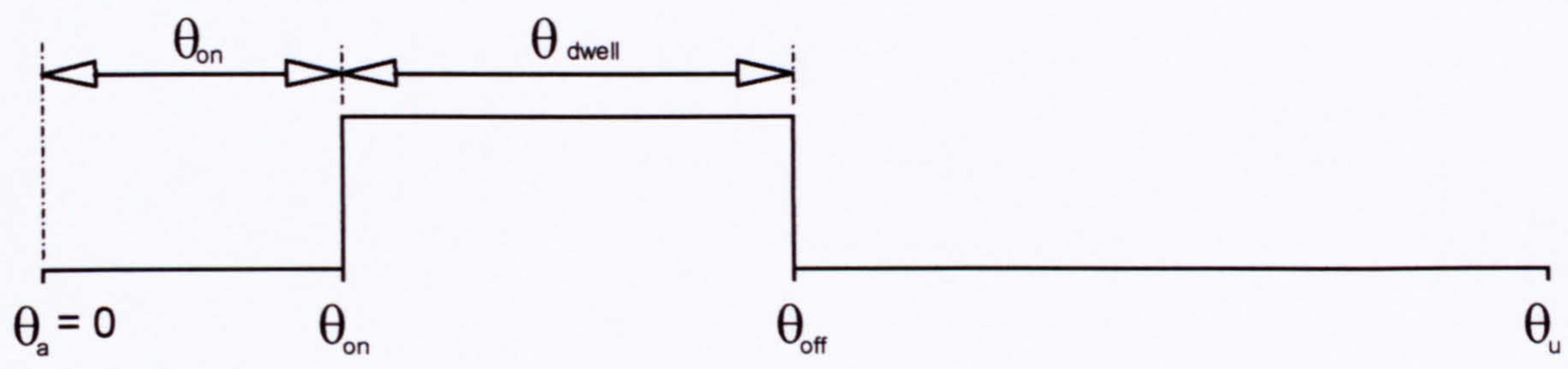

Figure 3.3 Commutation logic for high speed operation.

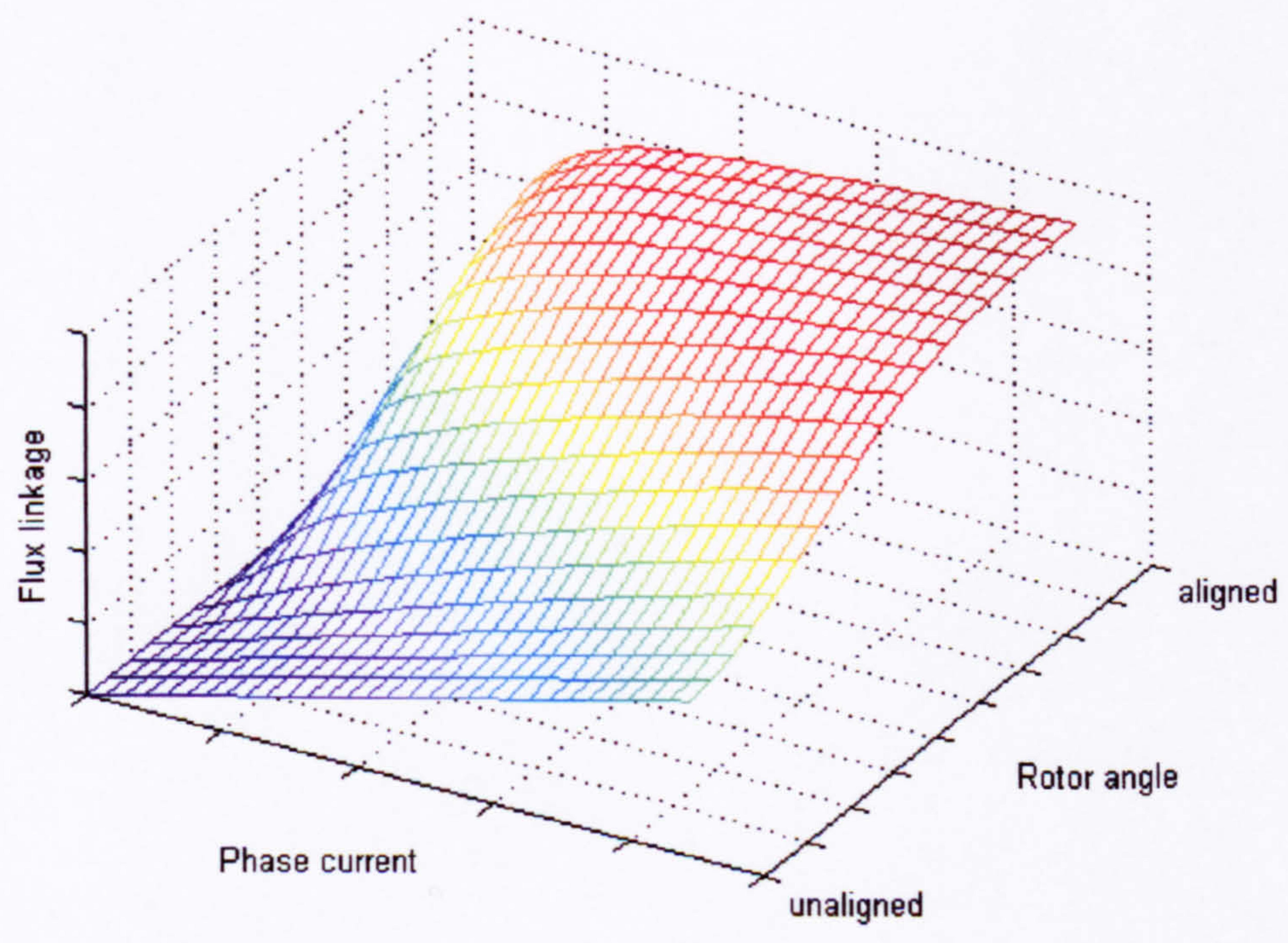

Figure 3.4. Variation of phase flux linkage with phase current and rotor position. 


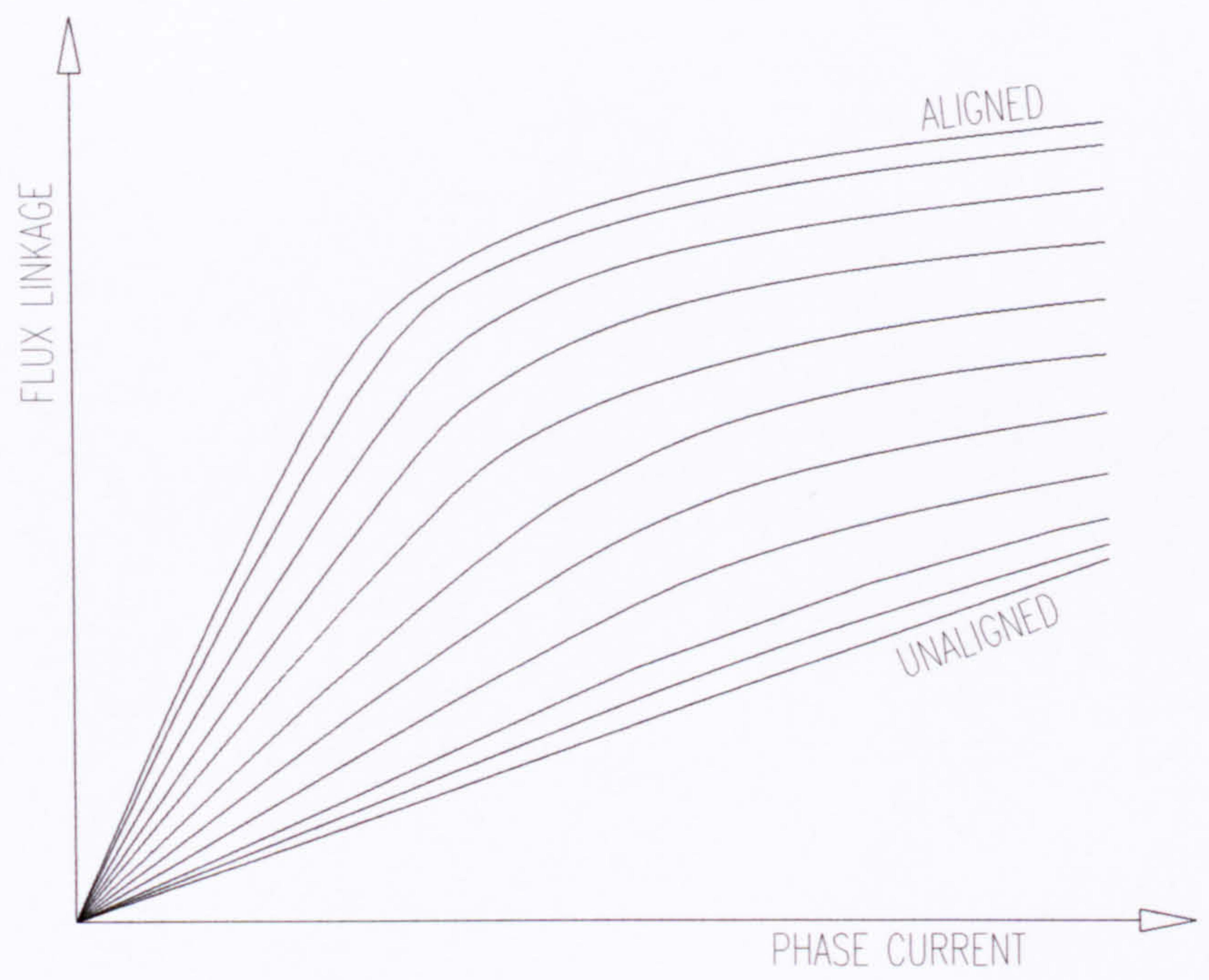

Figure 3.5a Flux linkage variation as a function of current at constant rotor position.

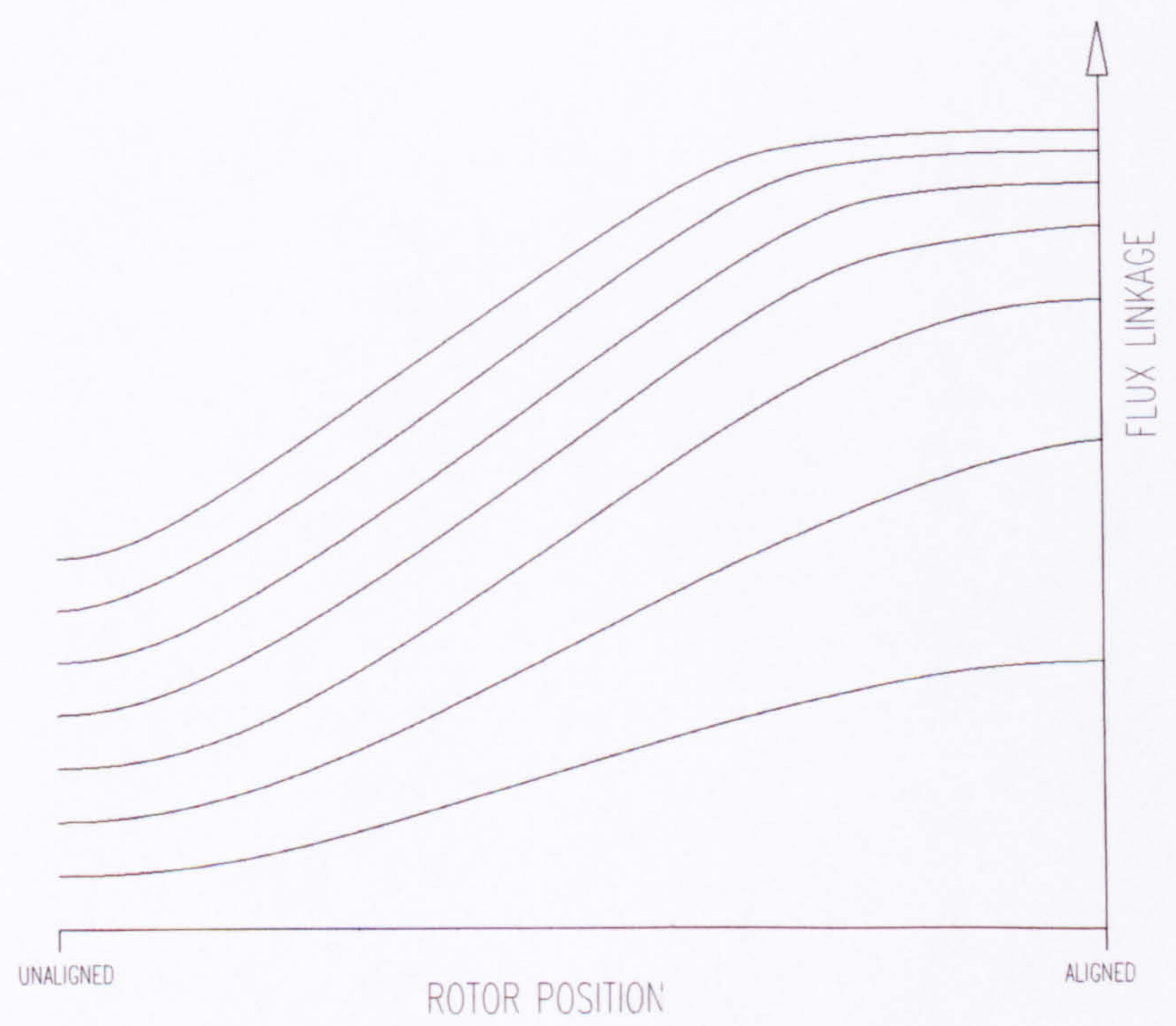

Figure $3.5 \mathrm{~b}$ Flux linkage variation as the rotor moves from the unaligned to the aligned rotor position at constant current. 


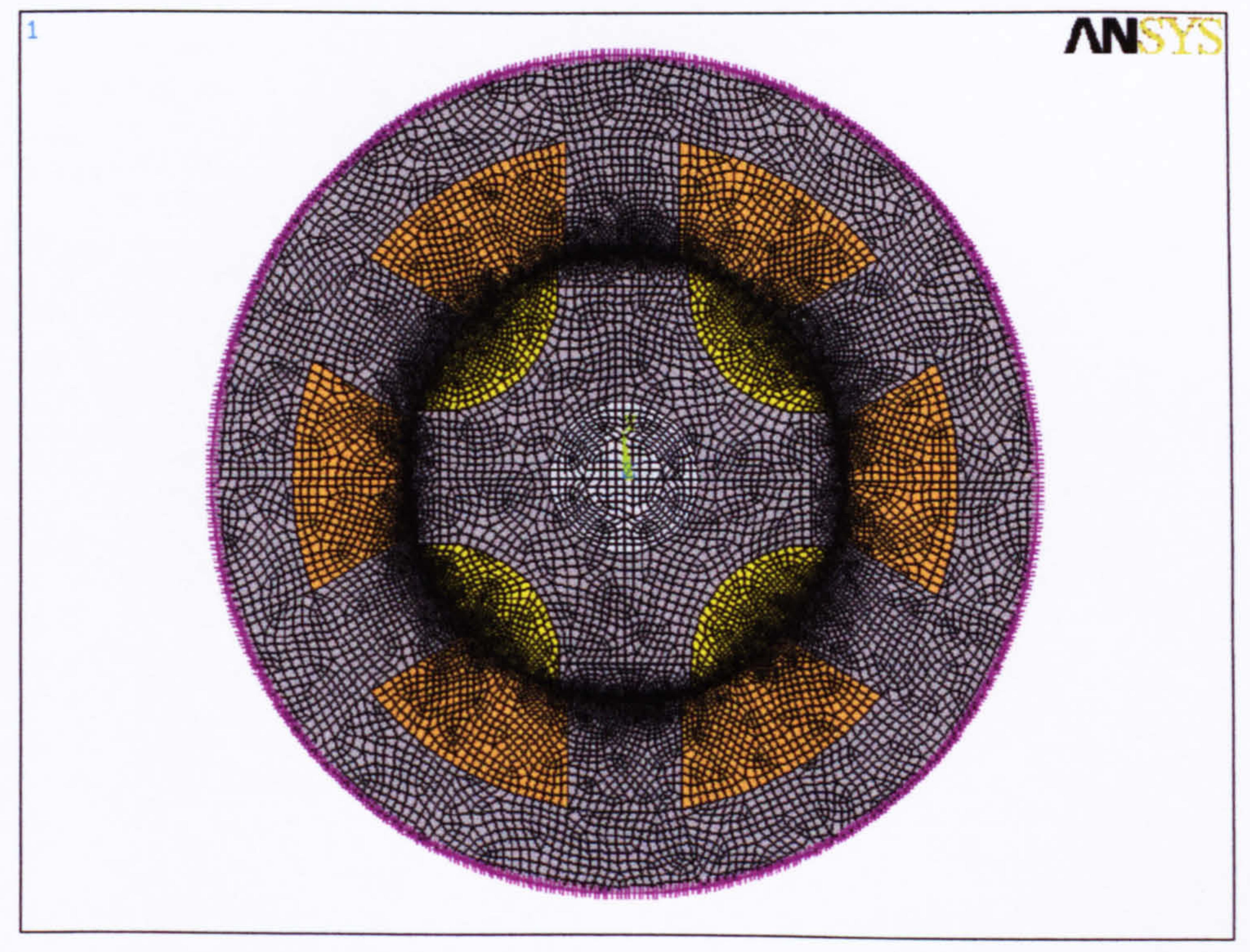

Figure 3.6a Finite element mesh of the SR machine consisting of 17468 second order elements and 52659 nodes.

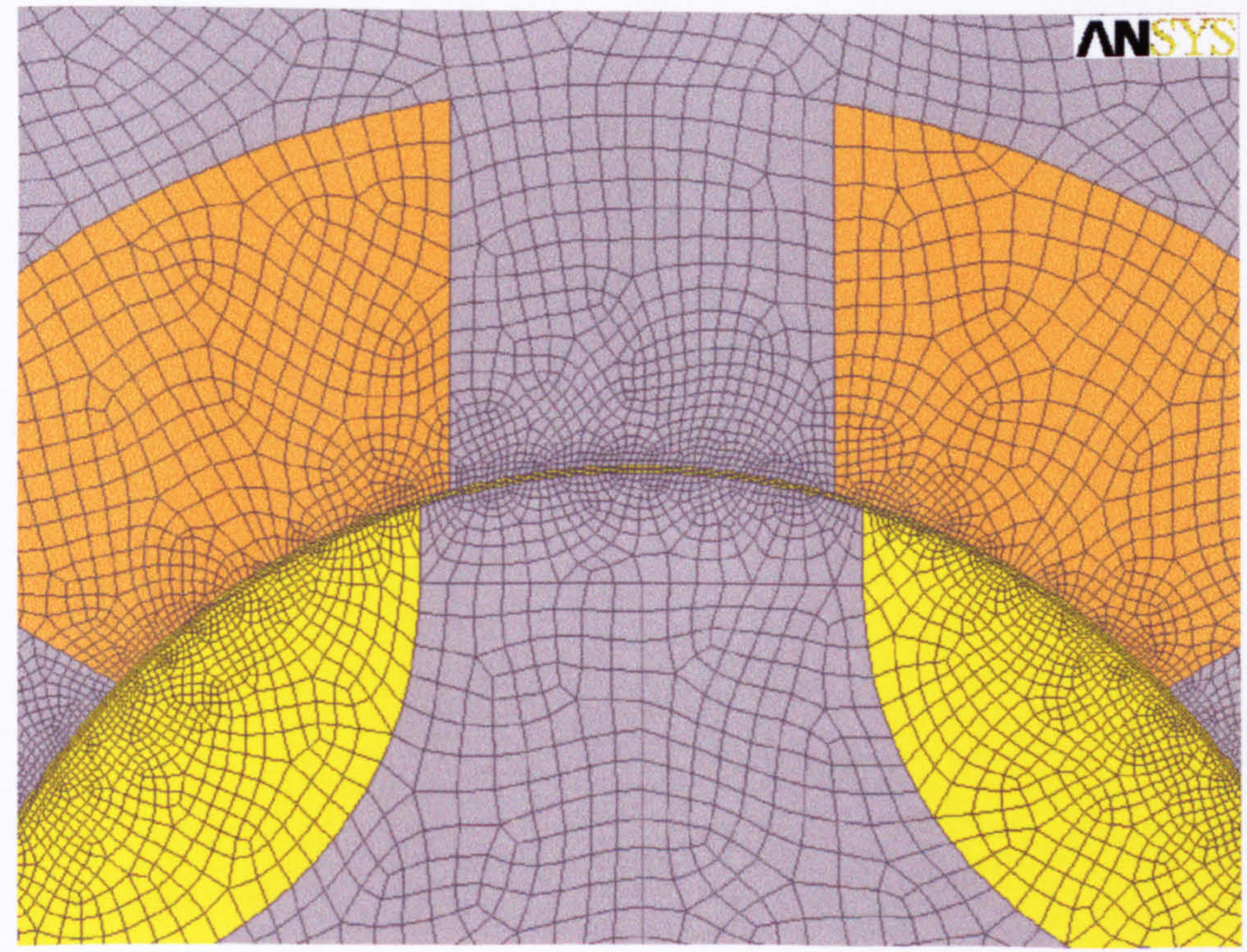

Figure 3.6b Detailed view of the finite element mesh of figure 3.6a. 


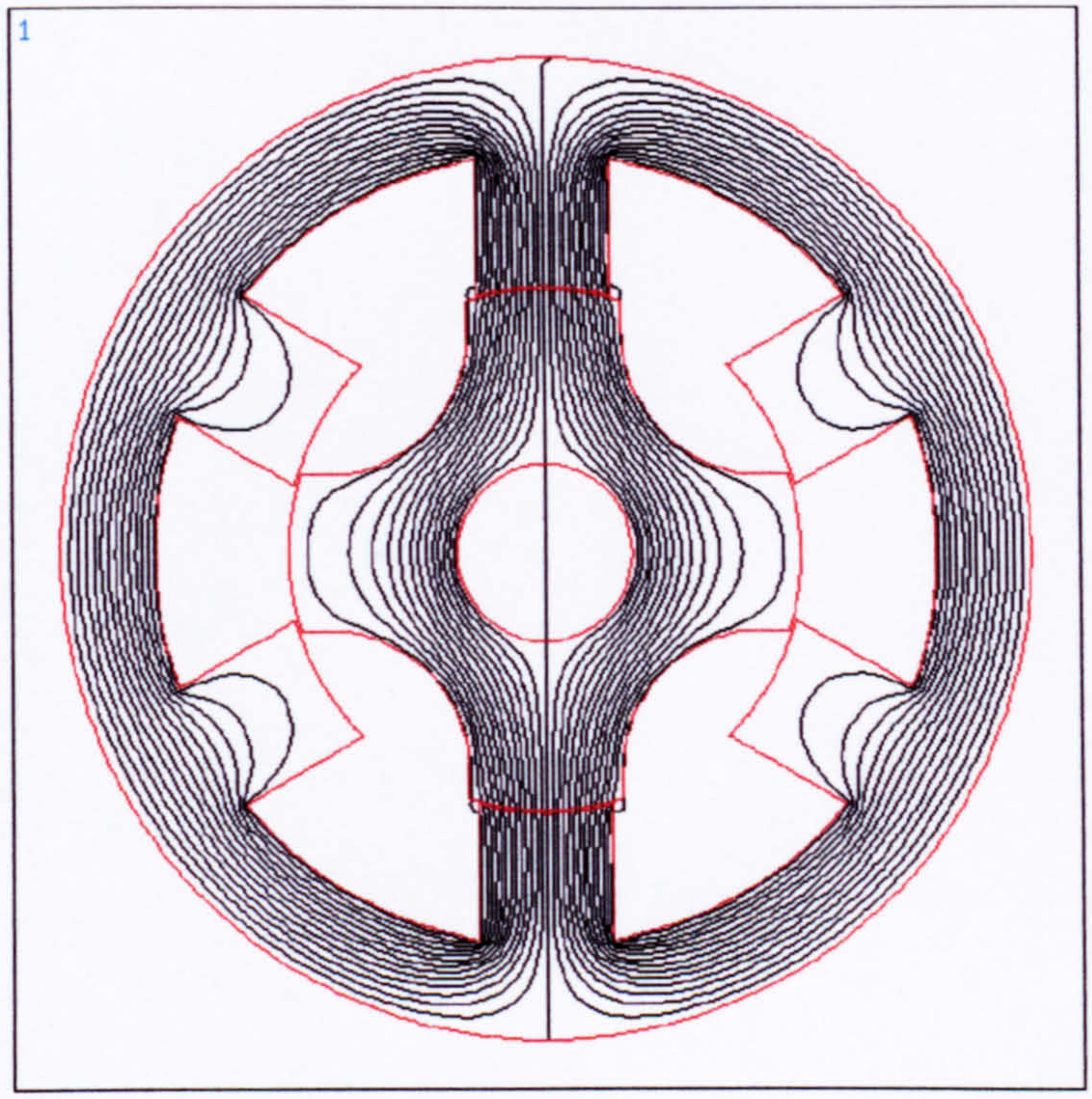

$$
\begin{aligned}
& \text { ANSYS } 5.4 \\
& \text { AUG } 32000 \\
& 10: 38: 28 \\
& \text { NODAL SOLUTION } \\
& \text { TEP-1 } \\
& \begin{array}{ll} 
& =1 \\
&
\end{array} \\
& \text { IME } \\
& \text { RSYS }=0 \\
& \text { SMN }=-.002462 \\
& \text { SMX }-.002462 \\
& -.002371 \\
& -.002189 \\
& -.002006 \\
& -.001824 \\
& -.001642 \\
& -.001277 \\
& -.001094 \\
& -.912 \mathrm{E}-03 \\
& -.730 \mathrm{E}-03 \\
& -.365 \mathrm{E}-03 \\
& -.182 \mathrm{E}-03 \\
& -.547 \mathrm{E}-07 \\
& .182 \mathrm{E}-03 \\
& .547 \mathrm{E}-03 \\
& \text { 729E-03 } \\
& .729 \mathrm{E}-03 \\
& .912 \mathrm{E}-03 \\
& .001094 \\
& .001641 \\
& .001824 \\
& .002006 \\
& .002371
\end{aligned}
$$

Figure 3.7 Flux lines for the finite element mesh of figure 3.6a

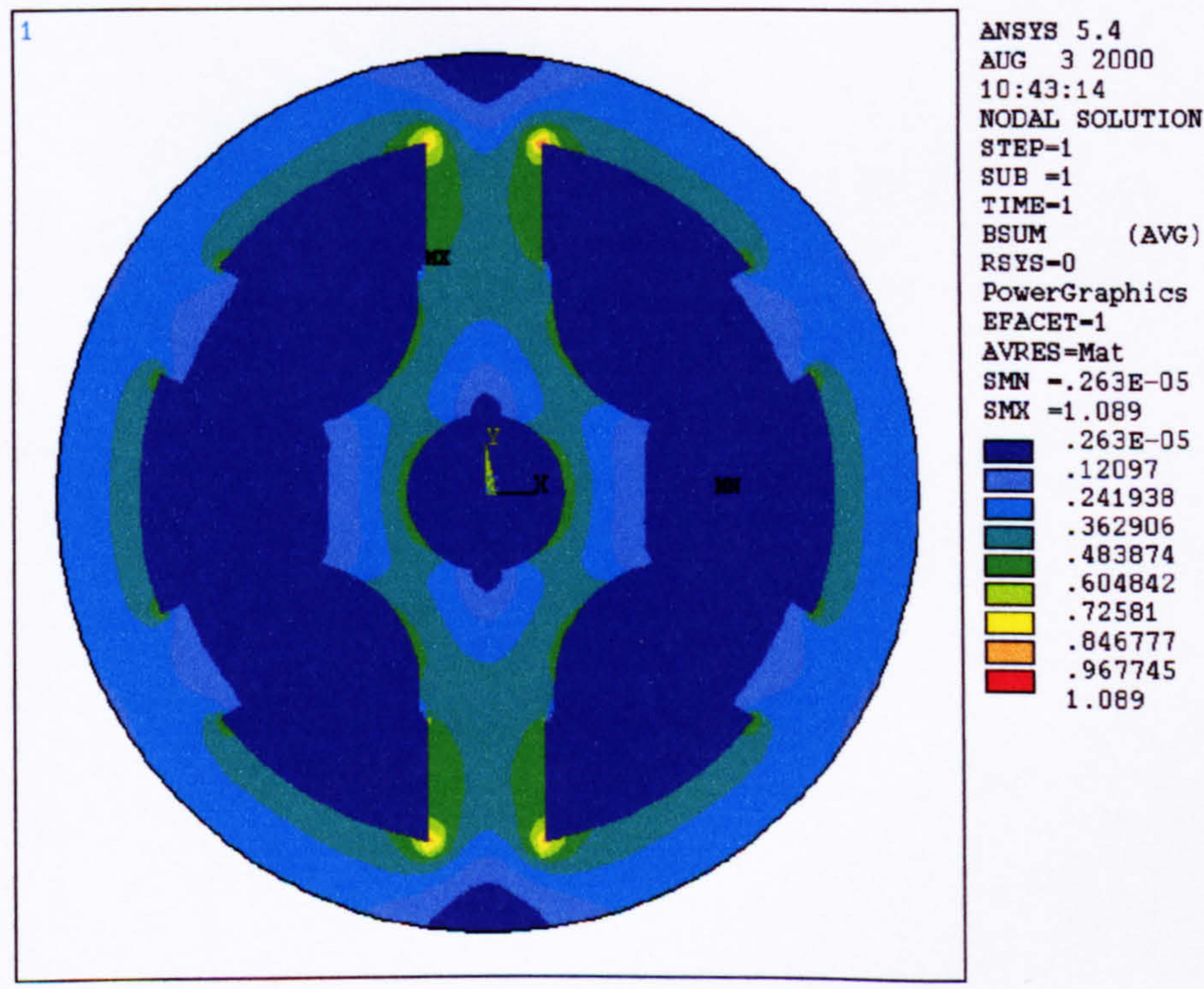

Figure 3.8 Flux density contours for the machine with a current density of $1 \mathrm{~A} / \mathrm{mm}^{2}$ applied to phase A. 


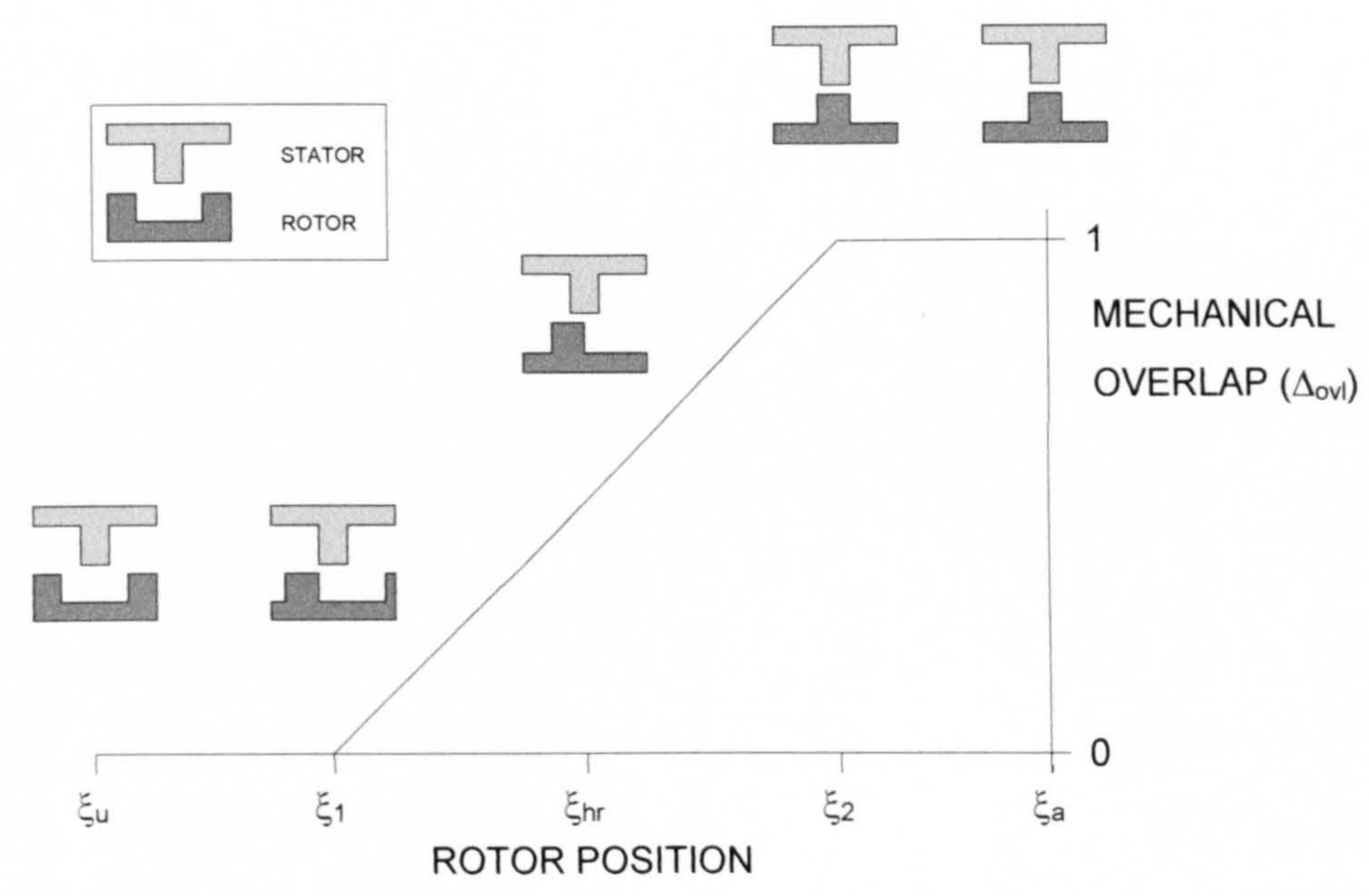

Figure 3.9 Degree of relative mechanical overlap of rotor and stator teeth as a function of rotor position.

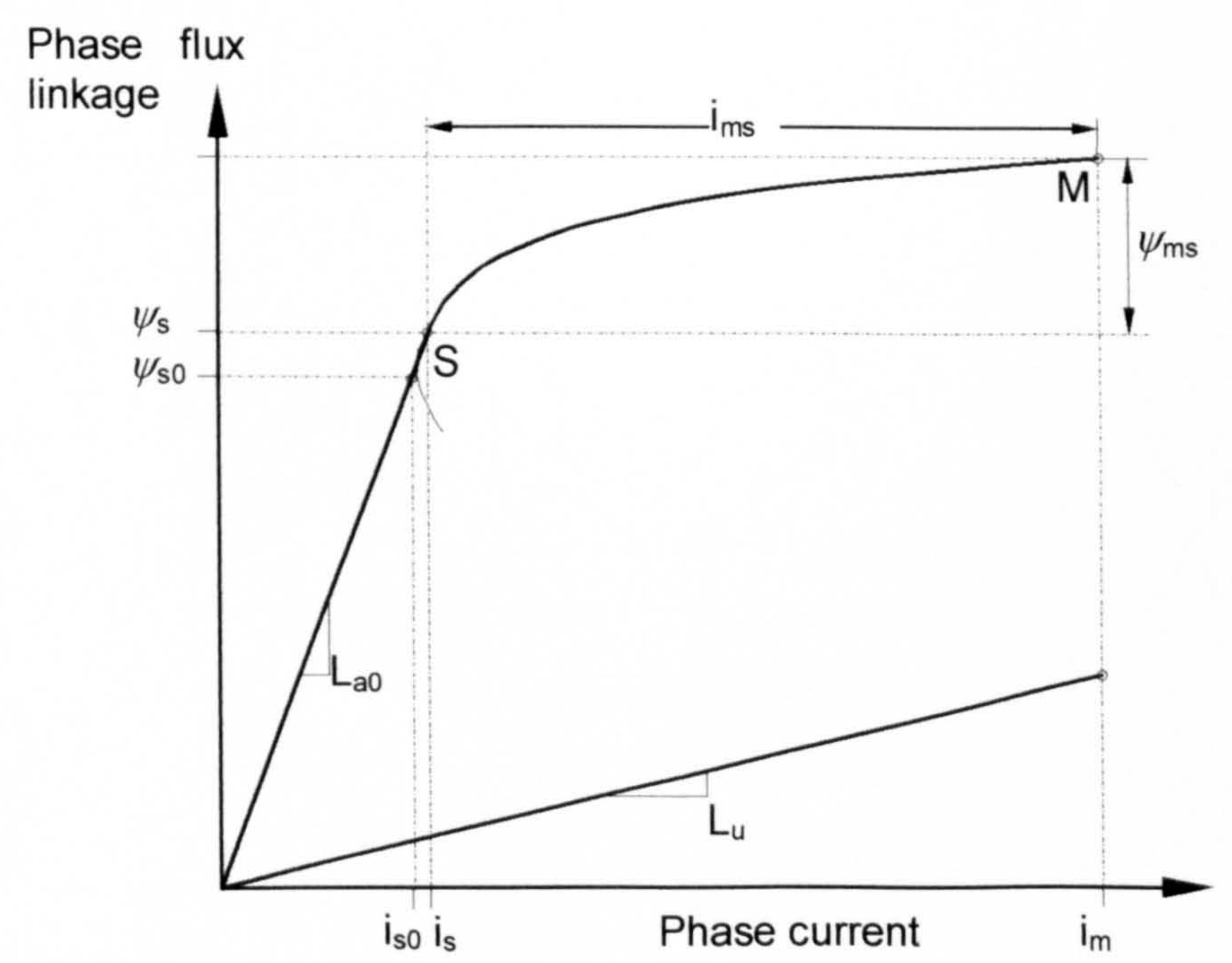

Figure 3.10 Parabolic curve fit of the aligned $\psi-i$ curve as described by Miller and McGilp [3.7]. 


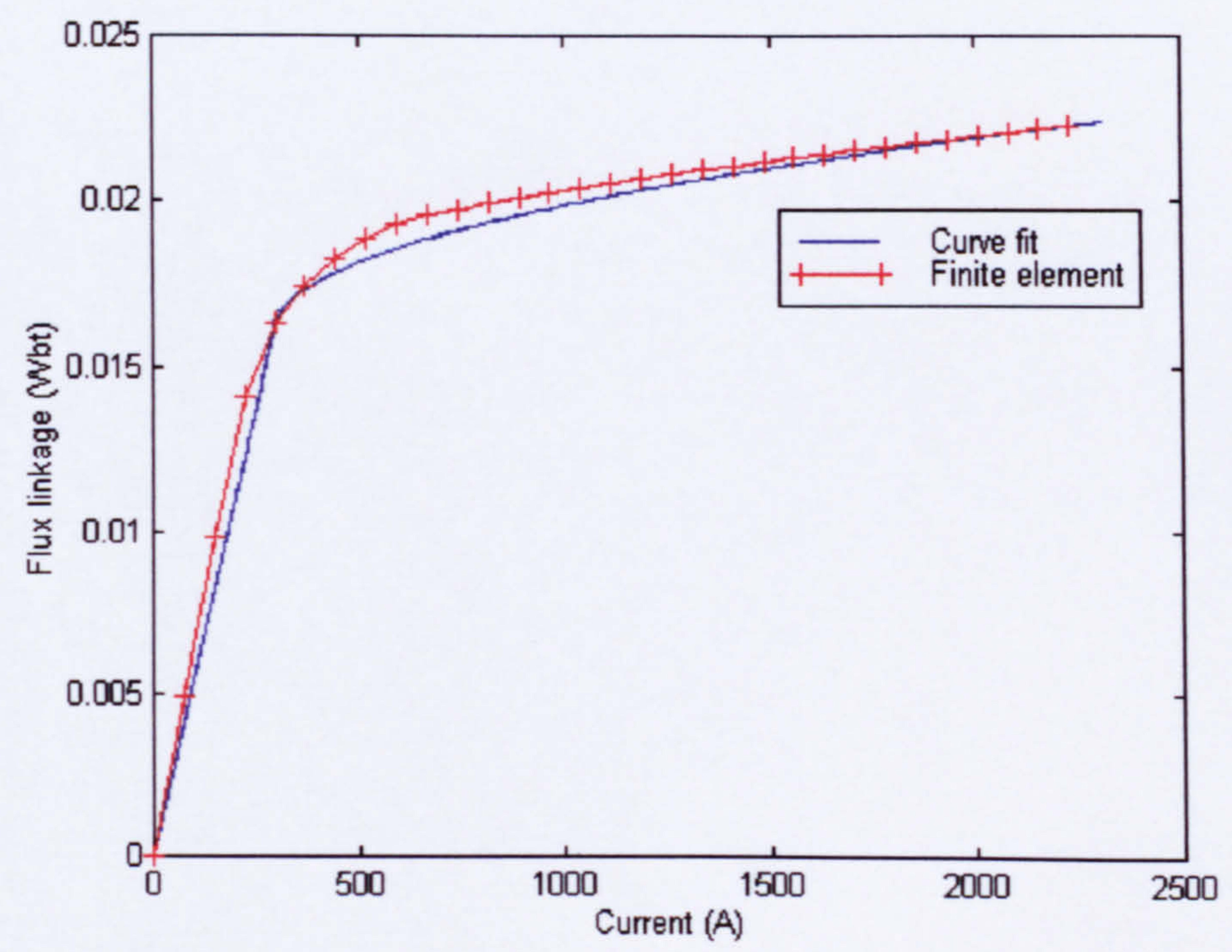

Figure 3.11 Aligned magnetisation curves calculated with Ansys and the applied parabolic curve fit.

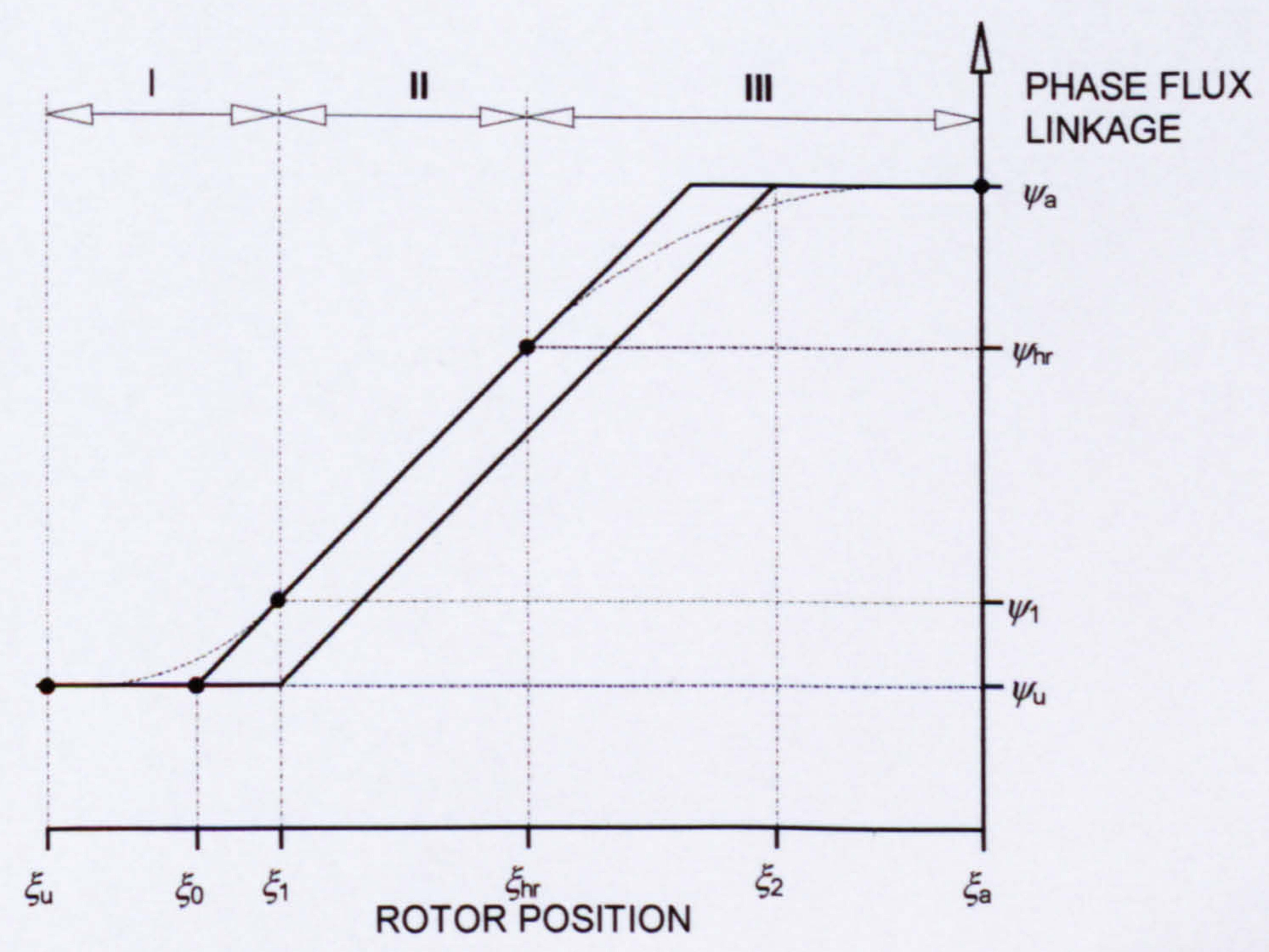

Figure 3.12. Schematic representation of the non-linear $\psi-\theta$ variation 


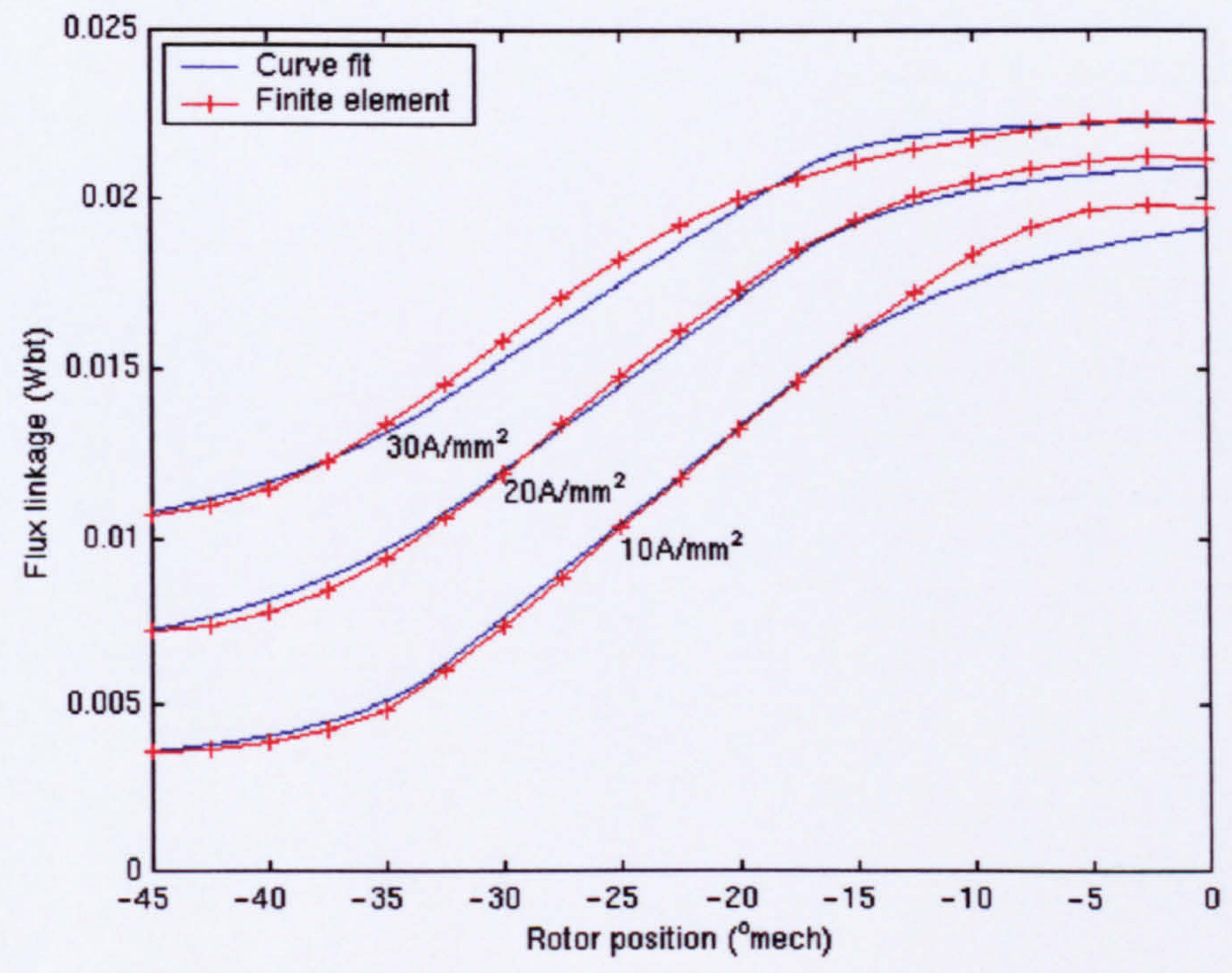

Figure 3.13. Gauge curves derived using the finite element method and the curve fitting functions described in appendix 3.3

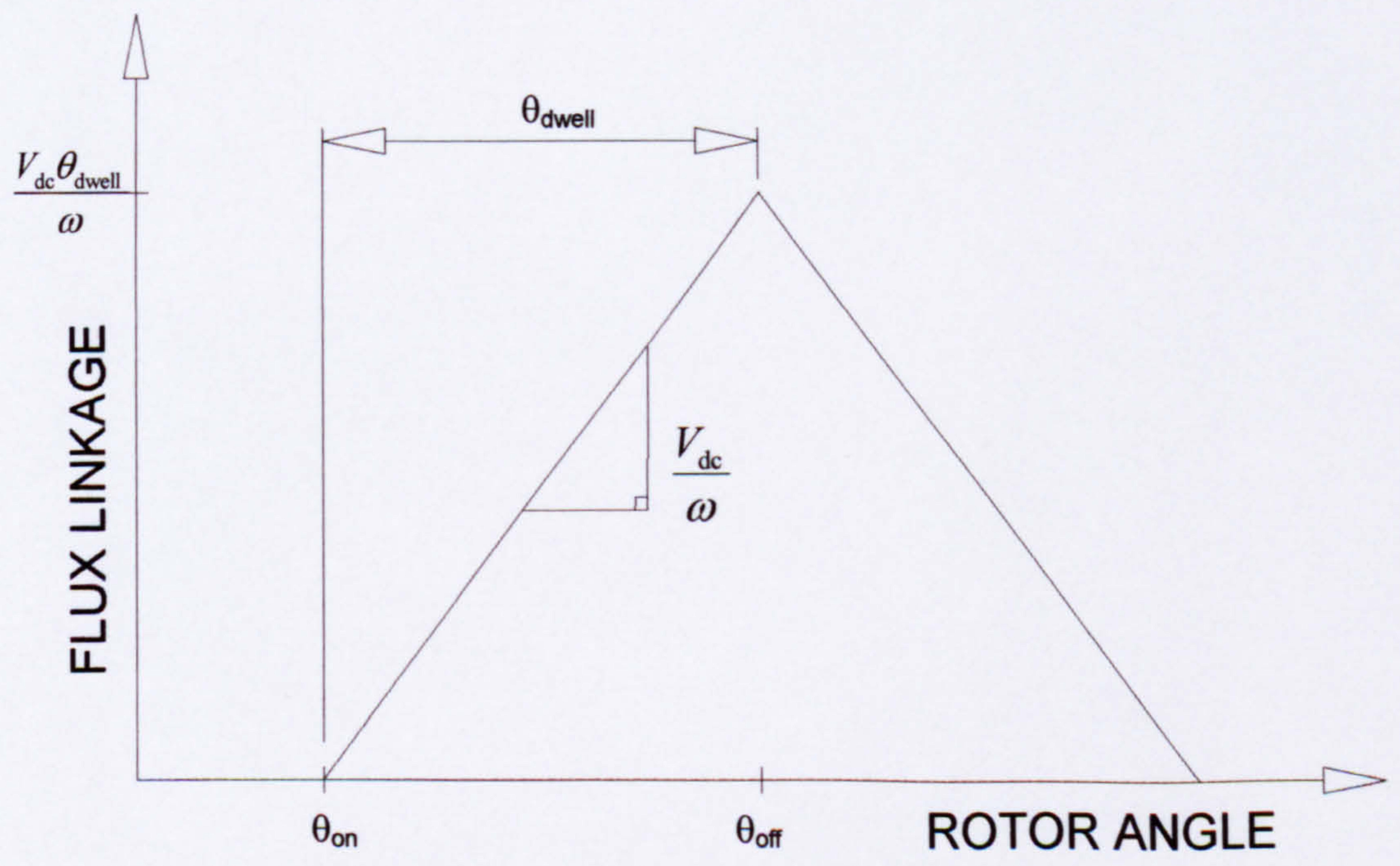

Figure 3.14 Flux density variation determined using a linear model of the SR machine. 


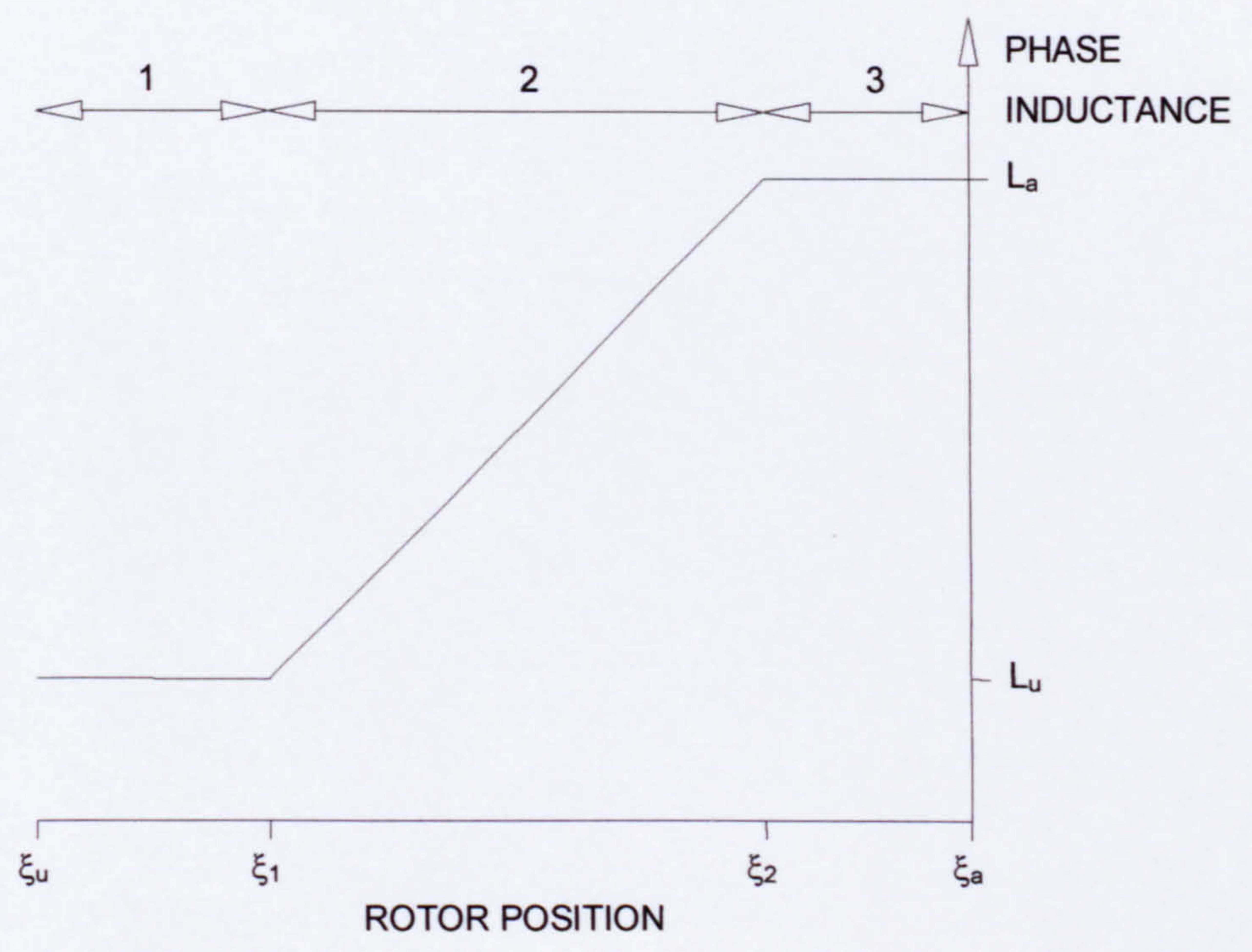

Figure 3.15 Simplified trapezoidal inductance variation as a function of rotor position.

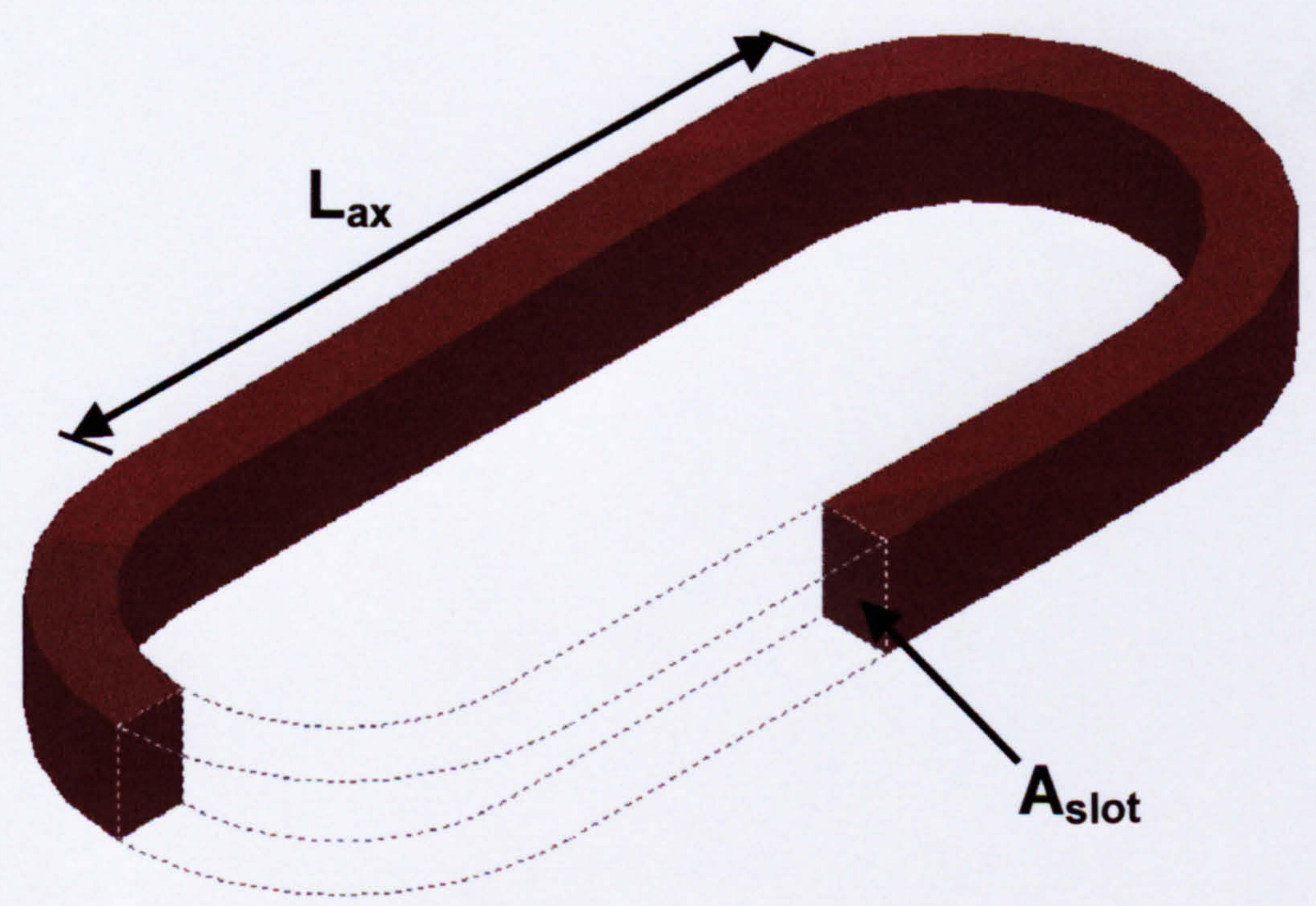

Figure 3.16. Basic geometric features of coil used to calculate phase resistance in non-linear model. 


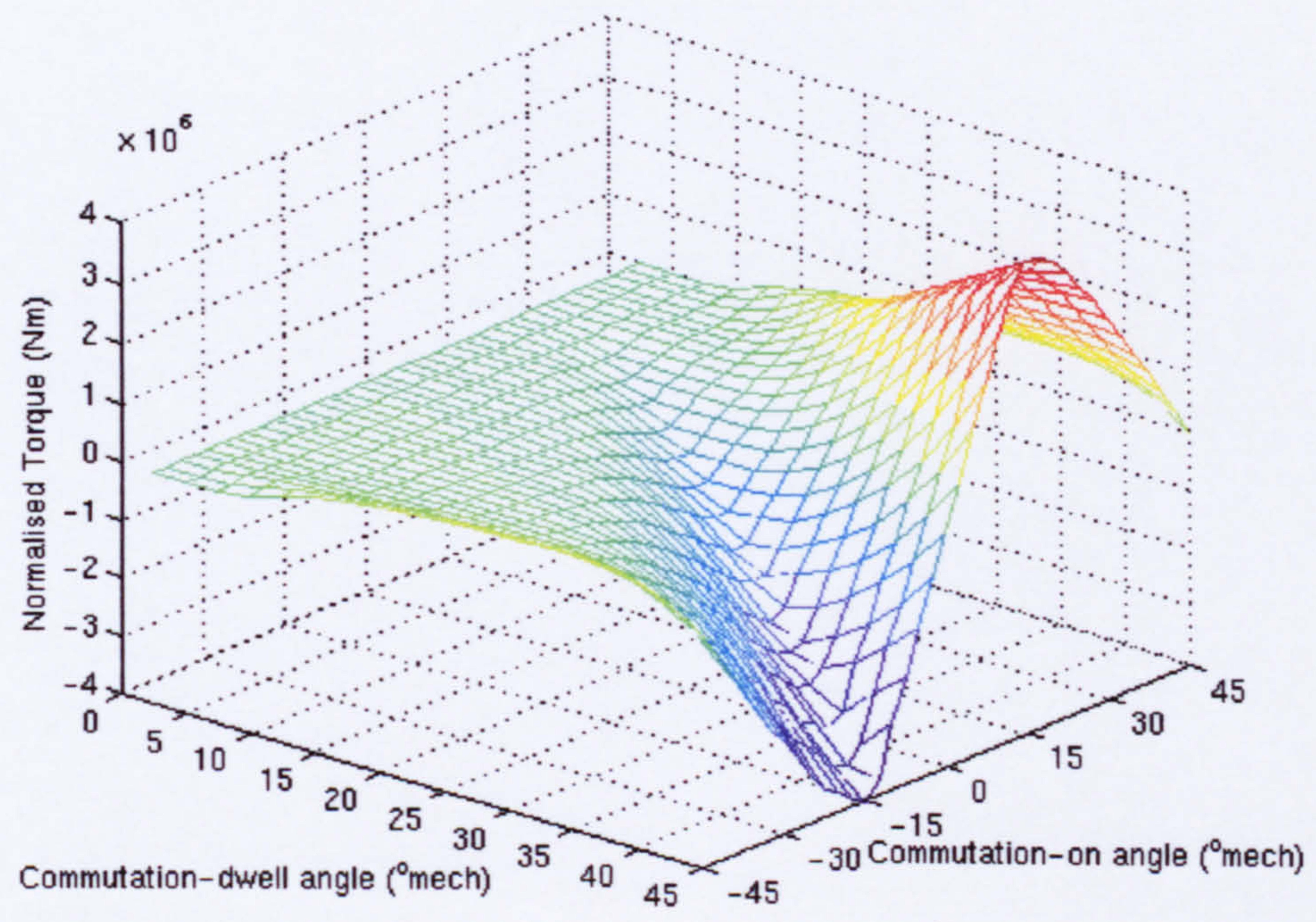

Figure 3.17 Normalised output torque as a function of the commutation conditions

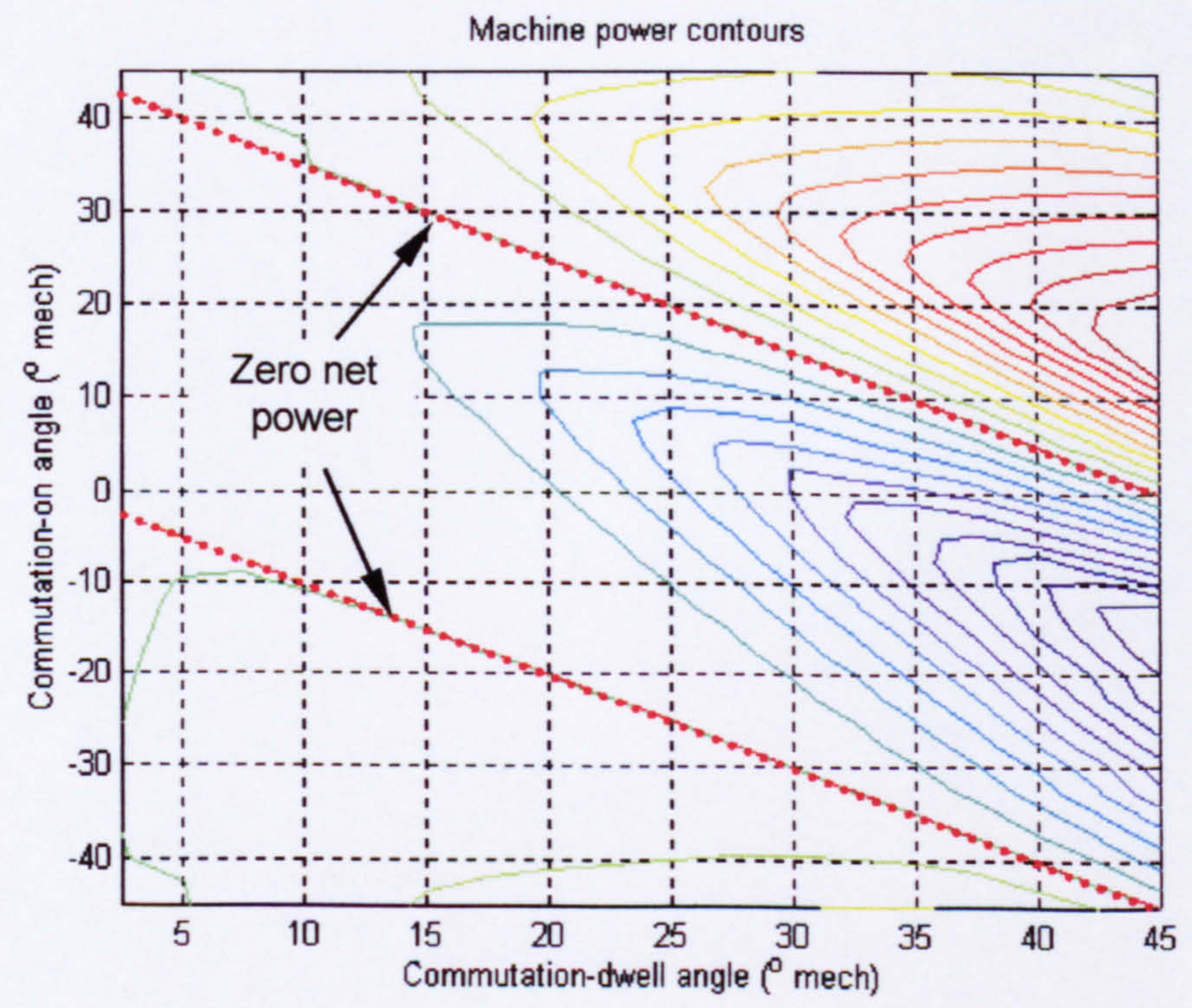

Figure 3.18 Contour map corresponding to the mesh of figure 3.17 showing the commutation angles yielding zero power. (increments of approximately $4 \times 10^{5}$ ) 


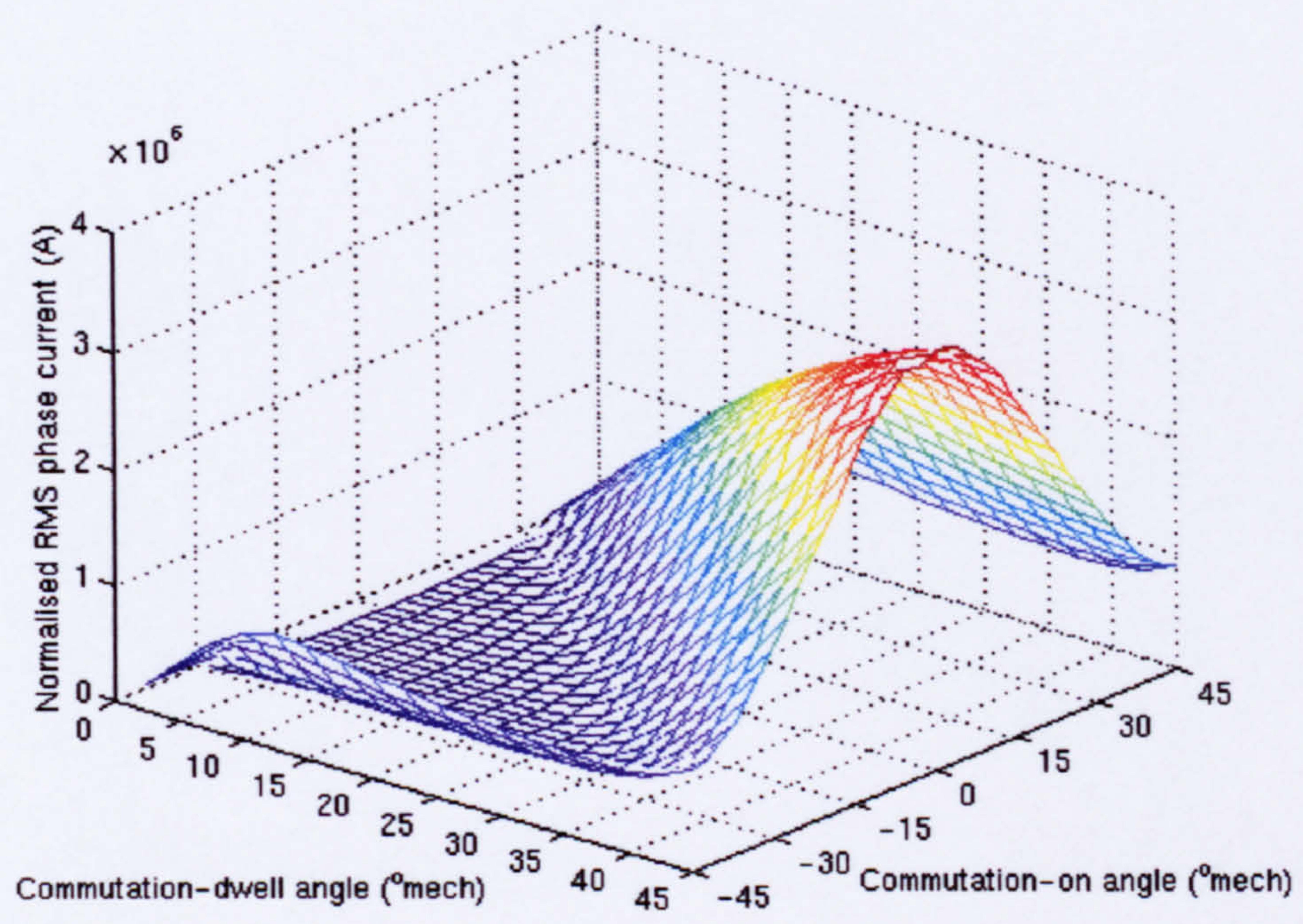

Figure 3.19 Normalised RMS current as a function of the commutation conditions.

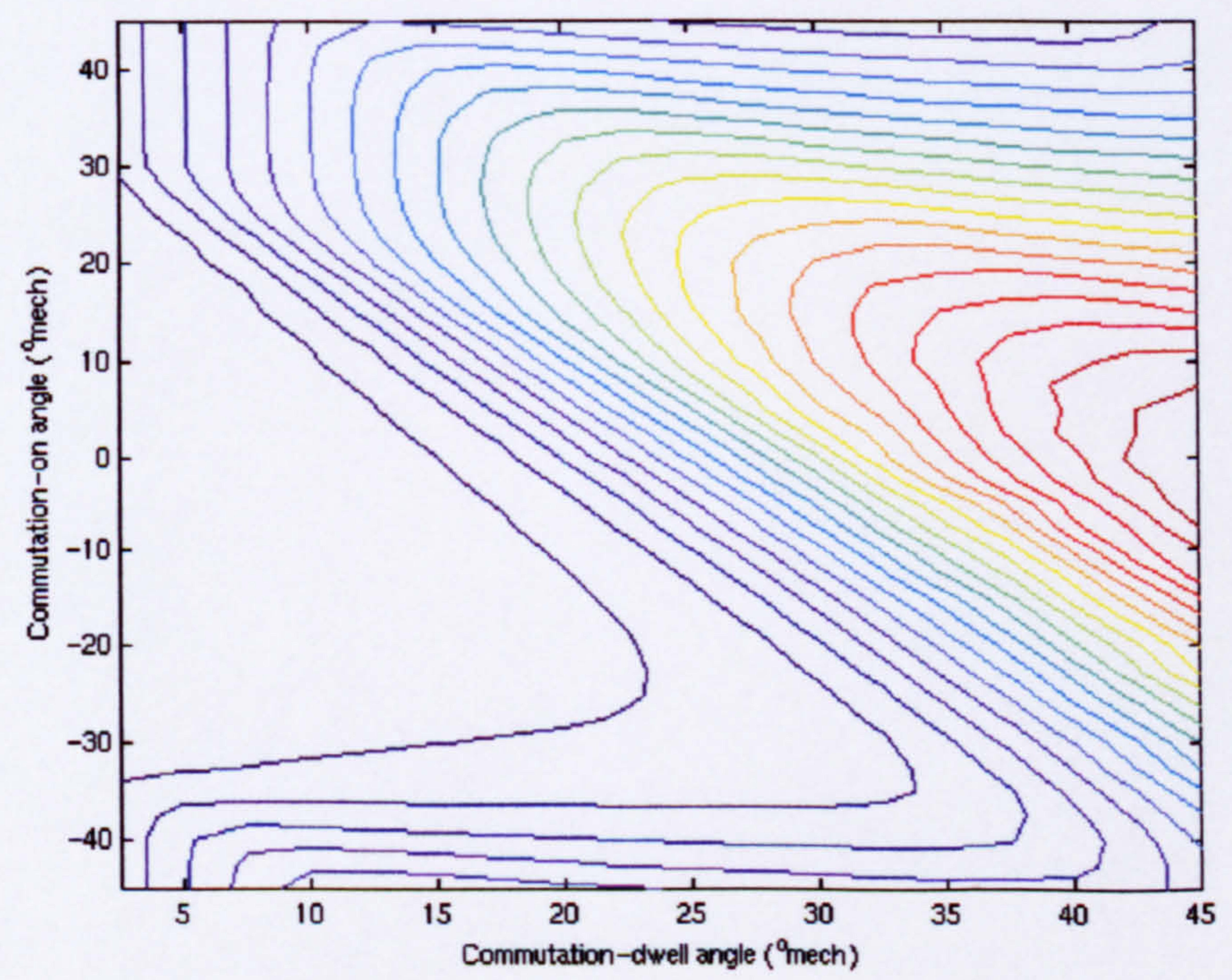

Figure 3.20. Normalised RMS current contours as a function of the commutation conditions (in increments of approximately $1.8 \times 10^{5}$ ). 


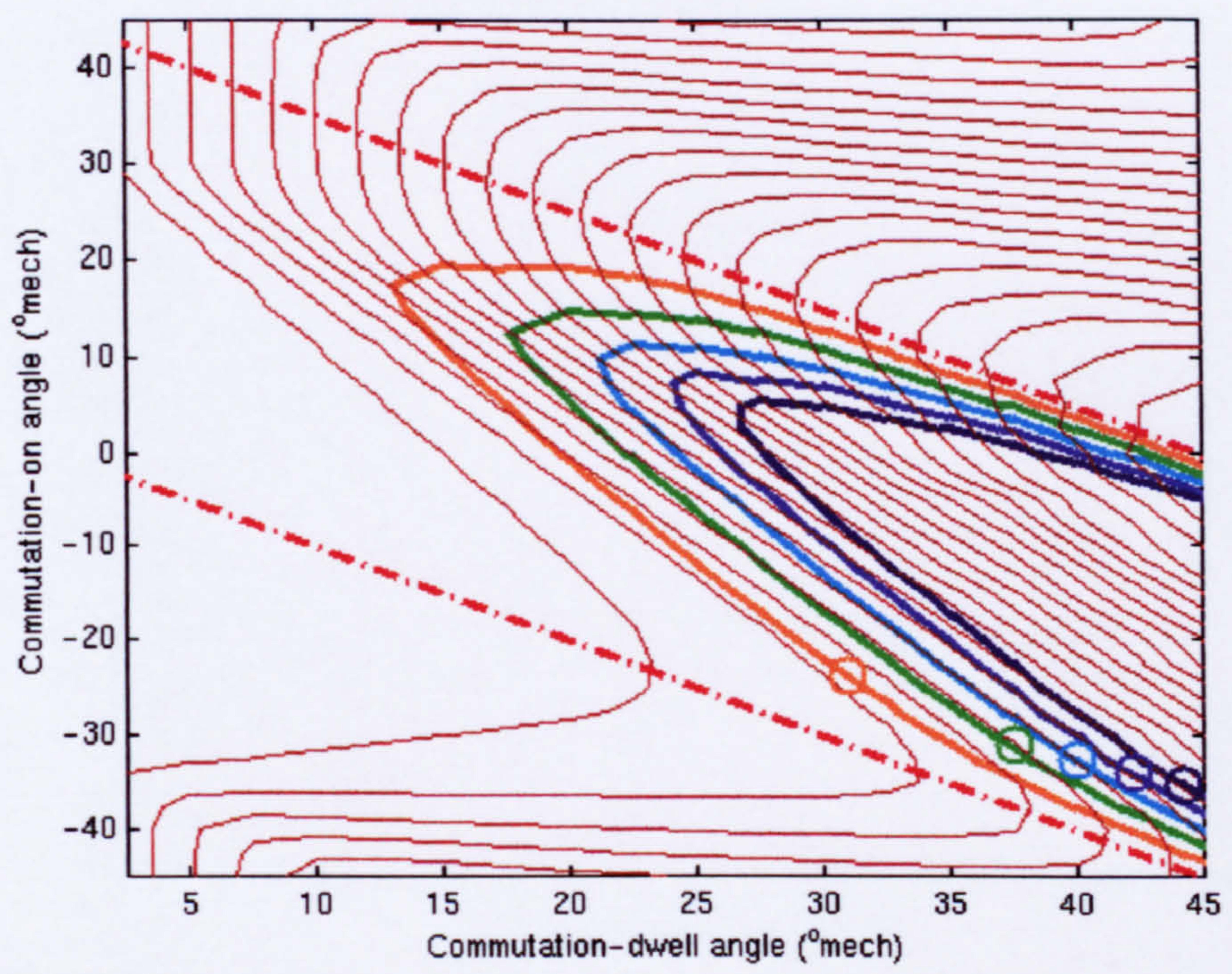

Figure 3.21. Superposition of constant power contours onto the contours of constant rms. phase current. The commutation conditions that minimise rms current for each of the power contours are highlighted with circles.

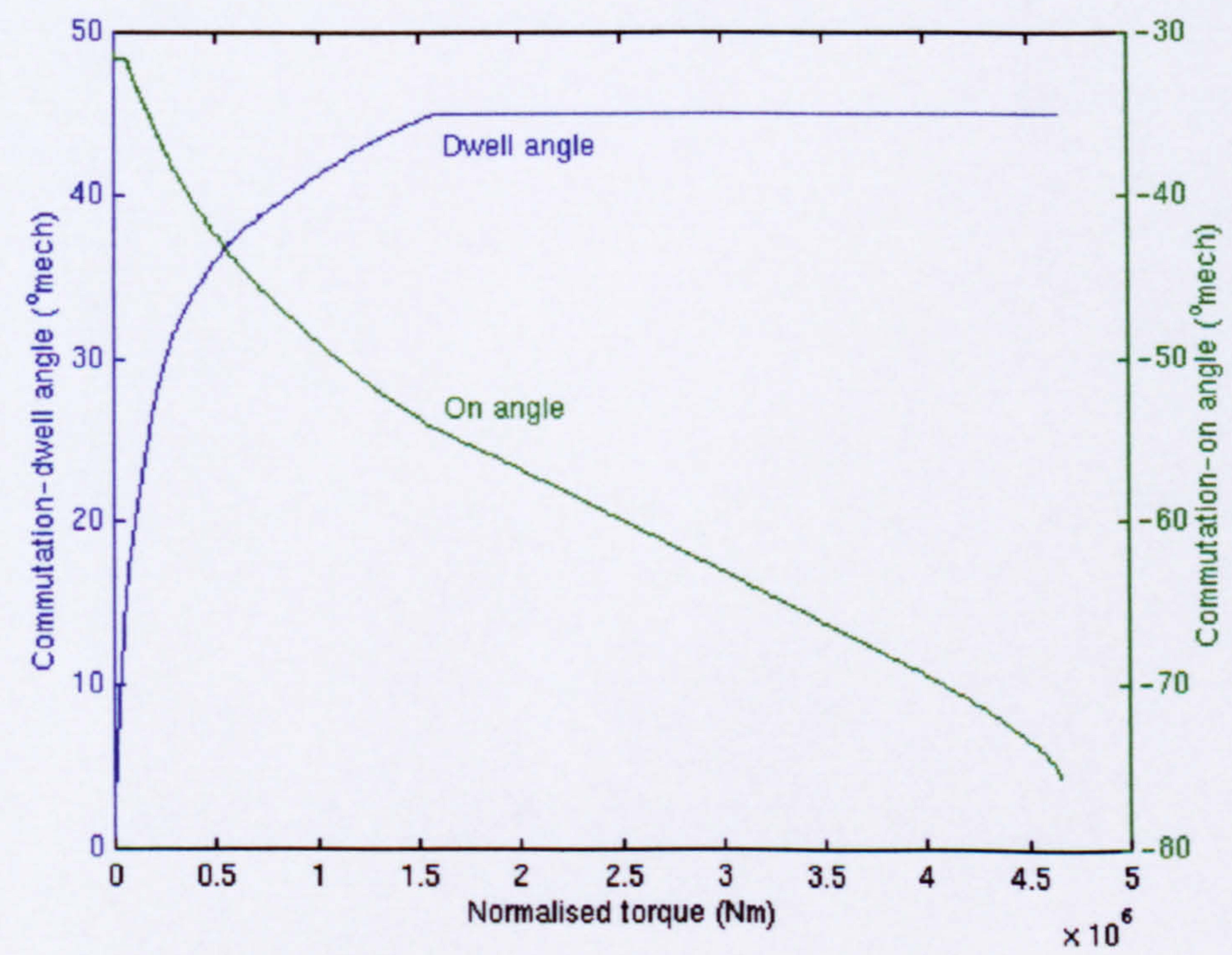

Figure 3.22. Commutation angles combinations as a function of normalised torque which yield the minimum copper loss. 


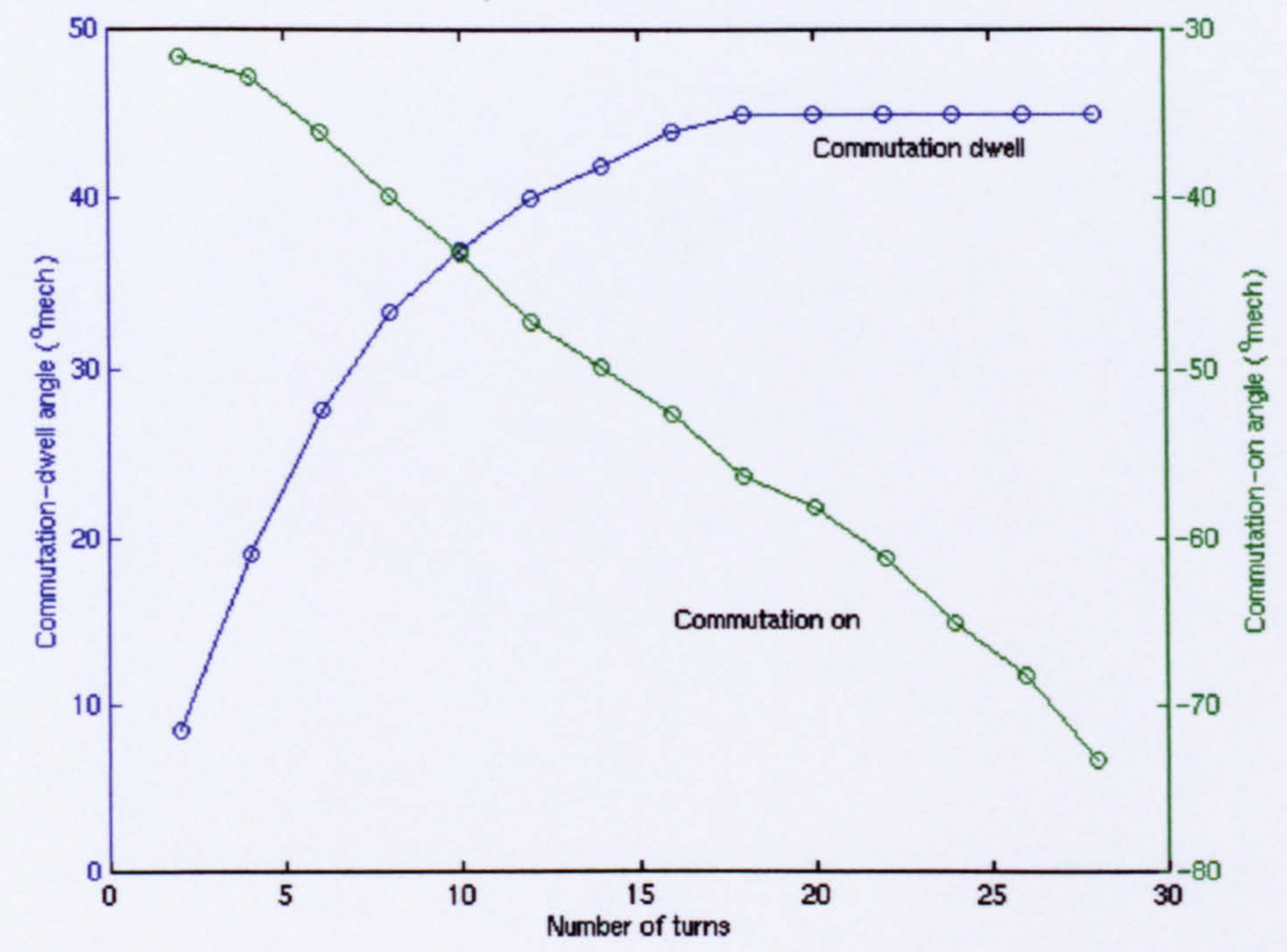

Figure 3.23. Optimum commutation conditions for $5 \mathrm{~kW}$ at 100,000 as a function of the number of turns on the winding.

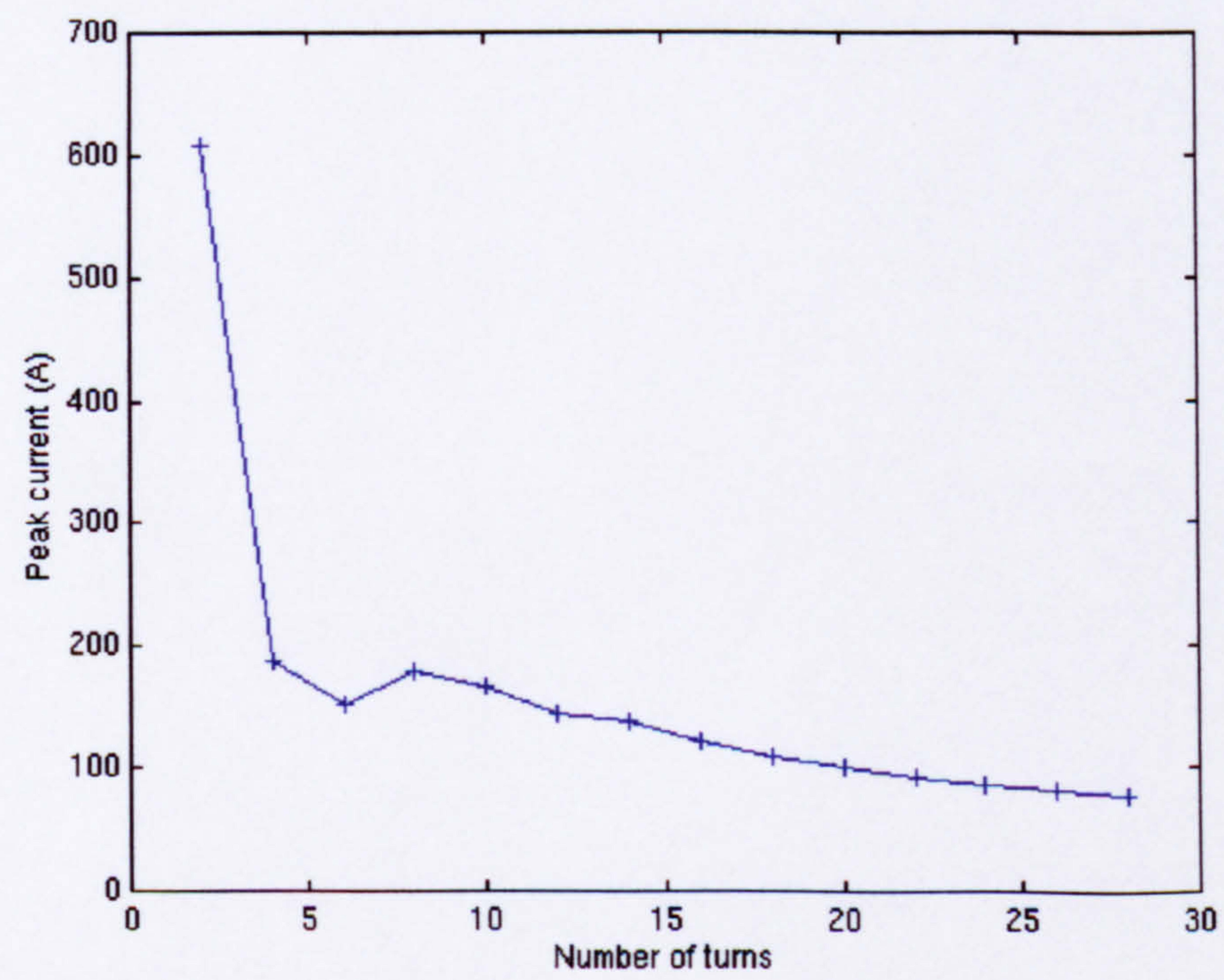

Figure 3.24. Peak winding current as a function of the number of turns on the winding for the $5 \mathrm{~kW}, 100,000 \mathrm{rpm}$ operating condition. 




Figure 3.25. Angular limits of conduction as a function of the number of turns on the winding for the $5 \mathrm{~kW}, 100,000 \mathrm{rpm}$ operating condition.

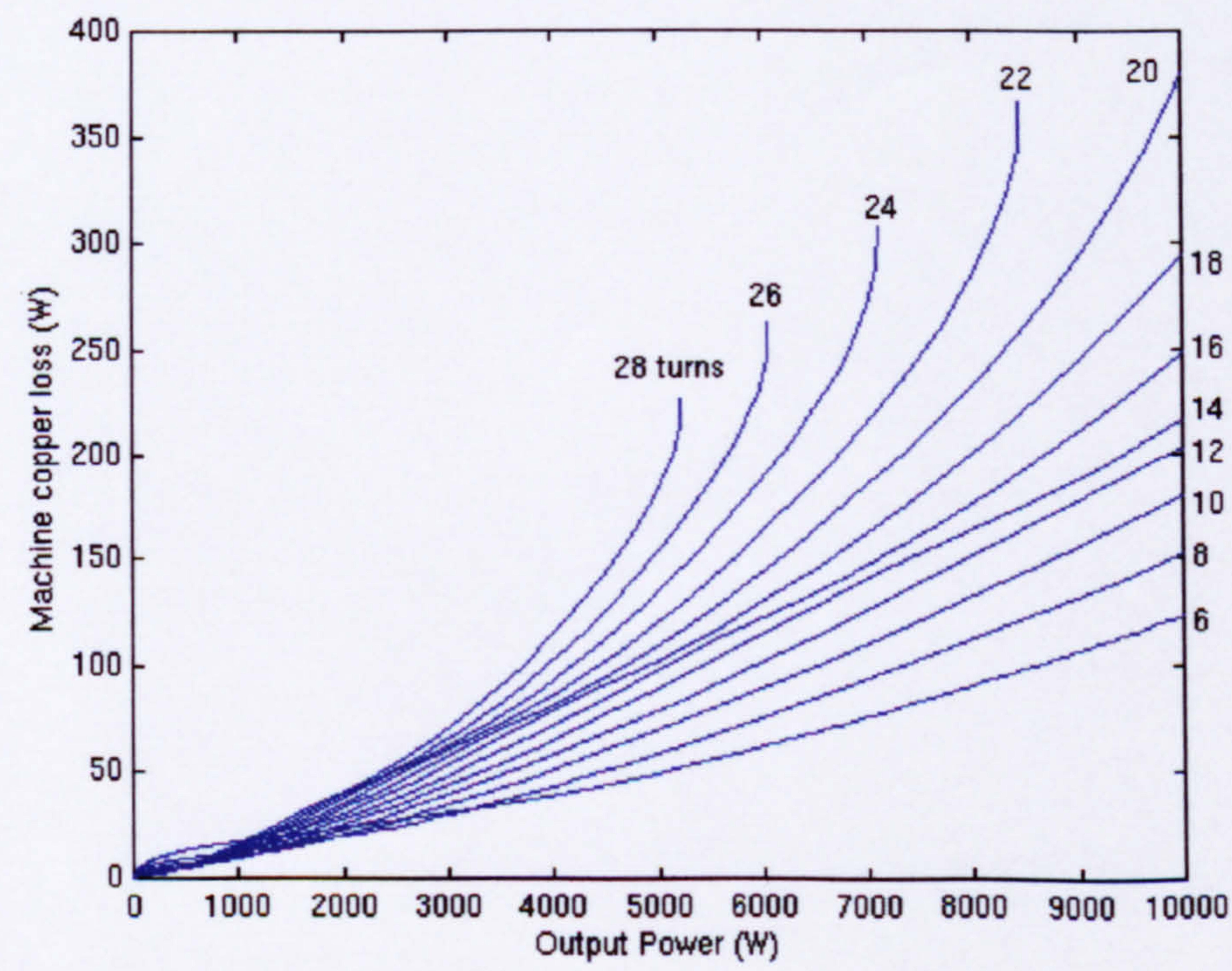

Figure 3.26. Copper loss as a function of the output power for windings comprising 8-28 turns. 


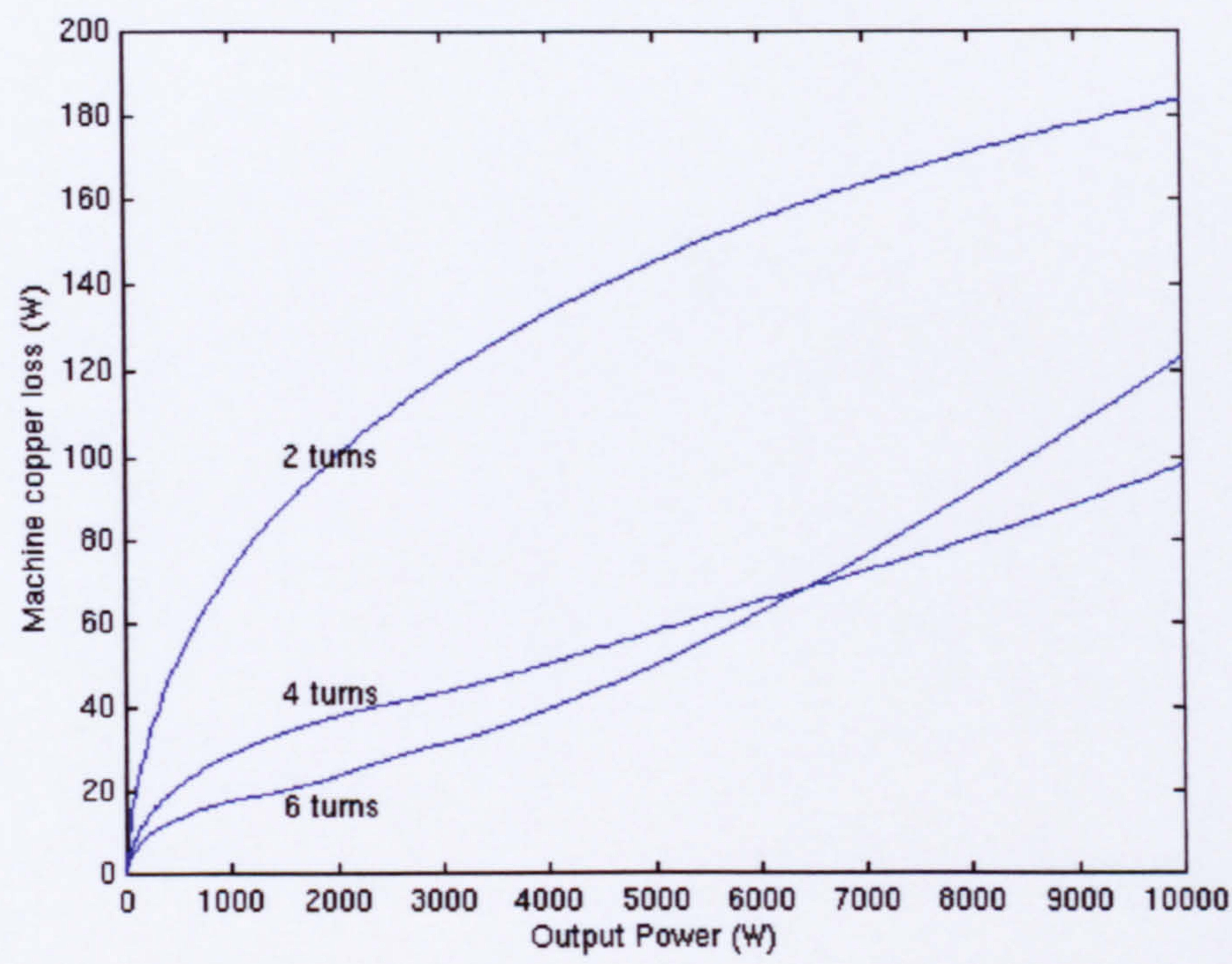

Figure 3.27. Copper loss as a function of the output power for windings comprising 2-6 turns.

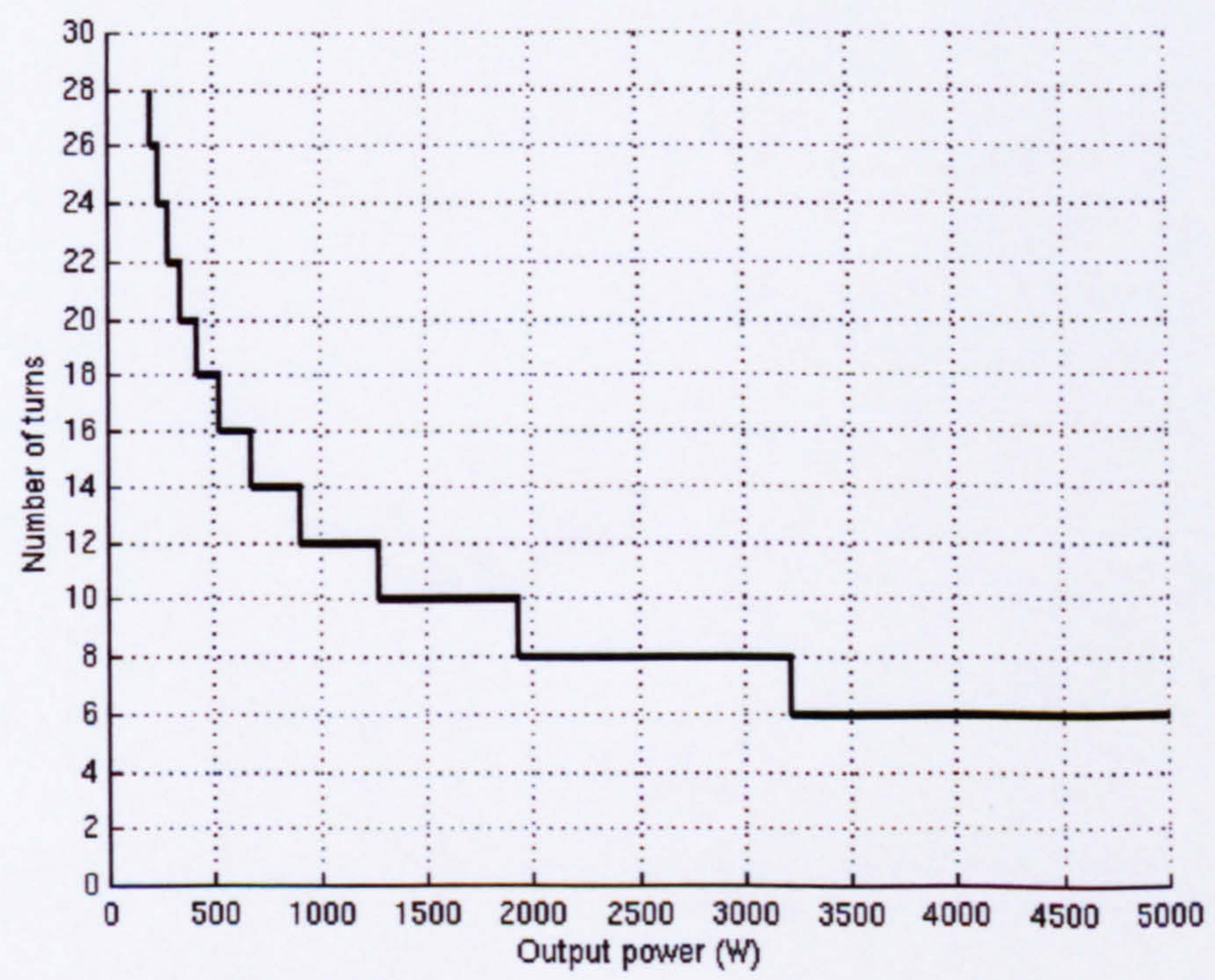

Figure 3.28. Copper loss optimised number of phase turns as a function of the output power at $100,000 \mathrm{rpm}$. 


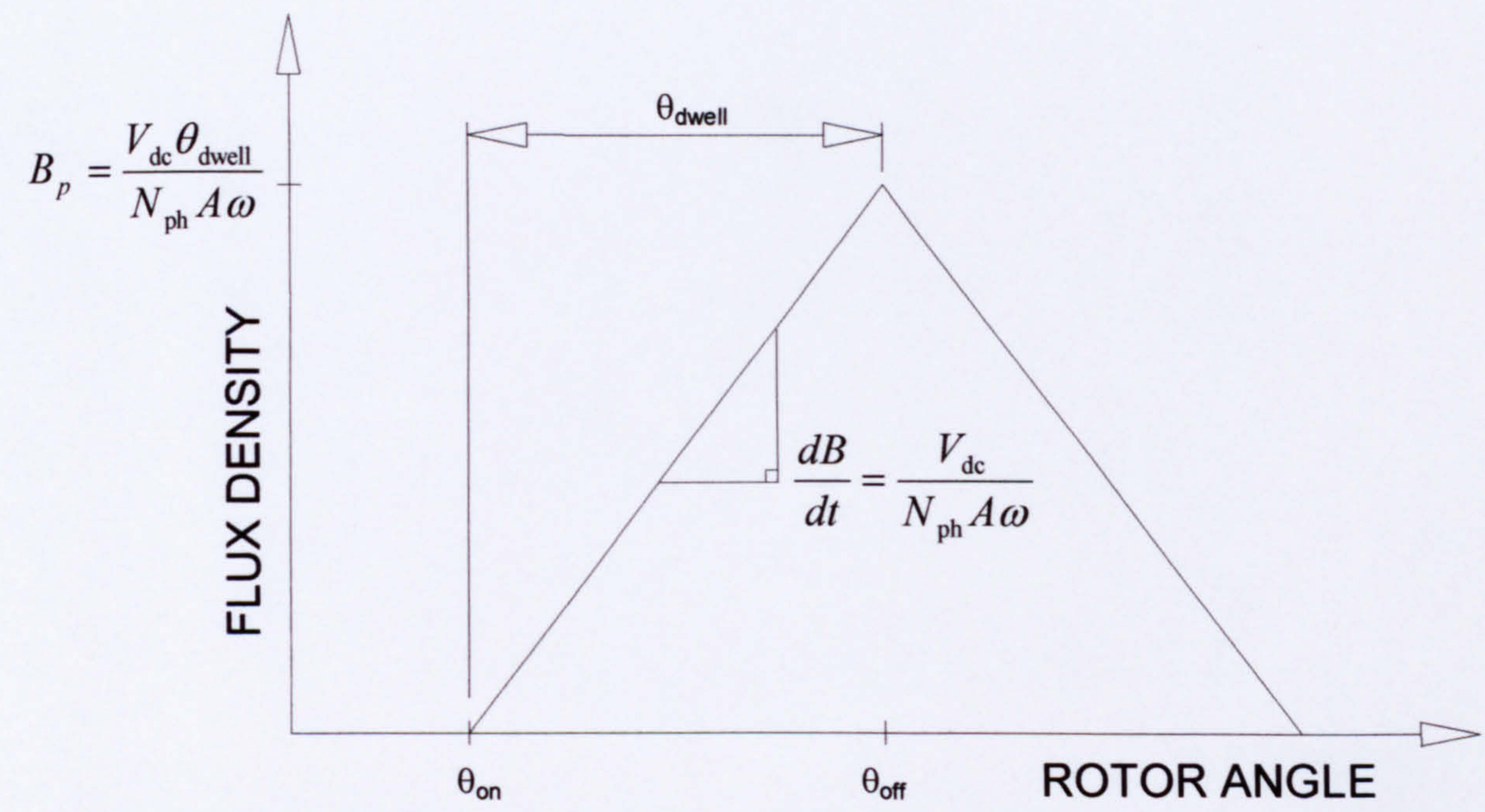

Figure 3.29. Flux density variation determined using a linear model of SR machine.

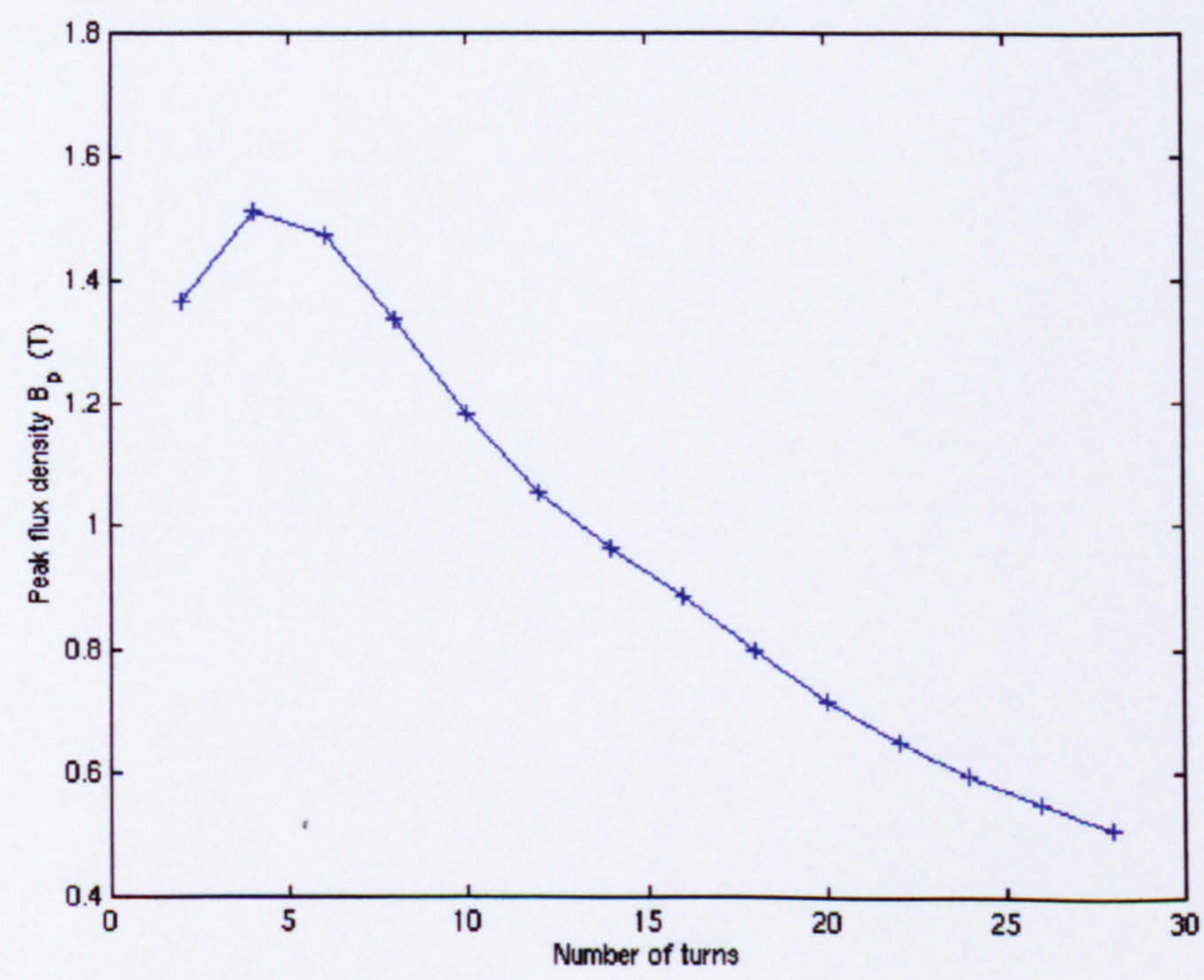

Figure 3.30. Variation of $B_{p}$ as a function of $N_{p h}$ based on the optimised values of $\theta_{\text {dwell. }}$ 


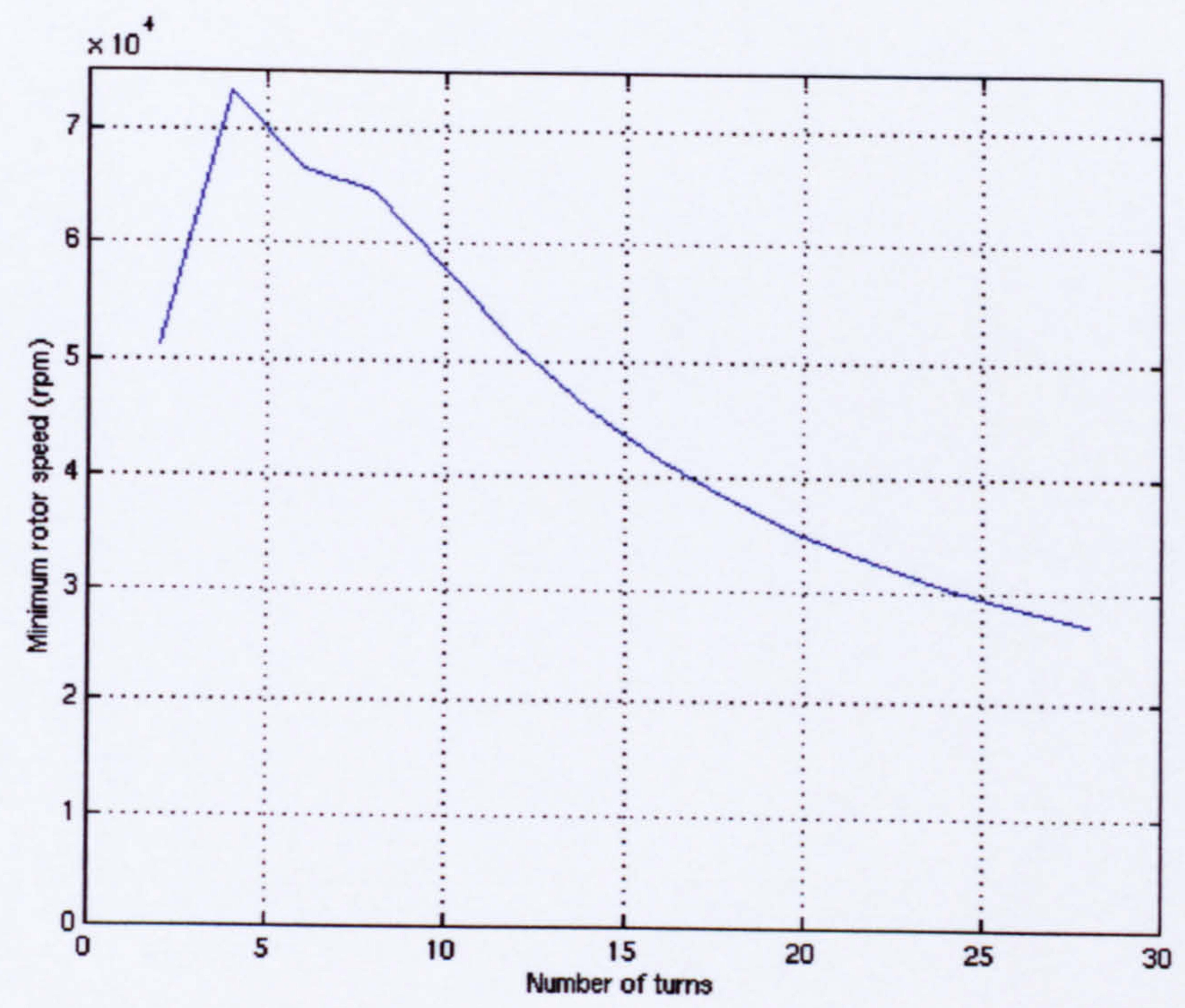

figure 3.31 Variation of the lower speed at which a $B_{p}$ of $1.8 \mathrm{~T}$ is achieved as a function of $N_{p h}$.

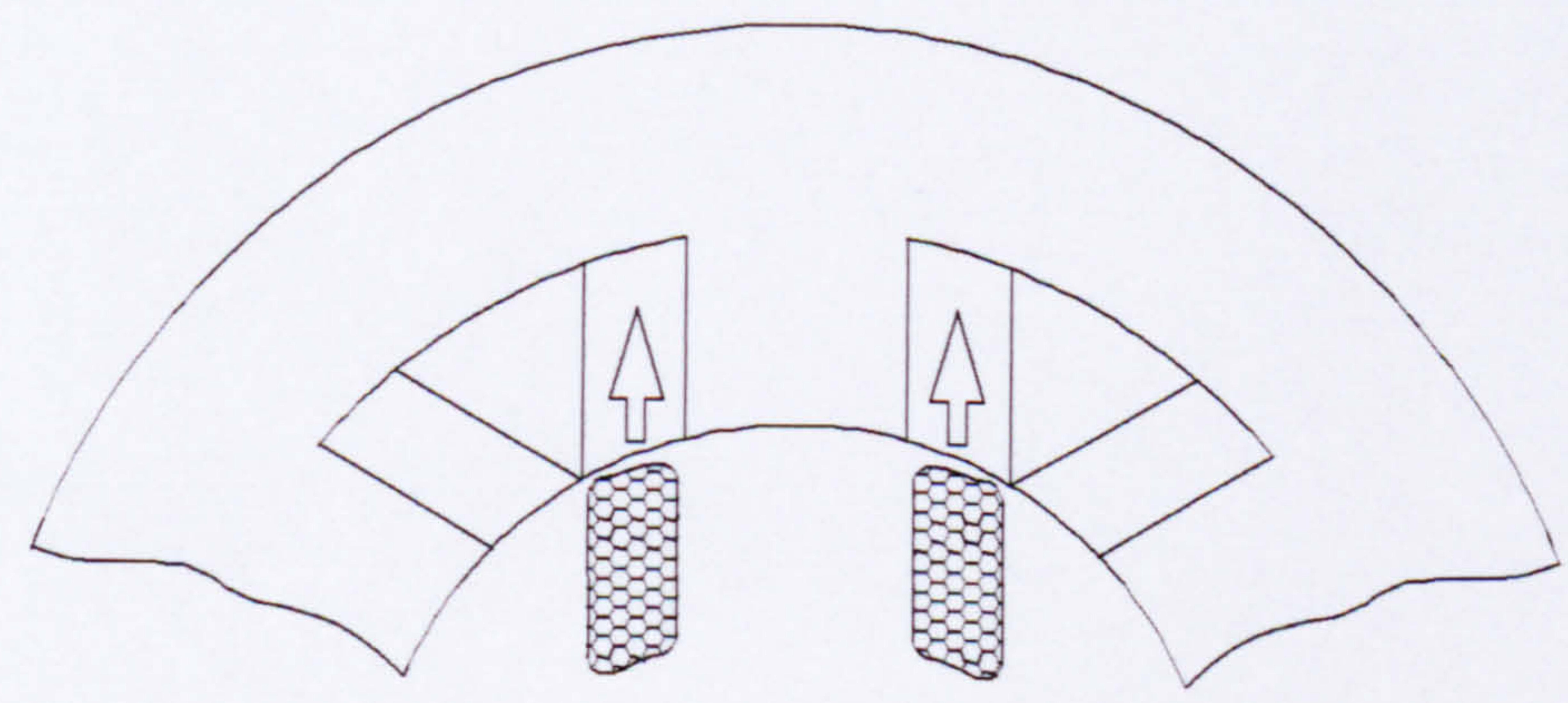

Figure 3.32 Pre-formed winding insertion . 


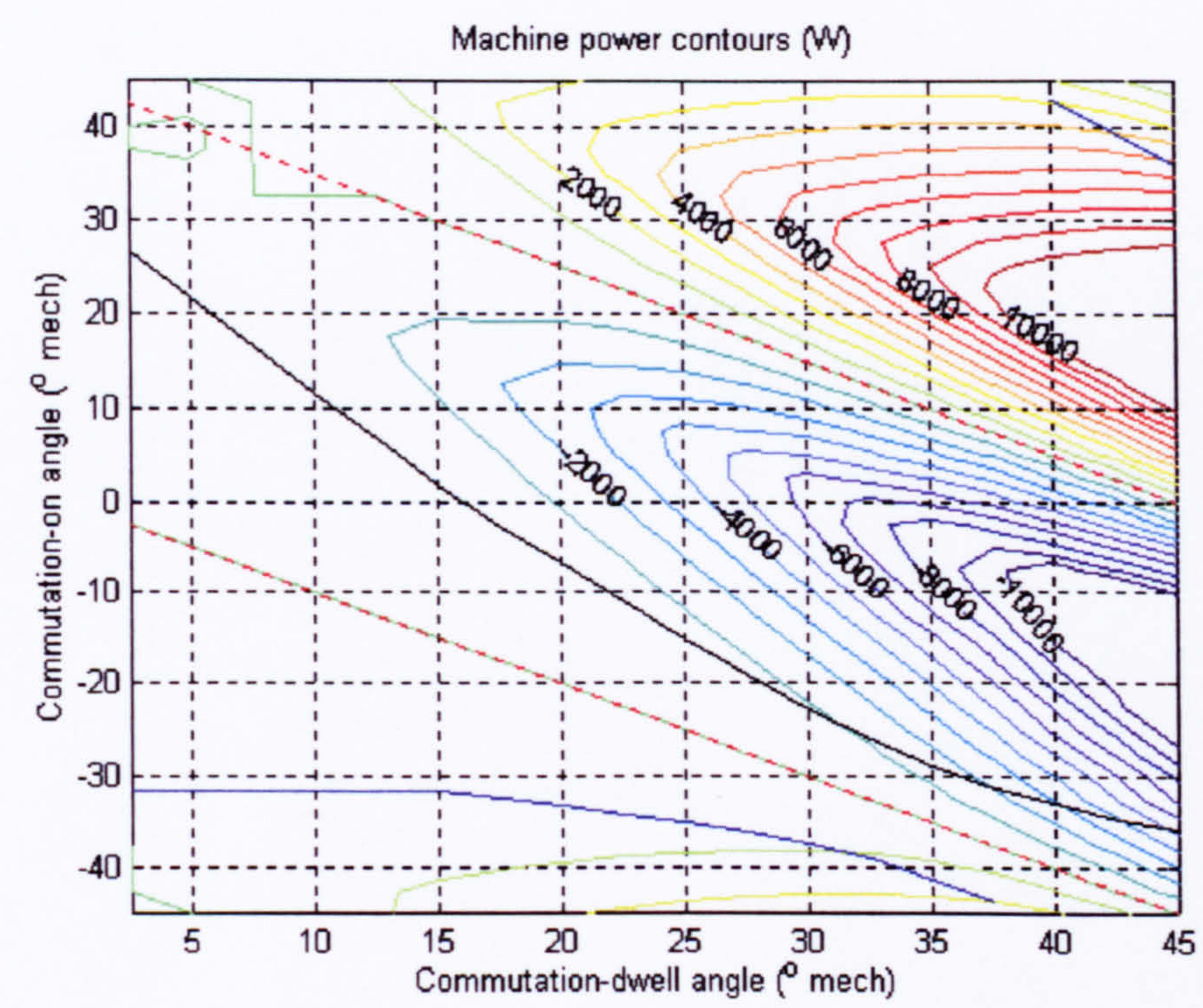

Figure 3.33 Power contours derived using the linear analytical model for the machine with 16 turns/phase.

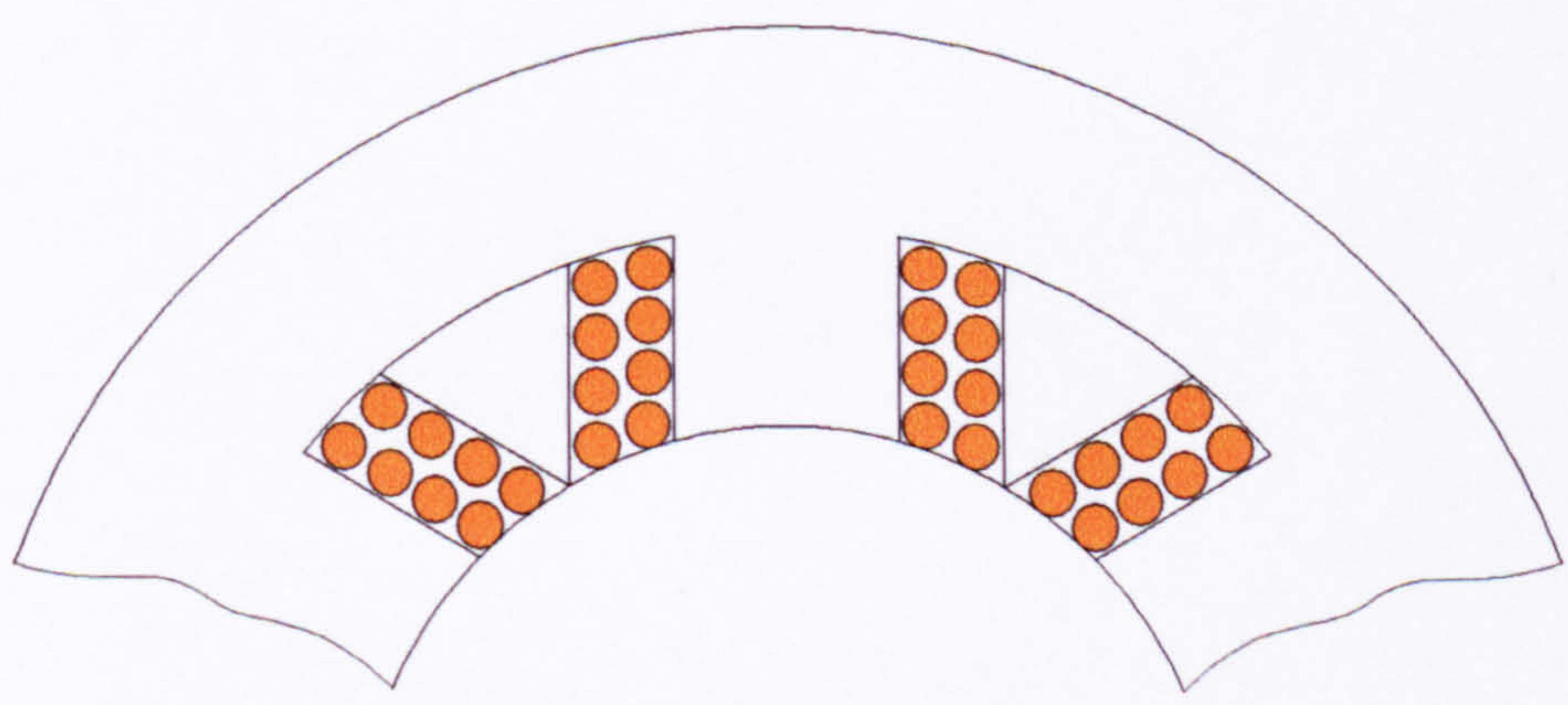

Figure 3.34 Winding design consisting of 8 turns/stator tooth. 


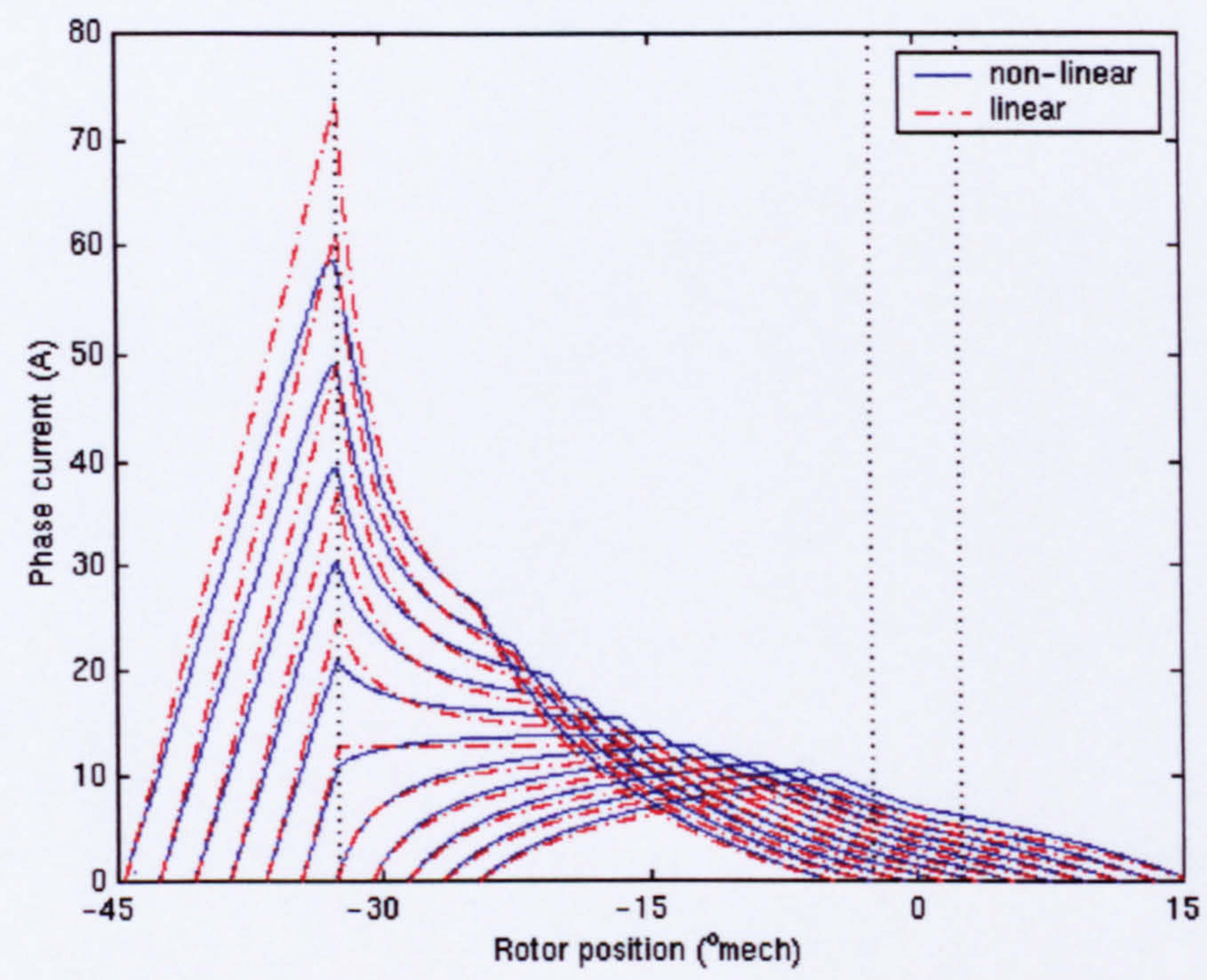

Figure 3.35. Current waveforms obtained using the linear and non-linear simulation techniques at various turn-on angles with a constant dwell angle of $20^{\circ}$ for the machine operating at $100,000 \mathrm{rpm}$.

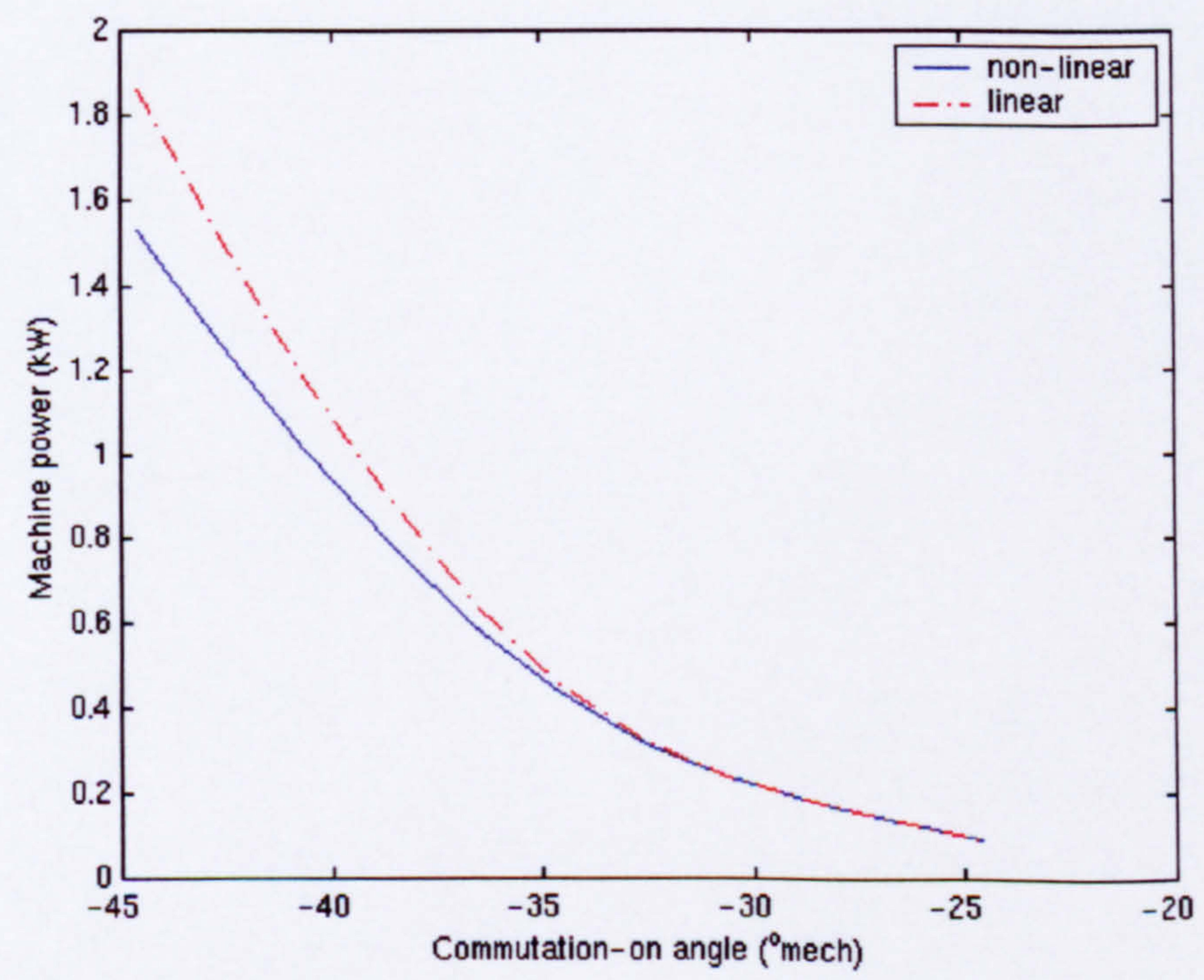

Figure 3.36. Average power derived from the operating conditions shown in figure 3.35 as a function of the turn-on angle. 


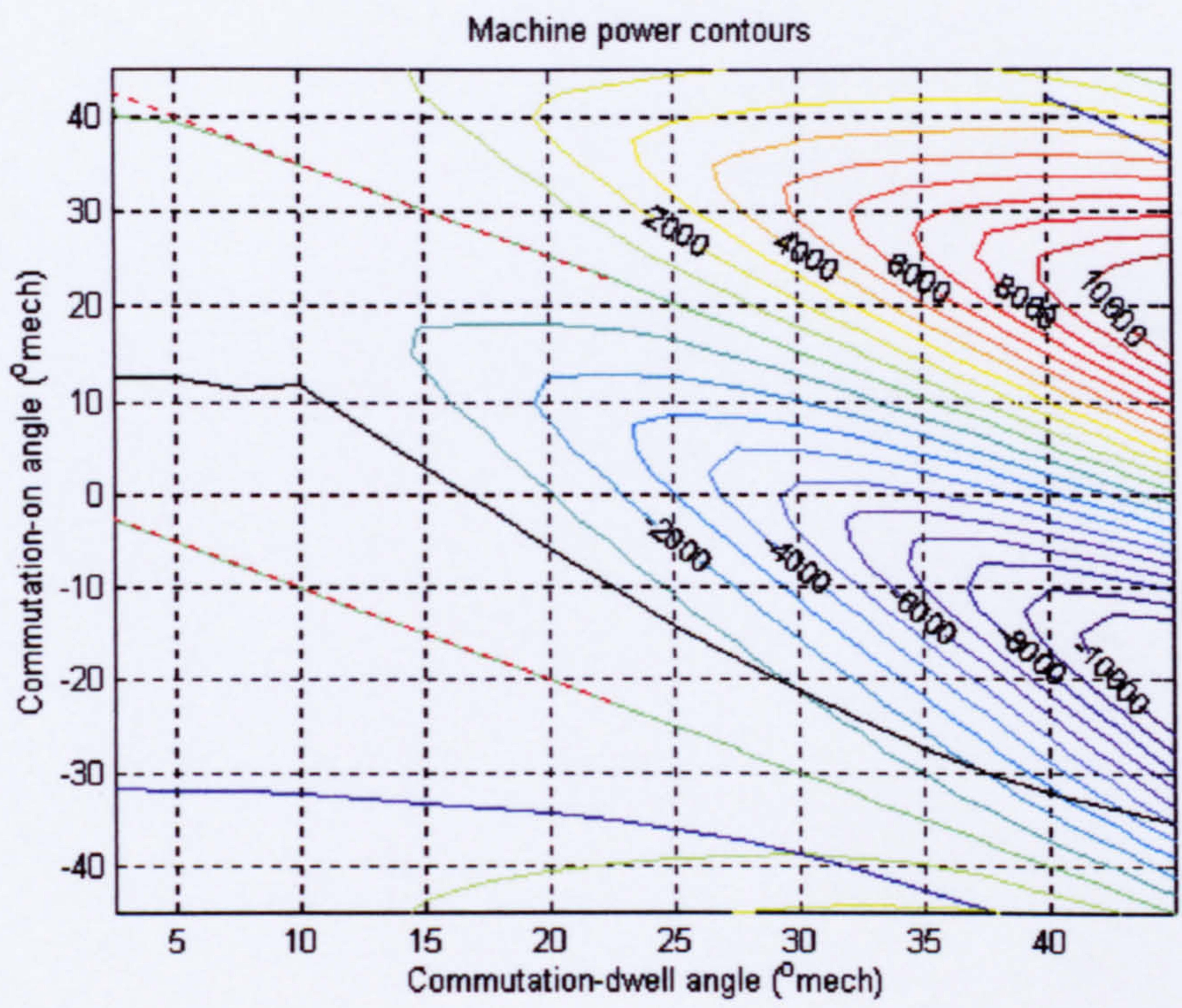

Figure 3.37. Performance map of the machine operating at $100000 \mathrm{rpm}$ calculated using the time stepped non-linear method.

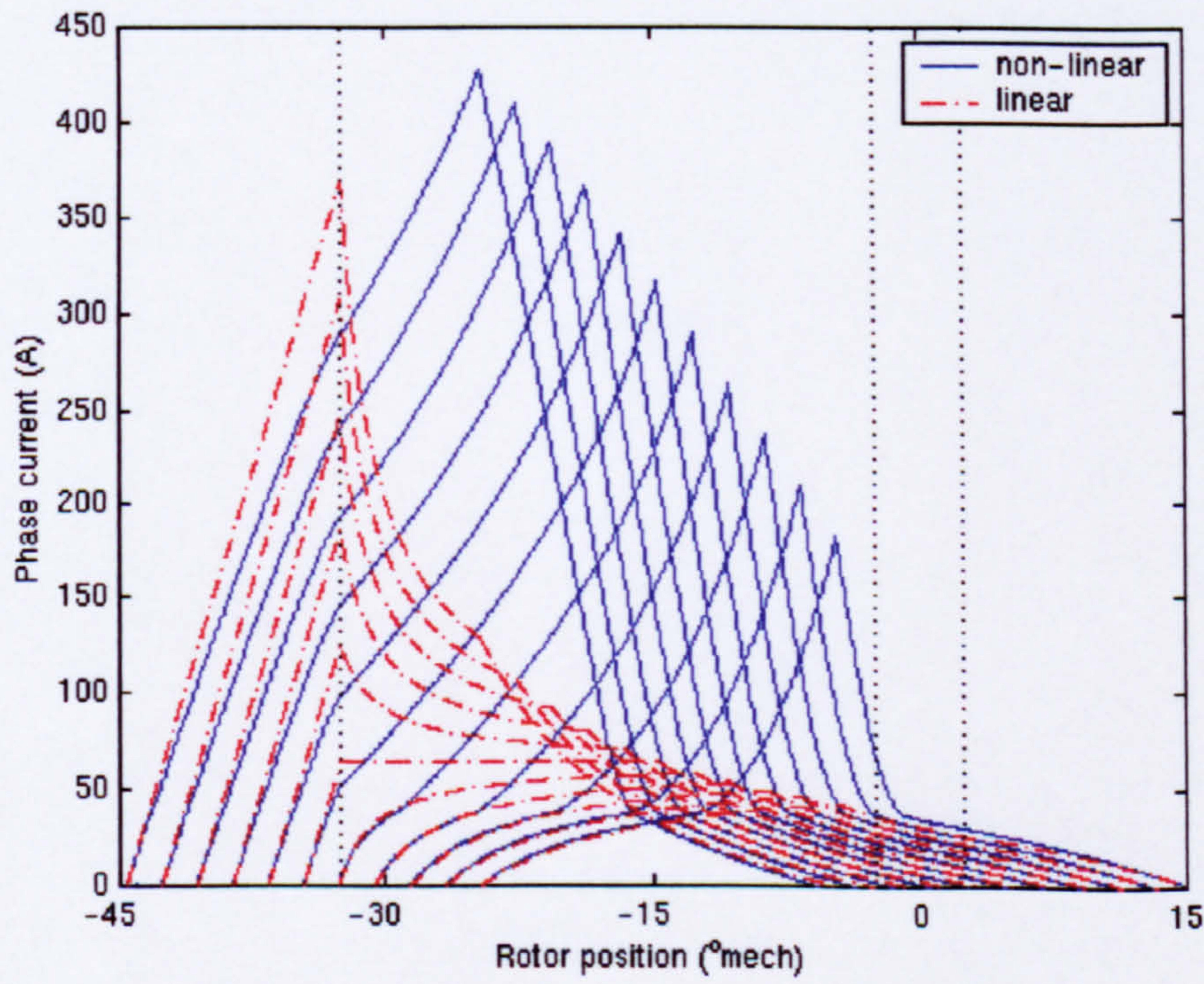

Figure 3.38. Current waveforms obtained using the linear and non-linear simulation techniques at various turn-on angles with a constant dwell angle of $20^{\circ}$ for the machine operating at $20,000 \mathrm{rpm}$. 


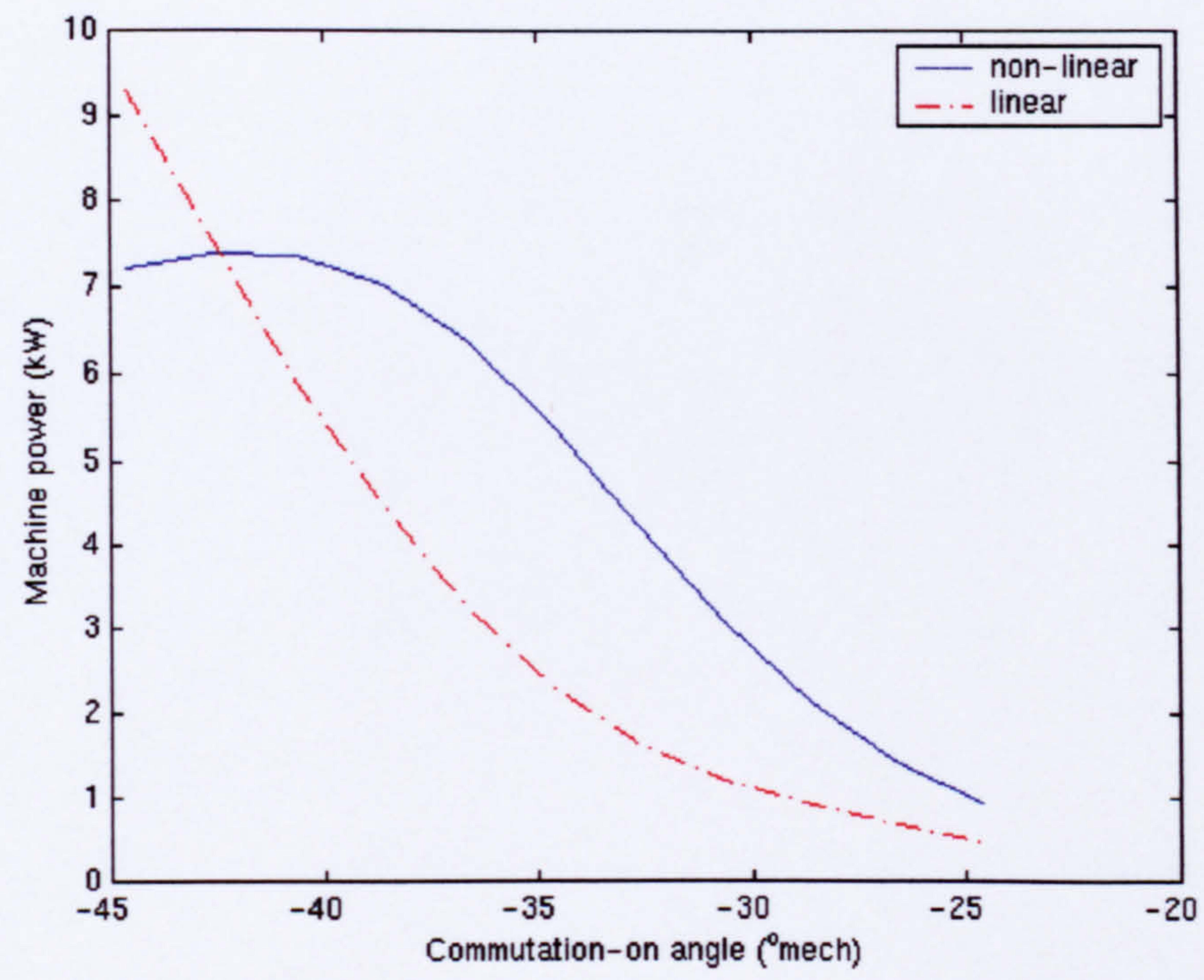

Figure 3.39. Average power derived from the operating conditions shown in figure 3.38 as a function of the turn-on angle

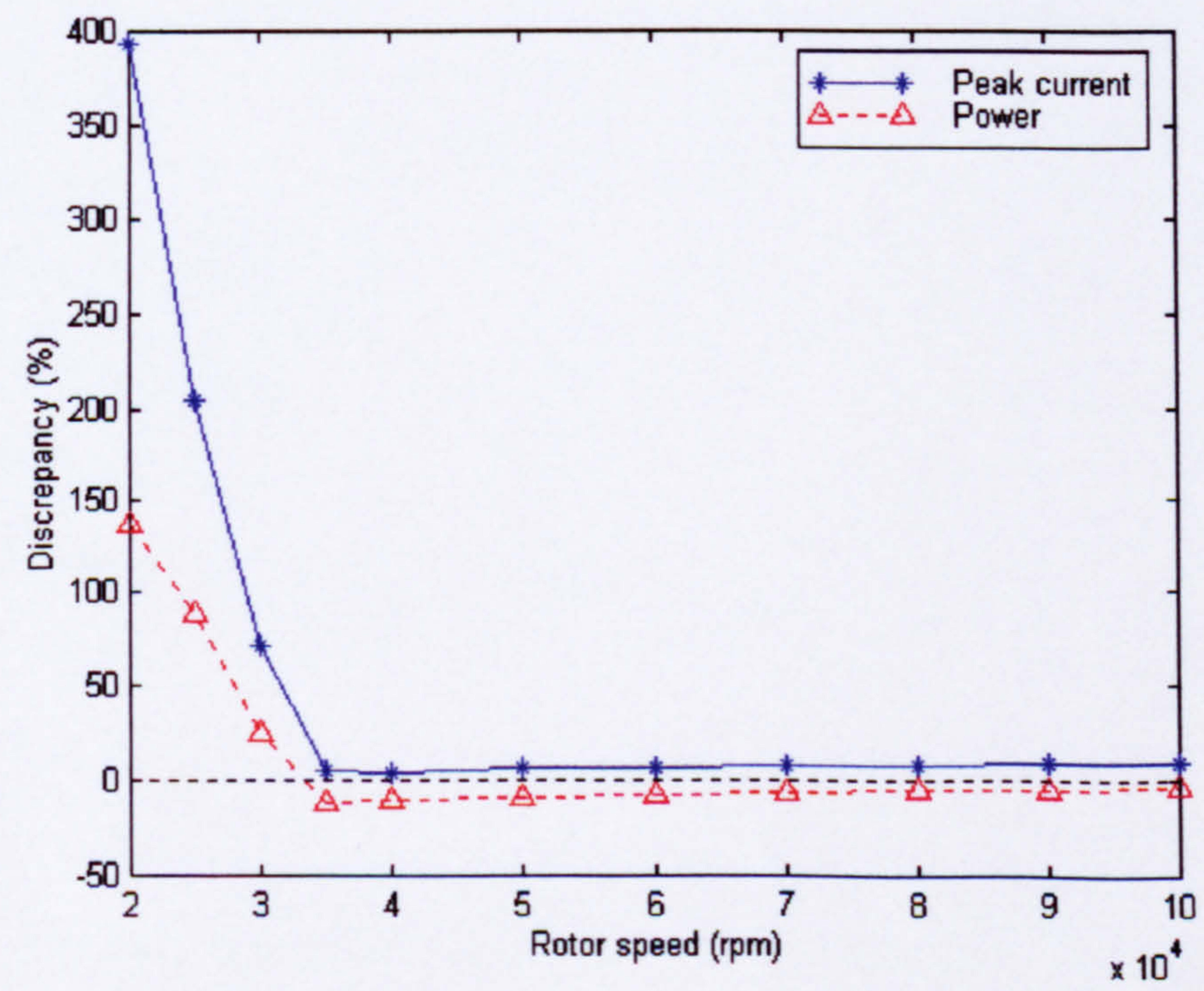

Figure 3.40. Discrepancy between the linear and non-linear model in the calculation of peak current and output power as a function of the rotor speed. 

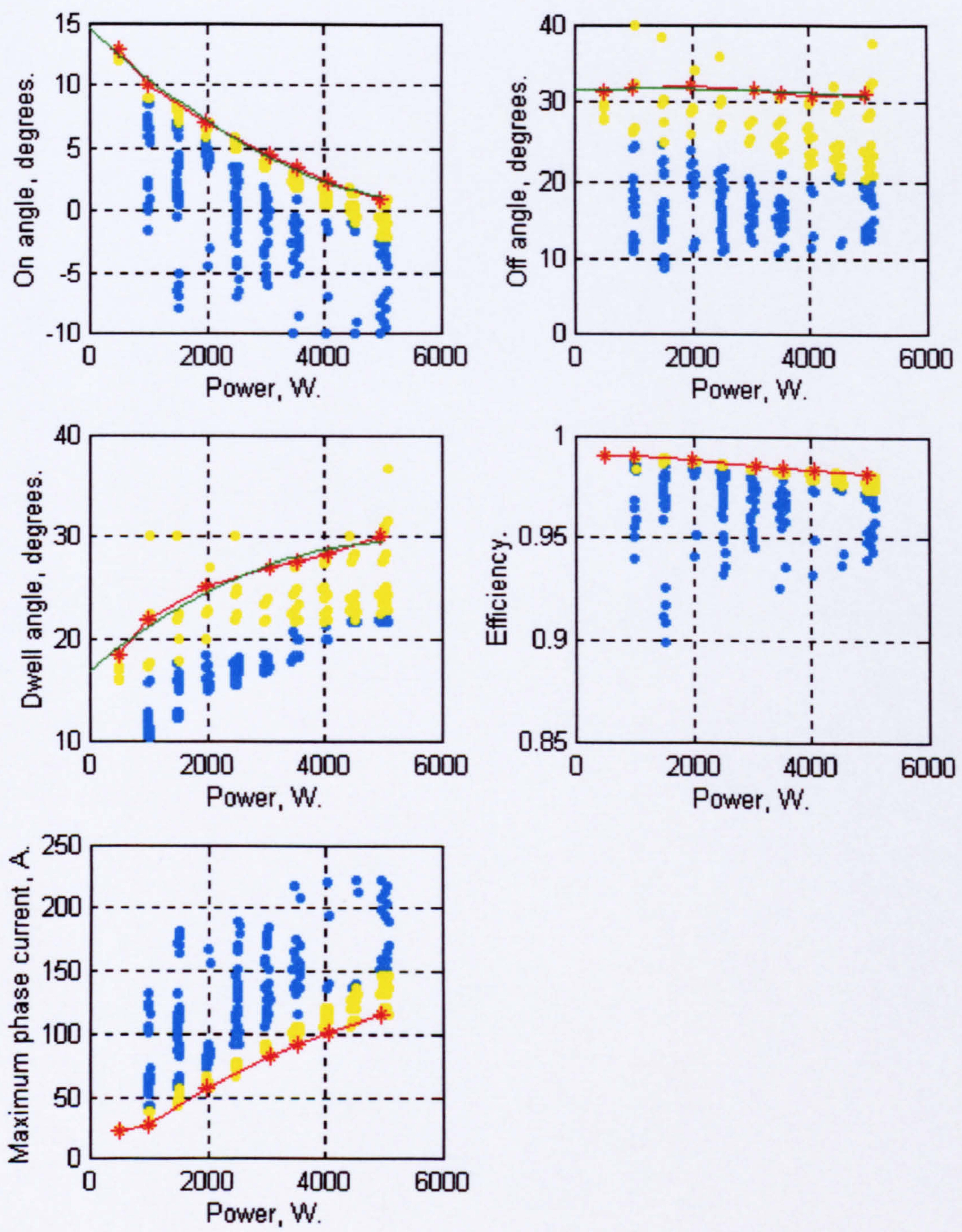

Figure 3.41. Sample Matlab GUI screen at 100,000rpm for selecting commutation angles. 


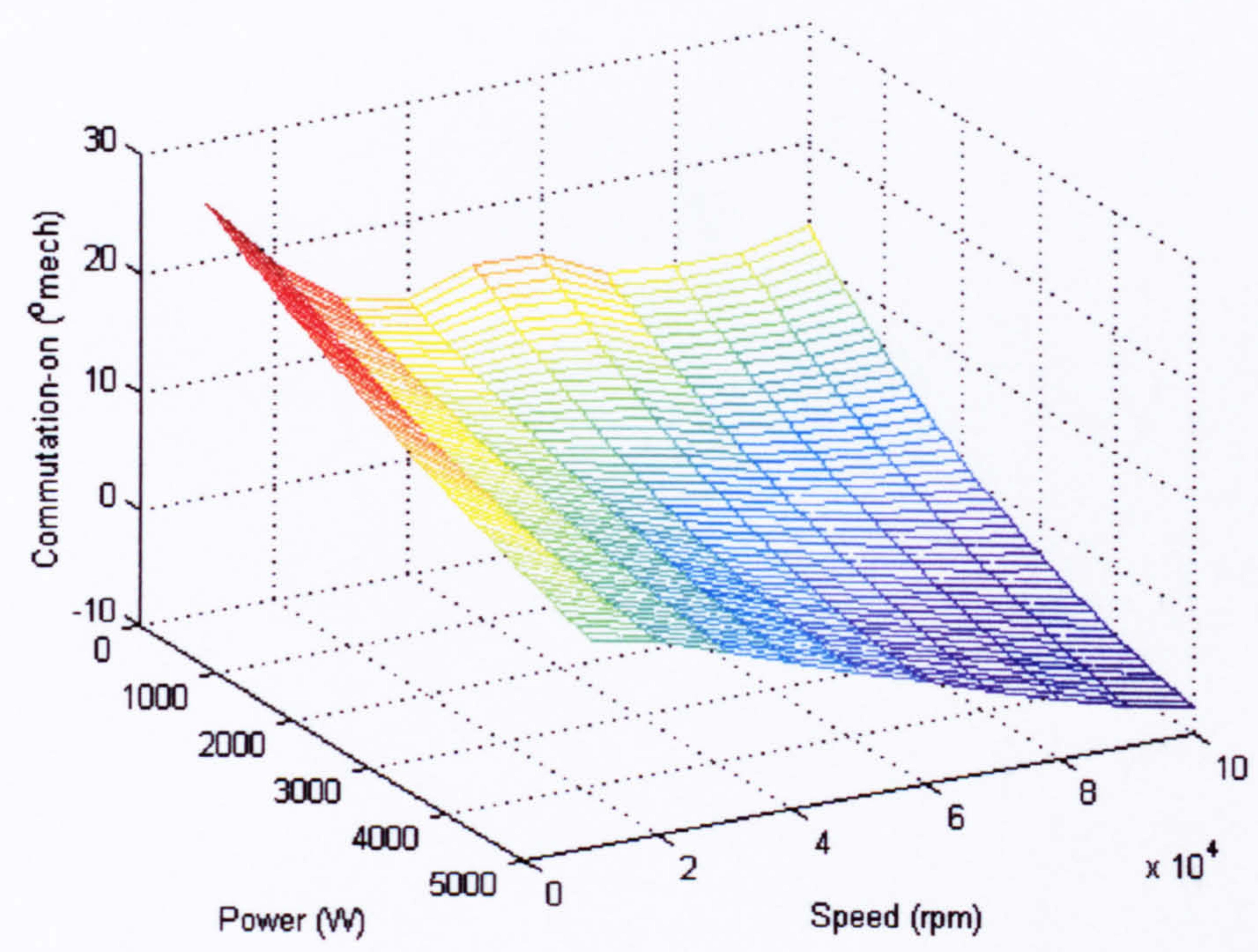

Figure 3.42a. Commutation-on angle map for motoring as a function of speed and power.

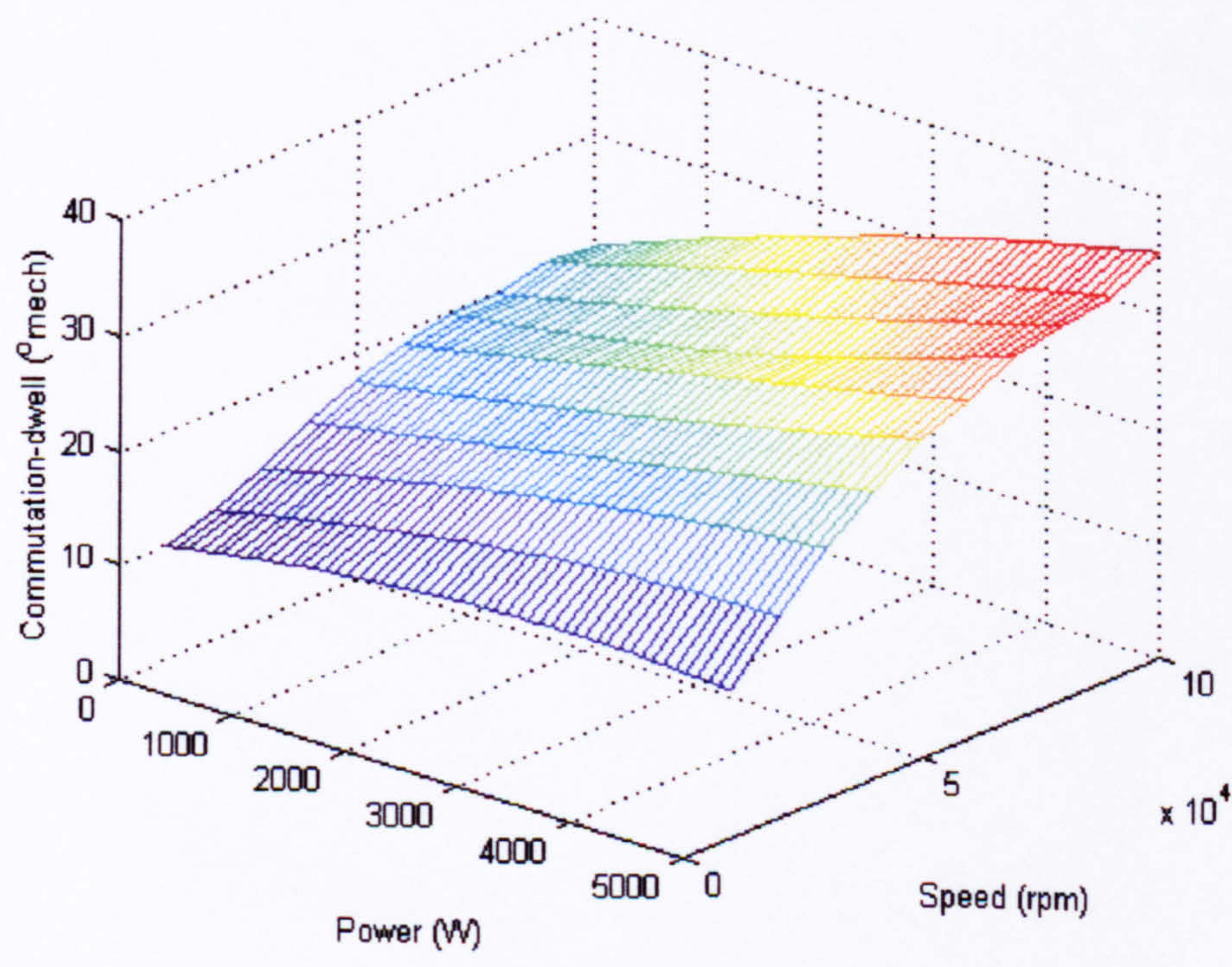

Figure 3.42b. Commutation-dwell angle map for motoring as a function of speed and power. 


\section{APPENDIX 3.1}

\section{POST PROCESSING FINITE ELEMENT SOLUTIONS TO CALCULATE WINDING FLUX LINKAGE AND INDUCTANCE.}

\section{Linear representation - Finite element calculation of inductance}

The inductance of the winding is calculated from the finite element field solution using:

$$
L_{\mathrm{ph}}=\frac{N_{\mathrm{ph}}^{2} L_{a x}}{2}\left[\frac{\sum_{i=1}^{m} A_{z, i} A e_{i}}{\sum_{i=1}^{m} A e_{i} \sum_{i=1}^{m} J_{i} A e_{i}}-\frac{\sum_{i=1}^{n} A_{z, i} A e_{i}}{\sum_{i=1}^{n} A e_{i} \sum_{i=1}^{n} J_{i} A e_{i}}\right]
$$

Where the integers $m$ and $n$ are the number of elements in the cross section of the winding defined in figure $\mathrm{A} 3.1, A_{z, i}$ is the vector potential of element $i$ calculated by Ansys, $A e_{i}$ is the cross sectional area of element $i$ and $J_{i}$ is the current density applied to element $i$.

\section{Non-linear representation - Finite element determination}

The finite element method discussed in the previous section may be extended to predict the variation of the flux linkage as the current is increased in the aligned and unaligned positions. Since the variation is no longer linear, the two characteristics (particularly the aligned) may no longer be represented as a single inductance and the full calculation of the $\psi-i$ curve is required. The current density applied to the mesh of figure 3.6, and a comparable mesh for the rotor in the unaligned position, was increased from 1 to $30 \mathrm{~A} / \mathrm{mm}^{2}$. The flux linking the phase for each case is calculated using 


$$
\psi_{\mathrm{ph}}=L_{\alpha x} N_{\mathrm{ph}}\left[\frac{\sum_{i=1}^{m} A_{z, i} A e_{i}}{\sum_{i=1}^{m} A e_{i}}-\frac{\sum_{i=1}^{n} A_{z, i} A e_{i}}{\sum_{i=1}^{n} A e_{i}}\right]
$$

Figure A3.2 shows the results of the finite element simulations for the aligned and unaligned case. It is apparent from figure A3.2 that for the range of current densities modelled the material shows a high degree of saturation in the aligned position, conversely however the characteristic remains linear throughout the range of current densities modelled for the rotor in the unaligned position. 


\section{APPENDIX 3.2}

\section{ANALYTICAL CALCULATION OF INDUCTANCE IN ALIGNED AND UNALIGNED POSITIONS.}

\section{Linear representation - Analytical inductance calculation}

The inductance is calculated separately for the aligned and unaligned case. The calculation of the inductance in the aligned position, $L_{a}$, is relatively straightforward, assuming the steel is infinitely permeable and the reluctance of the magnetic circuit is attributable solely to the airgap.

The flux density in the airgap may be determined using:

$$
B_{8}=\frac{\mu_{0} N_{\mathrm{ph}} i}{2 l_{8}}
$$

and the cross sectional area of the airgap is calculated from the smaller of the rotor or stator pole arcs (which in this case is the stator pole arc):

$$
A_{g}=\frac{\beta_{s}}{2} D_{g} L_{a x}
$$

The flux linking the phase is therefore:

$$
\psi=N_{\mathrm{ph}} \phi=N_{\mathrm{ph}} B_{g} A_{g}=\frac{\mu_{0} N_{\mathrm{ph}}^{2} i \beta_{g} D_{g} L_{a x}}{4 l_{g}}
$$

And hence the phase inductance is: 


$$
L_{\mathrm{ph}, \mathrm{a}}=\frac{\psi}{i}=\frac{\mu_{0} N_{\mathrm{ph}}^{2} \beta_{s} D_{g} L_{a x}}{4 l_{g}}
$$

Which for the current design yields an aligned inductance of $6.25 \mu \mathrm{H}$ with a winding comprising a single turn per stator tooth (i.e. $N_{p h}=2$ ).

Calculation of the unaligned inductance is however much more onerous than for the aligned case. Radun [3.3] uses conventional field theory to estimate the inductance of the rotor/stator design shown as a developed view in figure A3.3. For this particular geometry the inductance is given as a Fourier series thus:

$$
L_{\mathrm{ph}, \mathrm{u}}=4 \mu_{0} N_{\mathrm{ph}}^{2} L_{a x} l \sum_{n=\mathrm{odd}} \frac{\frac{\sin \left[\frac{n \pi \pi_{1}}{l}\right]}{l_{1}}+\frac{\sin \left[\frac{n \pi \pi_{1}}{l}\right]}{l_{2}}}{(\pi n)^{2} \tanh \left[\frac{n \pi h}{l}\right]}
$$

For the unaligned position where the interpolar region of the rotor lies perfectly aligned with the stator tooth then equation $\mathrm{A} 3.7$ becomes:

$$
L_{\mathrm{ph}, \mathrm{u}}=8 \mu_{0} N_{\mathrm{ph}}^{2} L_{a x} \cdot \frac{l}{l_{1}} \sum_{n=\mathrm{odd}} \frac{\sin \left[\frac{n \pi \pi_{1}}{l}\right]}{(\pi n)^{2} \tanh \left[\frac{n \pi h}{l}\right]}
$$

The rotor geometry adopted by Radun differs slightly from the current interpolar fillet design, see figure A3.4. Figure A3.5 shows a developed view of the current rotor with a fillet radius ratio of 0.275 . The interpolar fillet design adopted in the current application to minimise the stress concentration within the rotor structure is, geometrically, more complex than the design shown by Radun and the effective length of the rotor teeth $h$ is difficult to determine. Figure A3.6 shows the inductance variation calculated using equation A3.8 where the relative height of the rotor teeth 
(with respect to the interpolar spacing) is varied. It is apparent from figure A3.6 that the unaligned inductance is insensitive to this variable if it is greater than 1.5-2 times the circumferential spacing between the rotor and stator teeth, $l_{1,2}$. It is permissible therefore to determine the length of the rotor teeth for use in equation A3.8 based on the mean radius of the interpolar fillet, $r_{y}$, shown in figure A3.7. The mean radius of the interpolar fillet is calculated using equations A3.9 and A3.10. Where the angle, $\theta_{y p}$, between the yoke and the rotor pole is defined in figure A3.7.

$$
\gamma=\sqrt{\frac{2 r_{y}^{2}\left(\theta_{y p}\right)-2 \sqrt{2} D_{r} r_{y} \sin \left(\alpha_{p} / 2\right) \sin \left(\theta_{y p} / 2\right)}{D_{r}^{2}(4-\pi)}}
$$

Where,

$$
\theta_{y p}=\frac{\pi}{2}-2 \sin ^{-1}\left(\frac{D_{r} \sin \left(\alpha_{p} / 2\right)}{2 r_{y}}\right)
$$

Equation A3.9 cannot be inverted and must be solved iteratively to find the mean radius of the rotor yoke, which for the current rotor, where the fillet radius ratio $\gamma$ is 0.275 , is $13.19 \mathrm{~mm}$. The height of the rotor tooth for use in equation A3.9 is subsequently determined using

$$
h=\frac{D_{r}}{2}-r_{y}
$$

Using equations $\mathrm{A} 3.9$ - $\mathrm{A} 3.11$ it is possible to determine the unaligned inductance for rotors as a function of the fillet radius ratio. Figure A3.8 shows the variation in the unaligned inductance for the rotor with a diameter $38 \mathrm{~mm}$ with a single turn per stator pole where the fillet radius ratio is increased from zero to the maximum value of 0.326 . The inductance (for a winding comprising a single turn per pole) varies 
from a minimum value of $0.33 \mu \mathrm{H}$ when the fillet radius ratio is zero to a maximum of $0.46 \mu \mathrm{H}$ when the fillet radius ratio is 0.326 . The effective height $h$ of the rotor tooth for these cases is $10.92 \mathrm{~mm}$ and $4.93 \mathrm{~mm}$ respectively. For the current rotor the unaligned inductance is $0.41 \mu \mathrm{H}$ 


\section{APPENDIX 3.3}

\section{APPROXIMATING THE GAUGE CURVES USING ESTABLISHED CURVE FITTING TECHNIQUES}

The finite element analysis reported in the previous section highlighted two key areas in which the nature of the $\psi-\theta$ curve deviates from the simplified trapezoidal variation, viz. the constant offset, and the curvature of the variation at the beginning and upon completion (figure 3.12) and may be approximated by the following empirically derived formula:

$$
\xi_{0}=\xi_{1}-\frac{\psi}{\psi_{m}} \frac{\left(\xi_{a}-\xi_{u}\right)}{12}
$$

Where $\psi$ is the flux linking the phase winding at any instant and $\psi_{m}$ is the flux linkage at maximum excitation.

In order to represent the curvature of the $\psi-\theta$ variation the characteristic gauge curves are divided into the three regions, as shown in figure 3.12, mathematical functions and boundary conditions are defined for each region. Region II is characterised by a simple linear variation and it is appropriate to discuss this region in the first instance as it is used as a basis on which the boundary conditions for regions I and III may be defined.

\section{Region II $\left(\xi_{1}-\xi_{\text {hr }}\right)$}

The variation of flux linkage, at constant current, in this region is attributable to the increase in the effective area of the airgap as the degree of mechanical overlap is increased. It is appropriate to represent the flux linkage in the region as a linear variation, in a similar manner to the trapezoidal approach discussed 
above. The gradient $k_{a}$ of the straight line, which is bounded by the rotor positions $\xi_{l}$ and $\xi_{h r}$ is obtained using

$$
k_{a}=\frac{\psi_{a}-\psi_{u}}{\xi_{2}-\xi_{1}}
$$

The flux linkage at any rotor position within region II can be calculated from:

$$
\psi(\theta)=\psi_{u}+k_{a}\left(\theta-\xi_{0}\right)
$$

Which for convenience can be presented as

$$
\psi(\theta)=\psi_{u}+\frac{\left(\theta-\xi_{0}\right)\left(\psi_{a}-\psi_{u}\right)}{\left(\xi_{2}-\xi_{1}\right)}
$$

\section{Region I $\left(\xi_{\mathrm{u}}-\xi_{1}\right)$}

In the trapezoidal model, the flux linkage is assumed to be $\psi_{u}$ at all rotor angles where the mechanical overlap of figure 3.9 is zero. In practice the fringing of the flux before the rotor comes into mechanical alignment requires that the flux linkage variation is modelled as a non-linear function of rotor position. The most appropriate curve, identified by Miller [3.7], is that of a Fröhlich-type curve of the form

$$
\psi(\theta)=\psi_{1}+\frac{A\left(\theta-\xi_{1}\right)}{B-\left(\theta-\xi_{1}\right)}
$$

The imposition of two boundary conditions viz.: 


$$
\frac{d \psi\left(\xi_{1}\right)}{d \theta}=k_{a} \quad \text { and } \quad \psi\left(\xi_{u}\right)=\psi_{u}
$$

give a curve described by

$$
\psi(\theta)=\psi_{1}+\frac{k_{a}\left(\psi_{1}-\psi_{u}\right)\left(\xi_{1}-\xi_{u}\right)\left(\theta-\xi_{1}\right)}{\left(\psi_{1}-\psi_{u}\right)\left(\xi_{1}-\xi_{u}\right)-\left(\theta-\xi_{1}\right)\left[k_{a}\left(\xi_{1}-\xi_{u}\right)-\left(\psi_{1}-\psi_{u}\right)\right]}
$$

Again the flux linkage variation is presented as a function of known rotor angles and flux linkages.

\section{Region III $\left(\xi_{\mathrm{hr}}-\xi_{\mathrm{a}}\right)$}

The flux linkage variation in this region is again represented by a Fröhlich curve similar to that adopted in region I. The boundary conditions with which the specific curvature function is derived are:

$$
\frac{d \psi\left(\xi_{h r}\right)}{d \theta}=k_{a} \text { and } \psi\left(\xi_{a}\right)=\psi_{a}
$$

The curvature function is similar to that of region I and is given by:

$$
\psi(\theta)=\psi_{h r}+\frac{k_{a}\left(\psi_{a}-\psi_{h r}\right)\left(\xi_{a}-\xi_{h r}\right)\left(\theta-\xi_{h r}\right)}{\left(\psi_{a}-\psi_{h r}\right)\left(\xi_{a}-\xi_{h r}\right)-\left(\theta-\xi_{j h r}\right)\left(k_{a}\left(\xi_{a}-\xi_{h r}\right)-\left(\psi_{a}-\psi_{h r}\right)\right]}
$$

One convenient feature of this particular set of curve fitting functions and boundary conditions is that, apart from the determination of the aligned and unaligned magnetisation curves in the initial instance, the $\psi-\theta$ plane is fully defined for all levels of excitation and rotor angles. However, the inclusion of the flux linkage terms $\psi_{1}$ and $\psi_{h r}$ require that a time-stepped approach is used in order to simulate the machine with arbitrary excitation levels. 


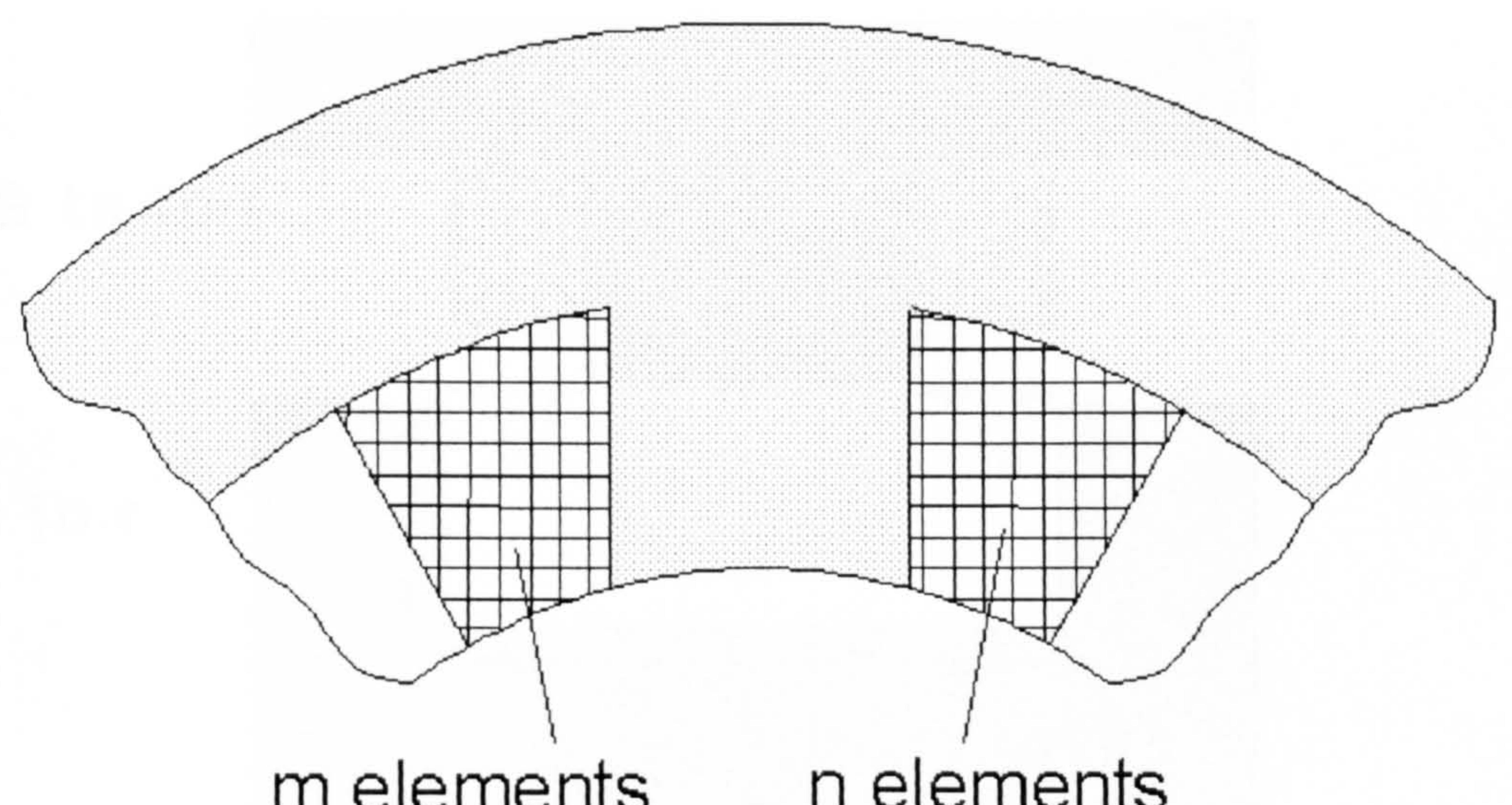

Figure A3.1 Elements used for the calculation of phase inductance.

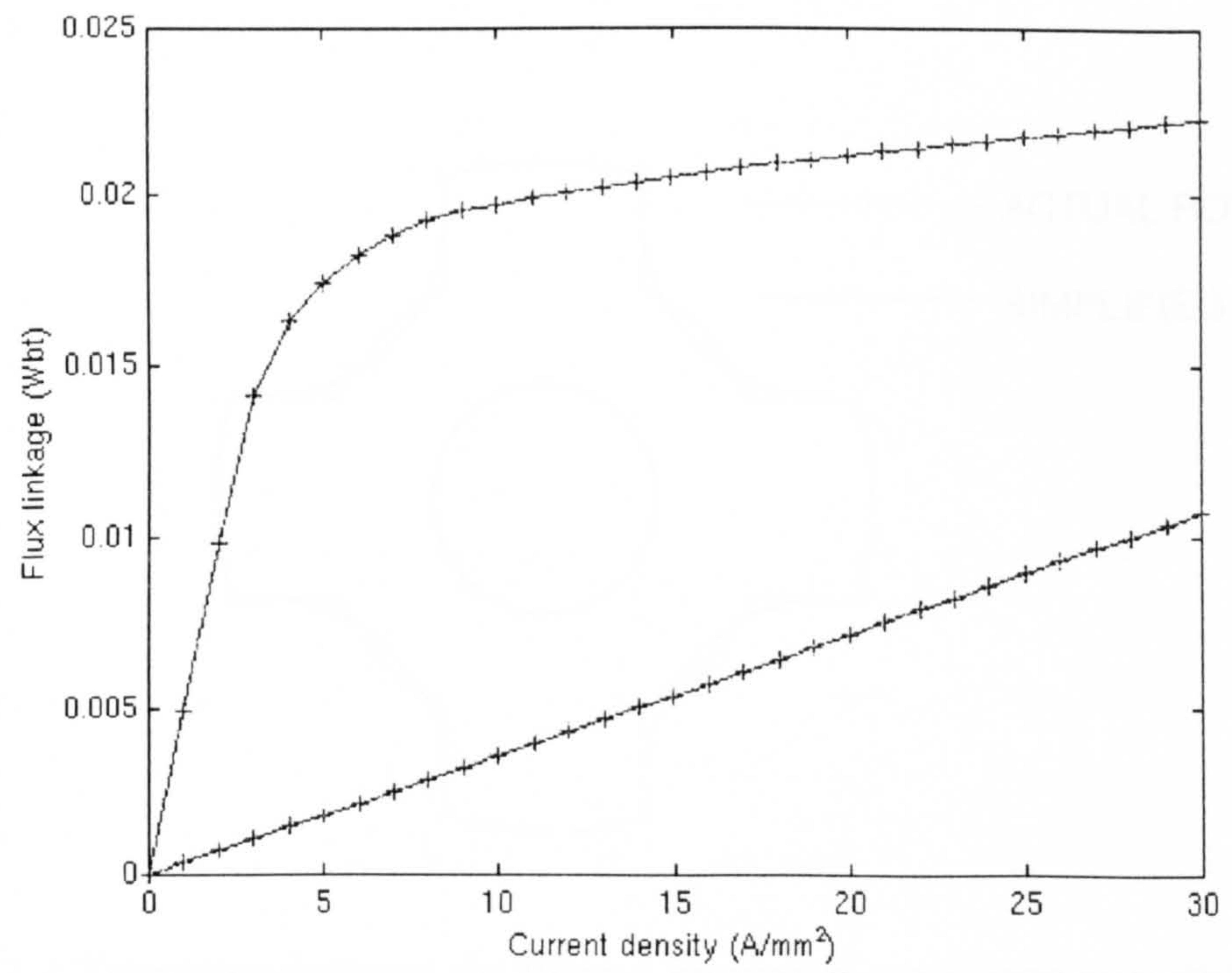

Figure A3.2. Aligned and unaligned $\psi-i$ characteristics calculated using Ansys 


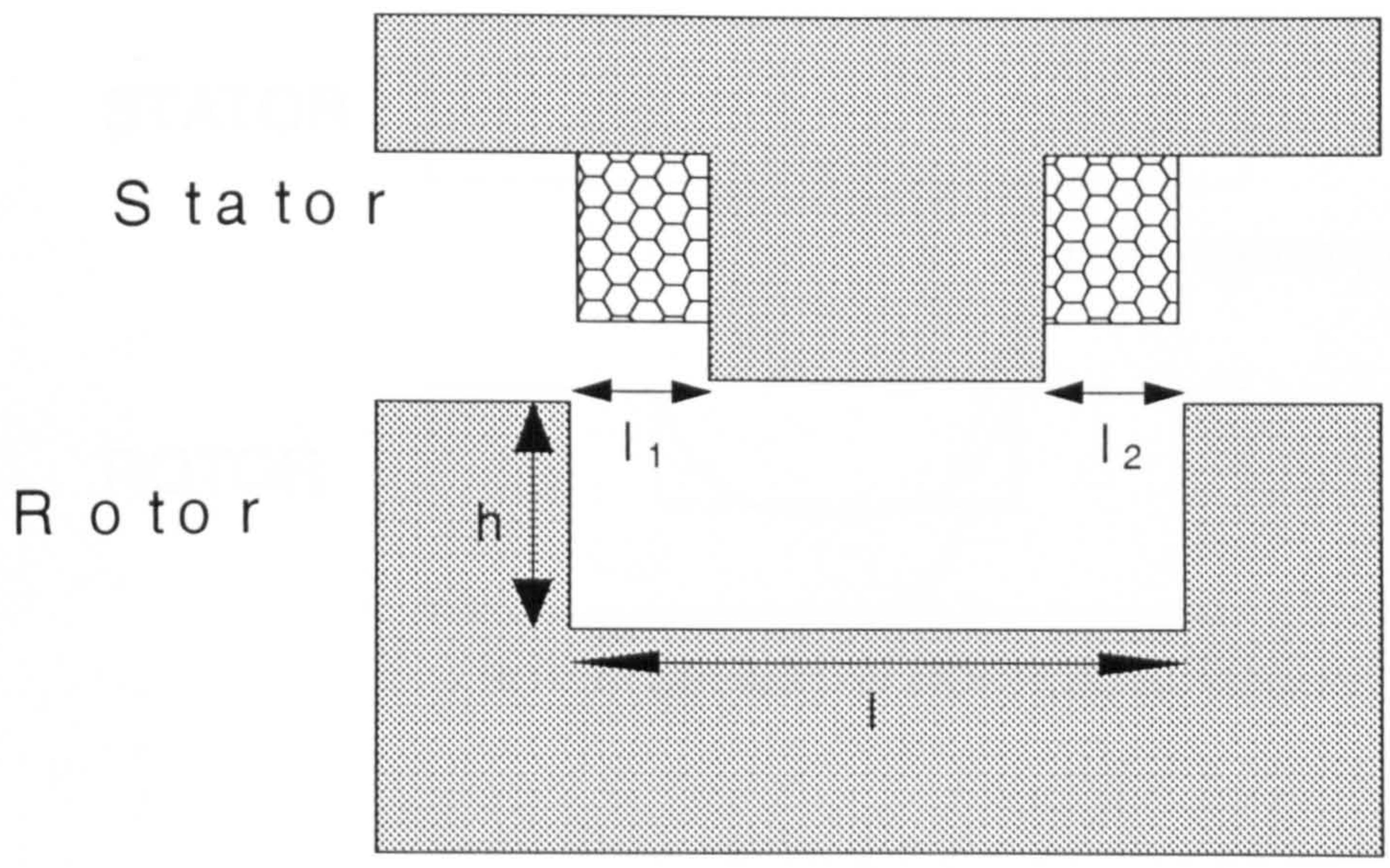

Figure A3.3 Simplified interpolar geometry of rotor for inductance calculation [3.3]

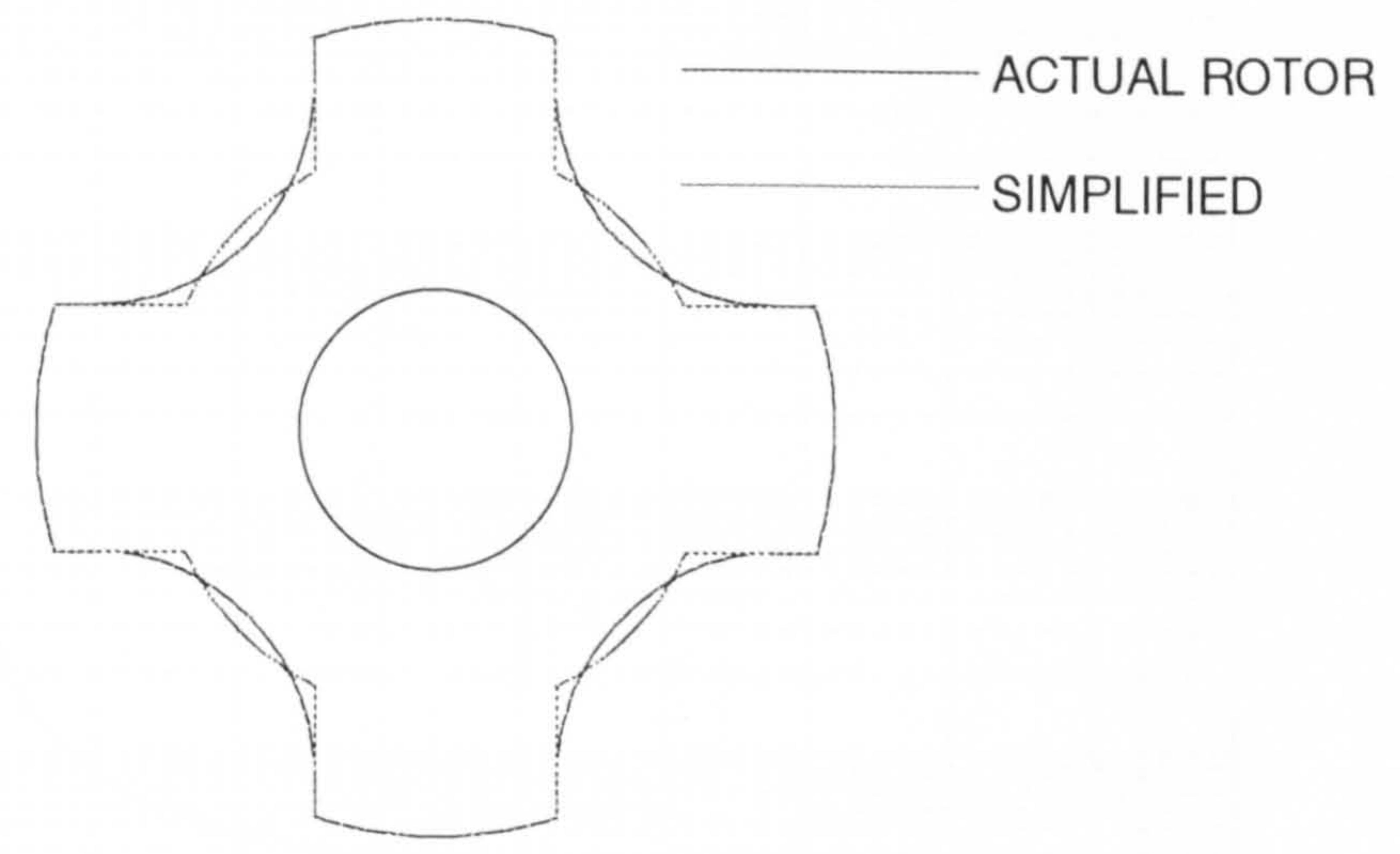

Figure A3.4 Two rotor designs: the filleted interpolar rotor geometry and a simplified geometry used by Radun [3.3]. 


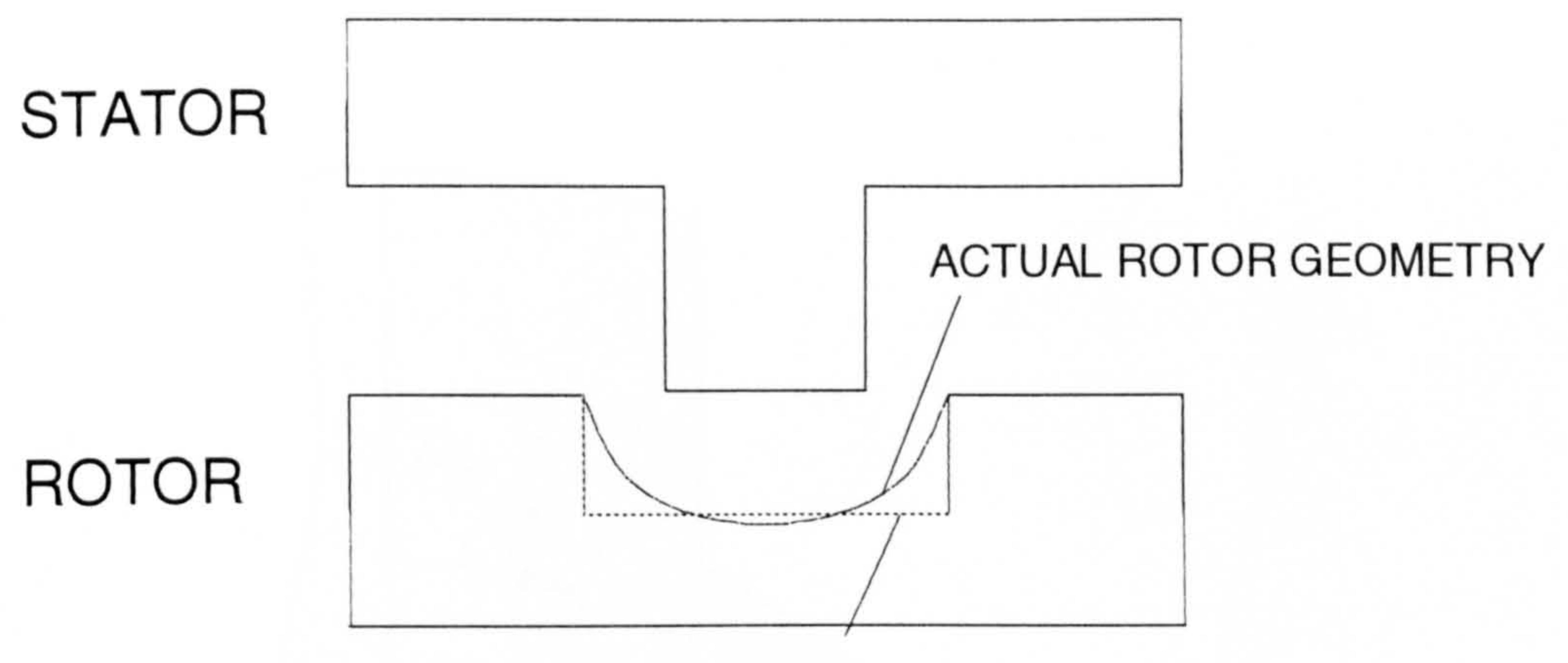

ROTOR GEOMETRY MODELED BY RADUN

Figure A3.5. Developed rotor and stator geometry for the rotor with a fillet radius ratio of 0.275 and the geometry modelled by Radun [3.3].

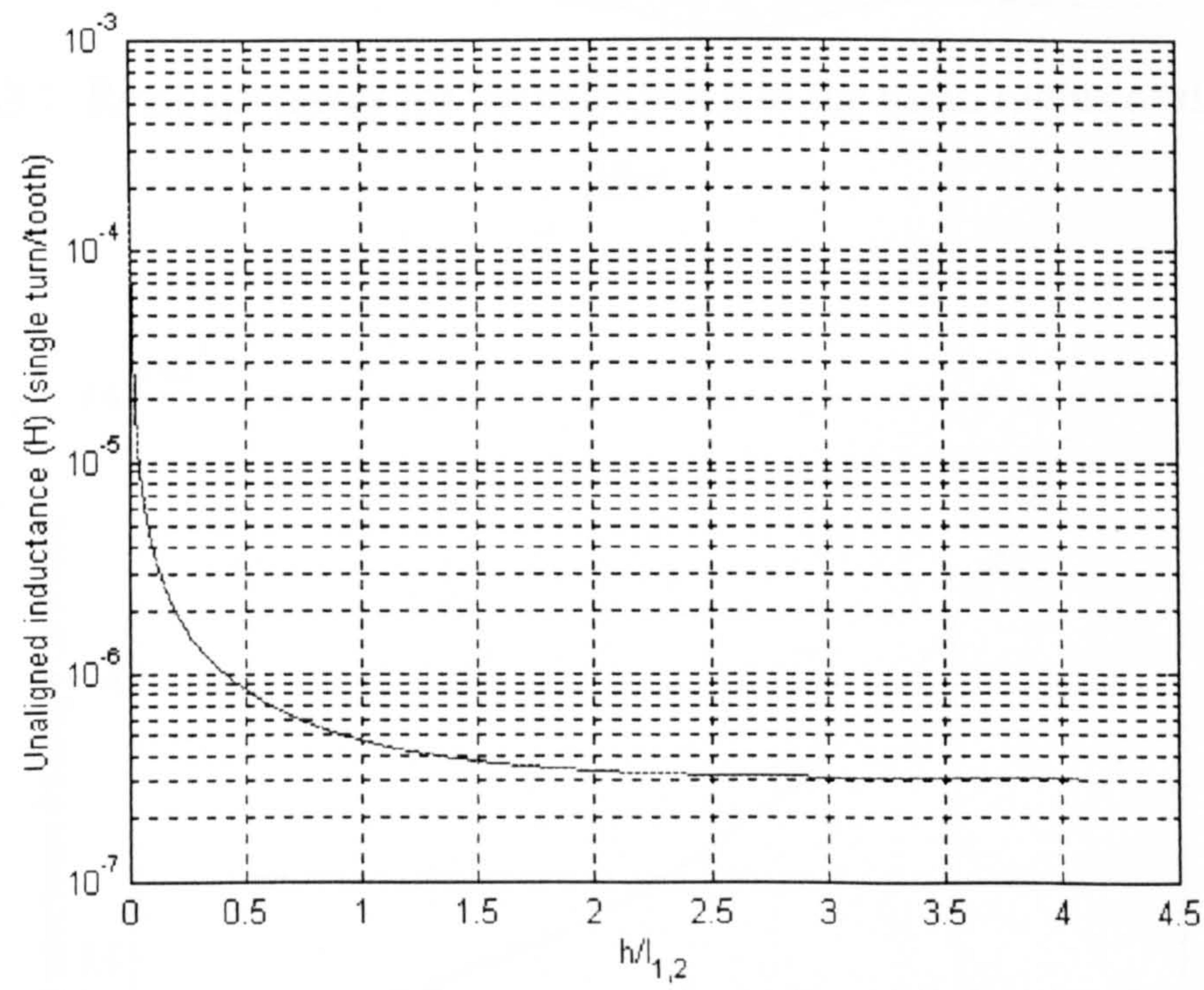

Figure A3.6 Variation of unaligned inductance as a function of the height of the rotor teeth. 


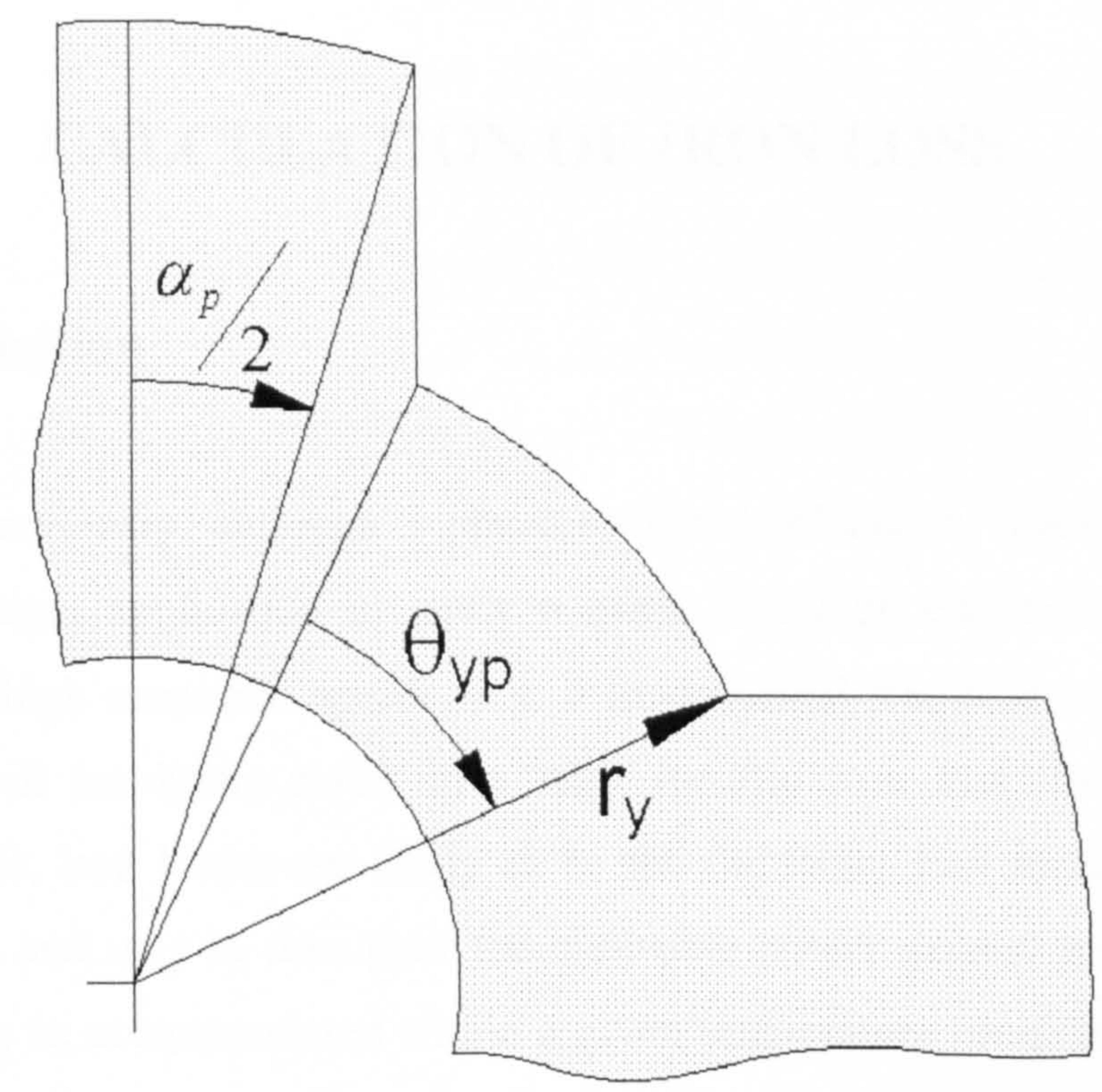

Figure A3.7. Rotor geometry for the calculation of the mean radius of the interpolar fillet.

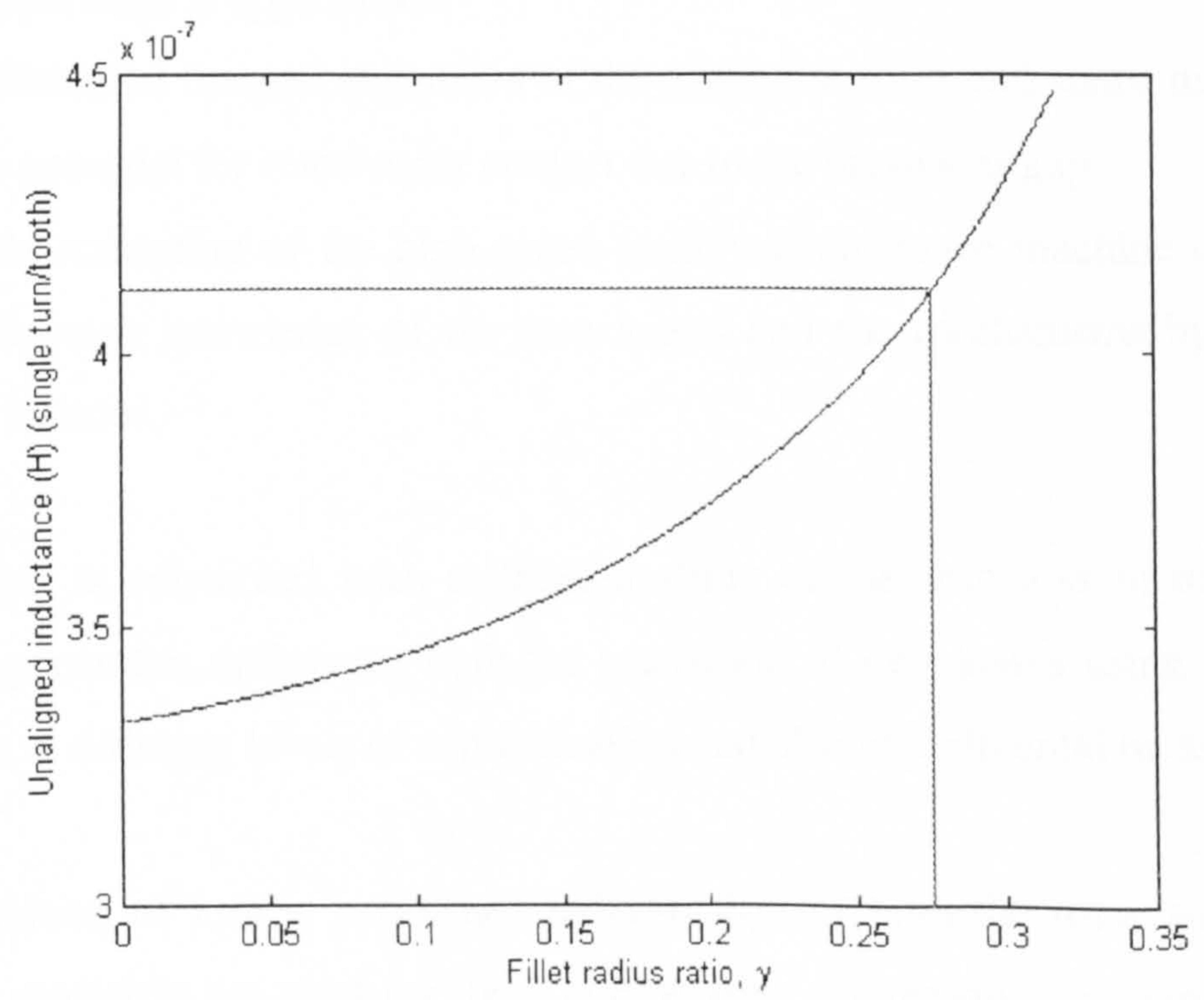

Figure A3.8. Unaligned inductance as a function of the fillet radius ratio 


\section{CHAPTER 4}

\section{CALCULATION OF IRON LOSS}

\subsection{Introduction}

As discussed previously, although 3 phase switched reluctance machines are well suited to the target application in many respects, the high excitation frequencies encountered at high rotational speeds (e.g. a fundamental frequency of $1.66 \mathrm{kHz}$ at $100000 \mathrm{rpm}$ ) will inevitably result in a high specific iron loss. Indeed, at high rotational speeds, iron losses are likely to be the dominant loss mechanism in SR machines [4.1], and may in turn limit the attainable power density and/or the duty cycle which can be accommodated within a given temperature limit. In this case the thermal limits of the machine may be defined by a combination of factors such as:

1. Conductor and lamination insulation thermal limits.

2. The reduced permeability of the soft magnetic material as the Curie temperature is approached.

3. Differential thermal expansion of the dissimilar rotor and stator materials and the potential for rotor/stator contact due to the narrow airgap.

The reliable utilisation of the high-speed switched reluctance machine can only be achieved through knowledge of the iron losses and the mechanisms by which the losses are induced.

This chapter is concerned with a detailed study of the iron loss in the proposed prototype generator, embracing both the prediction of iron losses using a variety of techniques at differing levels of sophistication and also experimental measurements.

The calculation of loss is performed individually for both the rotor and stator, as dissimilar materials are used for these components for reasons discussed in section 7.1.2 of chapter 7. Where any material or empirical constants are defined, the 
distinction between the two lamination materials is made. The calculation techniques are demonstrated initially for a reference operating condition of $5 \mathrm{~kW}$ electrical generation at 100,000 rpm. The specific operating parameters under these conditions are a DC link voltage of $96 \mathrm{~V}$ with a commutation on angle of $-13^{\circ}$ and a commutation off angle of $20^{\circ}$ (where $0^{\circ}$ is the aligned position). This operating condition is chosen as it represents the highest machine load at the highest fundamental flux frequency, and corresponds to the highest level of iron loss expected in the lamination material. (Motoring and generating conditions provide the same level of iron loss in switched reluctance machines [4.2]).

\subsection{Prediction of Iron Losses}

The iron loss in a given sample of soft magnetic material is a complex and material dependent function of excitation frequency and flux density. In the case of SR machines, different regions of the machine (viz. rotor teeth, rotor core, stator teeth and stator back iron) variously exhibit DC biased bi-polar, uni-polar alternating and rotating flux density waveforms. In this regard SR machines offer a particular challenge, as many of the current modelling techniques lack the degree of sophistication required to provide accurate results under these conditions.

In common with other machine types such as permanent magnet and induction machines, the prediction of iron loss in SR machines can be divided into two distinct stages:

i) The calculation of the temporal and spatial flux density variation within the machine at a given operating condition.

ii) The calculation of iron loss for the resulting flux density variation. 
The flux density waveforms in discrete machine regions can be established using a number of approaches:

i) Analytical - Direct analytical calculation of flux density waveforms in a limited number of lumped discrete regions such as tooth body, stator back-iron.

ii) De-coupled Finite Element Analysis - Calculation of a current waveform for a given operating condition using an electric circuit simulation such as that discussed previously, followed by a series of magneto-static de-coupled FE solutions with imposed current sources varying with rotor angle.

iii) Coupled Finite Element Analysis - The electric circuit equations and the electromagnetic field are solved simultaneously, where the field equations can be coupled to the electric circuit equations for a constant voltage supply [4.3]. This thesis is restricted to the consideration of the analytical and de-coupled finite element approaches as these methods can be integrated into an overall system simulation that is much more computationally efficient in terms of control strategy determination.

Having established localised flux density waveforms, either for a limited number of distinct regions of the machine (typically 20 or so for a 6/4 SR machines) or for a very large number of individual finite elements, the corresponding iron loss in a given material must be established. While techniques for the calculation of iron losses in materials exposed to symmetrical bi-polar flux density waveforms are relatively well established, (typically sinusoidal flux density waveforms with varying degrees of sinusoidal harmonic content), the calculation of iron loss for truly arbitrary flux density waveforms such as uni-polar waveforms and DC biased bipolar waveforms, remains unresolved in terms of a robust and fully generalised method. 


\subsection{Winding Configuration}

An important consideration in SR machines in which iron losses are likely to be the dominant loss is the connection of the windings. Since the torque generated by a phase winding is independent of the current polarity, then in the case of a 6/4 SR machine, either of the two possible winding connections defined as A and B in figures $4.1 \mathrm{a}$ and $4.1 \mathrm{~b}$ respectively can be employed. In winding connection $\mathrm{A}$, the coils are configured such that for a uni-polar current, three adjacent poles effectively act as $\mathrm{N}$ poles, and three as $\mathrm{S}$ poles. In the case of winding connection $\mathrm{B}$, the coils are configured such that the polarities of adjacent poles alternate. By virtue of the fact that the coupling between phases in a 6/4 SR machine is relatively low [4.4-4.6], there is very little difference between the two winding connections in terms of net machine torque capability. However, as will be demonstrated in this chapter, the winding connection does have an impact on the magnitude of the iron loss. Of the two alternative connections, published literature [4.1] suggests that winding arrangement $\mathrm{A}$ is likely to result in a lower iron loss, although no quantitative data was presented to support this assertion.

\subsection{Calculation of Flux Density Waveforms in the Machine}

\subsubsection{ANALYTICAL}

The analytical technique for the prediction of flux-linkage as a function of rotor position, which was described at length in section 3.5 of chapter 3 , can be extended to calculate the flux density variations in various regions of the stator and rotor iron. The flux in various regions such as the localised section of the stator back-iron can be calculated by appropriate summation of the tooth fluxes calculated in section 3.5 , with due account of the impact of the various current polarities on the flux distribution. It is necessary therefore to define a sign convention based on the direction of the flux in each of the regions to be used throughout the course of the chapter, as follows: 
Radially 'outwards'

Circumferentially clockwise
Positive

Positive

Published techniques [4.1, $4.7 \& 4.8]$ yield a generalised approach to calculating the flux waveforms using matrix analysis. In this thesis, a more specific formulation has been adopted, which is based on this well-established approach, and can clearly be shown to accommodate the differing winding connections detailed in figures $4.1 \mathrm{a}$ and $4.1 \mathrm{~b}$.

The flux variation in any region such as a section of the stator back-iron can be determined simply by appropriate addition of the individual tooth fluxes. In the general case, the flux density in region $i$, can be expressed in terms of the various winding flux linkages as:

$$
B_{i}=\frac{1}{G_{i} N}\left(k_{i A} \psi_{A}+k_{i B} \psi_{B}+k_{i C} \psi_{C}\right)
$$

Which in matrix form may be written

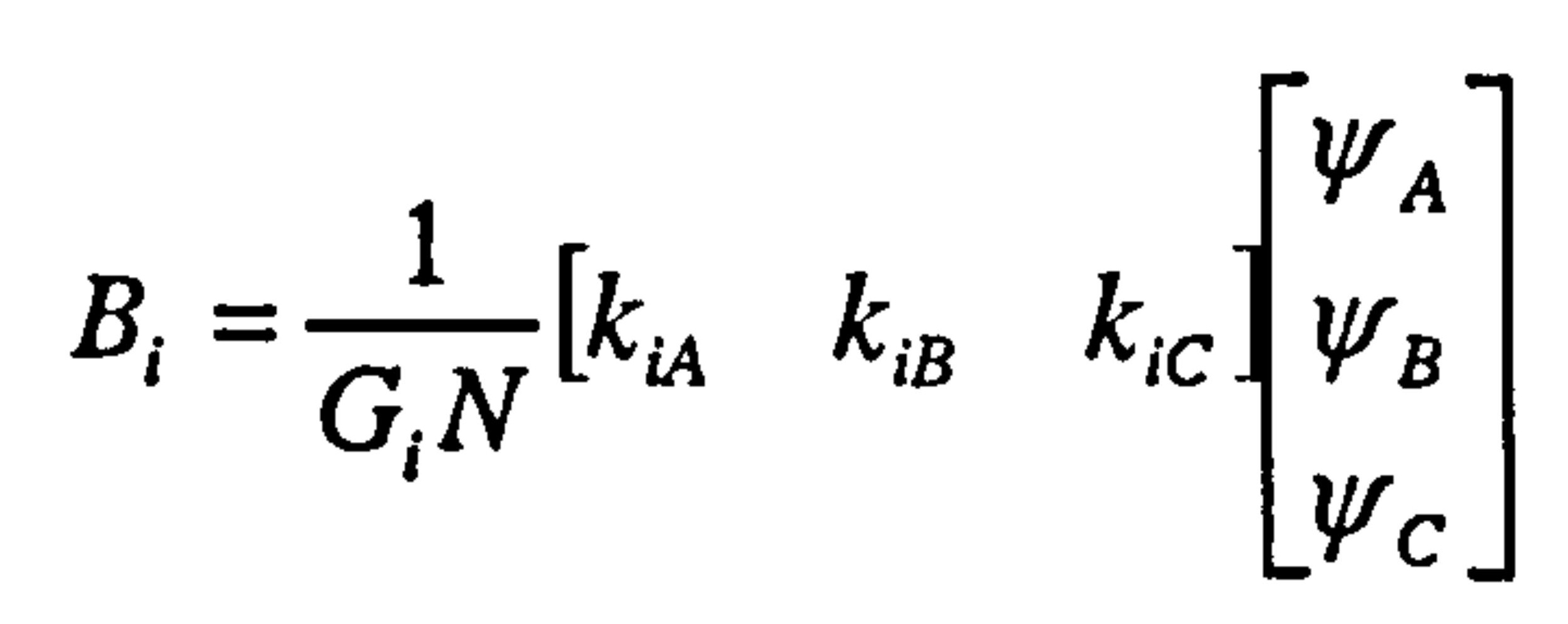

Where

$G_{i}$ is the effective cross sectional area of the region, incorporating the assumption that the flux produced in a given tooth by an excited phase winding is divided evenly in the corresponding back-iron region. Hence, $G_{i}=$ $A_{i}$ or $2 A_{i}$ in the pole and back-iron regions respectively.

$N$ is the number of series turns on each coil.

$\psi_{x}$ is the flux linking phase $x$ 
The coefficients of the $k$-matrix take values of 0,1 or -1 depending on the relative polarities of the individual flux linkages with respect to the location of region $i$ and the convention described.

For the 6 stator regions shown in figure 4.2, the coefficients of the $k$-matrix can be determined from table 4.1a for winding connection A (figure 4.1a) and table 4.1b for winding connection B (figure 4.1b). For example, by substituting the effective cross sectional area and the values in the $k$-matrix, equation 4.2 for region 1 with winding connection A becomes:

$$
B_{1}=\frac{1}{A_{1} N}\left[\begin{array}{lll}
-1 & 0 & 0
\end{array}\left[\begin{array}{l}
\psi_{A} \\
\psi_{B} \\
\psi_{C}
\end{array}\right]\right.
$$

and for region 4:

$$
B_{4}=\frac{1}{2 A_{4} N}\left[\begin{array}{lll}
-1 & -1 & +1
\end{array}\left[\begin{array}{l}
\psi_{A} \\
\psi_{B} \\
\psi_{C}
\end{array}\right]\right.
$$

\begin{tabular}{|c|c|c|c|}
\hline REGION & $k_{i A}$ & $k_{i B}$ & $k_{i C}$ \\
\hline 1 & -1 & 0 & 0 \\
\hline 2 & 0 & -1 & 0 \\
\hline 3 & 0 & 0 & -1 \\
\hline 4 & -1 & +1 & +1 \\
\hline 5 & -1 & -1 & +1 \\
\hline 6 & -1 & -1 & -1 \\
\hline
\end{tabular}

Table 4.1a. Coefficients of the $k$-matrix for the six stator regions detailed in figure 4.2 for winding connection $A$. 


\begin{tabular}{|c|c|c|c|}
\hline REGION & $k_{i A}$ & $k_{i B}$ & $k_{i C}$ \\
\hline 1 & -1 & 0 & 0 \\
\hline 2 & 0 & +1 & 0 \\
\hline 3 & 0 & 0 & -1 \\
\hline 4 & -1 & -1 & +1 \\
\hline 5 & -1 & +1 & +1 \\
\hline 6 & -1 & +1 & -1 \\
\hline
\end{tabular}

Table 4.1b. Coefficients of the $k$-matrix for the six stator regions detailed in figure 4.2 for winding connection $B$.

It is evident when comparing the coefficients in tables $4.1 \mathrm{a}$ and $4.1 \mathrm{~b}$ that the flux density waveform in region 6 for the two winding arrangements will differ. The regions 1-5 differ only in phase and overall polarity, and will exhibit, in the analytical case, identical iron loss densities.

The rotor regions are shown in figure 4.3, and the waveforms are calculated in a similar manner. However the angular position of the rotor in the stator determines the direction of the flux passing through the respective region. For example consider the case shown in figure 4.4a. Phase A produces flux in the rotor pole region highlighted radially inwards and, by convention, the flux density is negative. However if the rotor is rotated by $180^{\circ}$ the flux produced by phase A now acts radially outward in the rotor and is therefore considered positive.

The coefficients in the $k$-matrix are now dependent upon rotor position and change at $30^{\circ}$ intervals for the rotor pole (region 7) and $60^{\circ}$ intervals for the rotor back iron (region 8). It is possible however, to present the $k$-matrix, in both cases, at $60^{\circ}$ intervals if a further coefficient $k_{\tau}$ is introduced. This takes a value of 0 or 1 dependent upon the rotor angular position, such that $k_{\tau}=1$ for the first $30^{\circ}$ of each of the six $60^{\circ}$ intervals presented in tables $4.2 \mathrm{a}$ and $4.2 \mathrm{~b}$. 
For the rotor pole (region 7) the values for the $k$-matrix as a function of rotor angular position, $\theta$, (positive $\theta$ indicates clockwise rotation) for each of the winding connections detailed in figures $4.1 \mathrm{a}$ and $4.1 \mathrm{~b}$, are:

\begin{tabular}{|c|c|c|c|}
\hline Rotor angle & $k_{7 \mathrm{~A}}$ & $k_{7 B}$ & $k_{7 C}$ \\
\hline $0<\theta \leq 60$ & -1 & 0 & $+k_{\tau}$ \\
\hline $60<\theta \leq 120$ & $-k_{\tau}$ & -1 & 0 \\
\hline $120<\theta \leq 180$ & 0 & $-k_{\tau}$ & -1 \\
\hline $180<\theta \leq 240$ & +1 & 0 & $-k_{\tau}$ \\
\hline $240<\theta \leq 300$ & $+k_{\tau}$ & +1 & 0 \\
\hline $300<\theta \leq 360$ & 0 & $+k_{\tau}$ & +1 \\
\hline
\end{tabular}

Table 4.2a. Coefficients of the $k$-matrix for the rotor region 7 detailed in figure 4.3 for winding connection $\mathrm{A}$.

\begin{tabular}{|c|c|c|c|}
\hline Rotor angle & $k_{7 A}$ & $k_{7 B}$ & $k_{7 C}$ \\
\hline $0<\theta \leq 60$ & -1 & 0 & $+k_{\tau}$ \\
\hline $60<\theta \leq 120$ & $-k_{\tau}$ & +1 & 0 \\
\hline $120<\theta \leq 180$ & 0 & $+k_{\tau}$ & -1 \\
\hline $180<\theta \leq 240$ & +1 & 0 & $-k_{\tau}$ \\
\hline $240<\theta \leq 300$ & $+k_{\tau}$ & -1 & 0 \\
\hline $300<\theta \leq 360$ & 0 & $-k_{\tau}$ & +1 \\
\hline
\end{tabular}

Table 4.2a. Coefficients of the $k$-matrix for the rotor region 7 detailed in figure 4.3 for winding connection $\mathrm{B}$. 
And similarly the $k$-matrix for the rotor back iron (region 8 ) is presented in tables $4.3 \mathrm{a}$ and $4.3 \mathrm{~b}$.

\begin{tabular}{|c|c|c|c|}
\hline Rotor angle & $k_{8 A}$ & $k_{8 B}$ & $k_{8 C}$ \\
\hline $0<\theta \leq 60$ & +1 & -1 & -1 \\
\hline $60<\theta \leq 120$ & +1 & +1 & -1 \\
\hline $120<\theta \leq 180$ & +1 & +1 & +1 \\
\hline $180<\theta \leq 240$ & -1 & +1 & +1 \\
\hline $240<\theta \leq 300$ & -1 & -1 & +1 \\
\hline $300<\theta \leq 360$ & -1 & -1 & -1 \\
\hline
\end{tabular}

Table 4.3a. Coefficients of the $k$-matrix for the rotor region 8 detailed in figure 4.3 for winding connection $\mathrm{A}$.

\begin{tabular}{|c|c|c|c|}
\hline Rotor angle & $k_{8 A}$ & $k_{8 B}$ & $k_{8 C}$ \\
\hline $0<\theta \leq 60$ & +1 & +1 & -1 \\
\hline $60<\theta \leq 120$ & +1 & -1 & -1 \\
\hline $120<\theta \leq 180$ & +1 & -1 & +1 \\
\hline $180<\theta \leq 240$ & -1 & -1 & +1 \\
\hline $240<\theta \leq 300$ & -1 & +1 & +1 \\
\hline $300<\theta \leq 360$ & -1 & +1 & -1 \\
\hline
\end{tabular}

Table 4.3b. Coefficients of the $k$-matrix for the rotor region 8 detailed in figure 4.3 for winding connection $\mathrm{B}$.

Inspection of tables 4.1-4.3 shows that the difference in the winding connections is accommodated by the sign of the $k_{i B}$ coefficient, indicating the differing polarity of phase $B$ in the two arrangements. 
Given the geometry of the machine, as detailed in chapters 2 and 3 the effective cross-sectional area $A_{i}$ of each of the rotor and stator regions and hence the term $G_{i}$ can be determined as in table 4.4.

\begin{tabular}{|c|c|c|}
\hline REGION & $A_{i}\left(\mathrm{~m}^{2}\right)$ & $G_{i}\left(\mathrm{~m}^{2}\right)$ \\
\hline 1 & $5.02 \times 10^{-4}$ & $5.02 \times 10^{-4}$ \\
\hline 2 & $5.02 \times 10^{-4}$ & $5.02 \times 10^{-4}$ \\
\hline 3 & $5.02 \times 10^{-4}$ & $5.02 \times 10^{-4}$ \\
\hline 4 & $3.50 \times 10^{-4}$ & $7.00 \times 10^{-4}$ \\
\hline 5 & $3.50 \times 10^{-4}$ & $7.00 \times 10^{-4}$ \\
\hline 6 & $3.50 \times 10^{-4}$ & $7.00 \times 10^{-4}$ \\
\hline 7 & $5.71 \times 10^{-4}$ & $5.71 \times 10^{-4}$ \\
\hline 8 & $3.34 \times 10^{-4}$ & $6.68 \times 10^{-4}$ \\
\hline
\end{tabular}

Table 4.4. Effective cross sectional area of the rotor and stator regions defined in figures 4.2 and 4.3 .

The analytical waveforms in all the regions of the machine, with each winding connection, for the reference operating point selected in section 1 of this chapter are shown in figure 4.4.

\subsubsection{DE-COUPLED FINITE-ELEMENT METHOD}

The analytical approach described above, although offering a computationally efficient means of estimating the flux density waveforms, is nevertheless reliant on a number of simplifications in terms of machine and material behaviour as discussed in section 3.5 of chapter 3 .

In terms of the ability to accurately represent the flux conditions experienced in the machine, and hence provide a basis for subsequent iron loss calculation, the analytical method can be seen to be deficient in three ways: 
1. Insufficient spatial refinement. The analytical approach described requires the 3-phase SR machine to be divided into only 20 distinct regions. Due to the nature of the SR machine operation, significant spatial variation of flux density within the relatively coarse regions considered may be significant, particularly in the tooth tips as the rotor and stator teeth move into and out of mechanical alignment. As a consequence the prediction of iron loss, which is a non-linear function of flux density variation at fixed frequency may prove inadequate when based on the spatially averaged flux density waveforms.

2. Simplified representation of flux density conditions. The flux density waveforms calculated using the analytical approach must lie in either the radial or circumferential direction in the teeth and back iron regions respectively. Under these conditions no account can be taken of the flux density conditions in the transitional regions, where the principal flux direction may not lie in the radial or circumferential direction, or indeed a high rotational component may be present.

3. Poor resolution of field harmonics. Simplifications regarding the superposition of the flux in various regions of the machine represent a significant source of error in the analytical approach. As will be demonstrated in this chapter, the flux waveforms in all regions of the machine contain a significantly higher harmonic content than that suggested using the analytical approach

Non-linear finite element analysis by contrast is able to provide a more accurate representation of the harmonic content in the flux waveforms at a very high degree of spatial discretisation, which has considerable consequent benefit both in terms of predicting localised fields and providing the necessary spatial resolution for the subsequent calculation of iron loss. Nevertheless this method still requires several approximations:

1. The behaviour of the machine can be represented to an appropriate degree by a two-dimensional model, which inherently neglects end-effects and the presence of any axial field; this assumption becomes more appropriate as the 
ratio of machine length to diameter is increased. Whereas in the current application this ratio may not be large enough to prevent significant error of this type, the two-dimensional approach is adopted to reduce the problem domain, which is desirable given the high number of solutions required for the purpose of generating flux density waveforms.

2. Magnetic hysteresis within the stator and rotor lamination material is neglected in terms of its influence on the flux density waveforms for a given current excitation (although the effect of hysteresis is accounted for in the subsequent calculation of the iron loss for a given flux density waveform). Thus the non-linear behaviour of the stator and rotor lamination can be represented by a monotonic magnetisation curve.

3. The problem is assumed to be magneto-static i.e. the presence of any redistribution fields due to eddy currents within the laminations is neglected, although again eddy currents are considered in the subsequent calculation of iron loss.

4. The problem can be bounded at, or in close proximity to, the outer surface of the stator lamination by imposition of a Dirichlet boundary condition (where the flux is constrained to be tangential to the outer surface by the imposition of zero vector potentials on the boundary nodes) thus avoiding the need to model an extensive region of surrounding air, either by an extended mesh or by the use of specialised open-boundary conditions [4.9]

5. The stator and rotor laminations are modelled as being magnetically isotropic as they are nominally non-oriented materials. In practice, such 'non-oriented' materials exhibit some degree of anisotropy as a result of the rolling stage of manufacture [4.10], the effect of which on losses is discussed by Moses and Shirkoohi [4.11].

In order to achieve the flexibility required for the development of specific postprocessing programs for the calculation of iron loss, the mesh was constructed using in-house software. The mesh within the small airgap and tooth tips of the machine is highly dicretised, in order to accurately resolve the rapid spatial change in field distribution within this region. Consequently with the lack of any adaptive meshing 
capability the whole machine must be meshed with a very high level of discretisation, which is limited by data storage and computational requirements.

The resulting two-dimensional finite element mesh consisting of 23374 first order triangular elements (within which the rotor can be rotated about the axis at $1^{\circ}$ intervals) forms the basis of this investigation is shown in figure 4.5.

For the purpose of the finite element simulation, the generator operating point was again that described in section 1 of this chapter, viz. the generation of $5 \mathrm{~kW}$ at $100000 \mathrm{rpm}$. The input to the finite element model consists of the 3-phase current density waveforms that are shown in figure 4.6. Finite element calculations were performed for both the winding connections shown in figure 4.1a and $4.1 \mathrm{~b}$

The finite element calculation can be separated into three discrete stages, which are repeated at each rotor position:

1. Angular displacement of the rotor and the reconstruction of the mesh to accommodate the new rotor position

2. Imposition of specified current densities within the winding regions, the magnitudes of which for a given rotor position are determined directly from the waveforms of figure 4.6.

3. Solution of the finite element model using a non-linear Newton-Raphson type solver generated in-house [4.9] and subsequent data output for the purpose of the post-processing phase.

Although finite element solutions were performed for $1^{\circ}$ (mechanical) steps, by exploiting symmetry, only 90 solutions are required in the case of winding type A and 30 for winding type B. Figure 4.7 shows predicted field distributions for both winding connection types at $10^{\circ}$ intervals up to a rotor angle of $20^{\circ}$ (the angles being referenced relative to the $0^{\circ}$ datum which corresponds to the aligned position with respect to phase A) 
The finite element mesh comprises 7308 stator iron elements and 5164 rotor iron elements. The localised flux density within each element can be derived at each step, and hence a very extensive series of waveforms can be derived for the loss calculation. However, such an approach is computationally very demanding, given the need to calculate the hysteresis loss and numerically integrate the dynamic loss for each of the waveforms. Nevertheless, this exhaustive calculation was performed as a reference against which more computationally efficient methods could be benchmarked.

An alternative approach would be to group elements into a number of discrete subregions within the rotor and stator, each sub-region being assigned a single waveform which is derived from an average of the constituent elemental waveforms, with due weighting to accommodate different element areas within the region. Such an approach was also investigated in this study, with the following aims:

1. To establish the correlation between this more computationally efficient method and that based on elemental summation of losses, and hence establish guidelines for selecting the appropriate number and distribution of sub-regions.

2. To provide a more manageable number of waveforms for the experimental measurement of losses.

3. To significantly reduce the number of distinct waveforms, aiding the interpretation of the machine behaviour.

The initial division of the stator and rotor into sub-regions is shown in figures. 4.8 and 4.9 (shown with the division of only one pole to aid clarity). The numbering convention for the regions is such that it is consistent with that adopted for the division of the stator and rotor in the analytical method. It is evident when comparing figures 4.8 and 4.9 with figures 4.2 and 4.3 that although this approach will inevitably reduce the spatial resolution compared to that obtained using an elemental approach, it nevertheless offers considerably greater resolution than with the regions considered in the analytical model. Further, the field solutions from which the averaged flux-density waveforms are derived still benefit from the fine mesh discretisation. 
The increased spatial resolution of this finite element sub-region technique over the analytical method can be illustrated by comparing the flux density waveforms of figure 4.10, which correspond to the two extremes of the stator tooth tip, labelled as $T_{1}$ and $T_{2}$ in figure 4.9. It is apparent that the flux density in the trailing edge of the tooth is considerably higher than that in the leading edge under the particular specified operating conditions, viz. in generating mode, which is a direct consequence of the commutation interval of the current with respect to rotor position. As will be illustrated in section 4.6, an iron loss calculation based on the use of a single region to represent the entire stator tooth face is likely therefore to exhibit considerable error as compared to an approach based on a refined spatial discretisation.

Figure 4.11 shows finite element predicted flux density waveforms for 8 regions of the stator and rotor (from a total of 204 regions), together with the corresponding analytically derived waveforms. These 8 regions in the finite element model were selected to demonstrate the reasonable correlation with the analytical technique, while highlighting the higher order harmonic content of the finite element predicted waveforms.

\subsubsection{ROTATIONAL COMPONENTS OF FLUX DENSITY}

An important consideration in the calculation of the corresponding iron loss for a given flux density waveform is the approach adopted to accommodate flux density variation which are not unidirectional in nature, i.e. the direction of the flux density vector relative to a given localised reference frame changes as a function of rotor angle. This is illustrated by figure 4.12 which shows the flux-density vectors at the centroid of sub-regions $T_{1}$ and $B_{1}$ at $15^{\circ}$ intervals as the rotor passes the stator tooth. The rotation of the flux density vector can be illustrated by the Lissajous plots of the corresponding waveforms of radial and tangential flux density shown in figures 4.13 and 4.14. In region $T_{1}$, the flux density vector rotates through an angle of $25^{\circ}$, 
whereas in sub-region $B_{1}$ the flux density is almost purely rotational in nature and swings a total of $180^{\circ}$.

The accurate prediction of iron loss due to rotational flux densities is, in general, much more complex than the case of unidirectional flux density variation. The relatively poor understanding of rotational power losses can be attributed to the following:

1. The characterisation of material is problematic since precisely controlled rotational flux density waveforms are difficult to induce in test samples. Poor directional control of the flux density and significant leakage around the samples results in a high harmonic content, particularly at high induction [4.12]

2. Loss measurement techniques are difficult with standard power meters and recourse to calorimetric methods is often required [4.12]

3. The specification of parameters and constants fully defining the nature of the flux density waveforms in terms of loss producing variables is difficult [4.13]

The iron loss associated with a rotating flux density variation is generally much higher than that for a unidirectional waveform of the same peak magnitude. However, no rigorous model for predicting the iron loss due to rotational flux has been presented as yet, particularly when the waveforms are non-sinusoidal. Indeed, for the waveforms present in the SR machines, e.g. uni-polar, DC biased bipolar etc, considerable difficulty arises in relating to any data based on measurement of rotational losses with sinusoidal excitation. For example, relative phase can be readily established for purely sinusoidal waveforms however this is somewhat more ambiguous when considering typical SR machine waveforms. The development of a generalised model for calculating the iron losses associated with any arbitrary rotating waveform is beyond the scope of this thesis. As such, account was taken of the rotational element in the overall iron loss by means of a simplified technique based on the summation of the loss from the orthogonal components of flux density, an approach described and verified, albeit under limited operating conditions, by Yamaguchi and Narita [4.12]. 
The model has been validated using a wide range of electrical steels. However the summation of the two orthogonal losses becomes increasingly erroneous with highly rotating flux densities of relatively large magnitude. Figure 4.15 shows the range of all operating points where the error between the predicted and measured values is less than 5\%. The eccentricity, $a$, of the two orthogonal flux density waveforms, in figure 4.15 is given by:

$$
a=\frac{\text { Flux density in minor axis direction }}{\text { Flux density in major axis direction }}
$$

Hence eccentricities of unity represent a purely rotational flux density variation and zero a uni-axial variation.

It is evident from figure 4.15 that the model is valid for a large range of eccentricities and major-axis flux densities. However in SR machines, high values of flux density are often encountered in the tooth tips, e.g. figure 4.10. In such cases, large rotational components would, on the basis of the results in figure 4.15 , be likely to yield significant over estimates. It has been demonstrated however that the rotating flux density in the stator tooth tips is limited to some $25^{\circ}$, with similar behaviour observed in the rotor teeth. As a consequence, the limited values of eccentricity probably indicate that the approach remains reasonably valid despite the high flux density levels.

The method requires that the two orthogonal flux density waveforms be arranged on two principal axes, rather than some arbitrarily defined co-ordinate system. Indeed regions that have purely alternating flux density waveforms may have significant components in two orthogonal directions with respect to a given geometrical reference frame simply from their relative alignment and, under these circumstances, the iron loss calculated would be an overestimate. For the case of orthogonal sinusoidal waveforms of a given magnitude and relative phase, $\delta$, in a given co- 
ordinate system plotted in a Lissajous format in figure 4.16 shown in equations (4.6) and (4.7):

$$
\begin{gathered}
x=x_{0} \sin \omega t \\
y=y_{0} \sin (\omega t+\delta)
\end{gathered}
$$

the determination, and the alignment, $\alpha$, of the principal axes, shown in figure 4.16 is relatively straightforward and is given by:

$$
\alpha=\frac{1}{2} \tan ^{-1}\left(\frac{2 x_{0} y_{0} \cos \delta}{x_{0}^{2}-y_{0}^{2}}\right)
$$

From this the flux density component along the principal axes can readily be obtained under these simplified conditions.

In contrast however the determination of the attitude of the principal axes for arbitrary waveforms in two orthogonal planes is difficult to achieve due to the high harmonic content. This is highlighted in figures 4.13 and 4.14 where the alignment of the principal axis is not clear.

In order to minimise the error associated with calculations based on misaligned principal axes the flux density waveforms of the SR machine, for the purpose of subsequent loss calculations, are specified in polar co-ordinates. Inspection of the switched reluctance machine reveals that the flux is constrained largely to travel in either the radial or circumferential direction as the geometry of the teeth or back iron dictates. The flux densities reported in polar co-ordinates are therefore, by virtue of the specific machine geometry, representative of the principal flux densities. 


\subsection{Loss Model}

\subsubsection{INTRODUCTION}

Core losses in ferromagnetic materials can, in general, be separated into quasi-static or hysteresis component and a dynamic component.

$$
P_{T O T}=P_{H Y S}+P_{D Y N}
$$

The hysteresis component is attributed to the net energy loss associated with sudden irreversible changes within the domain structure as the magnetisation is increased. The nature of these so-called Barkhausen jumps implies the hysteresis loss per cycle is independent of the frequency of flux reversal and is influenced solely by the magnitude of the flux density. The magnitude of the hysteresis loss is determined from the area of the B-H loop obtained under quasi-static conditions.

The dynamic loss is somewhat more complex in nature and arises due to local internal damping fields within the material opposing changes in the external magnetic field. The dynamic component can be decomposed into classical eddy current and excess loss, discussed below, and hence equation (4.9) can be rewritten

$$
P_{T O T}=P_{H Y S}+P_{C L A}+P_{E X C}
$$

The calculation of iron loss within an electrical machine must include accurate representation of each of these components.

At present however only two of these components, viz. classical and excess losses are calculable for any arbitrary induction waveform, the third, hysteresis loss, lacks rigorous analysis for arbitrary induction waveforms including minor loops. However for the one dimensional case where the flux density is symmetrical about zero, i.e. oscillating about some value $\pm B_{m}$, it is possible to measure the relationship as $B_{m}$ 
varies. There is a requirement, however, for a degree of empirical data to be applied to any analytical model mainly to account for the hysteresis content when the waveforms contain local minima (minor loops) and induction about a non-zero value.

\subsubsection{HYSTERESIS}

Hysteresis loss within soft magnetic material is the result of irreversible 'jumps' within the material domain structure as the material is exposed to a varying field. The hysteresis loss per cycle, which is independent of frequency, is given in the general case by:

$$
W_{h}=\frac{1}{m_{v}} \oint B . d H \quad(\mathrm{~J} / \mathrm{kg})
$$

The integral in equation 4.11, represents the total area enclosed within the B-H loop traversed during one cycle. For a steady-state waveform in which each cycle comprises only one minimum and maximum, e.g. a pure sinusoid, saw-tooth, trapezoid etc, the loss per cycle is dependent only on the magnitude of the peak flux density. The effective area enclosed within the corresponding hysteresis loop, and hence the hysteresis loss, can be estimated to an acceptable degree of accuracy using a curve fit of loss as a function of peak flux density, typically using three material specific constants. Such an approach for the estimation of hysteresis loss as a function of peak flux density is well established and documented [4.1, $4.13 \& 4.14]$, although the material constants required are rarely published by manufacturers, and often must be derived from measured data for a particular grade of electrical steel.

However in some waveforms, including many of those commonly observed in SR machines, each electrical cycle contains numerous local minima and maxima. In terms of hysteresis losses, the presence of these so-called 'minor loops' will generate additional hysteresis loss. For a waveform with significant harmonic content, the loss associated with these minor loops may represent a significant proportion of the overall hysteresis loss in the machine, thus necessitating their inclusion within an 
iron loss model. In order to accommodate the presence of minor loops rigorously, it would be necessary to perform the numerical integration of equation 4.11 , in which the instantaneous $\mathrm{B}$ and $\mathrm{H}$ values were derived from a hysteresis model. Hysteresis models have been developed for predicting the hysteretic behaviour of magnetic materials, using either

1. A purely statistical approach with little physical basis [4.15],

2. Extensive characterisation of the material [4.16]

3. Physical based micro-magnetic models [4.17].

Although this latter approach appears promising in terms of providing a basis for predicting the behaviour of the material, the accuracy of the loss calculation is critically dependant on the precise determination of material parameters, which has been demonstrated to be difficult to achieve [4.18]

An alternative method, adopted in this thesis, is to separate the total hysteresis loss into two components. Firstly, the major loop loss associated with the excursion from $-B_{m}$ to $+B_{m}$, and secondly the individual contribution of each minor hysteresis loop. The former can be readily derived from established techniques, while a novel method for approximating the loss associated with minor hysteresis loops is presented in this thesis.

\subsubsection{Major loop hysteresis loss component}

The major loop component of the hysteresis loss can be curve-fitted as a function of the peak flux density, $B_{m}$, by the following relationship $[4.1,4.13,4.14]$ :

$$
W_{h}=k_{h} B_{m}^{a+b B_{m}} \quad(\mathrm{~J} / k g)
$$

Where the constants $k_{h}, a$, and $b$ are material specific and must be determined from experimental loss measurement and the peak flux density $B_{m}$ is specified in Tesla. To this end, hysteresis loss measurements were performed on both the rotor (CK26) and 
stator (V300) materials, using the computer-controlled test-rig described in Appendix 4.1. The characterisation was performed in both cases for magnetising field strengths up to $20,000 \mathrm{~A} / \mathrm{m}$, which gives rise to flux densities around $1.9 \mathrm{~T}$ in both materials. Figures $4.17 \mathrm{a}$ and $4.17 \mathrm{~b}$ show the measured major hysteresis loops for the rotor and stator material respectively. To aid clarity, and to illustrate the nature of the hysteresis loops, the loops shown are for magnetising fields up to only $1000 \mathrm{~A} / \mathrm{m}$. The values of the material constants obtained for magnetising field strengths up to $20,000 \mathrm{~A} / \mathrm{m}$ are shown in table 4.5 . These values were derived from a curve fit of equation 4.12 using the Nelder-Mead simplex algorithm [4.19].

\begin{tabular}{|l|c|c|}
\cline { 2 - 3 } \multicolumn{1}{c|}{} & $\begin{array}{c}\text { Rotor material } \\
(\mathrm{CK} 26)\end{array}$ & $\begin{array}{c}\text { Stator material } \\
(\mathrm{V} 300)\end{array}$ \\
\hline$k_{h}$ & $19.56 \times 10^{-3}$ & $17.90 \times 10^{-3}$ \\
\hline$a$ & 1.415 & 0.841 \\
\hline$b$ & 0.303 & 1.023 \\
\hline
\end{tabular}

Table 4.5. Experimentally determined hysteresis loss coefficients.

Figure 4.18 shows the predicted hysteresis loss per cycle calculated using equation 4.12 and the constants in table 4.5, together with measured data points. As is evident in figure 4.18 this simple model of hysteresis although having no direct physical basis, is able to represent the measured behaviour accurately over the range considered.

\subsubsection{Existing techniques for the estimation of minor hysteresis loop losses}

The methods considered in this investigation to calculate the additional loss associated with minor loops are all based on the individual and independent consideration of each reversal, of which there may be many within a given a cycle. 
One simple approach, which requires no additional material specific data is to estimate the major loop hysteresis loss from equation 4.12 , with $B_{m}$ set equal to half the minor loop traversal. However, as is demonstrated by figure 4.19 , which shows a series of $2 \mathrm{~T}$ peak to peak hysteresis loops for a range of different starting flux densities, the loop shape and hence the associated hysteresis loss varies significantly as a function of the starting flux density, particularly when saturation flux densities are approached. This so-called 'non-congruent' behaviour of hysteresis loops necessitates some modification of equation 4.12 to include the influence of the starting flux density on the loss associated with a given flux density excursion.

A straightforward method of applying a correction factor to equation 4.12 to represent this behaviour was proposed by Lavers et al [4.20]. The method can be illustrated by reference to figure. 4.20 , which shows a symmetrical $A C$ waveform with a peak magnitude of $B_{m}$ and one additional reversal within each half cycle of magnitude $\Delta B$. For the general case of a waveform with $N$ such reversals within one half cycle, the total hysteresis loss, $W_{h}$, can be estimated by [4.20]:

$$
W_{h}=\left.W_{h}\right|_{-B_{m}} ^{+B_{m}} \times\left(1+k \frac{1}{B_{m}} \sum_{i=1}^{N} \Delta B_{i}\right)
$$

The minor loop correction coefficient $k$ in equation 4.13 , will inevitably take different values depending on the flux densities at the limits of the minor loop and the material properties. However, Lavers et al demonstrated that over a limited range of operating conditions, adopting a $k$ value of between 0.6 and 0.7 provides a reasonable estimate of loss for a range of materials [4.20]. In terms of flux density waveforms, good correlation between measured and predicted hysteresis losses was demonstrated for peak flux densities between 1.0 and $2.0 \mathrm{~T}$ with relatively low ratios of $\Delta B_{i} / B_{m}$. 
A similar approach was described by Hayashi and Miller [4.1], but in this case the correction was restricted to the particular waveform shown in figure 4.21, in which $\Delta B_{i}$ is equal to $B_{m}$. For this waveform, the total hysteresis loss is estimated using:

$$
W_{h}=\left.W_{h}\right|_{-B_{m}} ^{+B_{m}}+\left.0.4 W_{h}\right|_{-B_{m}} ^{+B_{m}}
$$

The correction factor 0.4 in equation 4.14 was determined experimentally for a sample of electrical steel (Losil 400/50), for a range of $B_{m}$ values between 1.40 and 1.92T. On close inspection of equations 4.13 and 4.14 it is evident that equation 4.14 is in fact a particular case of the model presented by Lavers, in which $k=0.8$ and $\Delta B$ $=B_{m}$.

Both these published methods were experimentally validated, each demonstrating an acceptable degree of correlation with measurements, albeit over a limited range of excitation conditions. However, the techniques have a number of deficiencies in terms of their ability to predict hysteresis loss adequately over the entire range of operating conditions that are likely to be encountered in an SR machine. This is highlighted by the fact that the values of $k$ ranging from 0.6 to 0.8 have been proposed for different materials and conditions.

In order to establish the most appropriate value of minor loop scaling factor $k$, a series of hysteresis loss measurements were performed using the general flux density waveform of figure 4.20 , where only a single reversal is undertaken at the peak of each cycle.

The quasi-static conditions required to isolate the hysteresis component of the iron loss was achieved in practice by employing the waveform of figure 4.20 at a frequency of $0.25 \mathrm{~Hz}$. The negligible influence of dynamic effects at this test frequency was confirmed by comparisons with a waveform having a frequency of $1 \mathrm{~Hz}$. A total of 50 measurements were performed at three discrete values of $B_{m}$, viz. $1.00,1.25$ and $1.50 \mathrm{~T}$, and in each case, the magnitude of the flux reversal, $\Delta B$, was 
varied between zero and $2 B_{m}$ in approximately $0.1 \mathrm{~T}$ increments. Figures. $4.22 \mathrm{a}, \mathrm{b}$ and c, show the resultant measured total hysteresis losses (major loop loss + minor loop loss), together with the corresponding losses predicted using the methods of Hayashi and Miller and Lavers et al.

As is evident from figures $4.22 \mathrm{a}, \mathrm{b}$ and $\mathrm{c}$, the magnitude of $B_{m}$ influences the loss for a given $\Delta B$. By means of example, for a $\Delta B$ of $0.3 \mathrm{~T}$, the measured minor loop components of the hysteresis loss per cycle are $3.25 \mathrm{~mJ} / \mathrm{kg}, 4.40 \mathrm{~mJ} / \mathrm{kg}$ and $7.85 \mathrm{~mJ} / \mathrm{kg}$ for $B_{m}$ values of $1.00 \mathrm{~T}, 1.25 \mathrm{~T}$ and $1.50 \mathrm{~T}$ respectively.

From the definitions of $\Delta B$ and $B_{m}$ in figure 4.20 , it is apparent that a waveform in which $\Delta B=2 B_{m}$ is identical in terms of hysteresis loss to 3 independent cycles of peak to peak magnitude $2 B_{m}$. This fundamental reference condition provides one useful means of validating the generality of the loss models proposed by Lavers and Hyashi. However, as is evident from equations 4.13 and 4.14, this reference condition will only be satisfied by adopting a value of $k=1.0$. As a consequence, neither of the loss prediction methods is able to satisfactorily cater for this condition if the suggested values of $k=0.6$ to 0.8 are employed. For the best case, i.e. when $k=$ 0.8 , the calculated loss using equation 4.13 underestimates the loss calculated for 3 independent cycles by some $13 \%$.

A useful method of representing hysteresis losses for minor loops is to scale the flux density change $\Delta B$ as being a proportion of $B_{m}$, with the resulting loss per cycle normalised to the loss for the corresponding loop between $-B_{m}$ and $+B_{m}$. The normalised loss values will hence vary between 1 (for the case of $\Delta B=0$ ) and 3 (for the case of $\Delta B=2 B m$ ). The absolute loss data shown previously in figure 4.22, can be represented using this normalisation approach, as shown in figure 4.23.

In order to demonstrate the limitations of adopting a fixed value of the parameter $k$, it is useful to calculate values of $k$ at each operating point, such that the loss predicted using equation 4.13 would accurately represent the measured loss, i.e. rather than 
being regarded as a constant, $k$ becomes a function of both $\Delta B$ and $B_{m}$. Figure 4.24 shows the values of $k$ derived from measured hysteresis loss on a sample of CK26, including the two reference excitation conditions employed by Lavers et al. and Hyashi, viz. $\triangle B / B_{m}=0.08$ and 1.0 respectively. As shown, for these particular reference conditions, the fixed $k$ values proposed are indeed appropriate. However, over much of the operating envelope, particularly at low values of $\Delta B / B_{m}$ and for the lower values of $B_{m}$, the values of $k$ proposed by Lavers and Hyashi are inappropriate.

Given the limitations of these two published methods, an alternative technique for predicting minor loop hysteresis losses has been developed.

\subsubsection{Alternative method for estimating minor loop losses}

The complex and non-congruent nature of minor hysteresis loops makes it difficult to establish a simple algebraic relationship, particularly when magnetic saturation of the material is considered. Consequently there is a requirement to derive a relationship which incorporates non-linear measured material data. As with any method for determining the losses associated with minor loop hysteresis, there is a trade off between absolute accuracy, model complexity and material data requirements. The technique proposed in this chapter is based on an empirical relationship derived from measured major loops and the nature by which coercivity is developed in the material. The method is based on the relationship between the minor loop of magnitude $\Delta B$ traversing about zero and a loop of similar magnitude about some non-zero, arbitrary flux density such that:

$$
\left.W_{h}\right|_{B_{m}-\Delta B} ^{B_{m}}=\left.Q_{\text {minor }} W_{h}\right|_{\Lambda B / 2} ^{\Delta B / 2}
$$

The term $\left.W_{h}\right|_{-\Delta B / 2} ^{\Delta B / 2}$ can be readily calculated using the well proven approximation for symmetrical, bi-polar excursions of flux density about the origin, i.e. by using equation 4.12, with relevant material constants such as those in table 4.5. 
The term $Q_{\text {minor }}$ is not constant, as was assumed for the analogous $k$ term in Lavers' method, but rather is a non-linear function of $\Delta B$ and $B_{m}$. The variation of $Q_{m i n o r}$ can be deduced either from very extensive material characterisation for all combinations of $\Delta B$ and $B_{m}$, or as in the proposed method by reference to a single measured $\mathrm{BH}$ loop which extends from $-B_{\text {sat }}$ to $+B_{\text {sat }}$ and the measured dependency of the coercivity on the peak flux density. The exploitation of these non-linear, material dependant characteristics and their integration into the minor hysteresis loop correction factor, $Q_{\text {minor }}$, is discussed below.

1. Non-linear magnetisation characteristic of electrical steels - The influence of the inherent non-linearity of the magnetisation characteristic on the hysteresis loss can be illustrated by figure 4.25 , which shows a hysteresis loop of magnitude $\mathrm{B}_{\mathrm{m}}=1.25 \mathrm{~T}$ incorporating two minor loops of $\Delta \mathrm{B}=0.25 \mathrm{~T}$, about $1.125 \mathrm{~T}$ and $0 \mathrm{~T}$. The resulting magnetising field strength excursion, and hence hysteresis loss is considerably greater in the case of the minor loop about $1.125 \mathrm{~T}$. The variation of the area enclosed within a given major loop as a function of flux density, can be illustrated by figure 4.26. This shows a schematic representation of a B-H loop for the particular case of a fully saturated material (i.e. peak flux density of $B_{\text {sat }}$ ). The shaded areas $A_{1}$ and $A_{2}$ correspond to total areas enclosed by the saturation loop for two different flux density excursions, both of magnitude $\Delta B$ (one based around zero and the other about $B_{m}-\Delta B / 2$ ). Although these two areas do not represent the actual areas enclosed by the respective minor loops, the ratio of $A_{1}$ to $A_{2}$ can be used as one correction factor for the additional loss associated for a minor loop excursion about non-zero flux densities. Although the relationship between the two areas may itself be a function of the peak flux density of the outer loop, in order to minimise the material characterisation required, the behaviour exhibited by a loop that extends from $-\mathrm{B}_{\text {sat }}$ to $+\mathrm{B}_{\text {sat }}$ was assumed to be representative of the behaviour of other major loops. A useful and concise method of representing this area correction term is to calculate the cumulative area as a function of flux density, from which the ratio of two areas for a given 
flux density excursion can then be directly calculated. By means of example, figures 4.27 and 4.28 show the measured hysteresis loop (up to $15000 \mathrm{~A} / \mathrm{m}$ which represents an essentially saturated case), and the corresponding cumulative area enclosed within the loop as a function of flux density. A characteristic such as that of figure 4.28 , forms one contribution to the correction term $\mathrm{Q}_{\operatorname{minor}}$

2. Coercive Field - The area correction established above only partly corrects for the behaviour which is observed in experimental measurements of minor loops. Its major limitation is its inability to represent the partial filling of the areas bounded by the major hysteresis loop, a factor that is strongly coupled to the development of coercivity in the material. The coercive field strength that is developed in the material as a result of being magnetised is strongly dependent on the magnitude of the peak magnetising flux density, as illustrated by figure 4.29 which shows the measured variation in coercivity (i.e. $H$ at $B=0$ ), as a function of the peak flux density for CK26 Silicon Steel (in each case for previously demagnetised material). The coercive field established in the material clearly has an impact on the subsequent minor loop hysteresis loss, as is evident from figure. 4.25. For the particular case shown, the upper minor loop $\left(B_{m}=1.25\right.$, $-\triangle B=0.25 \mathrm{~T}$ ) almost completely fills the area available within the outer major loop, while the lower minor loop $\left(B_{m}=0.125,-\Delta B=0.25 \mathrm{~T}\right)$ only fills a relatively small proportion of the outer loop between $-\triangle B / 2$ and $\triangle B / 2$. Thus the calculation of the loss associated with the upper minor loop by the simple application of the area correction factor established above to the loss for the lower loop loss will clearly be an underestimate. Thus there is a requirement to incorporate some additional correction to account for the coercive field developed, a factor which is particularly important for low values of $\Delta B$. The dependency of the coercivity on $B_{m}$, such as the characteristic shown in figure 4.29 can be approximated by the equation:

$$
H_{0}=H_{c}\left(1-e^{-k_{c} B_{m}}\right)
$$


For the two material grades considered in this study, viz. CK26 and V300, the resulting curve-fit parameters are shown in table 4.6.

\begin{tabular}{|c|c|c|}
\cline { 2 - 3 } \multicolumn{1}{c|}{} & $\begin{array}{c}\text { Rotor material } \\
(\mathrm{CK} 26)\end{array}$ & $\begin{array}{c}\text { Stator material } \\
(\mathrm{V} 300)\end{array}$ \\
\hline$H_{c}(\mathrm{~A} / \mathrm{m})$ & 45.36 & 49.32 \\
\hline$k_{c}\left(\mathrm{~T}^{-1}\right)$ & 2.41 & 2.61 \\
\hline
\end{tabular}

Table 4.6. Curve fit parameters for use with equation 4.16.

The means by which this factor can be integrated into an overall correction factor for hysteresis loss was established by inspection of measured results. The exponential curve fits contained in equation 4.16 were found to provide a reasonable estimate of minor loop losses, although no physical relationship is directly evident. The resulting overall correction term, $Q_{\text {minor }}$ is given by:

$$
Q_{\text {minor }}=\frac{A_{1}}{A_{2}} \times\left(1-e^{-k_{c} \Delta B / 2}\right)\left(1+e^{-k_{c} \Delta B}\right)
$$

\subsubsection{Comparison between measured and predicted minor loop losses}

Figure 4.30a,b and c shows the calculated minor loop hysteresis factor $Q_{\text {minor }}$ and the correction factor required to provide minor loop losses consistent with the measured values for starting flux densities of 1.0,1.25 and 1.5T and with minor loops spanning the full range (i.e. $0<\Delta B<2 B_{m}$ ). Also shown in figure 4.30 is the equivalent value derived from the model proposed by Lavers with the correction coefficient of 0.8 employed. It is apparent from figure 4.30 that the method presented for calculating the minor loop losses, although more reliant on measured material data, provides a much higher degree of accuracy, particularly for low values of $\Delta B / B_{m}$, for the limited category of loops considered. 
Having established an approximate technique for estimating minor loop hysteresis losses, this can be used in conjunction with equation 4.10 to calculate the hysteresis loss for a given flux density waveform, a procedure that involves three distinct stages, i.e.:

1. Determination of local maxima and minima in the waveforms by consideration of zero crossings in the time derivative of the flux density.

2. Loop identification where:

a) A major loop is defined between the extremes of the flux density variation if the excursion is symmetrical about zero.

b) Subsequent minor loops are defined and specified using the parameters $\Delta B$ and $B_{m}$.

3. Application of the appropriate minor loop correction factor to each of the individual loops, and subsequent loss calculation.

\subsubsection{CLASSICAL EDDY CURRENT LOSS}

The classical eddy current loss represents the power loss component due to the joule effect heating of the induced eddy currents within the conducting electrical steel. The effect is well documented, with well-proven expressions for the loss in a lamination of thickness $d$, density, $m_{v}$, and resistivity $\rho$. The energy loss over an interval of time $T$ can be expressed as:

$$
W_{c l}=\frac{d^{2}}{\rho f 12 m_{v}} \cdot \frac{1}{T} \int_{0}^{T}\left(\frac{d B}{d t}\right)^{2} d t(\mathrm{~J} / \mathrm{kg})
$$

In the particular case of sinusoidal induction with a peak flux density $B_{m}$ and frequency $f$, the integral can be evaluated analytically and hence the eddy current loss, $\mathrm{W}_{\mathrm{cl}}^{\mathrm{s}}$, is given by:

$$
W_{c l}^{s}=\frac{(\pi d)^{2}}{6 \rho m_{v}} B_{m}^{2} f(\mathrm{~J} / \mathrm{kg} . \text { cycle })
$$


However, for complex waveforms such as those encountered in SR machines, there is often no direct analytical solution to the integral term of equation 4.18 over one cycle. In such cases, there are two alternative approaches that have been considered in this investigation. In the case where a relatively simplified representation of the waveforms is used, e.g. the analytical dynamic simulation discussed in section 3.7, one cycle can be divided into a series of intervals over which analytical integration can be performed, and hence the overall loss derived by a summation over these intervals. Alternatively for complex waveforms, such as those derived from finite element analysis, a piecewise numerical integration can be performed over one complete cycle.

\subsubsection{EXCESS LOSS - BERTOTTI MODEL}

Bertotti [4.21] derived a general theory of iron losses in which particular attention was given to the physical nature of the dynamic losses, in particular the distinction between classical eddy current and excess loss. An external magnetising field applied to a ferromagnetic material will attempt to produce a homogenous magnetisation pattern throughout the cross section of the material. Indeed in a homogenous material with no magnetic texture this will be realised and the resulting dynamic loss component will consist purely of classical eddy current loss. This situation is not realised in all practical materials due to the presence of a magnetic domain structure, separated by so-called Bloch walls (groups of which were termed "magnetic objects") interacting with themselves and other similar walls and hence "internal correlation" fields are observed [4.21]. These correlation fields, coupled with coercive and eddy current effects, act as a damping field, opposing any changes in the external magnetising field, and hence provide the excess loss mechanism. The resulting expression (4.20), summarised by Fiorillo [4.22], is derived from a statistical analysis of the number of magnetic objects active at any given magnetisation frequency and the change in the active number of magnetic objects brought about by the magnitude of the external field. 


$$
W_{e x c}=\frac{1}{f m_{v}} \sqrt{\frac{G V_{0} S}{\rho}} \frac{1}{T} \int_{0}^{T}\left|\frac{d B}{d t}\right|^{1 . S} \cdot d t(\mathrm{~J} / k g)
$$

Where $S$ is the cross section of the lamination, $G$ is a dimensionless damping coefficient and $V_{0}$ is a characteristic field, determining the ability of the applied field to increase the number of active magnetic objects with increasing frequency.

For the case of sinusoidal induction, evaluation of the elliptical integral of the second kind yields:

$$
W_{e x c}^{s}=\frac{8.76}{m_{v}} \sqrt{\frac{G V_{0} S}{\rho}} B_{m}^{1.5} \sqrt{f}(\mathrm{~J} / \mathrm{kg} . \text { cycle })
$$

The excess loss constant $\sqrt{G V_{0} S}$ is a material constant that can be determined experimentally. The total iron loss, comprising both static hysteresis and dynamic components, can be readily measured for bipolar induction waveforms using a fairly standard system similar to that described by Atallah [4.13] and which is shown schematically in figure 4.31. In such systems the waveform generator outputs a symmetrical bipolar sinusoidal demand waveform. Analogue feedback from the search coil is used to ensure that the material is exposed to a truly sinusoidal flux density variation. The magnitude of the induced emf in the search coil under these conditions is related to the magnitude of the flux density using Faraday's law, i.e.

$$
e=N_{s} A \frac{d B}{d t}
$$

Where $N_{s}$ is the number of turns on the secondary coil and $A$ is the cross sectional area of the sample. 
Under sinusoidal conditions the value $B_{m}$ is calculated using

$$
B_{m}=\frac{1}{N_{s} A} \int_{0}^{T} e . d t
$$

The total iron loss due to the alternating flux in a sample of material can be obtained by integrating the area of the dynamic B-H loop:

$$
P=\frac{1}{T} \int_{T} H \frac{d B}{d t} \cdot d t
$$

Which in terms of primary, measured data can be written:

$$
P=\frac{1}{T} \frac{N_{p}}{l N_{s} A} \int_{T} e I . d t
$$

Where $N_{p}$ is the number of turns on the primary coil and $l$ is the mean path length of the magnetic circuit calculated at the mean diameter of the toroid.

Since the total iron loss is the sum of three components of which hysteresis and eddy current are known as functions of the induction waveform, it is possible to determine the excess loss component simply by numerical subtraction. Equation 4.26 [4.23] shows the decomposed iron loss equation for sinusoidal induction:

$$
W_{\text {tot }}^{s}=W_{h}+\frac{(\pi \pi)^{2}}{6 m_{\nu} \rho} B_{m}^{2} f_{0}+\frac{8.8}{m_{\nu}} \sqrt{\frac{G V_{0} S}{\rho}} B_{m}^{1.5} \sqrt{f_{0}} \quad(\mathrm{~J} / \mathrm{kg} . \text { cycle })
$$

The material constant $G V_{o} S$ is the only unknown in equation 4.26 and can be determined as discussed. However the accurate determination of this material constant is very sensitive to the accuracy to which the total power loss and the hysteresis component can be measured. It is advantageous therefore to repeat the iron 
loss measurements over a range of frequencies and curve-fit the results. Figures 4.32 and 4.33 show the decomposition of total iron loss for the rotor and stator materials respectively over a range of frequencies, after the excess loss constant has been determined.

The excess loss constants derived using this experimental technique for the rotor and stator materials are $2.89 \times 10^{-4} \frac{N^{2} s \Omega}{m^{3} T^{3}}$ and $2.00 \times 10^{-4} \frac{N^{2} s \Omega}{m^{3} T^{3}}$ respectively.

\subsubsection{CALCULATION OF DYNAMIC COMPONENT OF IRON LOSS BASED ON THE ANALYTICAL METHOD}

The dynamic components of iron loss, i.e. classical eddy current and excess loss, can be calculated for any arbitrary waveform by the numerical evaluation of the integrals in equations 4.18 and 4.20 respectively. Alternatively, if the waveforms can be represented by an appropriate analytical expression, direct analytical integration is more convenient.

The analytical method used to predict the flux density in particular regions of an SR machine yields waveforms show many similarities with the trapezoidal waveforms of the type often observed in brushless dc permanent magnet machines. Amar [4.23] and Fiorillo [4.22] both use a loss decomposition method to estimate the core losses in ferromagnetic materials with arbitrary induction waveforms. In both cases, the simplest case presented was that of a rectangular voltage waveform of period $T$ and duty cycle $\tau / T$. The voltage and the resulting trapezoidal flux density waveform are shown in figure 4.34. The time derivative of the flux density is given by:

$$
\pm \frac{2 B_{m}}{\tau} \text { or zero. }
$$


The resulting integral terms in the classical eddy current loss equation, are hence given by:

$$
\begin{aligned}
\int_{0}^{T}\left(\frac{d B}{d t}\right)^{2} \cdot d t & =\int_{0}^{\tau / 2}\left(\frac{2 B_{m}}{\tau}\right)^{2} d t+\int_{\tau / 2}^{(T-\tau) / 2} 0 d t+\int_{(T-\tau) / 2}^{(T+\tau) / 2}\left(-\frac{2 B_{m}}{\tau}\right)^{2} d t+\int_{T+\tau / 2}^{T-\tau / 2} 0 d t+\int_{T-\tau / 2}^{T}\left(\frac{2 B_{m}}{\tau}\right)^{2} d t \\
& =2 \int_{0}^{\tau}\left(\frac{2 B_{m}}{\tau}\right)^{2} d t
\end{aligned}
$$

Hence the classical eddy current component of loss is given by:

$$
W_{c l}=\frac{1}{\rho f} \frac{t^{2}}{12 m_{v}} \frac{2}{T} \int_{0}^{\tau}\left(\frac{2 B_{m}}{\tau}\right)^{2} d t=\frac{1}{\rho f} \frac{t^{2}}{12 m_{\nu}} \frac{2}{T}\left[\frac{4 B_{m}^{2}}{\tau}\right]
$$

A similar approach to the excess loss component yields:

$$
W_{e x}=\frac{1}{f m_{v}} \sqrt{\frac{G V_{0} S}{\rho}} \frac{2}{T}\left[\frac{2^{1.5} B_{m}^{1.5}}{\sqrt{\tau}}\right]
$$

This approach of Amar [4.23] and Fiorillo [4.22] can be extended to cater for the more complex waveforms that are likely to be encountered in various regions of an SR machine, such as those shown previously in figure 4.4. These localised waveforms can be decomposed into a sequence of simple ramps and constant flux density intervals, with due account of the phase shift between individual phases, thus enabling the direct analytical evaluation of the dynamic components of iron losses under particular operating conditions. 
For a three phase machine, with each phase excited by a voltage pulse of duration $\tau$ (with appropriate phase shift between phases), the time derivative $\dot{B}$ of the flux density in a given region, $i$, of the machine can be either zero or one of six discrete values, viz.:

$$
\pm \frac{\phi_{m}}{G_{i} \tau}, \pm \frac{2 \phi_{m}}{G_{i} \tau} \text { or } \pm \frac{3 \phi_{m}}{G_{i} \tau}
$$

The maximum flux induced per phase, $\phi_{m}$ is calculated using the techniques discussed in section 3.7. For the benchmark operating condition of $5 \mathrm{~kW}$ (generating) at $100,000 \mathrm{rpm}$ and a DC link voltage of $96 \mathrm{~V}, \phi_{m}$ is $330 \times 10^{-6} \mathrm{~Wb}$. The effective cross sectional area, $G_{i}$, for various regions in the prototype machine are listed in table 4.4.

In the analytical simulation, it is assumed that a voltage pulse of duration $\tau$ is applied, followed by a reverse pulse of the same duration in order to de-flux the machine (although in practice the reverse pulse can be slightly shorter due to the effect of phase resistance). Each pulse pattern is separated between phases by $\pi / 6$ radians (mech.) inducing a flux density waveform with an arbitrary dc offset repeatable at intervals no greater than $\pi / 2$.

Having established that the various flux density waveforms in an SR machine can be considered as comprising the 6 distinct flux density variations defined in equation 4.31, the resulting values of the integrals for both classical eddy current and excess loss components can be evaluated for the 8 regions of the stator and rotor shown previously in figures 4.2 and 4.3. Since the flux density waveforms are influenced by the winding connection, one set of integrals can be derived for each of the winding configurations defined in figure.4.1. The resulting evaluated integrals are shown for these cases in Appendix 4.2. These expressions can be employed in order to estimate rapidly the iron loss associated with any given operating condition predicted using analytical simulation. Figure 4.35 shows a mesh of the dynamic components of iron 
loss calculated using the switching angles established in chapter 3 over the entire range of speed and power for winding connection $A$, where it is apparent that the dynamic component of iron loss increases with rotor speed and output power.

\subsection{Machine Level Predictions of Iron Loss}

The overall machine iron loss at the benchmark operating condition, i.e. $5 \mathrm{~kW}$ at $100,000 \mathrm{rpm}$, was calculated using three alternative sets of flux density waveforms for different degrees of spatial discretisation, viz.:

1. Analytical Waveform for the regions defined in figures 4.2 and 4.3 (20 regions)

2. Finite element generated waveforms for the regions defined in figures 4.8 and 4.9 (204 regions)

3. Finite element generated waveforms for each individual finite element $(12,472$ elements $)$

For the first case the dynamic components of the iron loss were calculated using the analytical expressions presented in Appendix 4.2, while for cases 2 and 3, discrete numerical integration of the flux density waveforms was employed. In the numerical cases, the time derivative was calculated based on the data points at angular intervals of $1^{\circ}$, with linear interpolation. The hysteresis loss, including minor loop losses, for each waveform was calculated using the method discussed in section 4.5.2.

A useful means of presenting the resulting localised loss distribution within the rotor and stator is to employ shaded contour plots of iron loss density. Figures 4.36, 4.37 and 4.38 show the iron loss density contours in the rotor and stator calculated using the three methods discussed for each of the two winding connection types respectively. Tables 4.7 and 4.8 summarise the total iron losses for each of the winding connection types for the machine operating at the benchmark condition. The 
relative contributions of classical eddy current, excess and hysteresis losses to these overall loss figures are contained in Appendix 4.3.

\begin{tabular}{|l|l|c|c|c|}
\cline { 3 - 5 } \multicolumn{2}{c|}{} & \multicolumn{3}{c|}{ Iron Loss (W) } \\
\cline { 3 - 5 } \multicolumn{2}{c|}{} & Method1 & Method 2 & Method 3 \\
\hline \multirow{3}{*}{ Stator } & Radial & 71 & 176 & 200 \\
\cline { 2 - 5 } & Circumferential & 265 & 380 & 395 \\
\hline Rotor & Radial & 163 & 250 & 320 \\
\cline { 2 - 5 } & Circumferential & 52 & 156 & 211 \\
\hline & TOTAL & $\mathbf{5 5 1}$ & $\mathbf{9 6 2}$ & $\mathbf{1 1 2 6}$ \\
\hline
\end{tabular}

Table 4.7 Iron loss components for winding connection type A.

\begin{tabular}{|l|l|c|c|c|}
\cline { 3 - 5 } \multicolumn{2}{c|}{} & \multicolumn{3}{c|}{ Iron Loss (W) } \\
\cline { 3 - 5 } \multicolumn{2}{c|}{} & Method1 & Method 2 & Method 3 \\
\hline \multirow{3}{*}{ Stator } & Radial & 71 & 183 & 203 \\
\cline { 2 - 5 } & Circumferential & 387 & 509 & 520 \\
\cline { 2 - 5 } & Radial & 184 & 250 & 304 \\
\cline { 2 - 5 } & Circumferential & 55 & 155 & 198 \\
\hline & TOTAL & $\mathbf{6 9 7}$ & $\mathbf{1 0 9 7}$ & $\mathbf{1 2 2 5}$ \\
\hline
\end{tabular}

Table 4.8 Iron loss components for winding connection type B.

For each of the three methods, the total predicted iron loss is lower for winding connection $\mathrm{A}$ than connection $\mathrm{B}$, for example with winding connection $\mathrm{A}$ the total iron loss is only $92 \%$ of that for winding connection B (at the highest level of dicretisation). This behaviour is consistent with that suggested by Hyashi [4.1]. This reduced loss is due mainly to the superposition (and resultant cancellation) of the time-varying component of the flux density in stator back-iron region 6 (as defined in figure 4.2), which in turn reduces the dynamic loss component, and with only very small flux traversals the hysteresis loss is also somewhat lower, as shown by loss 
contours of figure 4.36 which shows the total loss contours calculated using the analytical method. Indeed, the iron loss predicted in this region using the analytical technique is only $7 \mathrm{~W}$ for winding connection $\mathrm{A}$, as opposed to $129 \mathrm{~W}$ for winding connection $\mathrm{B}$. Winding connection $\mathrm{A}$ also gives rise to a slightly lower rotor iron loss, $215 \mathrm{~W}$ as compared to $239 \mathrm{~W}$, the major difference being the eddy current component. The relative merits of adopting winding connection $\mathrm{A}$ are apparent when considering figure 4.39 which shows the dynamic components of iron loss calculated using the equations listed in Appendix 4.2 plotted against the switch dwell angle up to a maximum value of $45^{\circ}$ for each of the winding connections.

Figure 4.37 shows the corresponding loss density contours due to the radial and circumferential flux density variations derived from the finite element method in which the stator and rotor are divided into a total of 204 regions. Also shown is the sum of the radial and circumferential loss densities, which is equal to the total loss density. The benefits in terms of the increased spatial discretisation are apparent particularly near the trailing edges of the rotor and stator teeth. The high flux densities observed in these areas yield much higher iron loss densities, a factor which, as discussed, cannot be accommodated using the analytical approach. Consequently the total iron loss predicted using this method is $74 \%$ and $57 \%$ higher than that predicted using the analytical approach for winding connections A and B respectively.

At the highest level of spatial discretisation, the iron loss was calculated from the flux density waveforms derived for each individual element in the mesh. Figure 4.38 shows the resulting loss contours for this method. As demonstrated by the total loss values in tables 4.7 and 4.8 above, this method predicts $17 \%$ and $12 \%$ greater loss than the less refined finite element method, and $104 \%$ and $76 \%$ higher than the analytical case for winding connection types $A$ and $B$ respectively.

These findings are consistent in terms of the major limitation of the analytical technique which was identified in section 4.4 .2 viz. its inability to account for any flux concentration during partial overlap, and hence its tendency to severely 
underestimate iron loss under such conditions. The discrepancy between the three methods will inevitably be a function of the particular operating conditions. For example, conditions in which the rise and fall of current occur predominantly during a period of limited mechanical overlap of the rotor and stator teeth are likely to exacerbate the relative errors between the methods.

The improved spatial discretisation obtained by a summation of the loss in each individual finite element will therefore provide a better estimate of iron loss than either an approach which averages the flux densities within a group of finite elements (method 2), or more particularly the analytical approach.

The large magnitude of the total iron loss, which is equivalent to an average value of $1.09 \mathrm{~kW} / \mathrm{kg}$ and $1.18 \mathrm{~kW} / \mathrm{kg}$ (for winding connections A and B respectively), coupled to the localised nature of very high loss densities (where specific losses as high as $13.24 \mathrm{~kW} / \mathrm{kg}$ and $13.26 \mathrm{~kW} / \mathrm{kg}$ are predicted for each of the winding connections) may have an influence on the validity of the assumptions made in the model, most particularly with respect to localised temperatures. The primary effect of the elevated temperature is likely to be the significant increase in resistivity of the lamination material, e.g. the resistivity of low alloy content steels typically increases by some $1 \%$ per ${ }^{\circ} \mathrm{C}$ [4.24], with a consequent reduction in dynamic components of iron loss. This behaviour gives rise to some degree of self-regulation of iron loss, and hence the calculation of iron loss based on a room temperature resistivity will be an overestimate. One further consequence of large temperature increases which is also neglected is the associated thermal expansion, which could in principle result in a reduction in the airgap and hence an increase in the flux density for a given level of excitation, and hence an increased loss. Further, in extreme cases, the temperature in the tooth tips could in principle approach or even exceed the Curie temperature and the operational characteristic of the machine could be significantly degraded.

An additional consideration that is neglected is the dependency of the iron loss on mechanical stress. Tensile stress in a material generally tends to decrease the specific iron losses, principally by increasing the permeability of the material and reducing 
the hysteresis and excess loss components [4.25] [4.26]. By means of example, figures $4.40 \mathrm{a}$ and $4.40 \mathrm{~b}$ show published variations of the measured loss components (hysteresis and excess loss respectively) in a sample of non-oriented Silicon Steel as a function of the stress level in the material [4.26]. Conversely compressive stress tends to increase the specific iron loss, indeed the effect of compressive stress on the two components of iron loss viz. hysteresis and excess, is much more pronounced than the effect of tensile stress. The presence of significant levels of tensile stress in the rotor at high speeds will therefore tend to produce an overestimate of the overall loss generated in the rotor, although it should be borne in mind that eddy current losses (which are largely unaffected by stress) are the dominant loss component at high speeds.

Hence, the accurate computation of iron loss in a machine operating at very high temperatures and at high speeds requires detailed knowledge of the temperature and stress distribution within the machine and the influence of temperature on the magnetic and electrical properties of the lamination material, and the stress dependency of iron loss. Although these limitations are recognised, the inclusion of such effects greatly increases the complexity of the iron loss prediction method and the material data required, and was deemed to be beyond the scope of this thesis.

\subsection{Dynamic Loss Measurement and Separation}

Having proposed a loss model (albeit one that includes a number of established elements), it is important to undertake appropriate experimental measurements. This is necessary in order to validate the loss model for the somewhat unusual excitation conditions encountered in SR machines. To this end, a series of loss measurements were performed on a sample of material. However, the limitations of the power amplifier and the data acquisition system available dictated that these loss measurements could not be performed at the high frequency of the benchmark machine operating condition. Whereas measurements at lower frequencies with the 
same waveform shape are useful in providing some validation of the losses that are likely to be encountered at $100,000 \mathrm{rpm}$ it should be noted that the relative contributions of the three loss components will be markedly different in both cases. Further, the very high frequencies encountered at $100,000 \mathrm{rpm}$ may give rise to a significant degree of eddy current re-distribution within the $0.5 \mathrm{~mm}$ thick rotor laminations, which is neither accounted for in the model nor is likely to be present in $50 \mathrm{~Hz}$ measurements.

\subsubsection{MATERIAL SAMPLES}

Several arrangements of the sample material may be used for the measurement of iron losses. The Epstein frame consists of four lengths of material arranged in a rectangular magnetic circuit with double overlapping corners. This configuration gives a high degree of repeatability, and requires minimal sample preparation. As a consequence it often finds favour in quality control environments for relative loss measurements. However the correlation in absolute terms with losses observed in practical devices is limited, since the inhomogeneity within the magnetic circuit caused by the corners provides a source of considerable error [4.27]. Hence a toroidal arrangement was preferred in this case, as it overcomes many of the errors associated with the Epstein frame, although it nevertheless is still based on using an approximate path length for the magnetic circuit. The sample preparation is considerably more onerous, since each material must be individually wound with toroidal excitation and search coils. The samples used have a $100 \mathrm{~mm}$ outer diameter and 90.45 inner diameter, giving a diameter ratio of 1.1:1 consistent with recommended measuring techniques [4.28]. The rotor material sample comprises eight laminations each $0.5 \mathrm{~mm}$ thick and the stator material 12 laminations each $0.35 \mathrm{~mm}$ thick, giving rise to approximately equal cross sectional areas. Two separate search coils were wound around the core, with 50 and 100 turns, to allow a range of measuring frequencies and flux densities to be measured. Similarly two excitation coils were wound around the samples (one with 600 and one with 200 turns) to allow a large range of fields to be applied to the sample, while making best use to the amplifier VA rating. 


\subsubsection{MEASURING THE LOSSES INDUCED BY ARBITRARY WAVEFORMS}

The measurement system described in section 4.5.4 employs analogue feedback of the emf induced in the search coil as a basis for the error correction. Such a system cannot accommodate flux density waveforms with a DC offset, such as those encountered in many regions of an SR machine. Further, analogue feedback systems have a limited range of stability, and frequent adjustment of the gain is often necessary if a large range of frequencies, flux densities and waveforms are to be catered for. This would be a very significant drawback for a system that may be required to measure several hundreds of arbitrary waveforms with minimal user input. As a consequence, a custom test-rig was designed using Labview 4.1 and a 12bit $1 \mathrm{MHz}$ data acquisition system. [4.29]. The system shares most of the features of the system described by Atallah [4.1] and which was shown previously in figure 4.31. However, instead of an analogue feedback loop driving a voltage controlled power amplifier, the new system uses a software-based iterative open-loop approach to generate a demand waveform for a current controlled power amplifier. The search coil emf and current waveform (measured using a Hall effect transducer) are captured by a data acquisition system. Figure 4.41 shows a schematic of the system architecture.

The software creates an array of values to output via a digital to analogue converter on the data acquisition system. The numerical data for the flux density waveform is obtained from the waveforms derived previously in this chapter. However given the flexibility of both the graphical programming language, Labview, and the data acquisition system, several additional features are incorporated within the waveform:

1. Soft-Start. The starting value of the flux density waveform is not constrained to be zero for a truly arbitrary waveform and hence the flux density variation includes a function which in essence provides a 'soft-start' that gives an initial flux density and emf of zero. If this function were not included, the large emf 
generated on initiation of the waveform would give rise to erroneous initial conditions in the integrator that would be carried through the entire calculation of flux density. The 'soft-start' function is given by:

$$
B(t)=\frac{B_{\text {init }}}{2}\left[\sin \left(\omega_{\text {ramp }} t-\pi / 2\right)+1\right]
$$

2. Finite number of repetitions of waveform. In order to eliminate any transient element that may be present in the system and to allow an average loss to be taken over a number of cycles the waveform can be repeated any number of times. While these factors indicate that a large number of cycles is appropriate, the requirement is balanced with the need to minimise the effect of integrator drift on the loss calculations and to reduce the heating of the sample. Typically 10-20 repetitions of the waveform were used and for the purpose of loss calculations the first and last 2-5 repetitions are neglected.

3. Smoothing of end conditions. Again, since the final value of the flux density waveform is not zero it is advantageous to ramp the flux density smoothly down to zero in a similar manner to that described by equation 4.32

4. Demagnetisation In order to ensure constant initial conditions for the following iteration the sample is fully demagnetised at the end of each output. Demagnetisation is achieved by applying an open-loop exponentially decaying sinusoidal current waveform calculated using equation 4.33 , to the sample at the end of each flux density waveform The system allows the independent specification of each of the variables of equation 4.33 in order to ensure the loss measurements are independent of these factors.

$$
i(t)=\hat{i} e^{-s t} \sin \left(\omega_{\text {demag }} t\right)
$$

An example of a complete waveform comprising all these elements is shown in figure 4.42 . 
The algorithm is based on the sequential output of a series of open-loop current waveforms, each of which is modified in response to two error signals based on the measured emf and the flux density respectively. Although the flux density may be used as a sole reference for the error calculation and subsequent current waveform modification, the inclusion of the emf improves the rate of convergence and adds stability to the process. Consequently, the stability of the process is less sensitive to the selection of the gain and hence a wide operating range can be achieved with the same specified gain, and indeed the gain can be readily adapted to ensure stability. The convergence of the process is accelerated by an initial estimate of the current waveform, which is derived from a nominal non-linear B-H characteristic and hence a high degree of non-linearity is introduced at the first iteration.

The point by point correction of the waveform for iteration $k$ is given by

$$
\Delta i_{k}=\frac{\left(\underline{e_{\text {ref }}}-\underline{\left.e_{\text {meas },(k-1)}\right)}\right) / K_{1}+\left(B_{\text {ref }}-\underline{\left.B_{\text {meas },(k-1)}\right)}\right.}{K_{2}}
$$

Where for this application the gains are initially set as

$$
K_{1}=100 \text { and } K_{2}=10
$$

As convergence is approached, the gain is reduced to prevent oscillation about the required conditions. When

$$
E_{\mathrm{emf}}=\frac{\sqrt{\left(\sum_{i=1}^{N}\left(e_{\mathrm{ref}, i}-e_{\mathrm{meas}, i}\right)^{2}\right)}}{N} \leq 0.05
$$

the gains are automatically adjusted to

$$
K_{1}=100 \text { and } K_{2}=100
$$


Since the correction method is implemented point by point, the data acquisition system must output the request current waveform while recording the emf and current waveform simultaneously. In order to achieve the simultaneous analogue output/input the software creates an internal trigger when commencing the output phase, upon which the input is timed to commence and hence, given the limiting time the data acquisition hardware requires to serve the channels, the input can be timed to coincide. System parameter identification [4.30] indicates that the limiting acquisition rate, where the error associated with non-coincident output/input is a minimum, lies around 45,000 data points per second, above which a consistent erroneous step is observed in the magnitude of the iron loss measured. Hence for finite element generated waveforms discretised at $1^{\circ}$ (mech) intervals the maximum operating frequency is $125 \mathrm{~Hz}$. In the current application the maximum measurement frequency is limited to $100 \mathrm{~Hz}$ to ensure the error is not encountered.

The open loop output and subsequent correction of the current waveform continues until suitable convergence is achieved In order to fully automate the process an automatic convergence detection routine was incorporated into the software. It is possible to determine convergence based upon error of the emf or flux density waveform or indeed by monitoring the rate of change of the iron loss calculated between iterations. For the current application the convergence detection is based on the change of $E_{\mathrm{emf}}$ described above in equation 4.36. Monitoring the rate of change in $E_{\text {emf }}$ rather than the absolute value allows a wider range of flux density waveforms to be used. Indeed absolute convergence of a specified waveform may be limited by the equipment performance, most notably the slew rate and maximum current capability of the power amplifier and the digital quantisation of the emf waveform. In order to prevent premature detection of convergence the emf error is averaged over ten iterations and subsequently a moving horizon type integration filter is applied as described by:

$$
M H_{k_{10}}=E_{\text {enf }}+0.95 M H_{k_{10}-1}
$$


Where $M H$ is the value of the moving horizon and the subscript $k_{10}$ indicates the average value over ten iterations. The threshold for convergence is again userspecified, and in the current application a value of

$$
M H_{k_{10}} \leq 0.0075
$$

is used to indicate satisfactory convergence. Figure 4.43 shows a simplified schematic of the measurement and waveform correction algorithm.

In order to allow a fully automated series of iron loss measurements to be undertaken for different waveforms under various operating conditions, the temperature of the sample is monitored. This prevents the sample from overheating to dangerous levels and further allows measurement to be restricted to a window of temperature to assist with repeatability (the system pausing where necessary to allow the sample to cool).

\subsection{Experimental Validation of Loss Model}

The iron loss was measured for the flux density waveforms shown previously in figure 4.4 which were derived using the analytical method described in section 4.4.1. The fundamental frequency of the waveforms used for the loss measurement was limited to $50 \mathrm{~Hz}$ because of the amplifier limitations discussed in section 4.7. The loss densities measured for each of the regions defined in figures 4.2 and 4.3 are summarised in table 4.9 , together with the corresponding values calculated using the loss model described in section 4.5. 


\begin{tabular}{|c|c|c|c|c|}
\cline { 2 - 5 } \multicolumn{1}{c|}{} & \multicolumn{4}{c|}{ Iron loss density (W/kg) } \\
\cline { 2 - 5 } \multicolumn{1}{c|}{} & Winding connection A & \multicolumn{2}{c|}{ Winding connection B } \\
\hline Region & Measured & Calculated & Measured & Calculated \\
\hline 1 & 1.22 & 1.33 & 1.23 & 1.33 \\
\hline 2 & 1.22 & 1.33 & 1.23 & 1.33 \\
\hline 3 & 1.20 & 1.33 & 1.23 & 1.33 \\
\hline 4 & 2.11 & 2.09 & 2.07 & 2.09 \\
\hline 5 & 2.07 & 2.09 & 2.07 & 2.09 \\
\hline 6 & 0.05 & 0.13 & 2.07 & 2.09 \\
\hline 7 & 1.51 & 1.84 & 2.11 & 2.20 \\
\hline 8 & 1.84 & 2.29 & 2.42 & 2.45 \\
\hline
\end{tabular}

Table 4.9 Comparison between the measured and calculated loss densities for the analytical waveforms of figures 4.4 .

It is apparent from table 4.9 that there is good agreement between the measured and predicted losses, although the measured results are, in general, slightly lower than those calculated, behaviour that can mainly be attributed to two factors, i.e.:

1. Simplifications in the loss model, in particular those made regarding the nature of the hysteresis loss. While the minor loss is accommodated using a simple model the truly arbitrary conditions observed even within the simplified waveforms are not rigorously accounted for in the model. This source of error is particularly prevalent at such low measurement frequencies since the relative contribution of the hysteresis is much larger than that at high speed. Indeed using the calculated results shown in appendix 4.3 for the case of $100,000 \mathrm{rpm}$, the relative contribution of hysteresis loss (albeit with the simplified model) to the overall loss is only $7.4 \%$ and $5.1 \%$ for winding connections A and B respectively.

2. The convergence of the loss measurement is based on a rate of change of error with respect to the reference and measured emf which is determined on 
a global basis using the rms error over the measured portion of the waveform Indeed convergence detected on an absolute error basis is inappropriate for the arbitrary waveform and is specific to the exact dimensions and winding design of a given toroid and the dynamic performance of the power amplifier. It is apparent therefore that when using this method convergence may be detected under conditions where the emf error is relatively large, and the induced flux density waveform does not truly reflect the reference waveform. Indeed a likely scenario is the requirement for a winding current with harmonic content above that that can be supplied by the power amplifier. Under these circumstances the peak rate of change of flux density may not be achieved and the measured loss is likely to be slightly lower than predicted due to the reduced dynamic loss components.

In order to validate the loss model for waveforms with a much higher harmonic content than those derived in the analytical model, the measurements were repeated (again at a fundamental frequency of $50 \mathrm{~Hz}$ ) for the flux density waveforms derived using the finite element method with winding connection A. In order to limit the number of individual measurements required the waveforms determined using the region method described in section 4.4.2 are used. The appropriate symmetry of the rotor and stator regions was exploited and out of the 204 machine regions a total of 84 independent rotor and stator regions were identified and hence a total of 168 waveforms (both radial and circumferential) were measured using the system. The equivalent loss derived by a summation of the measured losses in the various regions are summarised in table 4.10, together with values calculated using the loss model (in both cases at $50 \mathrm{~Hz}$ ). 


\begin{tabular}{|c|l|c|c|c|}
\cline { 3 - 5 } \multicolumn{2}{c|}{} & \multicolumn{2}{c|}{ Iron Loss (W) } & \multicolumn{1}{c|}{} \\
\cline { 3 - 5 } \multicolumn{2}{c|}{} & Measured & Calculated & Error (\%) \\
\hline \multirow{3}{*}{ Stator } & Radial & 0.50 & 0.58 & 14.1 \\
\cline { 2 - 5 } & Circumferential & 1.04 & 1.13 & 8.3 \\
\cline { 2 - 5 } & Radial & 0.50 & 0.58 & 13.4 \\
\cline { 2 - 5 } & Circumferential & 0.29 & 0.36 & 18.6 \\
\hline & TOTAL & $\mathbf{2 . 3 3}$ & $\mathbf{2 . 6 5}$ & $\mathbf{1 2 . 1}$ \\
\hline
\end{tabular}

Table 4.10 Measured and calculated iron loss in the rotor and stator of the machine using the flux density waveforms discussed in section 4.4 .2 for winding connection

A.

Some insight into the loss distribution, and the ability of the loss model to accommodate the very different conditions encountered with the machine, can be obtained using the graphical representations of total loss in figures $4.44 \mathrm{a}$ and $4.44 \mathrm{~b}$ respectively. Using these figures and the results listed in table 4.10, it is apparent that good agreement is demonstrated between the measured results and those determined using the iron loss model presented in this chapter. The overall discrepancy between the measured and predicted loss at $50 \mathrm{~Hz}$ is $12.1 \%$, with the measured results being lower than those predicted.

It should be noted that whereas the ability of the loss model to predict the loss associated with a given waveform has been demonstrated, this forms only one element of the strategy for predicting the loss in the machine (the other being the calculation of the current waveform and the resultant flux density waveforms). A machine level validation which embraces all three stages, is described in detail in section 7.3.4. 


\section{REFERENCES}

[4.1] Hayashi, Y. and Miller, T. "A new approach to calculating core losses in the SRM.” IEEE Trans. on Ind. Appl. Vol. 31, N 5, pp. 1039-1046, 1995.

[4.2] Hoang, G. et. al. "Enhanced accuracy method for magnetic loss measurement in switched reluctance motor." International conference on electrical machines (ICEM), vol 2, pp 437-442, 1994.

[4.3] Rodger, D. Leonard, P.J., Lai, H. C. and Allen, N., "Finite element modelling of coupled problems including circuits and movement." UK Magnetics Society Seminar on 'Advanced aspects of CAD in Magnetics' London, April $25^{\text {th }} 1996$.

[4.4] Jack, A. G., Mecrow, B. C. and Haylock, J. A. "A comparative study of permanent magnet and switched reluctance motors for high-performance fault-tolerant applications" IEEE Trans. on Industry Applications, Vol. 32, No 4 July 1996.

[4.5] Miller, T. J. E., and McGilp, M., 'Nonlinear theory of the switched reluctance motor for rapid computer-aided design.' Proc. IEE Vol. 137 Pt. B, Nov. 1990, pp337-347.

[4.6] Ray, W. F. and Davis, R. M. "Inverter drive for doubly salient reluctance motor: its fundamental behaviour, linear analysis and cost implications." IEE Trans on Electric Power Appl. Vol. 2, No 6. December 1979. pp 185-193.

[4.7] Materu, P. N., "Estimation of switched reluctance motor losses." IEEE Trans. on Ind. Appl., Vol. 28, No 3, pp 668-679, 1992.

[4.8] Lawrenson, P. J., Stephenson, J. M., Blenkinsop, P. T., Corda, J., and Fulton, N. N., 'Variable-speed switched reluctance motors.' Proc. IEE Vol. 127, Pt. B, July 1980. pp253-265.

[4.9] Low, W. F., "The computation of magnetostatic fields in permanent magnet devices." Ph.D., University of Sheffield, 1985.

[4.10] Landgraf, F. J. G. et al., "Anisotropy of the magnetic losses components in semi-processed electrical steels." Jnl Magnetism and Magnetic Materials, 196-197, pp380-381, 1999. 
[4.11] Moses, A. J. and Shirkoohi, G. H., "Iron loss in non-oriented electrical steels under distorted flux conditions.", IEE Trans. on Magnetics, Vol 23, No 5, pp 3127-3220, September 1987.

[4.12] Yamaguchi, T. and Narita, K., "Rotational power losses in commercial Silicon-Iron laminations." Electrical Eng. In Japan, Vol. 96, No 4, pp 15-21, 1976.

[4.13] Atallah, K. "Iron losses in brushless permanent magnet DC machines." Ph.D. thesis, University of Sheffield, 1993.

[4.14] Slemon, G. R. and Liu, X. "Core losses in permanent magnet motors." IEEE Trans. on Magnetics, Vol 26, No 5, pp1653-1655, September 1990.

[4.15] Lee, H. J and Hyun, D. S. "Hysteresis characteristics computation on PWM fed synchronous reluctance motor using coupled FEM and Preisach modeling." IEEE Trans. on magnetics, Vol. 36, No 4, July 2000. pp1209-1213.

[4.16] Jack, A. G. and Mecrow, B. C. 'Methods for magnetically nonlinear problems involving significant hysteresis and eddy currents.' IEEE Trans. on magnetics. Vol. 26, No 2, March 1990, p424-429.

[4.17] Jiles, D. C., and Atherton, D. L., "Theory of ferromagnetic hysteresis." Jnl.

Magnetism and Magnetic Materials, Vol. 61, pp48-60, 1986.

[4.18] Yunhe, Y., "Analytic and experimental studies of a wavelet identification of Preisach model of hysteresis." Jnl. Magnetism and Magnetic Materials, Vol. 208, pp255-263, 2000.

[4.19] The Mathworks Inc. Natick, MA, USA.

[4.20] Lavers, J. D. et al., "A simple method of estimating the minor loop hysteresis loss in thin laminations." IEEE Trans. on Magnetics, Vol. MAG-14, No 5, 1978.

[4.21] Bertotti, G., "Physical interpretation of eddy current losses in ferromagnetic materials. I. Theoretical considerations." Jnl. Appl. Physics Vol. 57, No 6, pp. 2110-2117, 1985. 
[4.22] Fiorillo F. and Novikov A., "Power losses under sinusoidal, trapezoidal and distorted induction waveform." IEEE Trans. on Magnetics, Vol. 26, No 5, September 1990, p 2559-2561.

[4.23] Amar M. and Kaczmareki R., "A general formula for the prediction of iron losses under nonsinusoidal voltage waveform" IEEE Trans. on Magnetics, Vol. 31, No 5, September 1995, p 2504-2509.

[4.24] Davies, J. and Simpson, P., "Induction heating handbook.", Mcgraw Hill, 1979.

[4.25] Ali, K., "Effect of mechanical stress on iron losses in permanent magnet brushless dc machines." MPhil thesis, University of Sheffield, Submitted August 1997.

[4.26] Lobue, M., Basso, V., Fiorillo, F. and Bertotti, G. "Effect of tensile and compressive stress on dynamic loop shapes and power losses of $\mathrm{Fe}-\mathrm{Si}$ electrical steels" Jnl. of Magnetism and Magnetic Materials, Vol. 196-197, May 1999, p372-374

[4.27] Sievert, J. D., "Determination of ac magnetic power loss of electrical steel sheet: present status and trends", IEEE Trans. on Magnetics, Vol. 20, No. 5p. $1702,1984$.

[4.28] Monkhouse, P. J., "Design and analysis of brushless dc drive." Ph.D. thesis, University of Sheffield, submitted December 1992.

[4.29] Calverley, S.D., Lister, J. D., Jewell, G. W. and Saunders R. J., "Measurement of iron losses in soft magnetic materials exposed to uni-polar and DC biased waveforms." UK Magnetics Society Seminar on 'Instrumentation for Magnetic Measurements', Warwick, June $14^{\text {th }} 2000$.

[4.30] Lister, J. D., "Computer controlled test-rig for the measurement of iron losses." BEng thesis, The University of Sheffield, May 2000. 


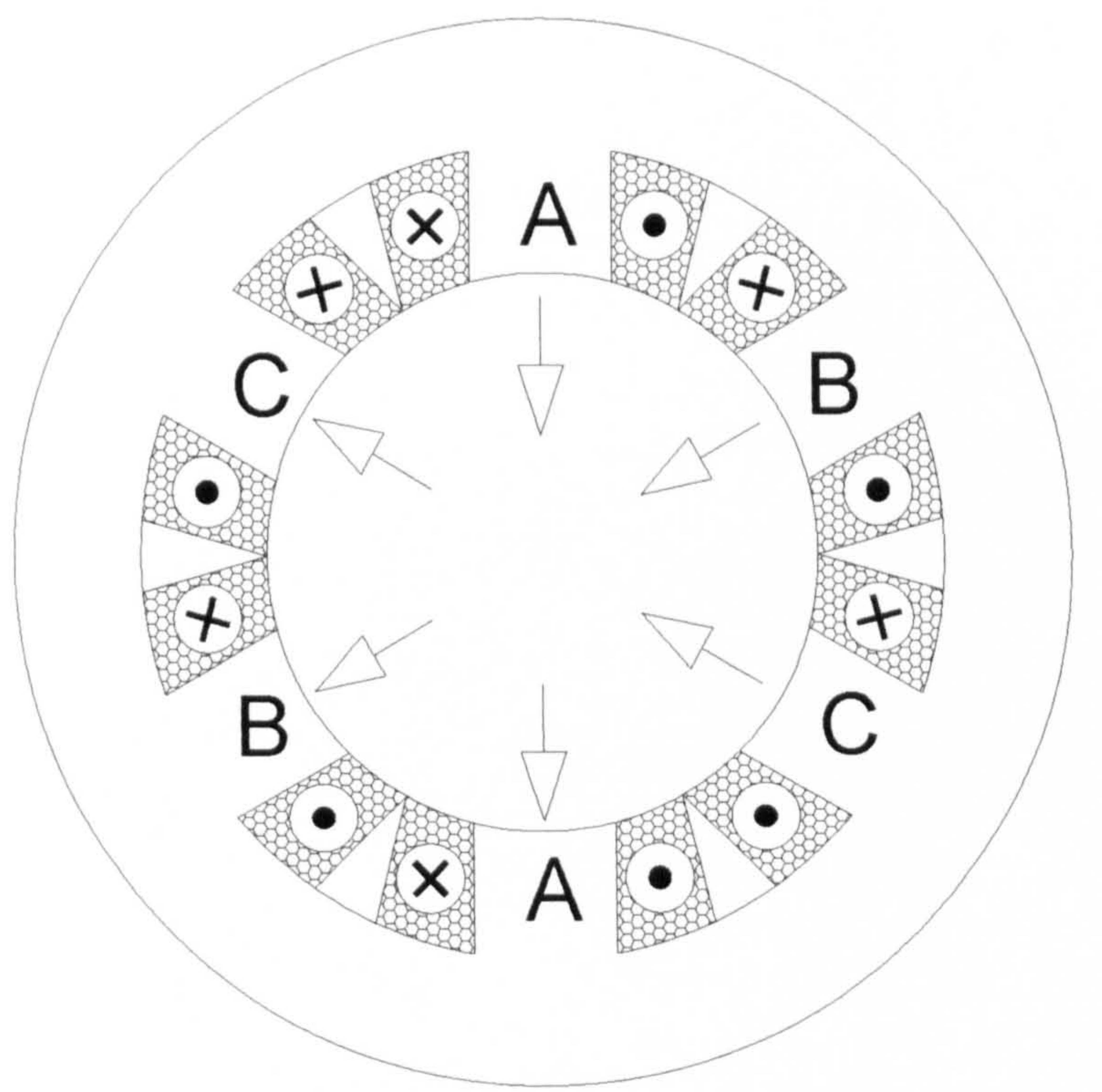

Figure 4.1a. Winding schematic of the $6 / 4$ machine with three adjacent poles having the same polarity (type $\mathbf{A}$ )

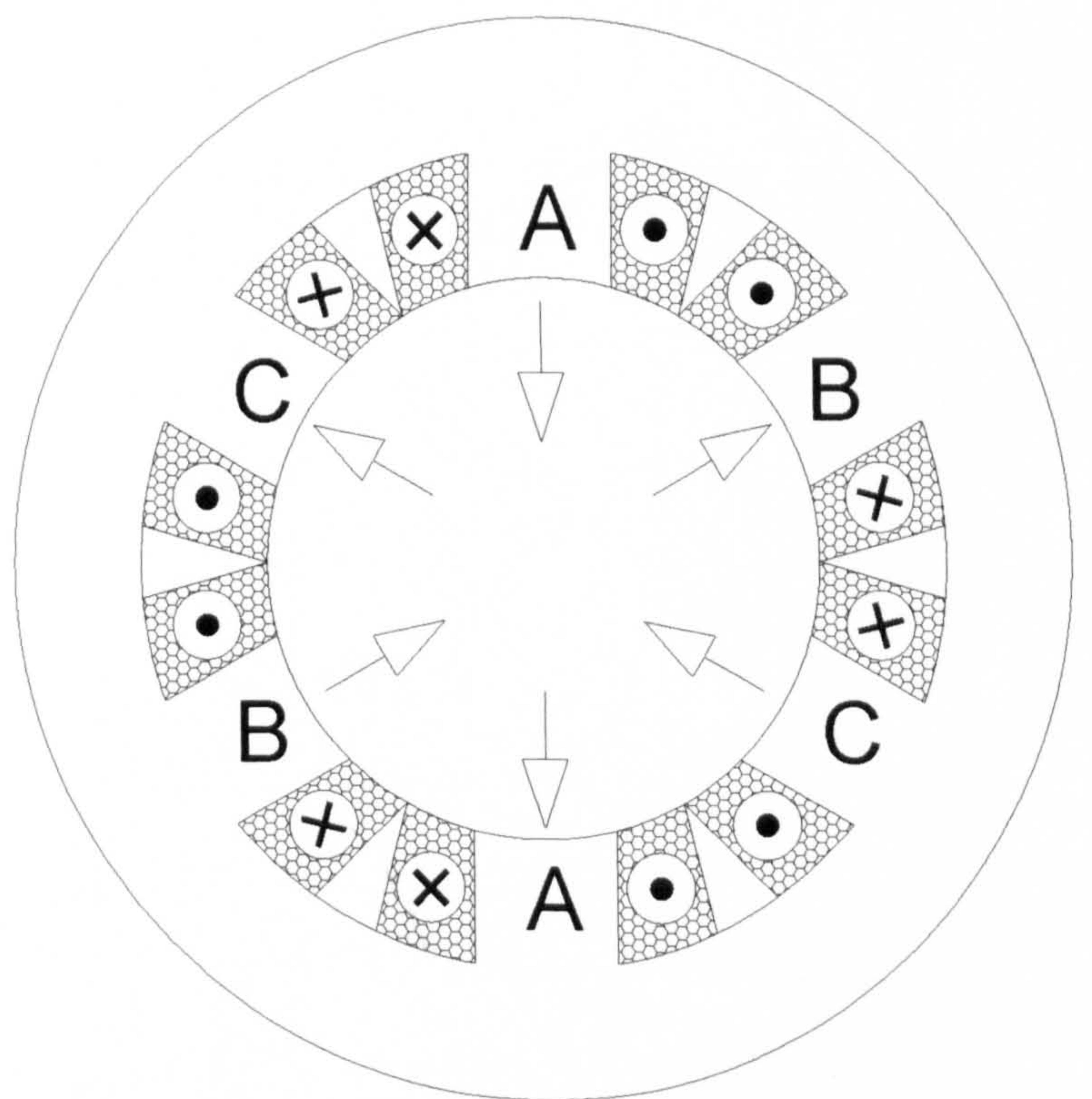

Figure 4.1b Winding schematic of the $6 / 4$ machine with adjacent poles of opposite polarity (type B). 


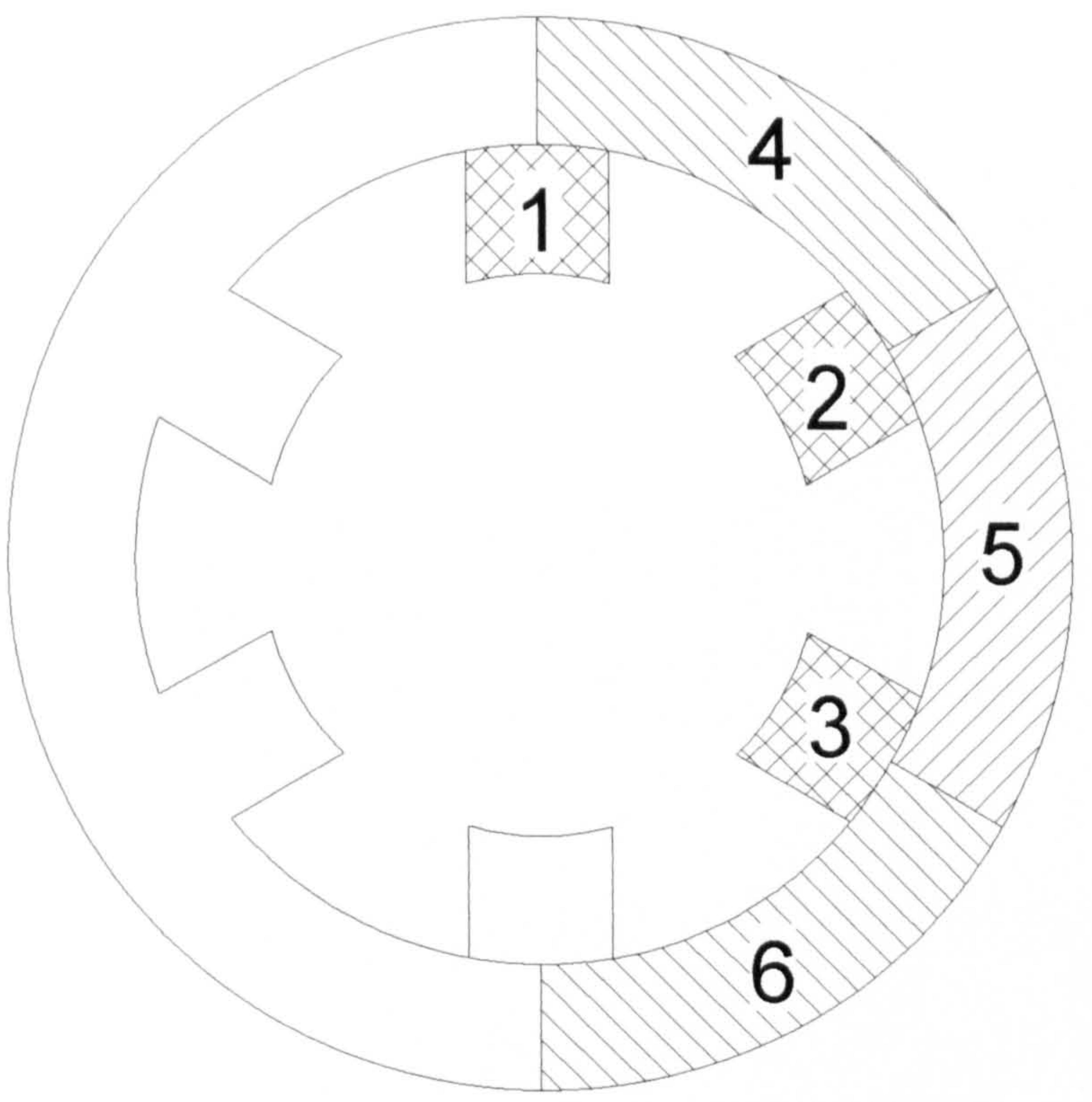

Figure 4.2. Discrete regions of the stator for the prupose of determining the flux density waveforms.

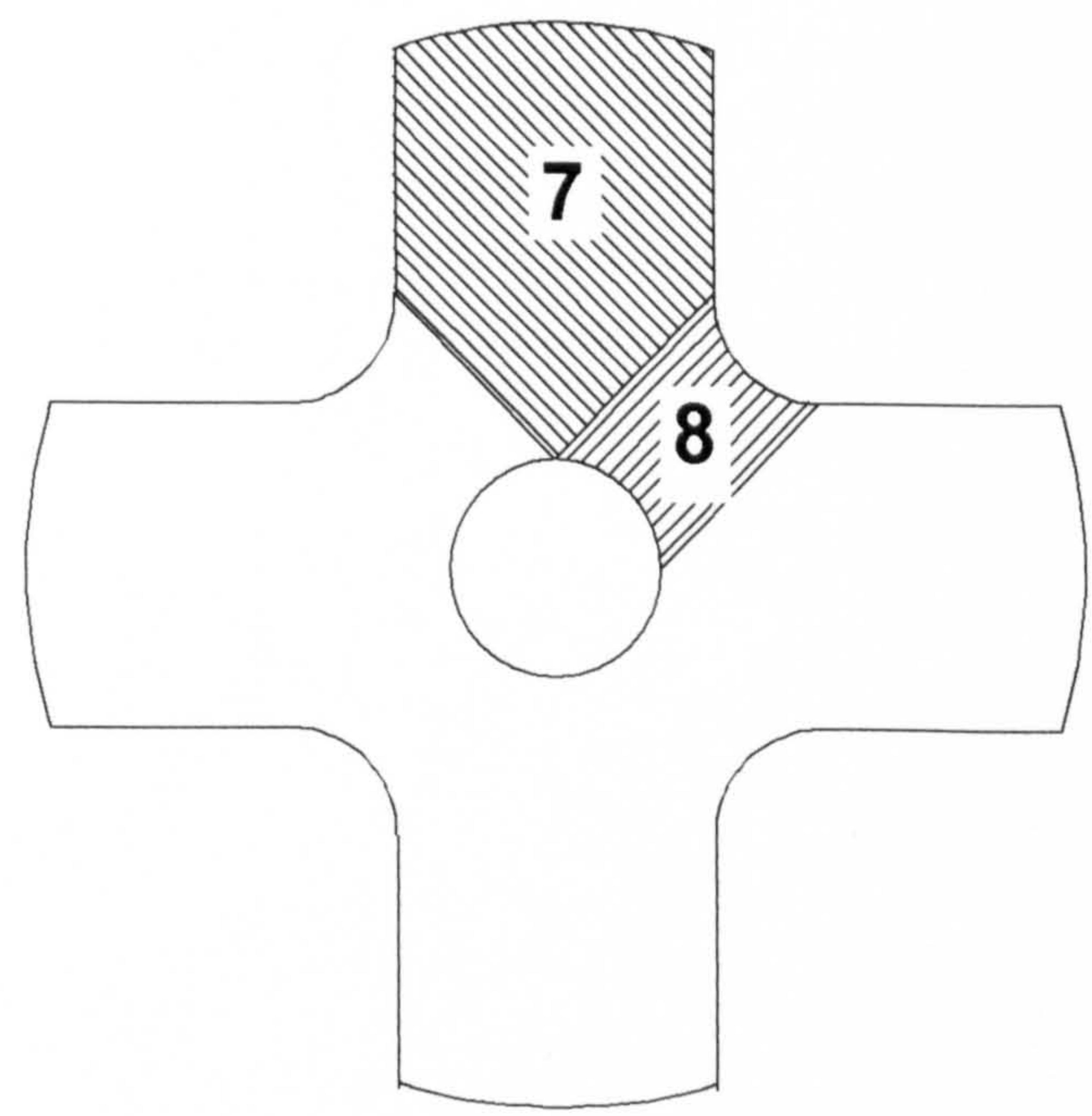

Figure 4.3. Discrete regions of the rotor for the prupose of determining the flux density waveforms. 
WINDING CONNECTION A

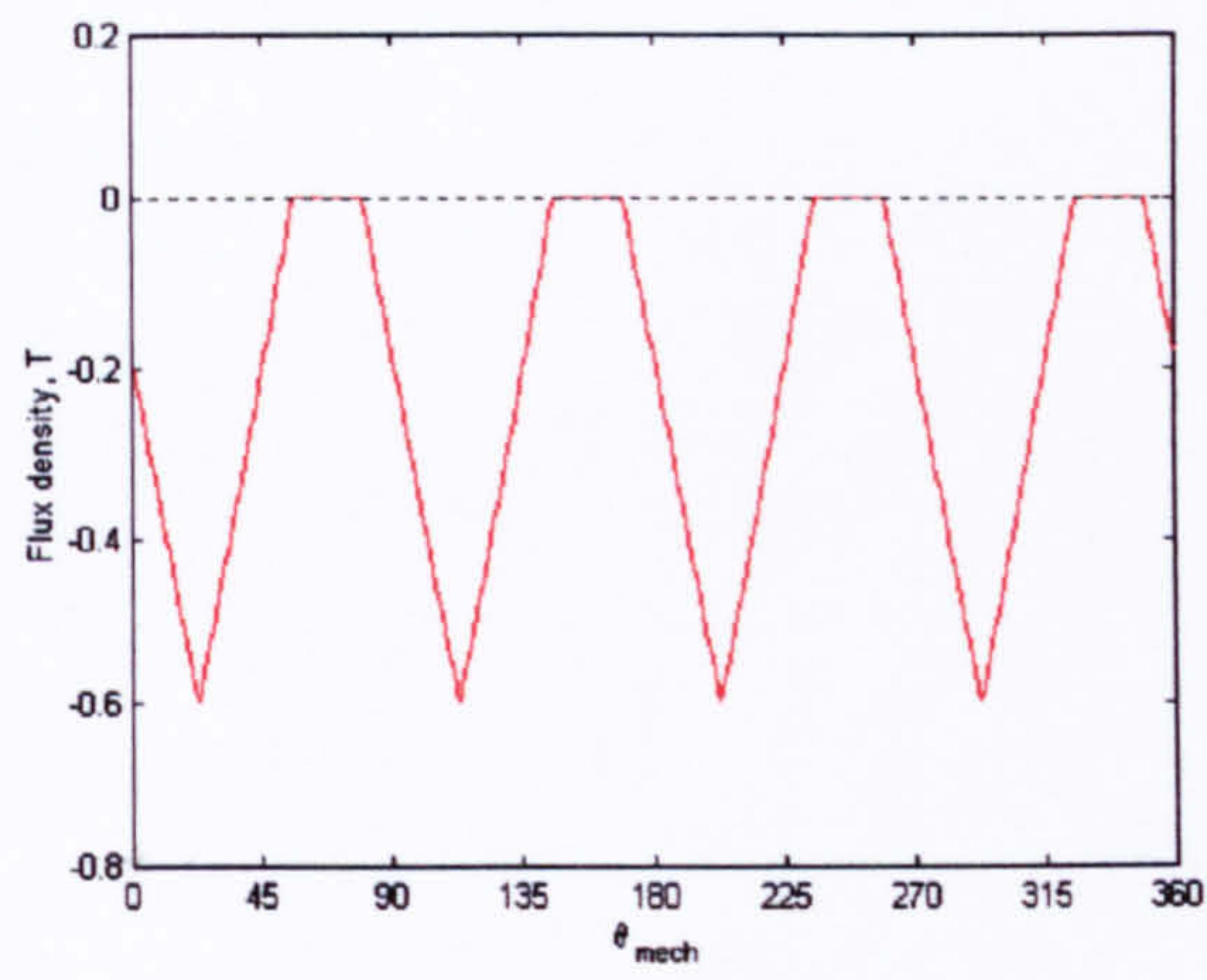

\section{WINDING CONNECTION B}

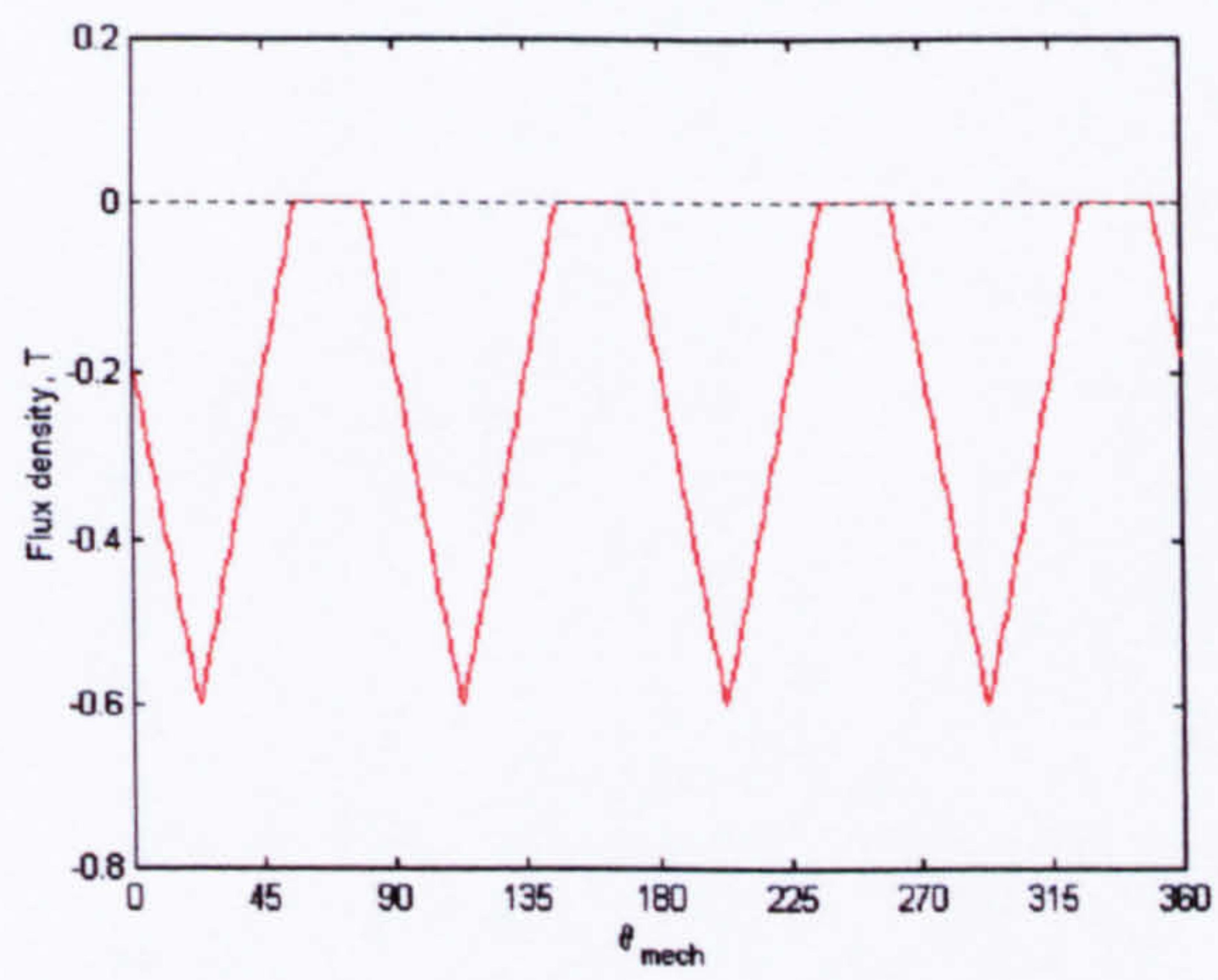

Figure 4.4a. Radial flux density in stator region 1.
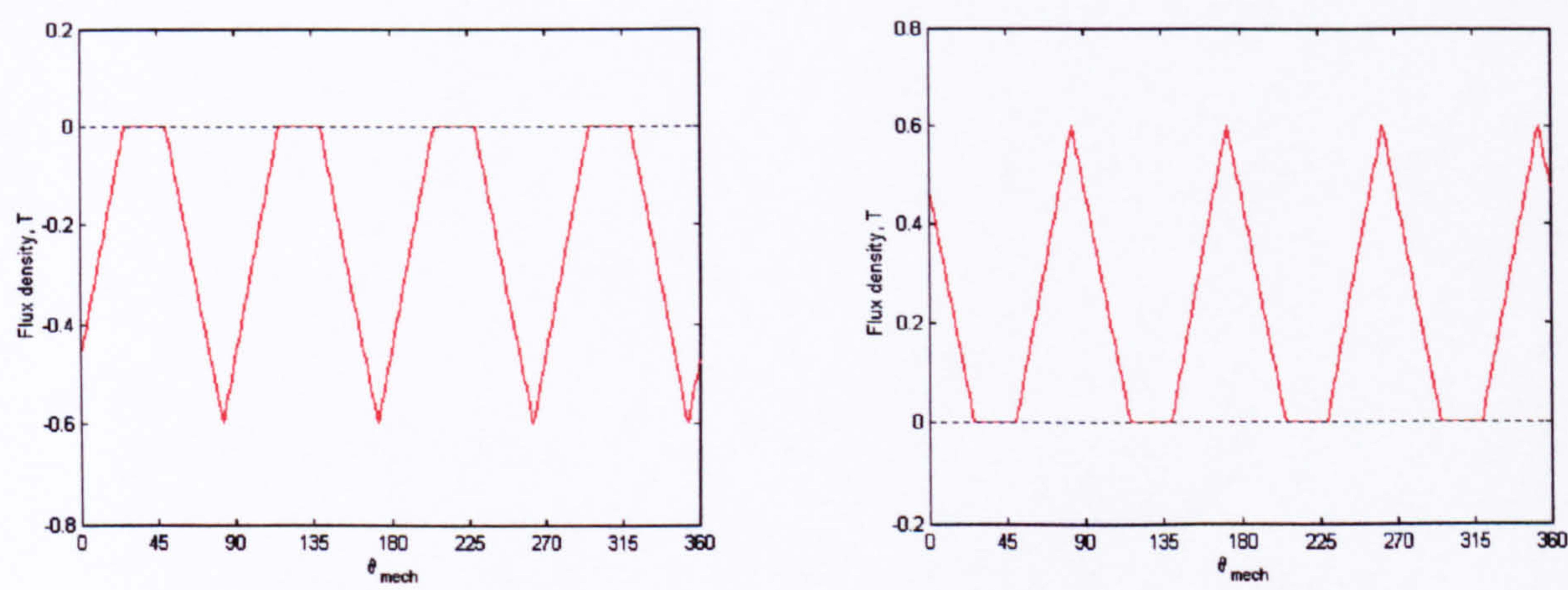

Figure 4.4b. Radial flux density in stator region 2
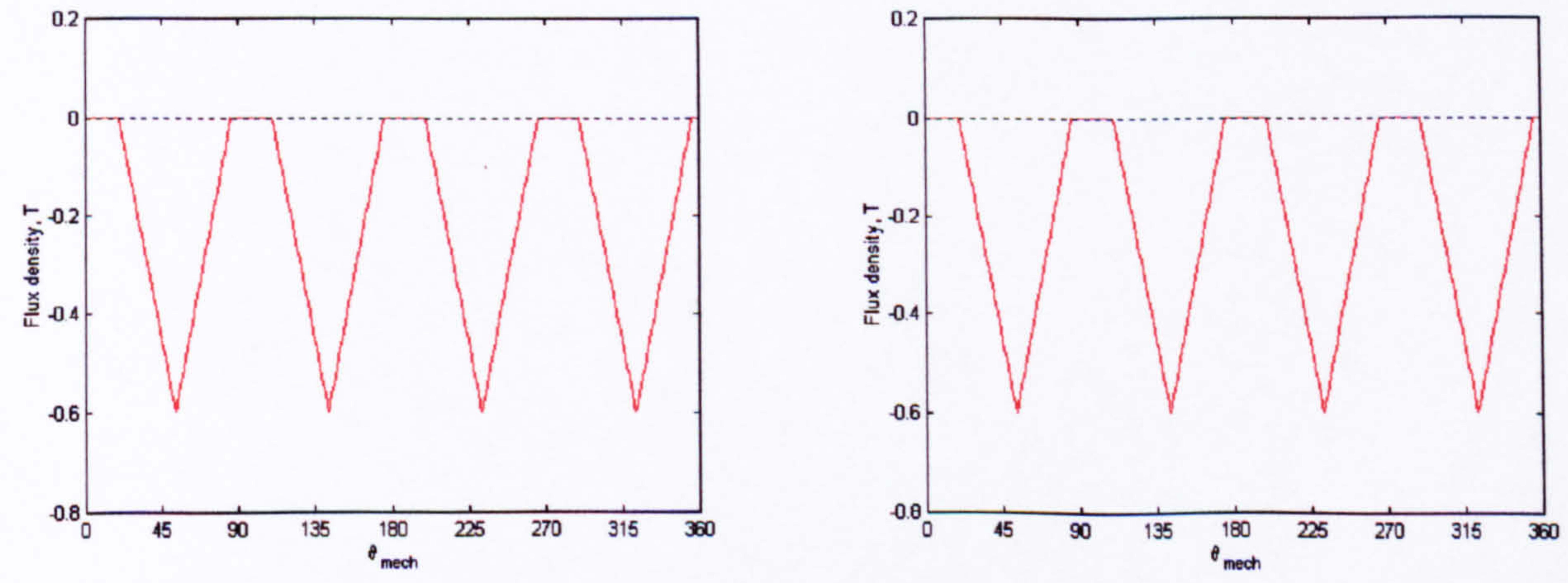

Figure 4.4c. Radial flux density in stator region 3 
WINDING CONNECTION A

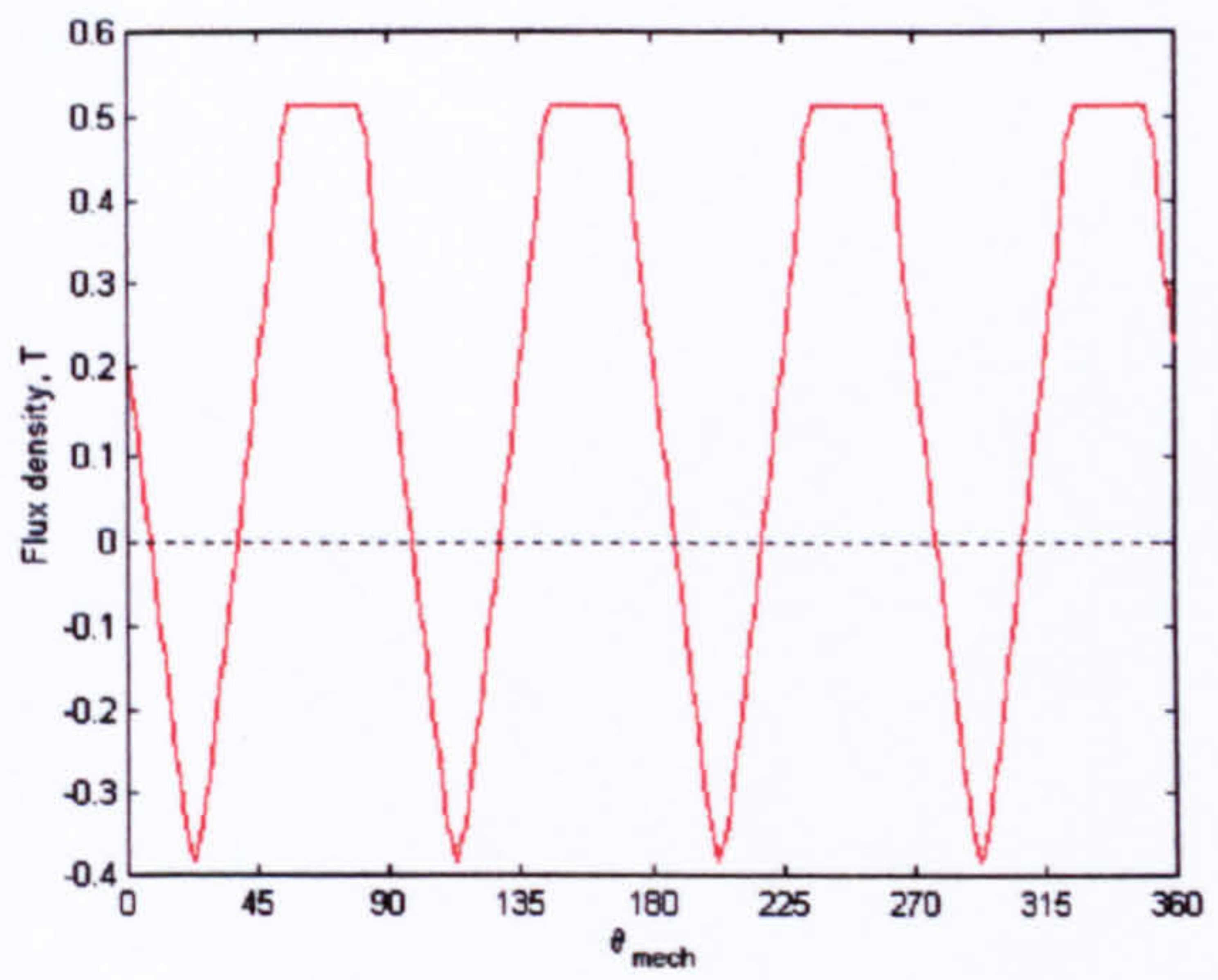

WINDING CONNECTION B

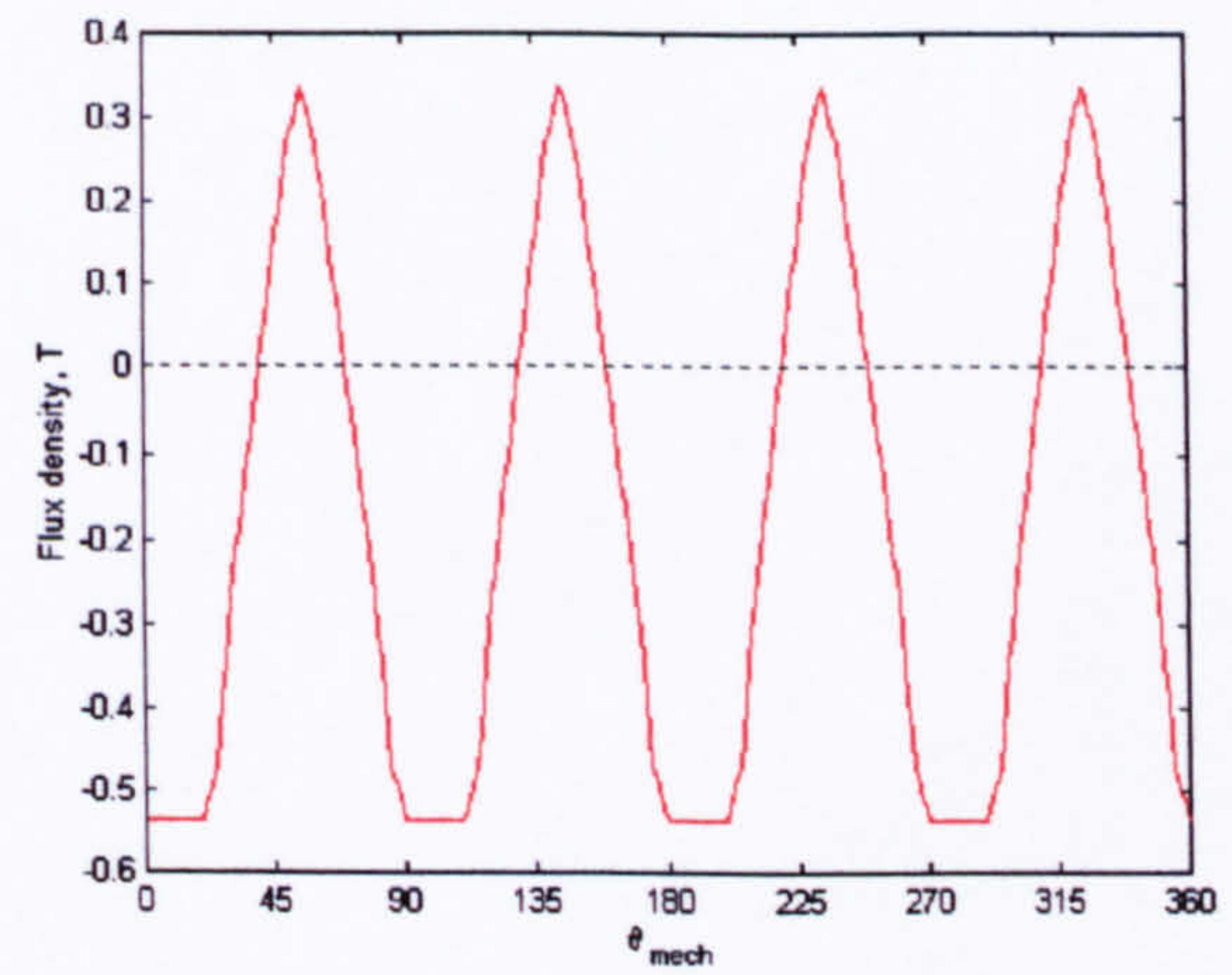

Figure 4.4d. Circumferential flux density in stator region 4
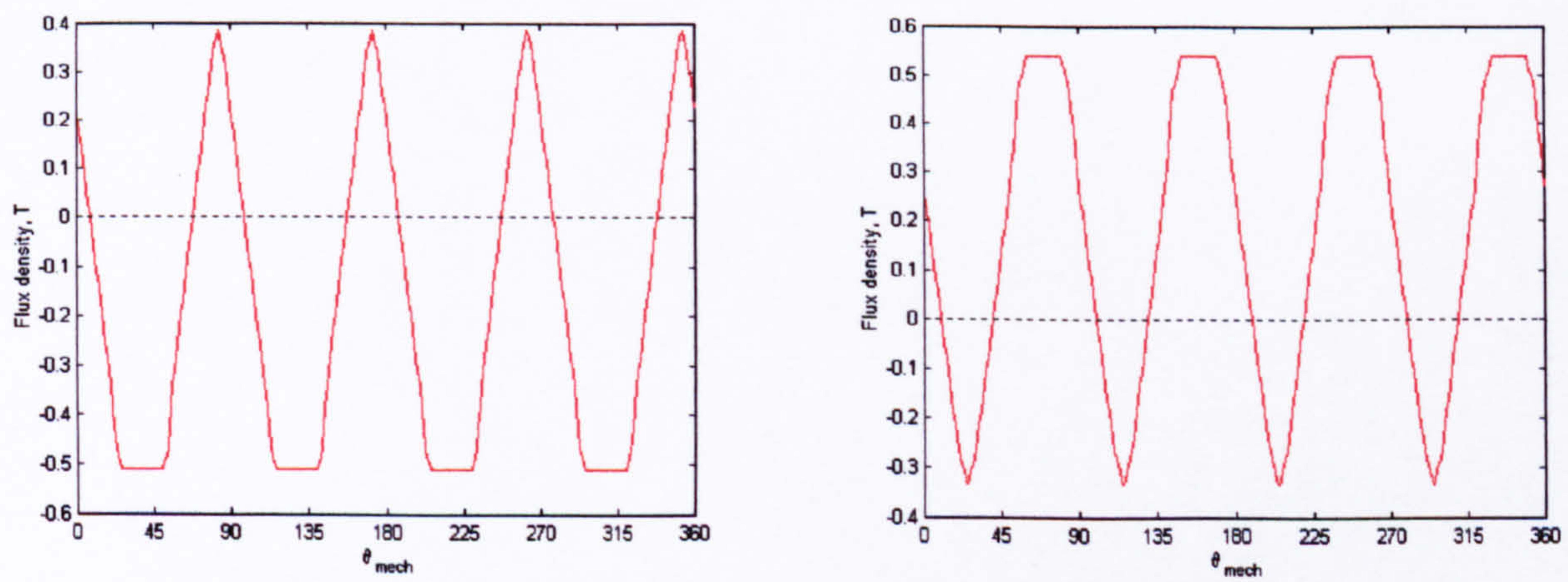

Figure 4.4e. Circumferential flux density in stator region 5
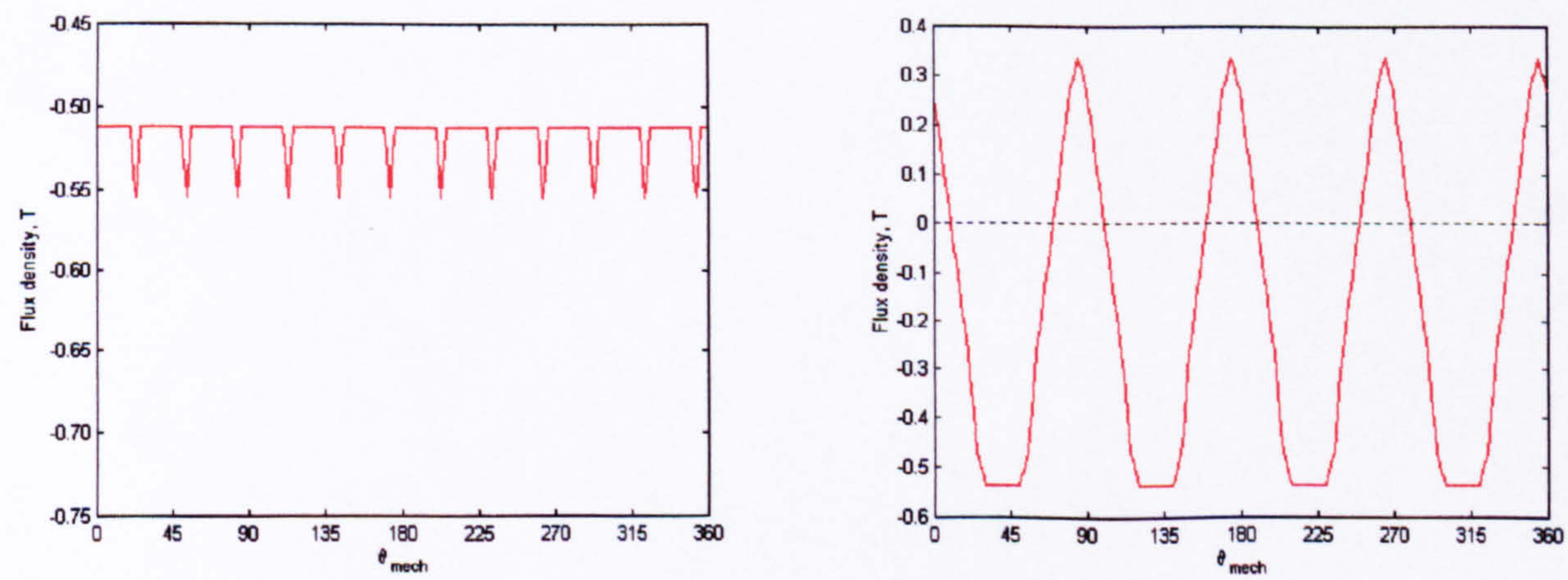

Figure 4.4f. Circumferential flux density in stator region 6 
WINDING CONNECTION A

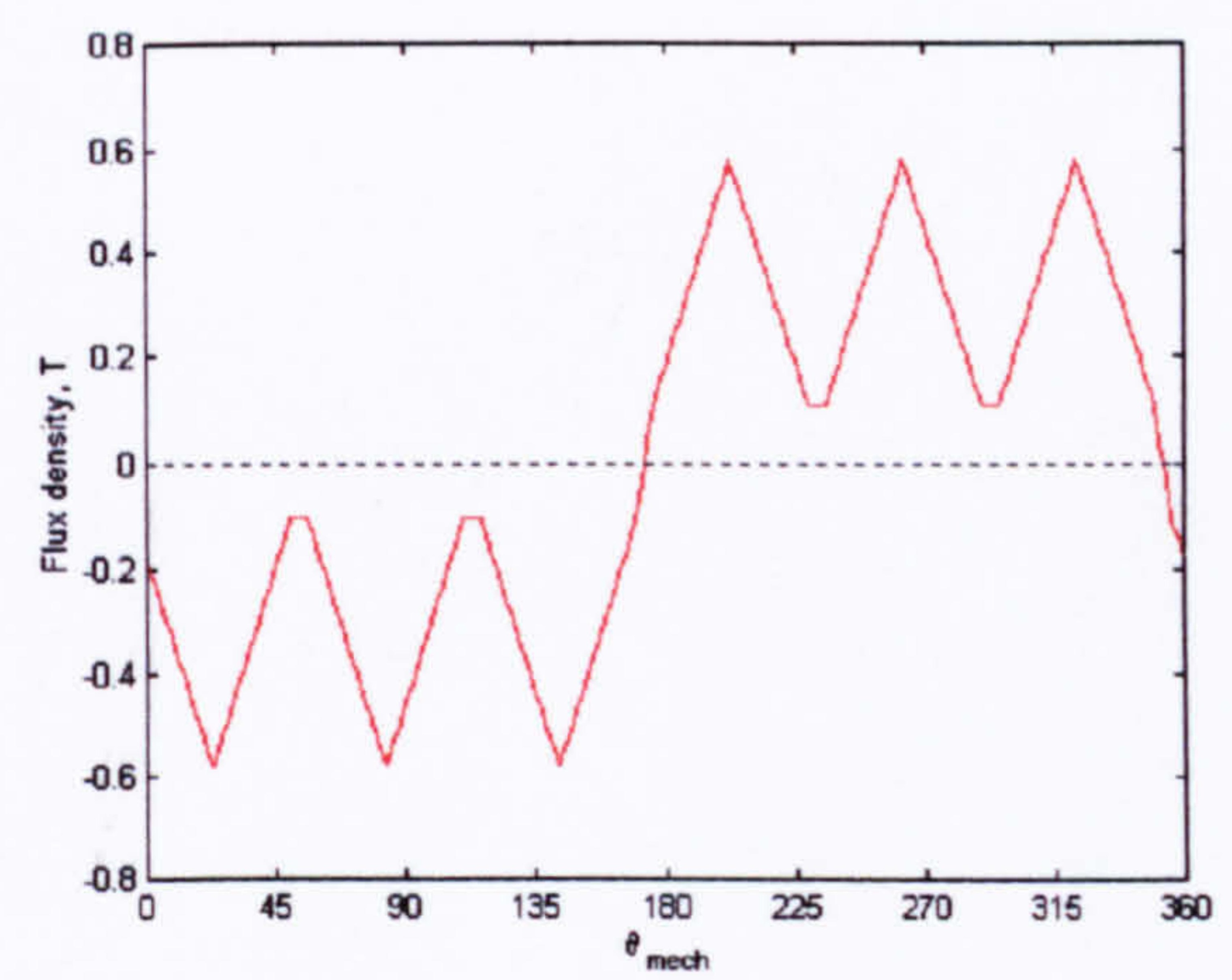

WINDING CONNECTION B

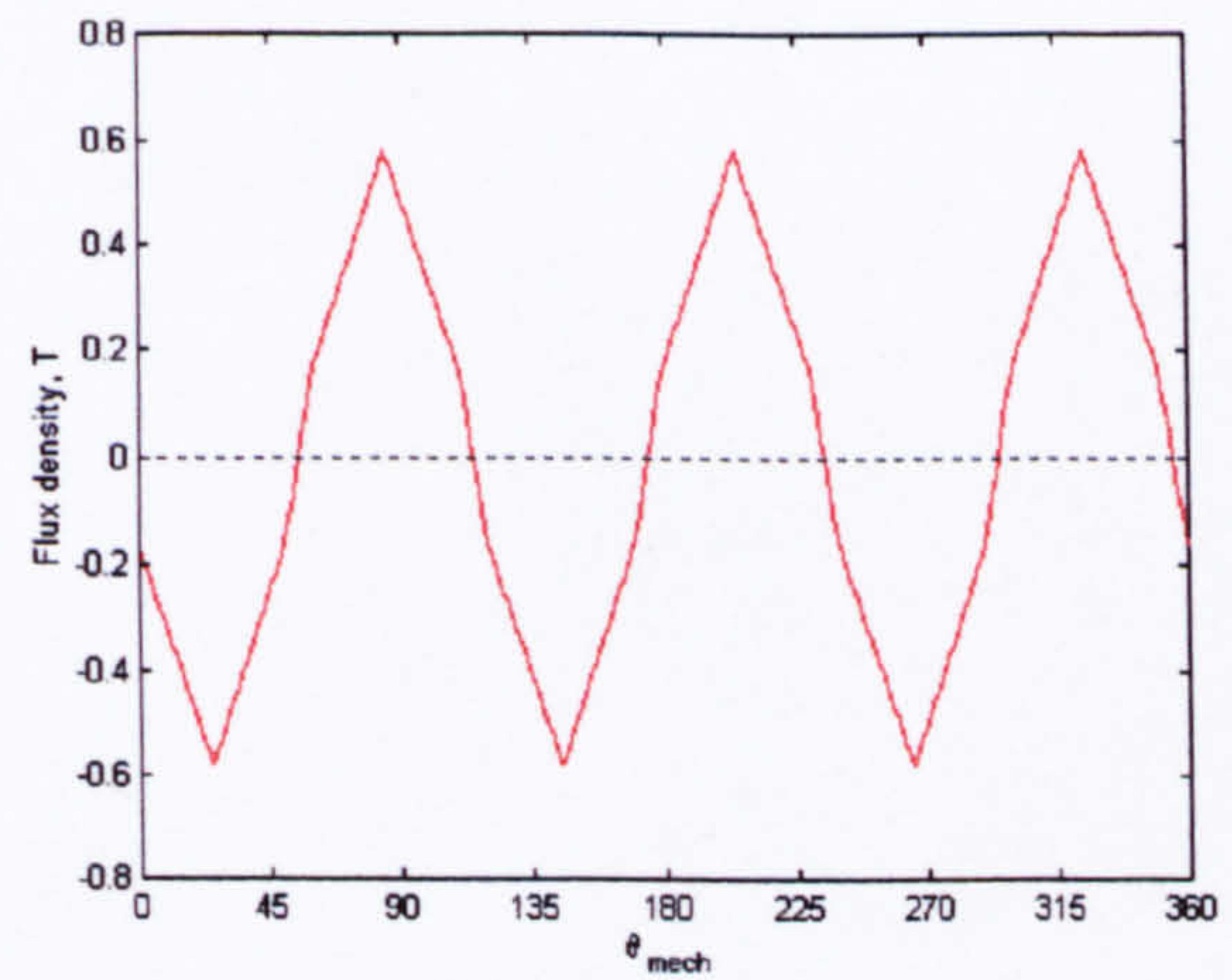

Figure 4.4g. Radial flux density in rotor region 7
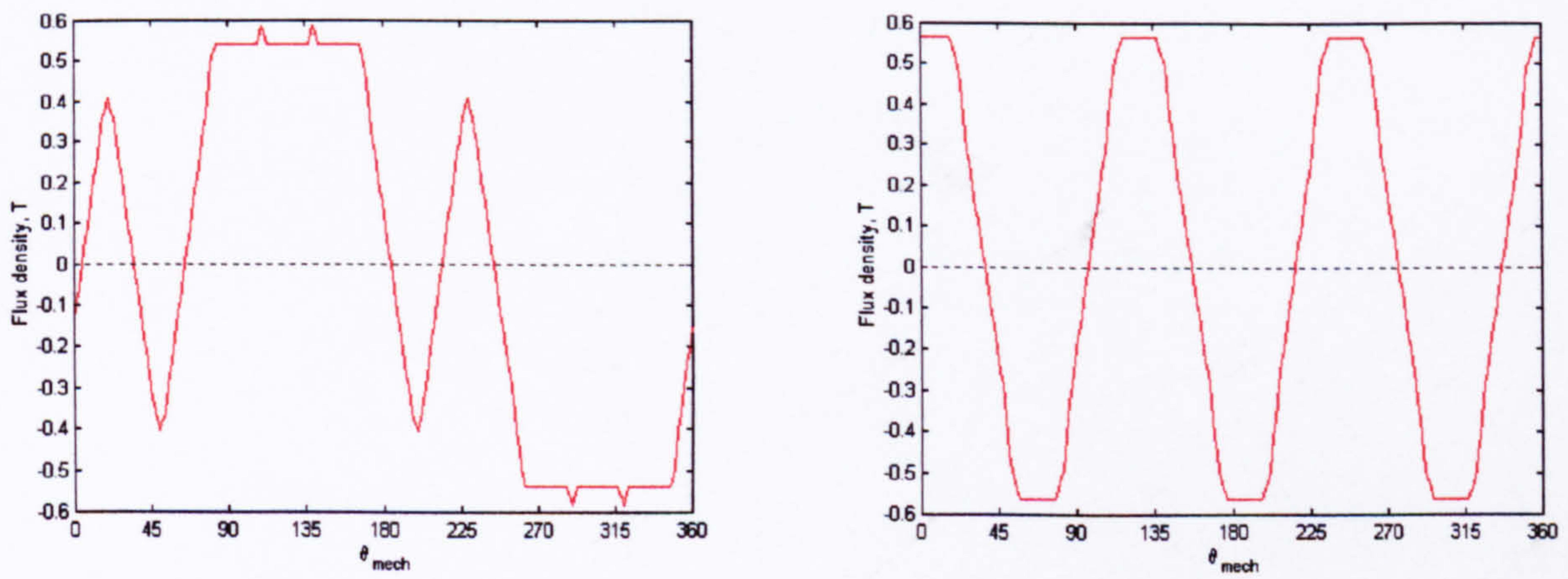

Figure 4.4h. Circumferential flux density in rotor region 8

Figure 4.4 Flux waveforms for various regions of the SR machine defined in figures 4.2 and 4.3 obtained using a simplified solution to the governing equation and a lumped magnetic circuit, employing two differing winding connection strategies defined in figure 4.1 . 

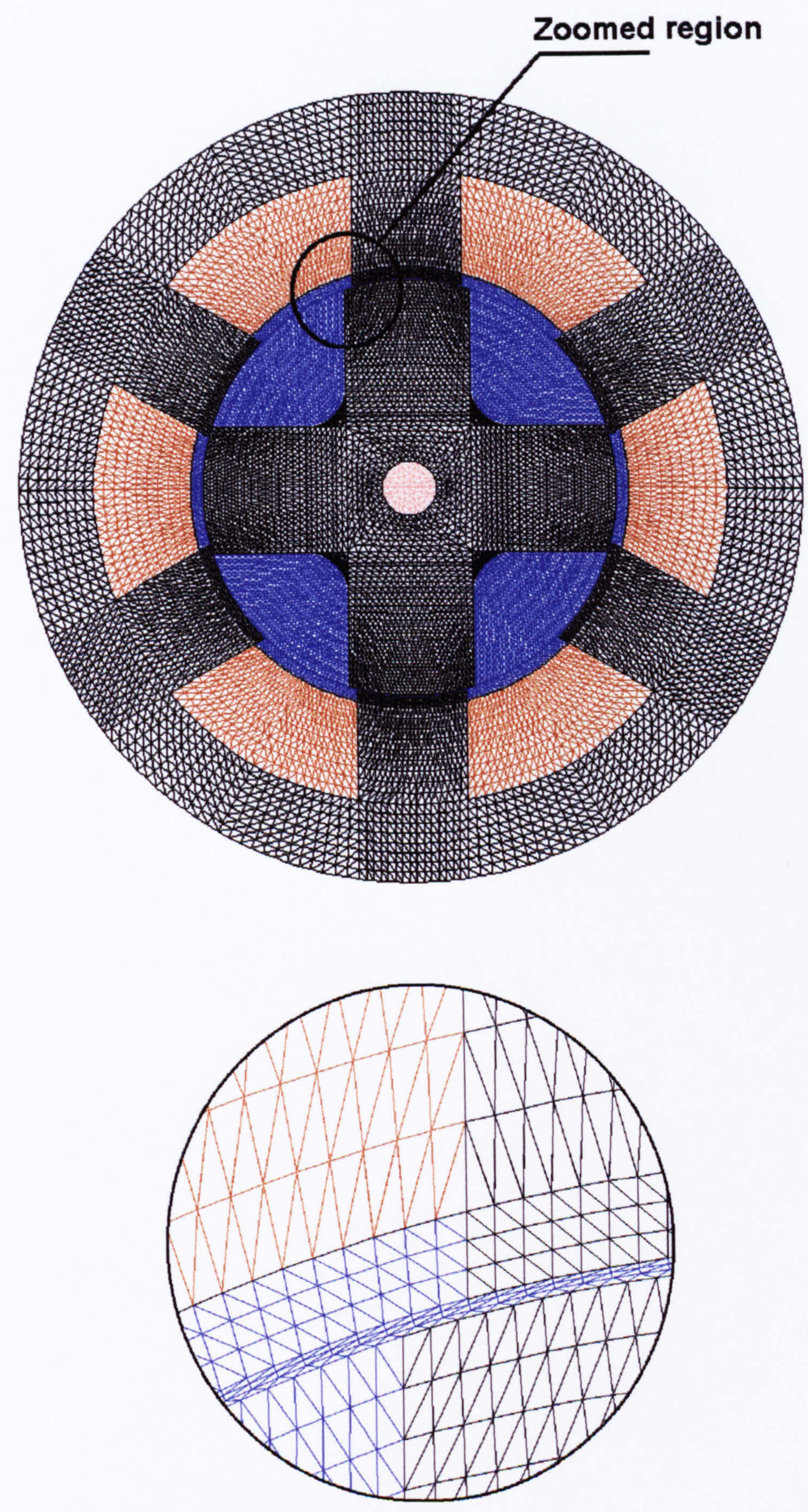

Figure 4.5 Finite element mesh consisting of 23374 first order triangular elements. 


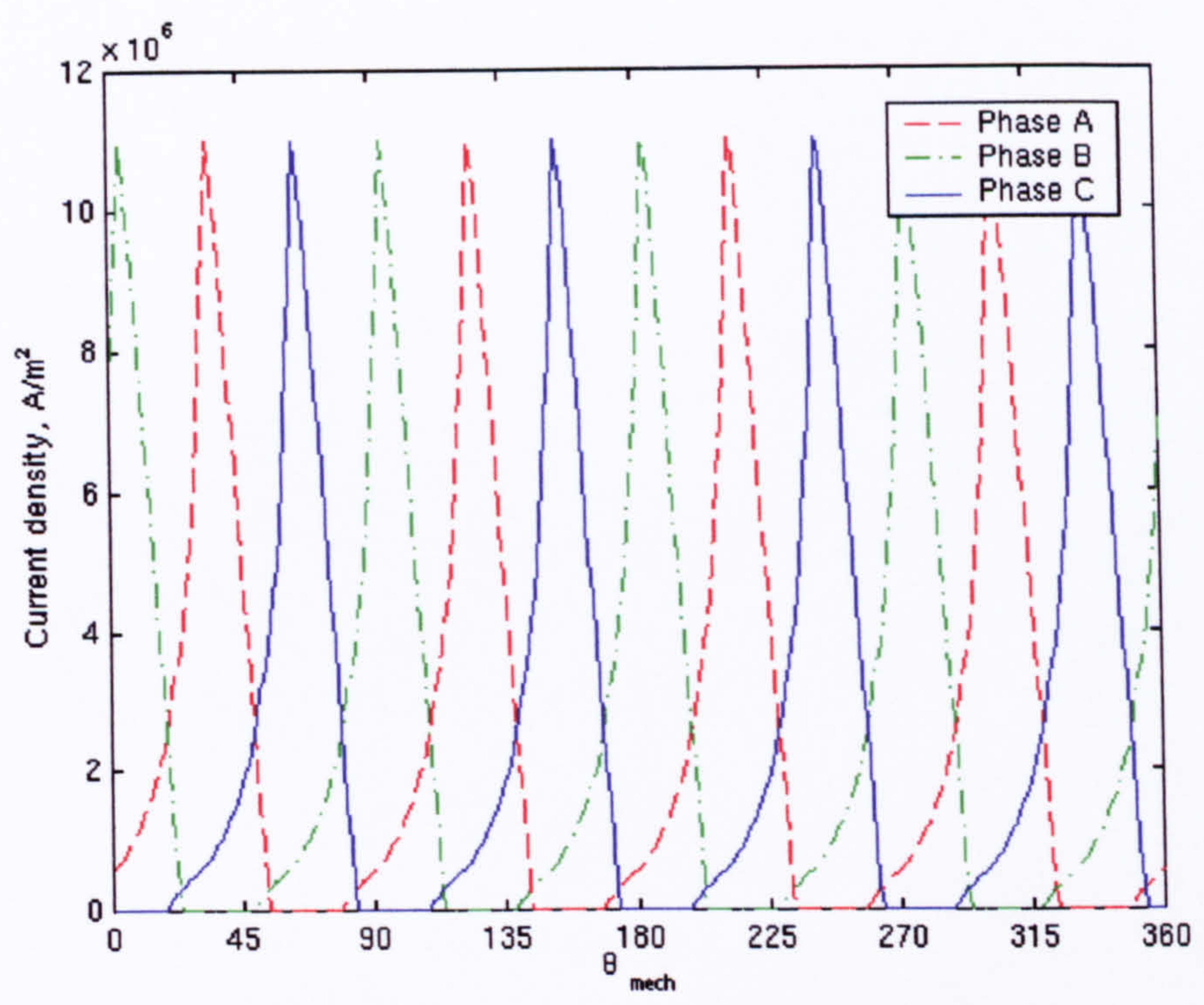

Figure 4.6. 3-phase current density waveform quantised and applied to the windings of the finite element model at $1^{\circ}$ intervals. 
WINDING CONNECTION A

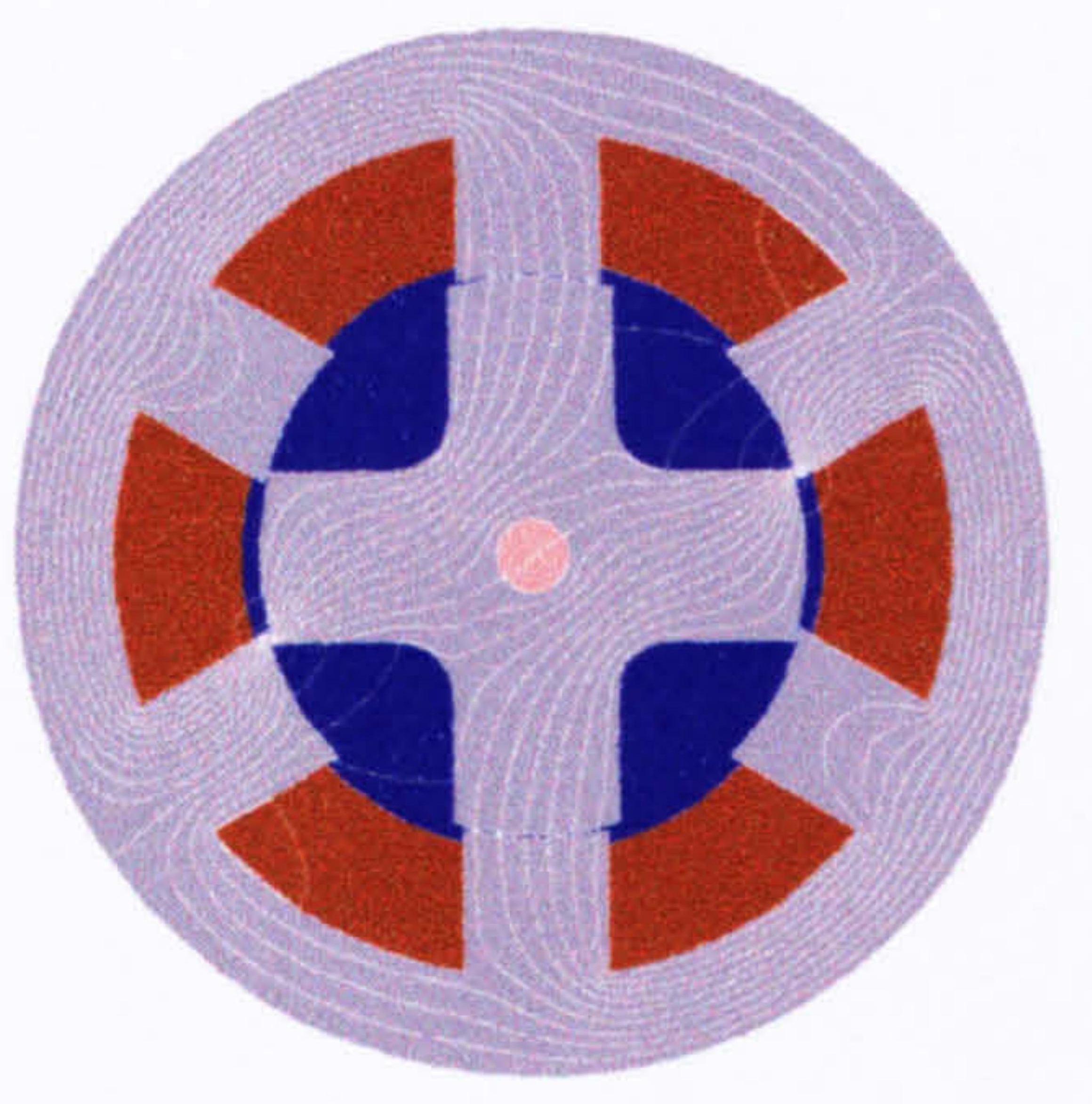

WINDING CONNECTION B

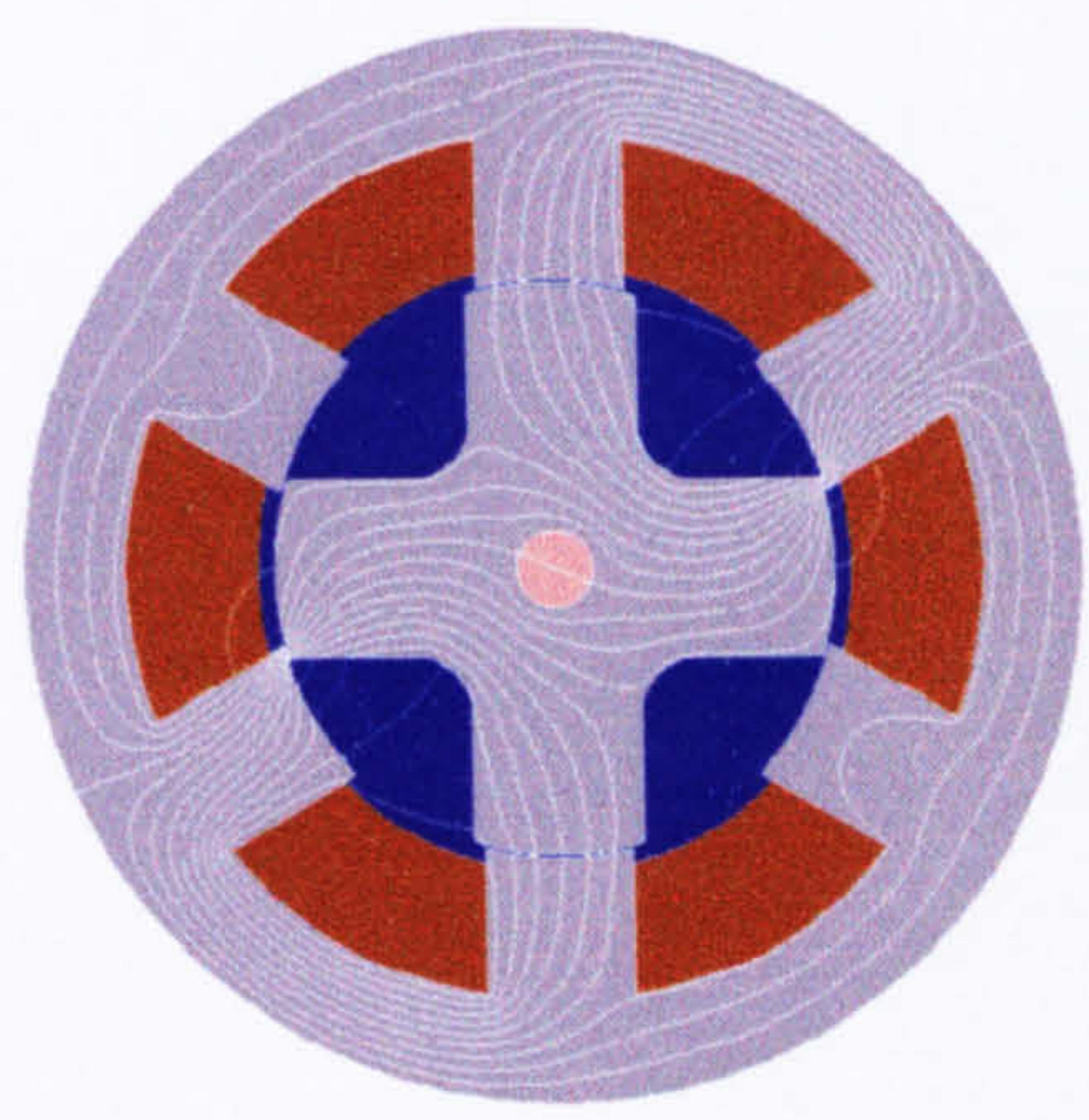

Aligned position
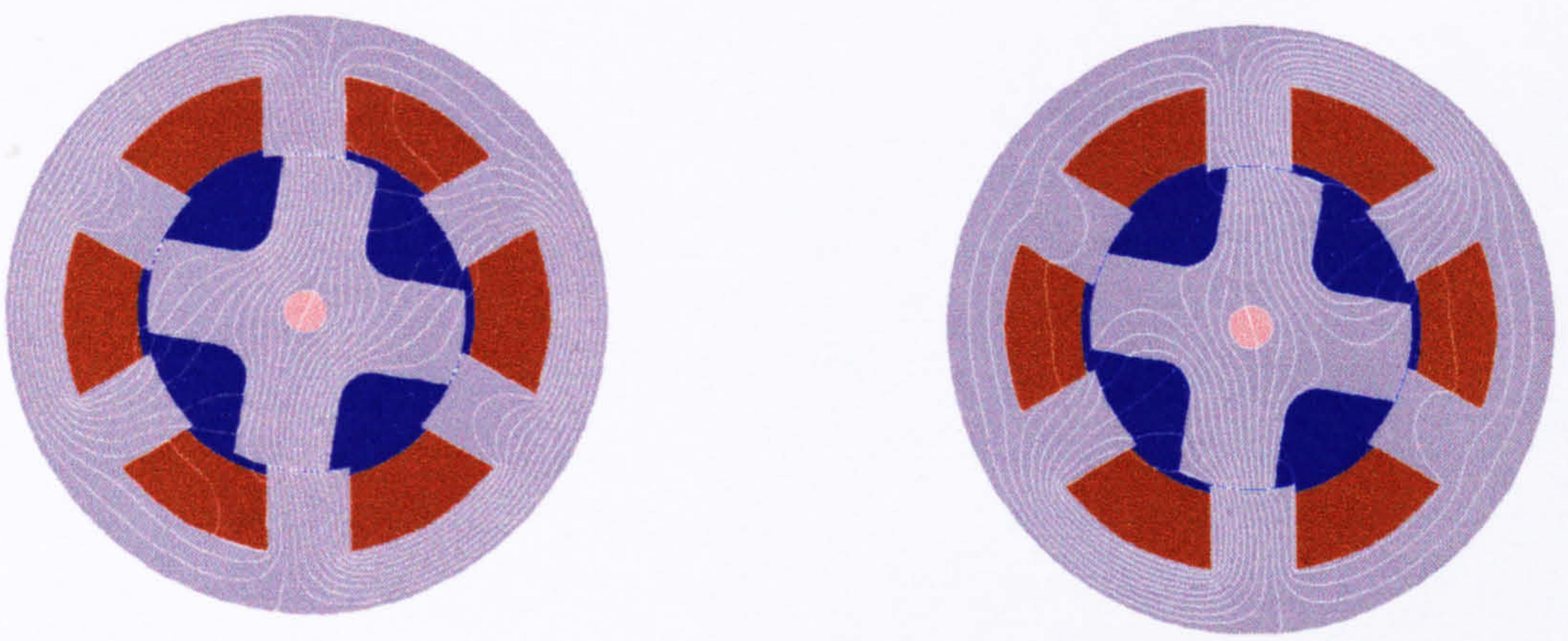

$10^{\circ}$ after alignment
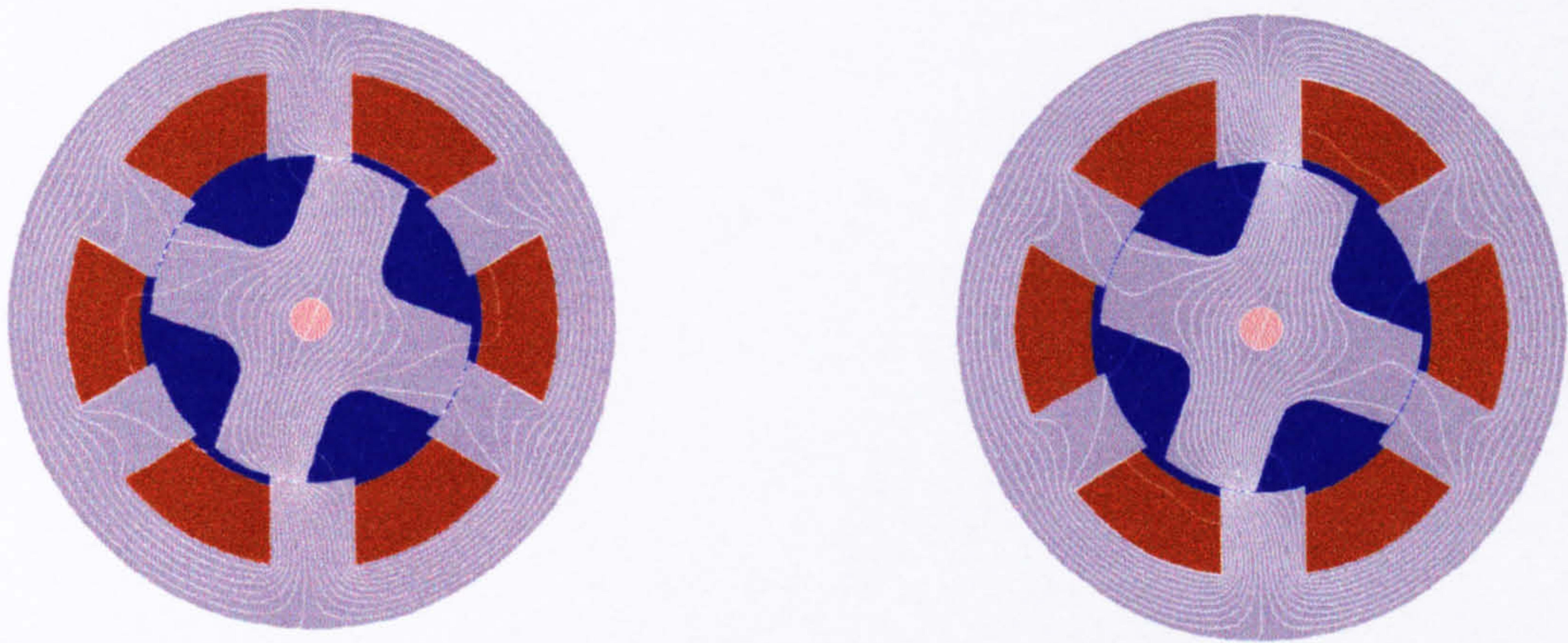

$20^{\circ}$ after alignment

Figure 4.7. Flux contour plots for the machine at $10^{\circ}$ intervals, highlighting the flux paths for the two winding connection types. 


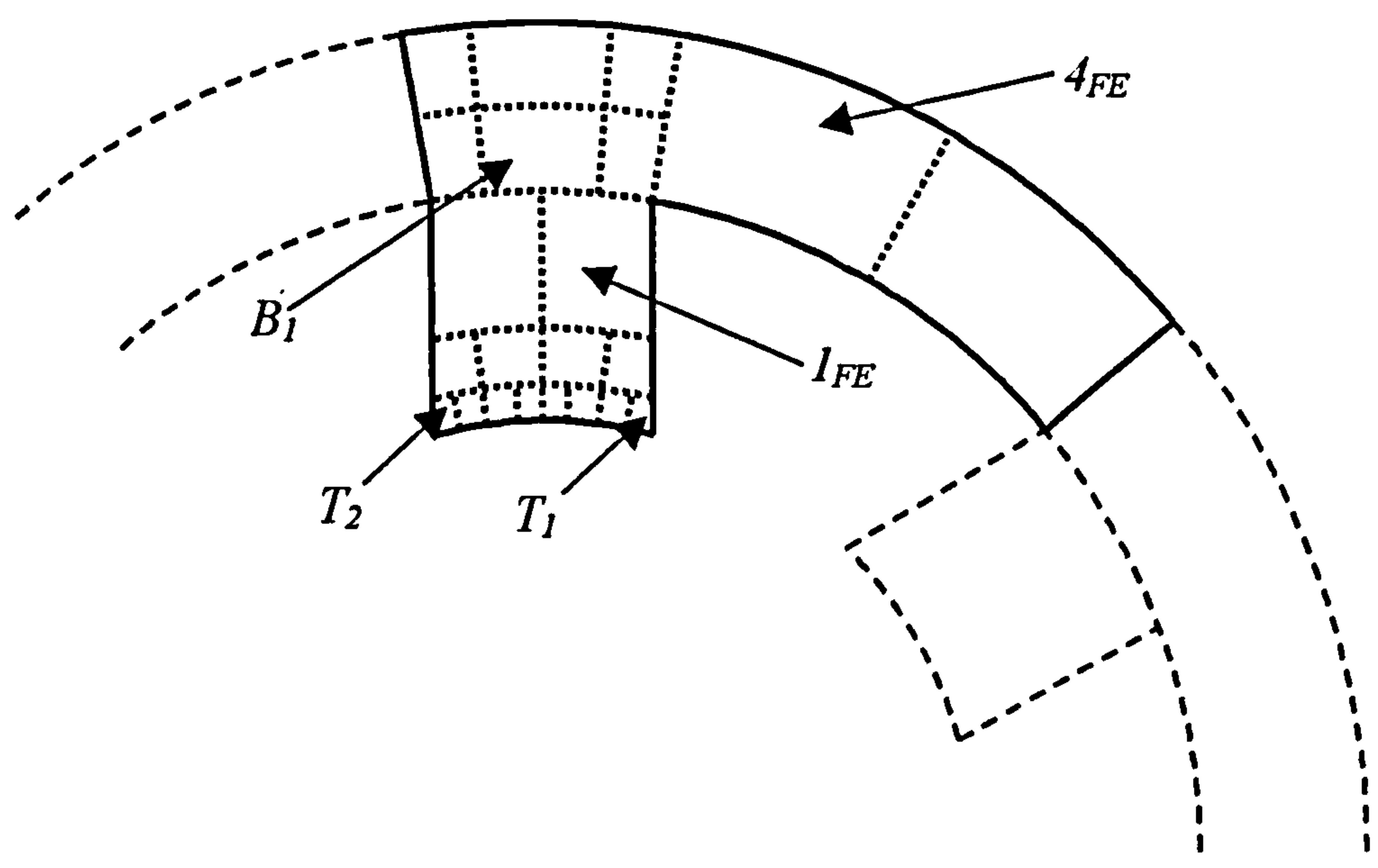

Figure 4.8. Discretisation of the stator into regions of various sizes for the computation of flux waveforms derived from the solution of a finite element model.

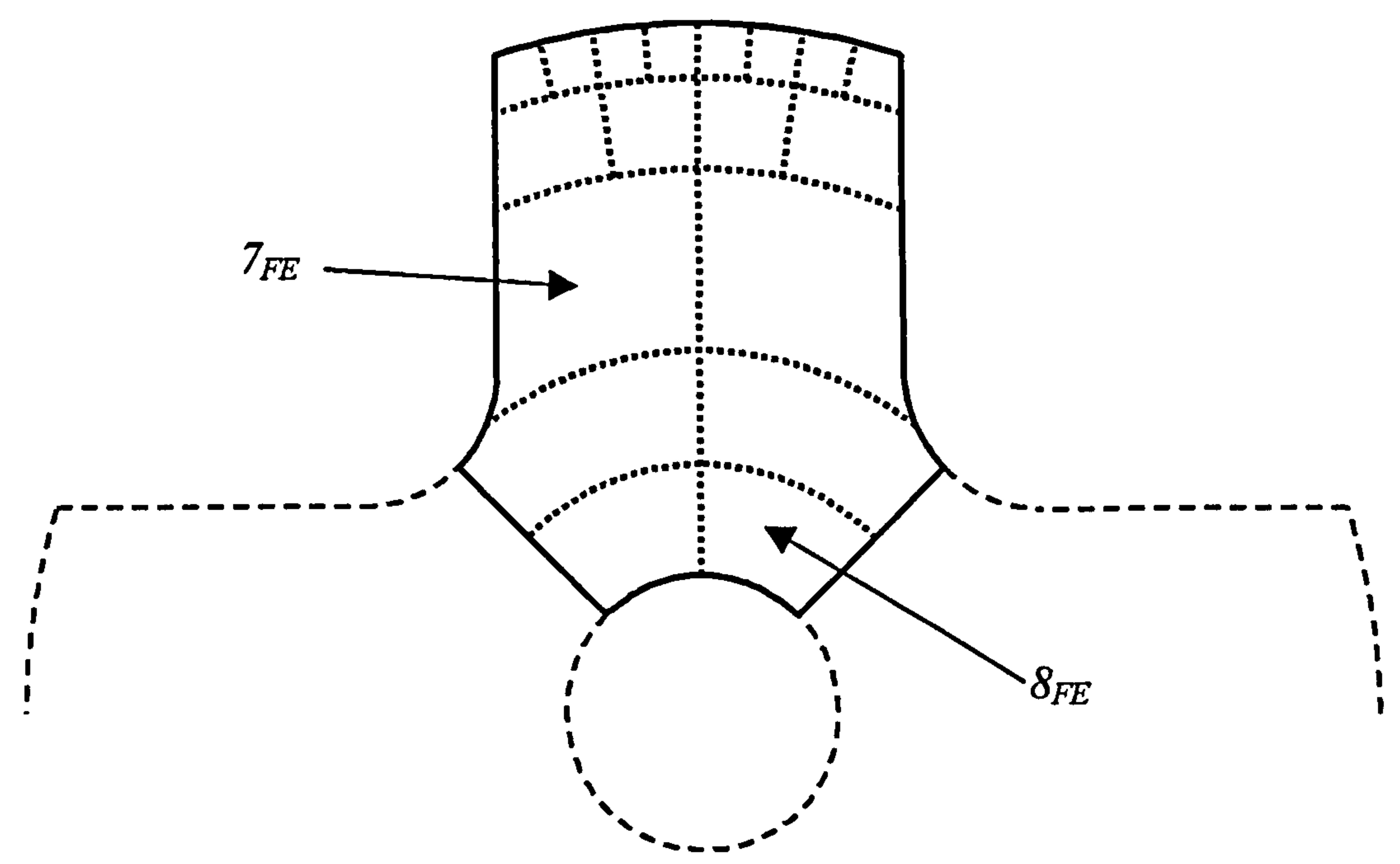

Figure 4.9. Discretisation of the rotor into regions of various sizes for the computation of flux waveforms derived from the solution of a finite element model. 


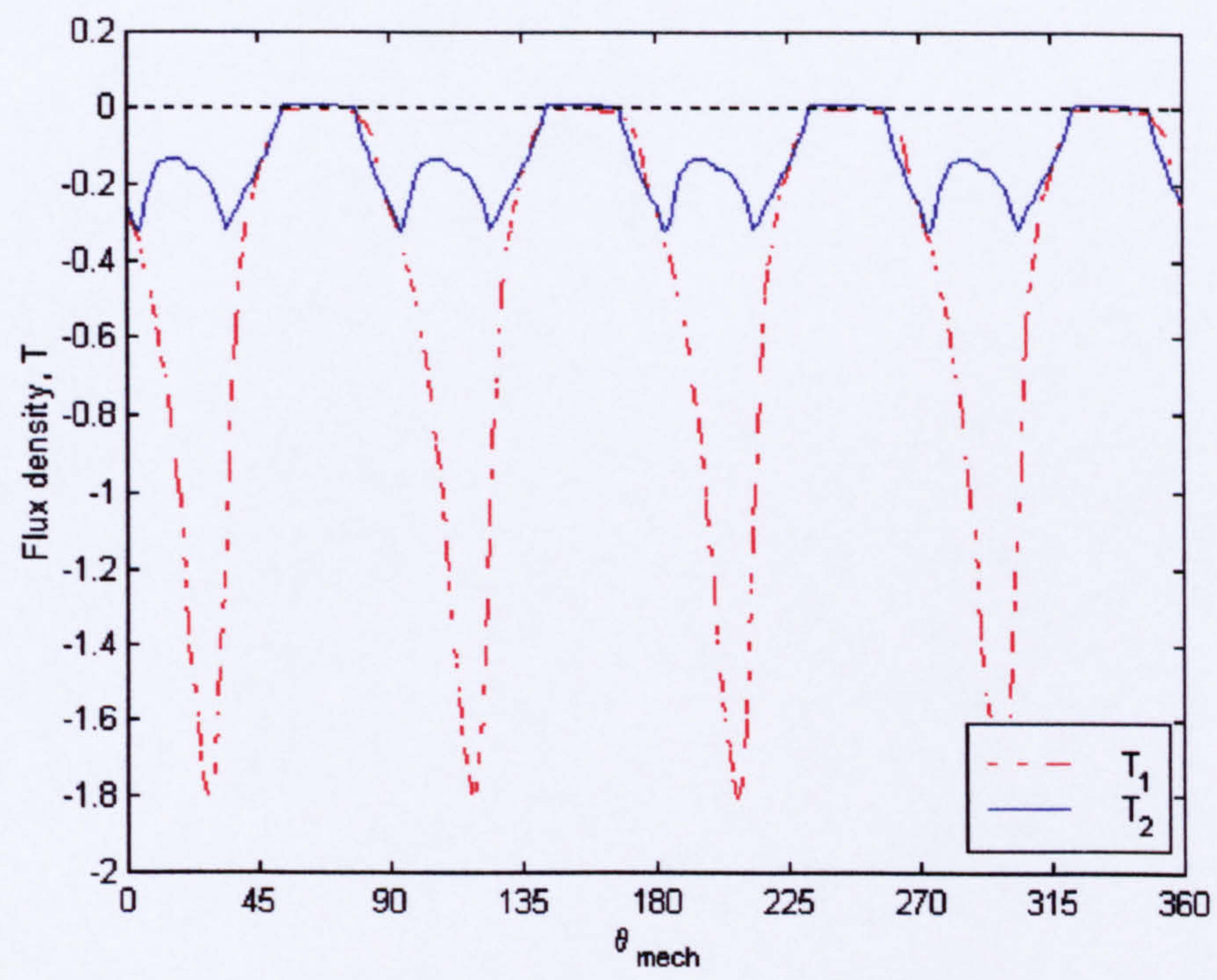

Figure 4.10. Radial flux density waveforms in regions T1 and T2 corresponding to the leading and trailing edge of the stator tooth defined in figure 4.8 for winding connection type A. 
WINDING CONNECTION A

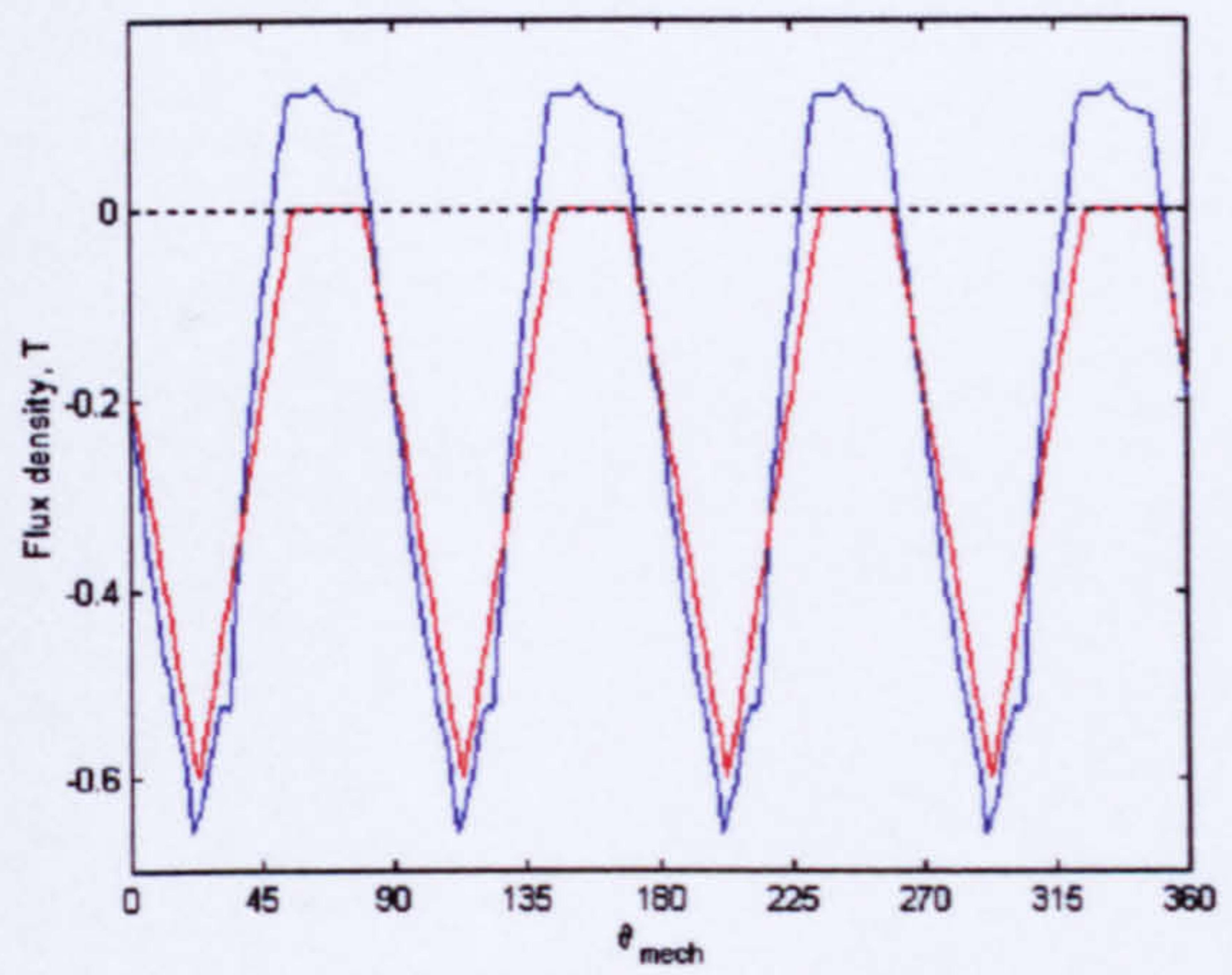

WINDING CONNECTION B

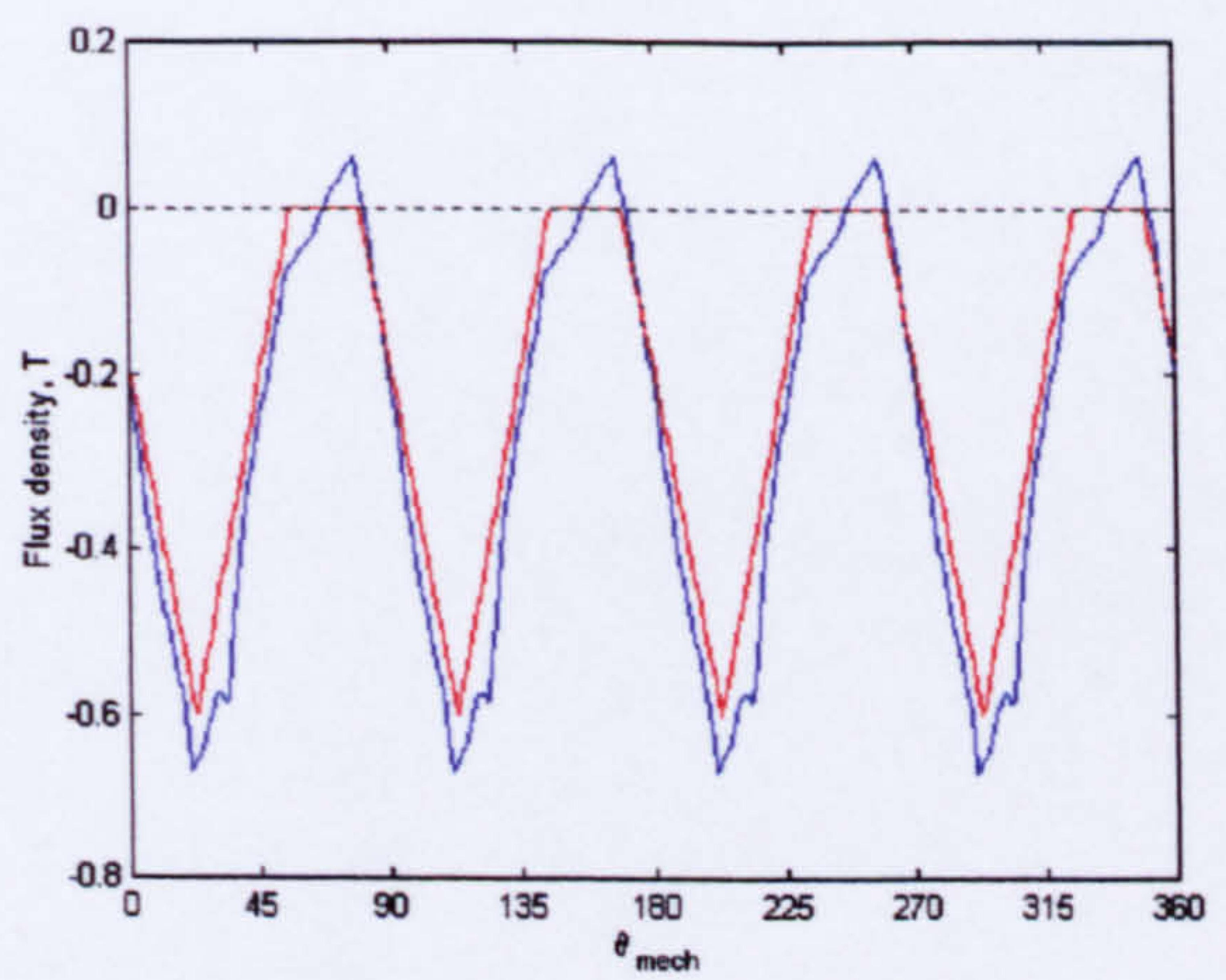

Figure 4.11a. Radial flux density in stator region 1 and $I_{F E}$
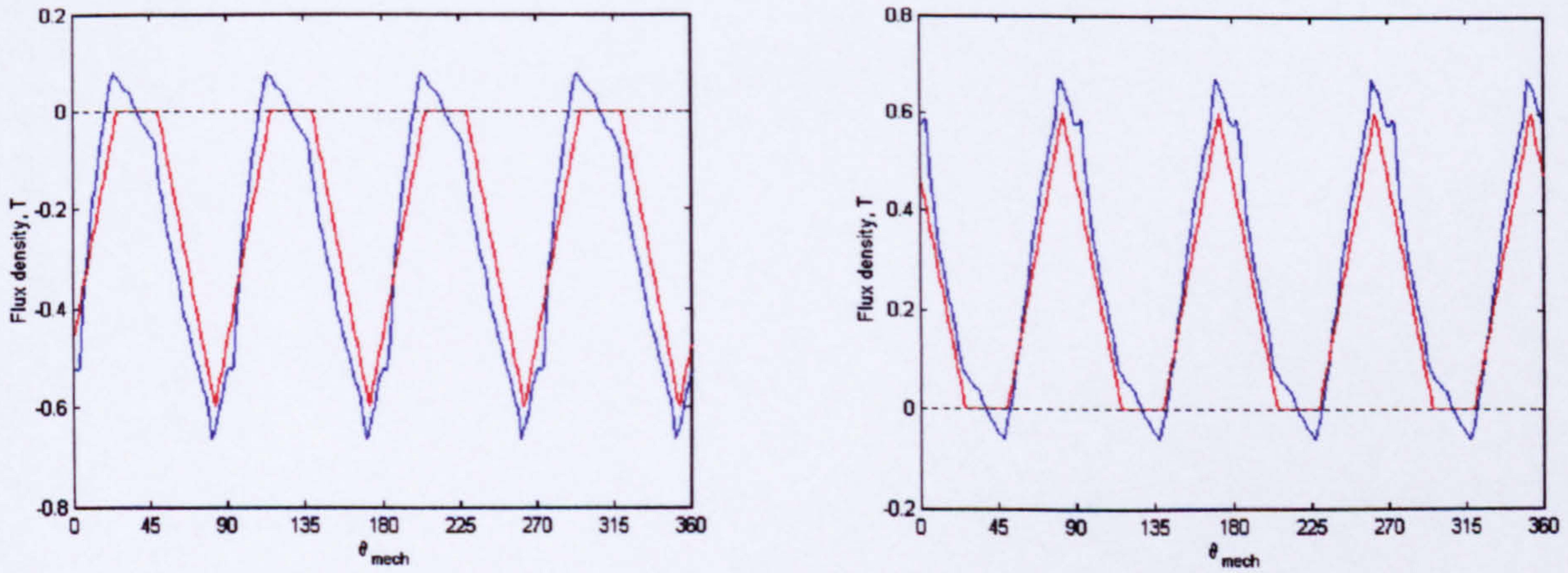

Figure $4.11 \mathrm{~b}$. Radial flux density in stator region 2 and 2 FE
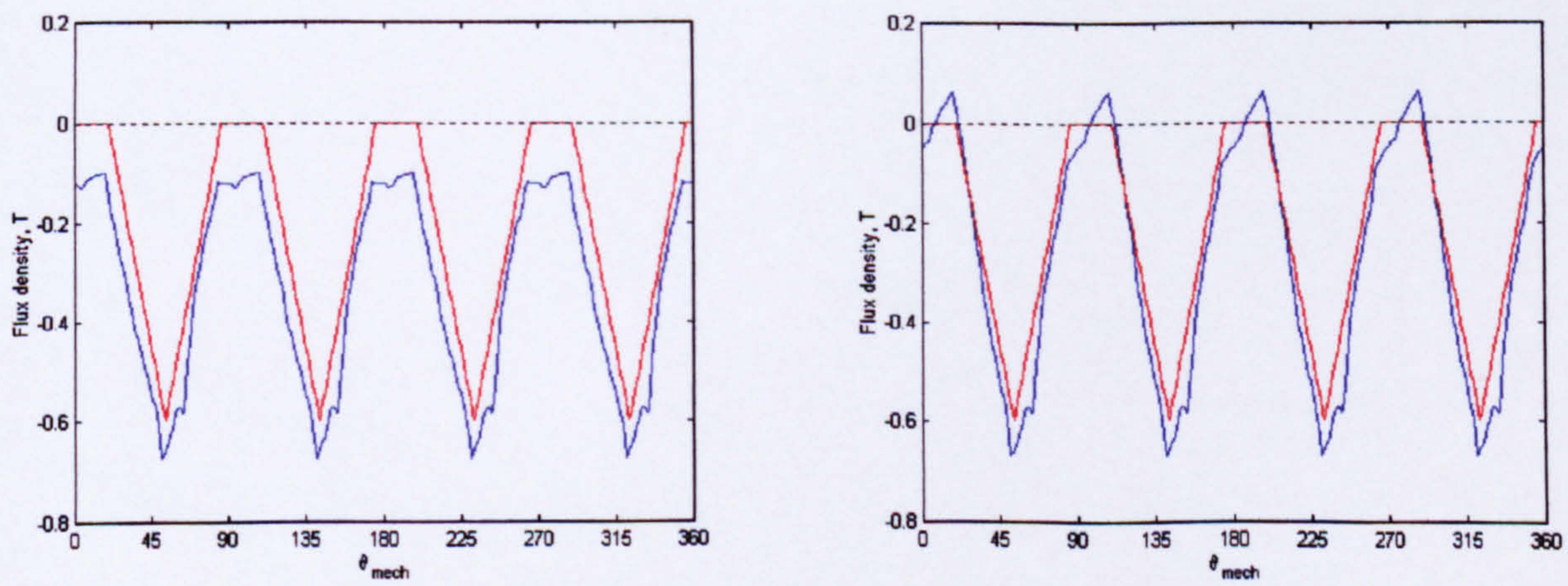

Figure 4.11c. Radial flux density in stator region 3 and $3_{F E}$ 
WINDING CONNECTION A

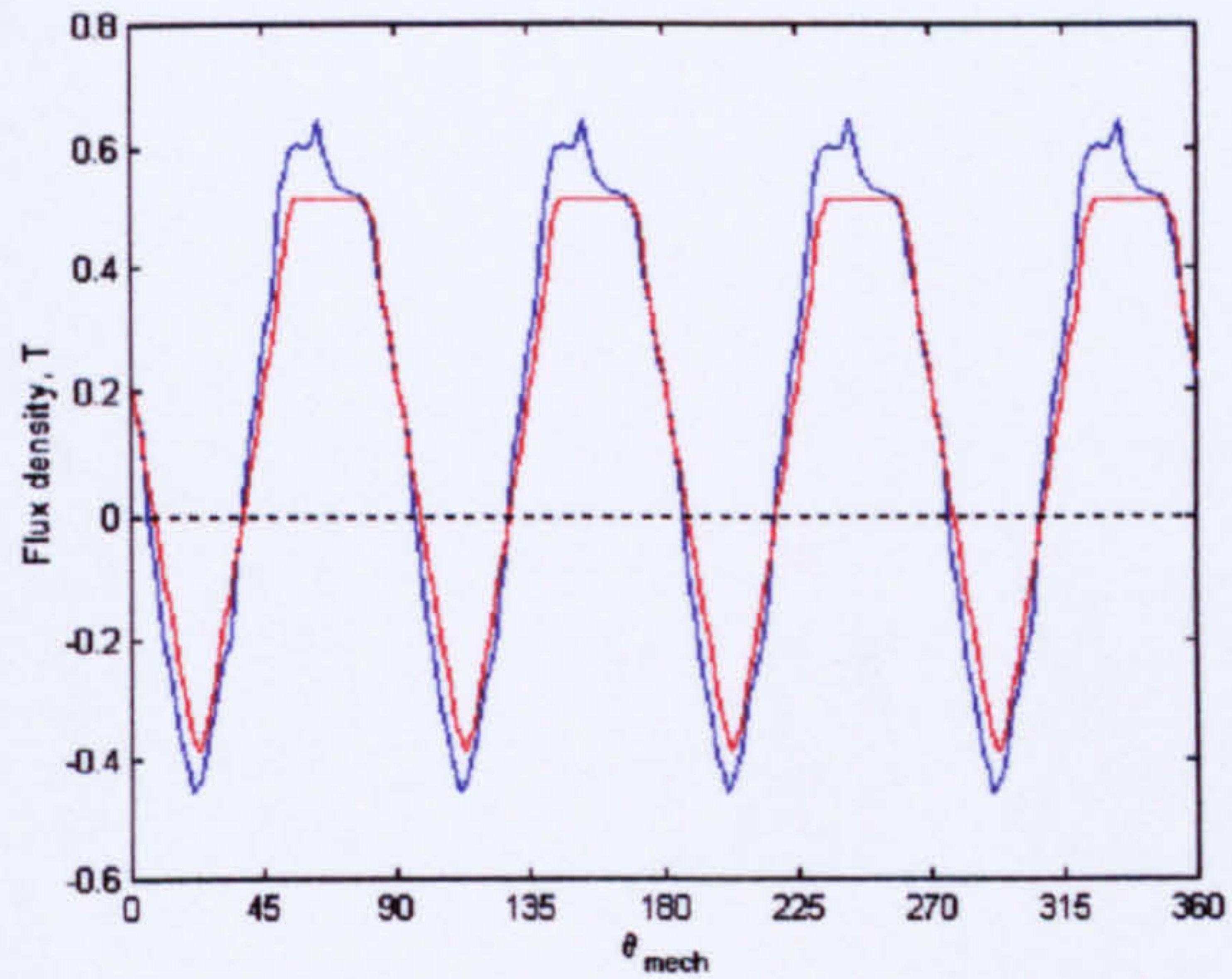

WINDING CONNECTION B

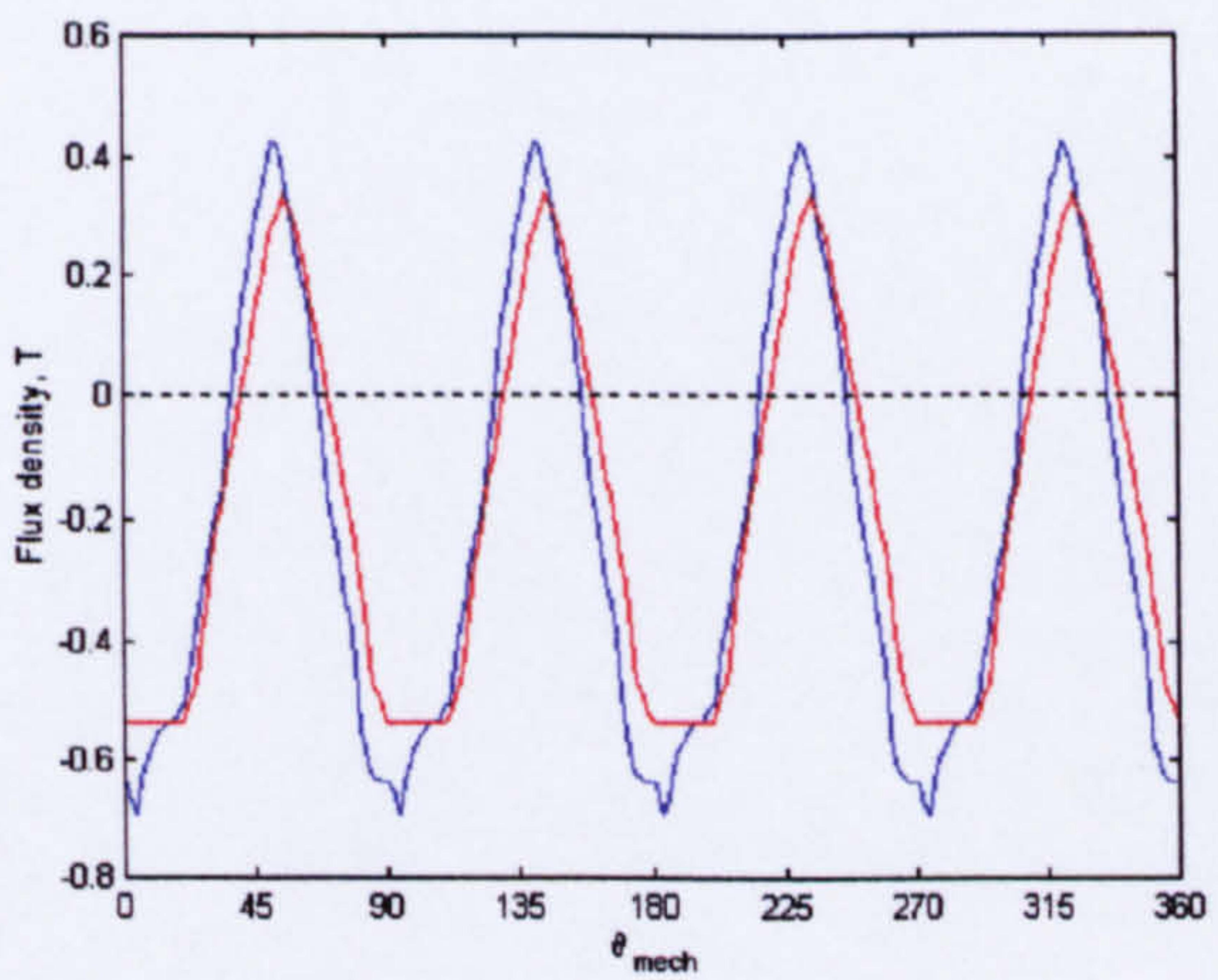

Figure 4.11d. circumferential flux density in stator region 4 and $4_{F E}$
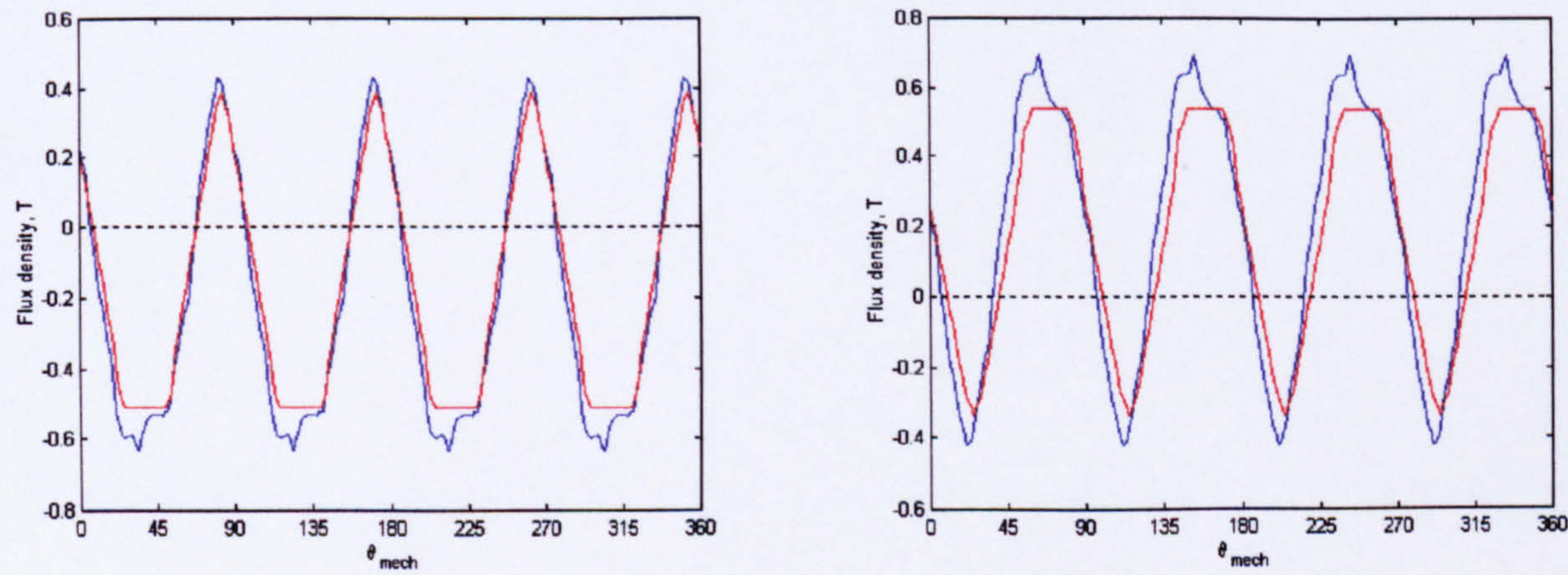

Figure 4.11e. circumferential flux density in stator region 5 and $5_{F E}$
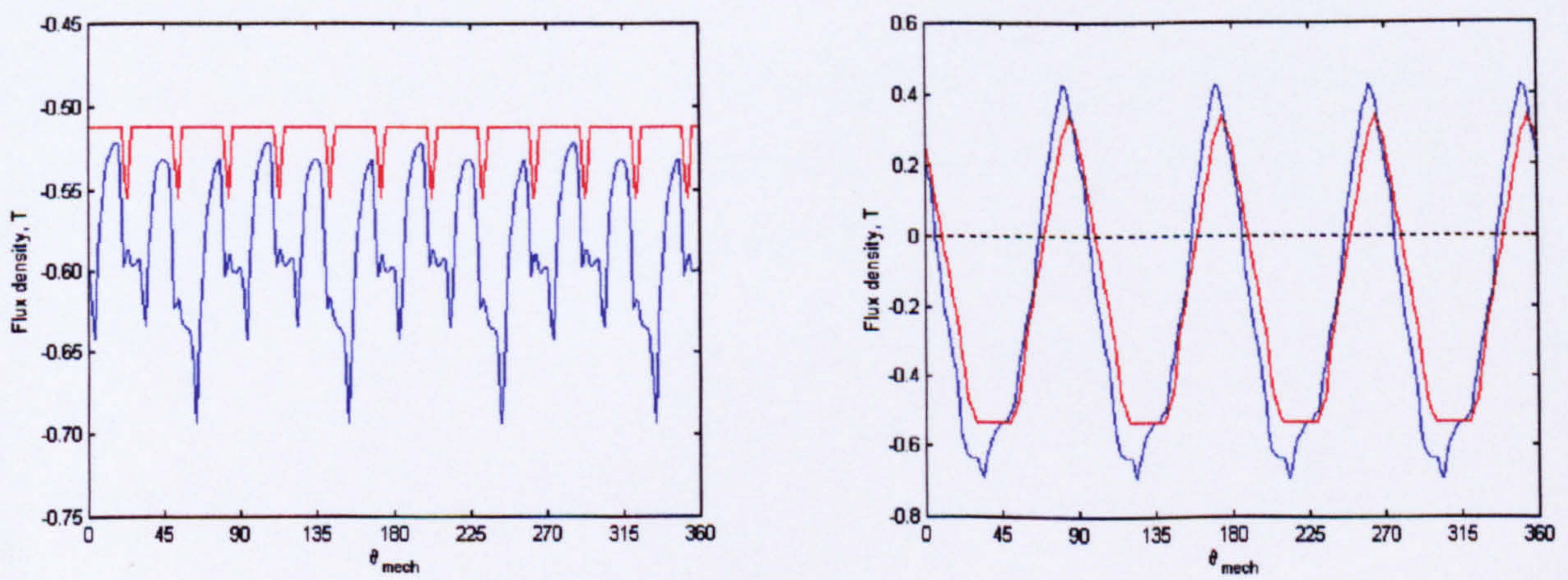

Figure 4.11f. circumferential flux density in stator region 6 and $6_{F E}$ 
WINDING CONNECTION A

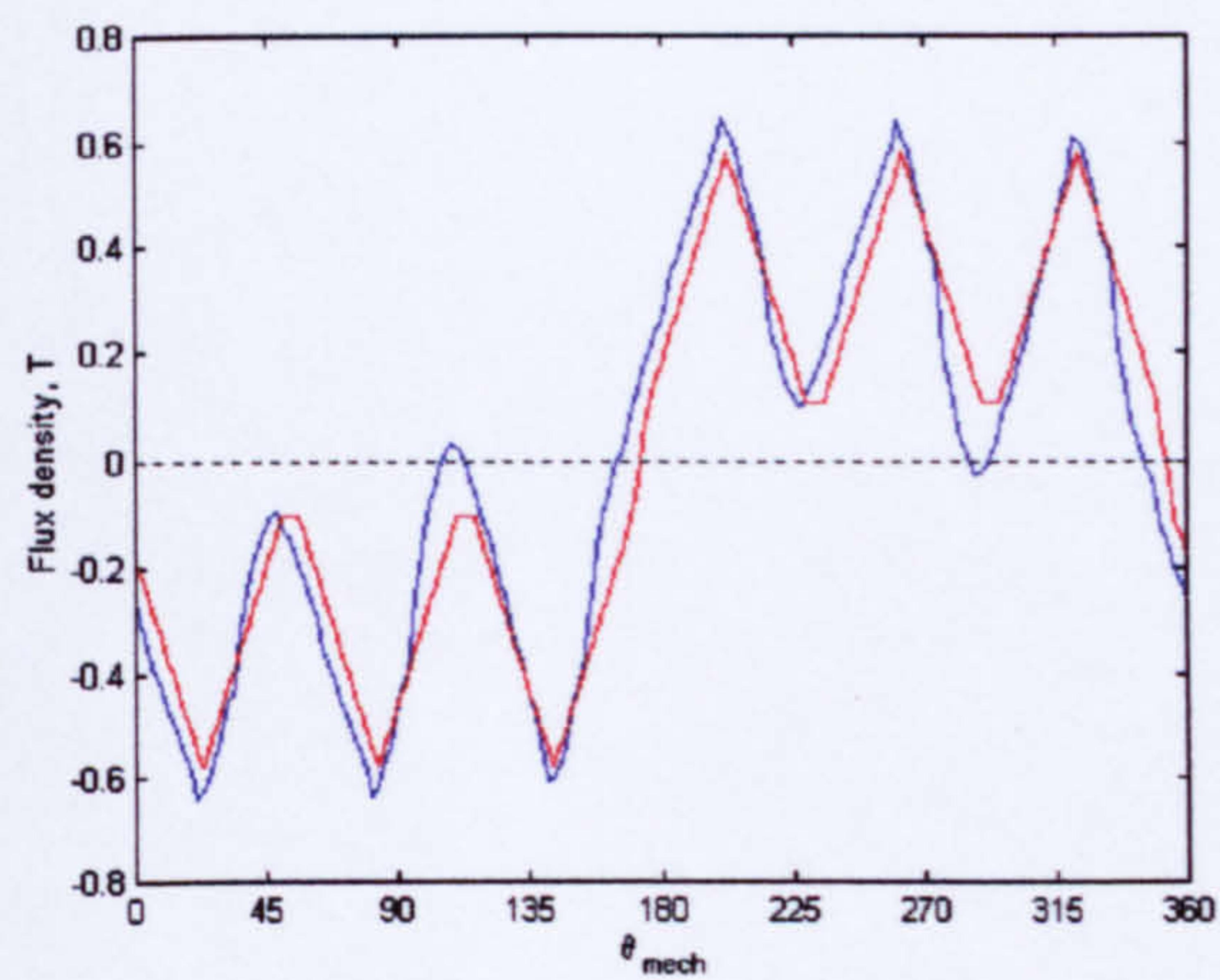

WINDING CONNECTION B

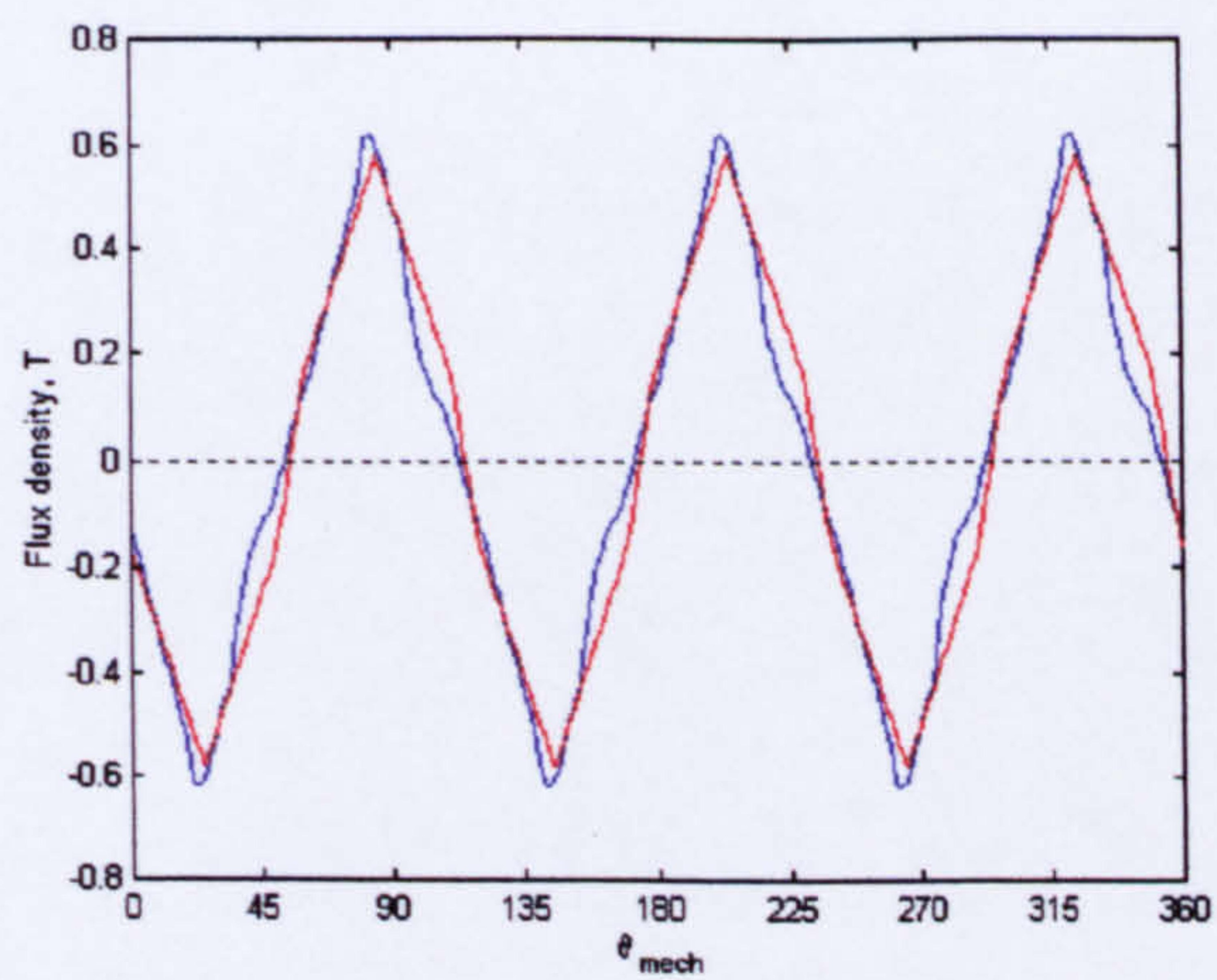

Figure 4.11g. Radial flux density in stator region 7 and $7_{F E}$
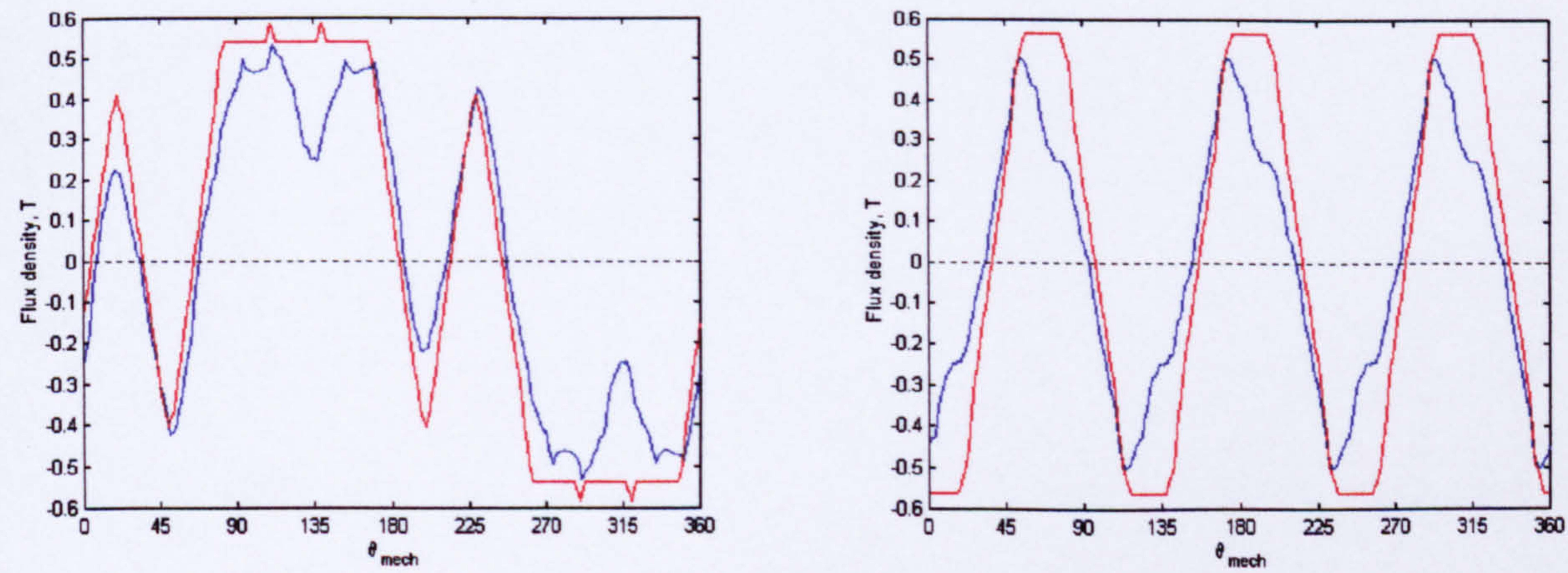

Figure $4.11 \mathrm{~h}$. circumferential flux density in stator region 8 and $8_{F E}$

Figure 4.11 Flux waveforms for the various regions defined in figures 4.8 and 4.9 with the analytical equivalent superimposed, employing two differing winding connection types defined in figure 4.1. 
$45^{\circ}$ before alignment

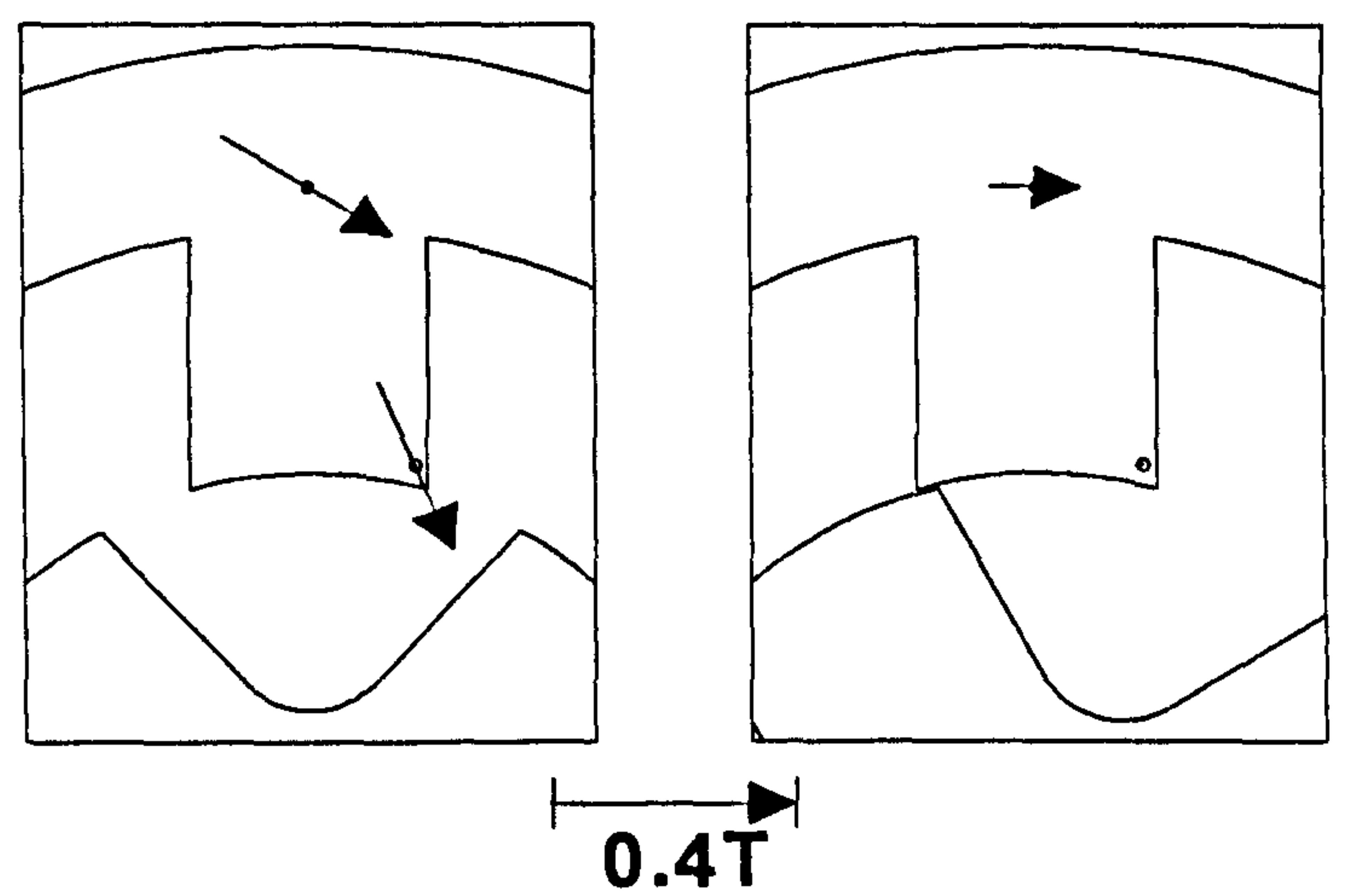

$30^{\circ}$ before alignment

$15^{\circ}$ before alignment

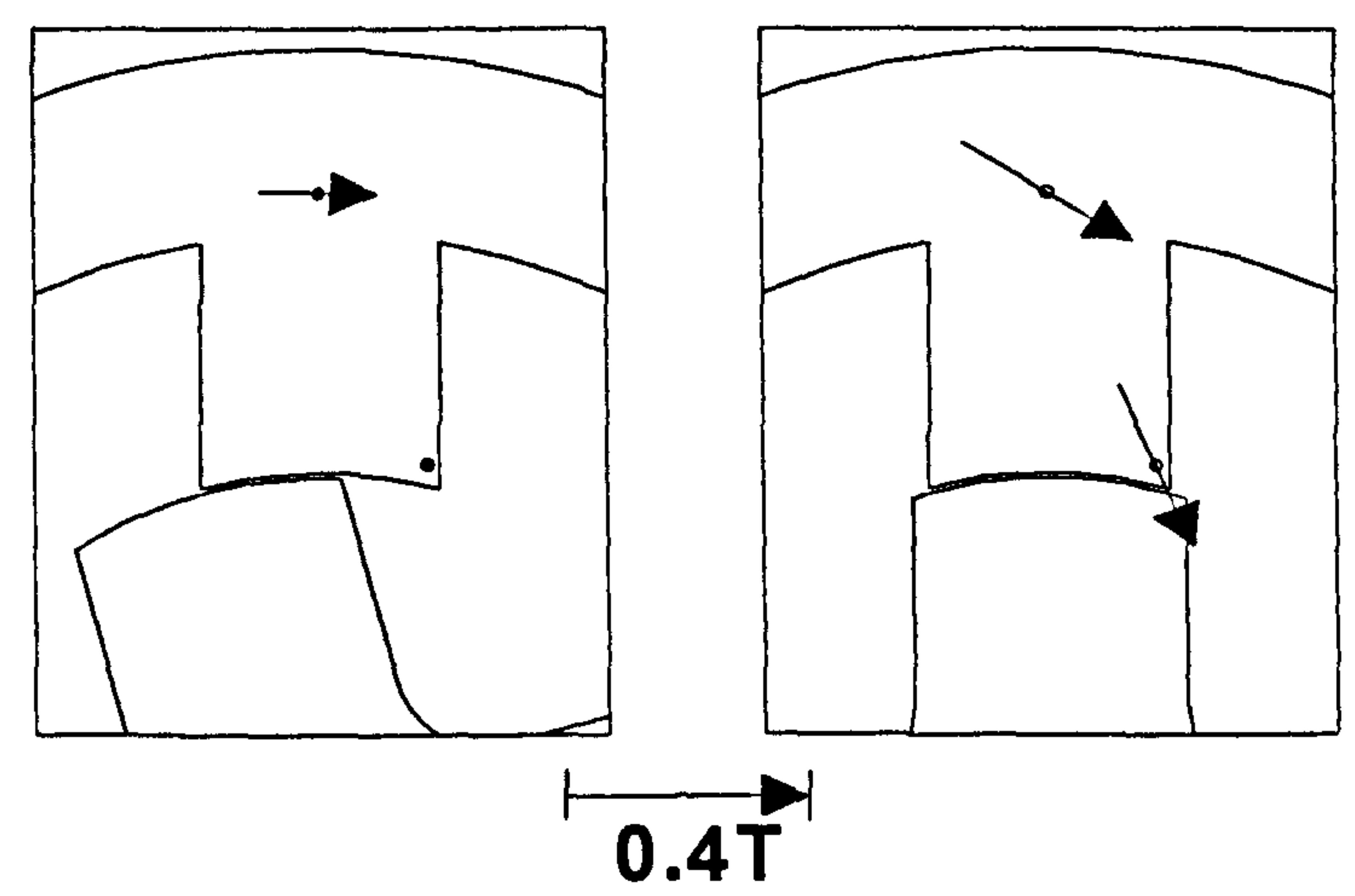

Aligned

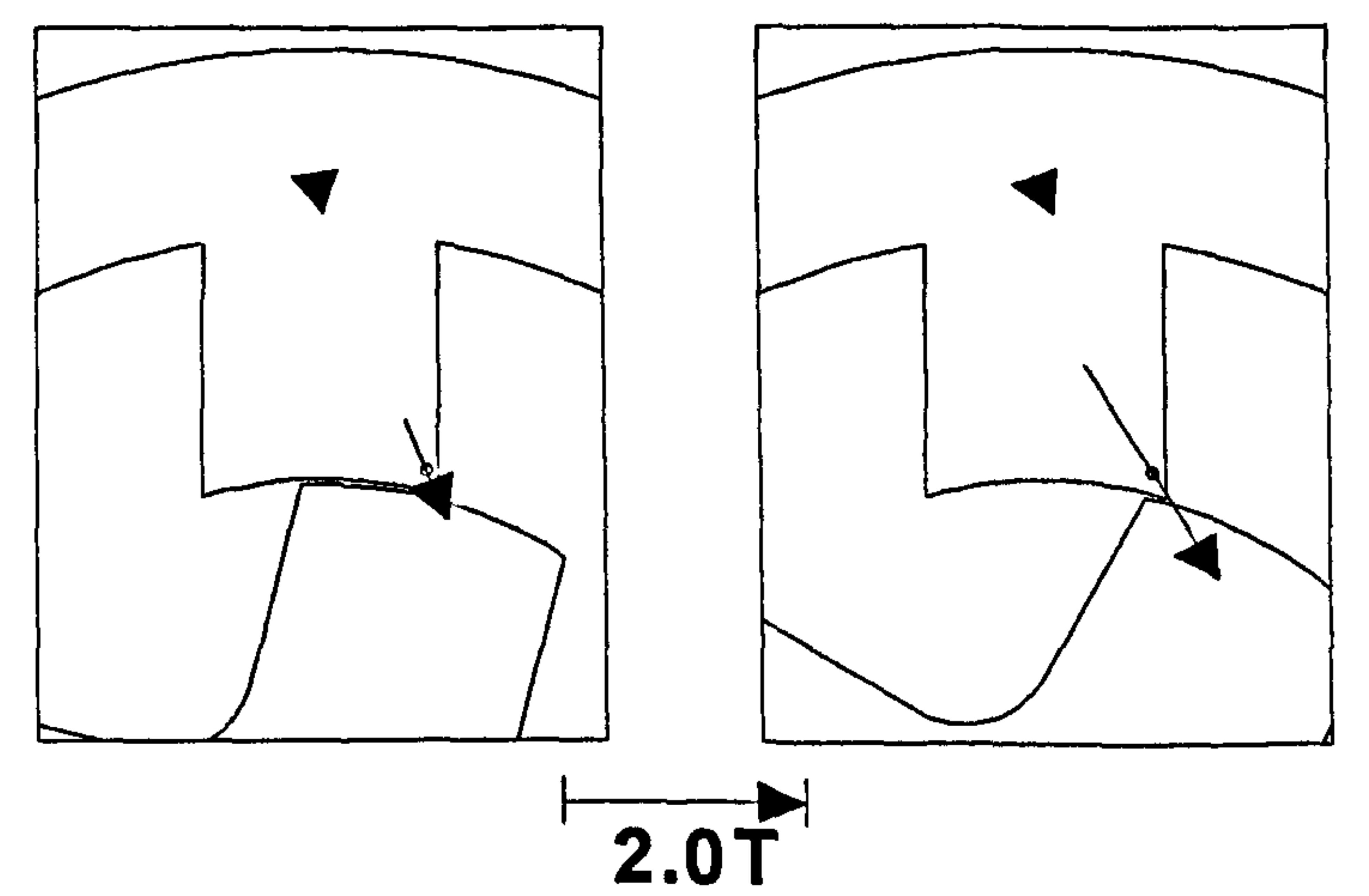

$30^{\circ}$ after alignment $15^{\circ}$ after
alignment 2.01

Figure 4.12 flux density vectors as the rotor passes phase $\mathrm{A}$ for winding connection A. 


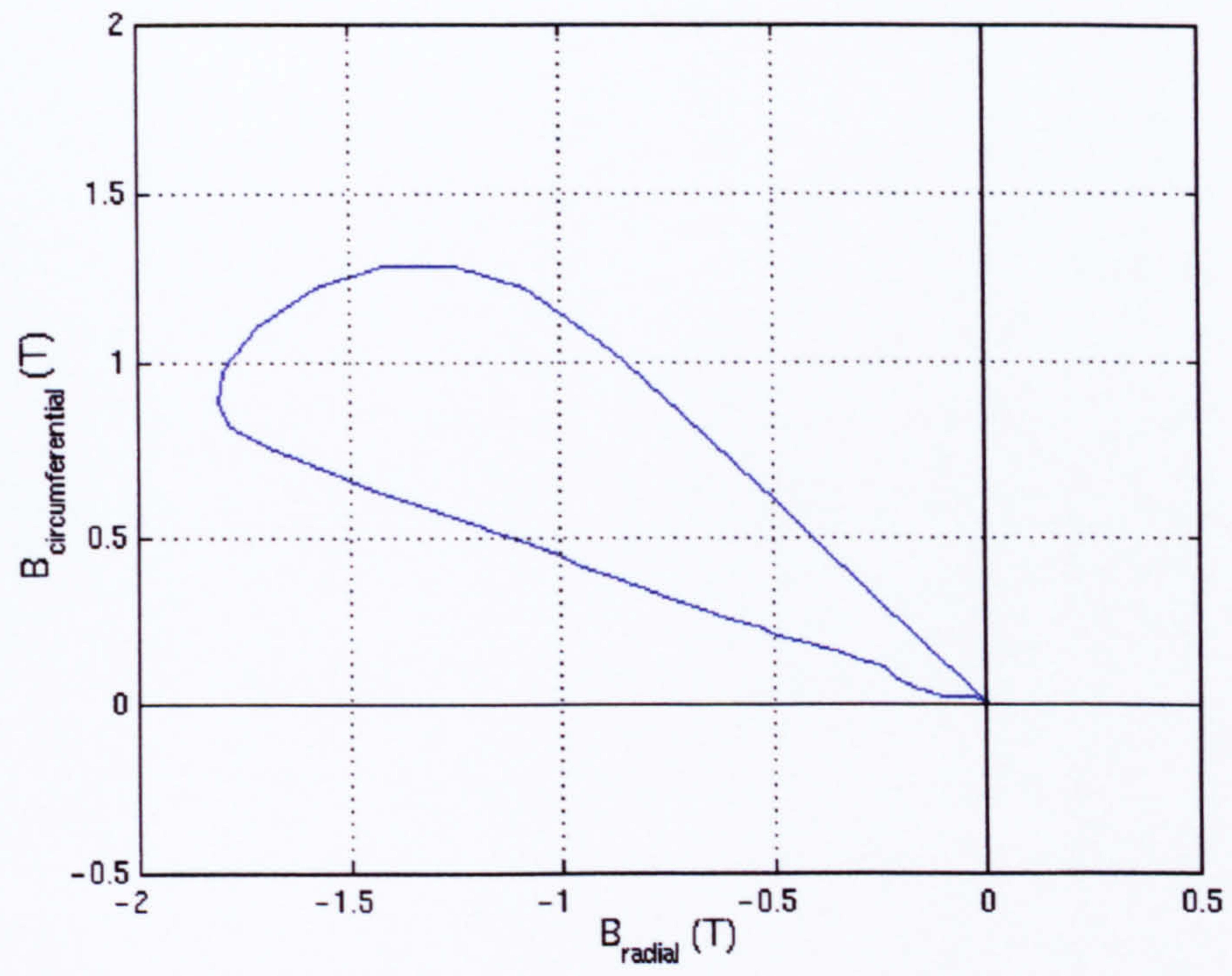

Figure 4.13 Radial and circumferential flux density loci in the stator tooth tip, region $T_{1}$.

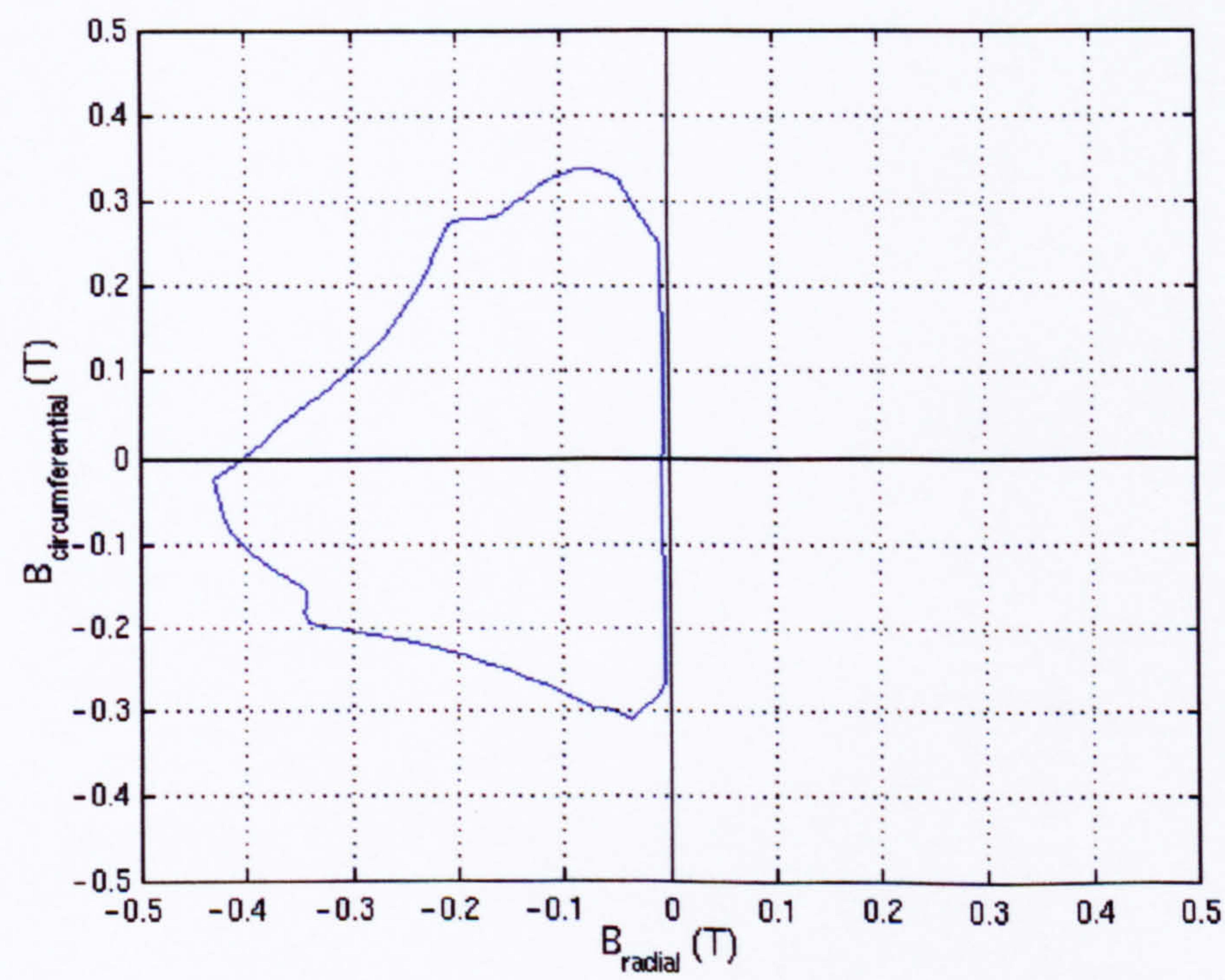

Figure 4.14 Radial and circumferential flux density loci in the stator back iron, region $B_{1}$. 


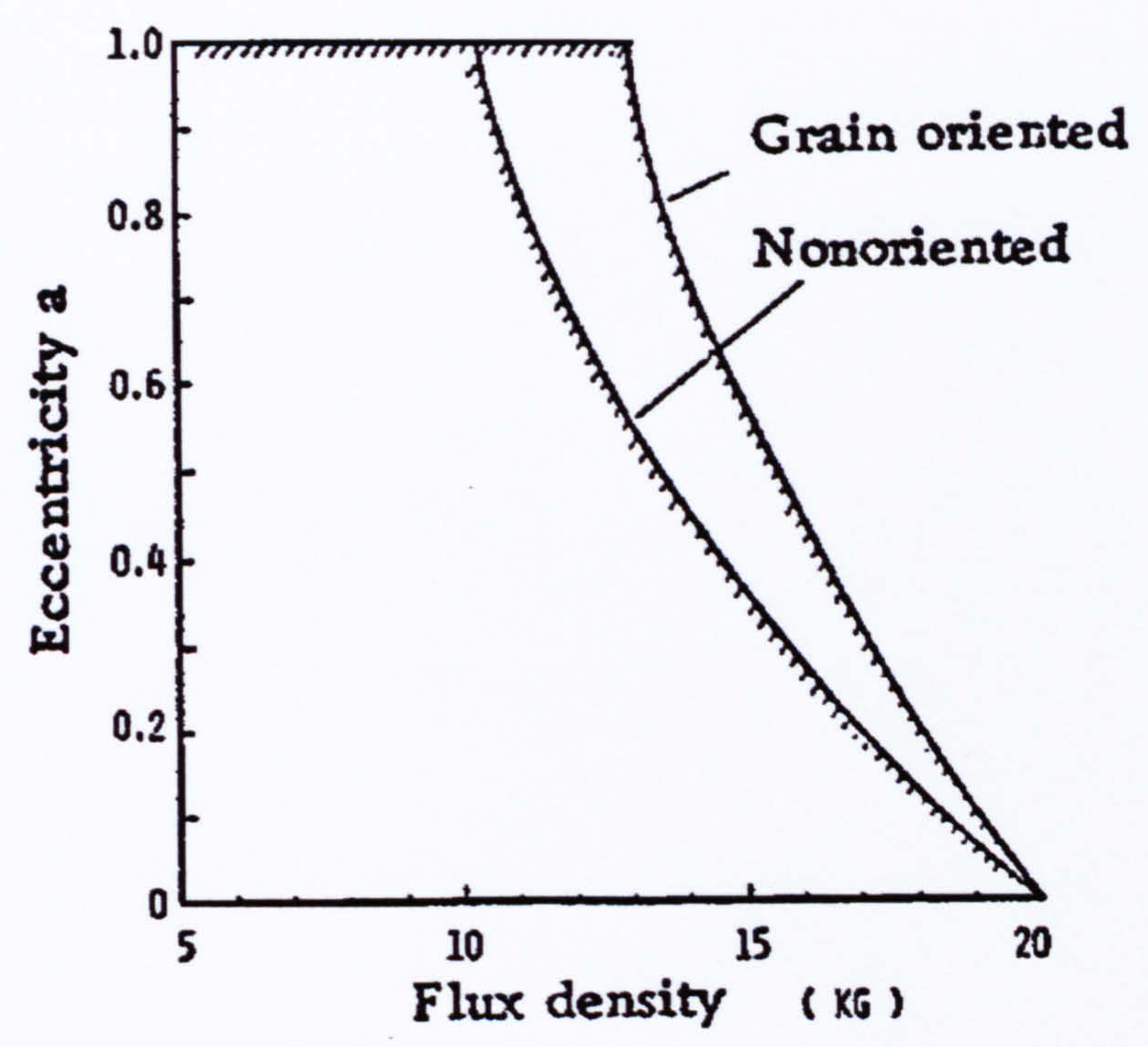

Figure 4.15 Locus of points where estimation of rotational power loss is applicable.

[4.12] $\left(10^{4} G=1 T\right)$

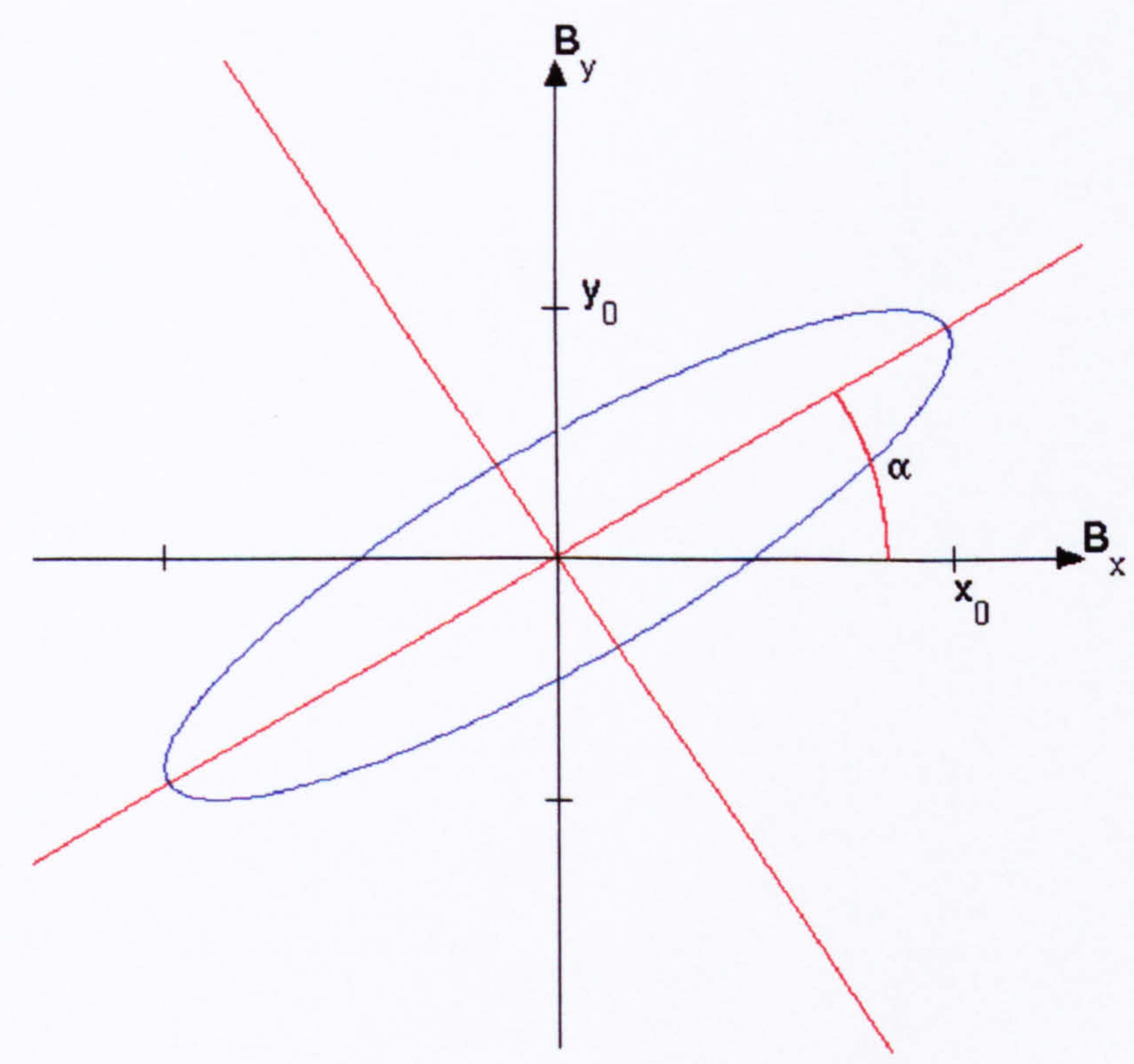

Figure 4.16 Lissajous representation of sinusoidal, orthogonal flux density variation with a constant phase difference showing alignment of principal axes and corresponding components of flux density. 


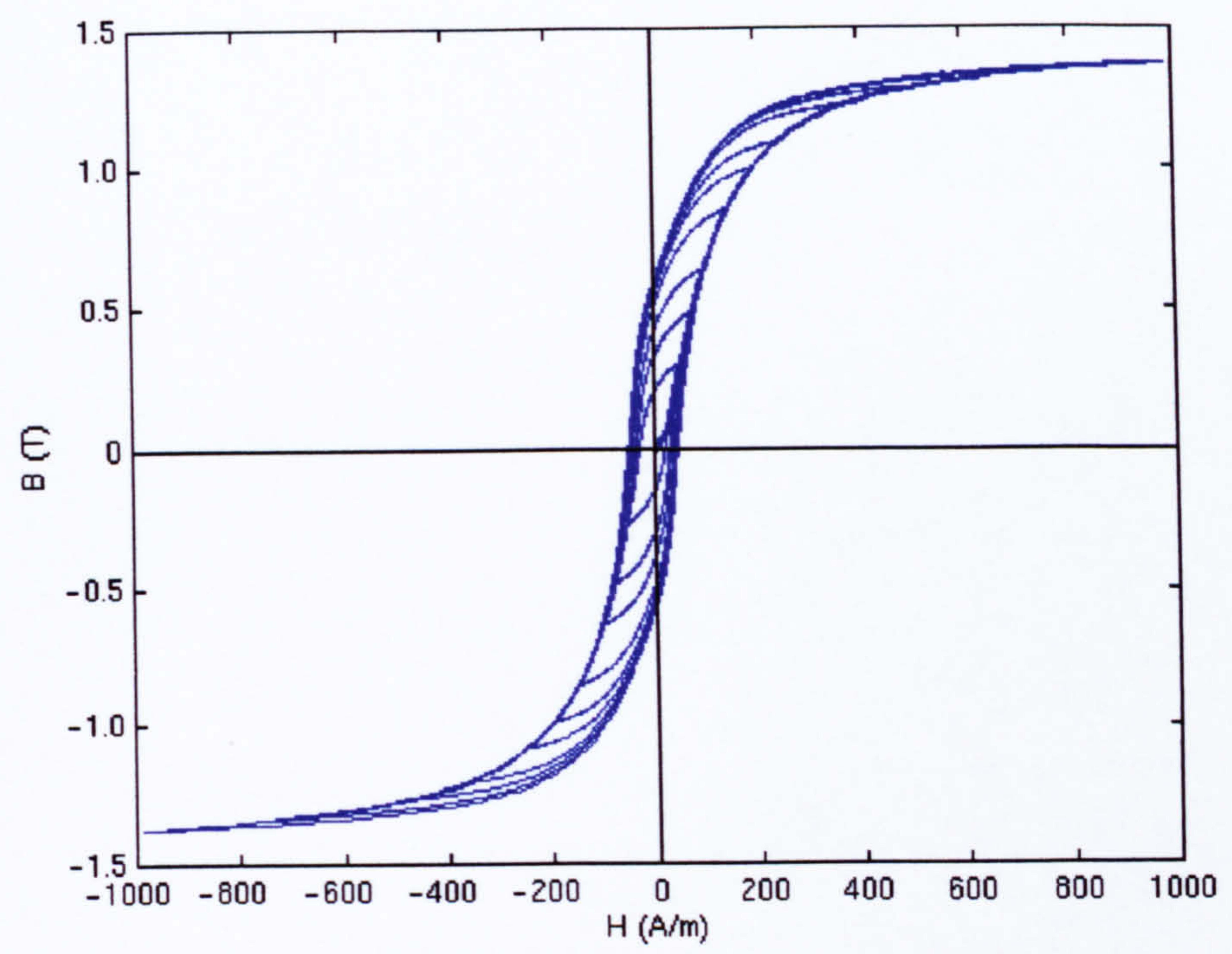

Figure 4.17a. Measured major hysteresis loops for the rotor material for fields up to $1000 \mathrm{~A} / \mathrm{m}$

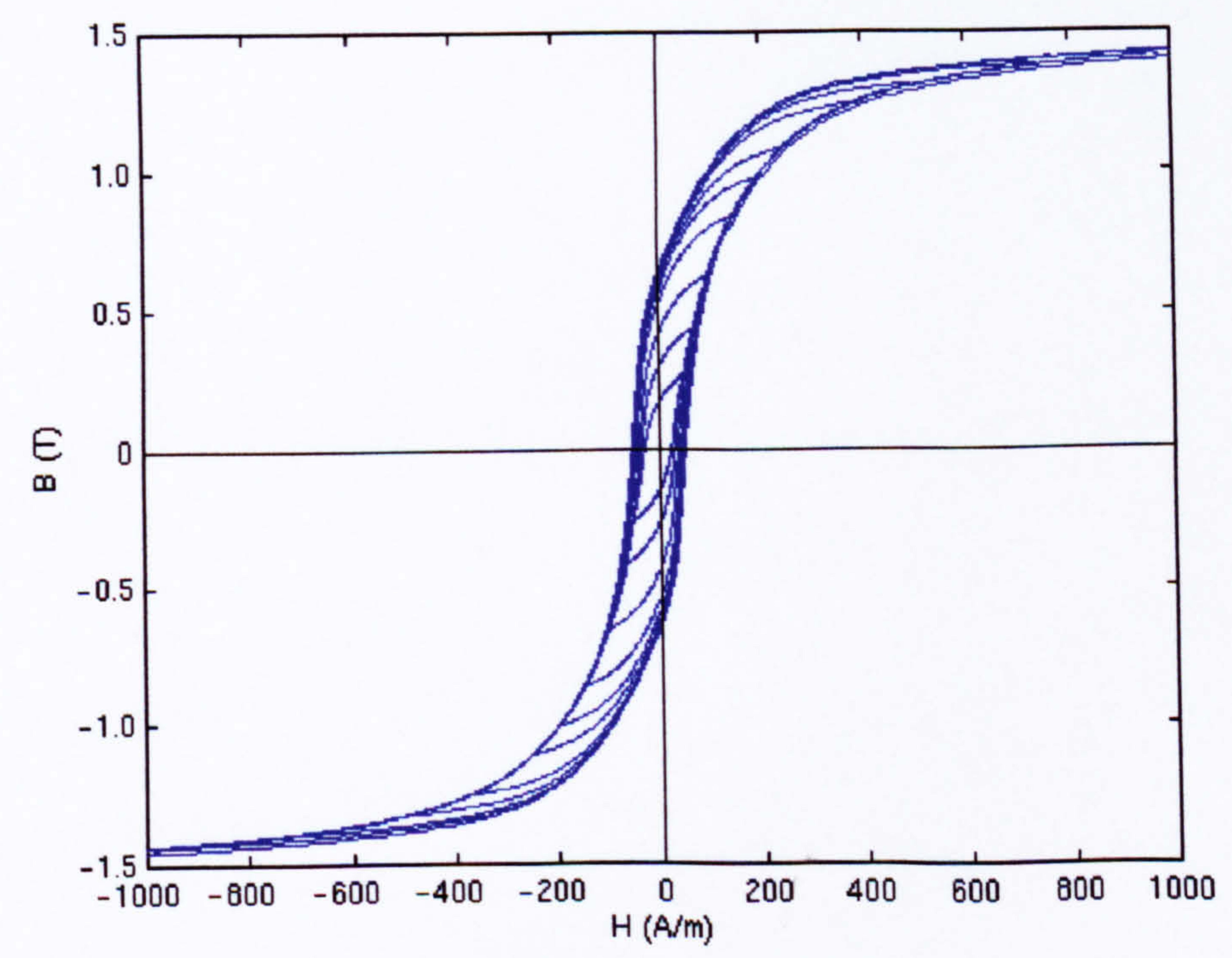

Figure 4.17b. Measured major hysteresis loops for the stator material for fields up to $1000 \mathrm{~A} / \mathrm{m}$ 


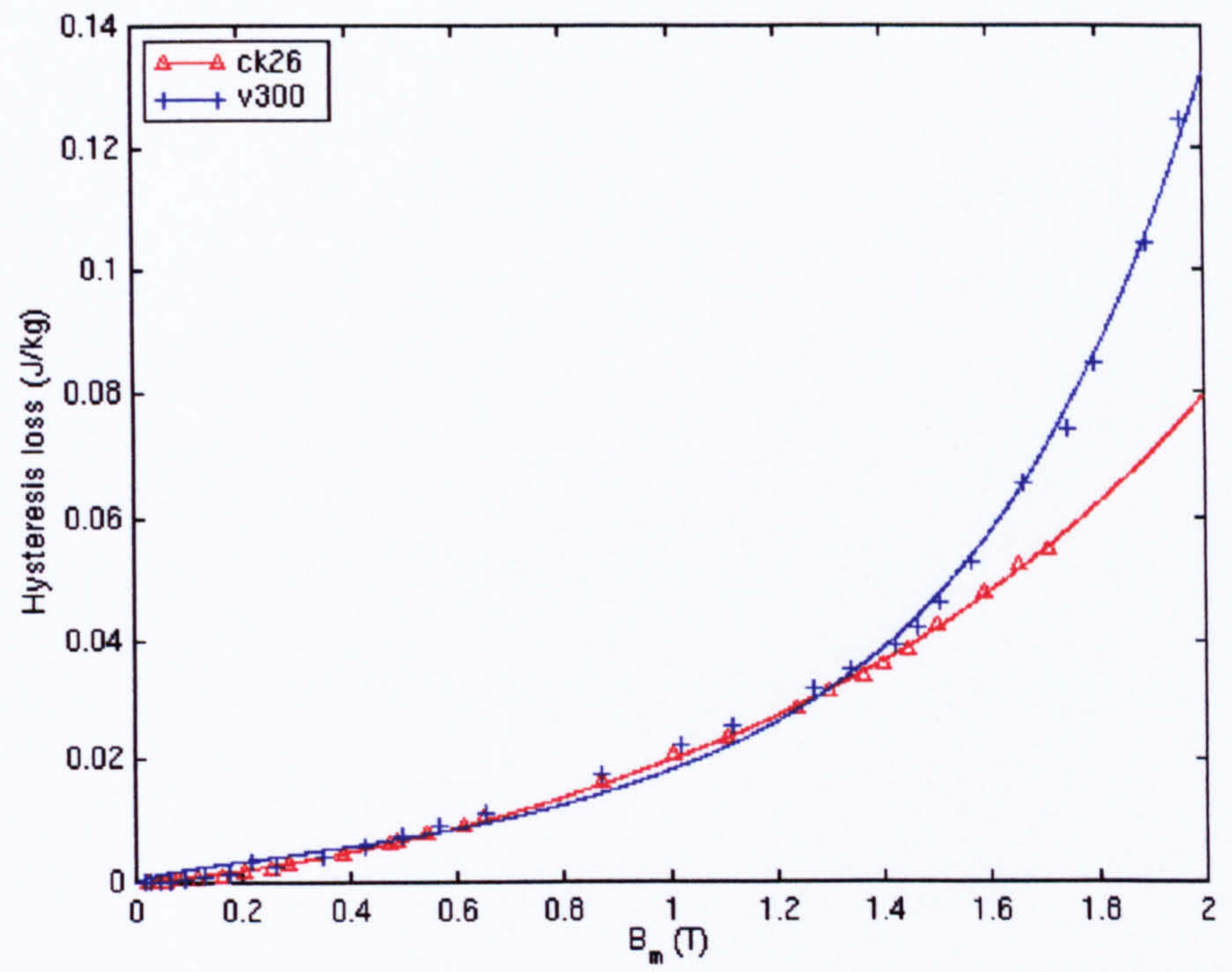

Figure 4.18. Measured hysteresis loss and curve-fit for the rotor and stator materials. 


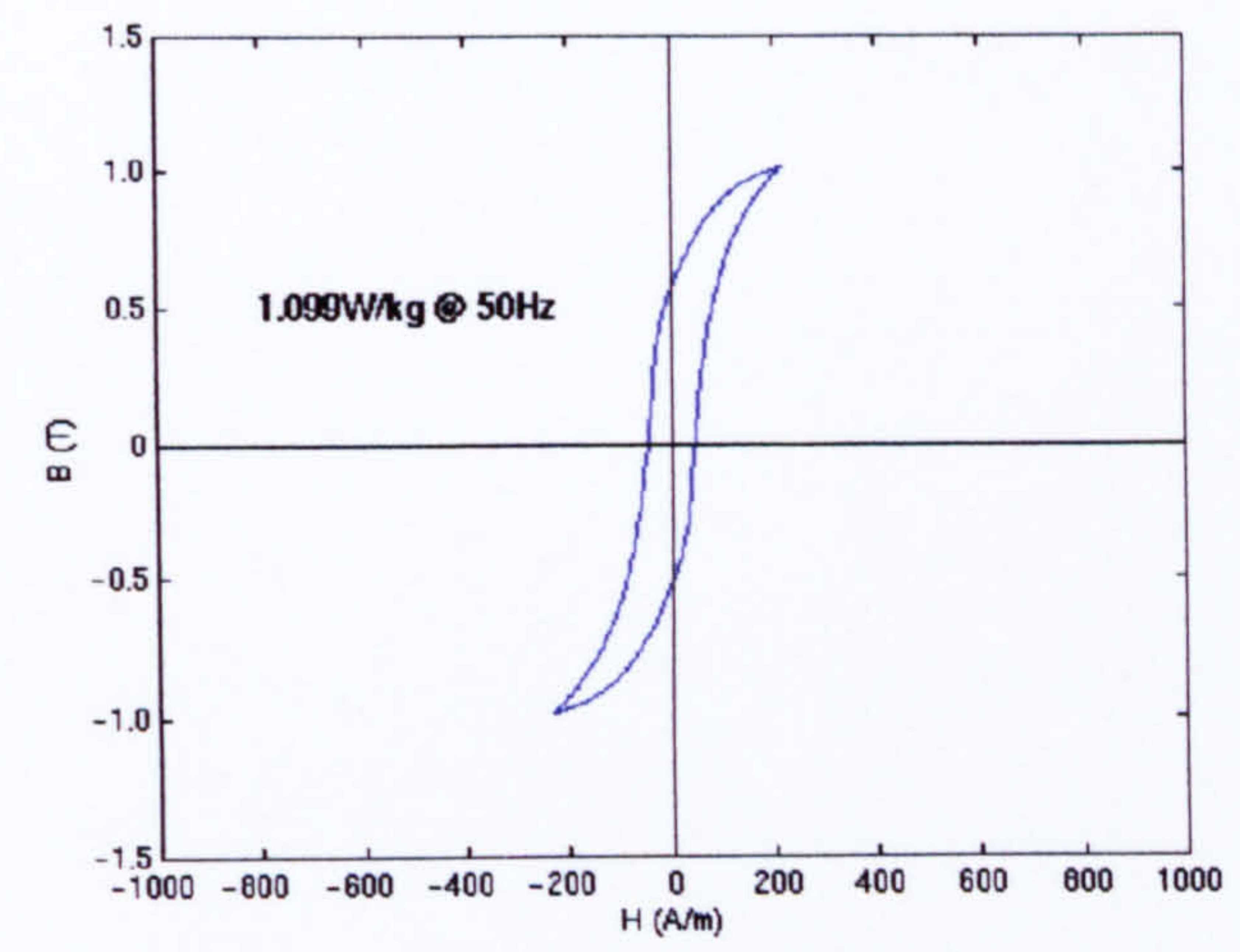

Figure 4.19a. Hysteresis loop for flux traversal 2T about zero Tesla (V300).

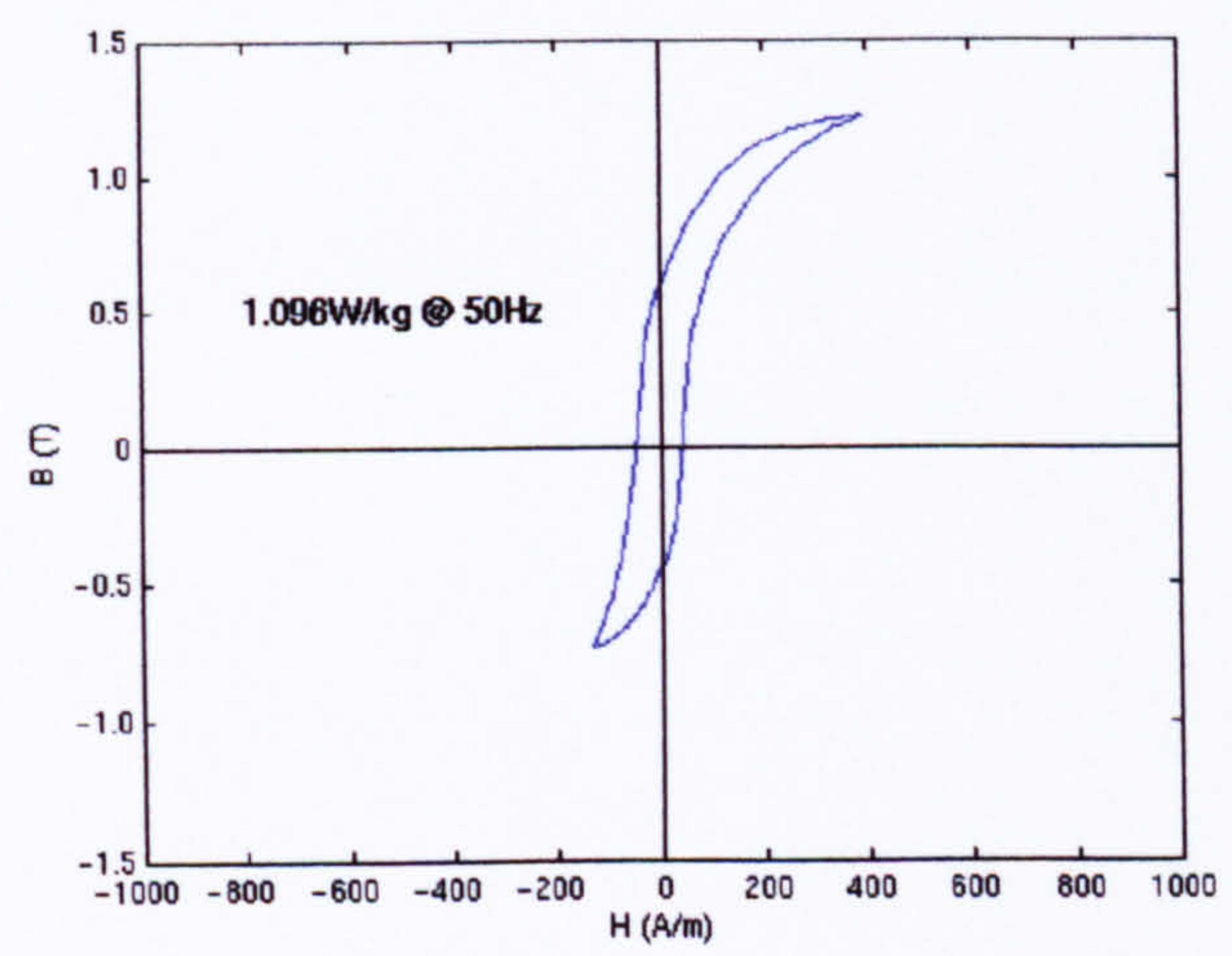

Figure 4.19b. Hysteresis loop for 2T traversal about 0.25T (V300).

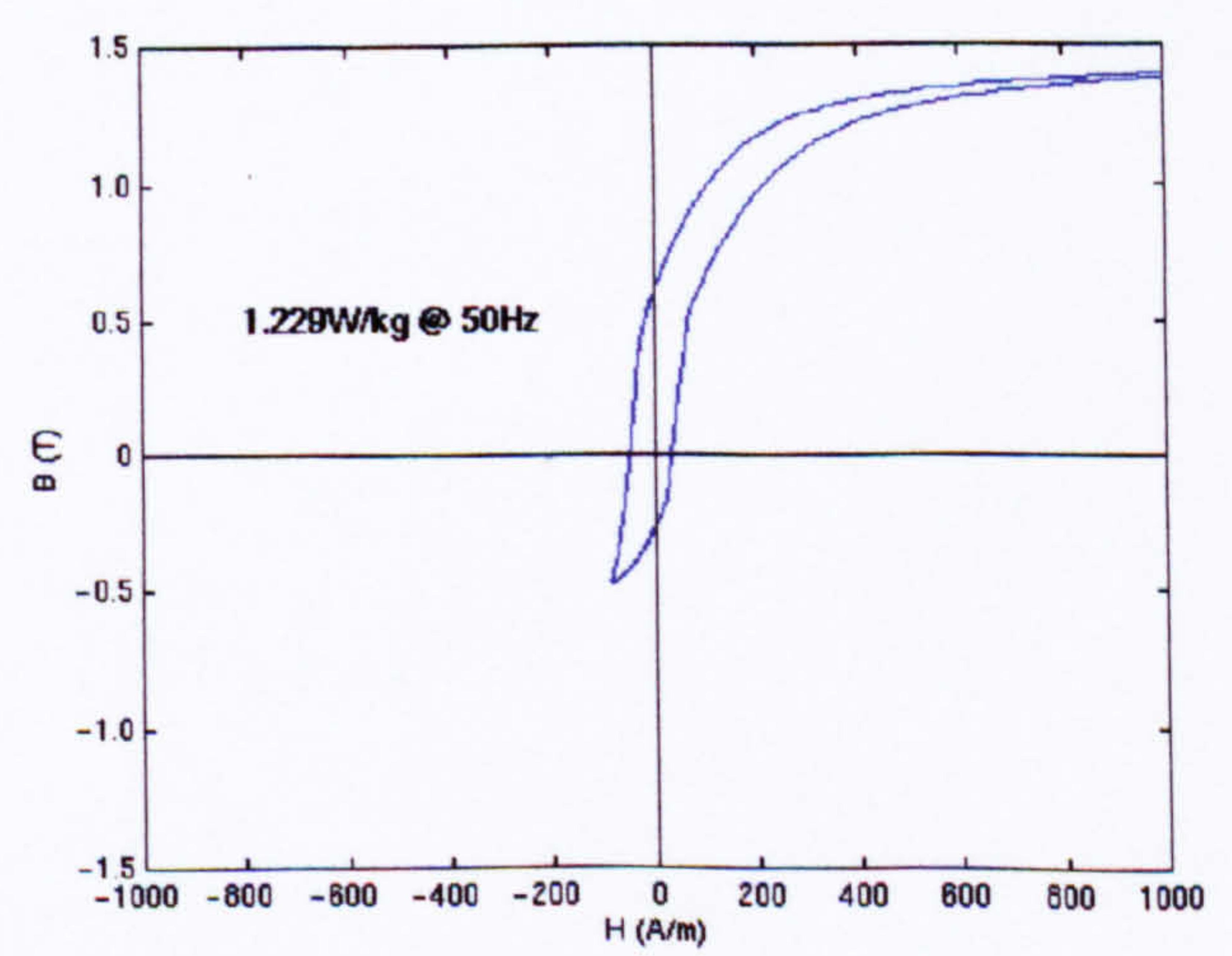

Figure 4.19c. Hysteresis loop for 2T traversal about 0.5T (V300). 


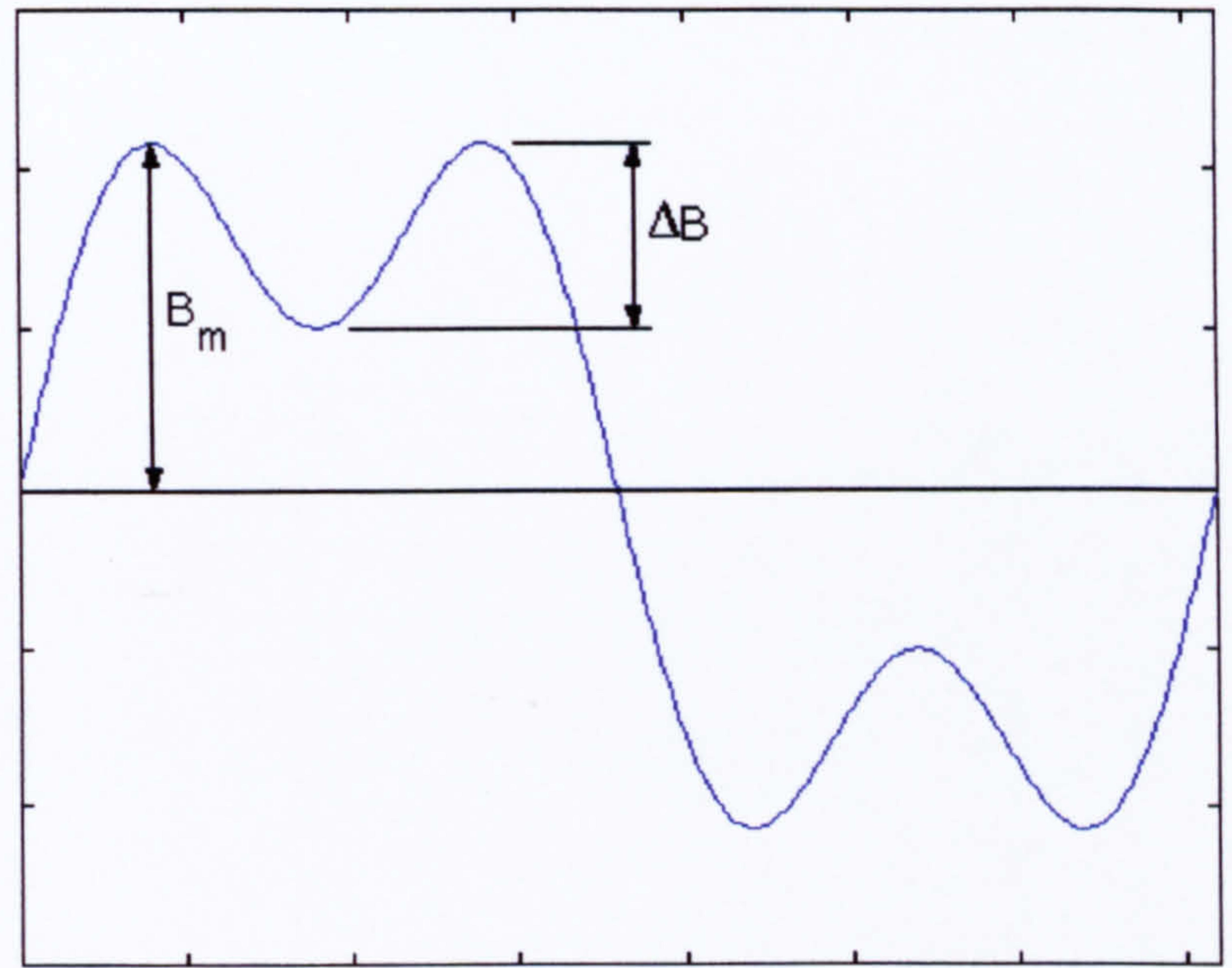

Figure 4.20. Induction curve including $3^{\text {rd }}$ harmonic distortion and subsequent minor loop of magnitude $\Delta B$.

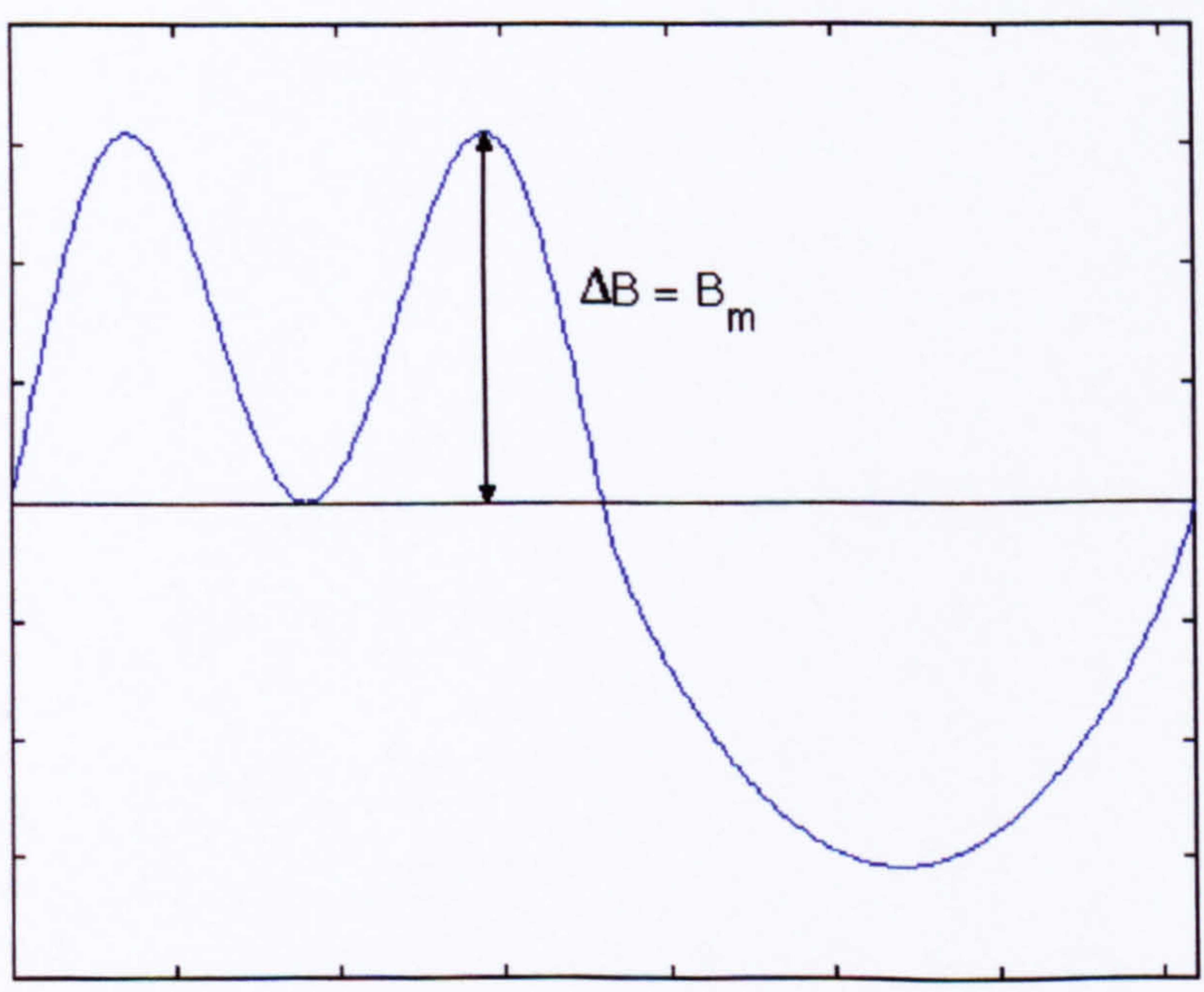

Figure 4.21. Induction curve including a single minor loop of magnitude $\Delta B=B_{m}$ 


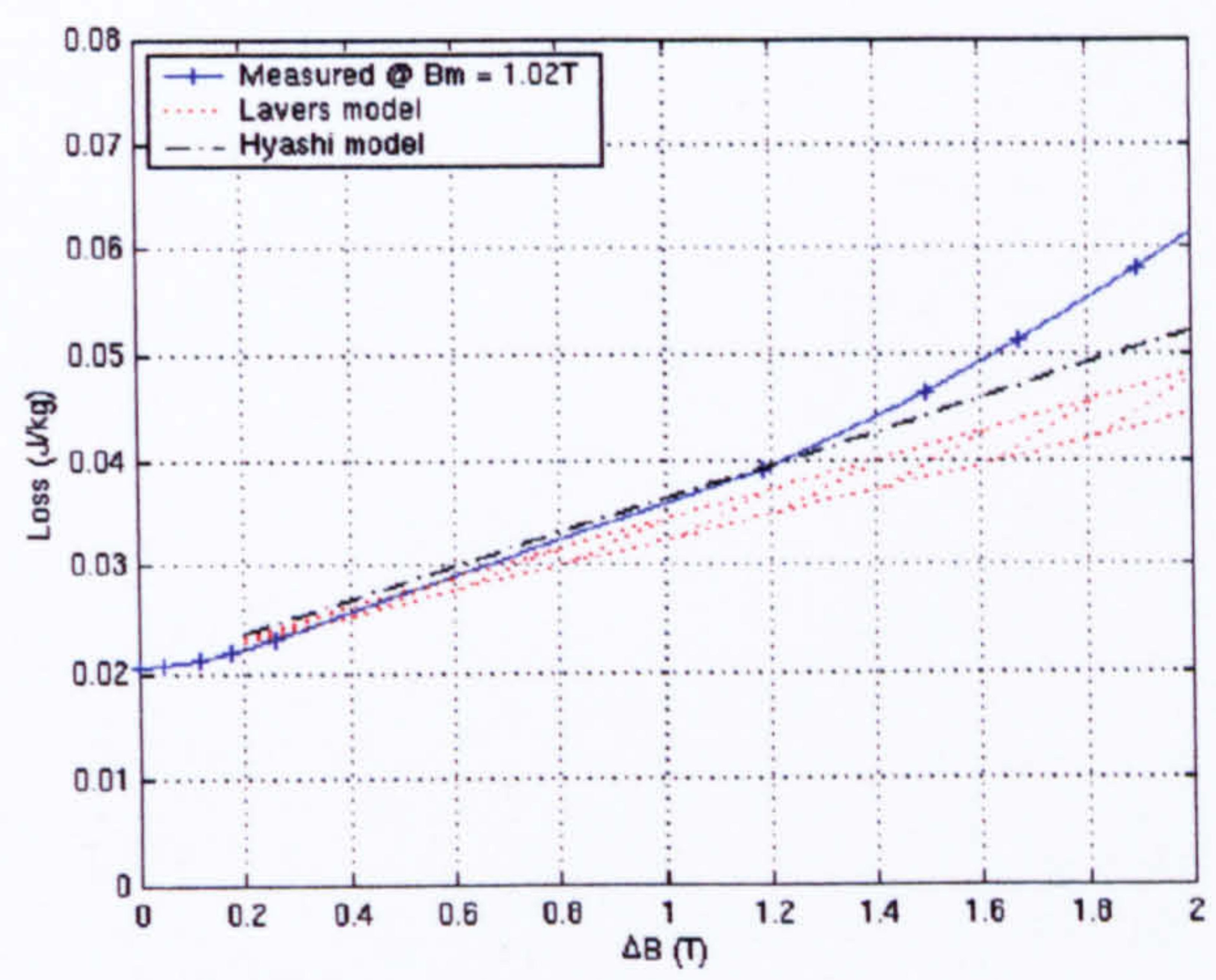

Figure 4.22a Hysteresis loss for major loops of $1 \mathrm{~T}$ with minor loops of magnitude $\Delta \mathrm{B}$.

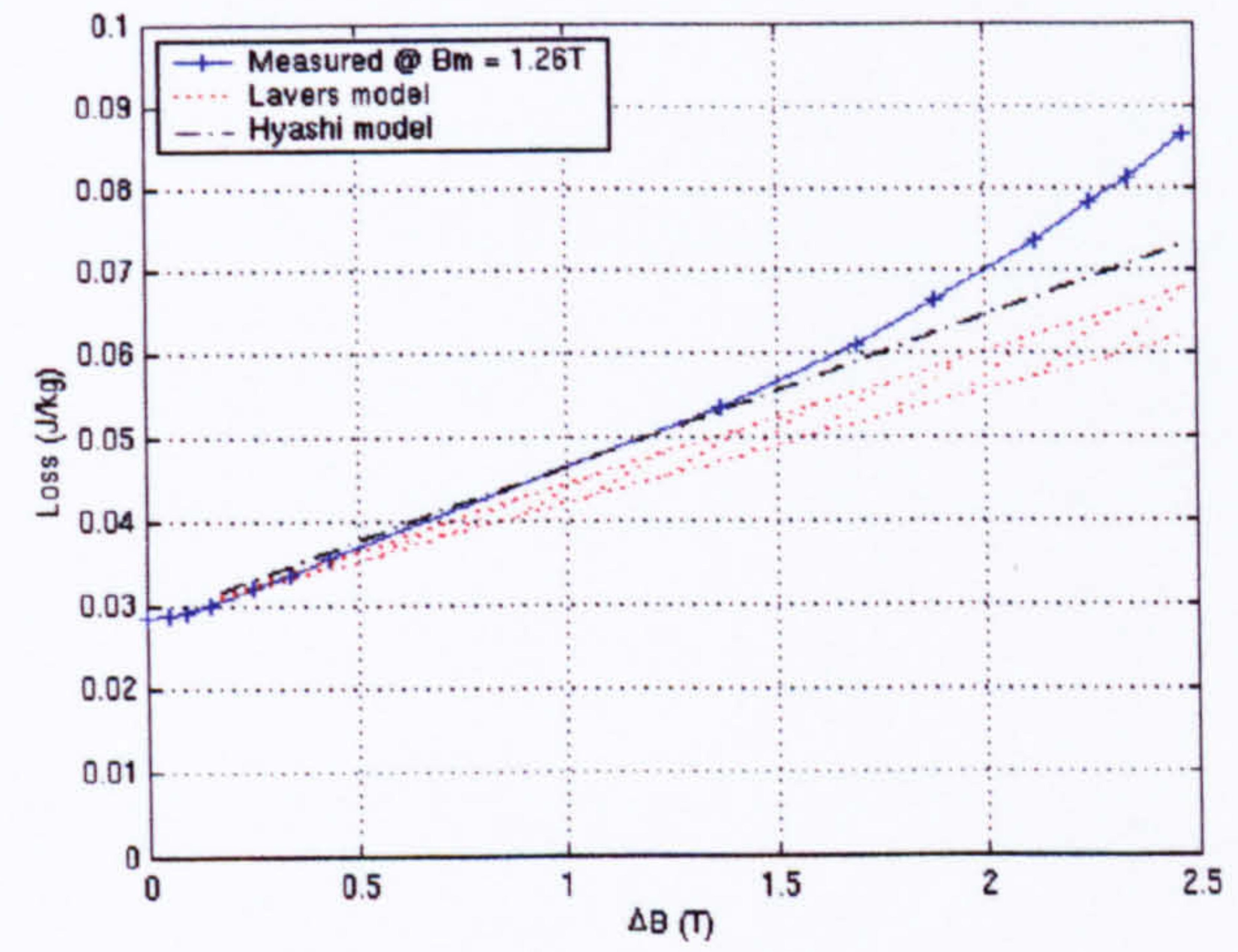

Figure 4.22b Hysteresis loss for major loops of $1.25 \mathrm{~T}$ with minor loops of magnitude $\Delta \mathrm{B}$.

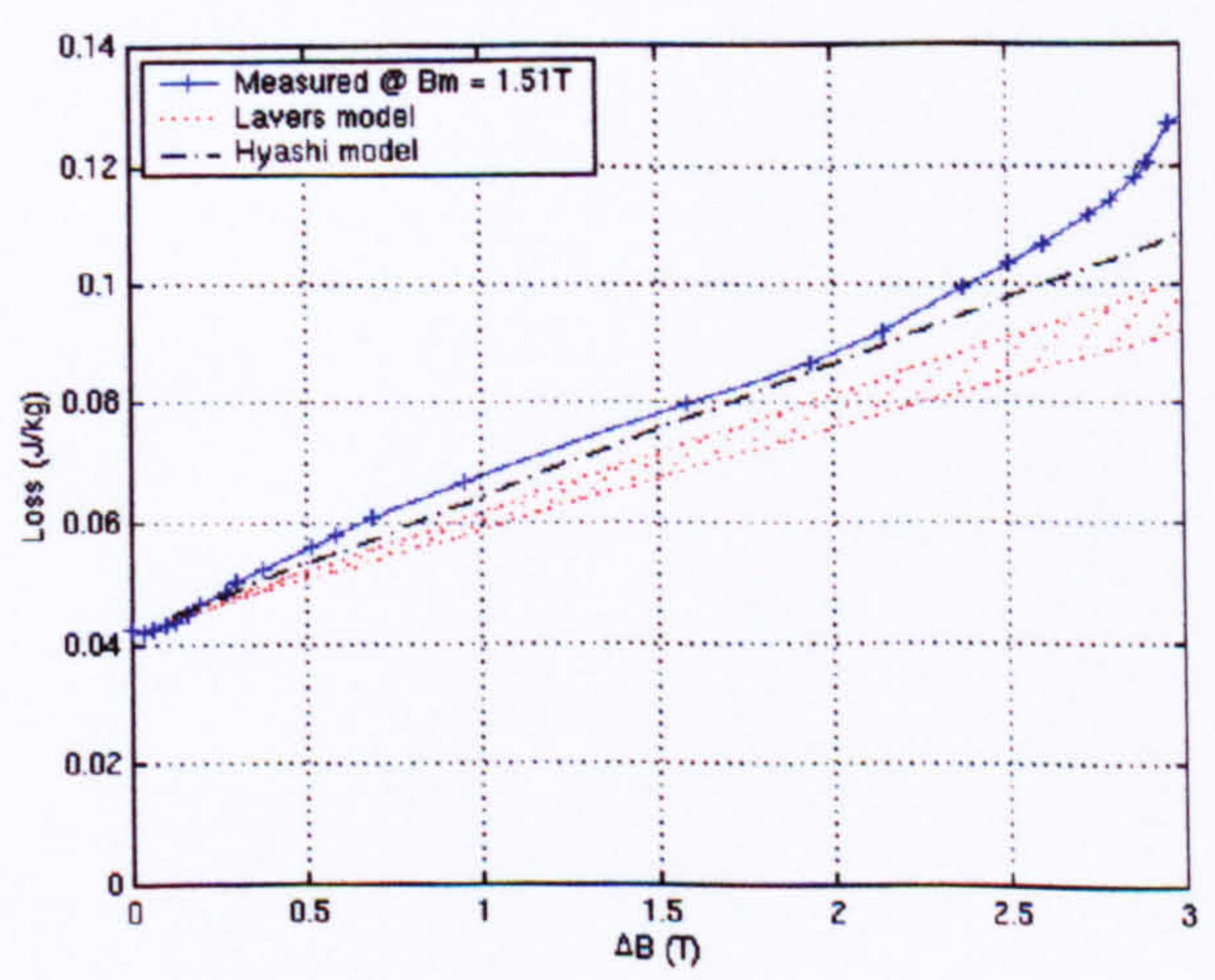

Figure 4.22c Hysteresis loss for major loops of $1.50 \mathrm{~T}$ with minor loops of magnitude $\Delta \mathrm{B}$. 


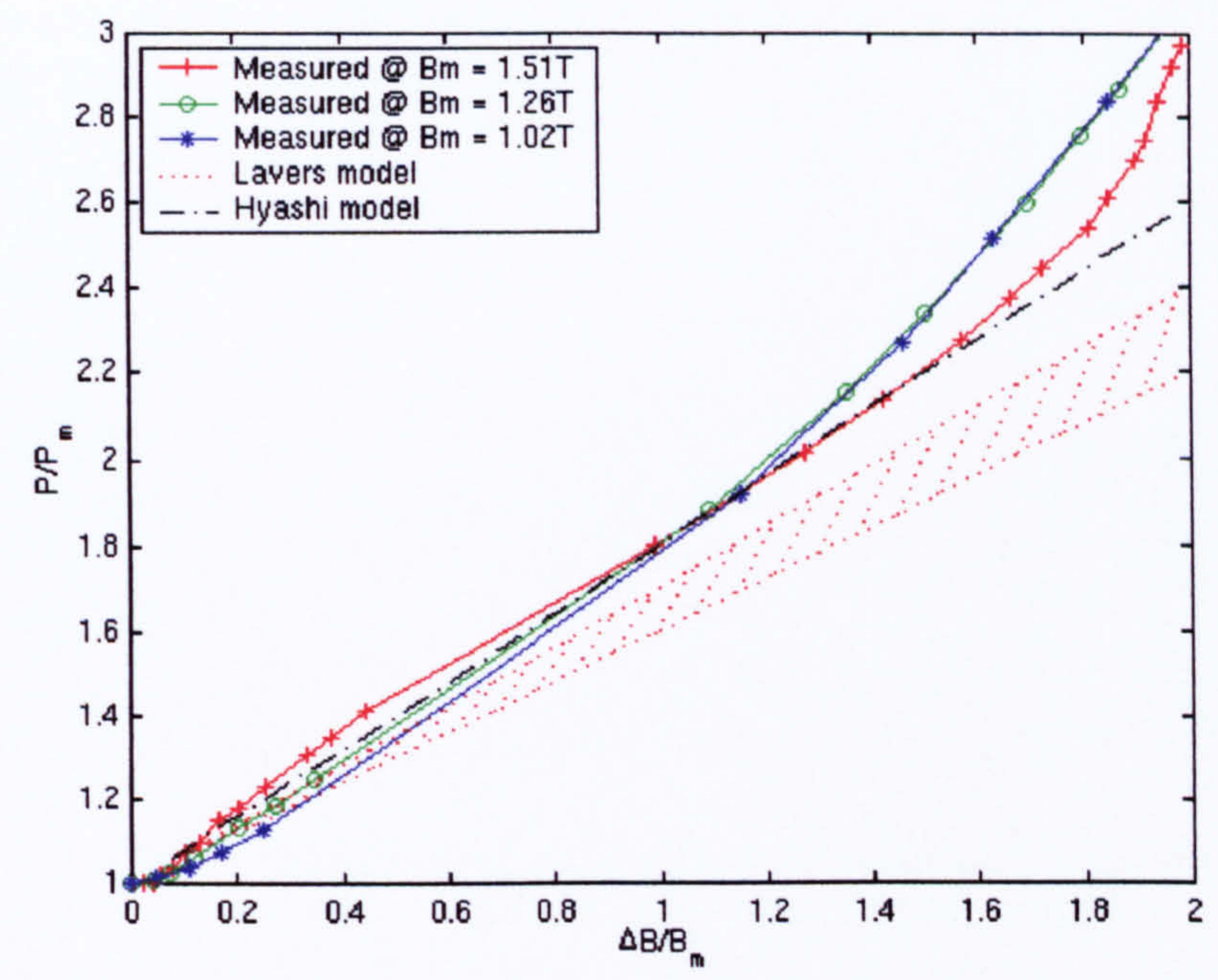

Figure 4.23. Normalised hysteresis loss for a flux density waveform with a peak induction of 1.51, 1.26 and 1.02T incorporating minor loops as in figure 4.20. Also shown are the results of the analysis presented by Lavers using material constants specified by Lavers [4.20] and Hyashi respectively [4.1].

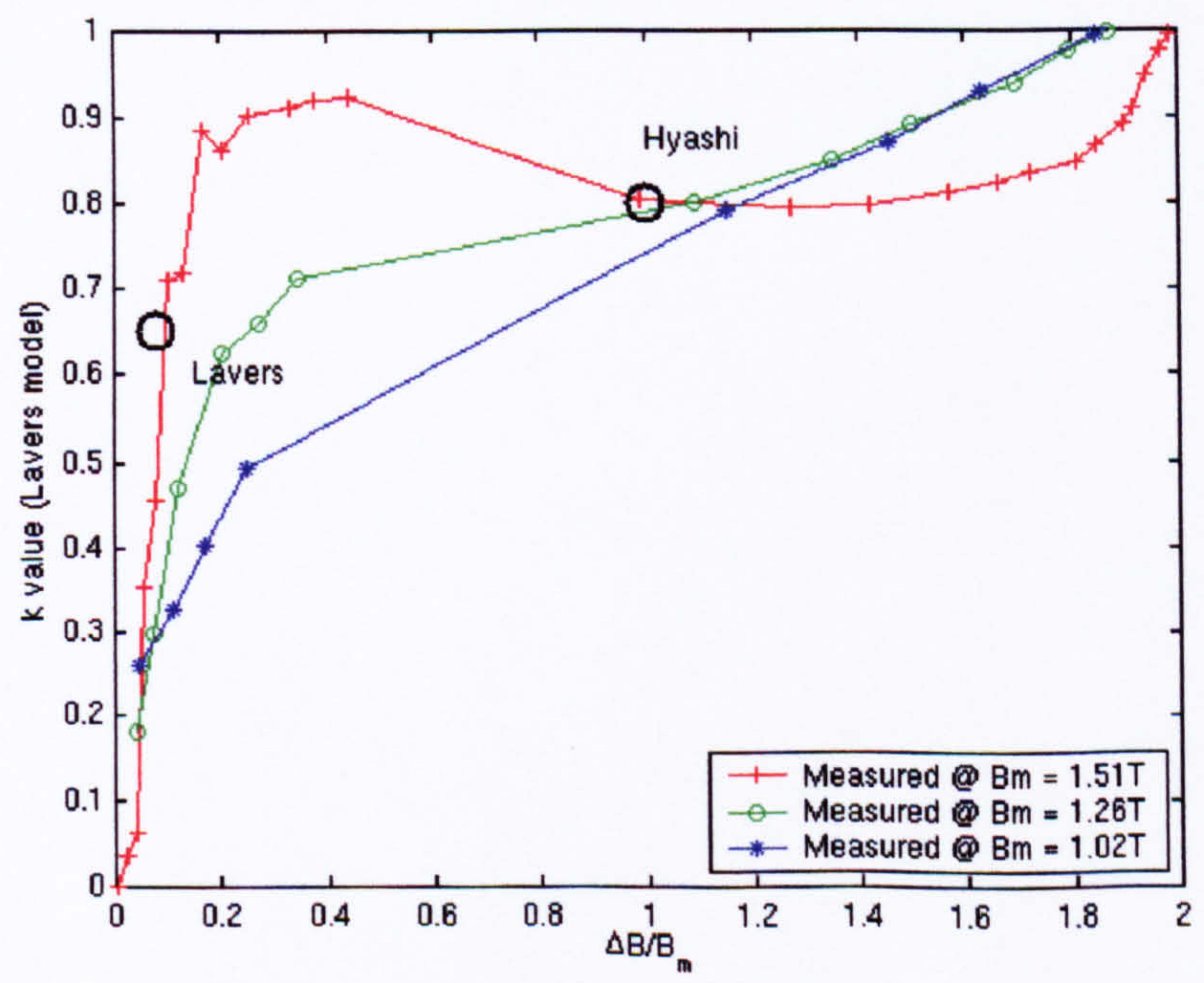

Figure 4.24. Variation of the constant $k$ applied in the model described by Lavers et al. as a function of the magnitude of the flux reversal and peak flux. Also shown are the specific points measured by Lavers $\left(\triangle B / B_{m}=0.08\right)$ and Hyashi $\left(\Delta B / B_{m}=1.0\right)$. 


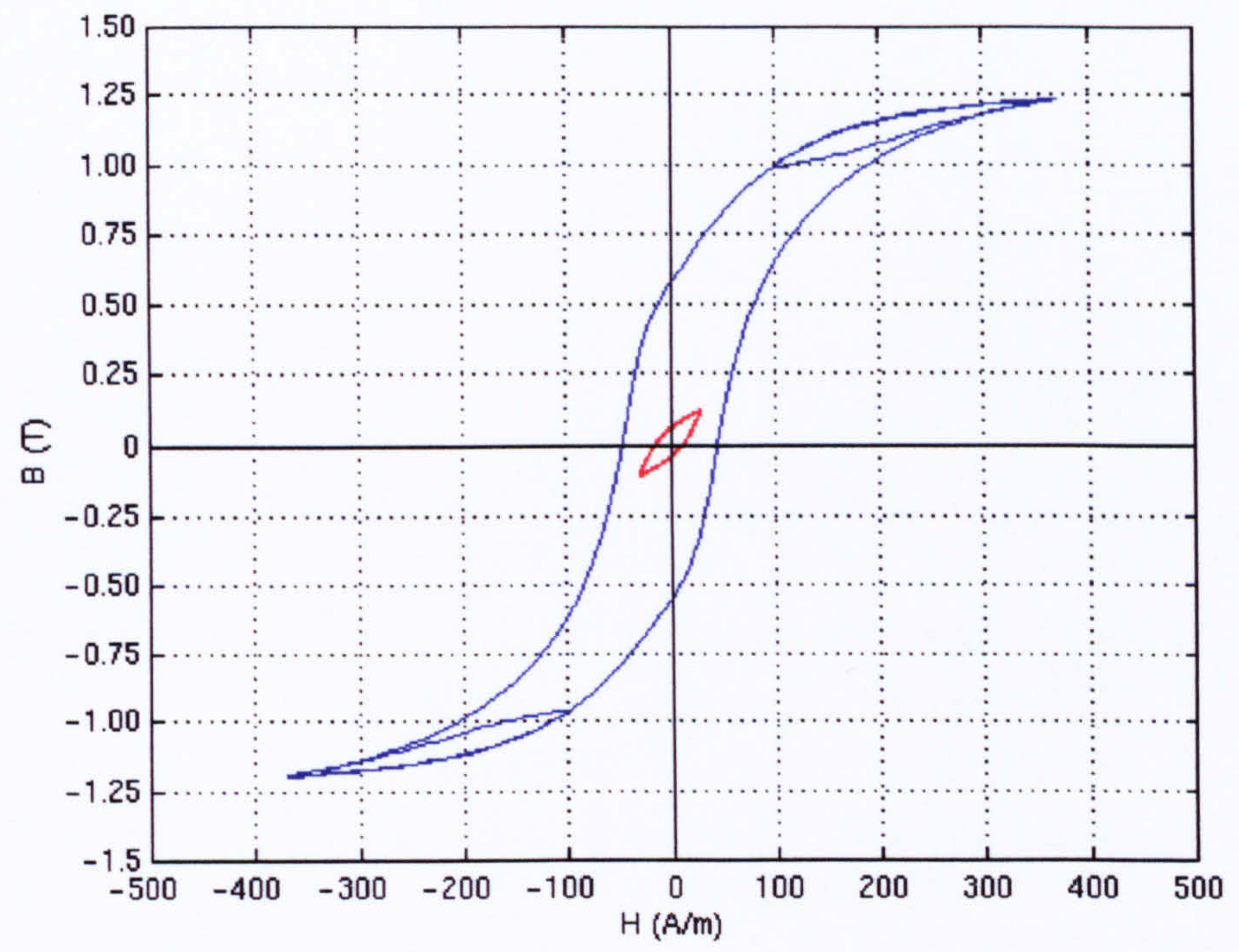

Figure 4.25. Measured loop of peak flux density $1.25 \mathrm{~T}$ incorporating minor loop of magnitude $0.25 \mathrm{~T}$ with equivalent major loop about the origin.

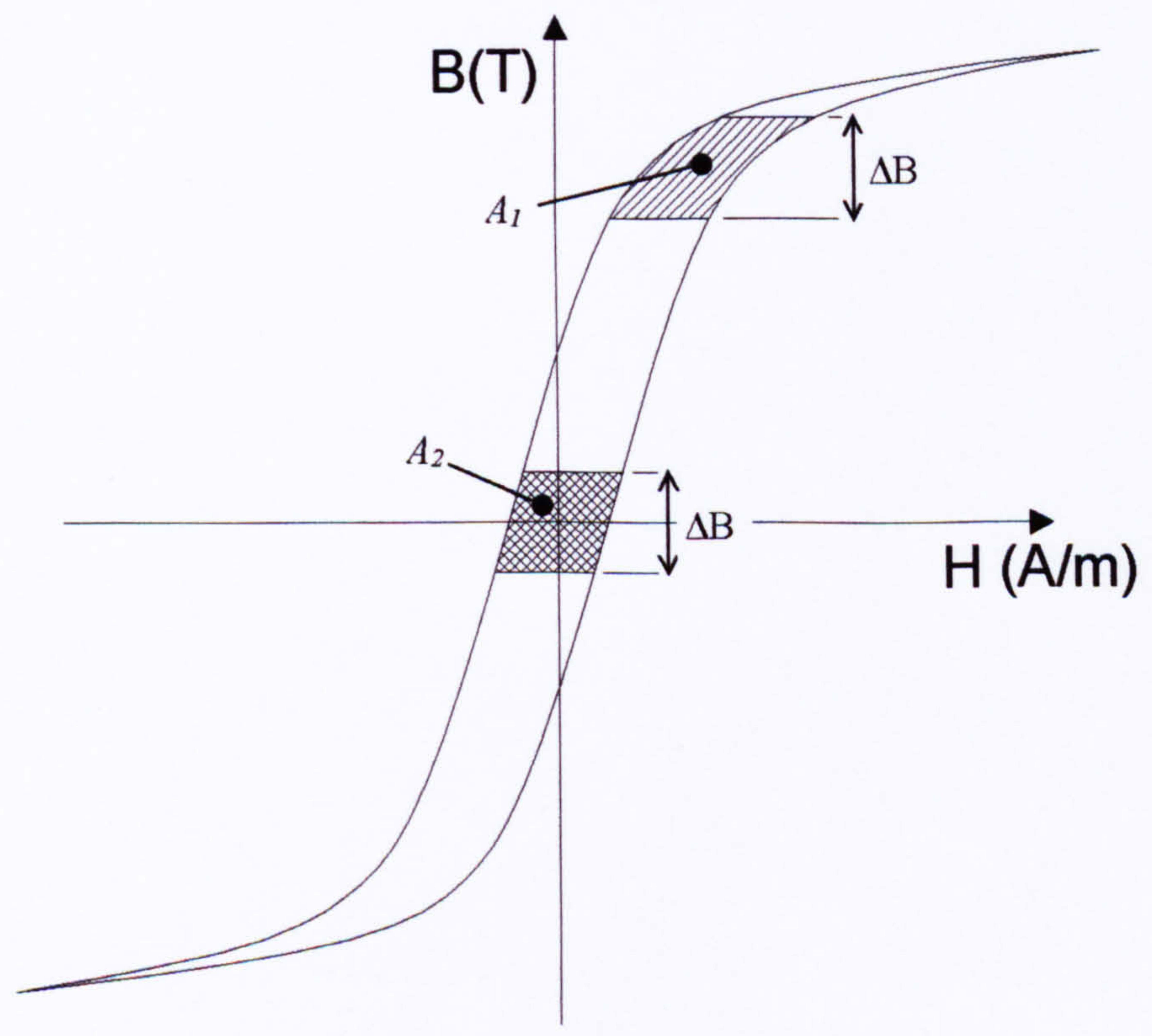

Figure 4.26. Schematic hysteresis loop showing the areas $A_{1}$ and $A_{2}$. 


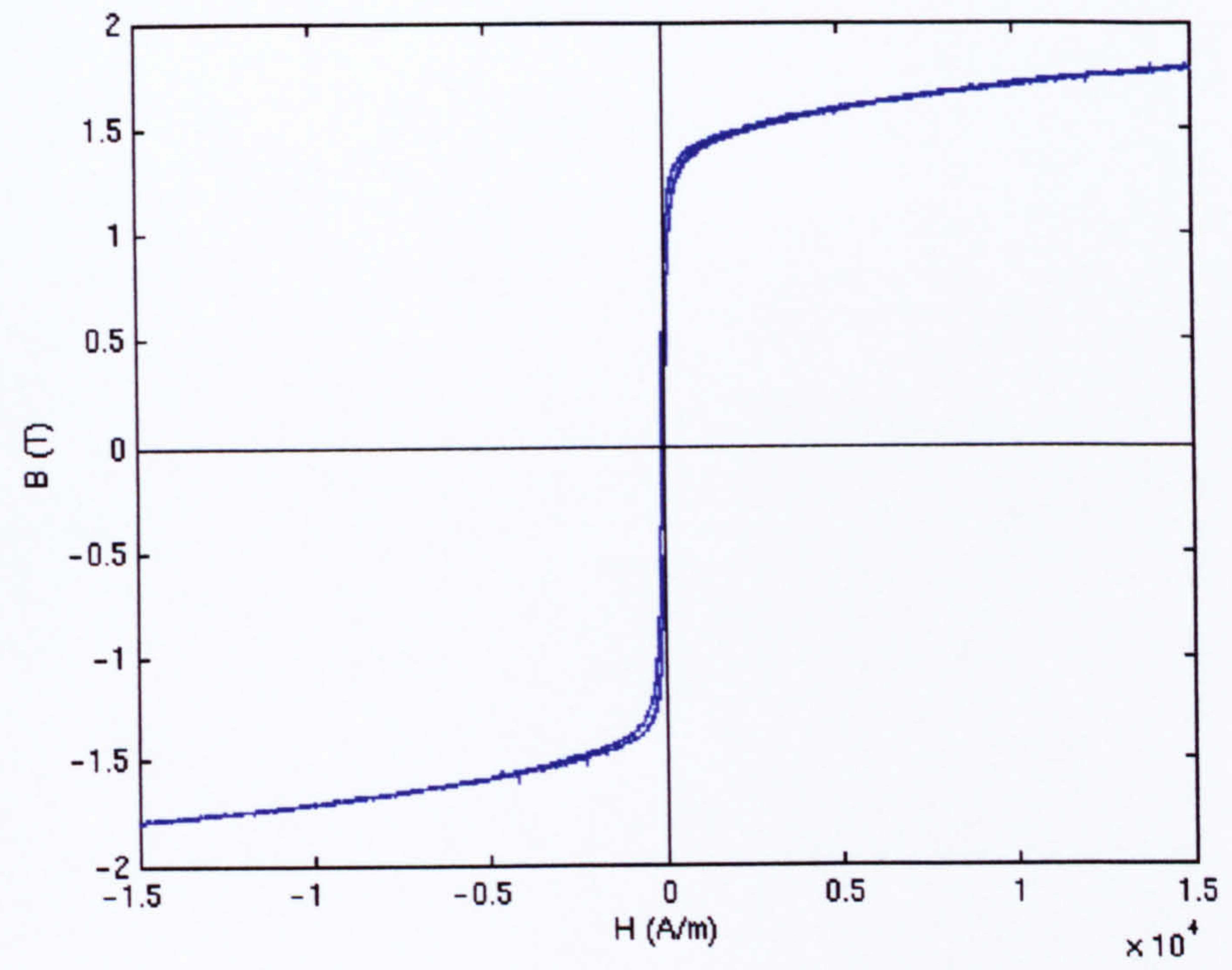

Figure 4.27. Hysteresis loop for the rotor material exposed to a magnetising field of $15000 \mathrm{~A} / \mathrm{m}$.

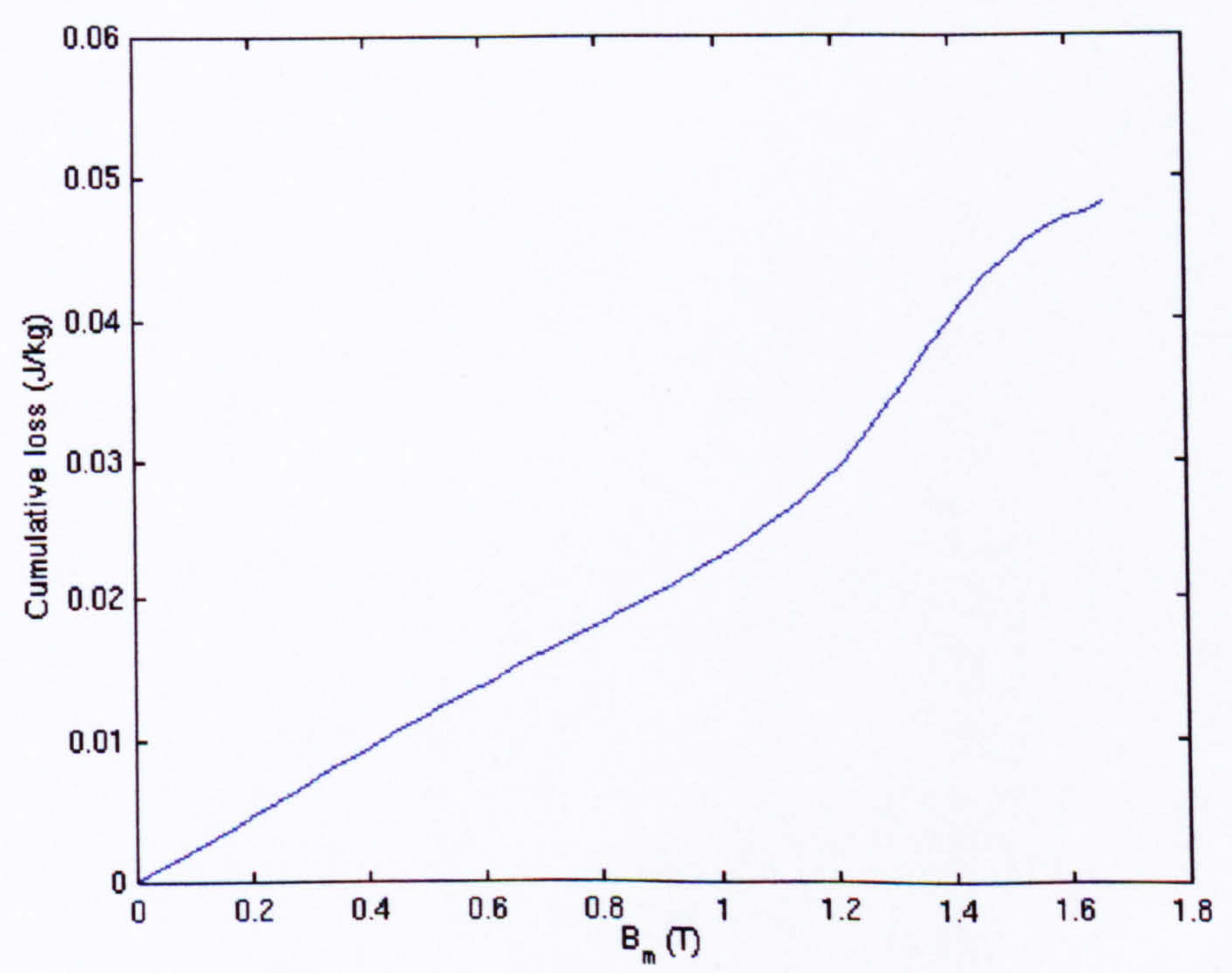

Figure 4.28 Cumulative loss function for the rotor material. 


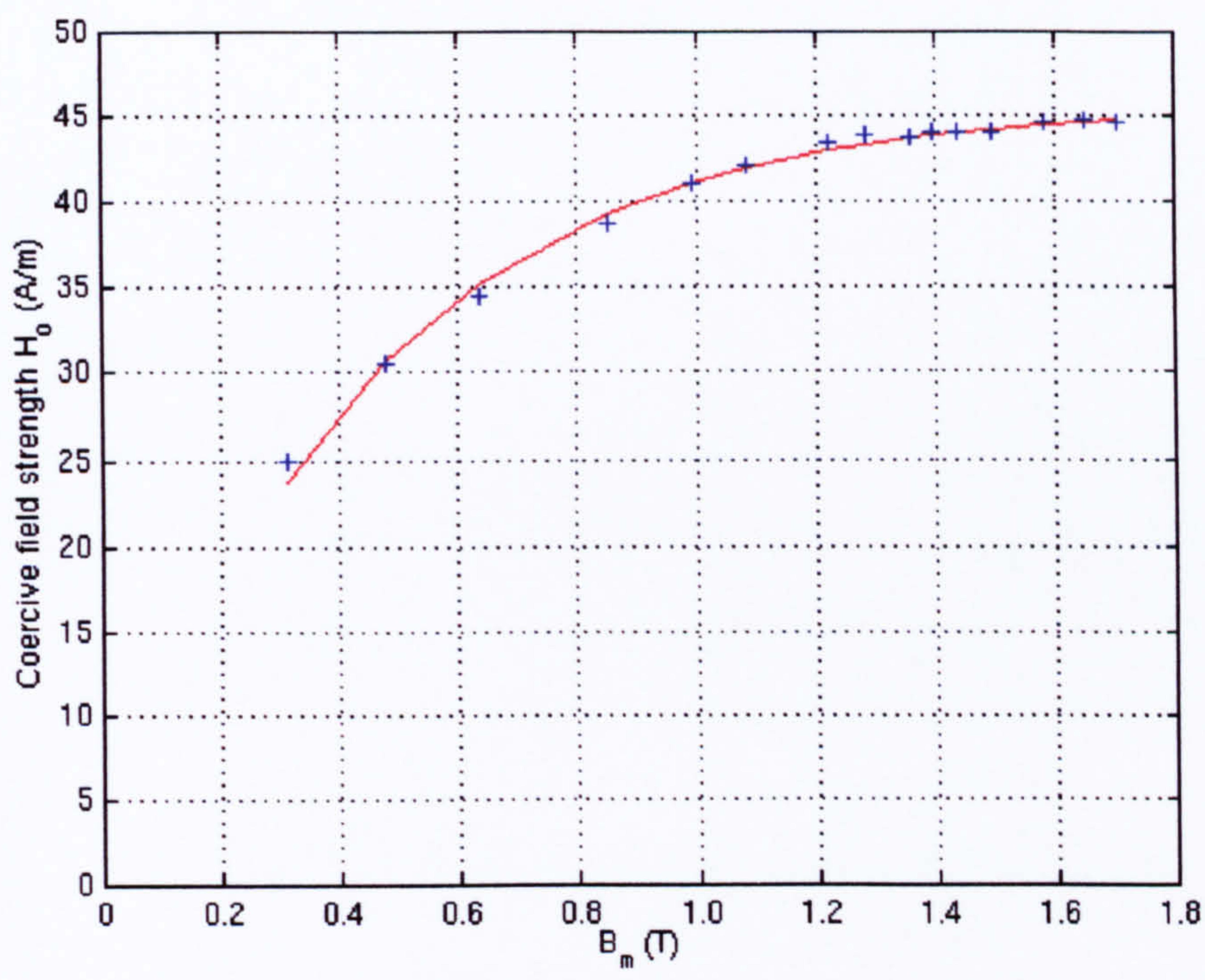

Figure 4.29 Coercive field strength of the rotor material as a function of magnetising flux density with applied curve fit. 

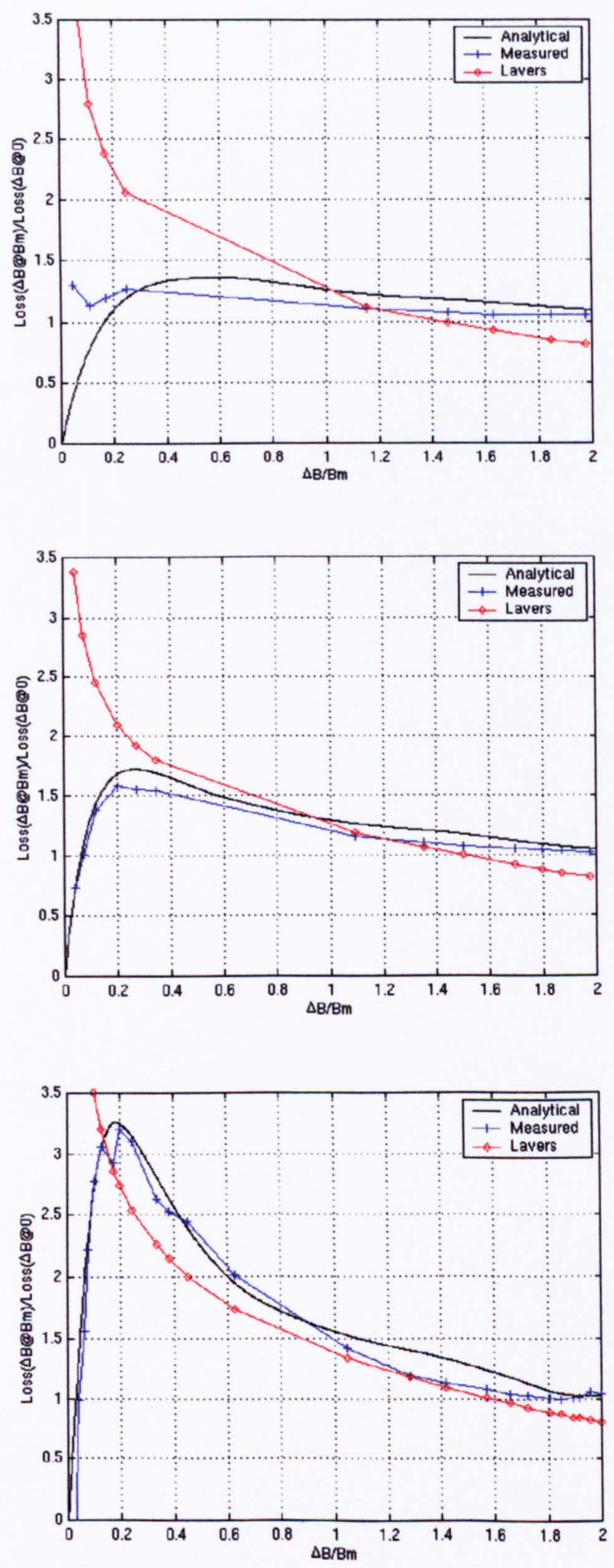

Figure 4.30 Calculated minor loop correction factor for the rotor material with starting flux densities of 1.0, 1.25 and 1.50T respectively. Also shown are the corresponding measured values, and those obtained using the model presented by Lavers with a coefficient of 0.8 . 


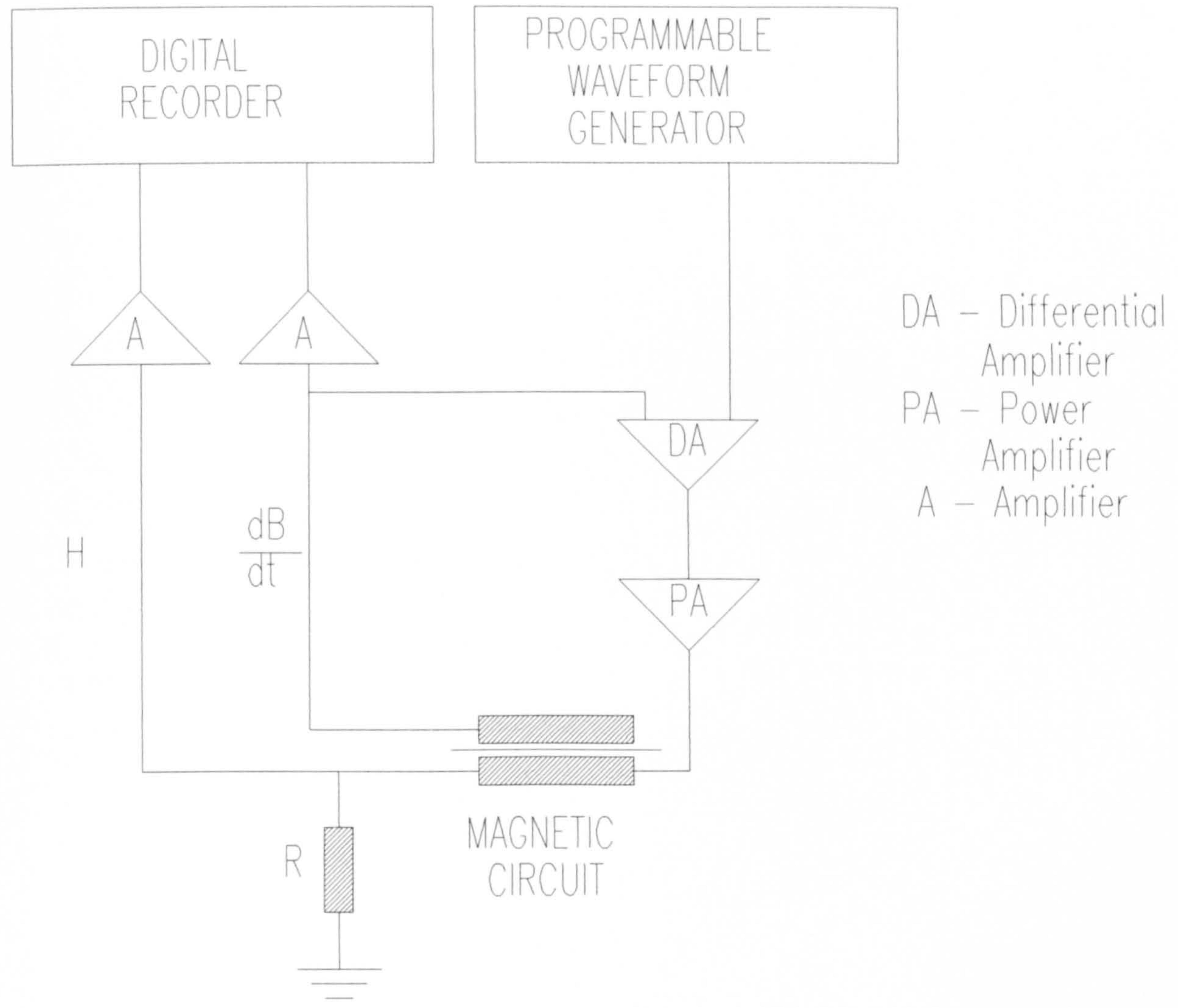

Figure 4.31. Schematic of iron loss measurement system for symmetrical bipolar waveforms. [4.13] 


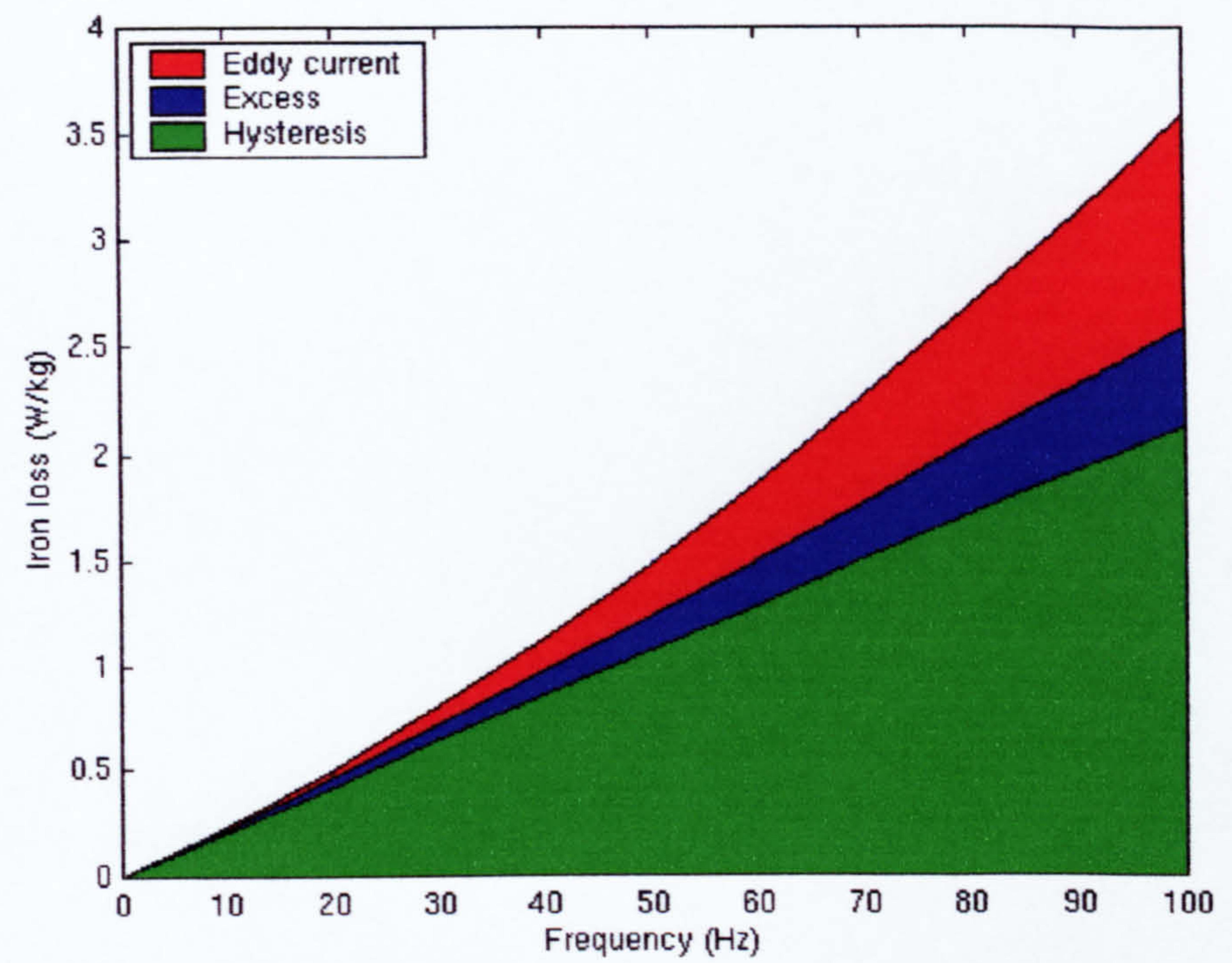

Figure 4.32. Loss components measured in the rotor material for (1T peak) sinusoidal flux density variation.

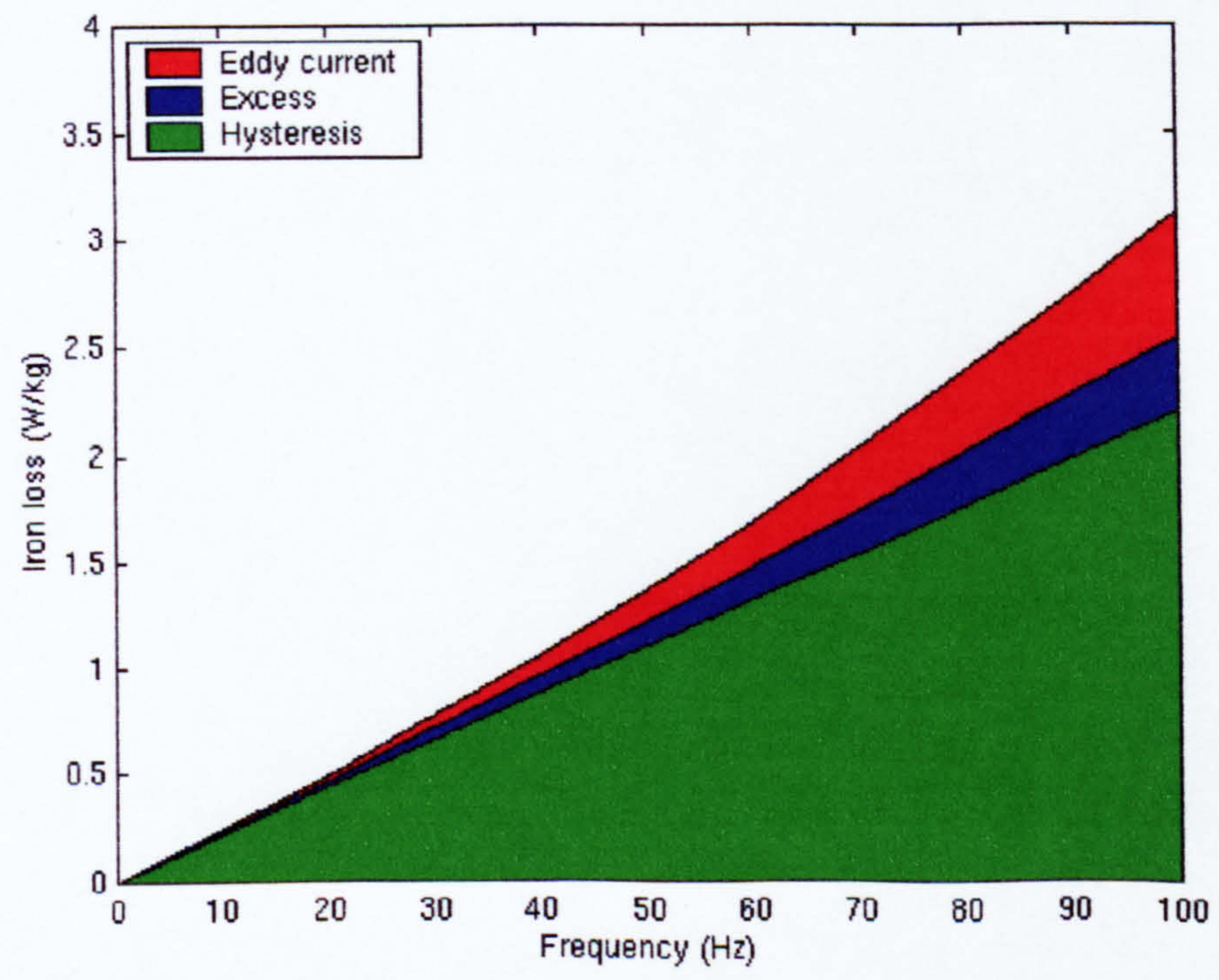

Figure 4.33. Loss components measured in the stator material for (1T peak) sinusoidal flux density variation. 


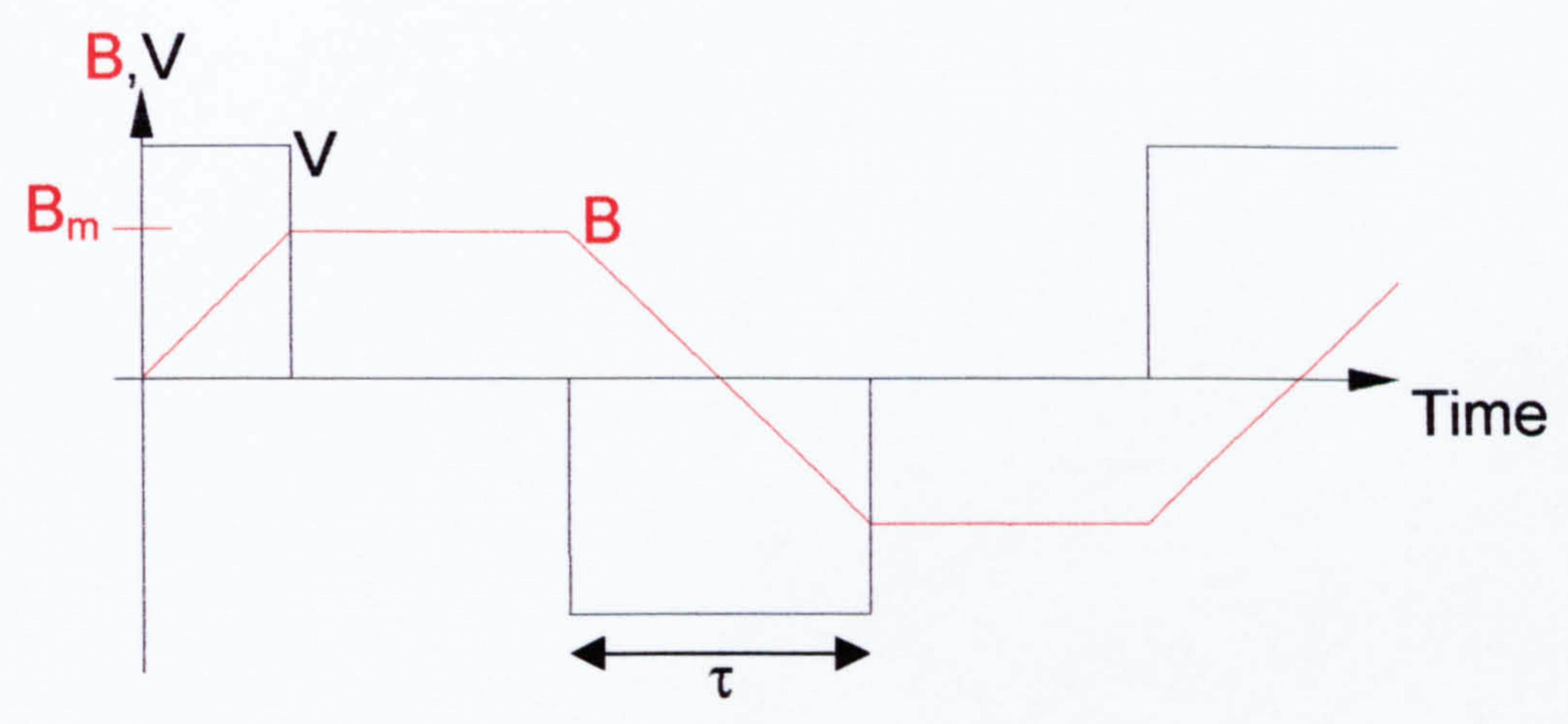

Figure 4.34. Schematic representation of square wave voltage variation and corresponding trapezoidal induction.

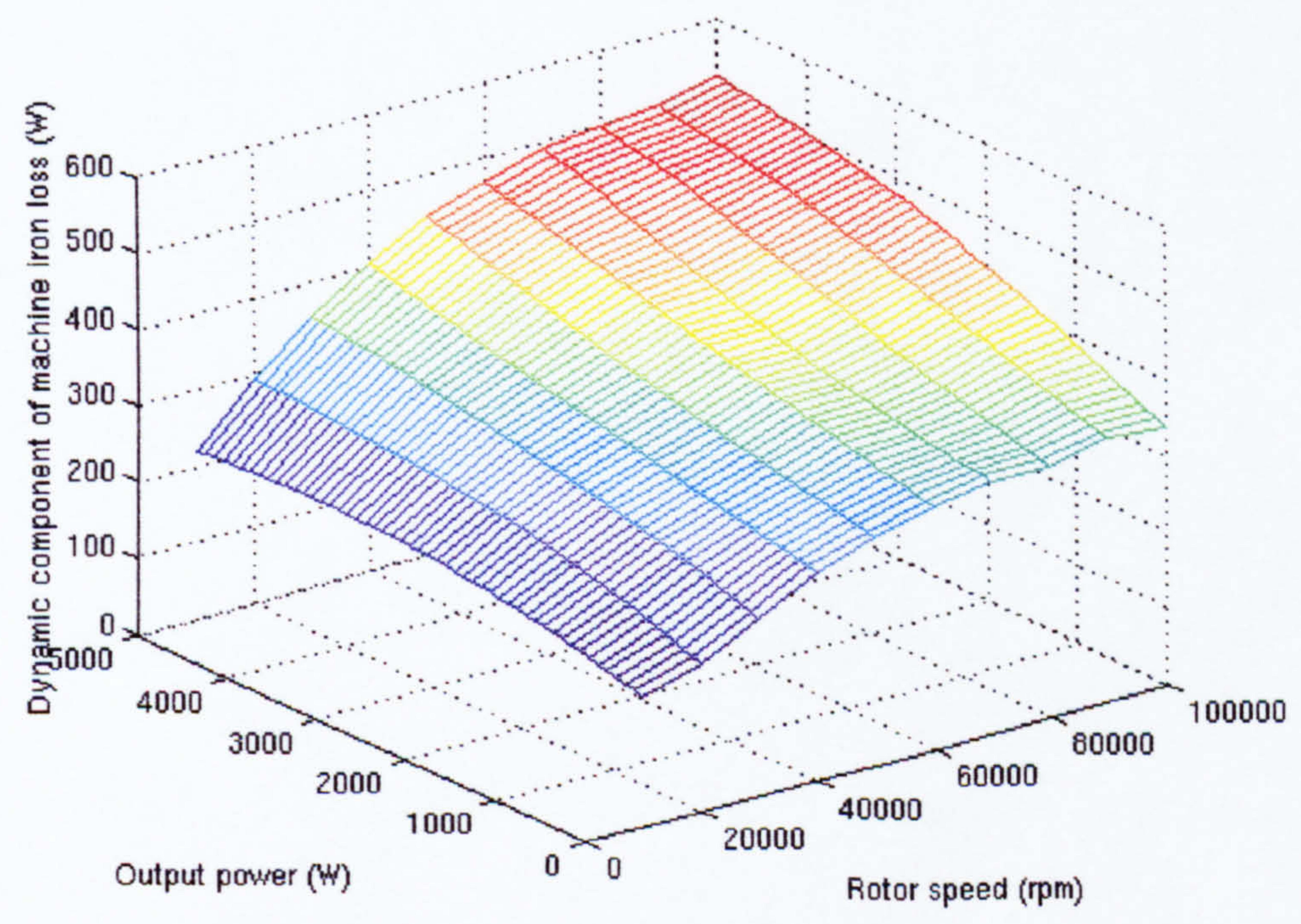

Figure 4.35 Dynamic components of iron loss as a function of rotor speed and output power for the switching angles established in chapter 3. 

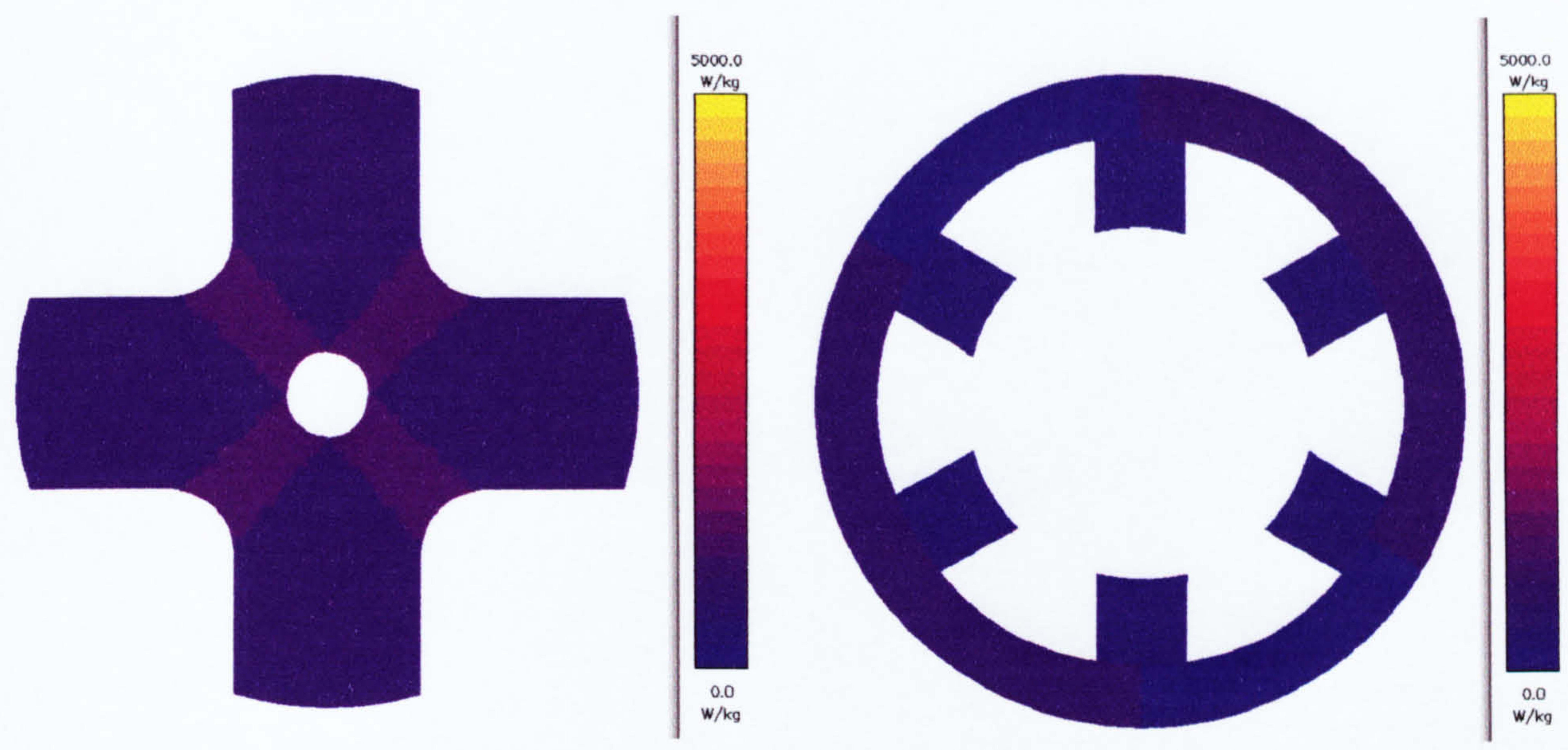

Figure 4.36a. Contours of total iron loss for the machine with winding connection A
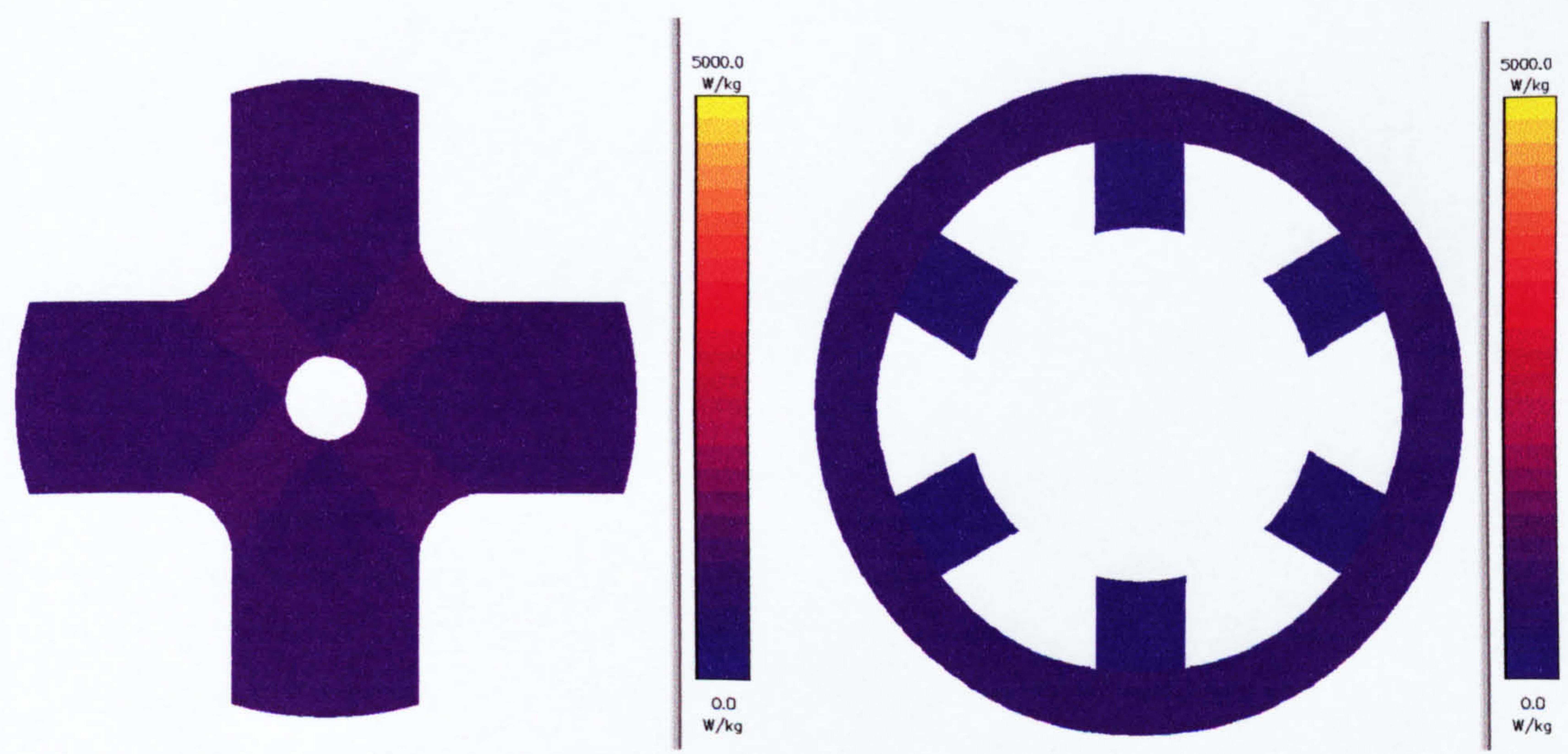

Figure 4.36b. Contours of total iron loss for the machine with winding connection $\mathrm{B}$

Figure 4.36. Iron loss contours calculated from analytically derived flux density waveforms. 


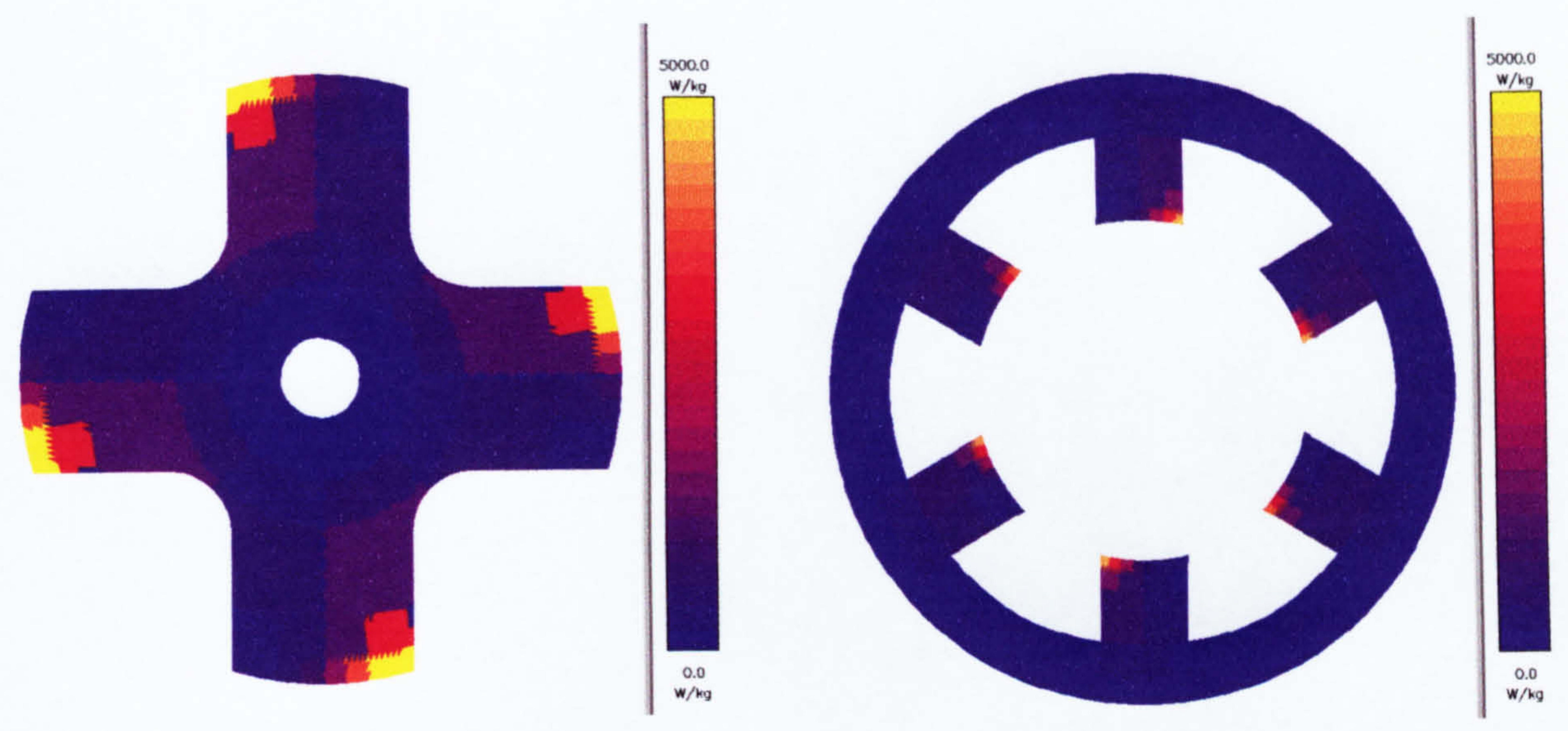

Radial loss contours

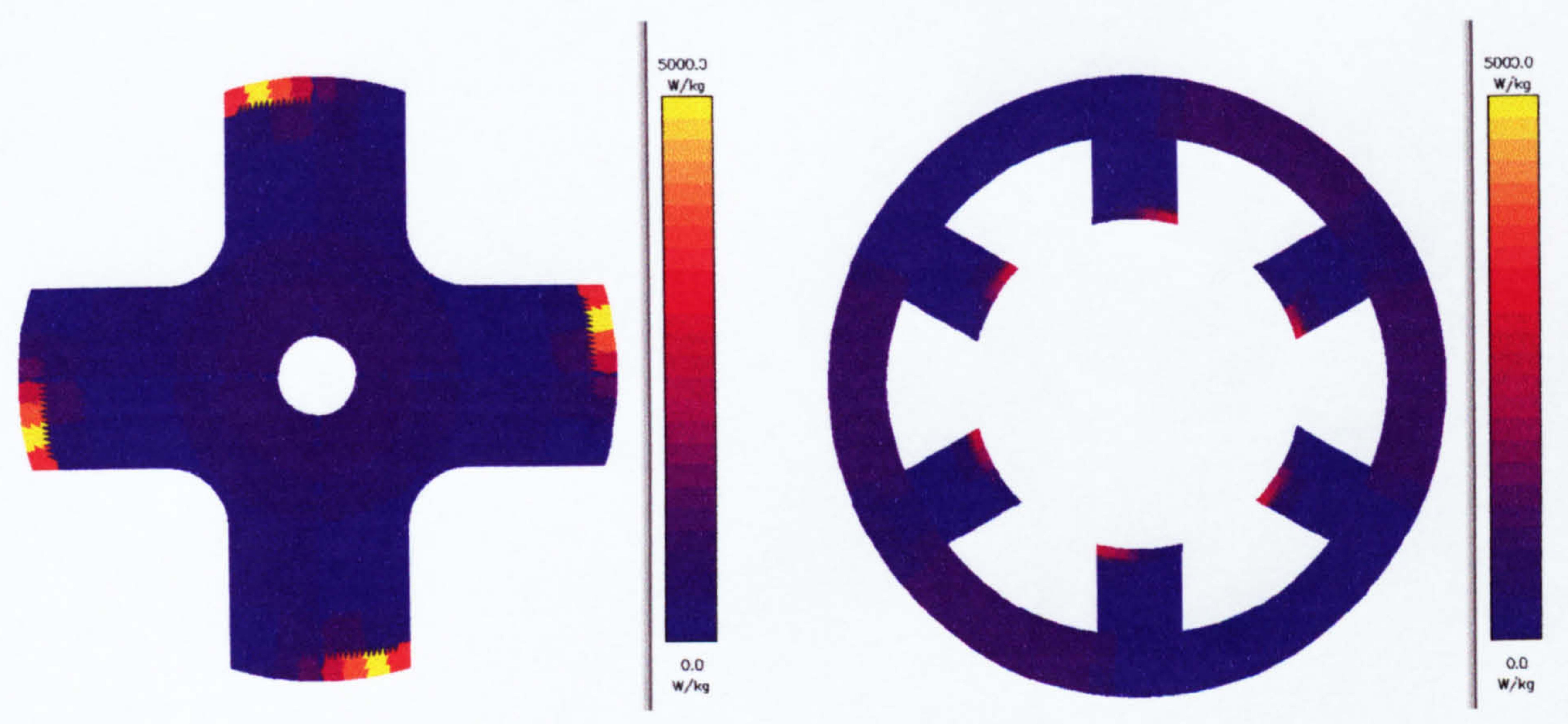

Circumferential loss contours

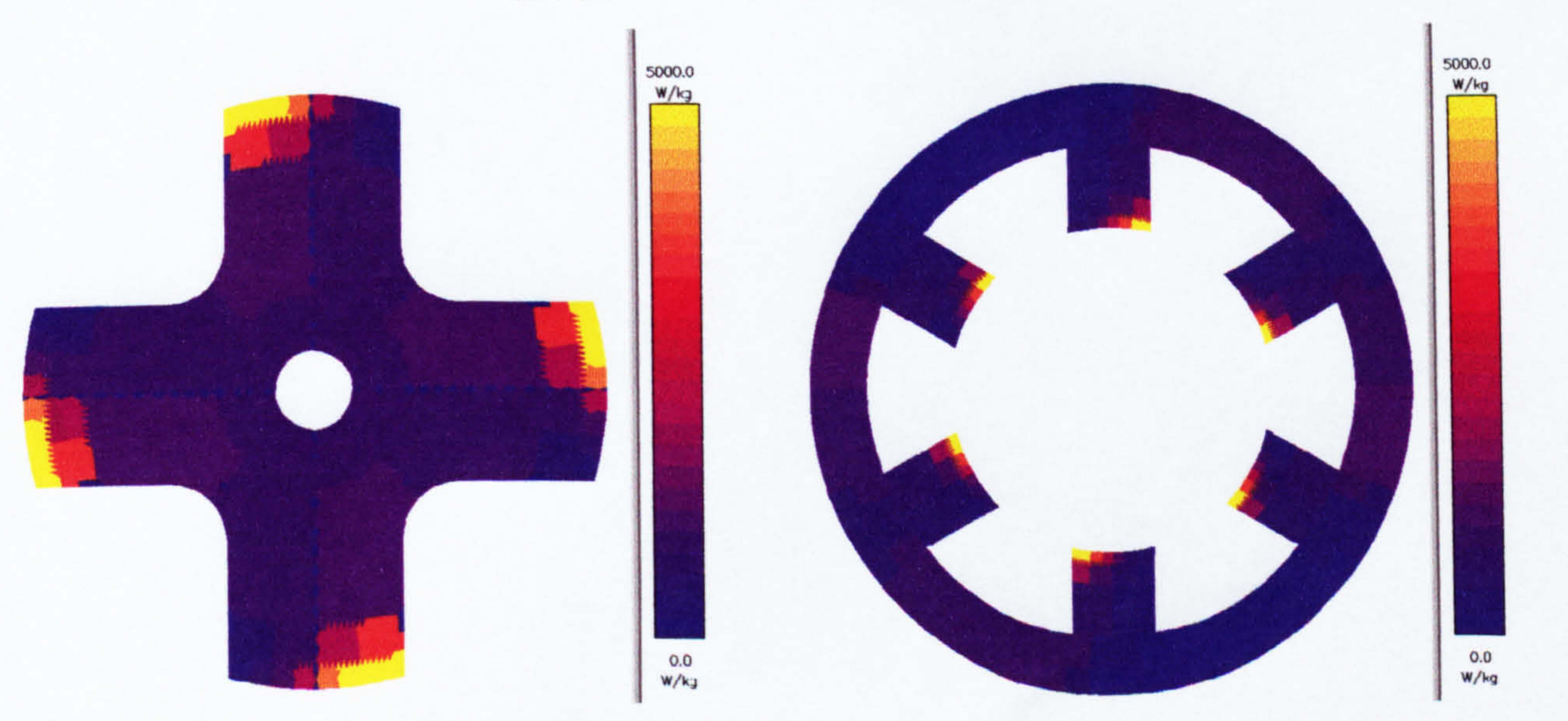

Total loss contours

Figure 4.37a. Iron loss contours for winding connection A - Region method 

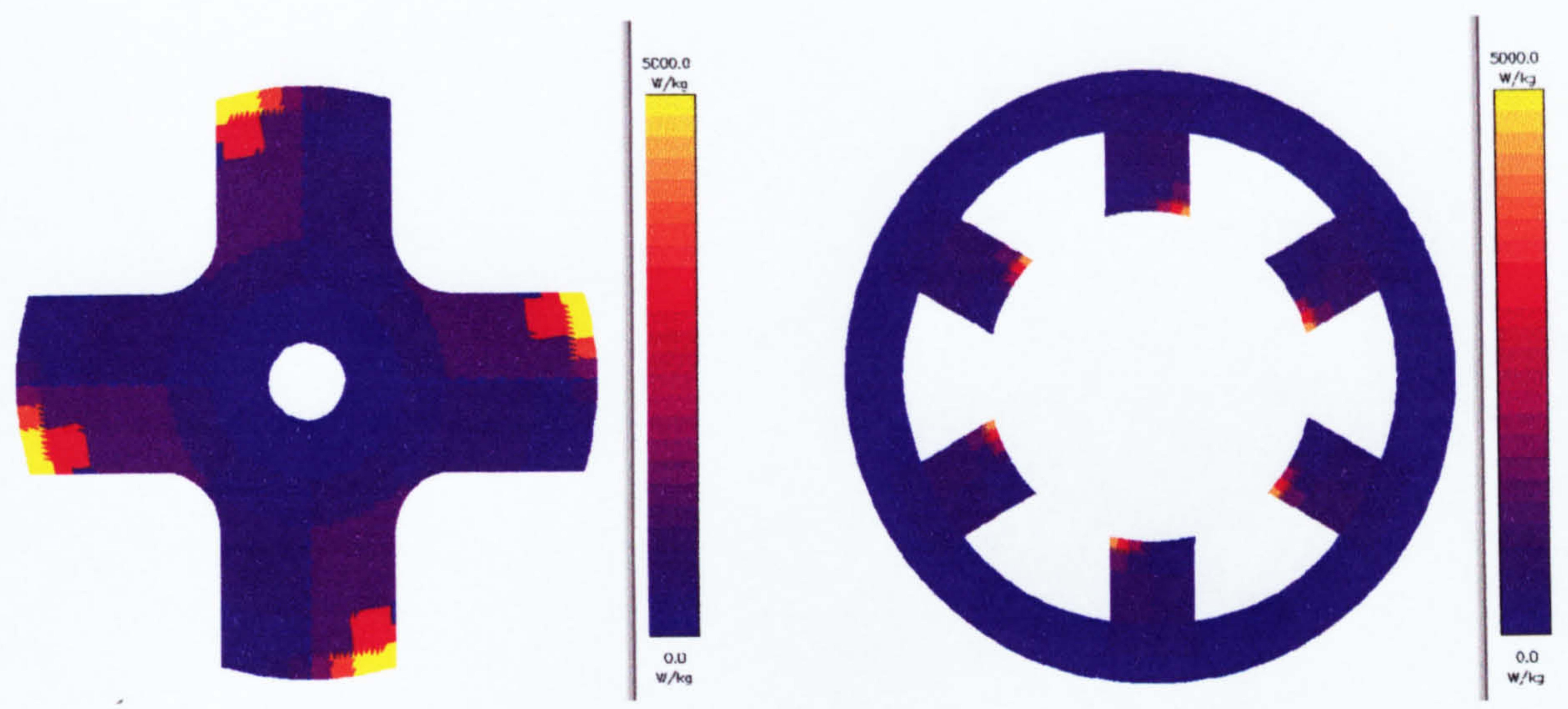

Radial loss contours
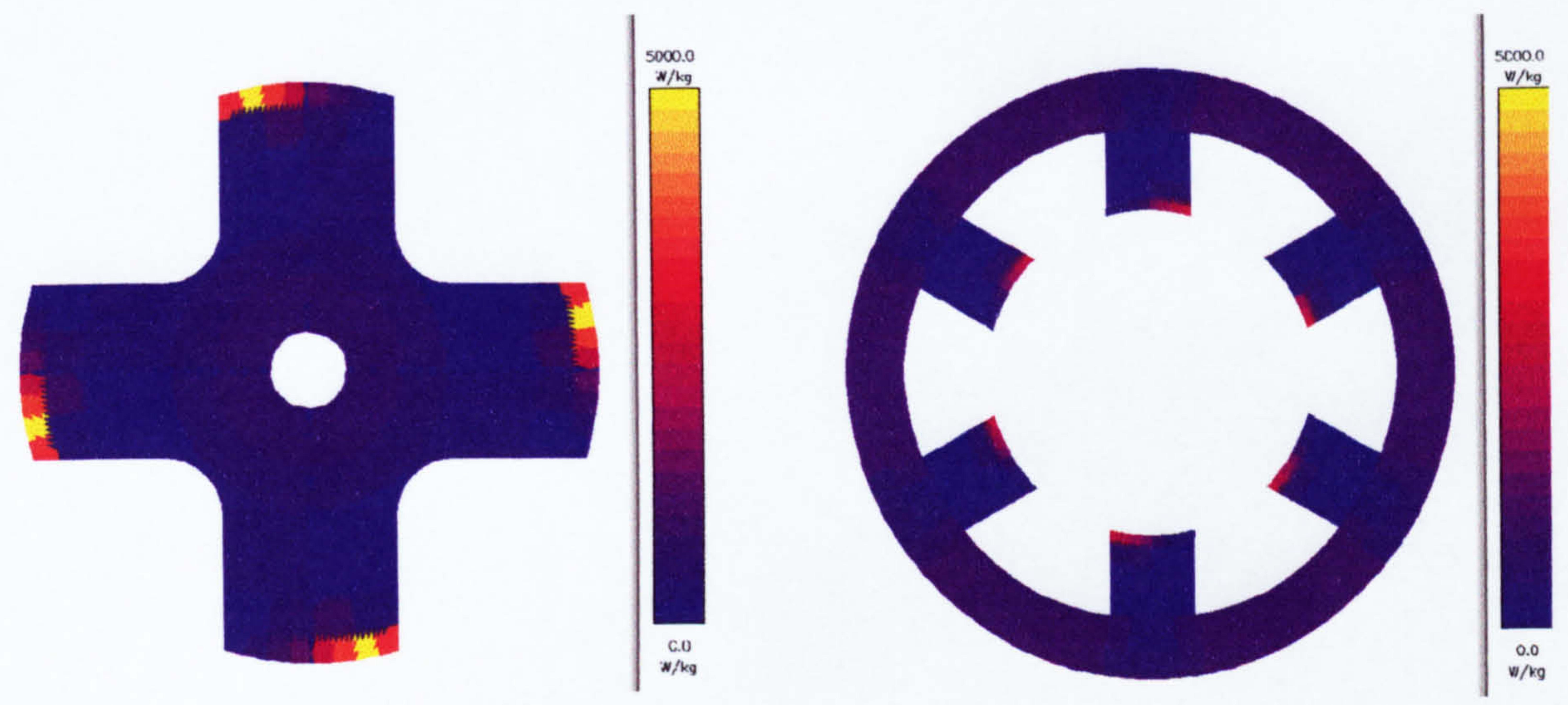

Circumferential loss contours
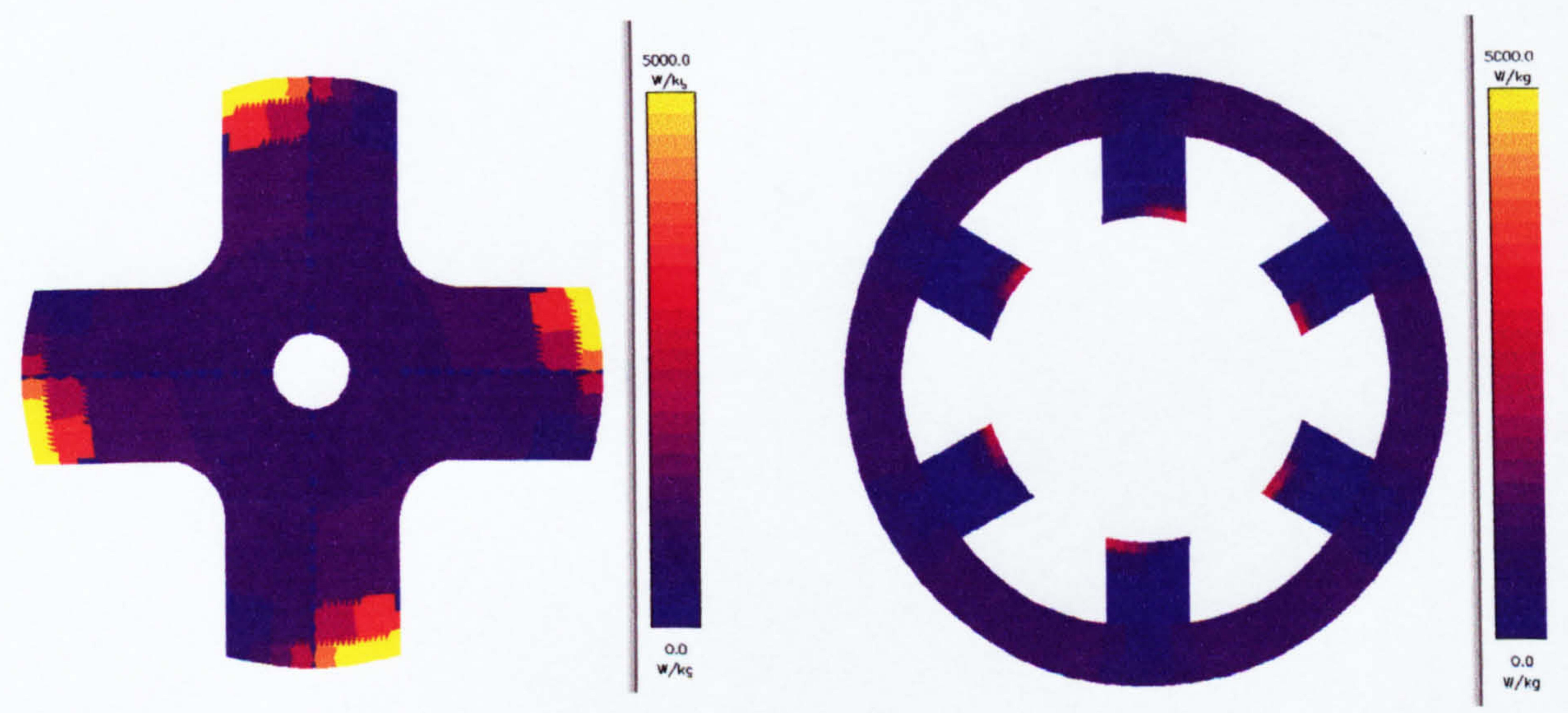

Total loss contours

Figure 4.37b. Iron loss contours for winding connection B - Region method. 

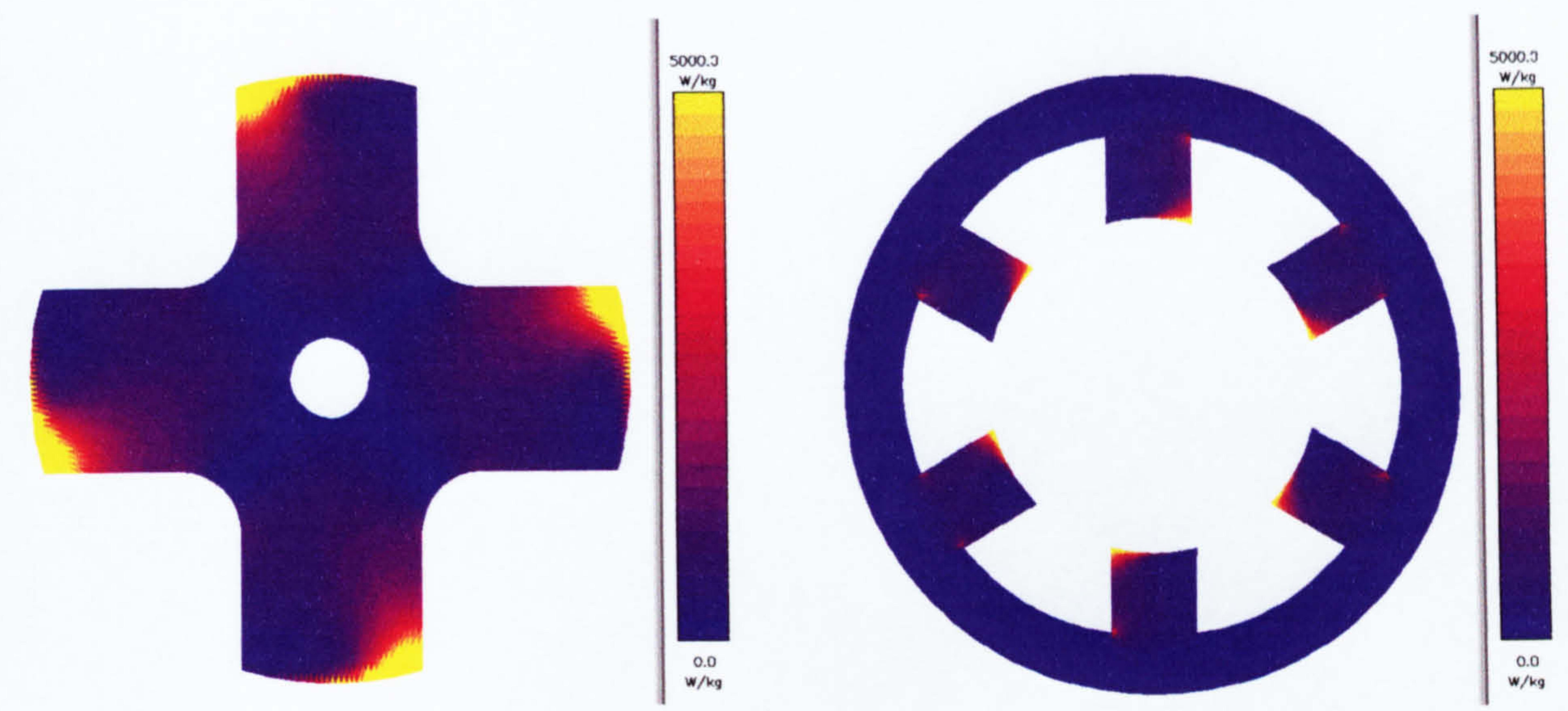

Radial loss contours

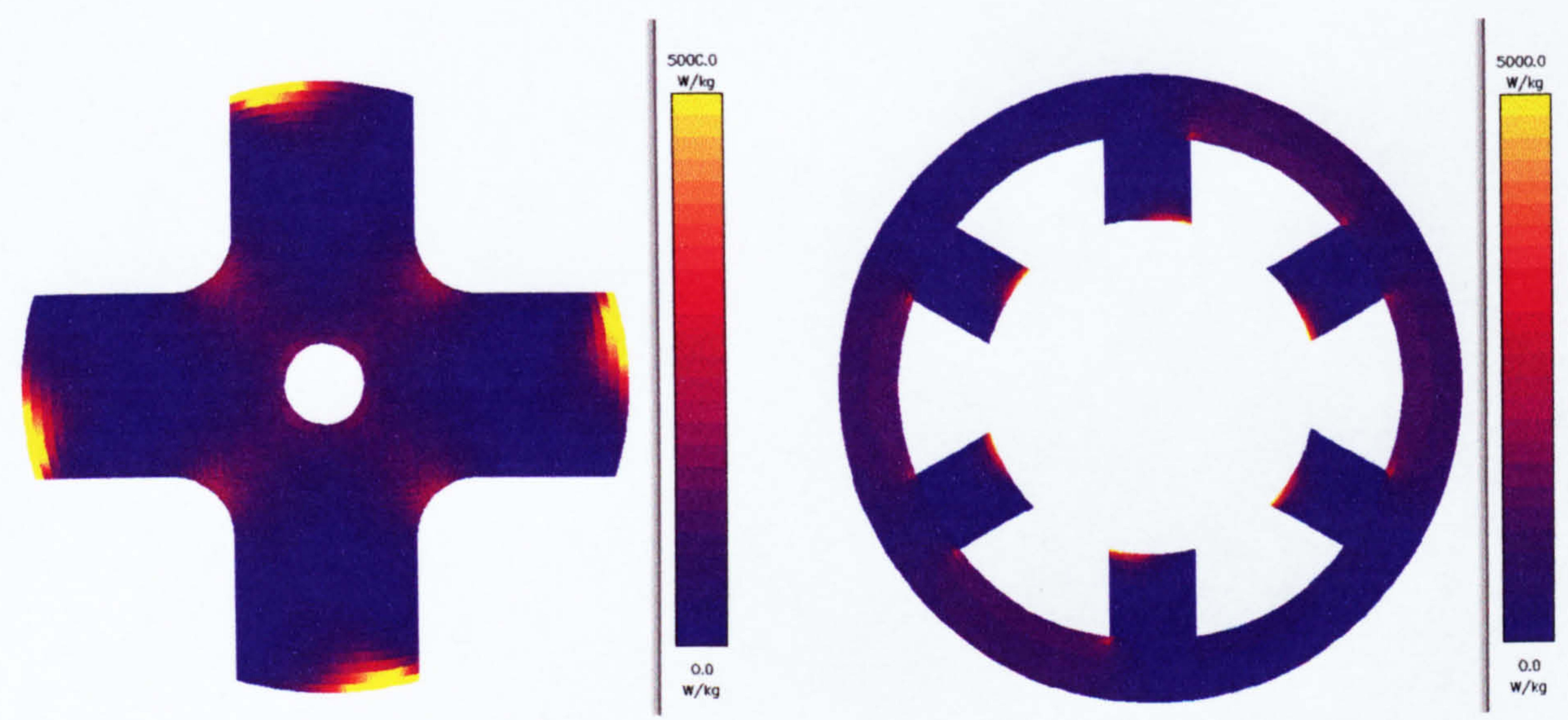

Circumferential loss contours

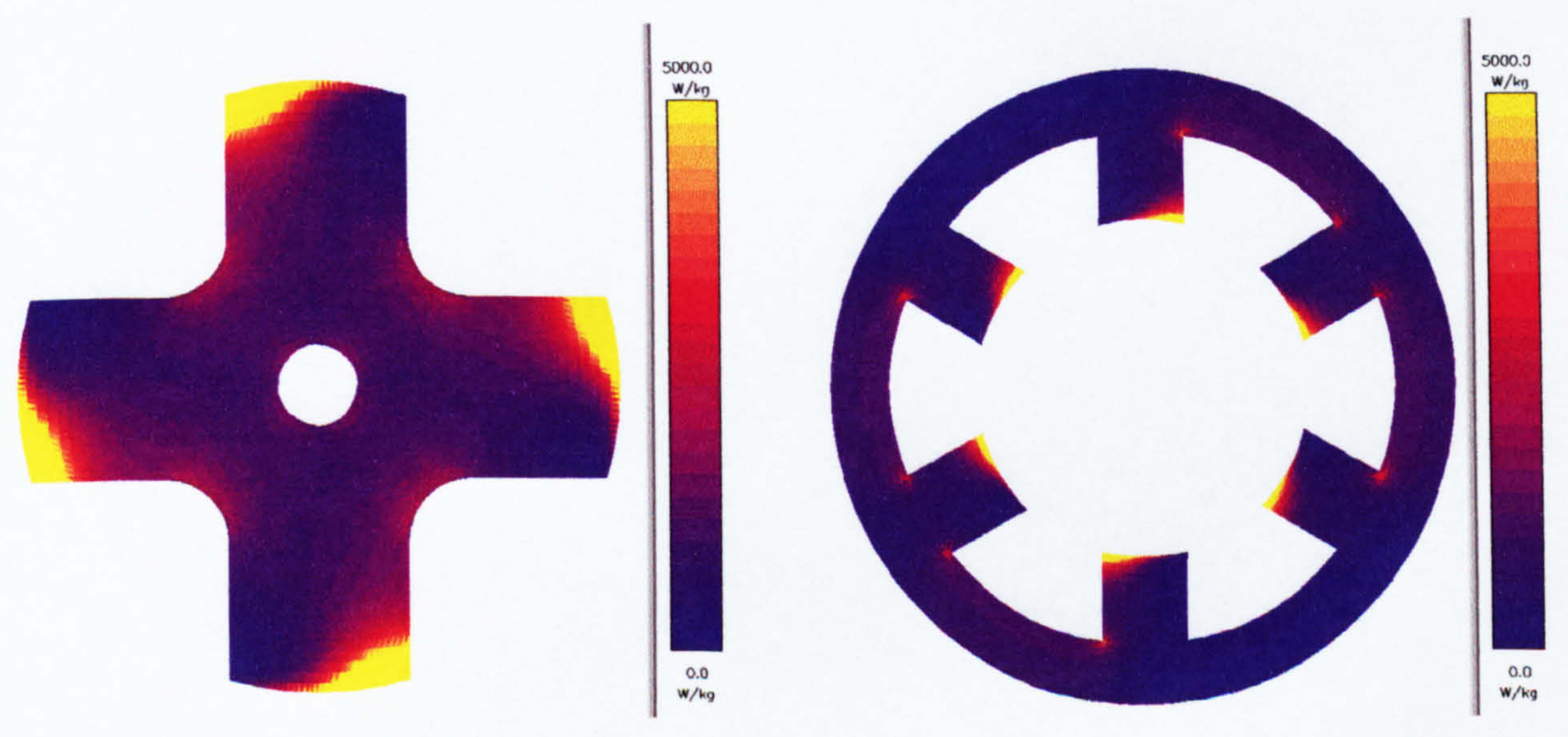

Total loss contours

Figure 4.38a. Iron loss contours for winding connection A - Element method 

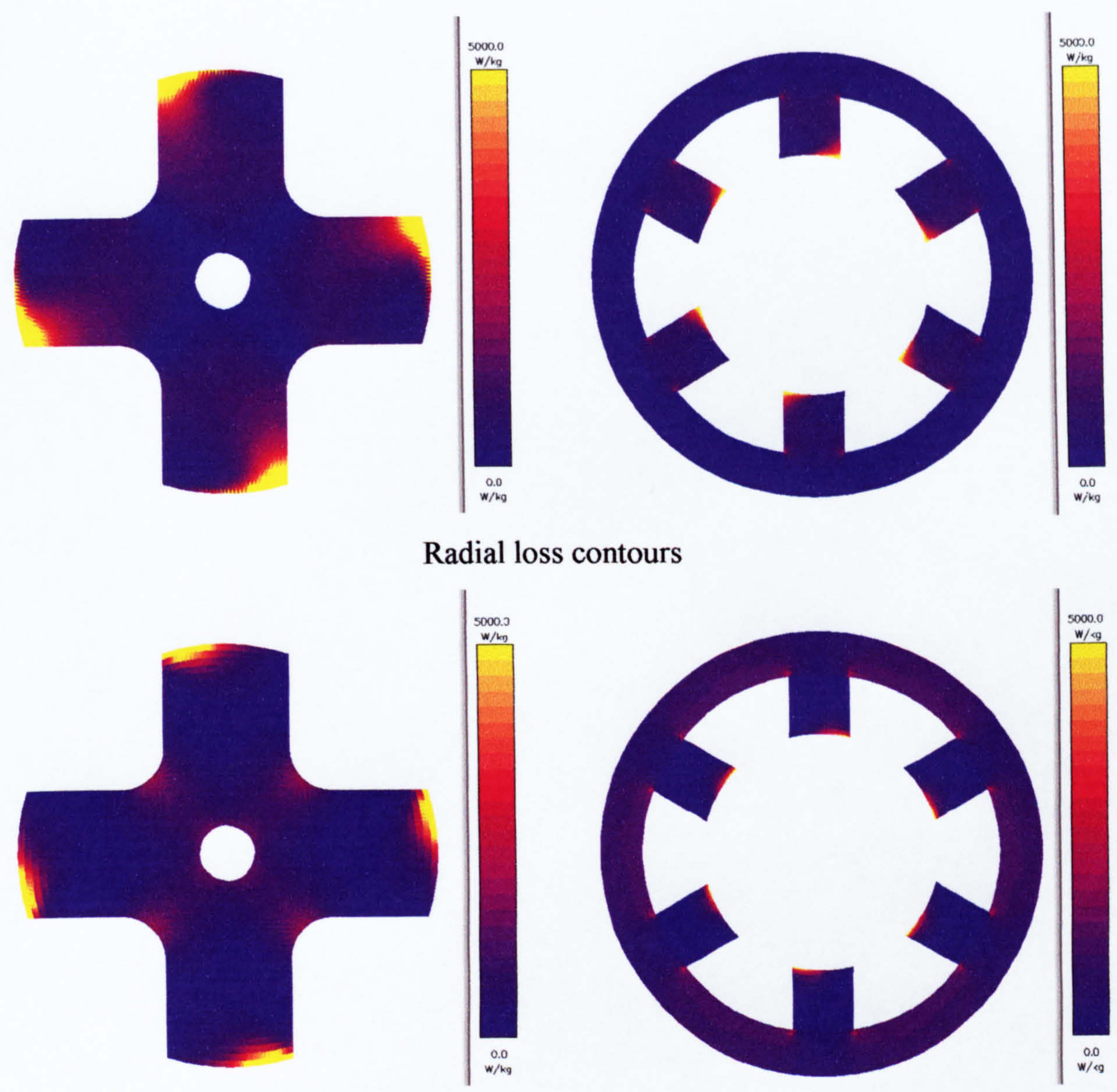

Circumferential loss contours

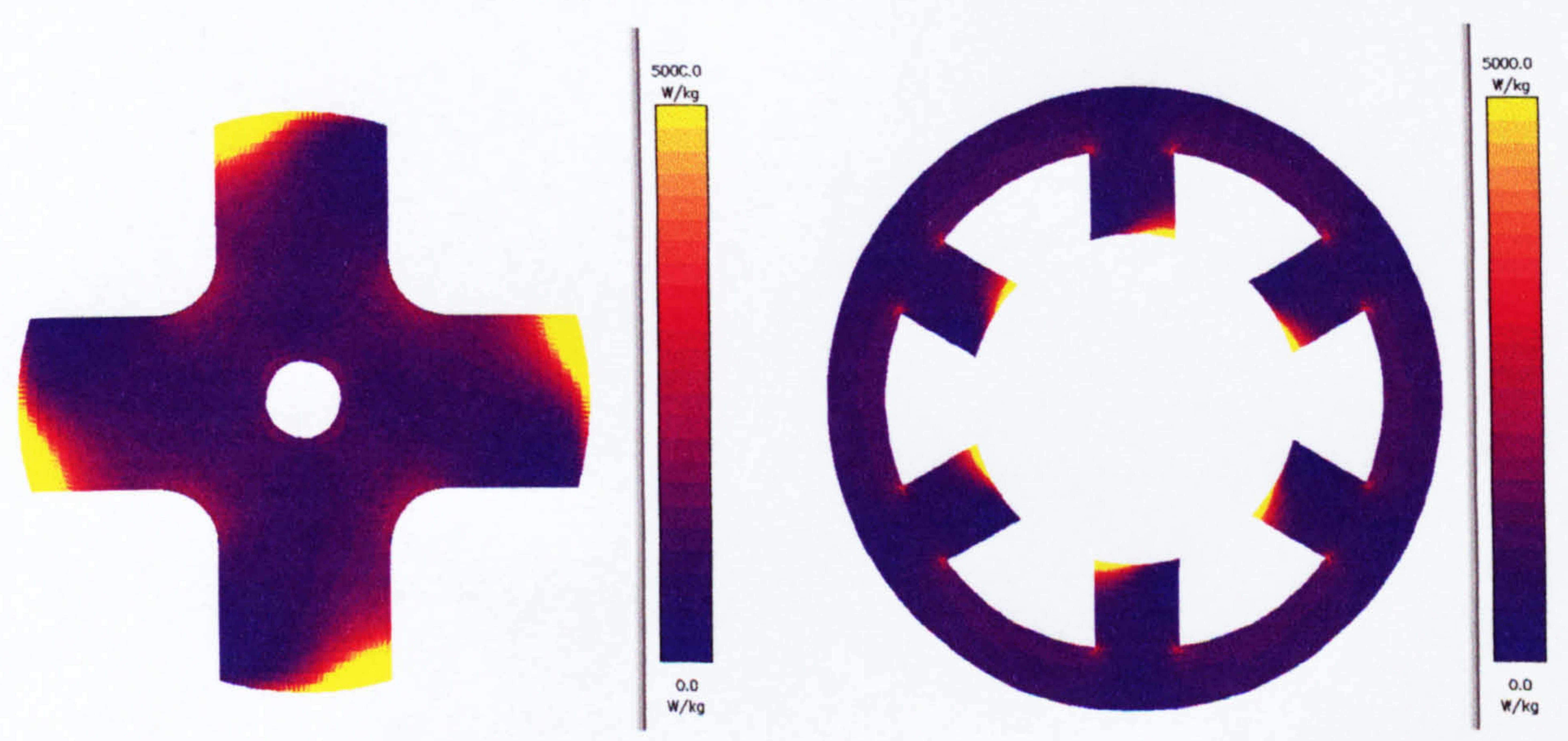

Total loss contours

Figure 4.38b. Iron loss contours for winding connection B - Element method. 


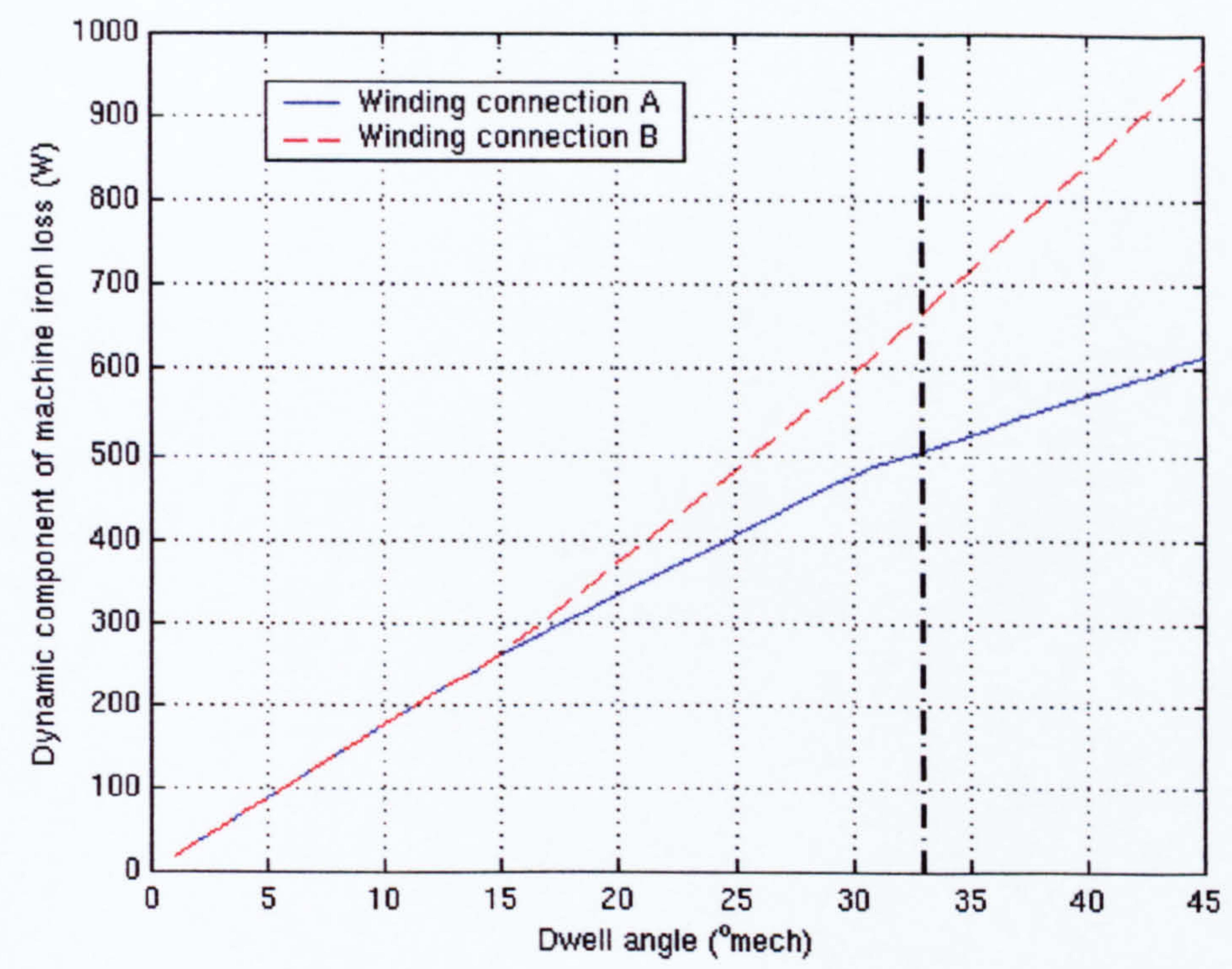

Figure 4.39 Dynamic components of total machine iron loss as a function of the switch dwell angle for the machine operating at $100,000 \mathrm{rpm}$ calculated using the equations listed in appendix 4.2 for the two winding connections. The reference operating condition, which corresponds to a dwell angle of $33^{\circ}$, is highlighted. 


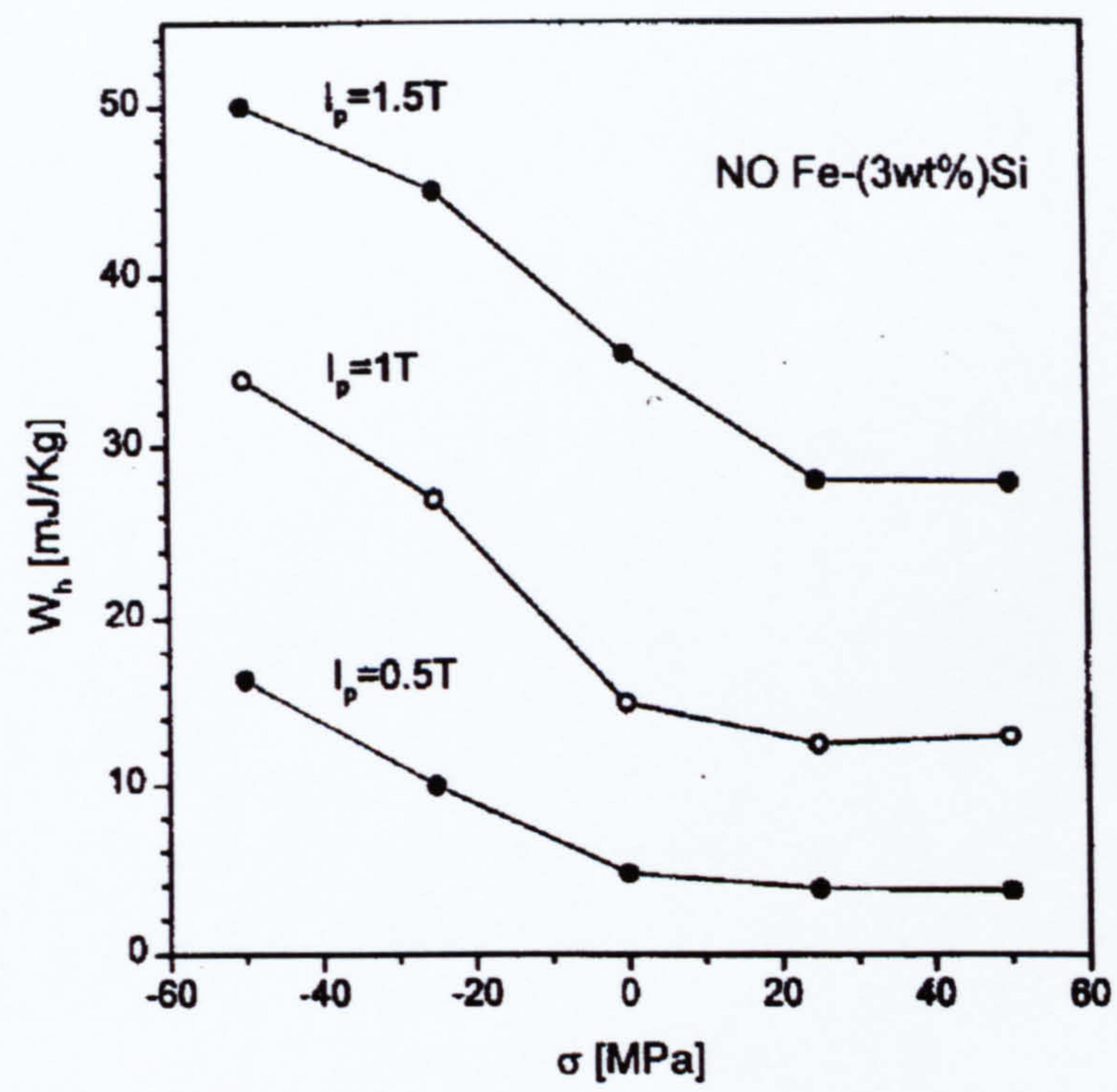

Figure 4.40a. Variation of hysteresis loss as a function of the applied stress. ( $\sigma>0$ indicates tensile stress) [4.26].

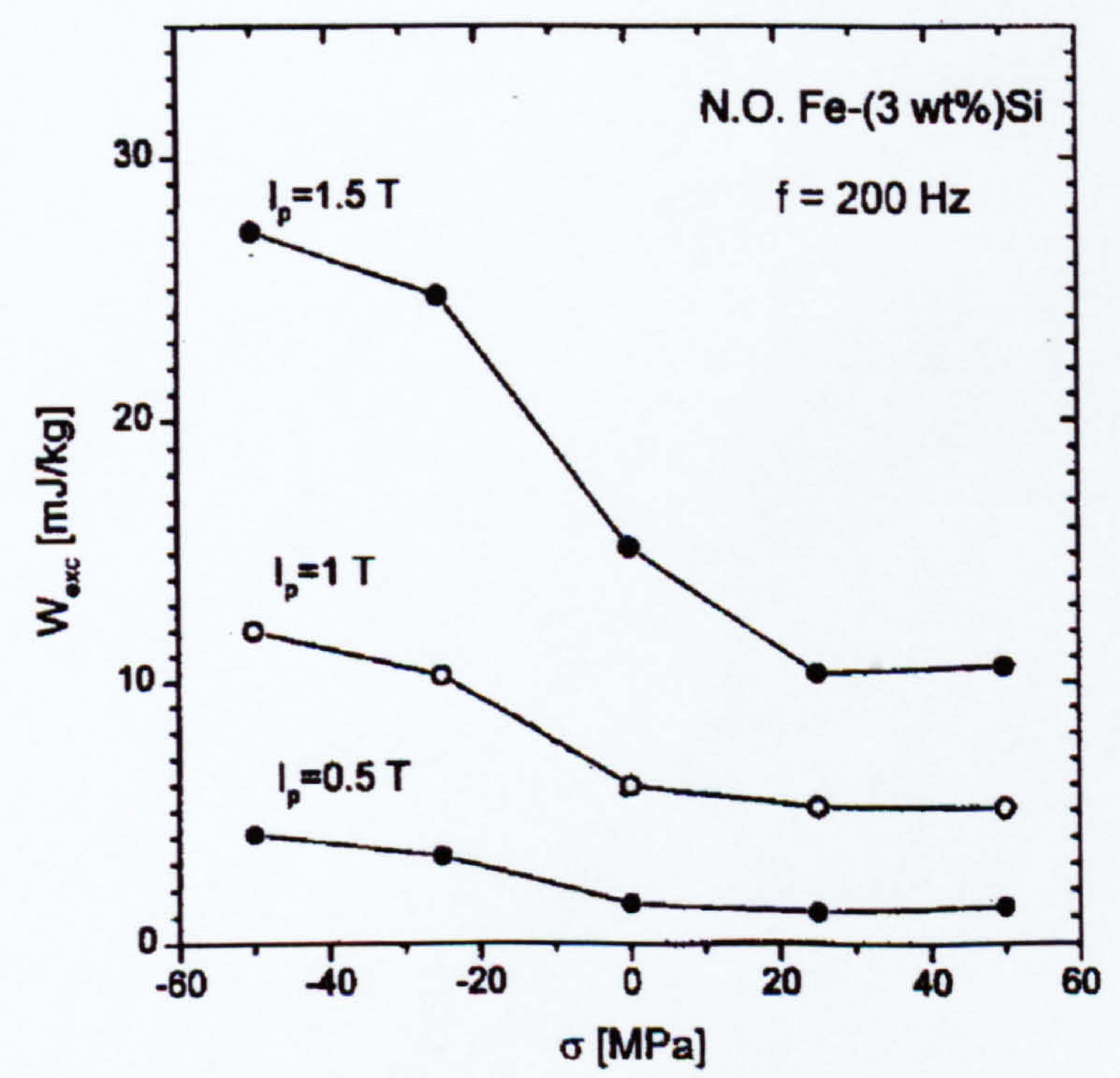

Figure $4.40 \mathrm{~b}$. Variation of excess loss as a function of the applied stress. $(\sigma>0$ indicates tensile stress) [4.26] 


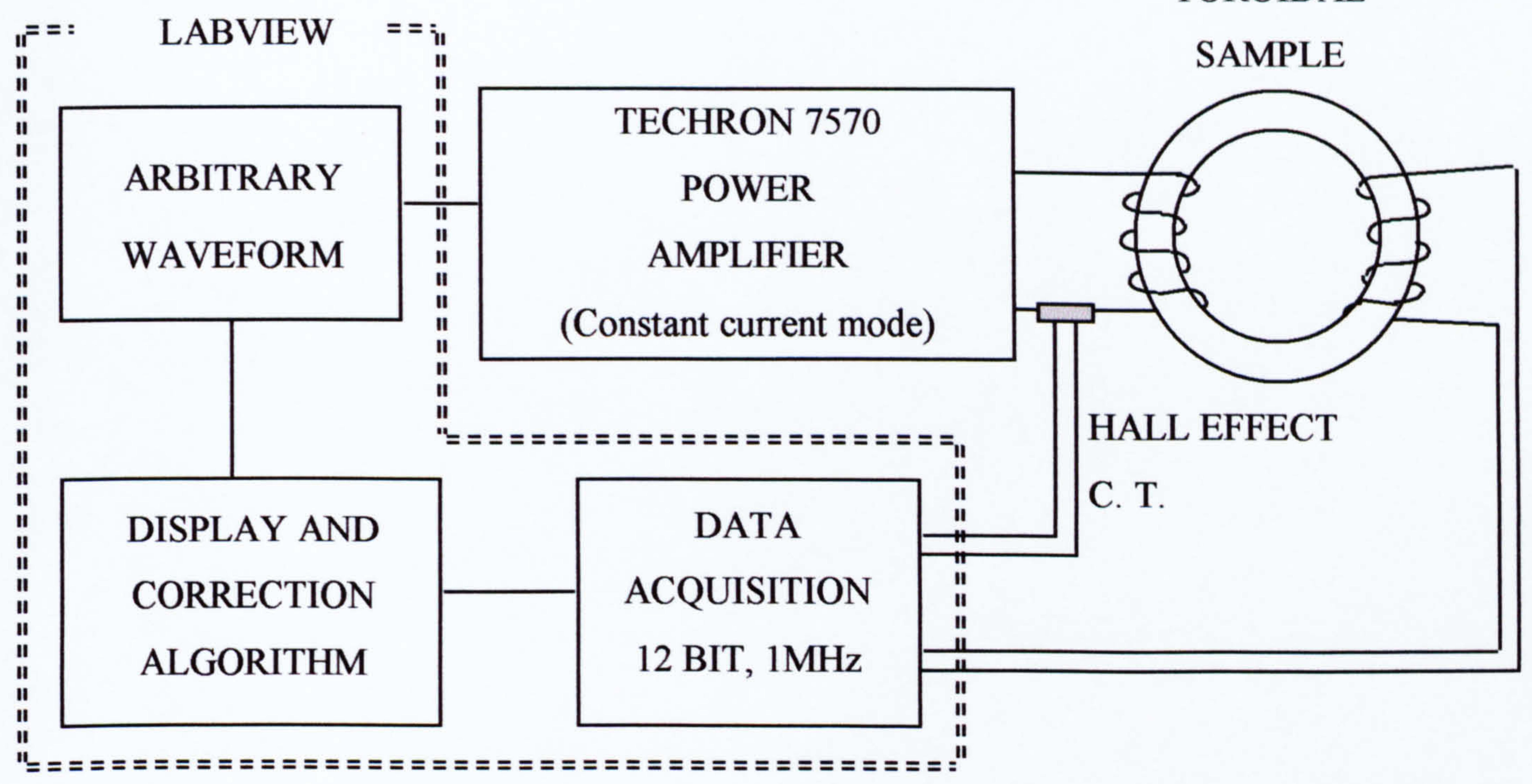

Figure 4.41. Schematic representation of the pseudo-closed loop iron loss test system ased on Labview.

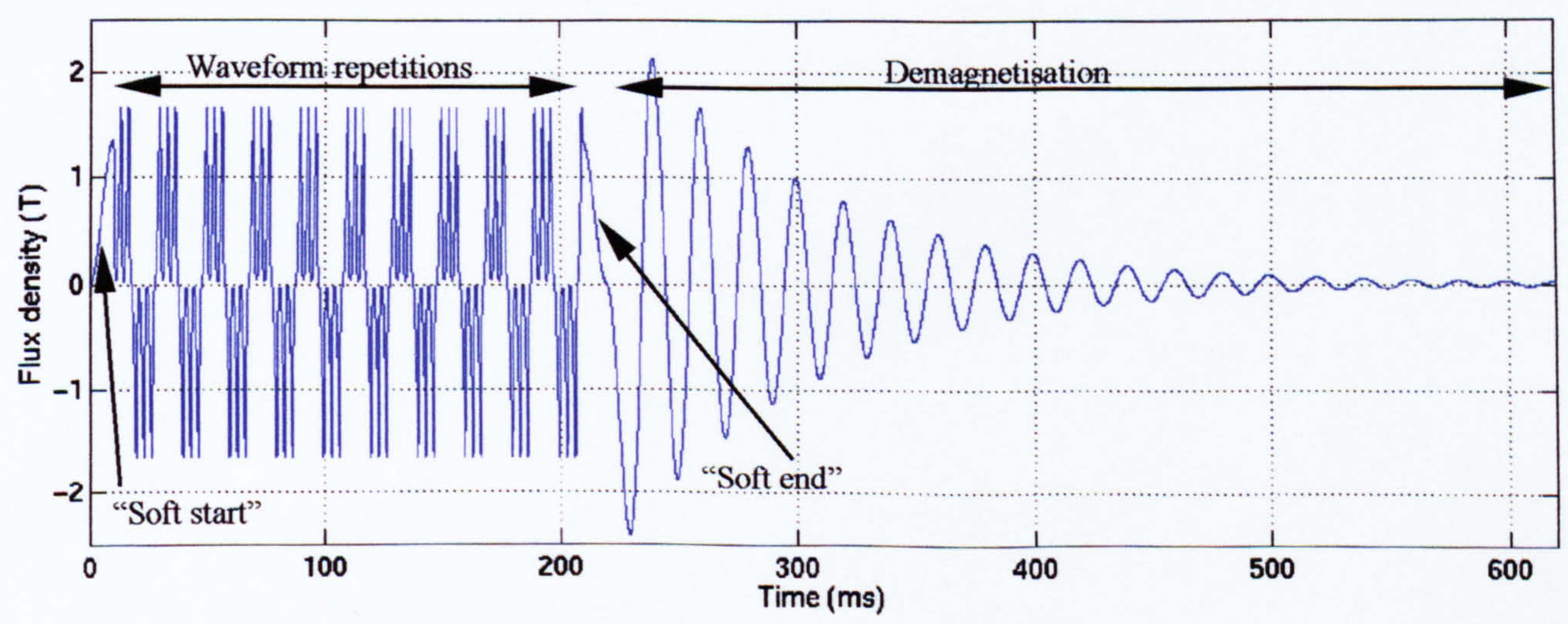

Figure 4.42. Sample flux density waveform consisting of a soft start, 10 repetitions of a $1.5 \mathrm{~T}$ peak sinusoid, a soft landing and an open loop demagnetisation profile. 


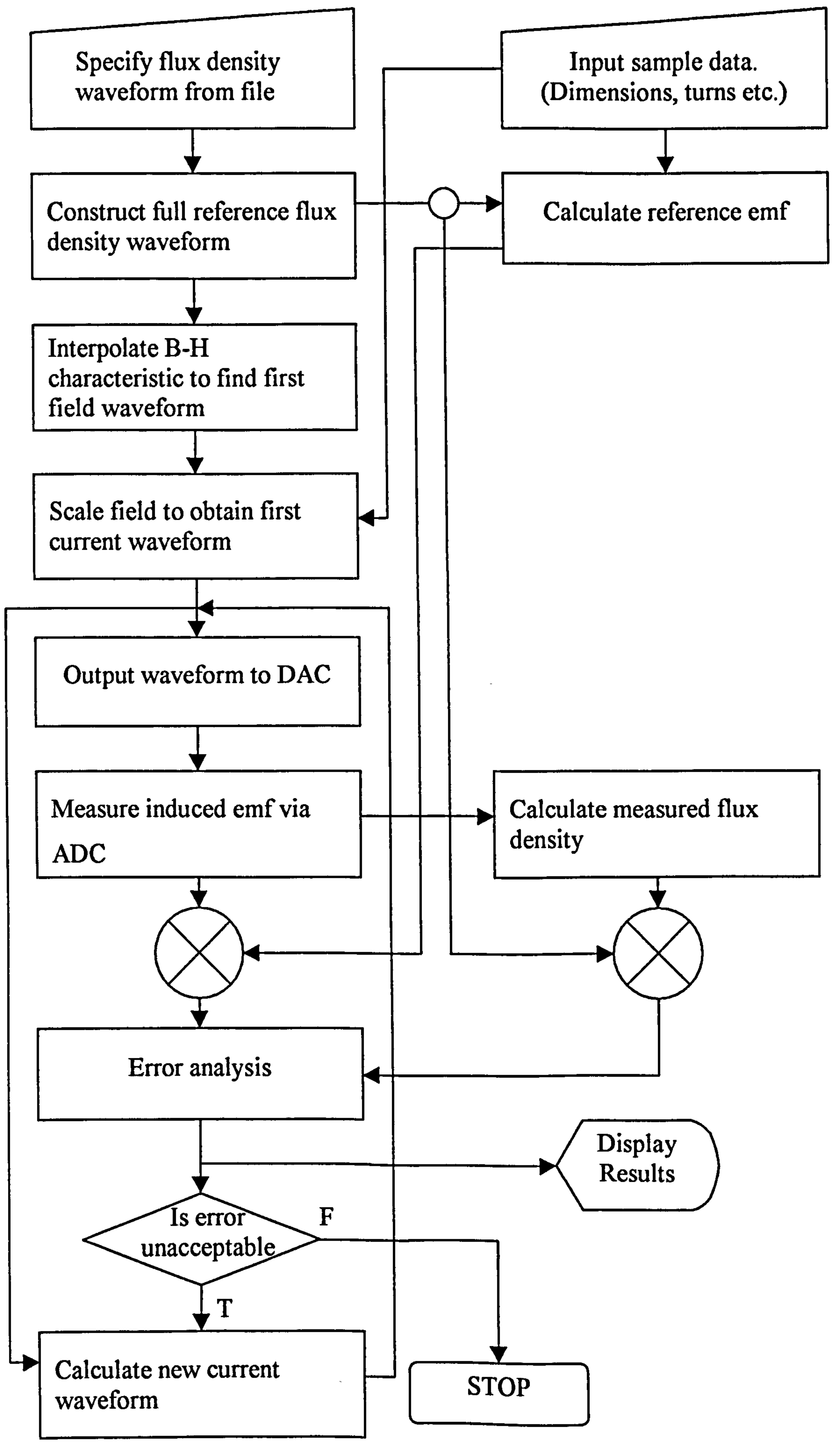

Figure 4.43. Flow diagram representation of iron loss convergence routine. 


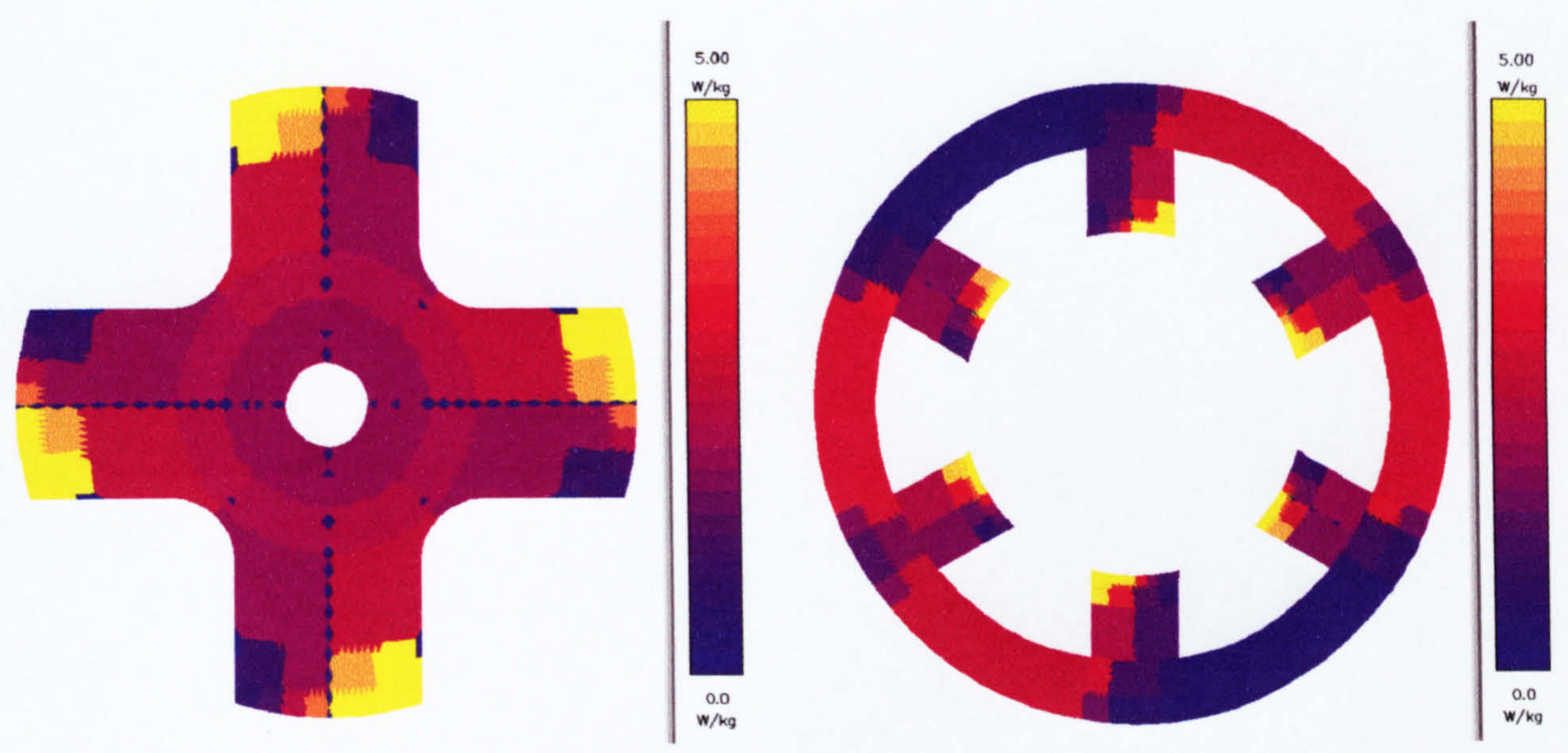

Figure 4.44a. Measured total loss contours at $50 \mathrm{~Hz}$

(measurements performed on toroidal samples)

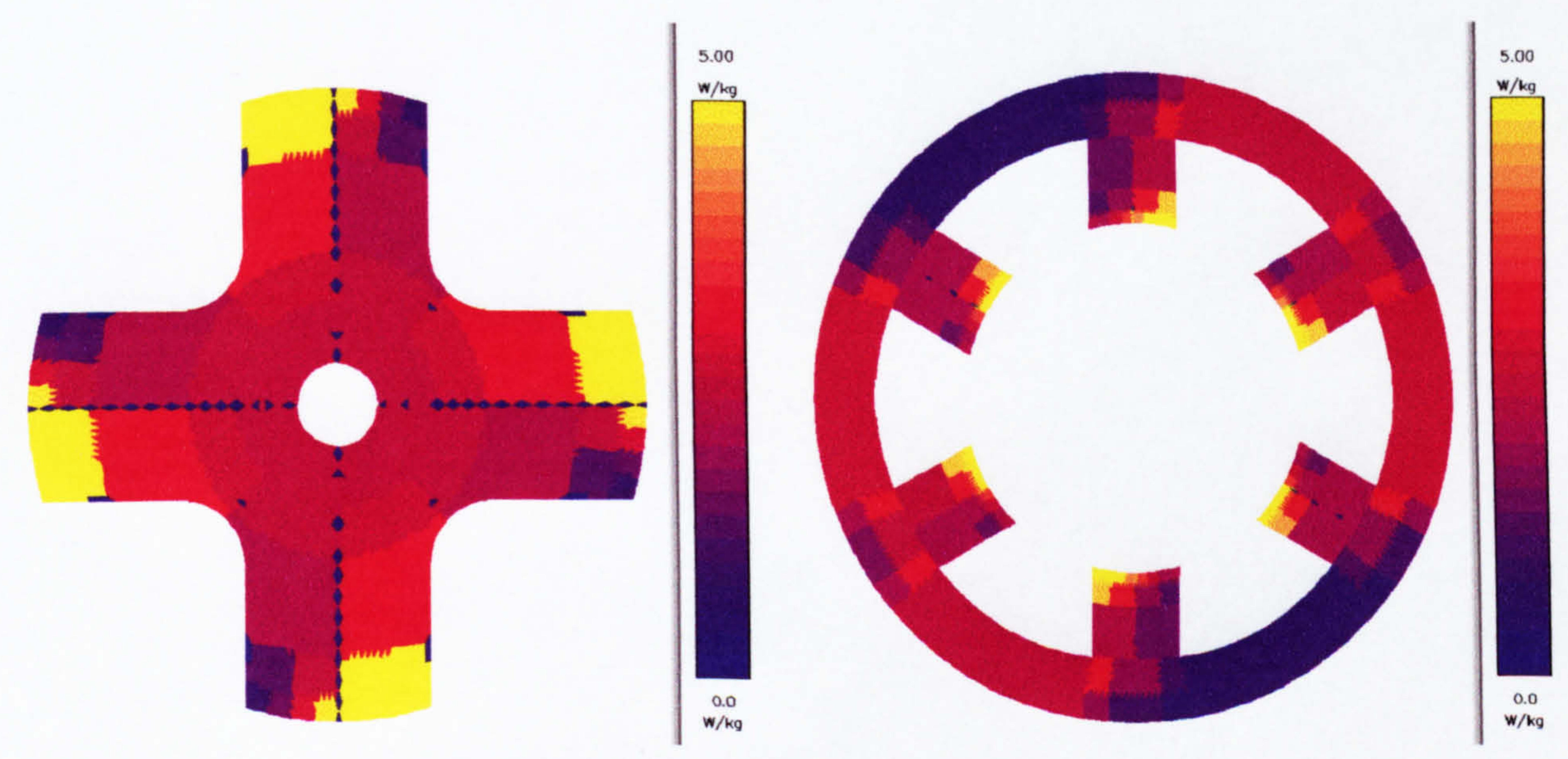

Figure 4.44b. Calculated total loss contours at $50 \mathrm{~Hz}$. 
APPENDIX 4.1. Hysteresis loss measurement equipment.

The static hysteresis loops of the rotor and stator materials are measured using a system comprising a $12 \mathrm{bit}, 1 \mathrm{MHz}$ data acquisition system mounted in a personal computer and software controlled using Labview v4.1. Modulating the demand signal of a Techron 7570 power amplifier in constant current mode controls the magnitude of the magnetic field applied to the sample. The flux induced in the sample is measured via a search winding wound close to the material core using a Magnetech integrating flux meter where the analogue output voltage proportional to the flux linkage is simultaneously recorded by the data acquisition system. The tests are conducted at frequencies typically in the range $0.25-1 \mathrm{~Hz}$ in order to maintain the dynamic loss component at a minimum, while reducing the potential drift of the integrating flux meter.

The system allows the magnitude of the magnetic field to be accurately controlled up to a limit defined by the size of the material sample, the number of turns on the excitation winding and the maximum current capability of the power amplifier. In this case the maximum magnetic field achieved was $20 \mathrm{kA} / \mathrm{m}$ and the maximum flux density induced in each sample was of the order of 1.9T. 
APPENDIX 4.2a. Evaluation of the integrals in the excess loss equation for winding connection type A.

$$
8 \frac{\phi_{m}^{1.5}}{\sqrt{\tau} G_{i}^{1.5}} \quad \frac{\pi}{0}<\tau \omega<\frac{\pi}{4}
$$

Stator regions 1, 2 and 3

$$
\begin{gathered}
24 \frac{\phi_{m}^{1.5}}{\tau G_{i}^{1.5}} \quad 0<\tau \omega<\frac{\pi}{12} \\
21.25\left[\frac{\phi_{m}^{1.5}}{\sqrt{\tau} G_{i}^{1.5}}\right]+1.37\left[\frac{\phi_{m}^{1.5}}{\tau^{1.5} \omega G_{i}^{1.5}} \frac{\pi}{6}\right] \frac{\pi}{12}<\tau \omega<\frac{\pi}{6} \\
4.31\left[\frac{\phi_{m}^{1.5}}{\sqrt{\tau} G_{i}^{1.5}}\right]-18.31\left[\frac{\phi_{m}^{1.5}}{\tau^{1.5} \omega G_{i}^{1.5}} \frac{\pi}{6}\right] \quad \frac{\pi}{6}<\tau \omega<\frac{\pi}{4}
\end{gathered}
$$

Stator regions 4 and 5

$$
\begin{gathered}
24 \frac{\phi_{m}^{1.5}}{\sqrt{\tau} G_{i}^{1.5}} \quad 0<\tau \omega<\frac{\pi}{12} \\
24\left[\frac{\phi_{m}^{1.5}}{\tau^{1.5} \omega G_{i}^{1.5}} \frac{\pi}{6}-\frac{\phi_{m}^{1.5}}{\sqrt{\tau} G_{i}^{1.5}}\right] \frac{\pi}{12}<\tau \omega<\frac{\pi}{6} \\
24\left[\frac{\phi_{m}^{1.5}}{\sqrt{\tau} G_{i}^{1.5}}-\frac{\phi_{m}^{1.5}}{\tau^{1.5} \omega G_{i}^{1.5}} \frac{\pi}{6}\right] \frac{\pi}{6}<\tau \omega<\frac{\pi}{4}
\end{gathered}
$$

Stator region 6

$$
\begin{gathered}
12 \frac{\phi_{m}^{1.5}}{\sqrt{\tau} G_{i}^{1.5}} \quad 0<\tau \omega<\frac{\pi}{6} \\
6.34\left[\frac{\phi_{m}^{1.5}}{\tau^{1.5} \omega G_{i}^{1.5}} \frac{\pi}{3}\right]-0.69\left[\frac{\phi_{m}^{1.5}}{\sqrt{\tau} G_{i}^{1.5}}\right] \frac{\pi}{6}<\tau \omega<\frac{\pi}{4}
\end{gathered}
$$

Rotor region 7 


$$
\begin{gathered}
24 \frac{\phi_{m}^{1.5}}{\sqrt{\tau} G_{i}^{1.5}} \quad 0<\tau \omega<\frac{\pi}{12} \\
7.03\left[\frac{\phi_{m}^{1.5}}{\tau^{1.5} \omega G_{i}^{1.5}} \frac{\pi}{6}\right]+9.94\left[\frac{\phi_{m}^{1.5}}{\sqrt{\tau} G_{i}^{1.5}}\right] \frac{\pi}{12}<\tau \omega<\frac{\pi}{6} \\
6.84\left[\frac{\phi_{m}^{1.5}}{\sqrt{\tau} G_{i}^{1.5}}\right]-10.13\left[\frac{\phi_{m}^{1.5}}{\tau^{1.5} \omega G_{i}^{1.5}} \frac{\pi}{6}\right] \quad \frac{\pi}{6}<\tau \omega<\frac{\pi}{4}
\end{gathered}
$$

Rotor region 8 
APPENDIX 4.2b. Evaluation of the integrals in the eddy current loss equation for winding connection type A.

$$
8 \frac{\phi_{m}^{2}}{\tau G_{i}^{2}} \quad \frac{\pi}{0}<\tau \omega<\frac{\pi}{4}
$$

Stator regions 1,2 and 3

$$
\begin{gathered}
24 \frac{\phi_{m}^{2}}{\tau G_{i}^{2}} \quad 0<\tau \omega<\frac{\pi}{12} \\
40\left[\frac{\phi_{m}^{2}}{\tau G_{i}^{2}}\right]-8\left[\frac{\phi_{m}^{2}}{\tau^{2} \omega G_{i}^{2}} \frac{\pi}{6}\right] \quad \frac{\pi}{12}<\tau \omega<\frac{\pi}{6} \\
16\left[\frac{\phi_{m}^{2}}{\tau G_{i}^{2}}+\frac{\phi_{m}^{2}}{\tau^{2} \omega G_{i}^{2}} \frac{\pi}{6}\right] \frac{\pi}{6}<\tau \omega<\frac{\pi}{4}
\end{gathered}
$$

Stator regions 4 and 5

$$
\begin{gathered}
24 \frac{\phi_{m}^{2}}{\tau G_{i}^{2}} \quad 0<\tau \omega<\frac{\pi}{12} \\
24\left[\frac{\phi_{m}^{2}}{\tau^{2} \omega G_{i}^{2}} \frac{\pi}{6}-\frac{\phi_{m}^{2}}{\tau G_{i}^{2}}\right] \quad \frac{\pi}{12}<\tau \omega<\frac{\pi}{6} \\
24\left[\frac{\phi_{m}^{2}}{\tau G_{i}^{2}}-\frac{\phi_{m}^{2}}{\tau^{2} \omega G_{i}^{2}} \frac{\pi}{6}\right] \frac{\pi}{6}<\tau \omega<\frac{\pi}{4}
\end{gathered}
$$

Stator region 6

$$
\begin{gathered}
12 \frac{\phi_{m}^{2}}{\tau G_{i}^{2}} \quad 0<\tau \omega<\frac{\pi}{6} \\
4\left[\frac{\phi_{m}^{2}}{\tau G_{i}^{2}}+\frac{\phi_{m}^{2}}{\tau^{2} \omega G_{i}^{2}} \frac{\pi}{3}\right] \frac{\pi}{6}<\tau \omega<\frac{\pi}{4}
\end{gathered}
$$

Rotor region 7

$$
\begin{gathered}
24 \frac{\phi_{m}^{2}}{\tau G_{i}^{2}} \quad 0<\tau \omega<\frac{\pi}{6} \\
12\left[\frac{\phi_{m}^{2}}{\tau^{2} \omega G_{i}^{2}} \frac{\pi}{2}-\frac{\phi_{m}^{2}}{\tau G_{i}^{2}}\right] \frac{\pi}{6}<\tau \omega<\frac{\pi}{4}
\end{gathered}
$$

Rotor region 8 
APPENDIX 4.2c. Evaluation of the integrals in the excess loss equation for winding connection type B.

$$
8 \frac{\phi_{m}^{1.5}}{\sqrt{\tau} G_{i}^{1.5}} \quad \frac{\pi}{0}<\tau \omega<\frac{\pi}{4}
$$

Stator regions 1,2 and 3

$$
\begin{gathered}
24 \frac{\phi_{m}^{1.5}}{\tau G_{i}^{1.5}} \quad 0<\tau \omega<\frac{\pi}{12} \\
21.25\left[\frac{\phi_{m}^{1.5}}{\sqrt{\tau} G_{i}^{1.5}}\right]+1.37\left[\frac{\phi_{m}^{1.5}}{\tau^{1.5} \omega G_{i}^{1.5}} \frac{\pi}{6}\right] \frac{\pi}{12}<\tau \omega<\frac{\pi}{6} \\
4.31\left[\frac{\phi_{m}^{1.5}}{\sqrt{\tau} G_{i}^{1.5}}\right]-18.31\left[\frac{\phi_{m}^{1.5}}{\tau^{1.5} \omega G_{i}^{1.5}} \frac{\pi}{6}\right] \frac{\pi}{6}<\tau \omega<\frac{\pi}{4}
\end{gathered}
$$

Stator regions 4,5 and 6

$$
\begin{gathered}
12 \frac{\phi_{m}^{1.5}}{\sqrt{\tau} G_{i}^{1.5}} \quad 0<\tau \omega<\frac{\pi}{6} \\
21.94\left[\frac{\phi_{m}^{1.5}}{\sqrt{\tau} G_{i}^{1.5}}\right]-9.94\left[\frac{\phi_{m}^{1.5}}{\tau^{1.5} \omega G_{i}^{1.5}} \frac{\pi}{6}\right] \quad \frac{\pi}{6}<\tau \omega<\frac{\pi}{4}
\end{gathered}
$$

Rotor region 7

$$
\begin{gathered}
24 \frac{\phi_{m}^{1.5}}{\sqrt{\tau} G_{i}^{1.5}} \quad 0<\tau \omega<\frac{\pi}{12} \\
9.94\left[\frac{\phi_{m}^{1.5}}{\sqrt{\tau} G_{i}^{1.5}}\right]+7.03\left[\frac{\phi_{m}^{1.5}}{\tau^{1.5} \omega G_{i}^{1.5}} \frac{\pi}{6}\right] \frac{\pi}{12}<\tau \omega<\frac{\pi}{6} \\
40.41\left[\frac{\phi_{m}^{1.5}}{\sqrt{\tau} G_{i}^{1.5}}\right]-23.44\left[\frac{\phi_{m}^{1.5}}{\tau^{1.5} \omega G_{i}^{1.5}} \frac{\pi}{6}\right] \quad 6<\tau \omega<\frac{\pi}{4}
\end{gathered}
$$

Rotor region 8 
APPENDIX 4.2d. Evaluation of the integrals in the eddy current loss equation for winding connection type $\mathrm{B}$.

$$
8 \frac{\phi_{m}^{2}}{\tau G_{i}^{2}} \quad \frac{\pi}{0}<\tau \omega<\frac{\pi}{4}
$$

Stator regions 1,2 and 3

$$
\begin{gathered}
24 \frac{\phi_{m}^{2}}{\tau G_{i}^{2}} \quad 0<\tau \omega<\frac{\pi}{12} \\
40\left[\frac{\phi_{m}^{2}}{\tau G_{i}^{2}}\right]-8\left[\frac{\phi_{m}^{2}}{\tau^{2} \omega G_{i}^{2}} \frac{\pi}{6}\right] \frac{\pi}{12}<\tau \omega<\frac{\pi}{6} \\
16\left[\frac{\phi_{m}^{2}}{\tau G_{i}^{2}}+\frac{\phi_{m}^{2}}{\tau^{2} \omega G_{i}^{2}} \frac{\pi}{6}\right] \frac{\pi}{6}<\tau \omega<\frac{\pi}{4}
\end{gathered}
$$

Stator regions 4,5 and 6

$$
\begin{gathered}
12 \frac{\phi_{m}^{2}}{\tau G_{i}^{2}} \quad 0<\tau \omega<\frac{\pi}{6} \\
36\left[\frac{\phi_{m}^{2}}{\tau G_{i}^{2}}\right]-24\left[\frac{\phi_{m}^{2}}{\tau^{2} \omega G_{i}^{2}} \frac{\pi}{6}\right] \frac{\pi}{6}<\tau \omega<\frac{\pi}{4}
\end{gathered}
$$

Rotor region 7

$$
\begin{gathered}
24 \frac{\phi_{m}^{2}}{\tau G_{i}^{2}} \quad 0<\tau \omega<\frac{\pi}{6} \\
72\left[\frac{\phi_{m}^{2}}{\tau G_{i}^{2}}\right]-48\left[\frac{\phi_{m}^{2}}{\tau^{2} \omega G_{i}^{2}} \frac{\pi}{6}\right] \frac{\pi}{6}<\tau \omega<\frac{\pi}{4}
\end{gathered}
$$

Rotor region 8 
APPENDIX 4.3. Components of iron loss for the 5kW, 100,000rpm operating condition.

\begin{tabular}{|c|c|c|c|c|}
\cline { 3 - 5 } \multicolumn{2}{c|}{} & \multicolumn{3}{c|}{ Iron Loss (W) } \\
\cline { 2 - 5 } \multicolumn{2}{c|}{} & Method 1 & Method 2 & Method 3 \\
\hline \multirow{3}{*}{ Stator } & Radial & 57 & 156 & 172 \\
\cline { 2 - 5 } & Circumferential & 227 & 331 & 340 \\
\hline \multirow{2}{*}{ Rotor } & Radial & 127 & 225 & 276 \\
\cline { 2 - 5 } & Circumferential & 39 & 140 & 180 \\
\hline & TOTAL & $\mathbf{4 5 0}$ & $\mathbf{8 5 2}$ & $\mathbf{9 6 8}$ \\
\hline
\end{tabular}

Table A4.1a Eddy current loss components for winding connection type A.

\begin{tabular}{|c|c|c|c|c|}
\cline { 3 - 5 } \multicolumn{2}{c|}{} & \multicolumn{3}{c|}{ Iron Loss (W) } \\
\cline { 2 - 5 } \multicolumn{2}{c|}{} & Method1 & Method 2 & Method 3 \\
\hline \multirow{3}{*}{ Stator } & Radial & 57 & 155 & 174 \\
\cline { 2 - 5 } & Circumferential & 331 & 439 & 450 \\
\hline \multirow{2}{*}{ Rotor } & Radial & 161 & 224 & 275 \\
\cline { 2 - 5 } & Circumferential & 50 & 138 & 179 \\
\cline { 2 - 5 } & TOTAL & $\mathbf{5 9 9}$ & $\mathbf{9 5 6}$ & $\mathbf{1 0 7 8}$ \\
\hline
\end{tabular}

Table A4.1b Eddy current loss components for winding connection type B. 


\begin{tabular}{|c|c|c|c|c|}
\cline { 3 - 5 } \multicolumn{2}{c|}{} & \multicolumn{3}{c|}{ Iron Loss (W) } \\
\cline { 3 - 5 } \multicolumn{2}{c|}{} & Method 1 & Method 2 & Method 3 \\
\hline \multirow{3}{*}{$\begin{array}{c}\text { Stator } \\
\cdot\end{array}$} & Radial & 7 & 15 & 15 \\
\cline { 2 - 5 } & Circumferential & 23 & 32 & 32 \\
\hline \multirow{3}{*}{ Rotor } & Radial & 12 & 16 & 18 \\
\cline { 2 - 5 } & Circumferential & 3 & 11 & 13 \\
\hline & TOTAL & $\mathbf{4 5}$ & $\mathbf{7 4}$ & $\mathbf{7 8}$ \\
\hline
\end{tabular}

Table A4.2a Excess loss components for winding connection type A.

\begin{tabular}{|c|c|c|c|c|}
\cline { 3 - 5 } \multicolumn{2}{c|}{} & \multicolumn{3}{c|}{ Iron Loss (W) } \\
\cline { 3 - 5 } \multicolumn{2}{c|}{} & Method1 & Method 2 & Method 3 \\
\hline \multirow{3}{*}{ Stator } & Radial & 7 & 15 & 16 \\
\cline { 2 - 5 } & Circumferential & 33 & 41 & 42 \\
\hline \multirow{3}{*}{ Rotor } & Radial & 15 & 16 & 18 \\
\cline { 2 - 5 } & Circumferential & 4 & 11 & 13 \\
\hline & TOTAL & $\mathbf{5 9}$ & $\mathbf{8 3}$ & $\mathbf{8 9}$ \\
\hline
\end{tabular}

Table A4.2b Excess loss components for winding connection type B. 


\begin{tabular}{|c|c|c|c|c|}
\cline { 3 - 5 } \multicolumn{2}{c|}{} & \multicolumn{3}{c|}{ Iron Loss (W) } \\
\cline { 3 - 5 } \multicolumn{2}{c|}{} & Method1 & Method 2 & Method 3 \\
\hline \multirow{3}{*}{ Stator } & Radial & 7 & 6 & 13 \\
\cline { 2 - 5 } & Circumferential & 15 & 18 & 23 \\
\hline \multirow{3}{*}{ Rotor } & Radial & 24 & 9 & 26 \\
\cline { 2 - 5 } & Circumferential & 10 & 5 & 18 \\
\hline \multirow{1}{*}{} & TOTAL & $\mathbf{5 6}$ & $\mathbf{3 8}$ & $\mathbf{8 0}$ \\
\hline
\end{tabular}

Table A4.3a Hysteresis loss components for winding connection type A.

\begin{tabular}{|c|c|c|c|c|}
\cline { 3 - 5 } \multicolumn{2}{c|}{} & \multicolumn{3}{c|}{ Iron Loss (W) } \\
\cline { 3 - 5 } \multicolumn{2}{c|}{} & Method1 & Method 2 & Method 3 \\
\hline \multirow{3}{*}{ Stator } & Radial & 7 & 13 & 13 \\
\cline { 2 - 5 } & Circumferential & 22 & 28 & 29 \\
\hline \multirow{2}{*}{ Rotor } & Radial & 9 & 10 & 11 \\
\cline { 2 - 5 } & Circumferential & 2 & 5 & 6 \\
\hline & TOTAL & $\mathbf{4 0}$ & $\mathbf{5 6}$ & $\mathbf{5 9}$ \\
\hline
\end{tabular}

Table A4.3b Hysteresis loss components for winding connection type B 


\section{CHAPTER 5}

\section{AERODYNAMIC LOSS}

\subsection{Introduction}

In many high-speed applications such as that considered in this thesis, aerodynamic or so-called 'windage' losses can become a key design consideration, since they can constitute a significant proportion of the overall drive system loss, in turn constraining the attainable performance. Hence, the design of an SR machine with a maximum speed of $100,000 \mathrm{rpm}$ should embrace aerodynamic considerations, since refinements which result in incremental benefits in terms of electromagnetic performance, may in turn have a dramatic and adverse effect on the aerodynamic losses. As a consequence, the ability to predict the magnitude of aerodynamic losses reliably is an important factor in the design of high-speed SR machines. To this end, a detailed computational fluid dynamics (CFD) study was undertaken in order to quantify the likely magnitude of the losses and to establish the influence of various design parameters. A cross-section through a typical three-phase 6/4 SR machine is shown in figure 5.1. There are three principal features of SR machines that influence the magnitude of the aerodynamic losses:

The airgap height - the radial clearance between the rotor and stator teeth when they are in the aligned position, denoted as $l_{g}$ in figure 5.1. In common with other singly excited electrical machines, SR machines are normally designed with relatively small airgaps. Indeed, as was discussed in section 3.1, the airgap selected for the prototype EATG was selected as $0.2 \mathrm{~mm}$

The geometry of the rotor - The regions between individual rotor teeth are recessed from the outer diameter in order to achieve the degree of rotor saliency necessary for torque production. As discussed in chapter 2, there are a number of conflicting mechanical and electromagnetic considerations in the selection of an 
appropriate fillet radius ratio. However, the depth of the recess between adjacent teeth will affect the air flow and hence the magnitude of the aerodynamic losses to some extent, as demonstrated later in section 5.3.1. All the rotors considered in this chapter have a pole arc of $35^{\circ}$.

The geometry of the stator bore - The stator lamination of an SR machine is by necessity a salient slotted structure. In the simplest case, the windings and associated insulation/impregnation materials in the machine do not fully fill the slot, resulting in a stepped airgap profile, which in turn can significantly influence the flow in the airgap region. However, a cylindrical stator bore can be achieved by incorporating specially designed non-magnetic slot closures, or by encapsulating the entire stator. Encapsulation using a high thermal conductivity resin is an attractive option for high-performance machines as it considerably enhances the heat transfer capability from the stator windings and provides additional protection to the windings. Although such an encapsulation technique was not eventually employed in the prototype, the aerodynamic study presented in this chapter was restricted to SR machines having cylindrical stator bores. This simplification was adopted to considerably reduce the computational and postprocessing requirements of the analysis, in that a single solution with an imposed velocity can be employed, rather than a transient time stepped approach.

\subsection{Simulation Techniques}

The general nature of the flow in a constant height airgap due to a rotating cylindrical rotor approximates closely to Couette type flow, provided that the airgap height (with respect to the overall rotor diameter) is relatively small. Analytical techniques for the prediction of turbulent Couette flow around a rotating cylinder, which generally employ a number of empirically determined constants, have been developed and comprehensively validated [5.1,5.2]. Such techniques have been applied to the prediction of the flow around cylindrical rotors [5.2]. However, because of the complex geometry of SR machines, the published research relating to losses 
associated with rotating smooth cylinders is of limited utility, and recourse to numerical modelling techniques is likely to be required in order to achieve a useful level of accuracy for design purposes. As a consequence, aerodynamic losses were calculated numerically using a CFD package (FLUENT Version 4.4).

A critical consideration in calculating the flow, is to establish whether the flow is laminar or turbulent. In the case of cylindrical rotors the flow is characterised by the Reynolds number:

$$
R e=\frac{u_{w} h_{g}}{v}
$$

Fully developed turbulent flow occurs for conditions which give rise to a Reynolds number greater than 2000 or so. In the case of a cylindrical rotor, this equation implies that many of the combinations of rotor diameter, airgap height and rotational speed considered in this chapter do not correspond to fully turbulent flow. However, it is clearly somewhat difficult to apply this formula directly to the complex geometry of an SR rotor. In this case the flow regime alternates between developing Couette type flow in the narrow airgap and a radial circulation between the rotor teeth, so that significant turbulence levels are likely to survive throughout the flow even at lower rotor speeds and small airgaps. As a consequence, for all the SR rotors considered the flow was modelled using a standard Reynolds Stress Model approach for turbulent flow at all values of rotational speed.

Alternative techniques available for turbulent flow, such as the standard and renormalisation group $\mathrm{k}-\varepsilon$ models, have a tendency to calculate spuriously large turbulence generation rates around flow impingers and corners (such as the sharp corners of the rotor teeth). This excess turbulent energy in turn overestimates the turbulent viscosity [5.3]. Further, the ability of the Reynolds stress method to accommodate two separate orthogonal components of turbulent viscosity lends itself to calculating the flow in SR machines, which is characterised by the presence of both a circumferential flow and a significant radial centrifugal acceleration. 
The flow distribution in close proximity to the rotor and stator surfaces was represented by using one of two wall functions depending on whether the cell centres lie within the laminar sub-layer. If the innermost cell centre lies within the laminar sub-layer, the standard Newtonian wall function is employed, whereas if the innermost cell centre lies outside the laminar sub-layer, a standard log-law of the wall is employed, the appropriate wall function being automatically selected. In all cases, zero-slip boundaries are enforced on the rotor surface and stator bore.

\subsection{Simulation Results}

\subsubsection{TWO-DIMENSIONAL ANALYSIS}

For a 4-pole SR rotor rotating within a cylindrical stator bore, the initial analysis was performed using a series of two-dimensional models, as this provides a means of establishing the influence of the various geometrical parameters with minimal computational effort.

Two rotors were modelled initially in order to investigate the influence of the fillet radius on the magnitude of the aerodynamic losses. These rotors were selected with the two extreme values of fillet radius ratio, viz. 0.025 and 0.3 , while both have the same outer diameter and airgap of $50 \mathrm{~mm}$ and $0.2 \mathrm{~mm}$ respectively. The rotors were modelled at rotational speeds up to $7000 \mathrm{rad} / \mathrm{s}$ (in $1000 \mathrm{rad} / \mathrm{s}$ increments). The rotational periodicity of the rotor was exploited to reduce the problem domain to one quarter of the machine section. Figures $5.2 \mathrm{a}$ and $5.2 \mathrm{~b}$ show the finite volume mesh employed in the analysis for fillet radii of 0.025 and 0.3 respectively.

The calculated power loss as a function of rotor speed for these two extreme values is shown in figure 5.3, which for comparison, also includes the corresponding loss for a (50mm diameter) cylindrical rotor with both laminar and turbulent flow assumptions. It is worth noting that the CFD predicted losses in figure 5.3 for the cylindrical rotor 
are in good agreement with previously published measured results for turbulent flow around a cylindrical rotor with a narrow airgap [5.1]. It is evident from figure 5.3 that using two-dimensional analysis, the loss predicted is slightly higher for the case of a rotor with a smaller fillet. Although the nature of the flow is influenced by the form of the inter-polar fillet, as shown in the flow vector diagrams (of the leading rotor edge) in figure 5.4a and 5.4, the resulting loss is nevertheless relatively insensitive to the fillet radius ratio, e.g. at $7000 \mathrm{rad} / \mathrm{s}$ the difference is only some $13 \%$ for these two extreme cases. Given the considerably greater influence that fillet radius ratio plays in determining centrifugal stress levels in high speed rotors, this is likely to be the overriding consideration in design, even if it involves some marginal compromise in the aerodynamic losses. On this basis, the subsequent CFD analysis was restricted to consideration of a rotor having a fillet radius ratio of 0.13 , which was established in chapter 2 as being the preferred ratio in terms of the utilisation of rotor material (albeit that the final design was modified somewhat by the practical rotor assembly requirements)

Although the airgap height has been fixed for the prototype EATG, it is useful to assess the aerodynamic loss over a wide range of airgaps in order to determine the nature of the trade-off between electromagnetic torque and aerodynamic loss. To investigate the influence of the airgap and the rotor diameter on the aerodynamic losses, 9 models were considered, embracing all inclusive combinations of diameters of 25,50 and $75 \mathrm{~mm}$ and airgaps of $0.1,0.2$ and $0.3 \mathrm{~mm}$

Using the loss figures generated by the two-dimensional CFD models, the variation in the calculated power loss per unit length as a function of rotational speed can be curve fitted to a reasonable approximation, by the equation:

$$
P=A \omega^{B} \quad(\mathrm{~W} / \mathrm{m})
$$

The calculated values of A and B for the 9 cases considered are listed in table 5.1. Over this range of diameters and airgaps, the aerodynamic losses increase with rotational speed with a relatively consistent exponent value of approximately 2.7 . It 
is interesting to compare this value with that for the corresponding cylindrical rotors and airgap, in which the losses increase with an approximate exponent of 2.2 for turbulent flow conditions.

\begin{tabular}{|c|c|c|c|}
\hline \multirow[b]{2}{*}{$\begin{array}{l}\text { Airgap height } \\
\text { (mm) }\end{array}$} & \multicolumn{3}{|c|}{ Rotor diameter } \\
\hline & $25 \mathrm{~mm}$ & $50 \mathrm{~mm}$ & $75 \mathrm{~mm}$ \\
\hline 0.1 & $\begin{array}{c}A=2.38 \times 10^{-8} \\
B=2.60\end{array}$ & $\begin{array}{c}A=2.41 \times 10^{-7} \\
B=2.61\end{array}$ & $\begin{array}{c}A=1.08 \times 10^{-6} \\
B=2.59\end{array}$ \\
\hline 0.2 & $\begin{array}{c}A=1.69 \times 10^{-8} \\
B=2.62\end{array}$ & $\begin{array}{c}A=1.68 \times 10^{-7} \\
B=2.62\end{array}$ & $\begin{array}{c}A=1.07 \times 10^{-6} \\
B=2.58\end{array}$ \\
\hline 0.3 & $\begin{array}{c}A=1.44 \times 10^{-8} \\
B=2.63\end{array}$ & $\begin{array}{c}A=3.70 \times 10^{-8} \\
B=2.79\end{array}$ & $\begin{array}{c}A=2.57 \times 10^{-7} \\
B=2.73\end{array}$ \\
\hline
\end{tabular}

Table 5.1. Calculated values of A and B for an SR rotor with a fillet radius ratio of 0.13

Figures $5.5 \mathrm{a}$ and $5.5 \mathrm{~b}$ show the variation of stator wall shear stress over one pole of the rotor for the case of a $50 \mathrm{~mm}$ diameter rotor with an airgap of $0.2 \mathrm{~mm}$ and a fillet radius ratio of 0.13 rotating at $1000 \mathrm{rad} / \mathrm{s}$ and $7000 \mathrm{rad} / \mathrm{s}$ respectively. Also shown for comparison in figures $5.5 \mathrm{a}$ and $5.5 \mathrm{~b}$ are the corresponding shear stresses calculated by CFD (using RSM) for a cylindrical rotor having the same airgap and rotating at the same angular velocity.

In both cases, there is a large peak in the shear stress distribution on the stator wall in the vicinity of the leading edge of the rotor tooth, with a reasonably constant value over the trailing two-thirds or so of the narrow airgap region. In the $1000 \mathrm{rad} / \mathrm{s}$ case, 
there is reasonable correlation in terms of magnitude between the near constant shear stress region within the airgap and the value calculated for a cylindrical rotor. However, for the $7000 \mathrm{rad} / \mathrm{s}$ case, the shear stress throughout the narrow airgap region is considerably higher than that calculated for a cylindrical rotor, which is evidence of the increasingly significant contribution of a pressure differential between the leading and trailing faces of the tooth as the rotor speed increases. As a consequence, although the shear stress generated within the narrow airgap region is the dominant contributor to the overall windage loss in SR machines, a simple geometrical scaling approach applied to published calculation techniques for cylindrical rotors with small airgaps is inappropriate, particularly at high speed.

\subsubsection{THREE-DIMENSIONAL ANALYSIS}

Two-dimensional analysis is useful in establishing the relative influence of the various geometrical parameters on the aerodynamic losses, and in providing an estimate of the loss per unit axial length. Three-dimensional analysis may however be necessary in many cases, particularly for rotors of relatively short aspect ratios for which both end-effects and axial pumping become significant. In order to investigate the relative merits of two and three-dimensional analyses, a series of threedimensional CFD calculations were performed. Given the considerably greater computational overhead involved in three-dimensional analysis, the investigation was restricted to 5 rotors, each having the same rotor cross section $(50 \mathrm{~mm}$ diameter with a fillet radius ratio of 0.13 and a radial airgap of $0.2 \mathrm{~mm}$ ) and axial lengths of 10 , $25,50,75$ and $100 \mathrm{~mm}$

In each case an axial clearance of $20 \mathrm{~mm}$ was included between the end of the rotor and the stationary wall representing the motor end-cap, in order to allow for the space required in practical machines to accommodate the overhanging stator end windings. In all cases, the stator was assumed to have a cylindrical inner bore. The finite volume mesh in each case was constructed using first-order hexahedral elements, the number of cells in each mesh being approximately 4000 . 
The predicted power loss for the various rotor axial lengths are summarised in table 5.2, together with equivalent values derived from scaling the power loss calculated from a two-dimensional model. The results in table 5.2 indicate a very large difference between the calculation techniques for the case of the shortest rotor, while even for the longest rotor, the error is still significant, being approximately $43 \%$ of the three-dimensional calculated value. The source of the significant errors between 2D and 3D analysis become apparent when the losses are segregated into three separate loss components on different regions of the stator wall, viz. the stator bore region directly above the active length of the rotor, the overhanging stator bore region above the shaft, and the end wall of the stator.

\begin{tabular}{|c|c|c|c|c|c|}
\hline $\begin{array}{c}\text { Rotor Axial } \\
\text { Length } \\
(\mathrm{mm})\end{array}$ & $\begin{array}{c}\text { Power loss } \\
\text { derived by } \\
\text { scaling 2D } \\
\text { analysis (W) }\end{array}$ & $\begin{array}{c}\text { Power loss } \\
\text { predicted by 3D } \\
\text { analysis(W) }\end{array}$ & \multicolumn{4}{|l|}{ Components of 3D calculated stator losses (W) } \\
\cline { 4 - 6 } & 21 & 109 & $\begin{array}{c}\text { Stator bore } \\
\text { region directly } \\
\text { above rotor }\end{array}$ & $\begin{array}{c}\text { Overhanging } \\
\text { stator bore } \\
\text { region }\end{array}$ & $\begin{array}{c}\text { Stator end } \\
\text { wall }\end{array}$ \\
\hline 10 & 52 & 154 & 63 & 64 & 20 \\
\hline 25 & 104 & 215 & 124 & 70 & 22 \\
\hline 50 & 155 & 276 & 188 & 67 & 22 \\
\hline 75 & 207 & 335 & 246 & 67 & 22 \\
\hline 100 & & & & & \\
\hline
\end{tabular}

Table 5.2. Comparison of two dimensional and three-dimensional predictions of aerodynamic losses for the reference SR machine at $7000 \mathrm{rad} / \mathrm{s}$ (Rotor O.D 50mm, $0.2 \mathrm{~mm}$ airgap and fillet radius ratio of 0.13 )

As shown in table 5.2, the component of the three-dimensional calculated losses in the region directly above the active section of the rotor are in adequate agreement with those calculated by scaling the two-dimensional losses by the rotor axial length. For each of the axial lengths, the difference between the calculated losses in this 
region is approximately $20 \%$. The contribution to losses from the overhanging stator bore region and the end wall remain relatively constant with increasing rotor axial length. This relatively constant 'end-effect' is well illustrated by figs. 5.6a and 5.6b, which show the calculated velocity vectors (relative to the rotating reference frame) immediately above the stator surface for both the $25 \mathrm{~mm}$ and $100 \mathrm{~mm}$ long rotors at $5000 \mathrm{rad} / \mathrm{s}$. As is evident from figs. $5.6 \mathrm{a}$ and $5.6 \mathrm{~b}$, the velocity vectors in both cases only depart from a purely circumferential flow over the end $5 \mathrm{~mm}$ or so of the rotor axial length. This is further evidenced by the stator wall shear stress contours shown in figures $5.7 \mathrm{a}$ and $5.7 \mathrm{~b}$.

\subsection{Experimental Validation}

In order to assess the accuracy of the various calculation techniques employed, aerodynamic losses were measured on a representative SR rotor using the test rig shown in figures 5.8 and 5.9. An SR rotor with an overall diameter of $50 \mathrm{~mm}$, fillet radius ratio of 0.13 , shaft diameter $11 \mathrm{~mm}$ and axial length $60 \mathrm{~mm}$, was manufactured from solid mild steel with a ground surface finish. The rotor was attached to a turbine assembly from a commercial automotive turbocharger (Holset WH1E). The assembled rotor structure was fitted with 50,000rpm rated high-speed precision ceramic ball bearings, and dynamically balanced to a grade of G2.5 as specified in ISO1940 [5.4].

The rotor assembly was mounted inside a smooth cylindrical Aluminium tube with a nominal radial clearance of $0.2 \mathrm{~mm}$ and an axial overhang at either end of $15 \mathrm{~mm}$.

The turbine was driven from a regulated compressed air supply of sufficient flow capability to allow rotor speeds up to $50,000 \mathrm{rpm}$ to be achieved. The reaction torque on the Aluminium tube was measured using a swinging frame arrangement with a torque arm acting on a $25 \mathrm{~g}$ load cell (Pioden UF1) located at a distance of $90 \mathrm{~mm}$ from the axis of rotation.

Fig. 5.10 shows the measured power loss (stator bore reaction torque $\times$ rotor speed) as a function of rotor speed up to 50,000rpm. Also shown, for comparison is the 
corresponding loss on the stator bore predicted by three-dimensional CFD, i.e. the component associated with the end-cap (which is typically some $10 \%$ or so) is subtracted from the total predicted loss. Reasonable correlation was achieved between the measured and predicted losses, with a maximum error of some $22 \%$ at the upper end of the speed range. The magnitude of the error between measured and predicted losses is consistent with published results for a rotating cylinder using a similar arrangement of apparatus and a similar CFD analysis [5.2]. It interesting to note that the measured losses in this case increase with angular velocity with an exponent of 2.97, as compared to values of 2.62 and 2.81 calculated using two and three-dimensional CFD analysis respectively. 


\section{REFERENCES}

[5.1] L.A Dorfman, "Hydrodynamic Resistance and the Heat Loss of Rotating Solids", published by Oilver and Boyd Ltd. 1963.

[5.2] P.M Wild, N. Djilali, G.W Vickers 'Experimental and Computational Assessment of Windage Losses in Rotating Machinery', Journal of fluids Engineering, Trans. of the ASME, 1996, Vol. 118, pp116-122.

[5.3] Fluent Users Manual, Version 4.4, Fluent Inc 1996

[5.4] 'Mechanical Vibration - Balance Quality Requirements for Rigid Rotors Part 1: Determination of Permissible Residual Unbalance', ISO 1940-1 Standard. 1986 (First Edition) 


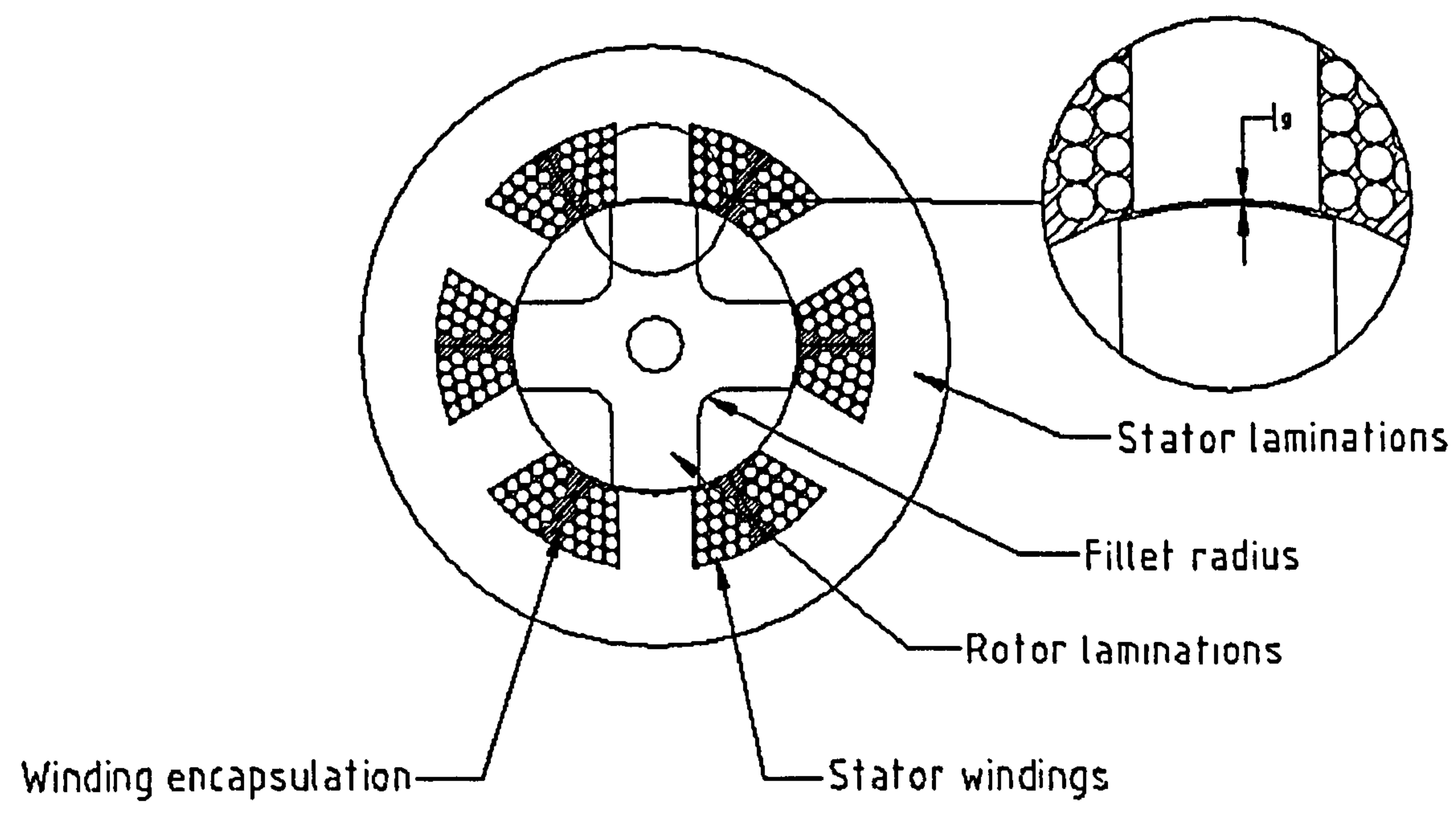

Figure 5.1. Cross-section of a three-phase 6-4 SR machine 


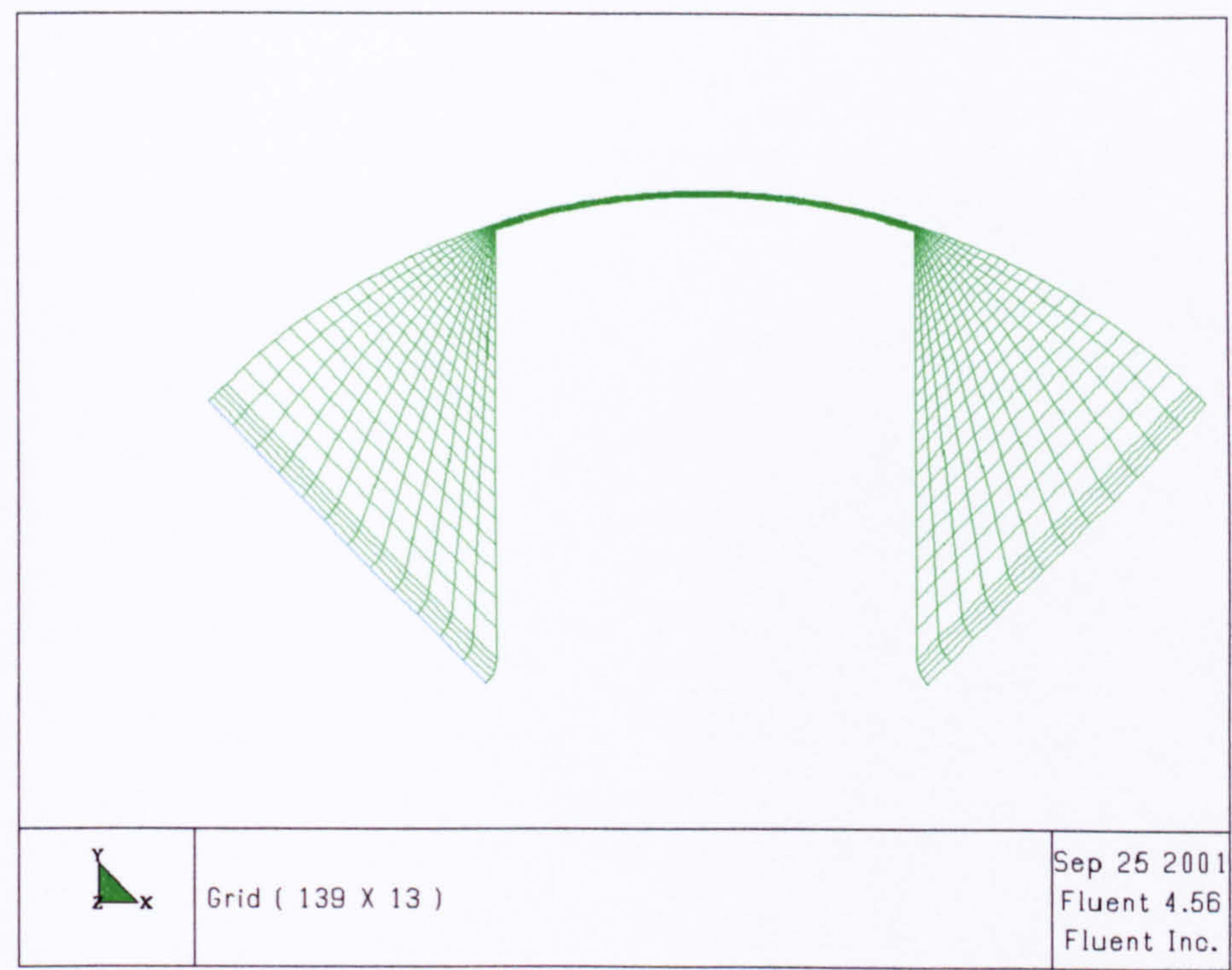

Figure 5.2a Finite volume mesh for rotor with fillet radius ratio of 0.025 with 1960 first order cells (rotor diameter $50 \mathrm{~mm}$, airgap height $0.2 \mathrm{~mm}$ )

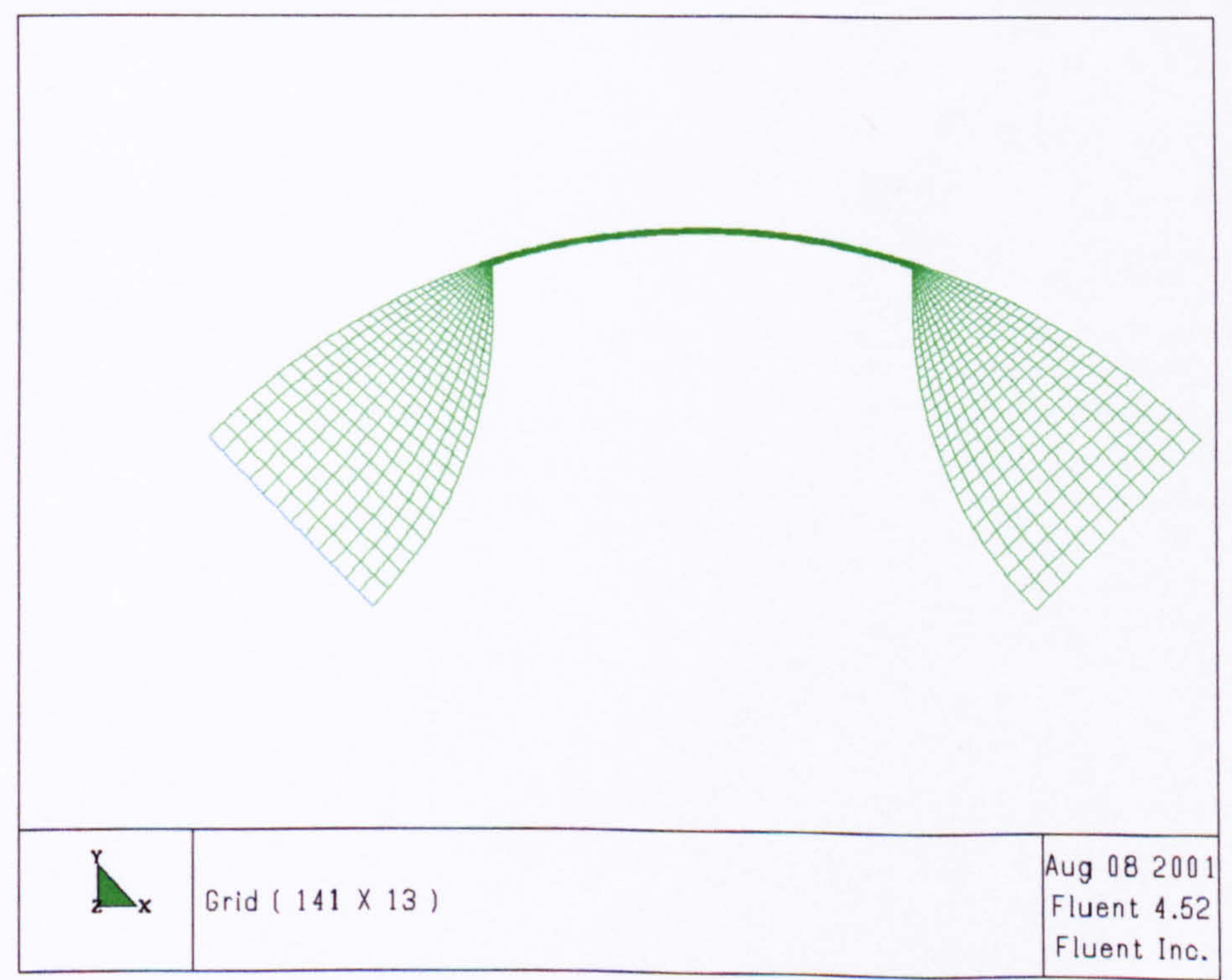

Figure 5.2b Finite volume mesh for rotor with fillet radius ratio of 0.3 with 1988 first order cells (rotor diameter $50 \mathrm{~mm}$, airgap height $0.2 \mathrm{~mm}$ ) 


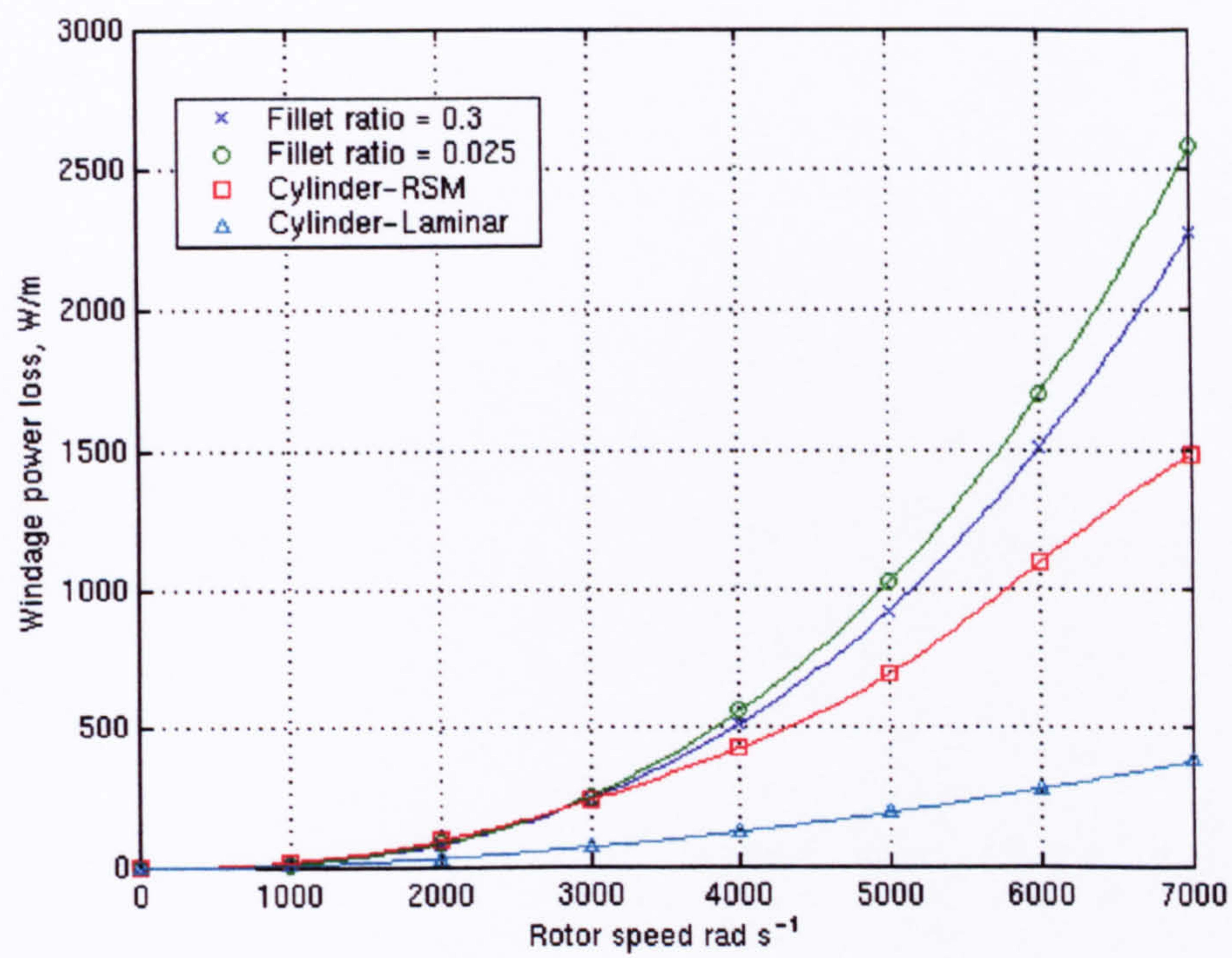

Figure 5.3 Calculated aerodynamic losses per unit axial length for SR rotors with fillet radius ratios of 0.025 and 0.3 and for a cylindrical rotor (airgap height $0.2 \mathrm{~mm}$; rotor diameter $50 \mathrm{~mm}$ ) 


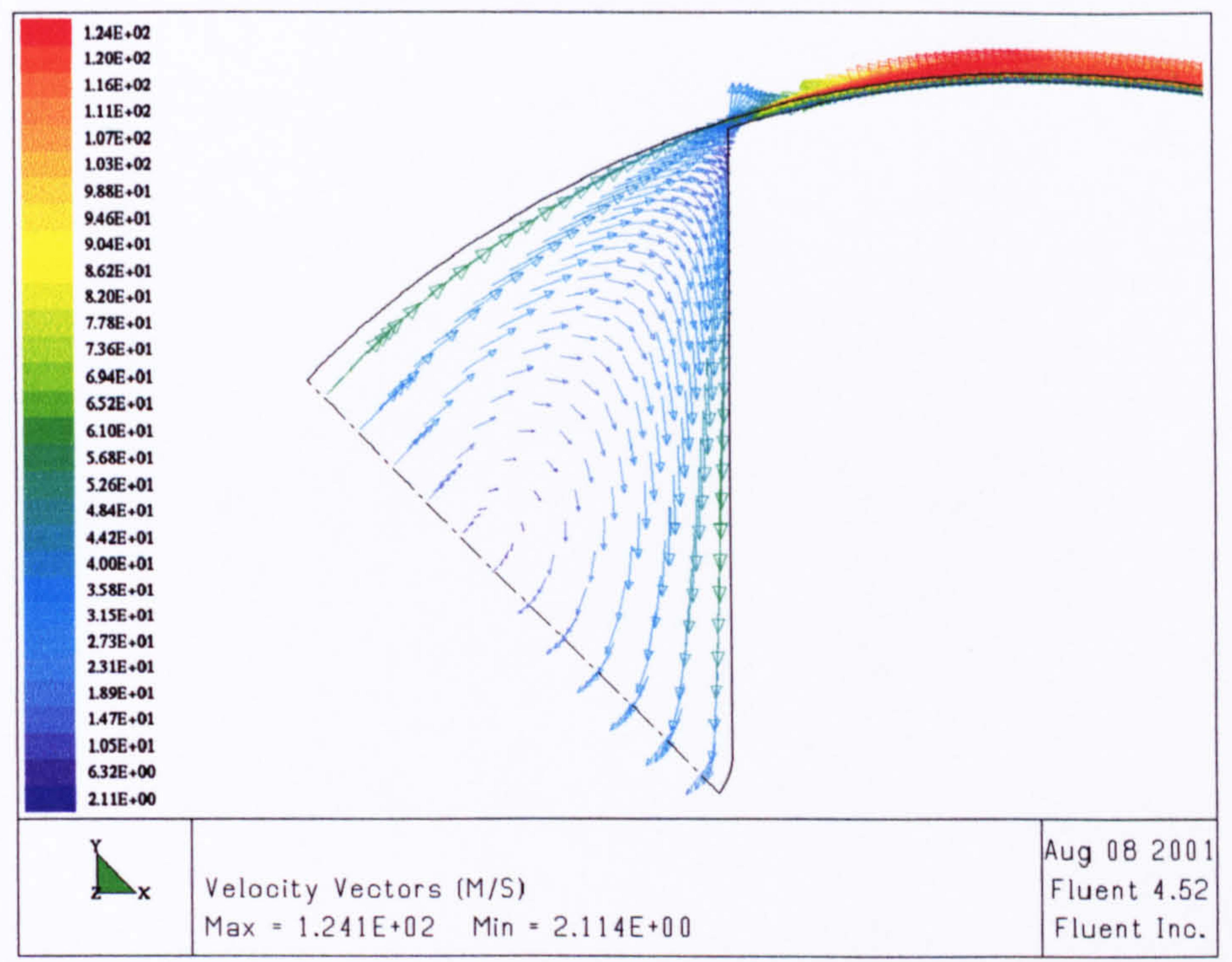

Figure 5.4a Two-dimensional velocity vectors relative to rotating reference frame $\left(0.2 \mathrm{~mm}\right.$ airgap, $50 \mathrm{~mm}$ rotor, fillet radius ratio of 0.025 at $\left.7000 \mathrm{rad} \mathrm{s}^{-1}\right)$.

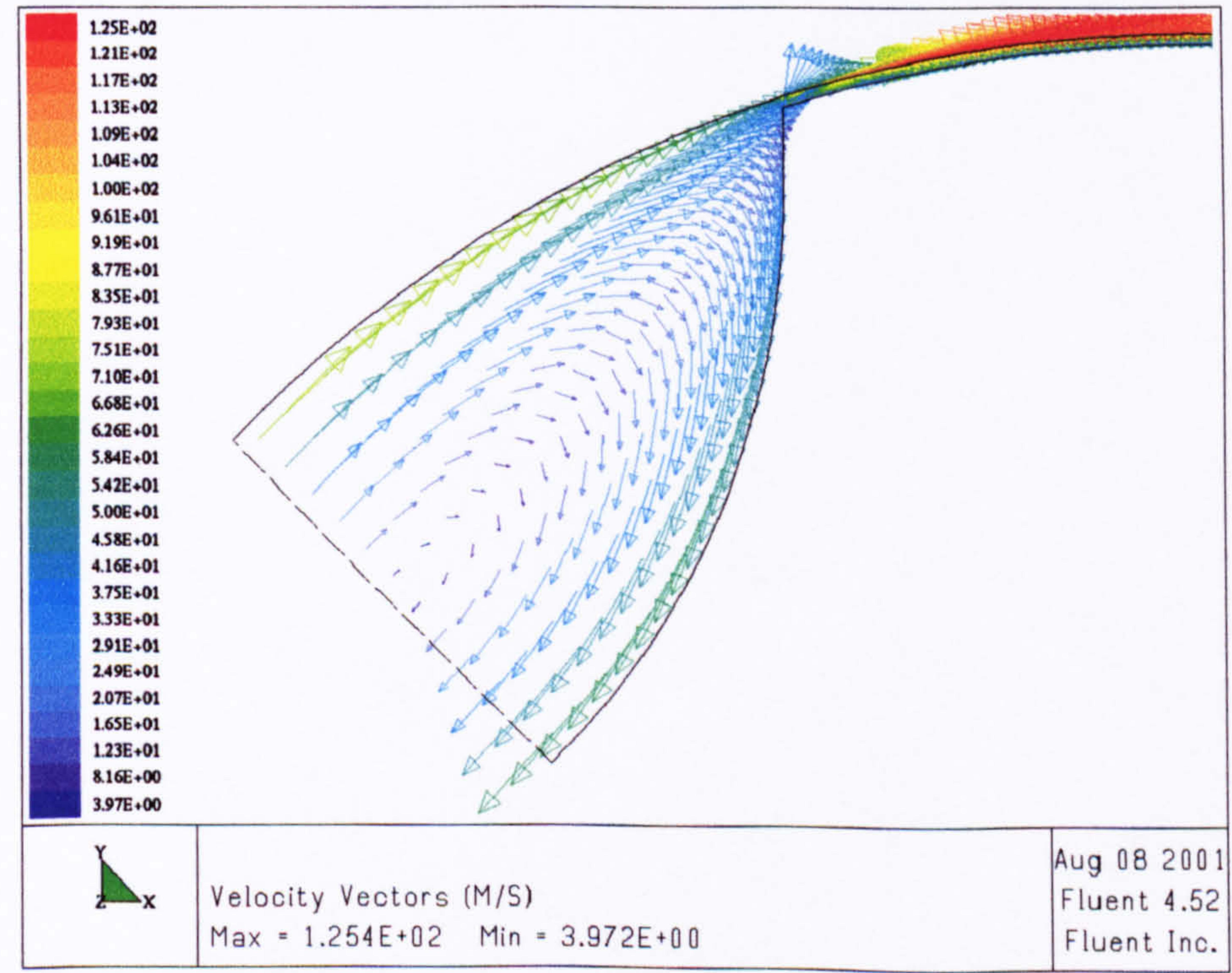

Figure 5.4a Two-dimensional velocity vectors relative to rotating reference frame $\left(0.2 \mathrm{~mm}\right.$ airgap, $50 \mathrm{~mm}$ rotor, fillet radius ratio of 0.3 at $\left.7000 \mathrm{rad} \mathrm{s}^{-1}\right)$. 


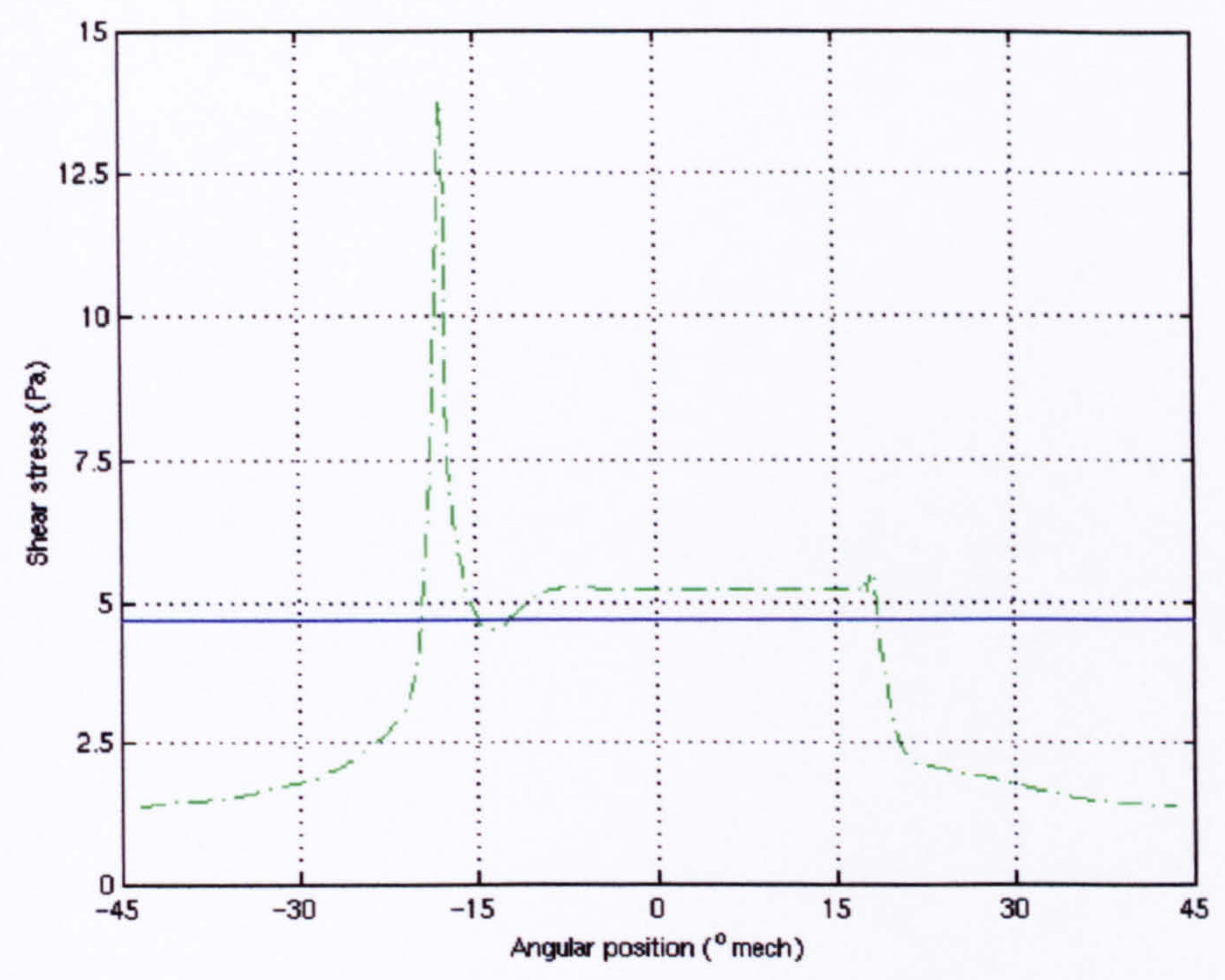

Figure 5.5a. Stator wall shear stress over one quarter of the machine circumference for the SR and cylindrical rotor at $1000 \mathrm{rad} \mathrm{s}^{-1}$. $(0.2 \mathrm{~mm}$ airgap; fillet radius ratio $0.13,50 \mathrm{~mm}$ diameter)

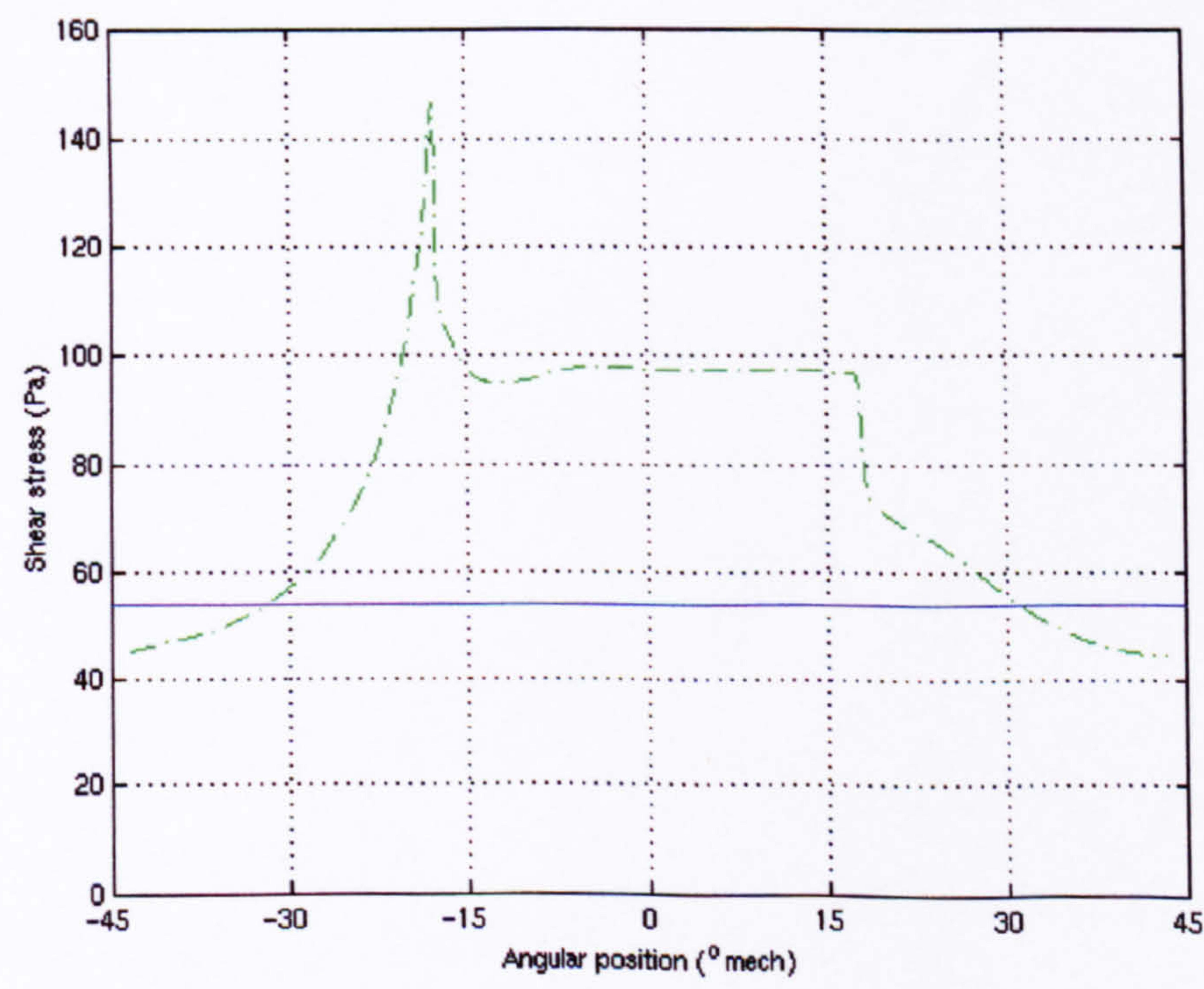

Figure 5.5b. Stator wall shear stress over one quarter of the machine circumference for the SR and cylindrical rotor at $7000 \mathrm{rad} \mathrm{s}^{-1}$. $(0.2 \mathrm{~mm}$ airgap; fillet radius ratio $0.13,50 \mathrm{~mm}$ diameter.) 


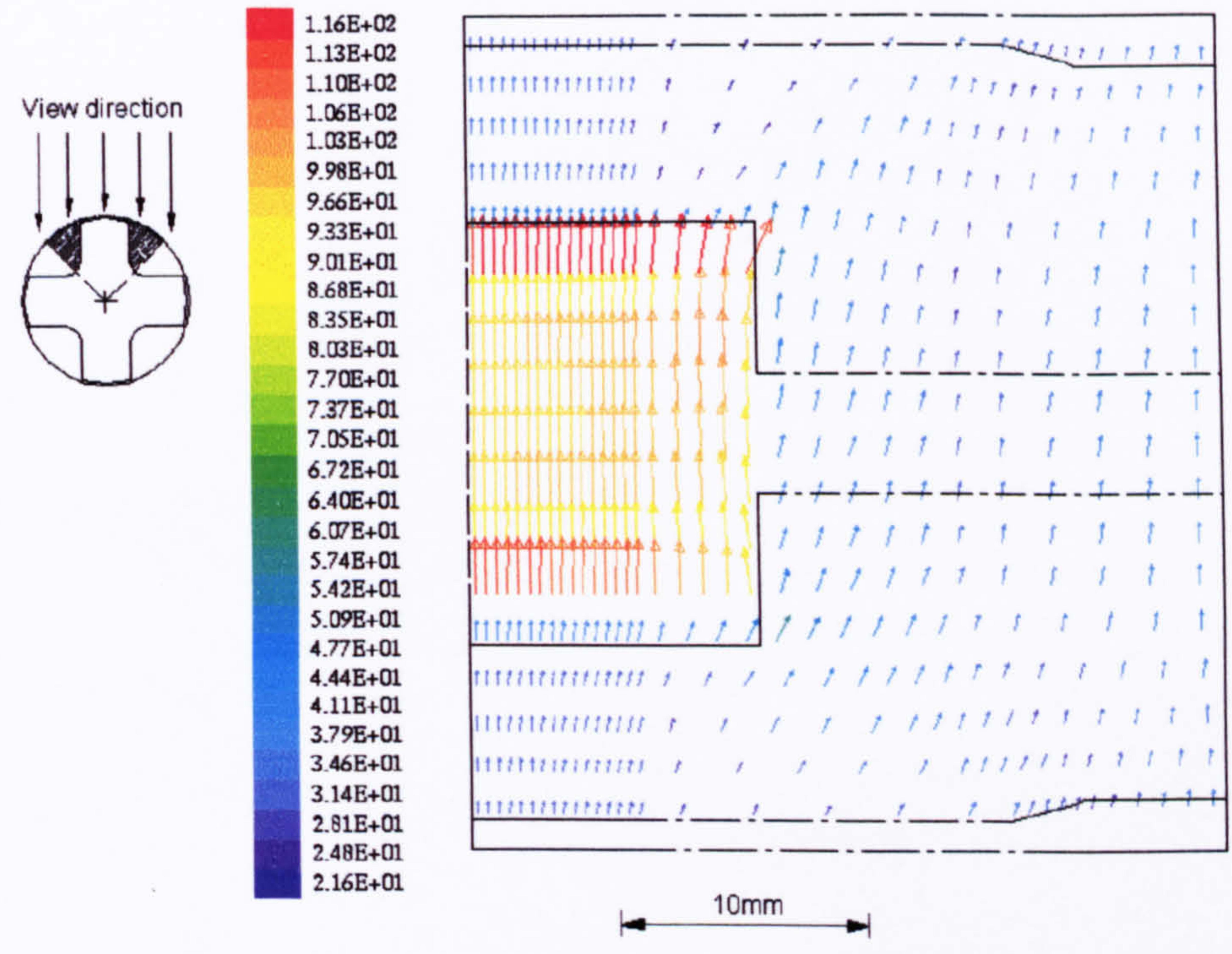

Figure 5.6a. Plan view of velocity vectors immediately adjacent to the stator wall in the three-dimensional model (axial length of $25 \mathrm{~mm}$ ).

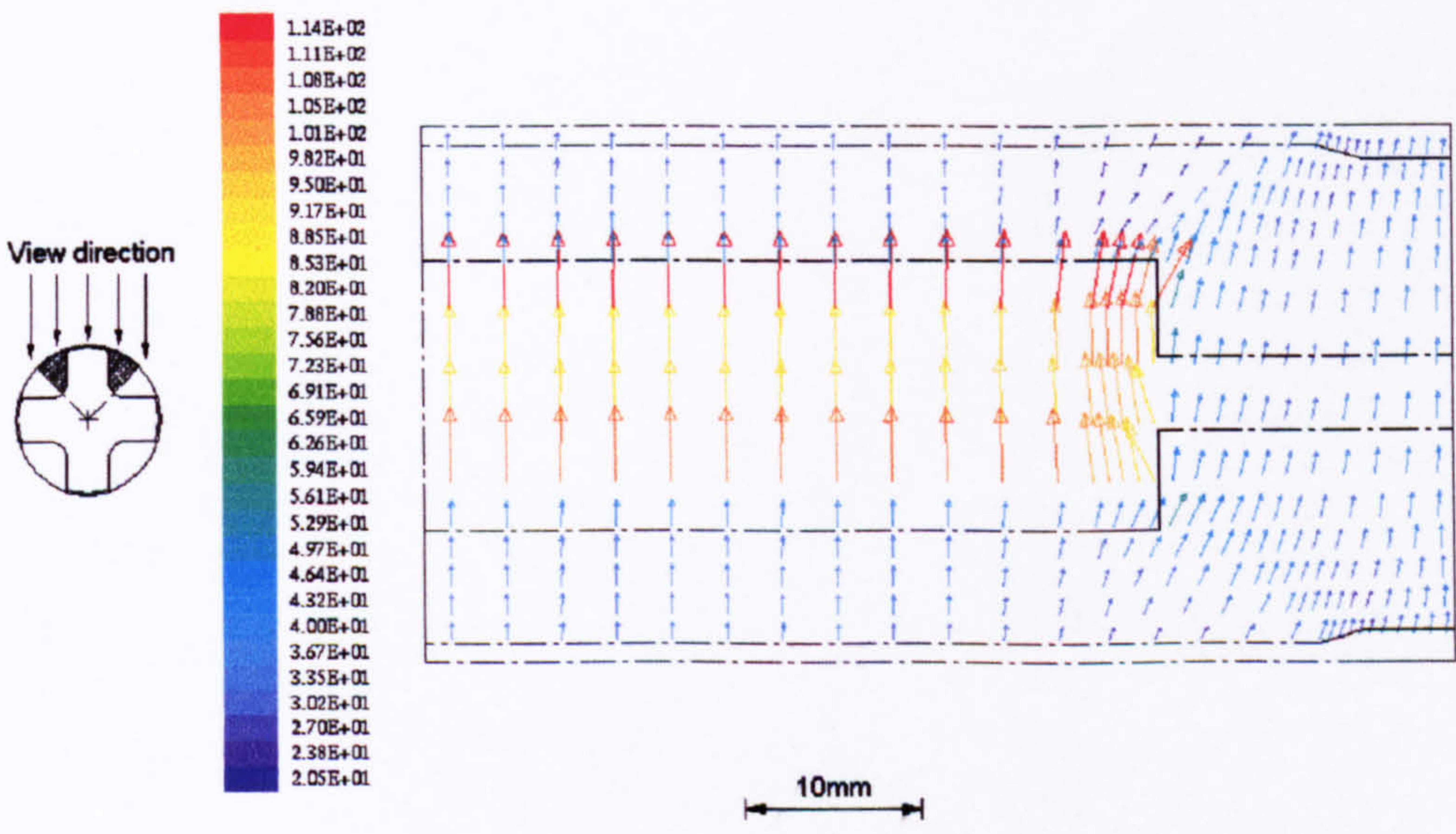

Figure 5.6b. Plan view of velocity vectors immediately adjacent to the stator wall in the three-dimensional model (axial length of $100 \mathrm{~mm}$ ). 


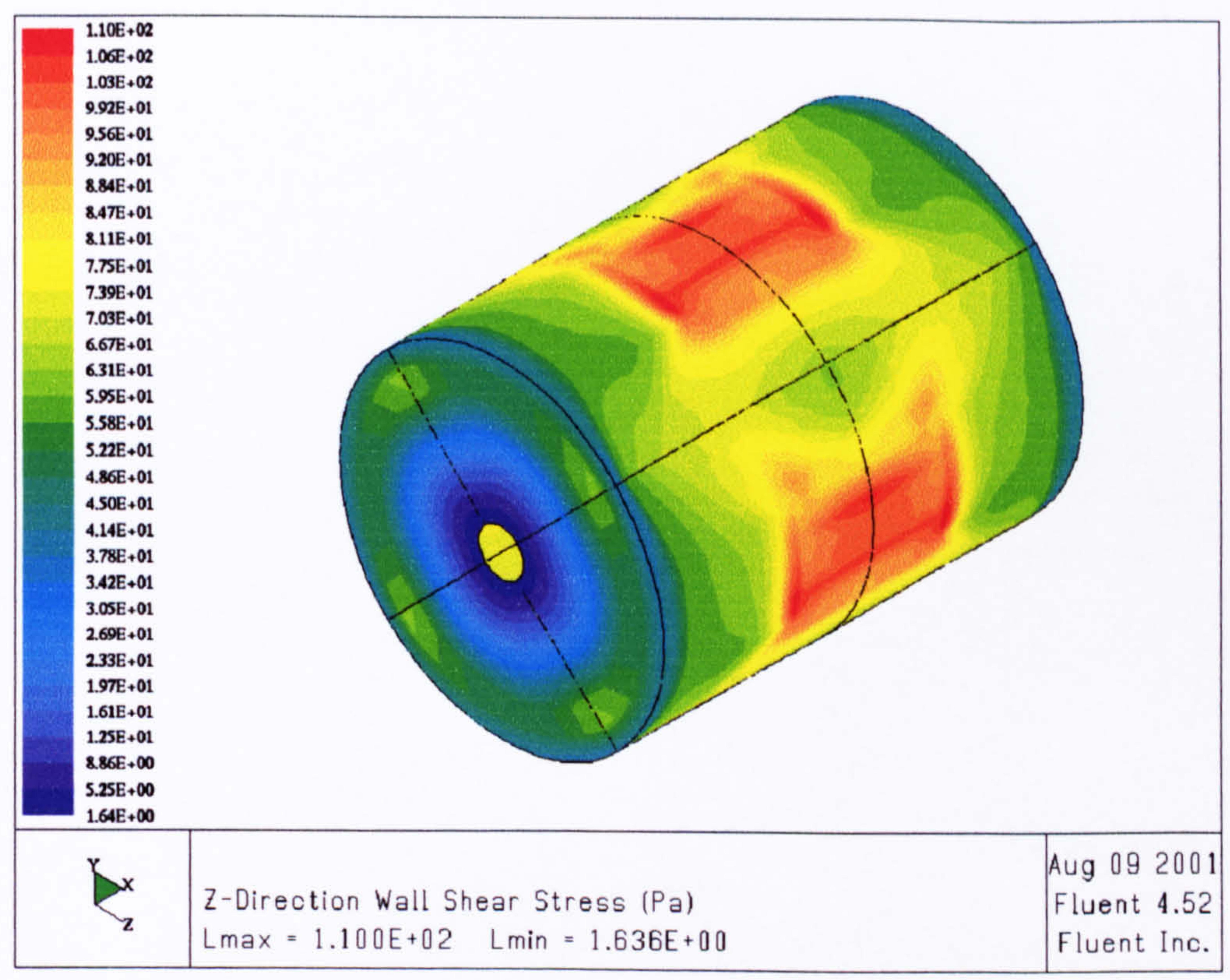

Figure 5.7a. Stator wall circumferential shear stress for the entire stator and end-caps (axial length $25 \mathrm{~mm}$ )

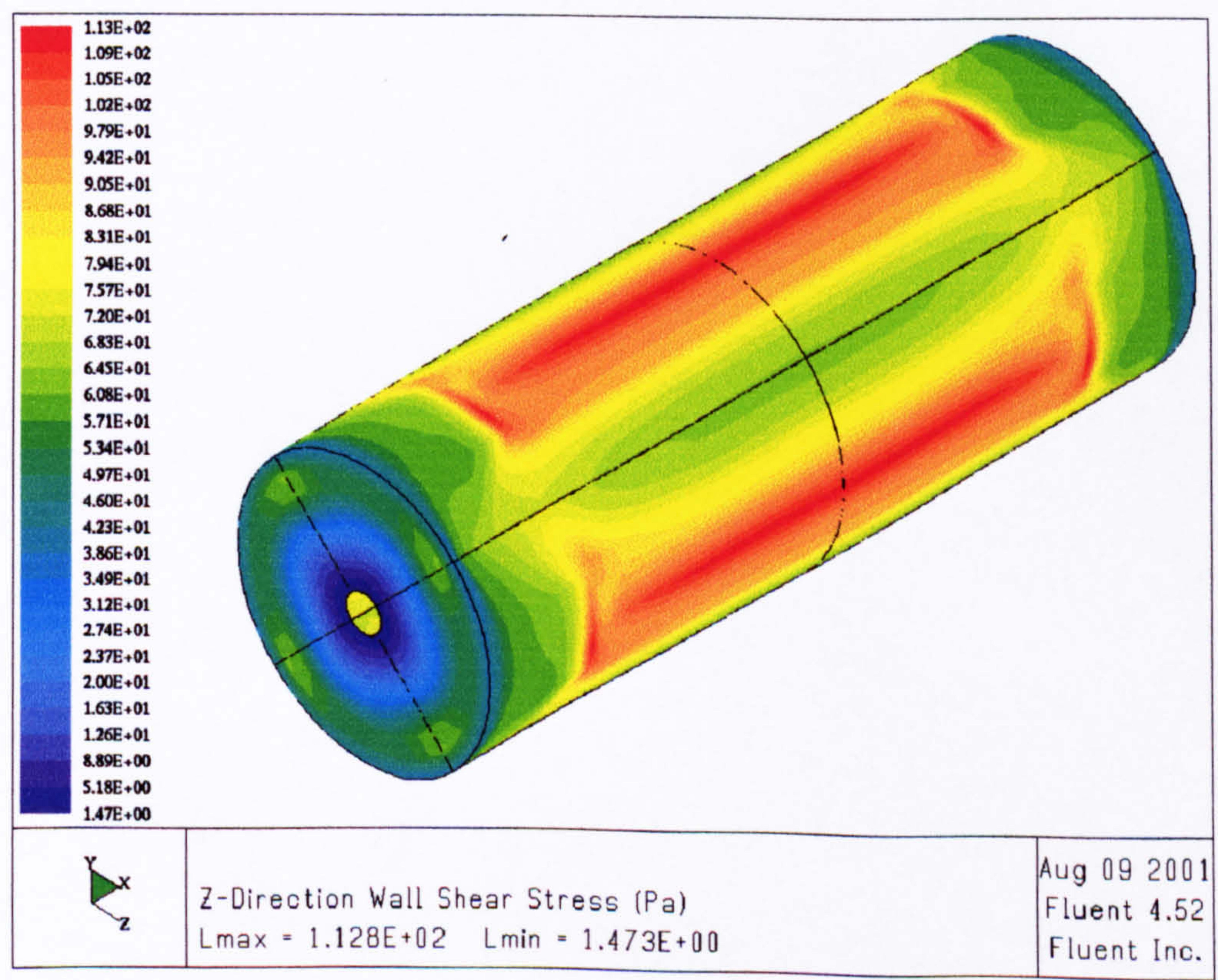

Figure 5.7b. Stator wall circumferential shear stress for the entire stator and end-caps (axial length $100 \mathrm{~mm}$ ) 


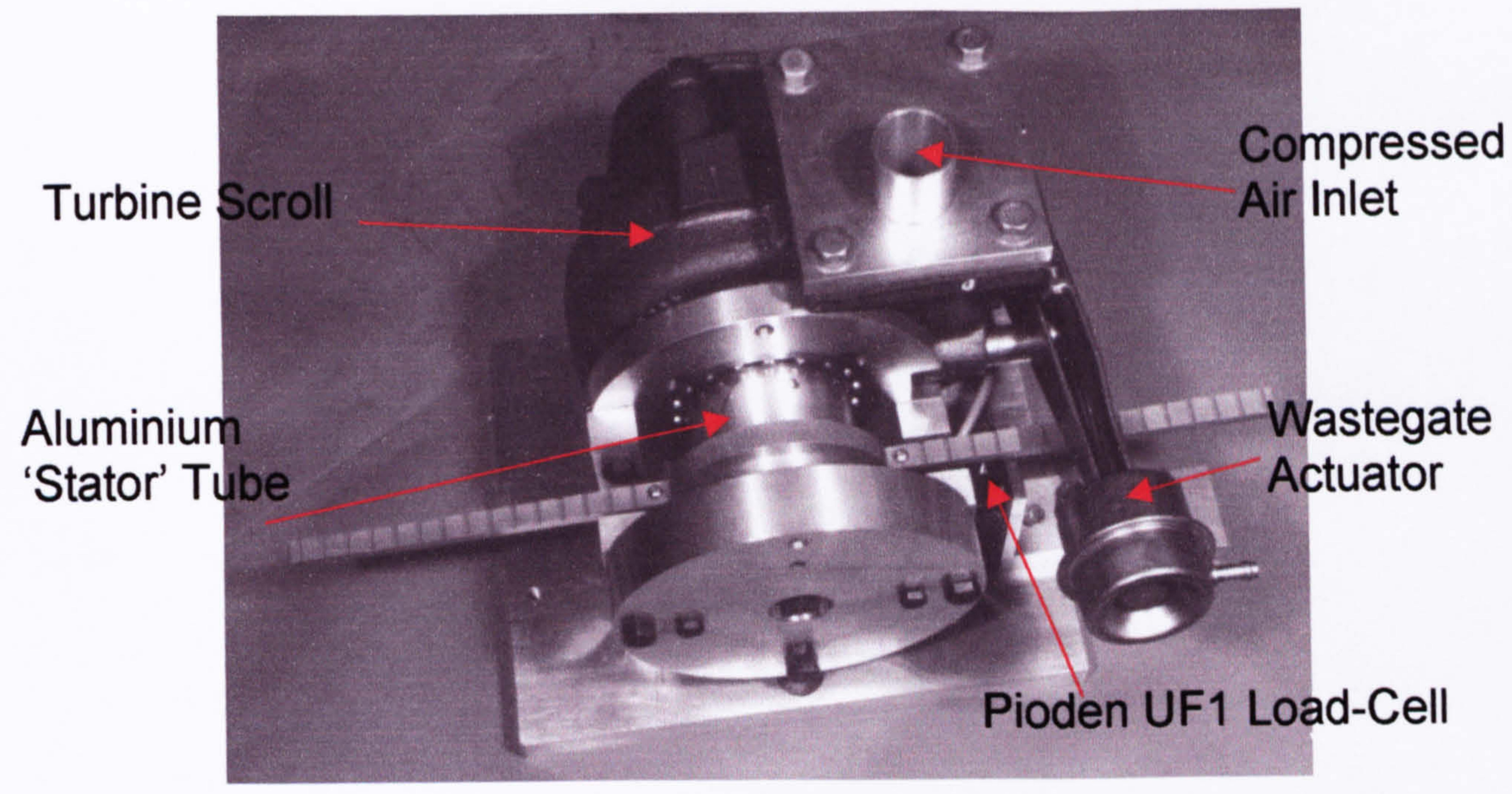

Figure 5.8. Photograph of the experimental apparatus

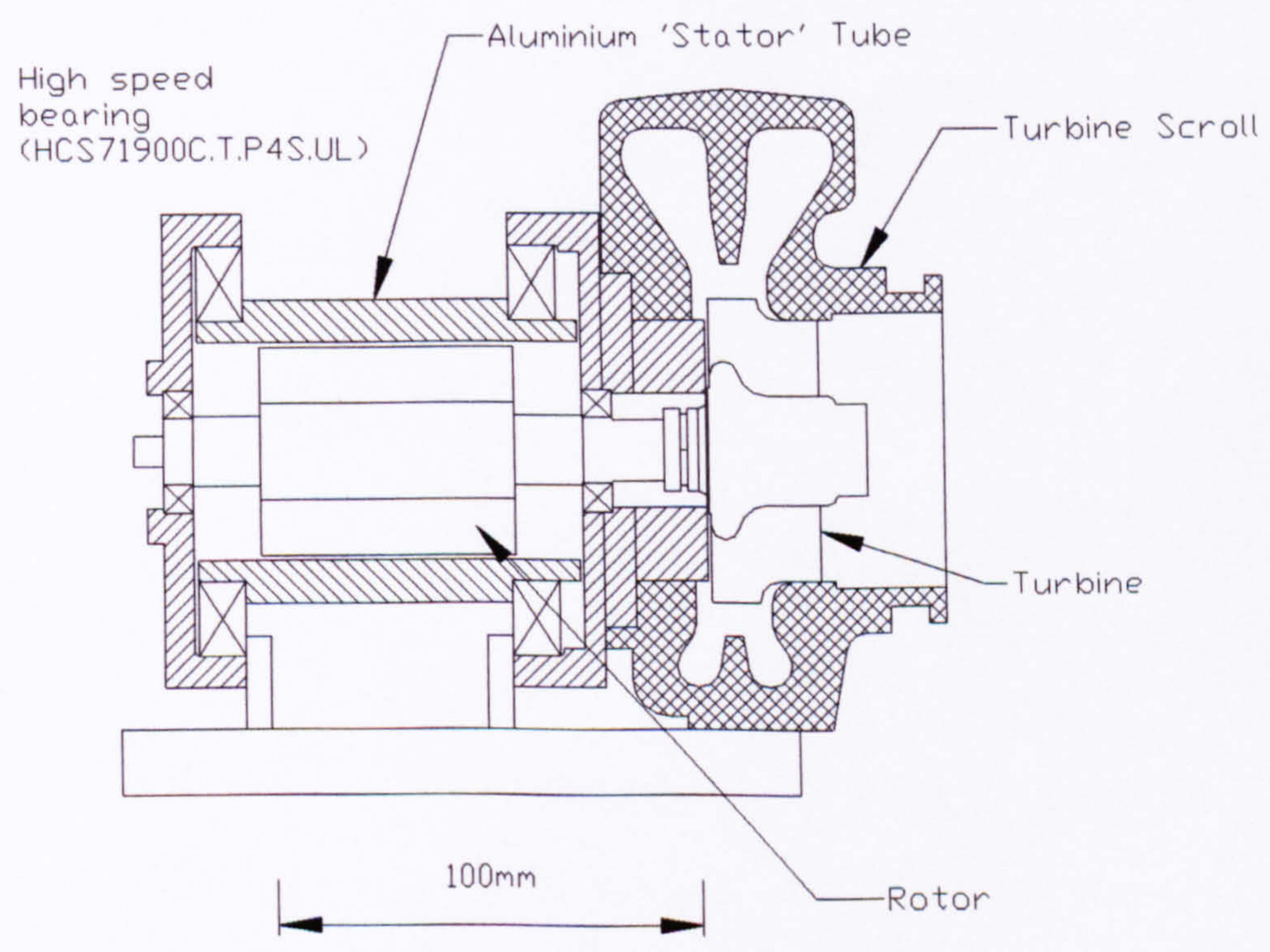

Figure 5.9. Cross section of the experimental apparatus. 


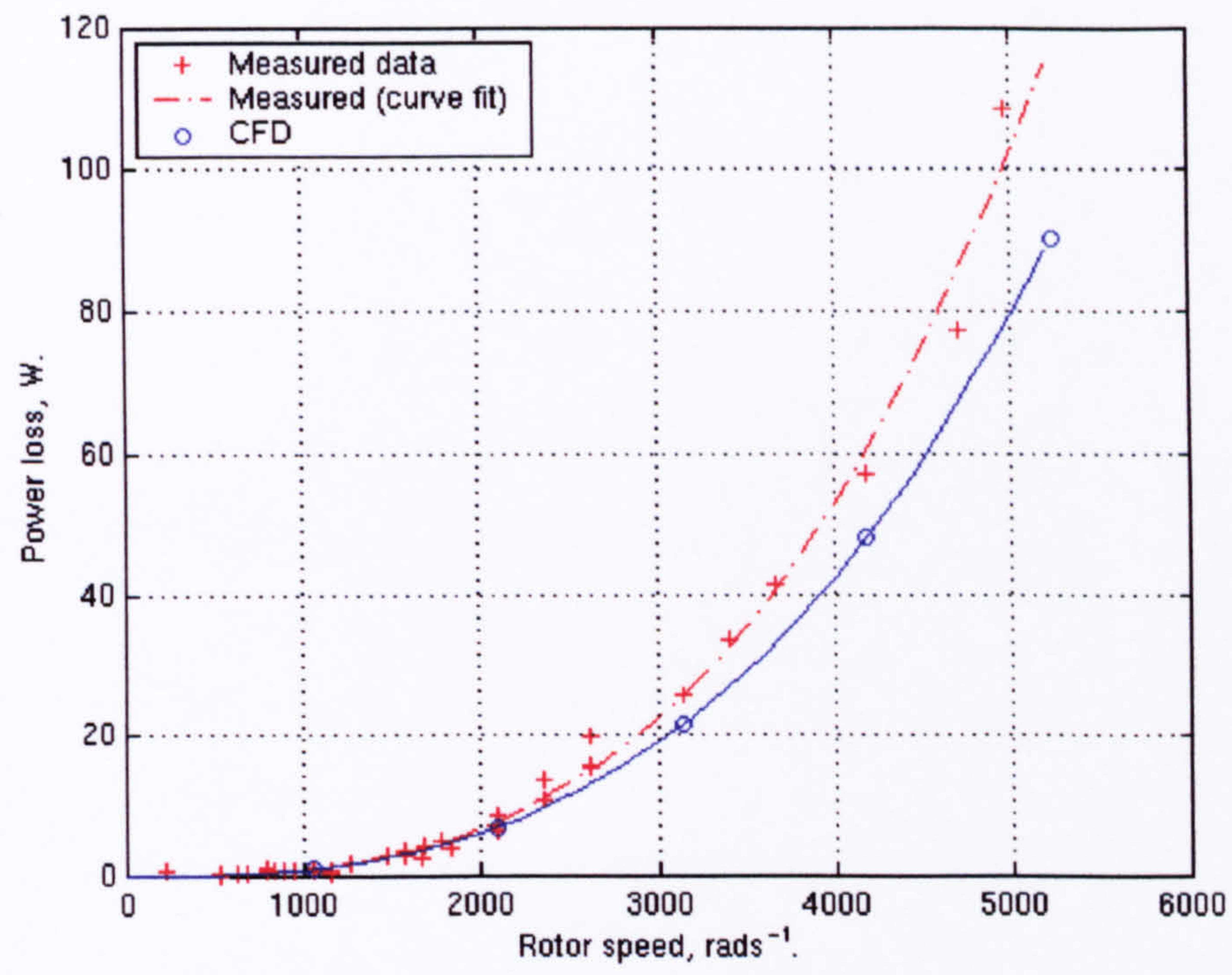

Figure 5.10. Measured power loss as a function of rotor speed, together with the corresponding values derived from CFD. 


\section{CHAPTER 6}

\section{BEARING SELECTION AND ROTORDYNAMIC ANALYSIS}

\subsection{Introduction}

The high specific powers and efficiencies exhibited by turbo-machinery are achieved mainly through operation at very high shaft speeds. Indeed, in order to achieve high efficiencies in radial flow turbines and centrifugal compressors, shaft speeds of the order of $150000 \mathrm{rpm}$ are commonplace. The design of these systems must embrace the many mechanical issues related to such high-speed operation, viz. the high inertial loads, shaft whirl, vibration and rotordynamic instability. In order to provide a basis on which the relative merits of various shaft/bearing arrangements can be assessed and to ensure the reliable operation of the prototype machine it is necessary to undertake appropriate dynamic analysis of the proposed system.

The main objective of dynamic analysis is the prediction of critical speeds (where the synchronous response of the rotor/bearing system to shaft unbalance is a maximum). This provides a framework to make modifications to the rotor and/or bearing system, so as to either move the critical speeds out of the operating speed range or to limit the response to acceptable levels, hence allowing the shaft to progress through the various critical speeds without damage. The full potential of a dynamic analysis can often not be realised prior to manufacture since the prediction of the amplitudes of the synchronous vibration due to rotor imbalance is difficult to achieve due to uncertainties arising from the exact distribution of mass imbalance along the rotor axial length, the rotor/bearing system damping and the typical highly non-linear stiffness. Further uncertainty arises when the dynamic parameters of the system are considered, since while static mass imbalance in all cases produces whirling which is synchronous with the shaft speed, not all shaft whirling is synchronous with shaft 
speed, and indeed the most destructive rotordynamic problems are associated with non-synchronous whirl [6.1]. The analysis must therefore be extended to consider the threshold speeds and causes of rotordynamic instability associated with nonsynchronous whirl, but for the purposes of this study the discussion of such instabilities is restricted to a qualitative analysis of the system.

\subsubsection{JEFFCOTT ANALYSIS}

A useful starting point in terms of analysing many rotor/bearing systems is to analyse the so-called Jeffcott rotor, which comprises a large single cylindrical disk mounted on a compliant shaft [6.2]. The Jeffcott analysis is useful in terms of highlighting some of the design issues associated with synchronous whirl, and in its simplest form this analysis provides three suggested methods of minimising the amplitudes of synchronous whirl i.e.,

- The rotor must be balanced to the highest tolerance to minimise the disturbing force.

- Critical speeds can be avoided by tailoring the material properties and/or rotor dimensions or by moving the operational speed range of the rotor.

- Damping should be added to the rotor/bearing system to limit the amplitude of vibration.

In some cases (examples cited include helicopter turbo-shaft engines and synchronous motors [6.1]) the geometry of the rotor is such that the system can be analysed to an adequate level using the simplified Jeffcott analysis. However, complications arise in many practical shaft systems because gyroscopic effects modify the critical speeds and the mass is often distributed along the shaft (i.e. more than one disk requiring multi-plane balancing). The applicability of the Jeffcott analysis is sometimes restricted further by the speed-dependent properties of hydrodynamic journal bearings, shaft seals and aerodynamic effects. In the case of a four-pole SR rotor, a further consideration is the non-uniform mass distribution and hence directional nature of the stiffness, which results from the salient rotor 
geometry. As a consequence, for the particular rotor/bearing system in the proposed prototype, the Jeffcott analysis provides little detailed insight into the likely system performance, although the general guidelines listed above remain pertinent. In the absence of a reliable analytical technique for this complex geometry, a structural finite element based approach was employed in order to determine the critical speeds to an appropriate accuracy,

\subsection{Bearing Configuration}

The geometry of the three components which are mounted on the rotor shaft, viz. the lamination stack, compressor and turbine, have been established with due account of the constraints of the centrifugal stress and/or electromagnetic performance requirements. However, there are numerous options in terms of the rotor configuration, i.e. the relative position of these three components and the bearings on the shaft, which in turn have consequences in terms of rotordynamic performance. Whereas rotordynamic performance is clearly a major factor in determining the preferred configuration, other factors such as mechanical complexity, aerodynamic efficiency and thermal limits of the bearings may also need to be taken into account. For a conventional turbocharger consisting of a turbine and compressor mounted on a single shaft, there are four possible bearing configurations as shown in figure 6.1 [6.3], each of which has been employed at some time in commercial devices.

The outboard design of figure 6.1a provides minimum radial loading on the bearings due to moments arising from mass unbalance and hence the bearings provide the maximum radial support for the shaft. The location allows easy maintenance of the bearings and adequate space for a self contained lubrication system and an oil or water-cooling jacket at the turbine end to maintain the bearing temperature within acceptable limits. The design is relatively complicated in terms of the number of individual components required on the stator, while the large bearing separation makes the system prone to misalignment due to the accumulation of tolerances. The design of figure $6.1 \mathrm{~b}$, which is referred to as an inboard bearing design, is very 
widely used in automotive turbochargers. It has the simplest construction and is the most compact of all the designs due to the low stator component count and the use of a single piece bearing housing which contains the two bearings and oil channels. The design is ideally suited to the radial flow turbine and centrifugal compressor combination since the bearings do not lie in the flow path and hence the induction housing is simple and efficient in construction. However this layout is more complex in terms of the rotor dynamics, in particular the gyroscopic effects associated with the relatively short distance between the bearings and the cantilevered arrangement of the compressor and turbine wheels.

A combination of these inboard and outboard designs is shown in figure 6.1c. In this case, one of the bearings, usually the compressor bearing, is mounted in the outboard sense and the turbine bearing is mounted inboard to alleviate the need to cool the bearing. This design however is relatively complex and costly (as it requires two bearing housings). The effects of the overhung, cantilevered turbine (which generally has a significantly higher mass than the compressor) remains a potential problem. The fourth design adopted by De Laval Co. [6.3] is shown in figure 6.1d, and has outboard bearings mounted at one end only (again the cooler compressor end). The bearing housing has very simple construction and provides good access for maintenance. The shaft in this case is generally operated above the first critical speed in bending in order to minimise the cantilevered rotor vibrations during normal running, and hence the stiffness and, perhaps more importantly, the damping of the rotor/bearing system must be carefully determined and not allowed to vary during the natural degradation of the system during the life of the turbocharger.

For the purpose of designing the turbogenerator prototype it was decided to adopt the inboard bearing design shown in figure 6.2 with the SR rotor mounted between the two bearings, as this is mechanically the simplest design, and is well suited to the incorporation of an SR rotor. This configuration is the same as that employed in the range of turbochargers manufactured by Holset, therefore allowing the greatest commonality with commercially available turbine and compressor components. However, as discussed above, this rotor/bearing configuration is not ideal in a 
rotordynamic sense due to the cantilevered masses. This is compounded by the longer (and hence lower stiffness) shaft, additional mass and the relatively low contribution to shaft stiffness made by the laminated SR rotor structure. In addition, the stress considerations discussed in chapter 2 resulted in a shaft diameter that is slightly smaller that in a standard turbocharger, further compromising the stiffness.

The $\mathrm{m}^{\text {th }}$ critical speed of the rotor $\omega_{m}$ is given by:

$$
\omega_{m}=2 \pi \sqrt{\frac{k_{m}}{m_{m}}}
$$

where $k_{m}, m_{m}$ are the modal stiffness and modal mass of the $m^{\text {th }}$ mode respectively.

Hence, the reduced stiffness of the turbogenerator rotor and its increased modal mass will inevitably reduce the first critical speed in bending as compared to the corresponding standard turbocharger rotor. Given that commercial turbochargers already operate at speeds that are a significant proportion of the first critical speed, it is likely that the proposed turbogenerator rotor will operate close to, or even above, the first critical speed. As a consequence, due care must be taken with regard to rotor and bearing design and manufacturing of the prototype.

One further challenge to the design is that the shaft is assembled from many individual components. It is desirable under such circumstances to ensure that the number of interfaces is kept to a minimum and the fits between the components are as precise and tight as possible. However, it is inevitable given the number of components in the prototype that manufacturing tolerances and the corresponding fits between components will impact to a marked degree on the absolute accuracy with which the rotor can be modelled using a-priori data, which essentially comprises nominal dimensions and material properties. 


\section{3 'Free-Free' Modal Analysis}

As discussed above, the inclusion of an SR rotor with the consequent increase in rotating mass and shaft length will inevitably impact on the rotor-dynamic behaviour. It is important, in terms of selecting bearings and/or the final mechanical design of the rotor, to establish quantitatively the extent to which the rotor dynamic performance is likely to be modified. Ultimately, the overall response is governed by the entire rotor and bearing system. However, a useful indication of the relative performance of a conventional turbocharger shaft assembly and one which includes an SR rotor can be obtained from the so-called 'free-free' modal analysis. In this analysis, the various modal frequencies of the two shaft assemblies can be deduced for the particular case of free vibration with no external constraints, i.e. in the absence of any bearings.

The leading dimensions of the turbogenerator rotor and the equivalent conventional turbocharger rotor are shown in figure 6.3. For the purposes of this modal analysis, it is desirable to represent the turbine and compressor as lumped masses (each exhibiting the same mass and polar and transverse inertia as the actual components) rather than very detailed models of the actual turbine and compressor blade geometries. This necessarily means that the modal vibrations of the blades themselves are neglected. However, these are common factors in both rotor designs and their inclusion within the model was deemed to be outside the scope of this thesis. The mass, inertia and axial length of the actual components are listed in table 6.1, where the transverse inertia is defined as that about the mass centre. 


\begin{tabular}{|l|c|c|}
\hline & Compressor & Turbine \\
\hline Mass, $\mathrm{kg}$. & 0.087 & 0.29 \\
\hline Polar Inertia, $\mathrm{kgm}^{2}$. & $2 \times 10^{-5}$ & $8 \times 10^{-5}$ \\
\hline Transverse inertia, $\mathrm{kgm}^{2}$. & $2 \times 10^{-5}$ & $6.6 \times 10^{-5}$ \\
\hline Axial length, mm. & 32.5 & 40 \\
\hline
\end{tabular}

Table 6.1 Mass and inertia of the turbine and compressor components utilised in the prototype design.

It is evident that since the ratio of the polar moment to the transverse moment is not simply 2 as is the case in a plain cylinder, then it is not possible to represent the turbine and compressor components with single cylinder equivalents. The turbine and compressor are both represented by a series of three cylinders spanning the axial length of the respective components, as shown in figure 6.4. The dimensions of the three cylinders in each case, $l_{1}, l_{2}, l_{3}, D_{1}, D_{2}$ and $D_{3}$ are determined using the following equations:

$$
\begin{gathered}
l_{\text {tot }}=l_{1}+l_{2}+l_{3} \\
m_{t o t}=m_{1}+m_{2}+m_{3}=\frac{\pi D_{1}^{2} l_{1} \rho}{4}+\frac{\pi D_{2}^{2} l_{2} \rho}{4}+\frac{\pi D_{3}^{2} l_{3} \rho}{4} \\
I_{p}=I_{p 1}+I_{p 2}+I_{p 3}=\frac{\pi}{32}\left(D_{1}^{4}-D_{0}^{4}\right) l_{1} \rho+\frac{\pi}{32}\left(D_{2}^{4}-D_{0}^{4}\right) l_{2} \rho+\frac{\pi}{32}\left(D_{3}^{4}-D_{0}^{4}\right)_{3} \rho \\
I_{t}=I_{t 1}+I_{t 2}+I_{t 3}=\frac{\pi}{64}\left(D_{1}^{4}-D_{0}^{4}\right) l_{1} \rho+\left(r_{c}-l_{1} / 2\right)^{2} m_{1}+\frac{\pi}{64}\left(D_{2}^{4}-D_{0}^{4}\right) l_{2} \rho+\left(r_{c}-l_{1}-l_{2} / 2\right)^{2} m_{2}+ \\
\frac{\pi}{64}\left(D_{3}^{4}-D_{0}^{4}\right) l_{3} \rho+\left(r_{c}-l_{1}-l_{2}-l_{3} / 2\right)^{2} m_{3}
\end{gathered}
$$


where the location of the mass centre, $r_{c}$, is defined as the axial distance from the rear face of cylinder 1 to the mass centre, and can be determined by taking moments, i.e.:

$$
r_{c}=\frac{l_{1}\left(m_{1}+2 m_{2}+2 m_{3}\right)+l_{2}\left(m_{2}+2 m_{3}\right)+l_{3} m_{3}}{2\left(m_{1}+m_{2}+m_{3}\right)}
$$

With only four independent equations and six variables, there is no direct solution for the three-disk approximation. Therefore, the dimensions of the three cylinders were established by an iterative approach (implemented using Matlab), in which a large number of combinations were systematically assessed, the final values being derived by reference to the relative errors. The resulting three-disk approximations for both the compressor and turbine are listed in table 6.2, the worst case error being less than $5 \%$.

\begin{tabular}{|l|c|c|c|}
\hline & Compressor & Turbine & Units \\
\hline$l_{1}$ & 2.167 & 8.0 & $\mathrm{~mm}$ \\
\hline$l_{2}$ & 4.875 & 4.0 & $\mathrm{~mm}$ \\
\hline$l_{3}$ & 25.458 & 28.0 & $\mathrm{~mm}$ \\
\hline$D_{1}$ & 75.0 & 56.0 & $\mathrm{~mm}$ \\
\hline$D_{2}$ & 32.2 & 46.0 & $\mathrm{~mm}$ \\
\hline$D_{3}$ & 31.0 & 22.0 & $\mathrm{~mm}$ \\
\hline Mass & 0.087 & 0.288 & $\mathrm{~kg}$ \\
\hline Polar Inertia & $22 \times 10^{-6}$ & $79 \times 10^{-6}$ & $\mathrm{kgm}^{2}$ \\
\hline Transverse inertia & $2.0 \times 10^{-5}$ & $6.6 \times 10^{-5}$ & $\mathrm{kgm}^{2}$ \\
\hline$r_{c}$ & 12.3 & 11.4 & $\mathrm{~mm}^{-5}$ \\
\hline
\end{tabular}

Table 6.2 Dimensions of three cylinders and corresponding masses and inertias.

The finite element model for the conventional turbocharger shaft and the turbogenerator shaft are shown in figures 6.5 and 6.6. The models consist of 3772 and 3096 structural elements and 4624 and 3718 nodes respectively. First order 
elements (of type SOLID 45 [6.4]) were employed, while the linear nature of modal analysis necessarily prevents any non-linear modelling of the interference between the individual components using for example contact elements. As a consequence, the individual components are rigidly fixed together, a reasonable approximation if a very close fit between the components is achieved during manufacture.

The material properties employed in the finite element analysis are summarised in table 6.3. Large values of elastic modulus were assigned to the compressor and turbine materials so as to effectively eliminate the flexural modes of these components from the analysis. The material properties of the laminated structure, in particular the shear modulii, are based upon values established experimentally by Long et al. [6.5].

\begin{tabular}{|c|c|c|c|c|c|}
\hline Sub-structure & Elastic modulus, GPa & Density, $\mathrm{kg} / \mathrm{m}^{3}$ & \multicolumn{3}{|c|}{ Shear modulus, GPa } \\
\hline \multirow[t]{2}{*}{ Laminations } & \multirow[t]{2}{*}{200} & \multirow[t]{2}{*}{7305} & $\overline{G_{x y}}$ & $\overline{\mathrm{G}_{\mathrm{yz}}}$ & $\overline{G_{2 x}}$ \\
\hline & & & 55 & 8 & 8 \\
\hline Compressor & 21000 & 2800 & & & \\
\hline Turbine & 21000 & 7800 & & & \\
\hline Shaft & 207 & 7850 & & & \\
\hline Nut, shaft sleeve & 207 & 7850 & & & \\
\hline
\end{tabular}

Table 6.3. Material properties of the components used in the finite element models.

Typical displacement plots of both rotors, illustrating the first bending mode, are shown in figures 6.7 and 6.8.

In order to validate these results an impulse type test was performed on the prototype rotor, using a modal hammer, an accelerometer and a dynamic signal analyser. A typical spectral response is shown in figure 6.9. For the case shown, the accelerometer was located mid-way between the turbine and the normal position of the turbine bearing (position $A$ in figure 6.3) and the impulse force was applied at the 
axial edge of the SR structure nearest the compressor (position $H$ in figure 6.3). A summary of the predicted and measured modal frequencies is contained in table 6.4. Each mode is categorised by the nature of vibration i.e. whether it results in torsion or bending, and the order of the mode shape, e.g. bending mode 2 gives rise to 3 nodes (i.e. position of zero displacement).

\begin{tabular}{|l|c|c|c|}
\hline \multirow{2}{*}{\multicolumn{1}{|c|}{ Mode }} & Conventional rotor & \multicolumn{2}{|c|}{ Turbo-alternator rotor } \\
\cline { 2 - 4 } & $\begin{array}{c}\text { Finite element } \\
\text { predicted }\end{array}$ & $\begin{array}{c}\text { Finite element } \\
\text { predicted }\end{array}$ & Measured \\
\hline Bending mode 1 & $989 \mathrm{~Hz}$ & $823 \mathrm{~Hz}$ & $720 \mathrm{~Hz}$ \\
\hline Torsional mode 1 & $1.24 \mathrm{kHz}$ & $1.22 \mathrm{kHz}$ & Not observed \\
\hline Bending mode 2 & $2.79 \mathrm{kHz}$ & $2.28 \mathrm{kHz}$ & $1.92 \mathrm{kHz}$ \\
\hline Torsional mode 2 & Not present & $3.15 \mathrm{kHz}$ & Not observed \\
\hline Bending mode 3 & $7.33 \mathrm{kHz}$ & $4.47 \mathrm{kHz}$ & $3.63 \mathrm{kHz}$ \\
\hline
\end{tabular}

Table 6.4 Natural frequencies of the rotors obtained using the finite element method and an impulse test on the prototype rotor.

In terms of the system response, the most important modes in this particular application are the first bending mode (likely to be excited by out-of-balance forces) and the first torsional mode (likely to be excited by torque ripple generated by the SR machine). As is evident, the inclusion of an SR rotor reduces the first bending mode frequency by some $17 \%$, while the torsional mode remains essentially the same (since the SR rotor is essentially located at a node in this mode). It is interesting to note that the inclusion of a third component on the shaft inevitably gives rise to a second torsional mode of vibration, although this is of considerably less concern in this application than the first mode.

There is considerable discrepancy between the predicted and measured modal frequencies of the turbo-alternator shaft, with an error of the order of 14-23\%, the measured value in each case being lower. This overestimate of the modal frequencies 
by a model in which the components are rigidly joined is consistent with the presence of some compliance between components in the practical shaft. Further contributions to the errors are likely to arise from the lamination material properties presented by Long et al. [6.5], which were necessarily somewhat specific to the geometry and fixing methods employed in their study. No attempt was made to arbitrarily modify the material to compensate for the observed error in subsequent analysis.

It is apparent from the finite element predictions that the addition of an SR rotor will not have a dramatic influence on bearing design/selection, from the point of view of its modal response as compared to a conventional turbocharger. However, as will be discussed in section 6.4, it does introduce complexities in terms of achieving comparable levels of mass balance. Thus, the requirements of the bearings in terms of stiffness and damping are likely to be very similar. Therefore in principle, the bearing technologies that are commonly employed in commercial turbochargers may well be suitable for the prototype turbo-alternator.

\subsection{Bearing Design}

In terms of selecting the most appropriate bearing technology for the prototype, it is useful to consider the principal features of the major bearing types employed in the high speed turbomachinery, viz. hydrodynamic journal and rolling element bearings. Operation at high speed is clearly reliant on the ability of the bearings to generate the necessary forces to give adequate stiffness and damping. In addition, the susceptibility of the bearings to de-stabilisation is likely to be crucial in this case, because of the additional factors which are likely to arise with the inclusion of an SR machine within the turbocharger, i.e. unbalanced magnetic pull, asymmetrical rotor stiffness, pulsating torque and increased mass. 


\subsubsection{HYDRODYNAMIC JOURNAL BEARINGS}

Many conventional turbochargers are operated with hydrodynamic journal bearings primarily because the high damping and relatively low stiffness is useful in terms of the rotordynamic performance. They are cost effective to manufacture and exhibit high reliability and durability if a consistent supply of contaminant-free lubricant is maintained [6.1].

The fluid support in hydrodynamic bearings, unlike hydrostatic bearings, is provided solely by the relative motion of the shaft in the bearing journal. The key design parameters in terms of realising a given performance are the shaft/journal clearance and the fluid viscosity (the supply pressure of the lubricating fluid is not critical and generally only needs to be sufficient to supply an adequate flow of lubricant to overcome leakage and prevent "touchdown"). The hydrodynamic support pressure is generated as the fluid is drawn into a converging 'wedge' produced by the eccentric displacement of the rotor, as shown in figure 6.10. The pressure in the converging wedge is higher than that in the diverging wedge, and hence a net force is generated which acts to oppose further rotor displacement.

The magnitude and direction of the restoring forces generated by a hydrodynamic bearing and hence the effective stiffness and damping can be determined using established analytical models for predicting the pressure field distribution throughout the circumference of the journal. The fluid film pressure distribution can be determined using the Reynolds equation in cylindrical co-ordinates [6.1]:

$$
\frac{\partial}{\partial \theta}\left[(1+\varepsilon \cos \theta)^{3} \frac{\partial p}{\partial \theta}\right]+R^{2} \frac{\partial}{\partial z}\left[(1+\varepsilon \cos \theta)^{3} \frac{\partial p}{\partial z}\right]=-6 \mu\left(\frac{R}{c}\right)^{2}[(\omega-2 \dot{\varphi}) \varepsilon \sin \theta-2 \dot{\varepsilon} \cos \theta]
$$

where $p=p(\theta, z)$ is the pressure distribution throughout the film in the circumferential and axial directions. The dimensionless eccentricity is defined as: 


$$
\varepsilon=e / c
$$

Where $e$ is the eccentricity and $c$ is the total journal clearance. $R$ is the radius of the journal, $\mu$ the viscosity of the lubricating fluid and $\omega$ and $\dot{\varphi}$ are the angular velocities of the rotor and the line of centres (defined in figure 6.10) respectively.

If the bearing is a plain cylindrical bearing of the type shown in figure 6.10 with axial length $L$ and a single entry oil supply, the boundary conditions for the solution of the Reynolds equation are [6.1]:

$$
\begin{aligned}
& p(\theta,-L / 2)=p(\theta, L / 2)=p_{a} \\
& p(0, z)=p(2 \pi, z)=p_{0}
\end{aligned}
$$

Where $p_{a}$ and $p_{0}$ are atmospheric pressure and fluid supply pressure or the fluid pressure at the region of maximum clearance respectively. In order to analytically integrate equation 6.7 , it is necessary to neglect one of the partial derivative terms in the left-hand side. In this case the second term was assumed to be zero which corresponds to a so-called long bearing where the axial variation in pressure field is negligible. This simplified form of the equation is valid for ratios of axial length to journal diameter of greater than 0.25 [6.1]. The resulting pressure distribution for a steadily loaded long journal bearing is given by [6.1]:

$$
p(\theta)=p_{0}+\frac{6 R^{2} \mu \omega}{C^{2}} \frac{\varepsilon(2+\varepsilon \cos \theta) \sin \theta}{\left(2+\varepsilon^{2}\right)(1+\varepsilon \cos \theta)^{2}}
$$

The solution to equation 6.10 is shown graphically in figure 6.11 for three different values of dimensionless eccentricity viz. $\varepsilon=0.25,0.5$ and 0.75 . The bearing and fluid parameters employed in these solutions are listed in table 6.5, and are representative of a small automotive turbocharger in which the lubricating fluid is SAE30 engine oil at $100^{\circ} \mathrm{C}$. 


\begin{tabular}{|c|c|c|}
\hline Parameter & Value & Units \\
\hline$p_{a}$ & $1 \times 10^{5}$ & $P a$ \\
\hline$\omega$ & 10000 & Rad.s \\
\hline$R$ & 7.5 & $m m$ \\
\hline$C$ & 50 & $\mu m$ \\
\hline$L$ & 10 & $m m$ \\
\hline$\mu$ & $1 \times 10^{-3}$ & $P a . s$ \\
\hline
\end{tabular}

Table 6.5. Summary of constants used in equation 6.10 .

One noticeable feature of the pressure distribution shown in figure 6.11 is that the calculated oil pressure distribution would appear to have a negative value in the diverging 'wedge' region. In practice, the oil film cavitates at a gauge pressure of around $-30 \mathrm{kPa}[6.1]$ in this region, and the bearing is referred to as partially filled or a $\pi$-film bearing indicating a film covering approximately half the journal circumference. The resultant force in the radial and tangential directions can be calculated by integrating the pressure distribution around the journal periphery. For a long $\pi$-film bearing, this gives rise to [6.1]:

$$
\begin{aligned}
& F_{r}=-6 \mu R L\left(\frac{R}{C}\right)^{2}\left[|\omega-2 \dot{\varphi}| \frac{2 \varepsilon^{2}}{\left(2+\varepsilon^{2}\right)\left(1-\varepsilon^{2}\right)^{2}}+\frac{\pi \dot{\varepsilon}}{\left(1-\varepsilon^{2}\right)^{3 / 2}}\right] \\
& F_{t}=\mu 6 R L\left(\frac{R}{C}\right)^{2}\left[(\omega-2 \dot{\varphi}) \frac{\pi \varepsilon}{\left(2+\varepsilon^{2}\right)\left(1-\varepsilon^{2}\right)^{1 / 2}}+\frac{4 \dot{\varepsilon}}{(1+\varepsilon)\left(1-\varepsilon^{2}\right)}\right]+2 R L P_{a}
\end{aligned}
$$

where the radial direction is defined as radially outwards parallel to the direction of the line of centres shown in figure 6.10 , and a positive tangential force is in the direction of rotation. Having established the magnitude of the bearing forces, the resulting stiffness characteristics can now be established at various eccentricities, using the standard approach of differentiating the bearing forces with respect to 
displacement [6.6-6.8]. Figure 6.12 shows the calculated bearing reaction force as a function of eccentricity, while figure 6.13 shows the corresponding variation of incremental stiffness, i.e. for example:

$$
K_{t r}=\frac{\partial F_{t}}{\partial r}
$$

It is useful in terms of assessing bearing performance, to compare the bearing reaction forces with the likely loads imposed during operation and to establish the resulting degree of eccentricity. For this purpose, the out-of-balance forces generated by the shaft assembly were calculated on the basis of the ISO 1940 (1986) standard [6.9], which specifies maximum acceptable mass eccentricities for a range of balance grades. For a rotor operating up to $100,000 \mathrm{rpm}$ which is balanced to a quality grade of G6.3 (a reasonably high precision grade typical of commercial turbochargers), the specified mass-eccentricity is approximately $1 \times 10^{-6} \mathrm{~kg} \mathrm{~m}$ per $\mathrm{kg}$ of rotor mass. At $100000 \mathrm{rpm}$ this gives a maximum radial out-of-balance force of approximately $100 \mathrm{~N}$. By inspection of figures 6.12 and 6.13 this would result in an eccentricity of some 0.75 (which is within an acceptable operating range) and a bearing stiffness of $1.16 \times 10^{7}$.

It is important to note that although the calculation of forces has been based on two orthogonal components, the cross-coupled terms, e.g. a radial displacement generating a tangential force, cannot be neglected since these forces give rise to the main destabilising mechanism observed in plain hydrodynamic journal bearings [6.1].

Having established that the free-free response of the prototype turbogenerator does not differ markedly from a conventional turbocharger shaft assembly in terms of the most significant vibration modes, i.e. $1^{\text {st }}$ bending modes of 822 and 989 for the prototype and standard rotors respectively, it is reasonable to conclude that at least in terms of direct stiffness and damping, that hydrodynamic bearings are likely to be suitable for the proposed prototype. However, in addition to standard consideration 
of a second order mass-spring-damper, in this case it is also necessary to pay particular attention to the various de-stabilising mechanisms specific to hydrodynamic bearings.

\subsubsection{Self-excited instability}

The partially filled oil-way and the resultant nature of the restoring forces in a plain hydrodynamic bearing can give rise to so-called "self-excited instability" or "oilwhip instability". This instability mechanism is extremely complex, and in some respects remains relatively poorly understood from a quantitative point of view [6.10]. However, some qualitative explanations can be proposed [6.10] by reference to figure 6.14, which shows the various operating modes of a plain journal bearing. Figure 6.14a shows the bearing operating under steady-state conditions with the film starting at the position of minimum clearance. If a small rotating force, such as that generated by shaft unbalance, is applied to the bearing, the locus of the journal centre whirls about the equilibrium position, shown in figure 6.14b. The magnitude and shape of the whirl orbit can be determined using the stiffness and damping coefficients of the bearing. If the disturbing force reaches a magnitude such that the whirl orbit (which has a frequency $F$ ) causes the position of minimum film thickness to rotate around the bearing journal, then the system departs from steady-state conditions. The relative speed of the whirl is $\omega-2 F$ (which is analogous with the $(\omega-2 \dot{\varphi})$ term in equation 6.11) and defines the relative position of the oil film with respect to the eccentricity. If this relative speed is positive the oil film tends to lag the vibration as shown in figure $6.14 \mathrm{c}$ while if the relative speed is negative the film leads (figure 6.14d). Under lagging film conditions, the reaction force of the bearing on the rotor (which is opposite in direction and magnitude to the force shown in figure 6.14c) exhibits a component in the same direction as the whirl and hence tends to increase the whirl, with the onset of self-excited instability. This provides one possible qualitative reason for the observed instability of this type of bearing undergoing oscillations with exciting forces that have frequencies at or below half 
the rotational speed [6.10]. For leading film conditions, as shown in figure $6.14 \mathrm{~d}$, the system remains stable with a tendency to reduce the whirl amplitude.

The tendency towards instability with sub-synchronous whirl implies that a shaft supported on plain hydrodynamic bearings must not be operated above twice the first critical speed in bending, since the rotor will always tend to vibrate at its own natural frequency. Indeed most turbocharger manufacturers that employ hydrodynamic bearings, aim to provide a large stability margin by operating at speeds below 60 $70 \%{ }^{1}$ of the first critical speed, and to avoid the large vibration amplitudes observed at the critical speed. This limitation on operating speed in commercial turbochargers is usually determined primarily with regard to avoiding self-excited de-stabilisation, since this is in general much more destructive than operating near or even above first critical speed.

\subsubsection{Parametrically excited instability}

Unlike self-excited instability, which is characterised by sub-synchronous whirl frequencies, so called 'parametrically excited' whirl can occur at any frequency. Examples in published literature of the causes of such parametric excitation are asymmetrical shaft stiffness [6.11], pulsating torque [6.12] and intermittent rotor/stator contact [6.13] - the first two being particularly relevant to the SR turboalternator.

The cross section of a 4-pole SR rotor has a "weak axis" where the stiffness is a minimum. Taylor and Schenectady [6.11] reported the behaviour of such shafts, but in their particular case, the shaft exhibited two distinct axes $90^{\circ}$ apart, whereas they are only $45^{\circ}$ apart in a 4-pole SR rotor. An asymmetrical shaft will exhibit two distinct critical speeds characterised by vibration in the direction of the two geometric axes. The rotor exhibits large amplitude vibrations at speeds equal to half

\footnotetext{
${ }^{1}$ Personal discussion with David Green of Holset Engineering Ltd.
} 
the lower critical speed due to the interaction of the varying geometry and unidirectional forces such as gravity [6.11]. Observations indicate that the rotor is unstable at speeds that lie between the two critical speeds, although unlike some "classical" instabilities this may be "driven through" without damage to the rotor bearing system [6.1]. In the case of an SR rotor whose principal axes lie $45^{\circ}$ apart, a similar consideration suggests that large amplitude vibrations will be observed at speeds equal to $1 / 4$ of the first critical speed in bending. It is noted however that the contribution to the stiffness by the lamination stack is not very large, and hence the "degree of dyssymmetry" discussed by Taylor and Schenectady [6.11] is small, and will be the frequency difference between the modes of vibration about the two axes.

Instability caused by pulsating torque may present a particular problem for a highspeed 6/4 SR machine. Figure 6.15 shows the simulated torque of the machine rotating at $100,000 \mathrm{rpm}$ and generating $5 \mathrm{~kW}$. The pulsating nature and high harmonic content of the torque waveform is clearly apparent. Eshleman and Eubanks [6.12] observed a region of instability when a small time-varying torque was superimposed on a constant torque. The region of instability (which was defined in the frequency domain) increases as the ratio of pulsating to constant torque increases (figure 6.16). This instability was characterised by shaft whirl at the first critical speed. Although no analysis was provided to enable the prediction of the region of stability, it was noted that the characteristic whirl is sub-synchronous at the first critical speed in bending and hence only occurs when the rotor is operated above its first critical speed. This again highlights the need to design the rotor with the first critical speed to be outside the operating range if possible.

In addition to the published instability mechanisms for plain rotors, one further factor that must be considered in the case of electrical machines is the presence of so-called 'unbalanced magnetic pull'. This is a particular concern for SR machines since the machine airgap is often very small in order to enhance efficiency and specific power (typically $200 \mu \mathrm{m}$ ), which is comparable with the radial clearance of $50-100 \mu \mathrm{m}$ in a typical hydrodynamic bearing. Although the radial force exerted on individual teeth 
is high in SR machines (and is a major factor in generating acoustic noise), the net unbalanced radial forces in $6 / 4$ SR machines, tend to be relatively small, since they arise from the second order effect of the flux density increasing in the smaller airgap due to the reduced fringing and leakage.

In order to determine the magnitude of these forces in the prototype machine, a series of magneto-static finite element solutions were undertaken. The rotor was modelled in the aligned position and the eccentricity varied between 0 and $60 \mu \mathrm{m}$, in steps of $10 \mu \mathrm{m}$ for the case of a nominal airgap of $200 \mu \mathrm{m}$. Two separate analyses were undertaken with current densities (assuming fully filled slots) of $5 \mathrm{Amm}^{-2}$ and $10 \mathrm{Amm}^{-2}$. A typical magnetic field distribution is shown in figure 6.17, for the case of an eccentricity of $60 \mu \mathrm{m}$ vertically downwards, and a current density specified of $10 \mathrm{Amm}^{-2}$. The reduced fringing and leakage between the lower poles as compared to the upper poles, gives rise to a net imbalance in the radial flux density and hence force. The resultant force on the rotor for a given current density and eccentricity was calculated, by a Maxwell stress integration around a circular path that passes over the surface of the rotor teeth. The radial and tangential components of force are given by [6.14]:

$$
\begin{gathered}
F_{n}=L \oint \frac{B_{n}^{2}-B_{t}^{2}}{2 \mu_{0}} d \theta \\
F_{t}=L \oint \frac{B_{n} B_{t}}{\mu_{0}} d \theta
\end{gathered}
$$

The vertically upward component of the force per unit area (i.e. in positive ydirection) around the rotor circumference is shown in figure 6.18. Clearly the total force on the top tooth is lower than on the bottom tooth, giving a net force in the direction of motion equivalent to a negative stiffness. The resultant force in the $y$ direction is shown in figure 6.19 for the range of displacements and current densities modelled. Over the range of displacements considered, the stiffness with a given 
current density does not vary with eccentricity. The radial stiffness in the aligned position for the current densities modelled are summarised in table 6.6.

\begin{tabular}{|c|c|}
\hline Current Density, $\mathrm{Amm}^{-2}$ & Stiffness, $\mathrm{kNm}^{-1}$ \\
\hline 5 & -36.7 \\
\hline 10 & -150 \\
\hline
\end{tabular}

Table 6.6. Stiffness of the rotor subjected to eccentric displacement in the aligned position.

The net stress in the $\mathrm{x}$-direction with the rotor in the aligned position is nominally zero. However, as the rotor rotates away from the aligned position, the net asymmetry in the geometry will give rise to a degree of cross-coupling between the eccentricity and the net force.

Although the magnitude of the unbalanced magnetic force is relatively low as compared to the force generated by mass imbalance in high-speed machines, the nature of the force may cause instability when operated with a plain hydrodynamic bearing. The particular problems that may arise with unbalanced magnetic pull can be illustrated by reference to a shaft operating as shown in figure 6.10 . With an externally applied force acting vertically downward displacing the shaft at some angle $\varphi$, the unbalanced magnetic pull exerts a force on the rotor in the direction of the eccentricity, where the bearing's ability to provide a restoring force is very low, which itself will tend to increase the angle $\varphi$. The direction of eccentric displacement will vary according to the relative magnitudes of the various synchronous and static components of force. The direction of the unbalanced magnetic pull generated by an SR machine is determined by the direction of eccentricity, the rotor alignment with respect to the stator and the instantaneous excitation applied by the stator windings. This complex behaviour, in which the unbalanced magnetic pull essentially rotates at twice the rotor frequency in the reverse direction, is likely to cause a destabilising force within the fluid film of the 
bearing. This behaviour is expected to be particularly problematic at speeds equal to half the first critical speed in bending. However, the scope for thorough quantitative and experimental research to assess the effects of this destabilising mechanism is beyond the scope of this thesis.

The potential for instability of the system can be reduced by using variations of the plain journal bearing such as the so-called lemon bore, dammed groove, offset halves, multi-lobed or tilted pad bearings. Each of these designs is aimed at providing a small converging section and hence a degree of stiffness in all directions regardless of the direction of rotor eccentricity. While these bearings can provide greater immunity to self excited instability, the design, manufacture and characterisation of such bearings is beyond the scope of the present work.

Whereas comparisons between the free-free modal spectra of the two rotors investigated in section 6.3 would suggest that the adoption of hydrodynamic bearings in the prototype turbo-alternator may be feasible, consideration of de-stabilising mechanisms has highlighted many potential problems, albeit qualitatively. As such the use of plain hydrodynamic bearings in the manufacture of the prototype machine without a thorough investigation is likely to be prone to problems. Indeed, even in prototype and low volume conventional turbochargers where the turbine and/or compressor design is modified from standard combinations, it is common practice to avoid hydrodynamic bearings in the first instance.

\subsubsection{ROLLING ELEMENT BEARINGS}

As discussed in section 6.1.1, it is desirable to have a bearing system that exhibits high damping and relatively low stiffness, so as to allow the rotor vibrations to be effectively accommodated. The oil film in hydrodynamic bearings satisfies these criteria very well. By contrast, shafts supported on rolling element bearings with no additional damping often exhibit very low damping of around $1.5-2.5 \%$ of critical damping [6.1]. Further, since most of this damping is generated in the shaft material itself under flexural conditions, this introduces a further destabilising mechanism, 
albeit one that is relatively minor. Rolling-element bearings in high-speed machinery are therefore often mounted on some form of external damper, the most common type being a squeeze film damper where a static oil film is present between the outer race of the bearing and the housing. This is usually coupled with a spring mechanism to provide mechanical support of the bearing. The spring device is generally either a cylindrical arrangement of thin ribs, termed a squirrel cage, cantilevered supports or curved beam segments around the outer race [6.1]. These mechanisms are often used in large turbomachinery where the space required to house the springs is available. In the turbo-alternator, the space envelope is highly restricted, a restriction which, when coupled with the indeterminate stiffness at elevated temperature, limits the scope to use such dampers. A further classification of damper consists of an elastomeric $\mathrm{O}$ ring placed around the outer race of the bearing. This provides low stiffness and moderate damping, however the fatigue and thermal degradation properties of these materials makes the performance of such dampers unpredictable, particularly at elevated temperatures [6.1]. The very high rotor and end-cap temperatures which are likely to be encountered (particularly on the turbine end of the shaft) and the highly constrained space envelope, again limit the scope for these dampers. As a consequence, it was decided to manufacture the prototype machine with rolling element bearings mounted directly into the end-caps.

The bearings used in the prototype are FAG high-speed, sealed, spindle bearings with ceramic balls. They were selected on the basis of their ability to operate at rotational speeds of up to $100,000 \mathrm{rpm}$ with a grease lubricant. The bearings are lubricated with Kluber Isoflex MBU15 grease specifically developed for ceramicsteel hybrid bearings. The leading dimensions of the bearing are defined in figure 6.20 and listed in table 6.7. 


\begin{tabular}{|l|c|}
\hline Bearing & HCS71900C.T.P4S.UL \\
\hline Outer diameter, $\mathrm{D}(\mathrm{mm})$. & 22 \\
\hline Inner diameter, $\mathrm{d}(\mathrm{mm})$. & 10 \\
\hline Width, $\mathrm{B}(\mathrm{mm})$. & 6 \\
\hline Diameter of rolling elements, $\mathrm{D}_{\mathrm{b}}(\mathrm{mm})$. & 2.381 \\
\hline Pitch diameter, $\mathrm{D}_{\mathrm{m}}(\mathrm{mm})$. & 16 \\
\hline Contact angle, $\alpha$ (degrees). & 15 \\
\hline Number of elements, $\mathrm{Z}$. & 13 \\
\hline
\end{tabular}

Table 6.7 Rolling-element bearing dimensions

The rolling elements are manufactured from hot isostatically pressed Silicon Nitride $\left(\mathrm{Si}_{3} \mathrm{~N}_{4}\right)$, and the raceways are manufactured from bearing steel 52100 to withstand the high Hertzian stresses associated with high-speed operation. The properties of the materials are summarised in table 6.8 [6.15].

\begin{tabular}{|l|c|c|}
\hline & Ceramic $\mathrm{Si}_{3} \mathrm{~N}_{4}$ & Bearing steel 52100 \\
\hline Elastic Modulus, GPa. & 310 & 207 \\
\hline Poisson's Ratio & 0.26 & 0.3 \\
\hline Density, $\mathrm{Kg} / \mathrm{m}^{3}$. & 3200 & 7800 \\
\hline
\end{tabular}

Table 6.8. Material properties of bearing components

One important limitation of the rolling element bearings in the prototype machine is the thermal constraints imposed on the operating conditions by the bearing cage. The operating temperature of the bearing at the turbine end is likely to be high in this application, although this can be alleviated to some extent by oil-cooling the endcaps. The standard fibre reinforced phenolic cage used in the FAG high-speed spindle bearing range has a maximum continuous operating temperature of $150^{\circ} \mathrm{C}$. Although the use of a brass cage (with a maximum operating temperature of around 
$330^{\circ} \mathrm{C}$ ) or indeed a "cageless" bearing will alleviate the thermal constraints, in this case these were not available as an 'off-the-shelf' item in small quantities. In an attempt to limit the maximum operating temperature of the turbine bearing, it was positioned further from the turbine than would be the case with a conventional hydrodynamic bearing in a standard turbocharger design. One notable consequence of this modification in the prototype design is that the critical speeds of the rotor/bearing system will be further reduced beyond those encountered in an equivalent standard turbocharger, due to the increased bending moment of the cantilevered turbine.

\subsubsection{Rolling element bearing stiffness}

In order to perform a quantitative rotordynamic analysis of the rotor/bearing system it is necessary to determine the effective radial stiffness of the rolling element bearing. The basis of stiffness calculations for rolling element bearings is the socalled 'Hertzian contact theory' [6.16]. In this type of analysis, the deflection of the inner and outer races are neglected, and any deflection is assumed to occur as a result of deformation of the balls and the race surface. The influence of the bearing supports and the external structure (e.g. end-caps etc) are also neglected since any contribution is highly dependent on the mechanical fit between components and is difficult to establish to any degree of accuracy due to compound manufacturing tolerances. It should be borne in mind that the stiffness predicted using Hertzian contact theory will inevitably result in a slightly higher value than can be achieved in a practical device where the bearing support structure will have a finite compliance. However, the associated error in a well designed and rigid end-cap will generally be within acceptable limits [6.16].

The total deflection of the shaft centre is the sum of the deflections with respect to both the inner and outer races respectively, i.e.:

$$
\delta_{n}=\delta_{i}+\delta_{o}
$$


For point contact, the Hertzian deformation is given by [6.16]:

$$
\delta_{n}=K_{n} Q^{2 / 3}
$$

Where $Q$ is the radial force and

$$
K_{n}=\left[\left(\frac{2 K(e)}{\pi m_{a}}\left(\sum \rho\right)^{1 / 3}\right)_{i}+\left(\frac{2 K(e)}{\pi m_{a}}\left(\sum \rho\right)^{1 / 3}\right]_{0}\right] \sqrt[3]{\frac{1}{8}\left[\frac{3}{2}\left(\frac{1-v_{1}^{2}}{E_{1}}+\frac{1-v_{2}^{2}}{E_{2}}\right)\right]^{2}}
$$

The parameters of equation 6.17 are defined such that:

$\sum \rho$ is the so-called 'curvature sum' of the bearing [6.16], which for the inner and outer races is given by:

$$
\begin{aligned}
& \sum \rho_{i}=\frac{1}{D_{b}}\left(4-\frac{1}{f_{i}}+\frac{2 \gamma}{1-\gamma}\right) \\
& \sum \rho_{o}=\frac{1}{D_{b}}\left(4-\frac{1}{f_{o}}-\frac{2 \gamma}{1+\gamma}\right)
\end{aligned}
$$

$D_{b}$ is the diameter of the rolling element, $f_{i / o}$ is the groove radius coefficient for the inner and outer raceways defined as:

$$
f=\frac{r}{D_{b}}
$$

$r$ is the radius of curvature of the raceway, and typically has a value between 0.515 and 0.525 for both raceways. The term $\gamma$ in equation 6.18 is defined as: 


$$
\gamma=\frac{D_{b} \cos \alpha}{D_{m}}
$$

$\alpha$ is the contact angle of the bearing and $D_{m}$ is the pitch diameter of the bearing.

The values $K(e)$ and $m_{a}$ for the contact with the inner and outer raceway are tabulated by Changsen [6.16] and determined using the curvature function $F(\rho)$ for the given raceways, given by:

$$
\begin{gathered}
F\left(\rho_{i}\right)=\frac{\frac{1}{f_{i}}+\frac{2 \gamma}{1-\gamma}}{4-\frac{1}{f_{i}}+\frac{2 \gamma}{1-\gamma}} \\
F\left(\rho_{o}\right)=\frac{\frac{1}{f_{o}}-\frac{2 \gamma}{1+\gamma}}{4-\frac{1}{f_{i}}-\frac{2 \gamma}{1+\gamma}}
\end{gathered}
$$

The corresponding values of these parameters for the bearings used in the prototype machine are listed in table 6.9.

\begin{tabular}{|l|c|c|}
\hline & Inner & Outer \\
\hline$f$ & 0.515 & 0.515 \\
\hline$\gamma$ & 0.1437 & 0.1437 \\
\hline$\sum \rho, \mathrm{mm}^{-1}$ & 1.006 & 0.759 \\
\hline$F(\rho)$ & 0.951 & 0.935 \\
\hline$K(e)$ & 3.74 & 3.59 \\
\hline$m_{a}$ & 4.15 & 3.71 \\
\hline
\end{tabular}

Table 6.9 Dimensional constants and curvature functions. 
For this bearing, the calculated value of $K_{n}$ is $2.83 \times 10^{-7}$. The total bearing deflection due to contact stresses on both the outer and inner raceways can now be calculated. The resulting force-displacement characteristic calculated using equation 6.16 is shown in figure 6.21, together with the variation of the stiffness (defined as $\frac{F_{r}}{r}$ and not $\left.\frac{d F_{r}}{d r}\right)$.

In terms of employing an equivalent bearing stiffness in a finite element modal analysis of a rotor and bearing system, it is necessary to establish a single value of stiffness to approximate the non-linear variation of stiffness shown in figure 6.21. As discussed in section 6.4.1, the likely out-of-balance forces are of the order of $100 \mathrm{~N}$. Using this representative load, which gives rise to a deflection of $6 \mu \mathrm{m}$, a reasonable estimate of operating stiffness is $1.64 \times 10^{7} \mathrm{~N} / \mathrm{m}$ which compares to an approximate stiffness of $1.16 \times 10^{7} \mathrm{~N} / \mathrm{m}$ for an equivalently sized hydrodynamic bearing.

\subsection{Critical Speed Analysis}

Having established that rolling element bearings will be used in the prototype, and having derived a representative equivalent stiffness, the critical speeds of the entire rotor and bearing assembly can be calculated (assuming perfectly rigid end-caps). Critical speeds (which are essentially analogous to natural frequencies) are those speeds at which the rotor vibrations are synchronous with the speed, and hence the magnitudes of vibration are at a maximum. One important limitation in the use of modal analysis in the prediction of rotor critical speeds is the assumption that the rotor whirl is indeed synchronous with the shaft rotation. The presence of unstable non-synchronous rotor whirl cannot be conclusively determined with this type of analysis.

It has been demonstrated in the preceding discussion that the stiffness of the rolling element bearing is a function of the radial deflection, which is itself dependent on the 
mass-eccentricity and rotational speed of the rotor. This dependency of the critical speeds on the rotor speed can in principle be plotted using a Campbell diagram [6.1]. However the reliable prediction of this behaviour would require very extensive finite element characterisation to cater for range of stiffness values encountered at each speed, which themselves would be functions of the exact distribution of the mass imbalance in the prototype rotor.

As a consequence, the speed dependency of the natural frequencies was neglected in this study, thus enabling a simple modal analysis to be used to establish the rotor/bearing system critical speeds. However, whereas an estimate of the rolling element bearing stiffness has been derived in section 6.4.2.1, a sensitivity study was undertaken to establish the influence of bearing stiffness on the critical speeds.

In the finite element model of the rotor/bearing system, the same mesh was employed to represent the rotor as was the case in the 'free-free' analysis of section 6.3 , while the bearings were represented using two orthogonally mounted, radial spring elements (of type COMBIN14 [6.4]). It should be noted that this simplified representation of the bearings in effect introduces a degree of torsional stiffness that is not present in the real bearing system (these being essentially free to rotate). Hence the predicted torsional mode frequencies with this model are not representative of those that would be encountered in a practical system. As a consequence, the torsional mode frequencies previously calculated using the 'free-free' analysis are considered to be more reasonable estimates of the likely behaviour.

A modal analysis was initially performed using a spring stiffness of $1.64 \times 10^{7} \mathrm{~N} / \mathrm{m}$ (which corresponds to the approximate stiffness for a $100 \mathrm{~N}$ load and a $6 \mu \mathrm{m}$ displacement). Further solutions were then undertaken using other stiffness values in logarithmic steps between $10^{5}$ and $10^{12} \mathrm{~N} / \mathrm{m}$. A further final solution was undertaken using the maximum allowable spring stiffness of $10^{44} \mathrm{~N} / \mathrm{m}$, in order to represent an essentially rigid system 
The findings of the finite element analysis are summarised in figure 6.22. The natural frequencies of the various modes are shown for a range of spring stiffness, while the form of the solid body and bending mode shapes are illustrated graphically by means of the radial displacement of the central nodes along the axial length of the rotor. The solid body modes generally correspond to conical whirl orbits of the shaft in the compliant bearing structure, while the bending modes correspond to high degrees of flexure of the shaft, and as such are generally regarded as the more destructive of the two vibration modes. As was the case with the 'free-free' analysis, the various bending modes are grouped with respect to their order. As is evident from figure 6.22, the categorisation of solid body modes, particularly at high stiffness, necessarily involves some degree of ambiguity, since there is considerable flexure in these modes, e.g. the mode that has been categorised as solid body mode 2 with a stiffness of $1 \times 10^{9}$.

Figure 6.23 shows the variation in the natural frequencies of the two solid body modes and the first four bending modes as a function of bearing stiffness over the range $1 \times 10^{5}$ to $1 \times 10^{12}$. As would be expected, the frequency of the solid body modes increases with increasing bearing stiffness. However as the bearing stiffness increases to values of the order of $10^{9} \mathrm{~N} / \mathrm{m}$ a degree of flexure is observed in the shaft as the bearing stiffness approaches that of the shaft, and the frequency of vibration increases at a diminishing rate as the spring stiffness is increased.

In terms of bending modes, at low bearing stiffness, i.e. below $10^{6} \mathrm{~N} / \mathrm{m}$ or so, the bearings provide insufficient additional stiffness to significantly modify the behaviour from that observed in the 'free-free' case. As the bearing stiffness is increased further (to values of around $10^{7}$ ) the contribution of bearing stiffness to the overall system stiffness becomes comparable to that of the rotor stiffness and hence the frequencies of the modal vibration tend to increase. Ultimately for bearing stiffness greater than $10^{10}-10^{11}$ or so, any incremental increase in bearing stiffness has little effect on the frequency or form of the mode shape since the modal vibration is such that a node appears at the location of each bearing. A comparison between the 
predicted modes with a stiffness of $10^{44} \mathrm{~N} / \mathrm{m}$ (maximum allowable by ANSYS for this model) and $10^{12} \mathrm{~N} / \mathrm{m}$ (the highest value shown in figure 6.23) confirmed that a value of $10^{12} \mathrm{~N} / \mathrm{m}$ provides a very good representation of an infinitely stiff system.

For the particular calculated rolling element bearing stiffness of $1.64 \times 10^{7}$, the frequencies of the various vibration modes, and the corresponding critical rotor speeds are summarised in table 6.10 .

\begin{tabular}{|l|c|c|}
\hline & Frequency (Hz) & Critical speed (rpm) \\
\hline Solid body & 304 & 18,200 \\
\hline Solid body & 908 & 54,500 \\
\hline Bending mode 1 & 1428 & 85,700 \\
\hline Bending mode 2 & 2581 & 155,000 \\
\hline Bending mode 3 & 4670 & 280,000 \\
\hline Bending mode 4 & 13318 & 799,000 \\
\hline
\end{tabular}

Table 6.10 Natural frequencies of the rotor obtained using the finite element method incorporating bearings of stiffness $1.64 \times 10^{7}$.

It is apparent from table 6.10 that for the prototype machine many of the critical speeds lie within the proposed operating range of the turbogenerator. While the solid body modes occur at relatively low frequencies viz. 18,200 and 54,500 rpm respectively, the nature of this type of vibration is generally less destructive than the bending modes and can be 'driven through' with no severe consequences. The first critical speed in bending occurs at $85,700 \mathrm{rpm}$ and is characterised by large vibration amplitudes and internal rotor stress. While examples can be found in literature of rotor/bearing systems that are operated above the first critical speed in bending [6.1 \& 6.3] it is generally desirable to avoid this critical speed. It should be noted that this value of $85,700 \mathrm{rpm}$ was derived on the basis of an approximate bearing stiffness figure and the finite element model of the rotor assembly. As has been demonstrated by the 'free-free' analysis, the actual prototype rotor has lower measured first natural 
frequency in bending than that predicted by finite element analysis, with a consequent reduction in the first critical speed in bending from the $85,700 \mathrm{rpm}$ value in table 6.10.

With this in mind, the scope to achieve significant increases in the first critical speed in bending by refinement of the mechanical design of the rotor was investigated. A useful starting point is to model a conventional turbocharger unit, shown previously in figure 6.6, with the same rolling-element bearings. This will provide an upper limit on the shift in first critical speed that can be achieved. A summary of the resulting modal analysis is contained in table 6.11 .

\begin{tabular}{|l|c|c|}
\hline & Frequency (Hz) & Critical speed (rpm) \\
\hline Solid body & 440 & 26,400 \\
\hline Solid body & 840 & 50,400 \\
\hline Bending mode 1 & 2139 & 128,300 \\
\hline Bending mode 2 & 3468 & 208,100 \\
\hline Bending mode 3 & 7388 & 443,300 \\
\hline
\end{tabular}

Table 6.11 Natural frequencies of a commercial turbocharger obtained using the finite element method incorporating bearings of stiffness $1.64 \times 10^{7}$.

As is evident from table 6.11, the margin between the first critical speed in bending and the maximum operating speed of $100,000 \mathrm{rpm}$ is likely to be adequate in the case of the standard turbocharger, albeit on the basis of an approximate representation of the bearing stiffness. As a consequence, there may some limited scope to increase the first critical speed in bending of the prototype by modifying the bearing location. The current bearing location is based on the need to avoid overheating of the phenolic cage of the turbine end bearing. Reducing this separation will have the effect of reducing the moment of the cantilevered turbine mass, which as shown in figure 6.22 appears to strongly influence the mode shapes. One other possible modification is to reduce the axial length of the relatively compliant laminated SR rotor. However, this 
has a proportionate impact on the output power capability of the SR machine. In order to determine whether sufficient benefits in terms of an increased first critical speed in bending can be achieved to offset the performance penalties, two further finite element models were analysed, viz.:

1. The separation between the turbine end bearing and the turbine wheel, i.e. $d_{t}$ in figure 6.3 , was reduced from $29.5 \mathrm{~mm}$ to $19.5 \mathrm{~mm}$.

2. The axial length of the lamination stack was reduced by $20 \%$

The natural frequencies of these modified rotor models were calculated with a bearing stiffness of $1.64 \times 10^{7} \mathrm{~N} / \mathrm{m}$ and categorised in a similar manner to those in the preceding analysis. Table 6.12 summarises the various critical speeds of the standard and modified rotor designs. Clearly, both of the proposed modifications will also impact on the mass of the rotor as well as the stiffness, thus further modifying the critical speeds.

\begin{tabular}{|l|c|l|c|l|c|}
\cline { 2 - 6 } \multicolumn{1}{c|}{} & Standard rotor & \multicolumn{2}{|l|}{ Short SR rotor } & \multicolumn{2}{l|}{$\begin{array}{l}\text { Reduced } \\
\text { separation }\end{array}$} \\
\cline { 2 - 6 } & Critical speed & $\begin{array}{l}\text { Critical } \\
\text { speed }\end{array}$ & $\begin{array}{l}\text { \% change } \\
\text { wrt standard }\end{array}$ & $\begin{array}{l}\text { Critical } \\
\text { speed }\end{array}$ & $\begin{array}{l}\text { \% change } \\
\text { wrt standard }\end{array}$ \\
\hline Solid body 1 & 18,200 & 17,900 & -1.64 & 22,400 & 22.70 \\
\hline Solid body 2 & 54,500 & 54,800 & 0.66 & 54,800 & 0.66 \\
\hline Bending 1 & 85,700 & 92,600 & 8.05 & 87,000 & 1.61 \\
\hline Bending 2 & 155,000 & 168,900 & 9.07 & 166,000 & 6.90 \\
\hline Bending 3 & 280,000 & 315,800 & 12.70 & 344,000 & 22.70 \\
\hline Bending 4 & 799,000 & 877,000 & 9.72 & 809,000 & 1.27 \\
\hline Mass & 0.774 & 0.715 & -7.62 & 0.767 & -0.90 \\
\hline
\end{tabular}

Table 6.12 Comparison of the critical speeds and rotor mass of the standard and modified rotor designs. 
It is interesting to note that although the case of a short SR rotor does exhibit a 8.05\% increase in the first critical speed in bending, this is of comparable order to the mass reduction, suggesting that a significant proportion of the observed increase is actually due to the reduced modal mass. The modification to the solid body critical speeds is marginal, with only a $1.6 \%$ decrease in the frequency of the first mode.

The reduced bearing/turbine wheel separation has a marginal effect on the first critical speed in bending, and is unlikely to yield sufficient benefits to warrant its adoption given the constraints on the bearing temperature. The most notable effect of reducing the bearing/turbine wheel separation is observed in the modes where a considerable degree of flexure of this portion of the shaft is encountered. Indeed, the third bending mode and the first solid body mode (which despite being categorised as a solid body mode includes a high degree of flexure) both increase by $23 \%$.

For the purpose of constructing a prototype machine the original shaft design was maintained, since the relatively small benefits that accrue from the proposed modifications in terms of the increased critical speeds in simple bending do not offset the penalties of the increased temperature loading of the bearings and the reduced power capability.

The rotordynamic studies in this chapter have illustrated, albeit with some simplifications regarding bearing performance and rotor construction tolerances, that the inclusion of an SR rotor compounds the difficulties of operating turbo-machinery at such high speeds. Indeed, the margin commonly employed in commercial turbochargers has been severely eroded, such that problematic critical speeds may well lie within the operating speed range. However, this was deemed as being acceptable at this stage of the machine development, even if the prototype speed range has to be limited in the first instance. Ultimately, it is likely that any commercial devices based on this prototype would employ custom designed hydrodynamic bearings, which would offer a tailored combination of stiffness and more particularly damping to accommodate the various modes of vibration. Further, the current design has been severely constrained by the need to use standard 
turbocharger components and manufacturing set-ups, in particular the relatively slender shaft that could be friction welded to the turbine. A custom designed shaft (together with the appropriate friction welding capability) could yield considerable dividends in terms of increasing shaft stiffness. 


\section{REFERENCES}

[6.1] Vance, J. M., "Rotordynamics of Turbomachinery." John Wiley \& Sons, 1988.

[6.2] Jeffcott, H. H., "The lateral vibration of loaded shafts in the neighbourhood of a whirling speed: The effect of want of balance." Philosophical Magazine, Series 6, No. 37. 1919.

[6.3] Watson, $\mathrm{N}$ and Janota, M. S., "Turbocharging the internal combustion engine." Macmillan Press Ltd, 1982.

[6.4] "Ansys users manual" Revision 5.0. Published by Swanson Analysis Systems Inc. Houston PA, USA.

[6.5] Long, S. A., Zhu, Z. Q. and Howe, D., "Vibrational Behaviour of Switched Reluctance Motors" Proc. International Conference on Electrical Machines, Sept. 2-4 1998 Istanbul, Turkey.

[6.6] Lund, J. W. and Saibel, E., "Oil Whirl Orbits of a Rotor in Sleeve Bearings", Journal of Engineering for Industry, pp 813-823, Nov. 1967.

[6.7] Kirk, R. G. and Gunter, E. J., "Stability and Transient Motion of a Plain Journal Mounted in Flexible Damped Supports", Journal of Engineering for Industry, pp576-592, May 1976.

[6.8] Holmes, R., "The Vibration of a Rigid Shaft on Short Sleeve Bearings" Journal of Mechanical Engineering Science, No 2, Part 4, pp337-341, 1960.

[6.9] "Mechanical Vibration - Balance Quality Requirements for Rigid Rotors Part 1: Determination of Permissible Residual Unbalance", ISO 1940-1 Standard. 1986 (First Edition)

[6.10] Smith, D. M., "Journal Bearings in Turbomachinery", Chapman and Hall, 1969.

[6.11] Taylor, H. D and Schenectady, N. Y., "Critical Speed Behaviour of Unsymmetrical Shafts", Journal of Applied Mechanics, pp A-71-A79, June, 1940.

[6.12] Eshleman, R. L. and Eubanks, R. A., "Effects of Axial Torque on Rotor Response: An experimental Investigation", ASME 70-WA/DE-14, Presented at the Annual Winter Meeting, New York, November 29-December 3, 1970. 
[6.13] Childs, D. W., "Fractional Frequency Rotor Motion Due to Nonsymmetric Clearance Effects", ASME 81-GT-145, Proc. of Gas Turbine Conference, Houston, March 9-12. 1981.

[6.14] Tärnhuvud, T., Reichert, K. "Accuracy Problems of Force and Torque Calculation in FE-Systems", IEEE Trans. on Magnetics, Vol. 24, No 1, Jan. 1998.

[6.15] Dowers, A., "The Pursuit of Higher Rotational Speeds; Developments in Bearing Design and Materials", IEE Colloquium on High Speed Bearings for Electrical Machines, April 1997.

[6.16] Changsen, W., "Analysis of Rolling Element Bearings", Mechanical Engineering Publications, London, 1991. 


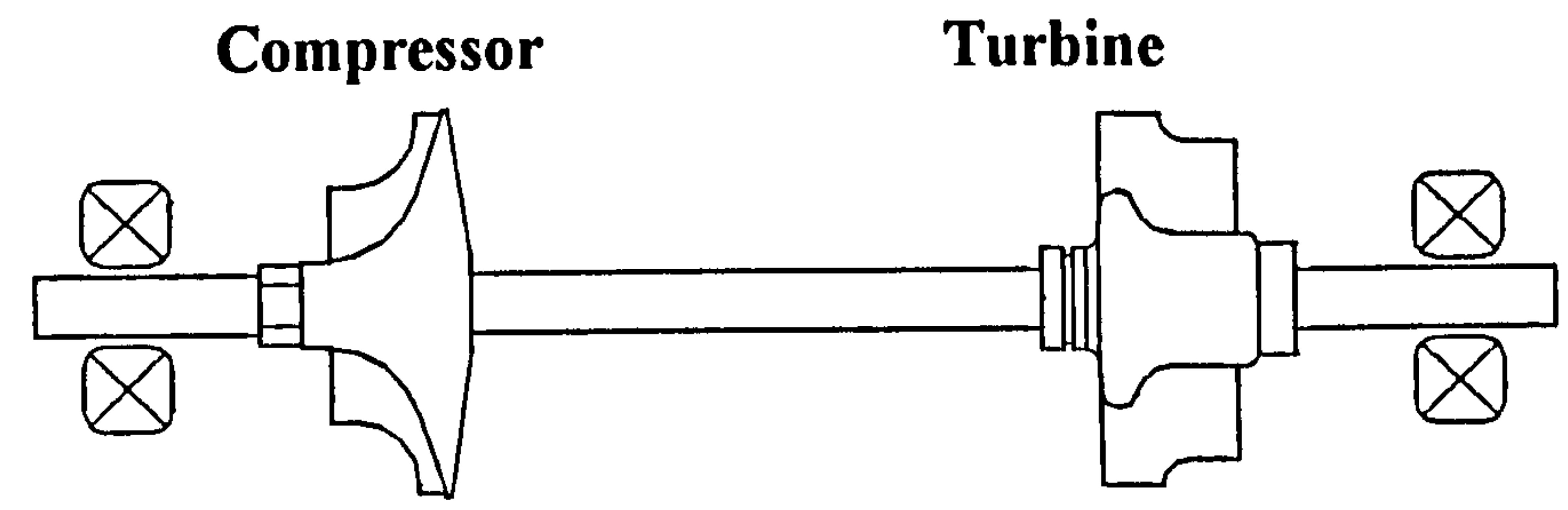

Figure 6.1a.

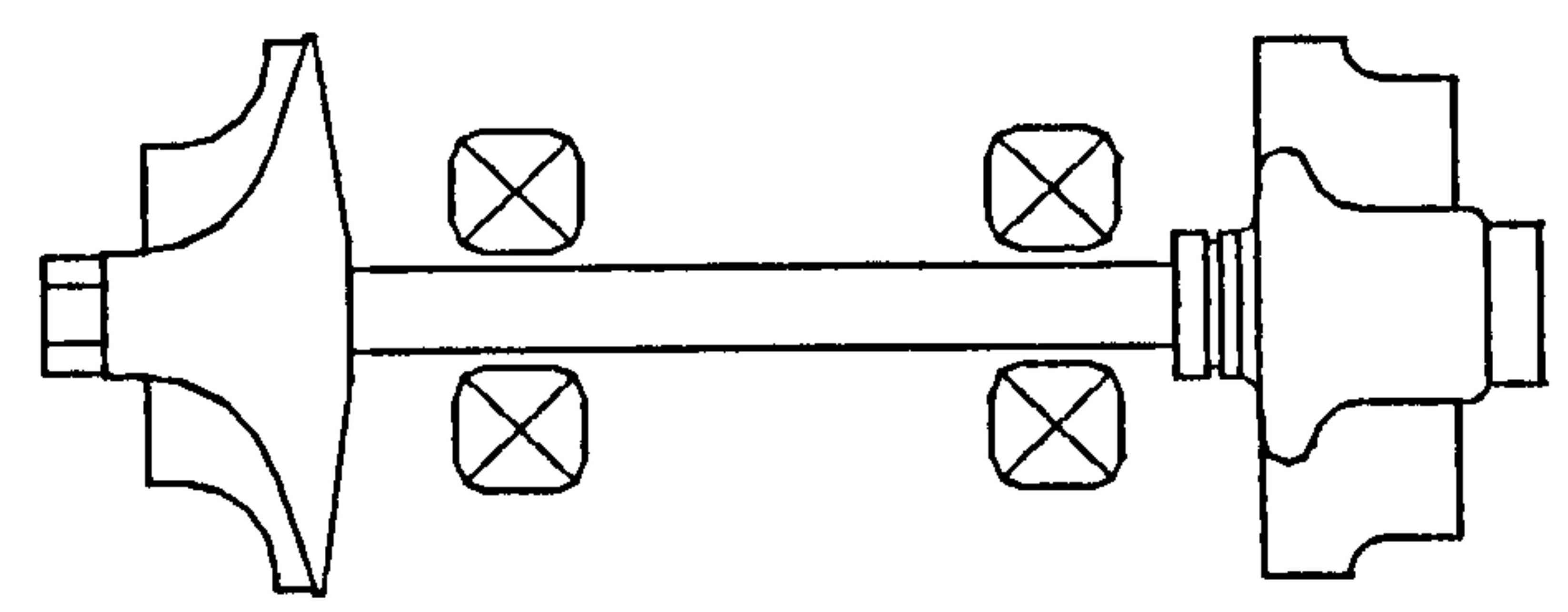

Figure 6.1b.

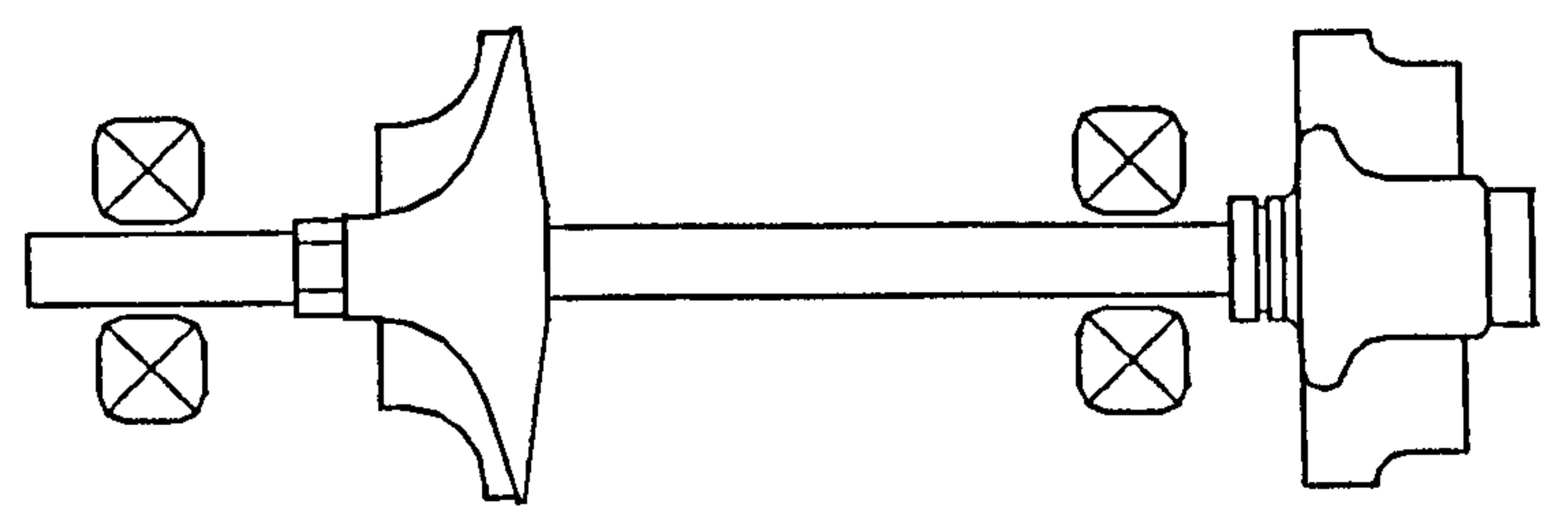

Figure 6.1c.

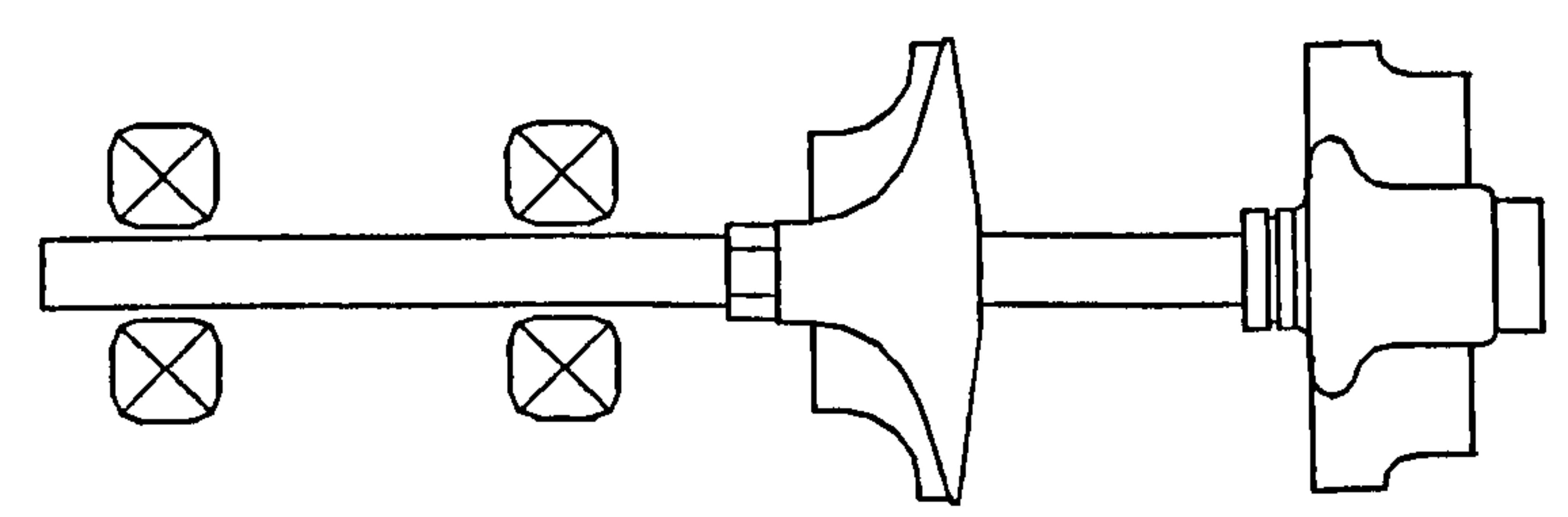

Figure 6.1d

Figure 6.1. Schematic of possible bearing configurations [6.3]. 


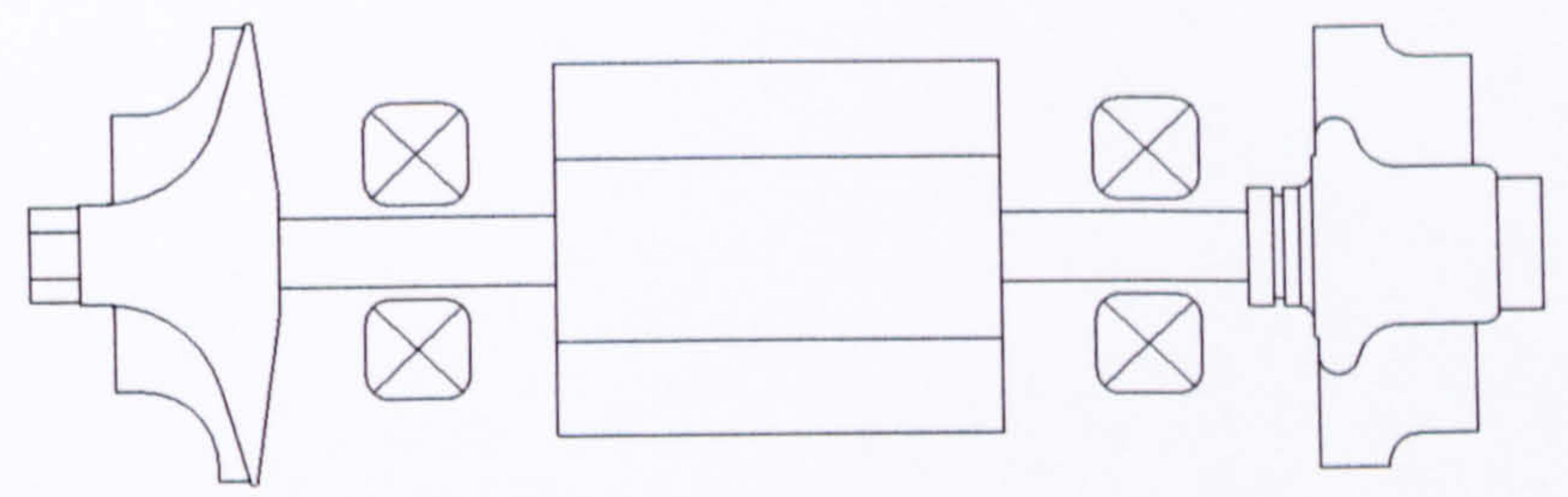

Figure 6.2. Bearing configuration with addition of SR rotor
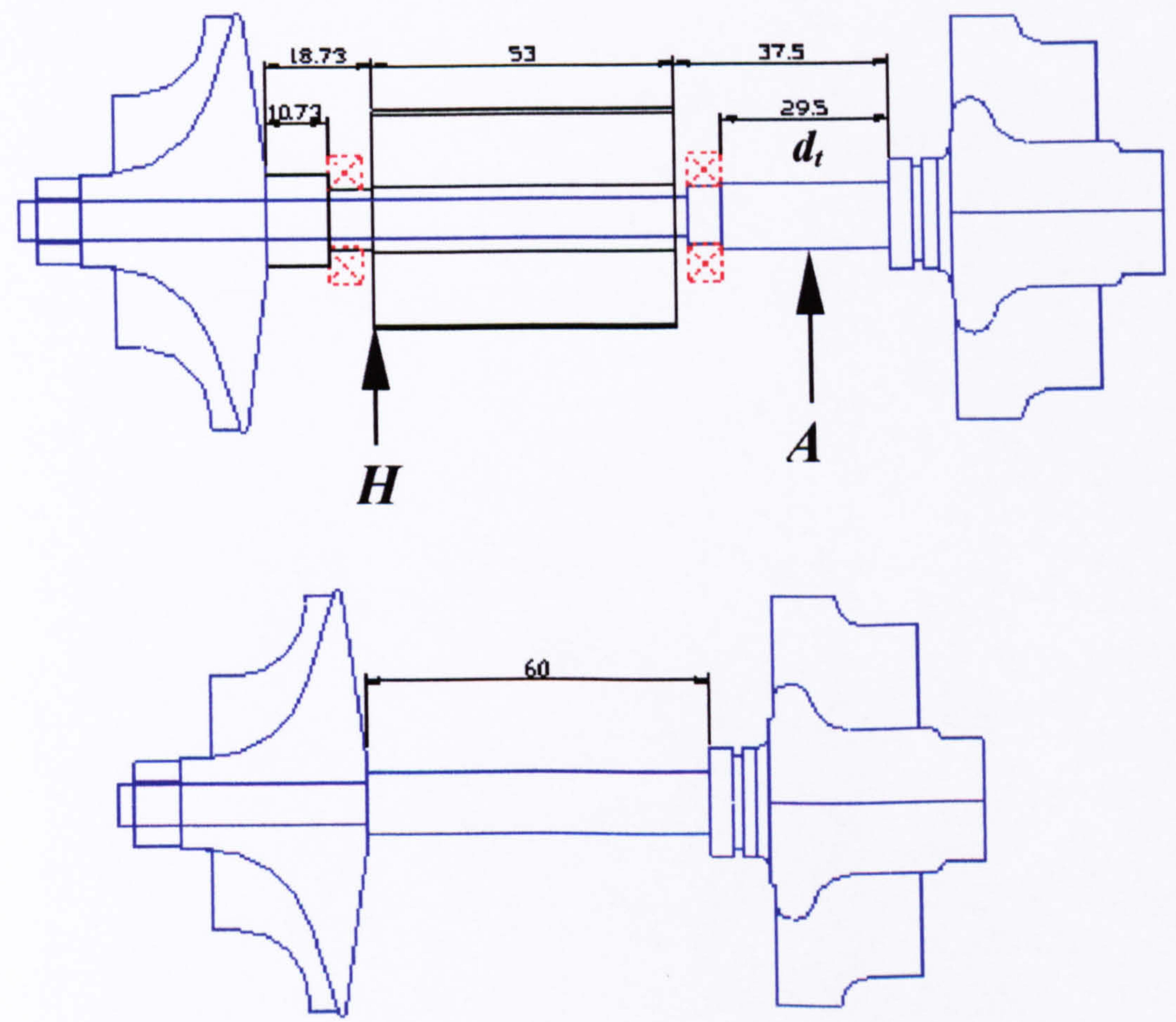

Figure 6.3 Basic assembly of the standard turbocharger and prototype turbogenerator rotors with principal dimensions in $\mathrm{mm}$. 


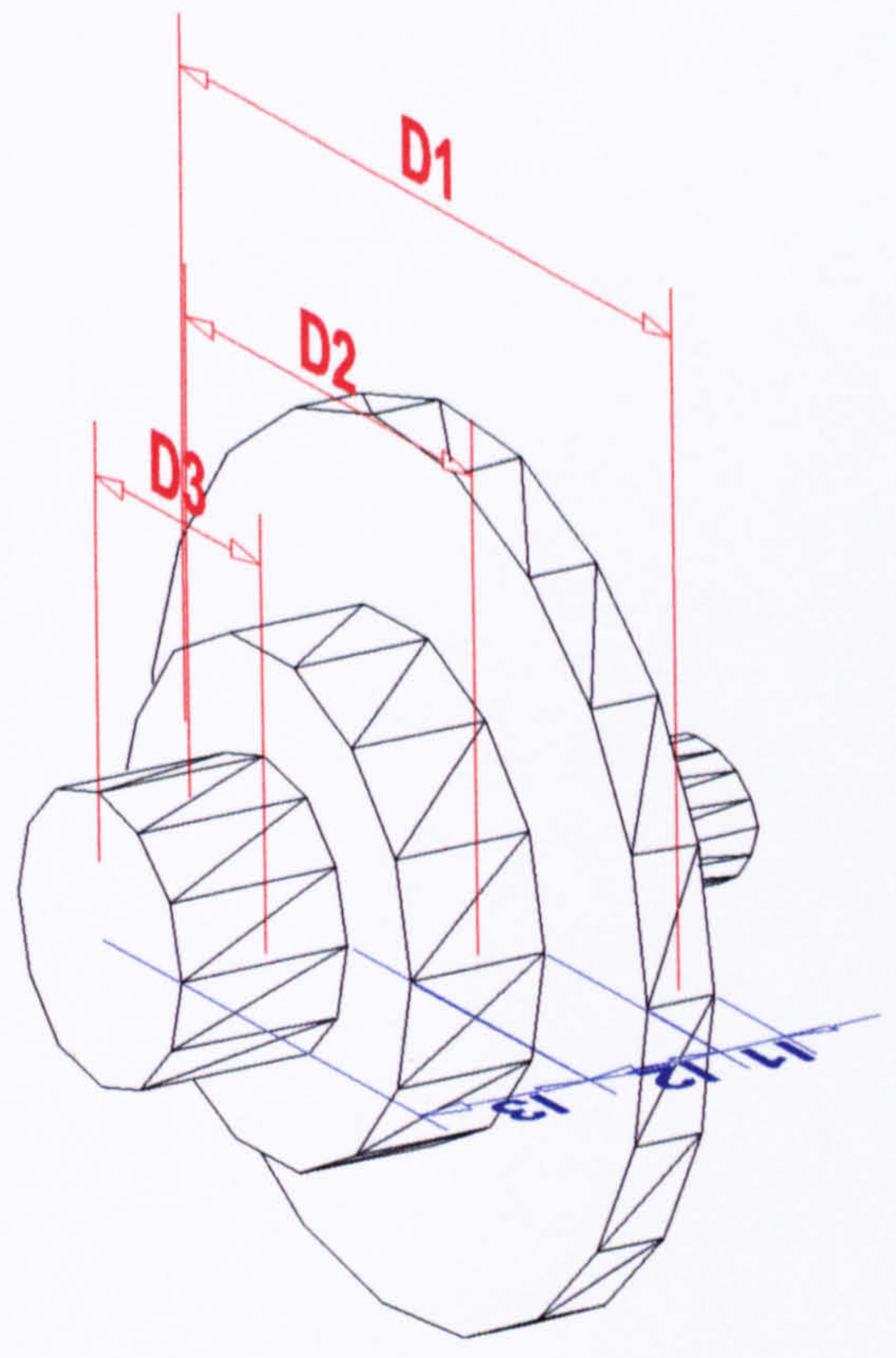

Figure 6.4 Principal dimensions of the three cylinder approximation to the turbine and compressor. 


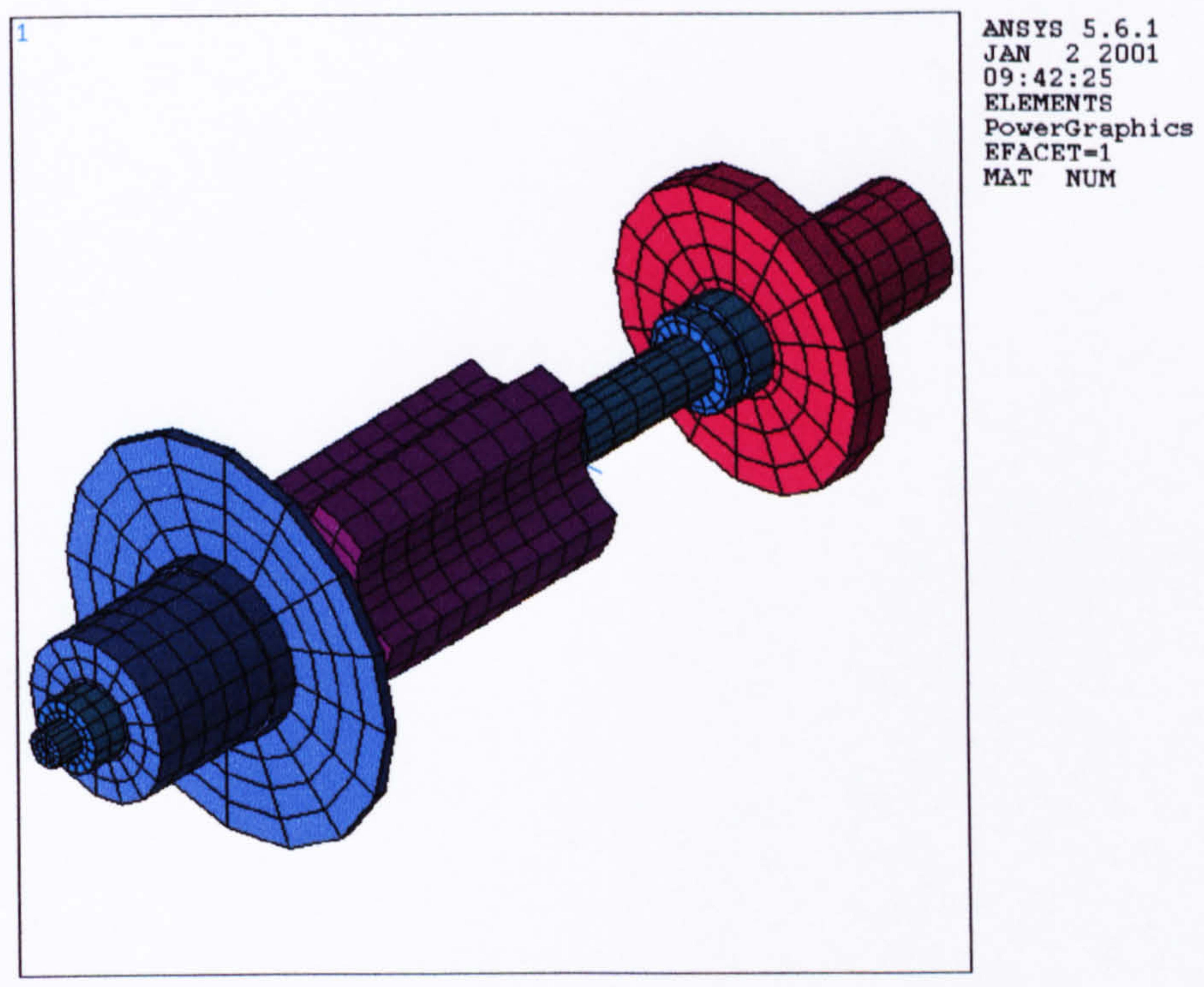

Figure 6.5 Finite element mesh of the prototype rotor consisting of 3772 first order structural elements and 4624 nodes.

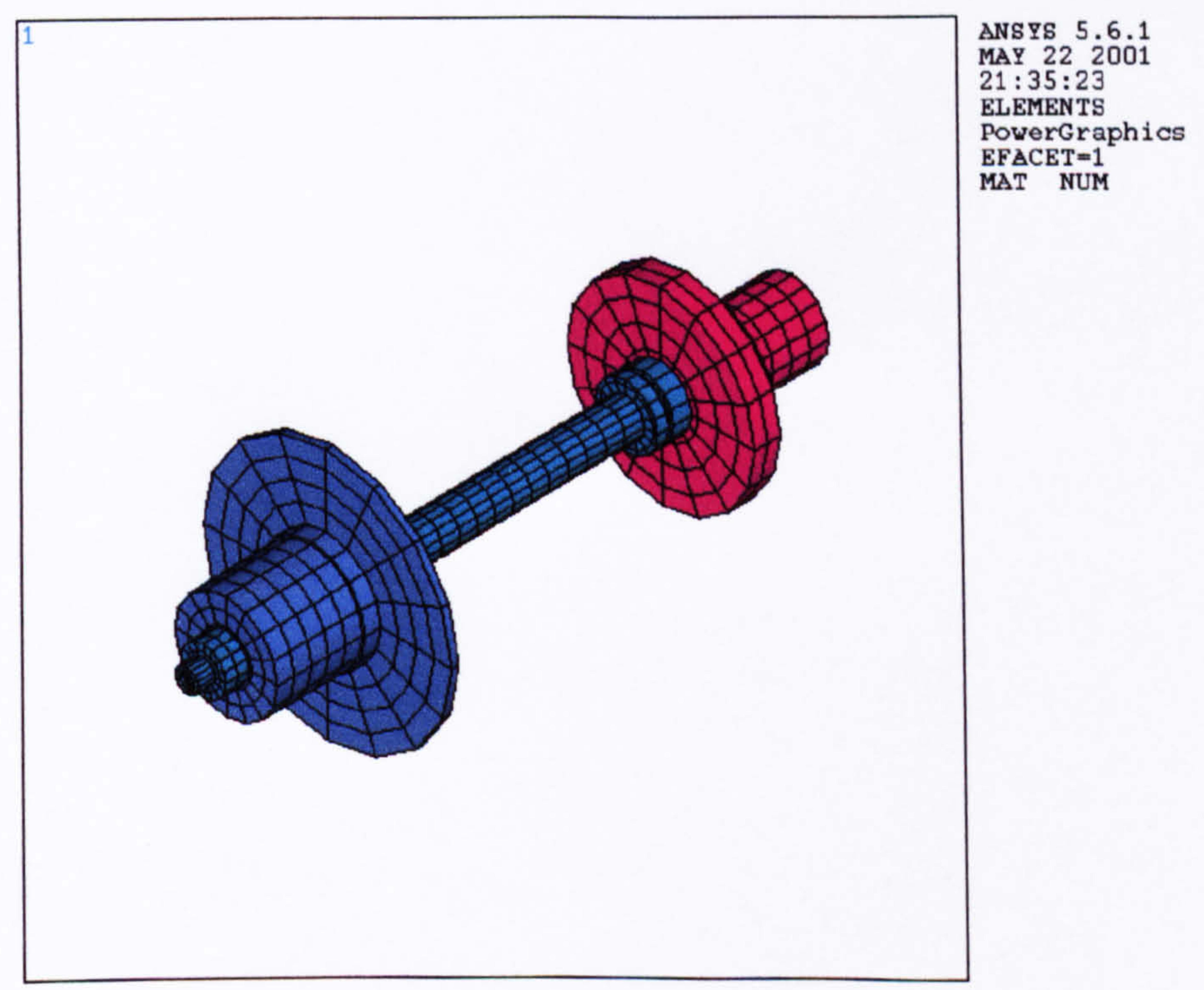

Figure 6.6 Finite element mesh of the standard rotor consisting of 3064 first order structural elements and 3718 nodes. 


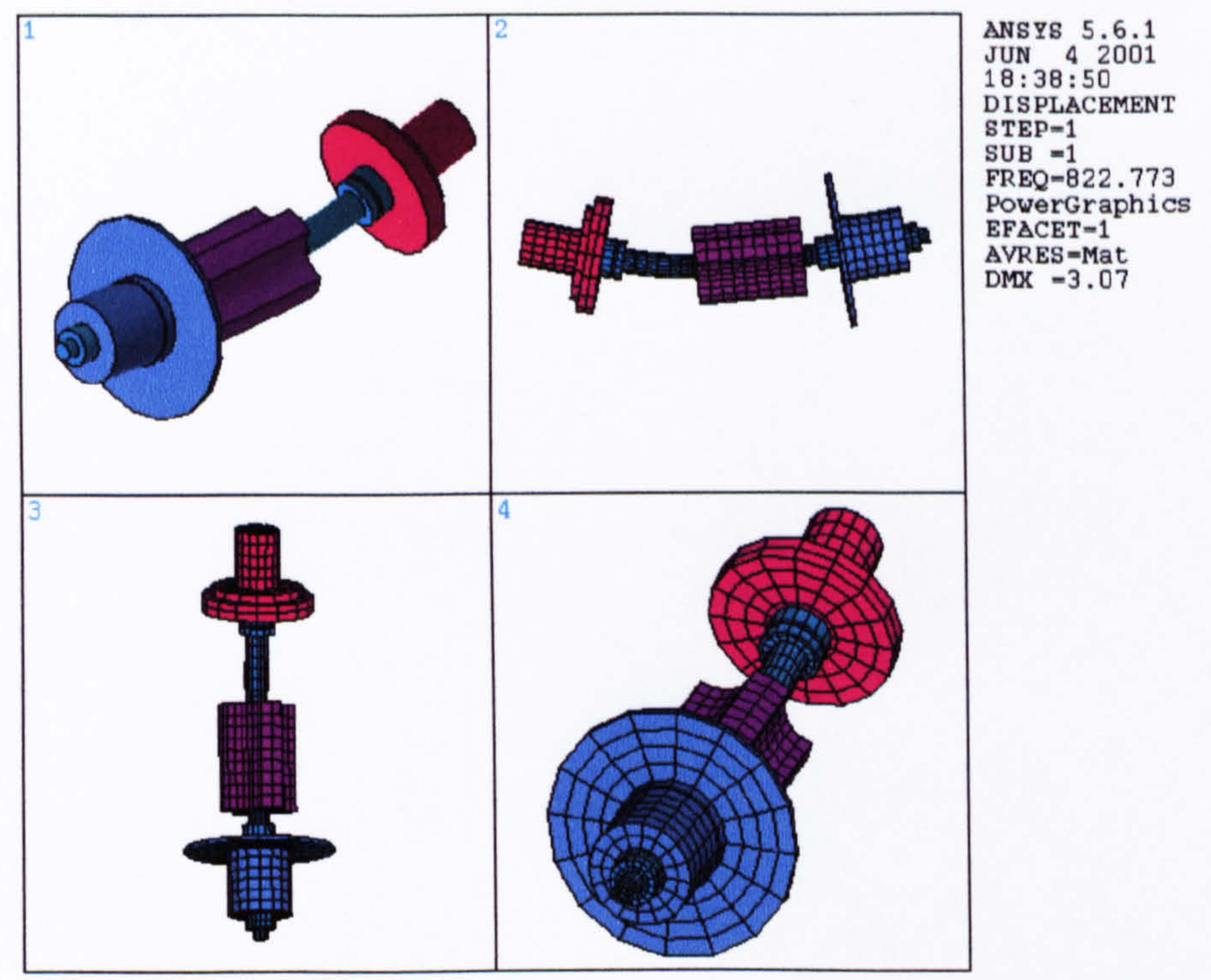

Figure 6.7. Free-free modal analysis showing the first bending mode of vibration of the prototype rotor.

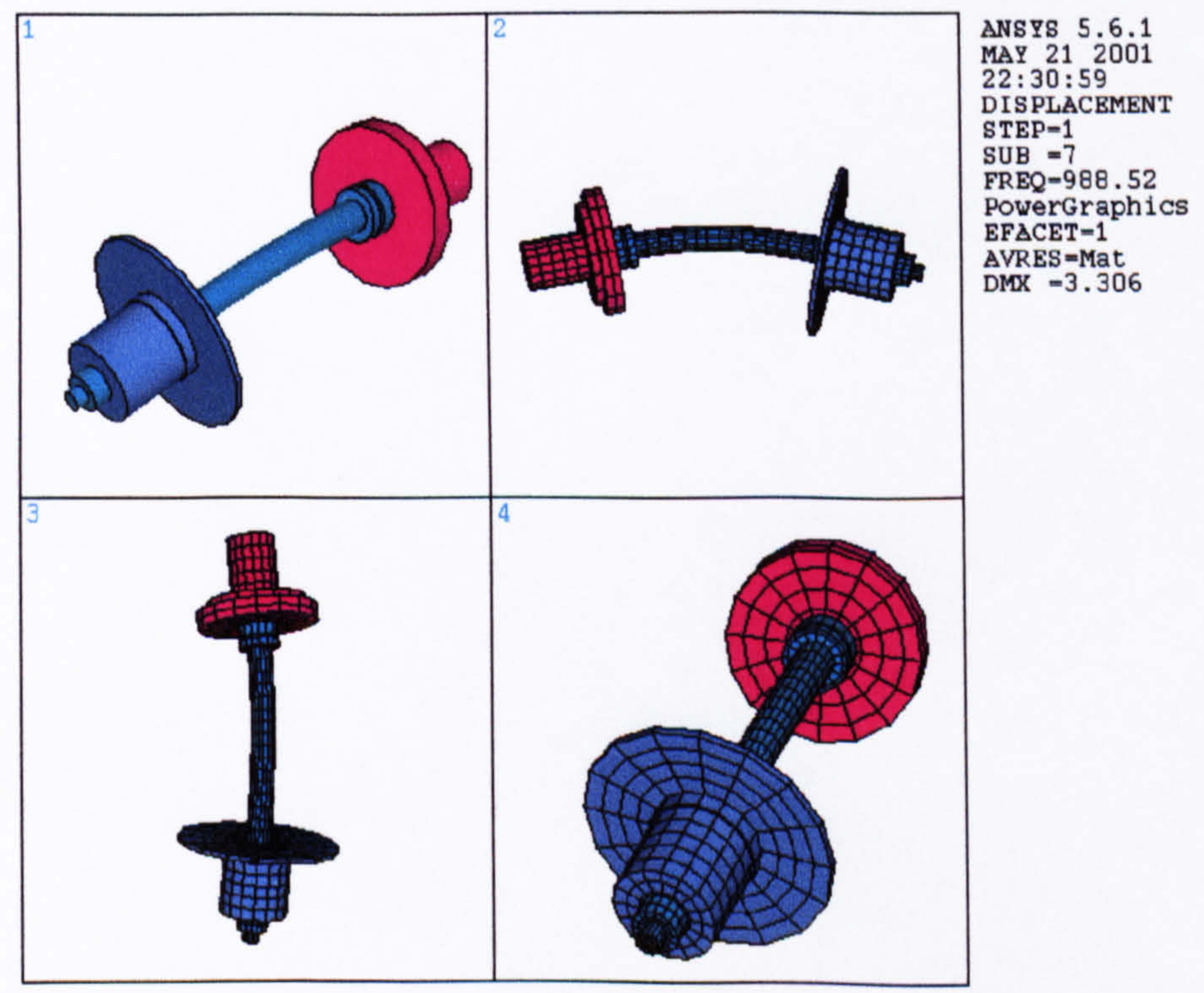

Figure 6.8. Free-free modal analysis showing the first bending mode of vibration of the standard turbocharger rotor. 


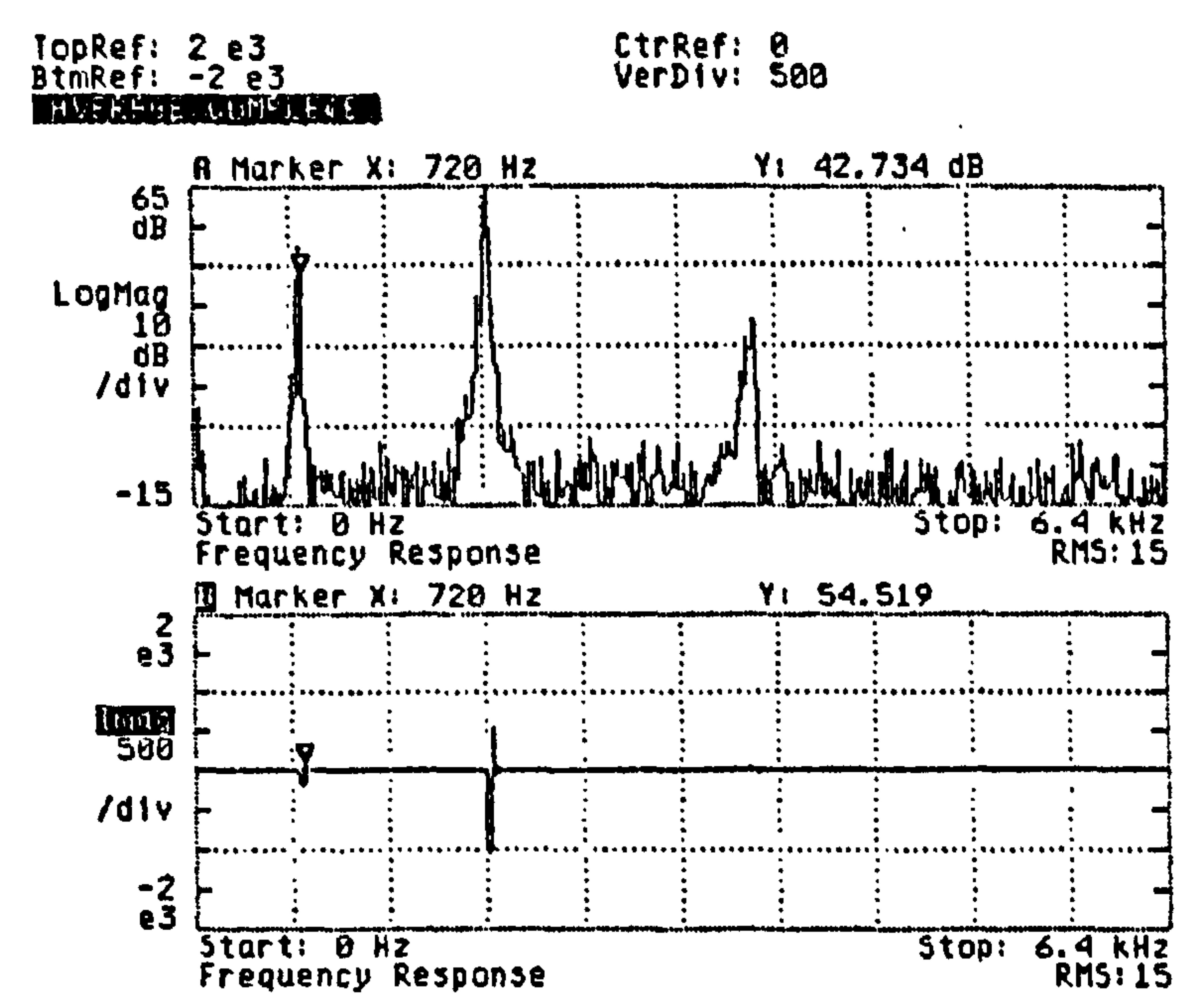

Figure 6.9. Measured free-free response spectra of the prototype rotor.

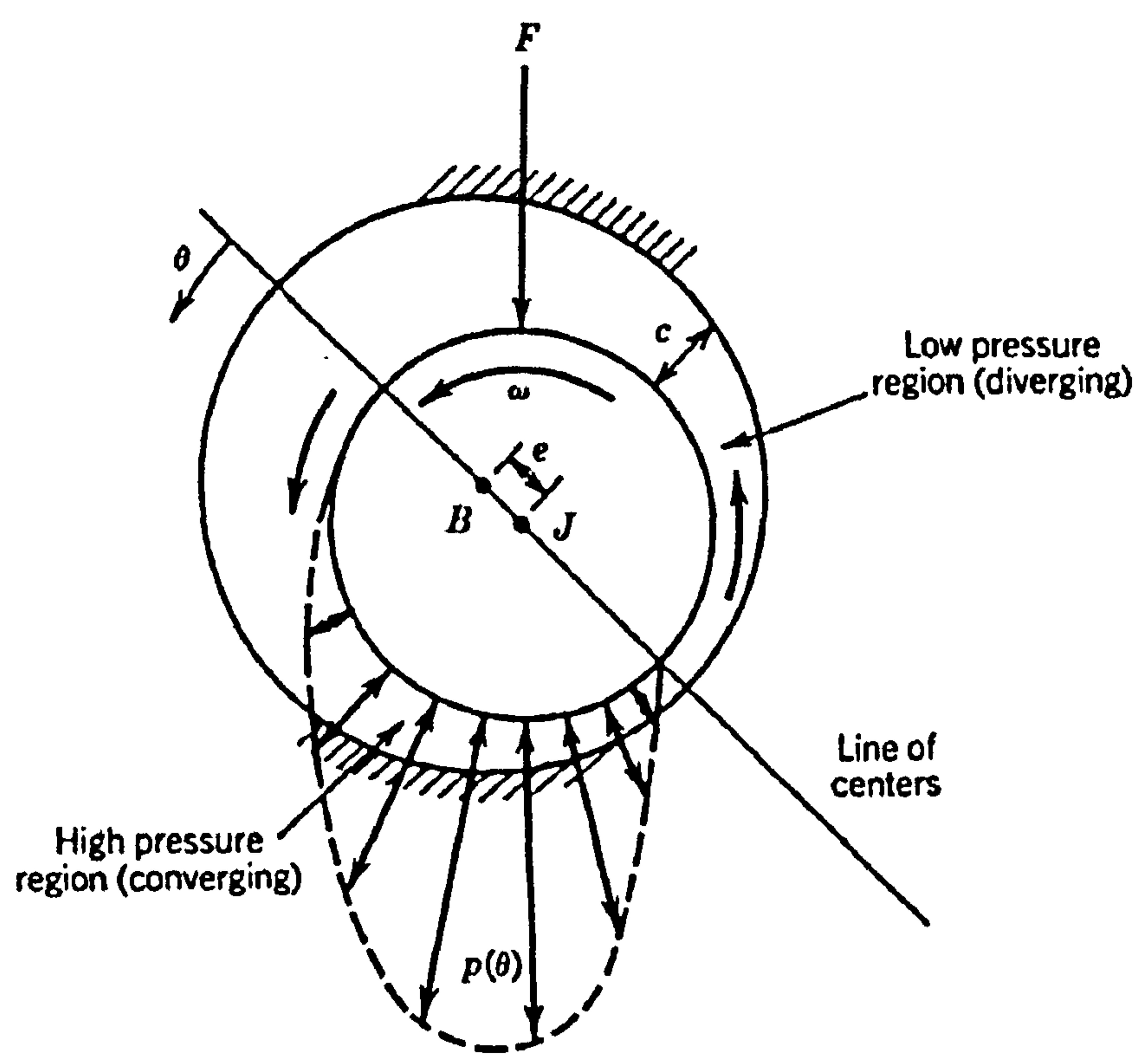

Figure 6.10. Hydrodynamic pressure field generated by an eccentric rotor. [6.1] 


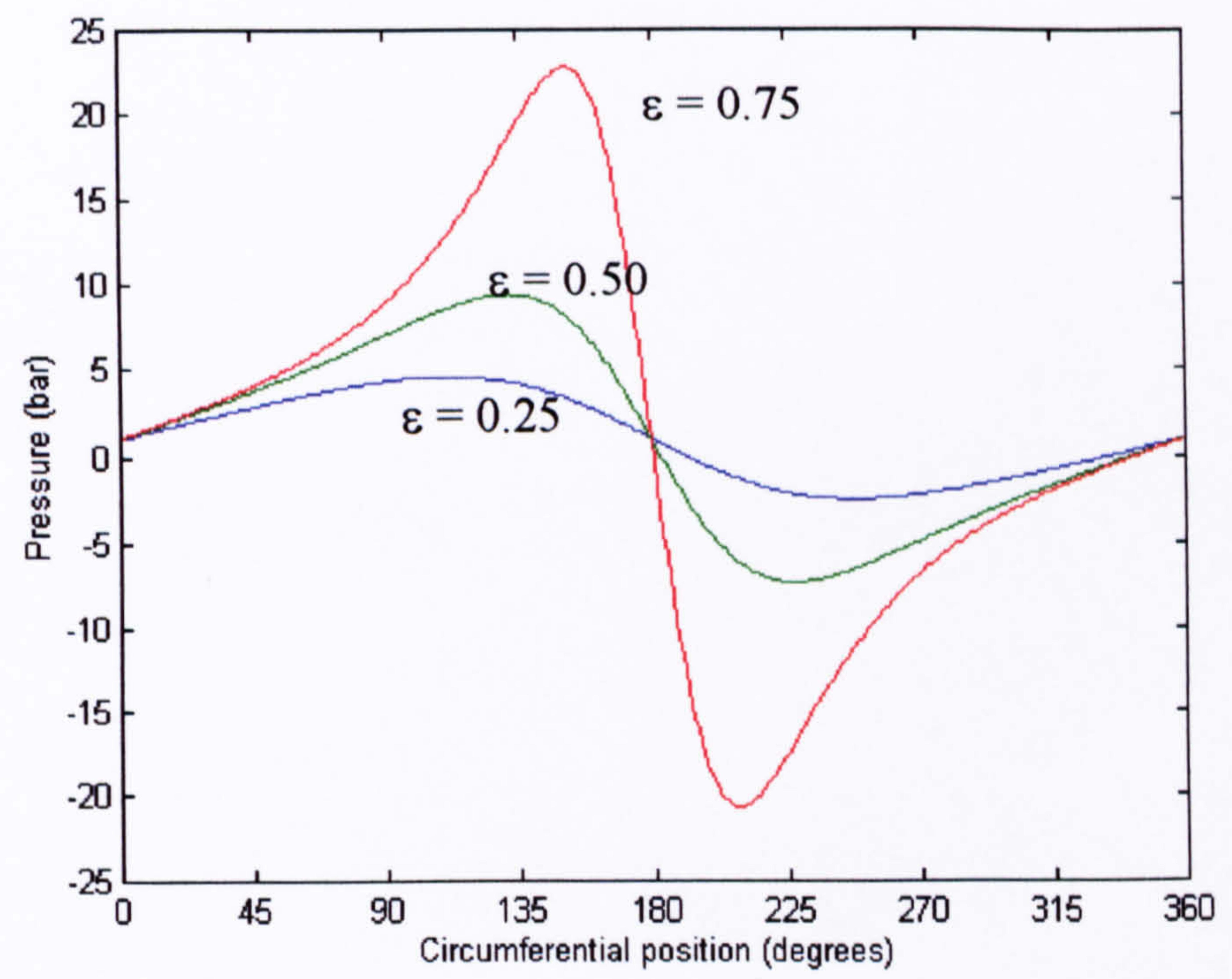

Figure 6.11. Hydrodynamic pressure distribution around the journal of a "long" automotive size bearing at differing eccentricities.

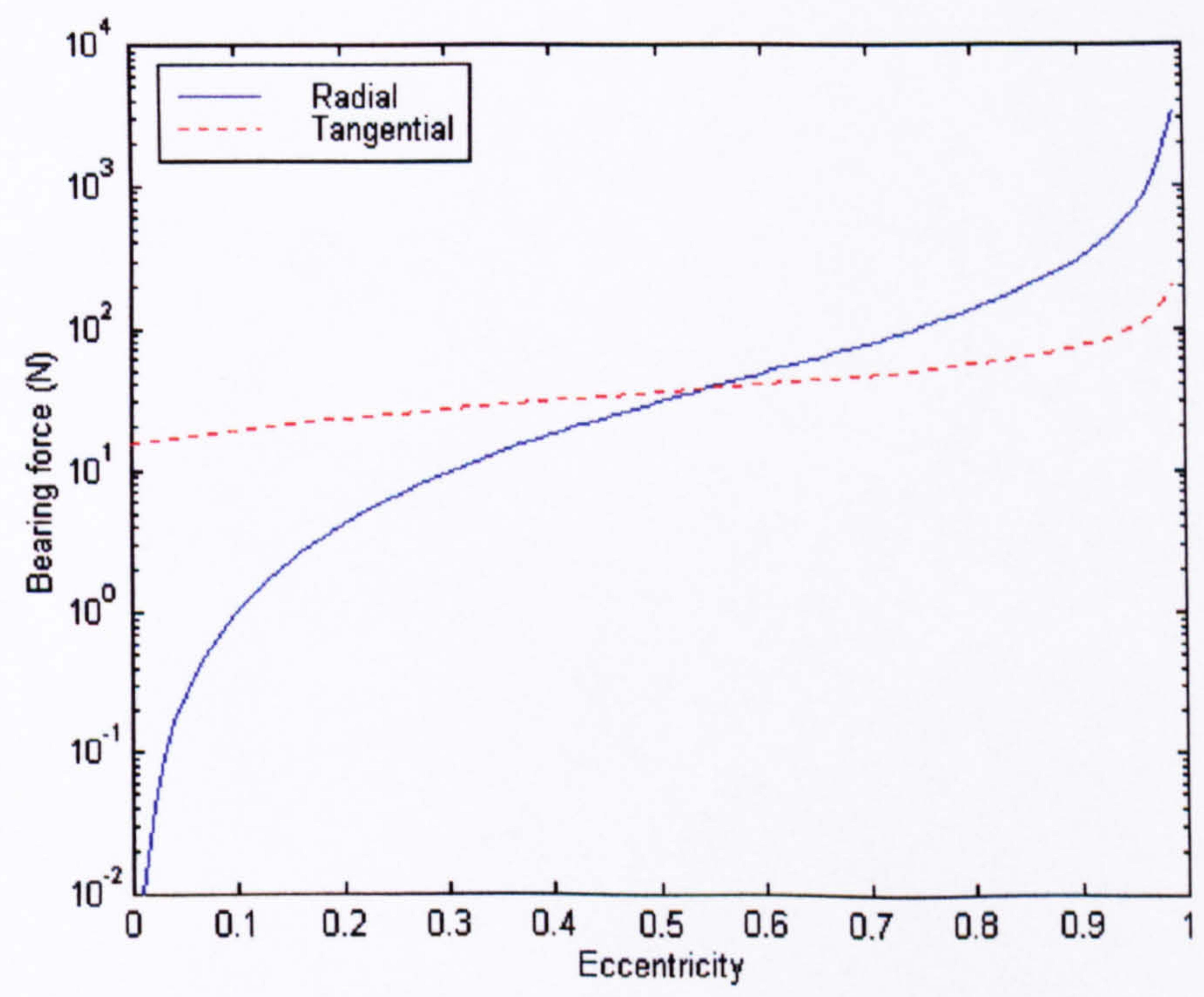

Figure 6.12. Radial and tangential components of bearing force of a typical automotive hydrodynamic bearing as a function of the rotor eccentricity. 


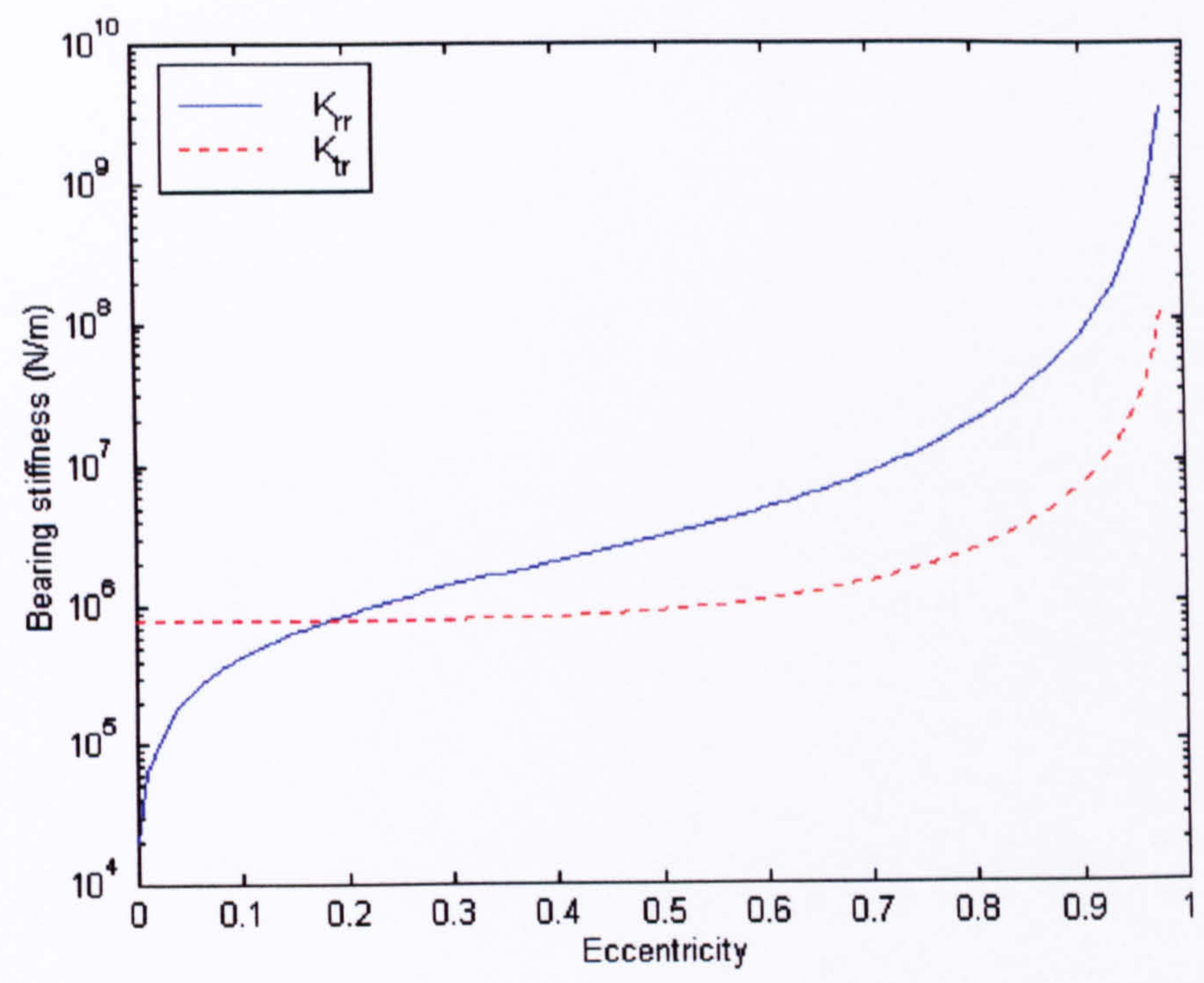

Figure 6.13. Incremental direct and cross-coupled stiffness of a typical automotive hydrodynamic bearing as a function of the rotor eccentricity.

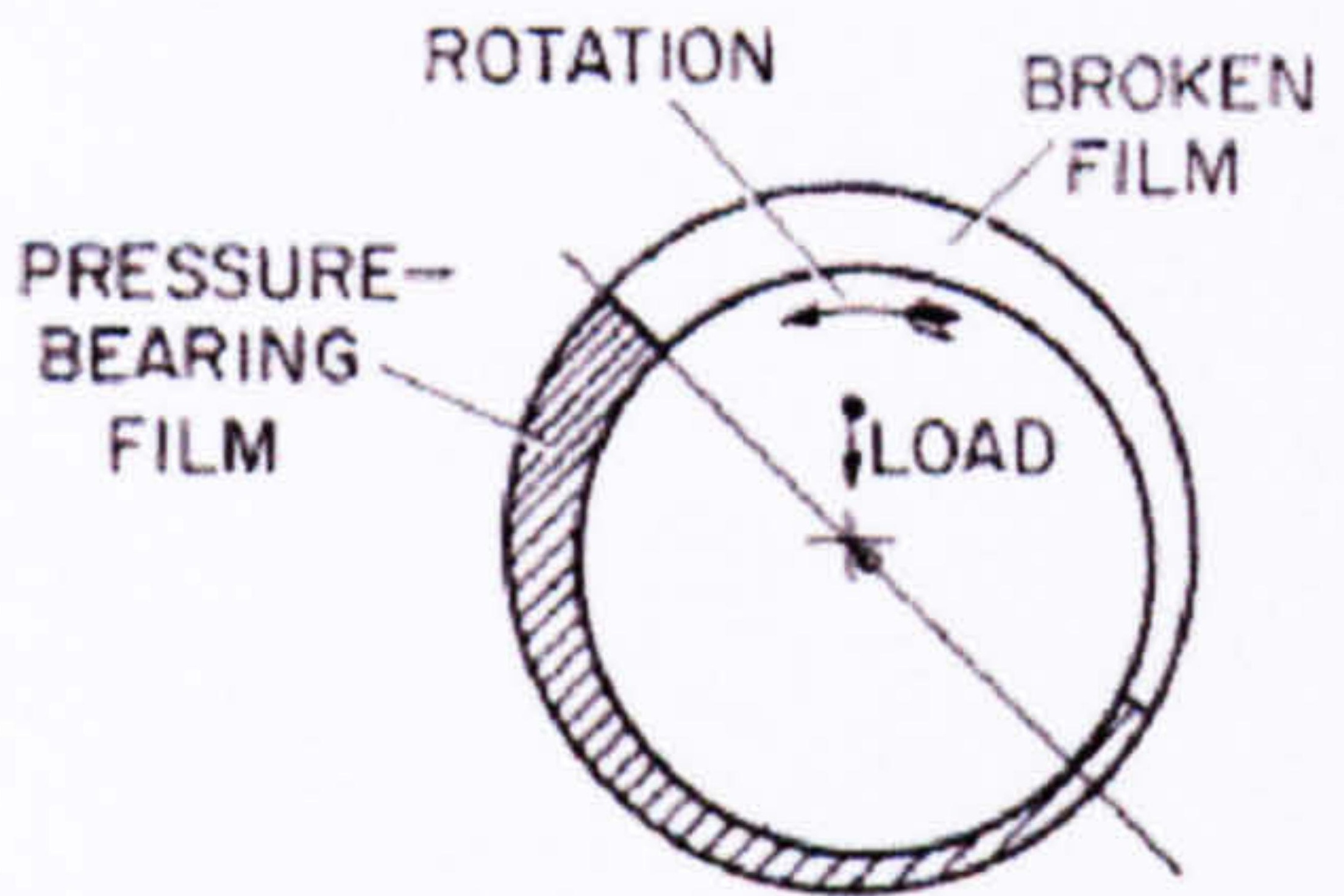

(a) STEADY STATE

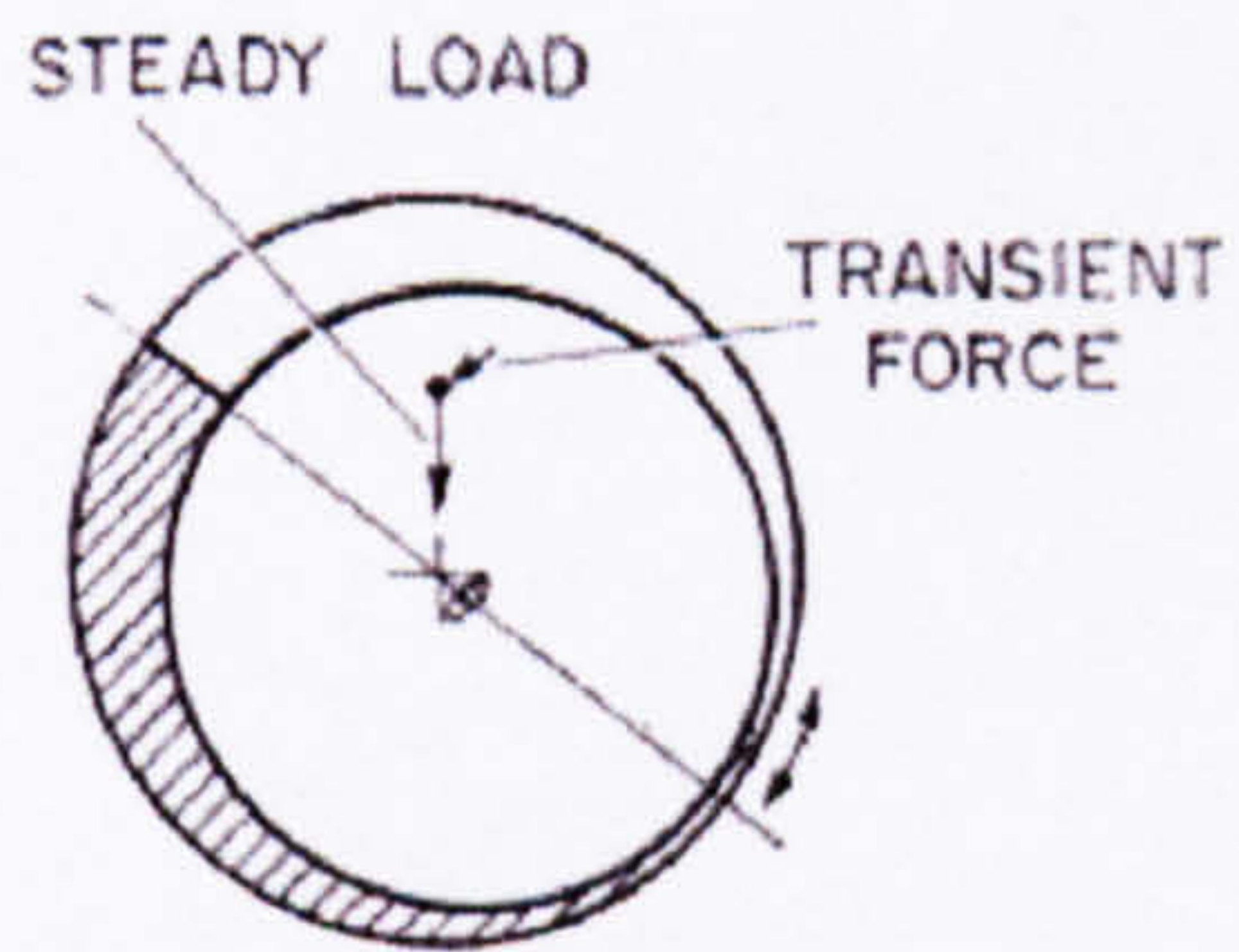

(b) OSCILLATING FILM

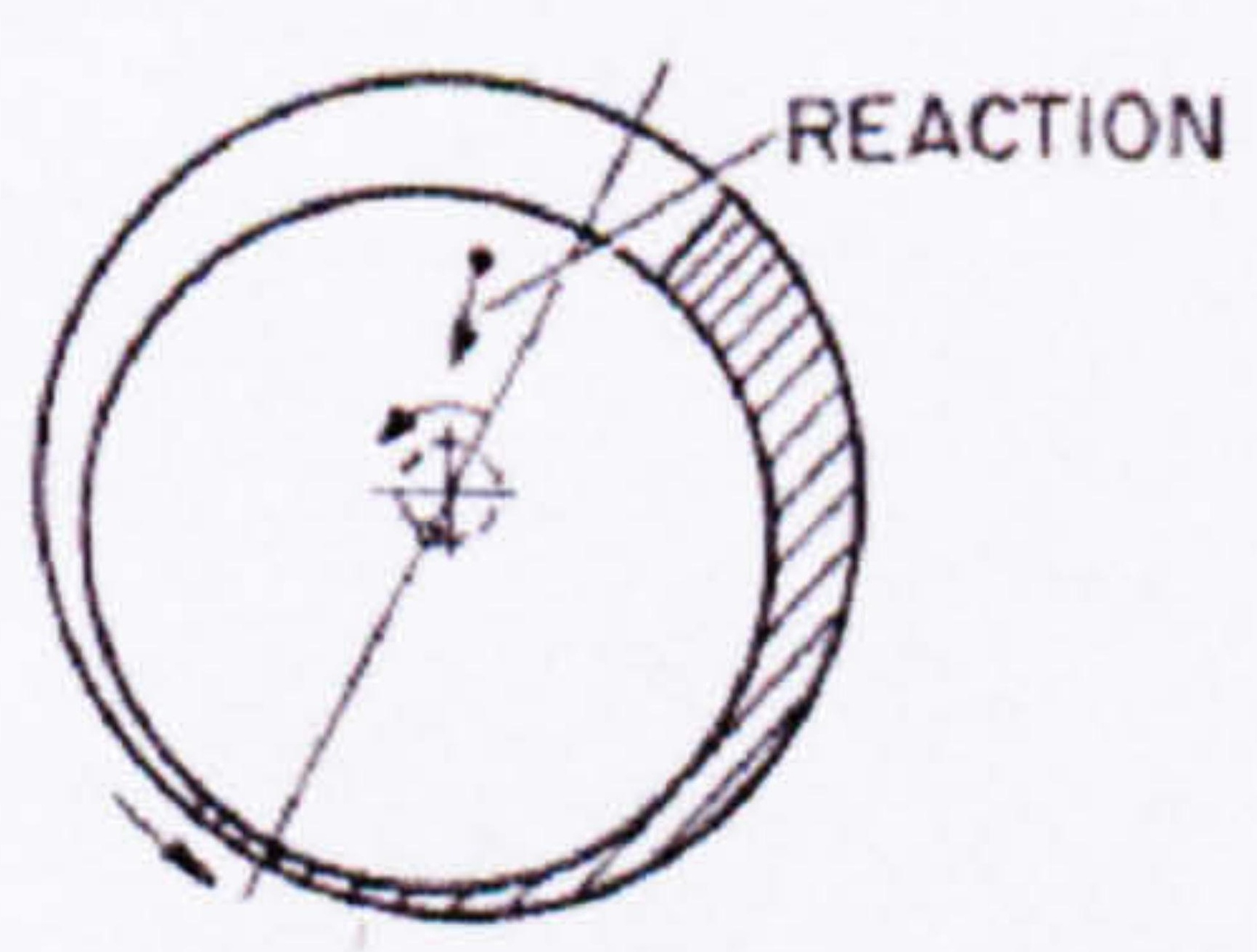

d) ROTATING LEADING FILM

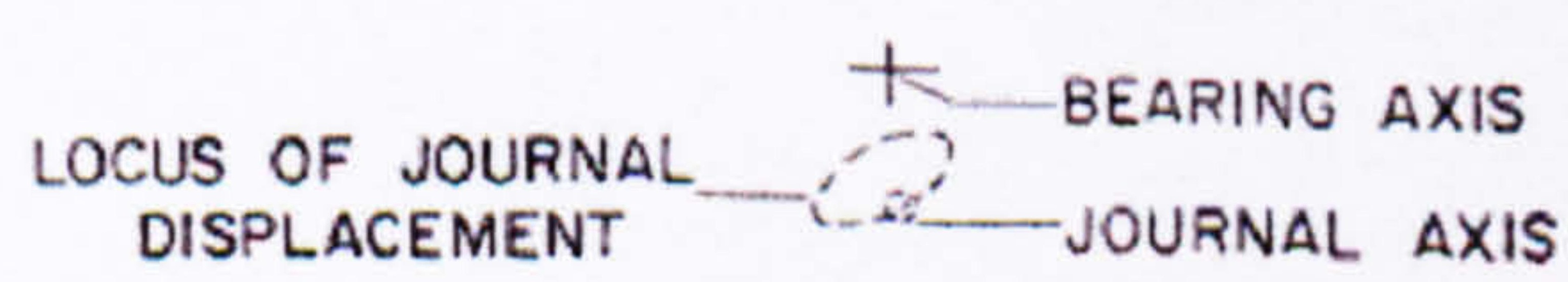

Figure 6.14. Various operating modes of plain hydrodynamic bearings. [6.10] 


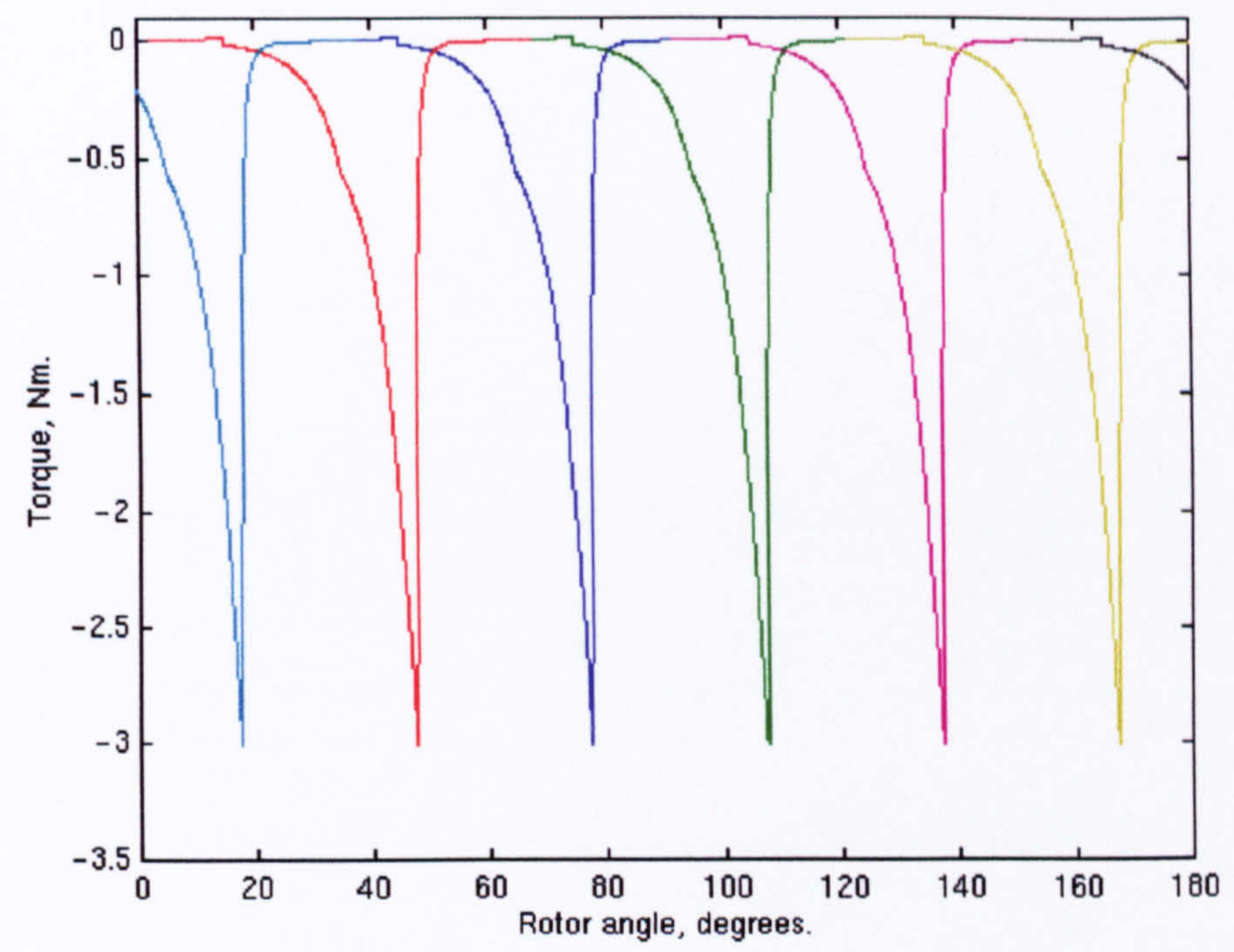

Figure 6.15. Individual torque pulsations for the machine operating at $100000 \mathrm{rpm}$ generating $5 \mathrm{~kW}$.

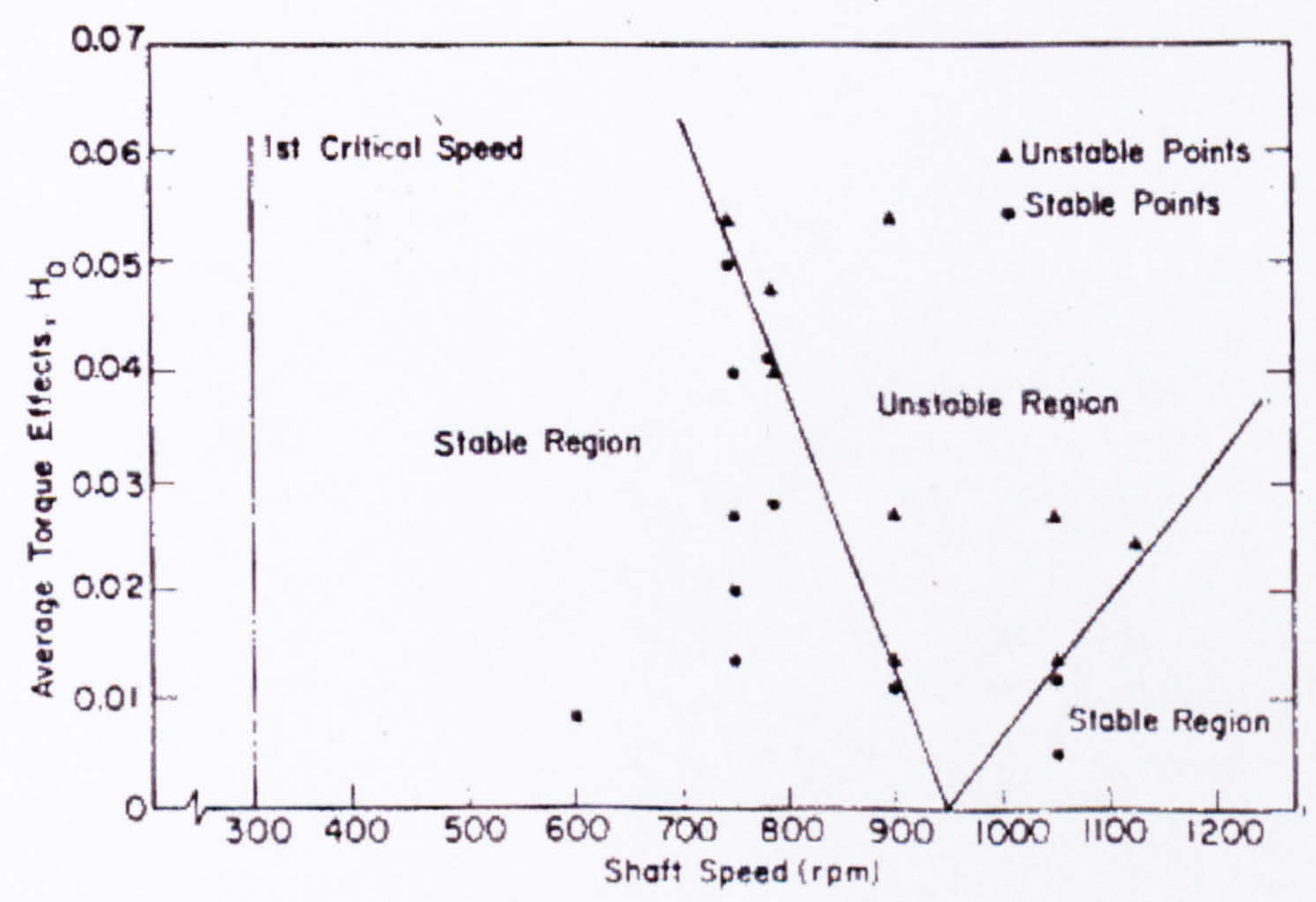

Figure 6.16. Instability of a rotor/bearing system induced by pulsating torque [6.12]. 


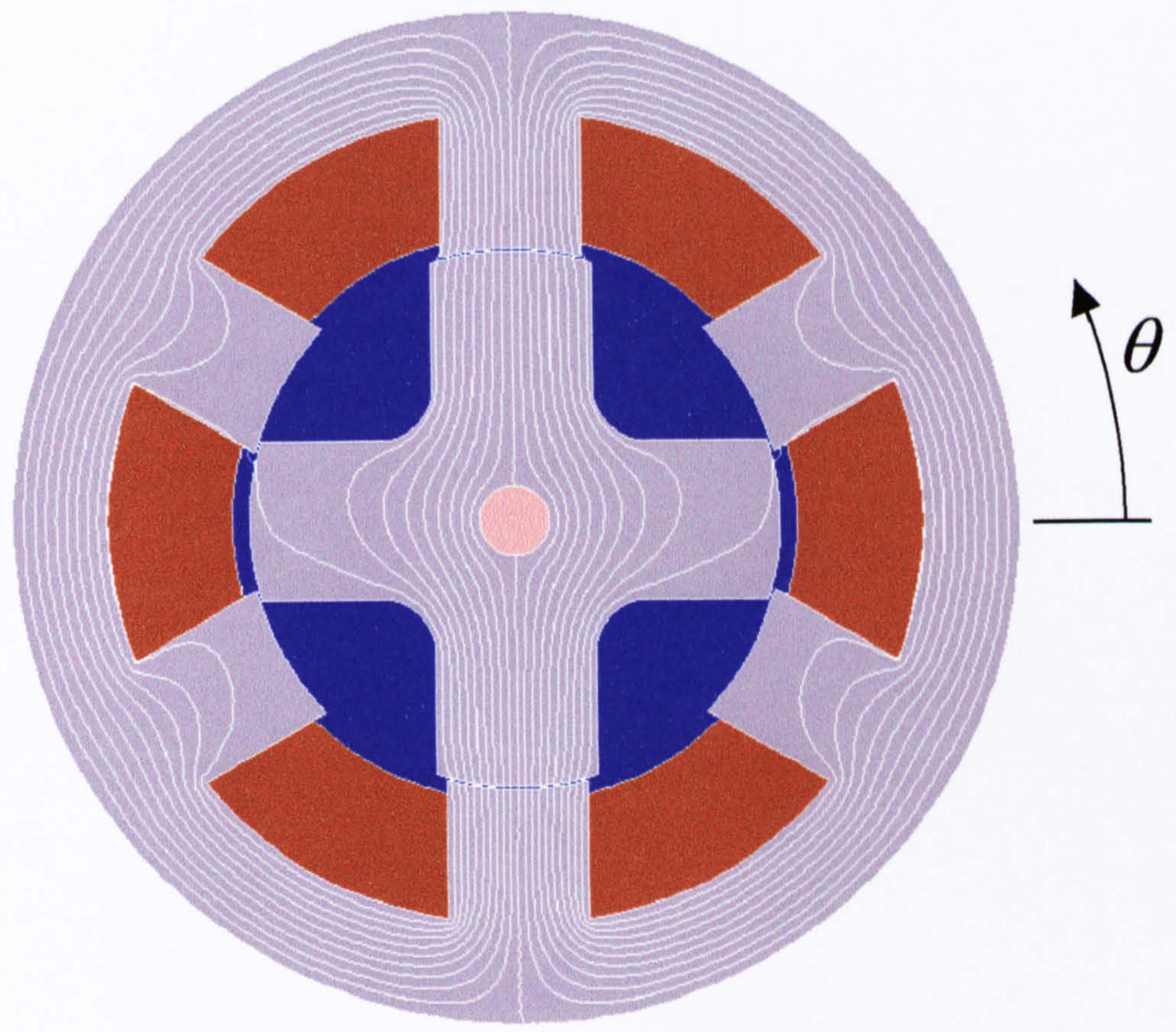

Figure 6.17 Field distribution with a rotor eccentricity of $60 \mu \mathrm{m}$ and current density of $10 \mathrm{Amm}^{-2}$.

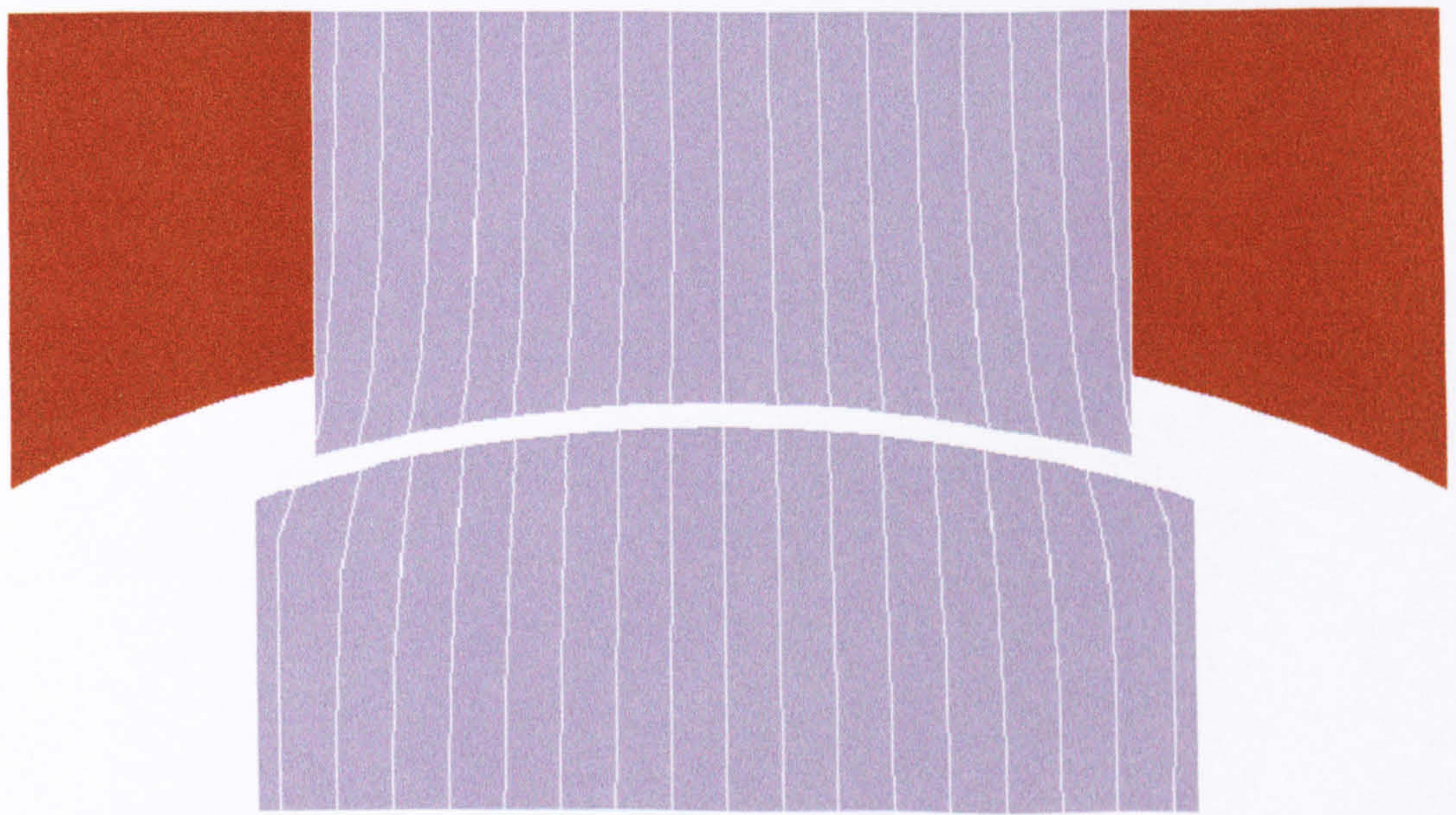

Figure 6.17a. Close up of the top rotor pole airgap showing fringing at edges. 


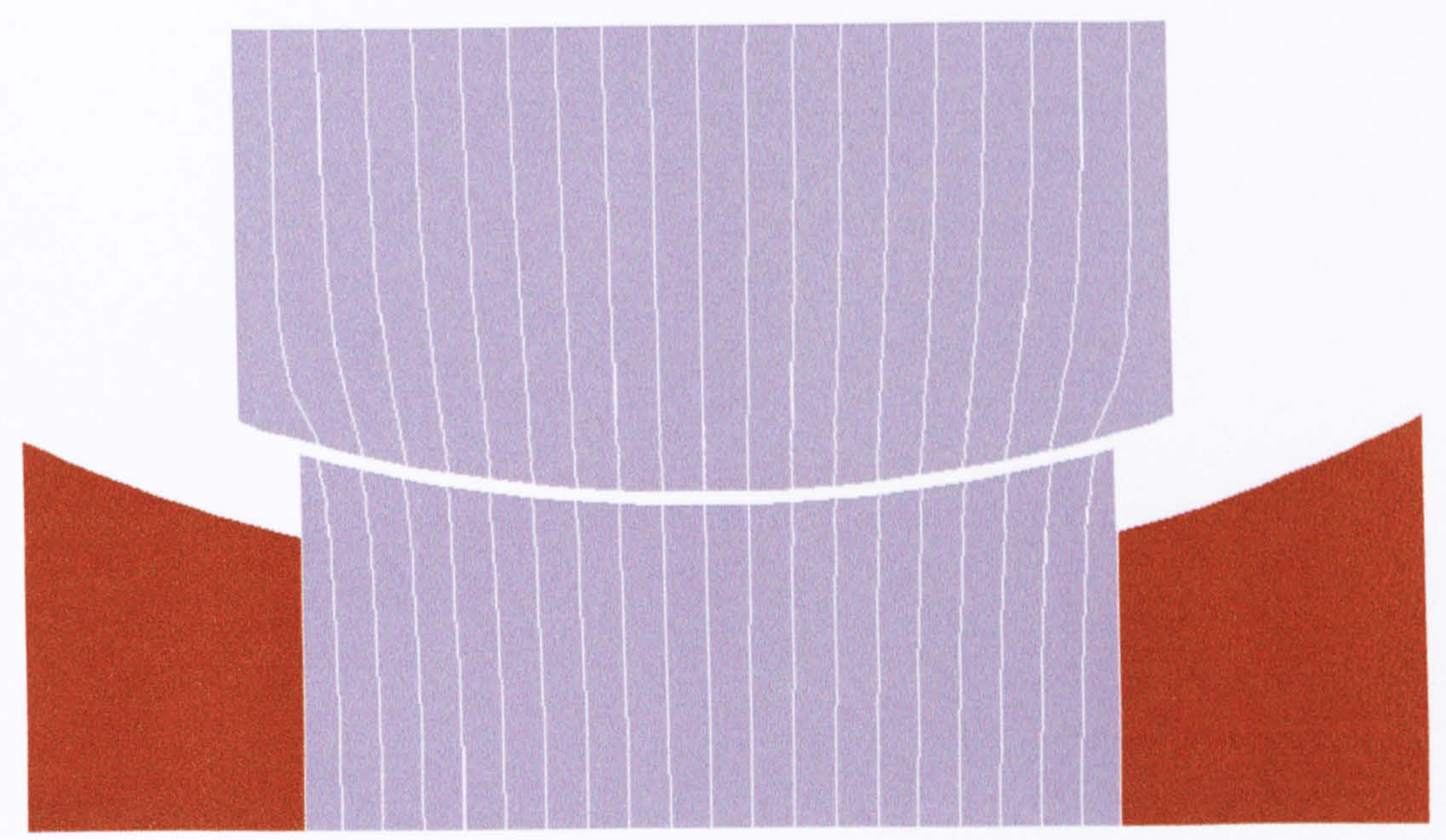

Figure $6.17 \mathrm{~b}$. Close up of the bottom rotor pole airgap with denser flux lines.

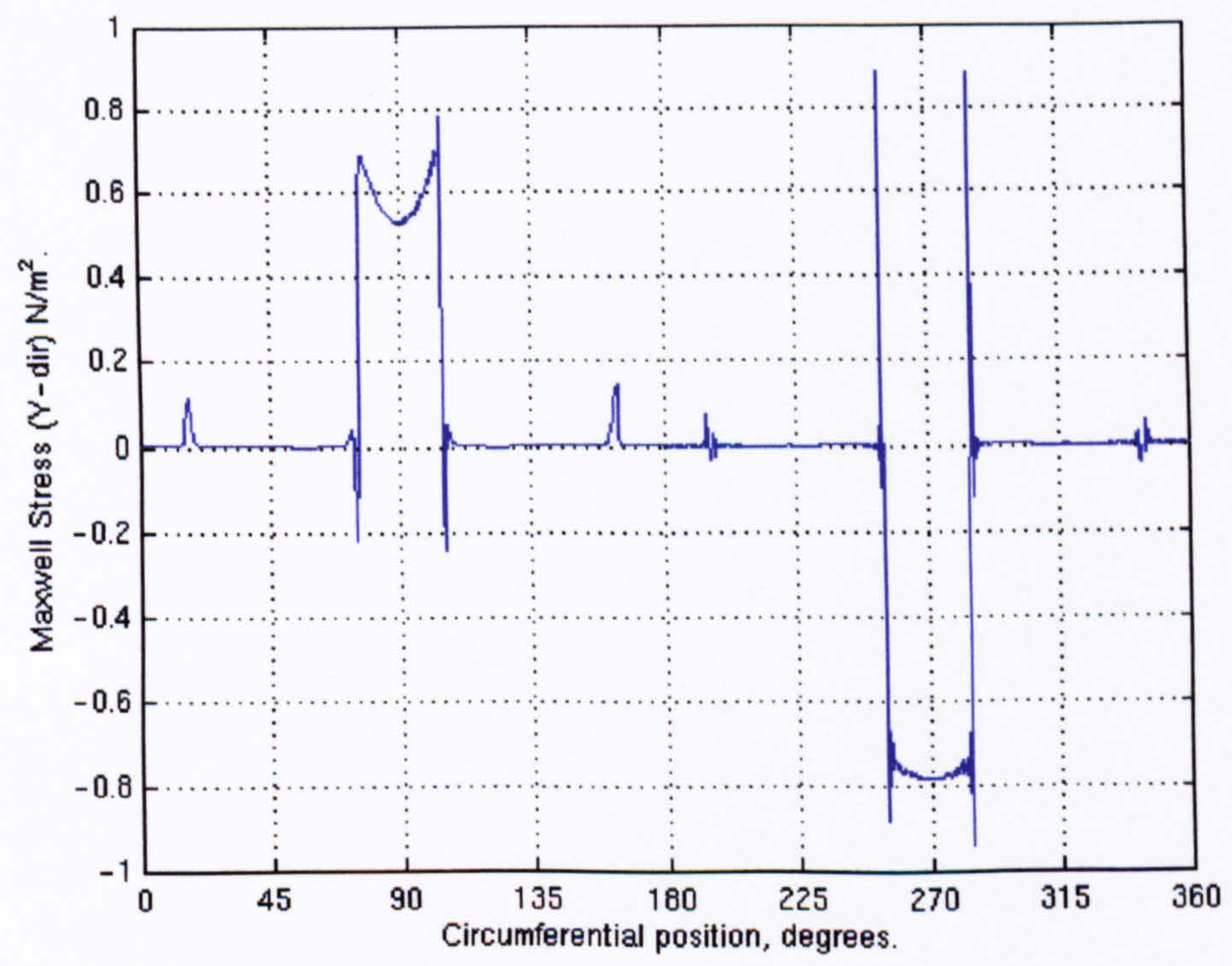

Figure 6.18. Maxwell stress distribution around the circumference of the path defined at the rotor radius. 


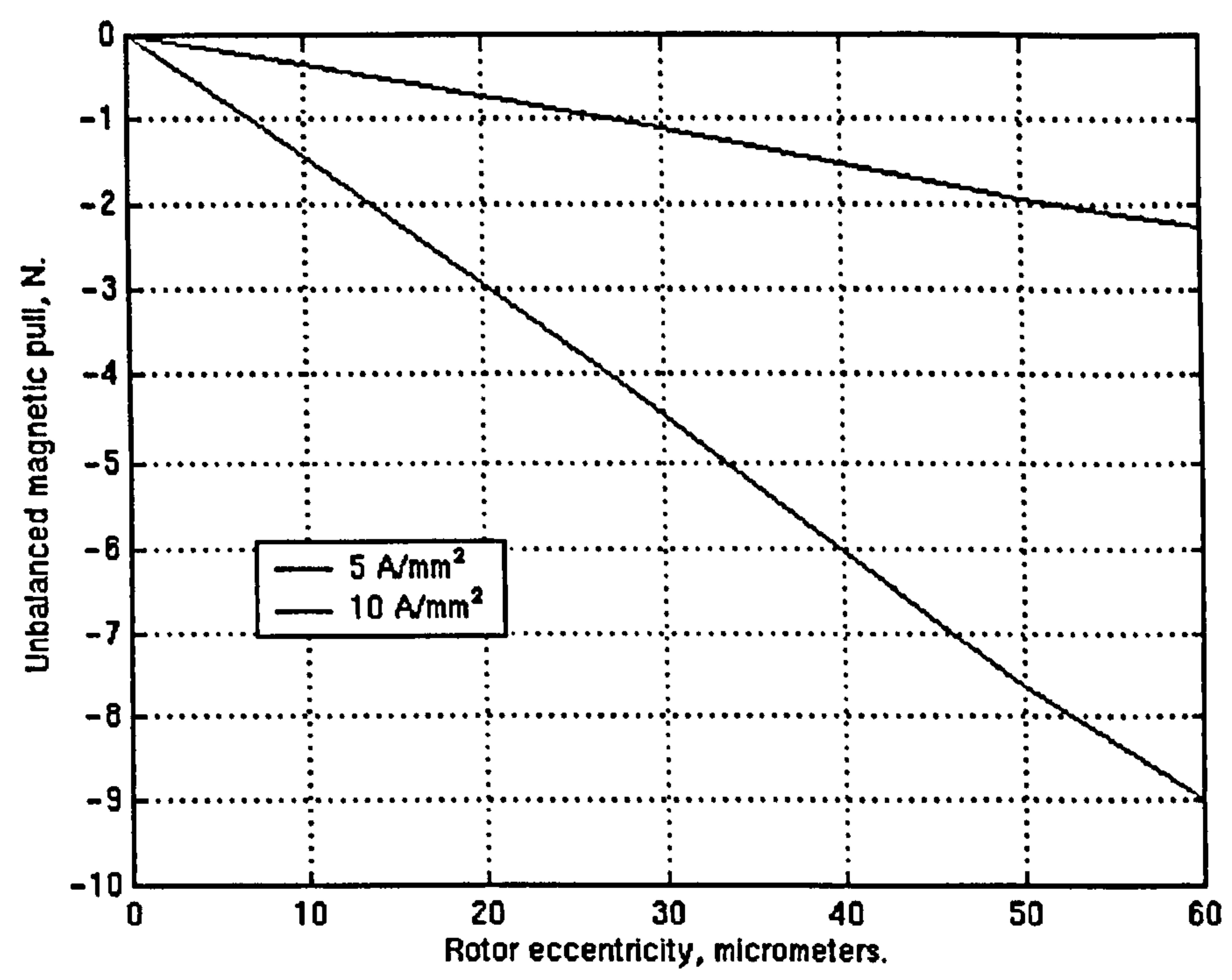

Figure 6.19. Unbalanced magnetic pull force on the rotor as a function of eccentricity and current density.

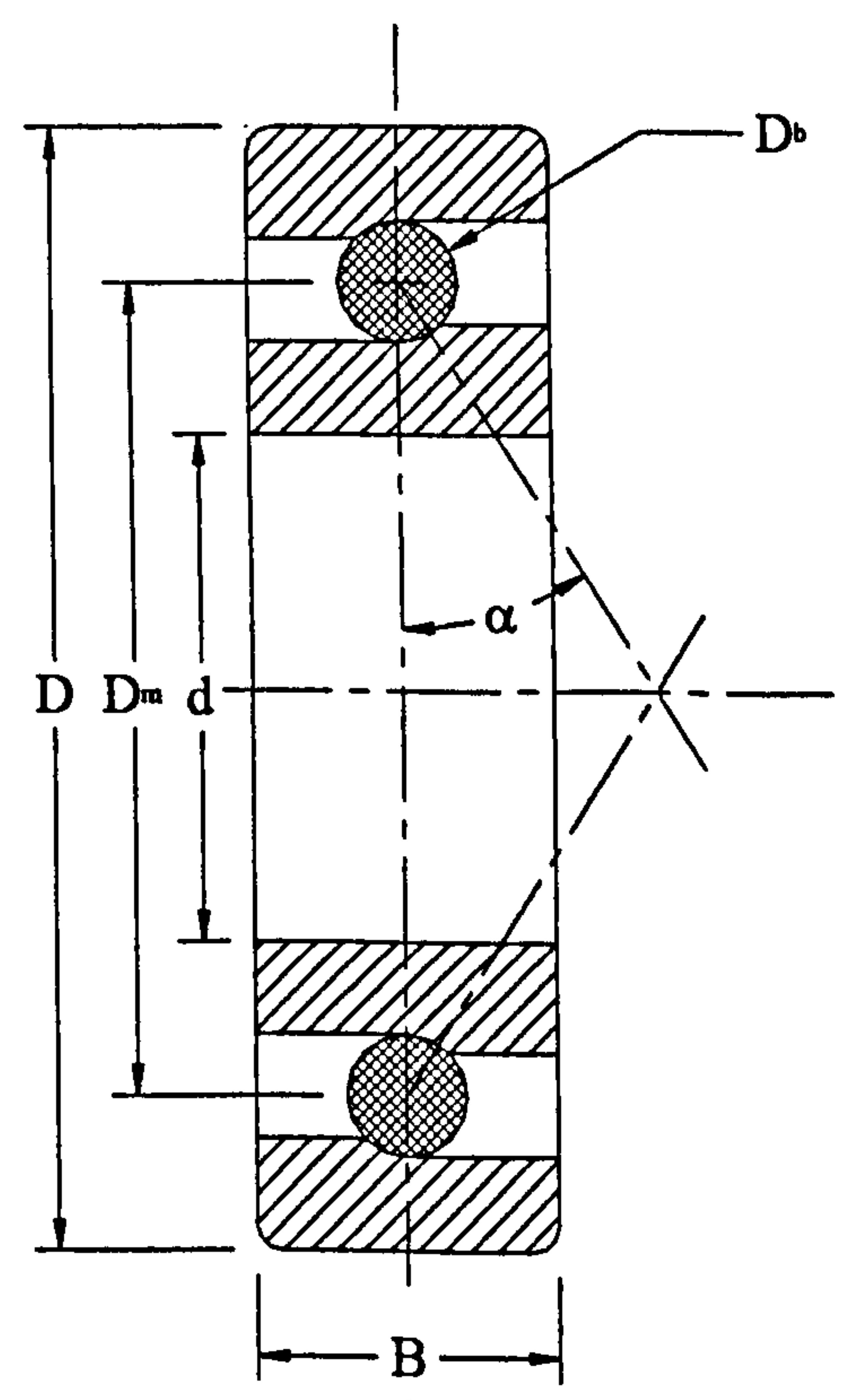

Figure 6.20 Schematic diagram of ceramic rolling element angular contact spindle bearing 


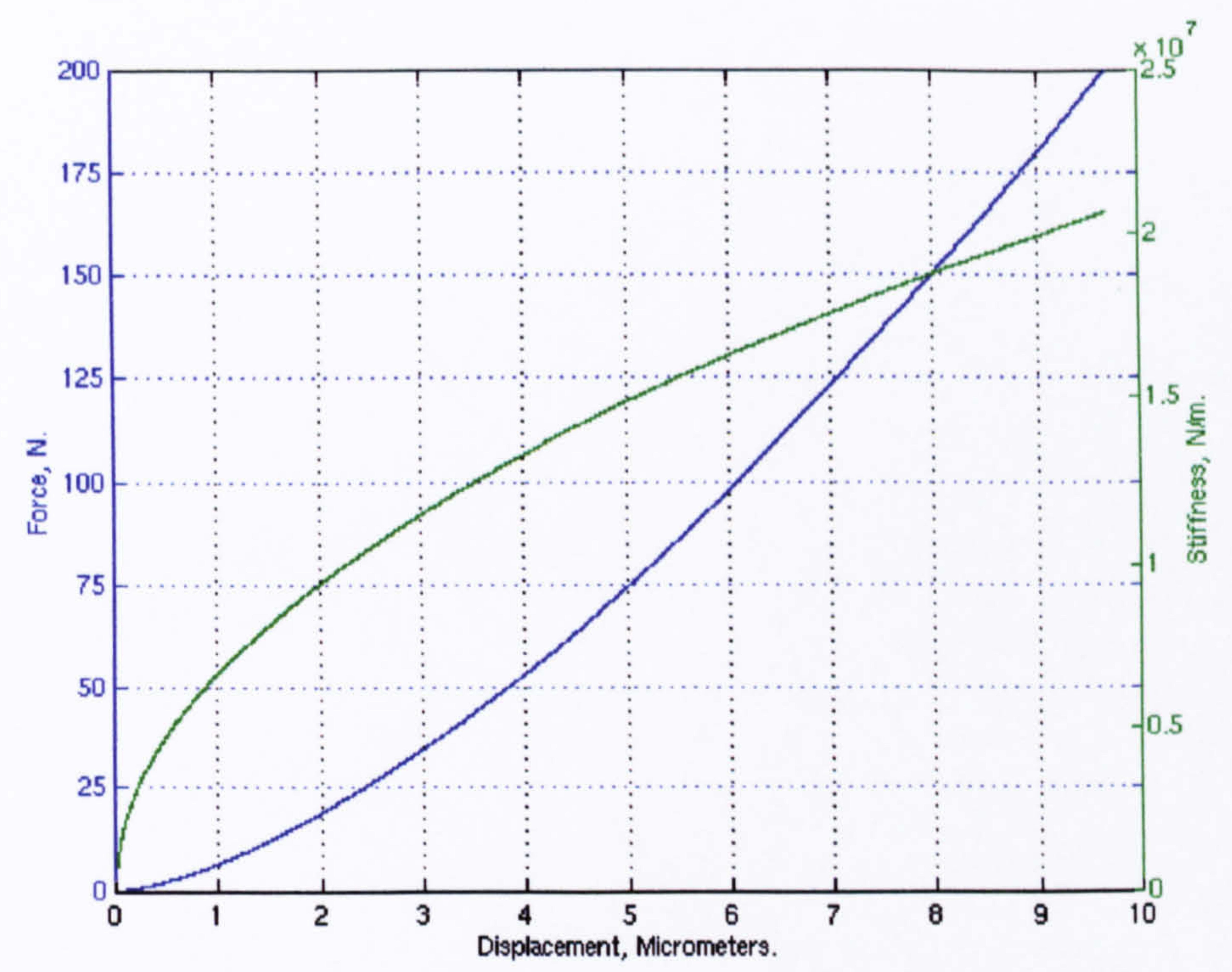

Figure 6.21. Force-displacement relationship and average stiffness for the ceramic rolling-element bearing. 


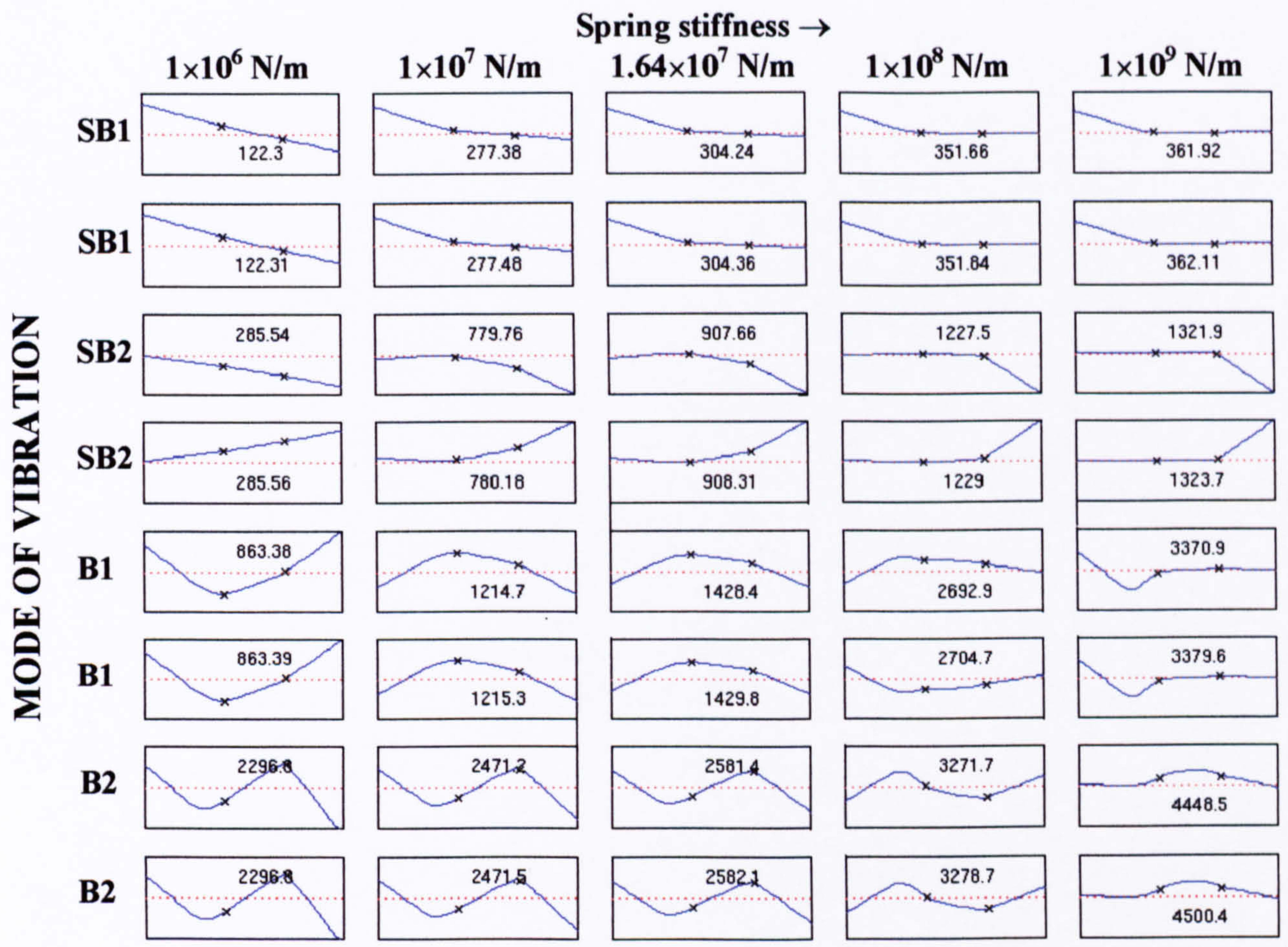

Figure 6.22. First four rotor modes of vibration as the bearing stiffness is increased from $1 \times 10^{6}$ to $1 \times 10^{9}$ (also shown are the mode shapes for the calculated bearing stiffness of $\left.1.64 \times 10^{6}\right)$.

\section{KEY}

SB1 - solid body mode 1

SB2 - solid body mode 2

B1 - bending or flexural mode 1

B2 - bending or flexural mode 2

All frequencies quoted in $\mathrm{Hz}$. 


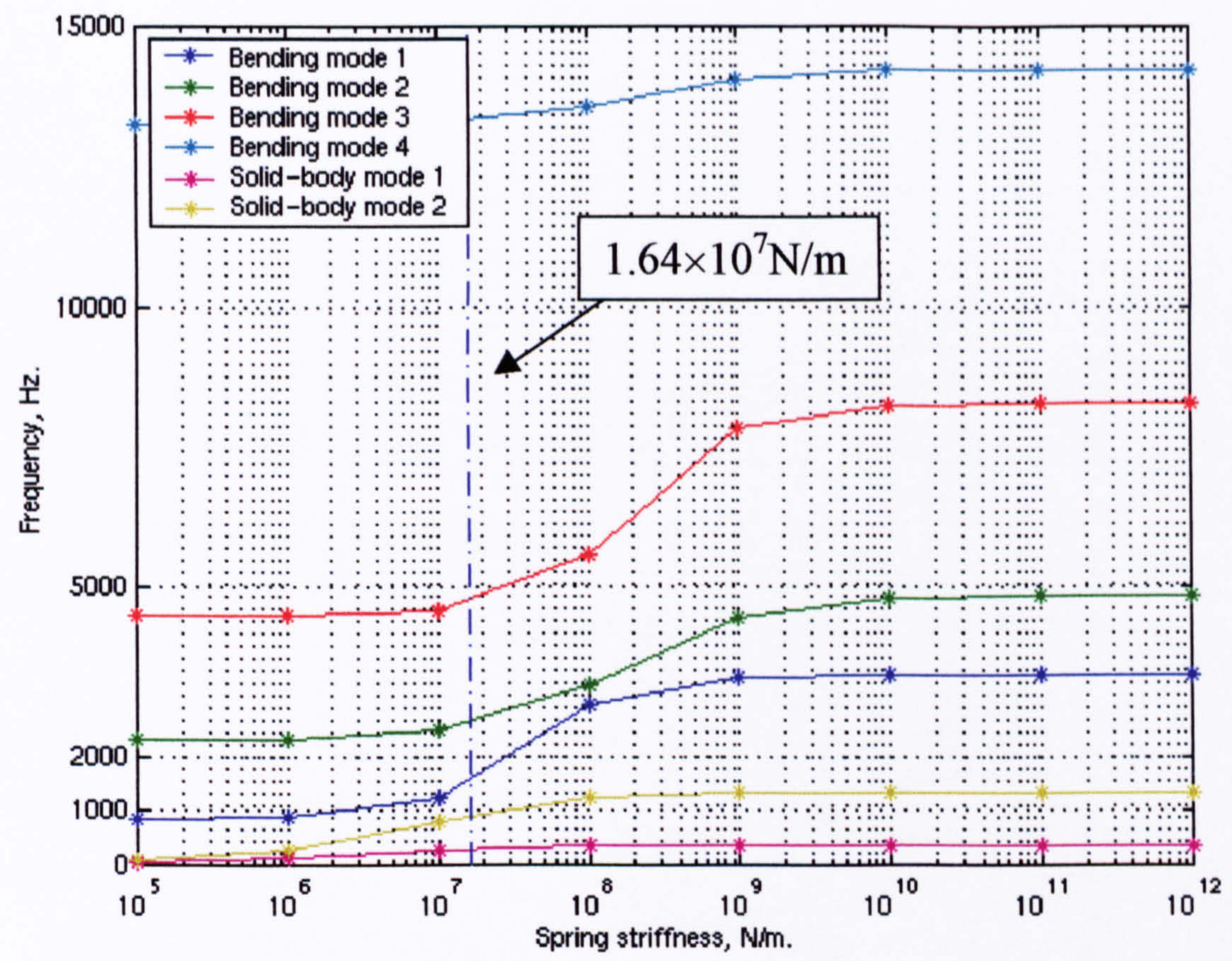

Figure 6.23. Variation of the rotor natural frequencies as a function of the bearing stiffness. 


\section{CHAPTER 7}

\section{PROTOTYPE EATG CONSTRUCTION AND EXPERIMENTAL VALIDATION OF FINDINGS}

\subsection{Prototype Machine Construction}

\subsubsection{INTRODUCTION}

The construction of the prototype high-speed SR machine, and in particular the coupling of the SR rotor to the turbine and compressor of a standard turbocharger presents many technical challenges. In order to highlight some of these challenges it is useful stage to briefly discuss the mechanical design of the various components.

\subsubsection{DESIGN AND MANUFACTURE OF THE ROTOR ASSEMBLY}

For the design of the prototype machine the scope to include any special features is constrained by the findings of the previous chapters, i.e.:

- The diameter, cross section and axial length of the SR rotor are limited by the centrifugal stress considerations detailed in Chapter 2 and the rotor dynamics as discussed in chapter 6.

- The compressor and turbine wheels are standard Holset commercial components.

- The overall layout of the shaft was largely determined in by the rotor-dynamic considerations of chapter 6 section 2 and the single-ended assembly procedure enforced by the friction welded turbine design.

The lamination stack was constructed from 100 individual CK26 Silicon Iron laminations each $0.5 \mathrm{~mm}$ thick. These are relatively thick laminations as compared to the more conventional $0.35 \mathrm{~mm}$ laminations that are generally employed in high- 
performance machines, which in turn will impact on the rotor loss. However, they were the only thickness available in this particular high-strength grade at the time of prototype construction. The lamination stack was welded along its length. Welding directly into the inter-polar fillet region would minimise the mass unbalance caused by the weld bead since it is placed on the minimum possible radius of the rotor. However, it was demonstrated in chapter 2 that this region of the material is likely to encounter the highest levels of tensile stress, and the potential introduction of material voids and high thermally induced residual stresses is likely to be undesirable. Since the welding process results in a localised electrical short-circuit between adjacent laminations and hence the eddy current component of iron loss may be significantly increased within the weld region. It was established in chapter 4 that the tips of the rotor teeth are likely to exhibit a high degree of localised eddy current iron loss, and hence it is inappropriate to position the weld in this location. Furthermore, welding both sides of the rotor teeth would create a circular current path around the teeth which will again allow a high level of eddy currents to be induced in this region due to the normal component of flux density acting radially in the rotor tooth. As a consequence, the weld was positioned mid-way down one side of the rotor pole, as shown in figure 7.1.

The bearings used in the prototype are FAG high-speed spindle bearings as described in detail in section 6.4.2. The inner diameter of these bearings is $10 \mathrm{~mm}$, and hence two collars were constructed to slide onto the $7 \mathrm{~mm}$ diameter shaft. In order to minimise the amount of exhaust gas leaking into the SR machine a piston-ring type gas seal was incorporated into the turbine end of the shaft as detailed in figure 7.2. Further, beyond this gas-seal are a series of three radial holes were incorporated into the turbine end-cap in order to vent any leakage exhaust gases, also shown in figure 7.2 .

The assembled rotor structure (i.e. compressor, turbine, shaft, bearings and SR rotor) was dynamically balanced by Holset in two planes corresponding the ends of the lamination stack by removal of material from the end laminations. The rotor was balanced at $2000 \mathrm{rpm}$ to tolerance of $1.4 \times 10^{-6} \mathrm{kgm}\left(2 \times 10^{-3}\right.$ oz-in Imperial units), a 
specification that is routinely employed for prototype turbo-machinery. The measured output from the balancing machinery for each of the balancing planes is shown in figure 7.3. One important consequence of the balancing operation is that a relatively large volume of material was removed from the edges of one pole piece of the rotor. In the prototype machine where the rotor position sensing is achieved using the end-face of the SR rotor, the resulting asymmetry posed some problems for the rotor position feed-back, which in turn required some modification of the position detection algorithm, as discussed later in section 7.2.1.

\subsubsection{STATOR LAMINATIONS}

The stator was constructed from $0.35 \mathrm{~mm}$ thick V300 Silicon Iron laminations. Most conventional electrical machines use an external case to house the stator laminations, often using a shrink fit assembly method or an arrangement of key-ways. This approach was deemed to be inappropriate for this prototype for the following reasons:

- The high levels of dissipation predicted in the SR machine, in particular iron losses (chapter 4), necessitates a low thermal resistance path from the laminations and the windings to the external ambient to avoid excessive temperatures. To this end, the removal of the case is advantageous.

- The small radial $(0.2 \mathrm{~mm})$ airgap between the rotor and the stator, requires a positive location of the rotor-bearing end-caps onto the inner bore of the stator. When a conventional case design is used the airgap between the rotor and stator is subject to the non-concentricity between the stator inner and outer bores, the outer case and the radial location of the outer case onto the end-cap with respect to the bearing recess centre position. Therefore the airgap in such a design is subject to an accumulation tolerances on these dimensions. In order to minimise the accumulation of radial tolerances a small shoulder was incorporated on each of the end-caps which locates directly into the inner bore of the stator laminations as shown in figure 7.2. The shoulder is machined at the same time as the bearing 
recess and hence the concentricity deviation between the rotor and stator is minimised.

As a consequence, the stator was constructed with the laminations being selfsupporting with no external case. Four tie bolts were employed to attach the end caps to the lamination pack, although as discussed above the concentric location was achieved using a shoulder in the end-cap and the inner bore of the laminations, rather than by the tie-bolts. The tie-bolts were placed in pre-machined holes in the lamination stack, where the pitch circle diameter (PCD) was selected on the basis of the standard practice in commercial compressor end-caps. Since the PCD of the mounting bolts is considerably larger than the outer diameter of the stator, the stator laminations were extended to incorporate four lugs to accommodate the mounting bolts. There may be some incremental benefits in increasing the back iron thickness in terms of reducing the flux density and hence iron loss in this region. However, in this case, the region between the bolt lugs was used to incorporate a series of radial fins to improve heat dissipation from the stator. To further enhance the heat dissipation capability, a degree of oil cooling was included in the stator lamination stack. This was achieved by incorporating a series of oil-channels in the stator laminations at locations where the removal of material is likely to have the least detrimental effect on the flux carrying capacity of the stator. Having established in chapter 4 that a high degree of iron loss is likely to be encountered in the tips of the stator teeth it is desirable to provide a cooling channel as close to this location as possible without adversely affecting the flux paths in the stator. Hence, an array of oil-ways were cut into the stator back-iron at the base of the stator teeth as shown in figure 7.4. In order to avoid any localised saturation, the width of the regions on either side of these oil-channels was maintained as being half of the stator tooth width. However it was recognised that with multiphase operation, the flux distribution is not as simple as the idealised symmetrical distribution which is often assumed in initial sizing of the lamination, and hence some marginal degradation in terms of increased saturation and iron loss may be introduced. The radial thickness of the back-iron was maintained in the vicinity of the oil channels as being the $7 \mathrm{~mm}$ 
selected in the initial design (section 3.2) by locally increasing the outer diameter of the stator.

A second array of oil-channels was incorporated in the stator laminations in the region between adjacent windings, a region that normally forms part of the stator slot in a conventional design, as shown in figure 7.5. Although this will promote some degree of additional slot leakage, it is an approach that has been used commercially for improving the dissipation from the coils with minimal detriment to the torque capability. The final stator cross section design employed in the prototype machine is shown in figure 7.6. A series of 12 radial holes (each $3 \mathrm{~mm}$ in diameter) were drilled in the stator lamination pack to allow connection of the stator windings. These holes were staggered axially by $5 \mathrm{~mm}$ to prevent any regions of the lamination being separated and are shown in figure 7.6. In order to assess the potential performance degradation in terms of increased slot leakage and unaligned inductance, a finite element model of the finned stator was constructed and solved using Ansys. Figure 7.7 shows the mesh for the rotor displaced $30^{\circ}$ from the aligned position that consists of 11,323 second order elements and 34,632 nodes. Figure 7.8 shows the flux density contours and the lines of constant vector potential for the mesh solved with a current density of $15 \mathrm{~A} / \mathrm{mm}^{2}$ applied to phase A. One notable feature of the flux density distribution shown in figure 7.8 is that the addition of the radial fins has little effect on the flux density distribution in the stator back iron, a feature which allows considerable savings in terms of computational effort in calculating the iron loss as discussed in section 7.3.4. Figure 7.9 shows the $\psi-i$ characteristic for the rotor in the aligned and unaligned positions and for two intermediate positions $\left(15^{\circ}\right.$ and $30^{\circ}$ from alignment). Also shown are the results calculated using the standard mesh presented in section 3.4.1. It is apparent from this figure that the inclusion of the additional features to aid the cooling of the machine has little detrimental effect on the limits imposed by the aligned and unaligned characteristics. The intermediate characteristics show a reduced level of induced flux at a given level of excitation, however this will have little effect on the specific torque production of the machine but may serve to slightly modify the commutation angles determined in chapter 3 . 


\subsubsection{TURBINE END-CAP}

The turbine end-cap essentially forms an interface between the SR machine and the turbine. As well as a providing mechanical location and the appropriate oil channels, it also has a useful role in shielding the SR rotor and the bearing from the high temperatures encountered at the turbine. It must also include a gas seal to prevent the exhaust gas leaking into the SR machine.

The oil circuit in the end-cap is a manifold type arrangement, as shown in figure 7.10 , where each of the stator oil-channels is fed from the same source in the endcap, i.e. the stator oil-channels are effectively in parallel. The oil-channel is fed from three equi-spaced external oil feeds in an attempt to provide a uniform flow down the stator oil-channels. The end-cap also incorporates a series of recesses to accommodate the stator end-windings in order to achieve a very compact assembly and hence minimise the axial length of the machine (consistent with the findings reported in chapter 6).

Having established the end-cap design for the interface with the SR machine it is necessary to consider its interface to the turbine. The specification of the mechanical interface in terms of the overall diameter, hole location and clamp design is governed by the geometry of the standard Holset turbine scroll. In all turbo-charger designs, the need to avoid excessive heat-soak from the turbine is an important consideration, and necessitates the inclusion of a mild steel heat shield, such as that shown in previously figure 7.2, which comes into contact with the exhaust gas and leaves a void between itself and the bearing housing. In addition, the constant oil-feed which is provided for the hydrodynamic bearings provides an effective means of cooling the bearing housing and limiting the thermal conduction down the shaft. However, for the prototype EATG, the use of rolling element bearings in the turbine end-cap and the close proximity of the machine end-windings require that the minimisation of heat transfer from the exhaust gas to the end-cap is likely to be critical. To this end, 
the turbine end-cap was manufactured from two parts with an oil circulation chamel milled into the interfacing components as shown in figure 7.11 and figure 7.12. The final assembled turbine end-cap including the standard heat shield was shown previously in figure 7.2 .

\subsubsection{COMPRESSOR END-CAP}

The SR machine interface to the compressor end-cap is similar to the turbine endcap, with a matching oil channel to return the oil to the external reservoir. Since the SR rotor is compressed axially using the compressor end retaining nut and no positive angular-location such as a key-way is incorporated in the shaft, the angular position of the lamination stack cannot be reliably registered with respect to other rotor components. In order to provide reliable position signals to the drive it is necessary therefore to sense the position of the rotor directly. In order to facilitate this measurement, the compressor end-cap includes three equi-spaced axially drilled mounting holes for three position sensors. The technical specifications of the Hall sensors is discussed in section 7.2. It would be desirable to set the PCD of the sensor mounting holes to obtain a pulse train with a $50 \%$ duty, i.e. at a radius which corresponds to rotor iron subtending an angle of $45^{\circ}$, as shown in figure 7.13. However, the outer diameter of the rotor bearing and the stator locating shoulder dictated that the PCD of the Hall sensors was such that a pulse train of $52 \%$ duty should be generated from a simple consideration of rotor geometry. This can however accommodated, along with any asymmetry in the rotor teeth, within the control algorithm, as discussed in section 7.2.1.

Figure 7.14 shows a cross section of the complete prototype EATG and figure 7.15 shows a photograph of the components prior to assembly. 


\subsection{Prototype Converter Design and Construction}

In order to test the prototype machine, a 3-phase power electronic converter was designed and constructed. It should be noted that the primary aim in the design of this converter was to realise a simple and robust drive purely for testing purposes rather than a packaged and highly optimised drive for integration into a vehicle. This inevitably resulted in the use of highly over-specified power electronic switching components, and interfaces and control systems that would be unlikely to be costeffective for a mass-production system.

The drive electronics are based around so-called "intelligent power modules" (IPMs) of type 7MBP300RA060 manufactured by Fuji Electrical Ltd. Each IPM consists of 6 individual 300A, 600V IGBTs internally connected as shown in figure 7.16 (a seventh lower Volt-Amp rated IGBT is also included in the IPM for braking if required). These IPMs are very convenient for the rapid prototyping of drives, since they incorporate pre-drivers for the IGBT gates that include a degree of protective circuitry to guard against short circuit, drive under-voltage, over current and IGBT over-heating.

However, the considerable advances which have occurred in IPMs over recent years has been driven principally by the demands 3-phase induction motors and to a lesser extent 3-phase brushless machines. As a consequence, IPMs for 3 phase machines are almost exclusively configured as shown in figure 7.16 for "star" connected AC machines. In order to accommodate SR machines it is necessary to couple 2 IPM's as shown in figure 7.17. Thus, although they offer an attractive approach for prototyping SR converters by virtue of their integrated gate drives and thermal management, they necessarily result in the redundancy of 6 of the switches, although their associated anti-parallel diodes are used as the free-wheel diodes in the circuit. The gate drive inputs of IPMs were connected to the DSP control system via an array of optical fibres (HFBR-0400 series manufactured by Hewlett Packard) in order to protect the DSP system from potentially destructive voltages that may occur under 
fault conditions. The use of optical fibres to transmit the commutation signals also provides additional benefits in terms of immunity to electromagnetic interference.

The rotor position is sensed by a series of self-calibrating, differential Hall sensors (ATS612LSB manufactured by Allegro Microsystems Inc.) which are located in the compressor end-cap. Each sensor comprises a small Samarium-Cobalt magnet and a pair of matched Hall sensors. By appropriate alignment of the position sensors, the passing of the edge of a rotor tooth will generate a difference in flux density at each Hall device, thus providing a mechanism for rotor detection. The output signals from the position sensors are also electrically isolated from the DSP system using highspeed inverting opto-isolators (H11N1). In order to minimise any spurious triggering, a low-pass filter was constructed with manufacturer's recommended parameters in order to provide some immunity from supply line noise. The circuit shown in figure 7.18 was constructed using surface mount components in order to enable the entire circuit to be mounted on the rear of the hall effect sensor. The sensor contains a selfcalibrating circuit that modifies the internal gain of the device to minimise the effect of airgap variation.

As discussed in section 7.1.2, the balancing of the rotor resulted in some asymmetrical removal of material from the end-face of the rotor lamination stack, as shown in figure 7.19. The amount of material removed is relatively significant with respect to the nominal $1.5 \mathrm{~mm}$ axial clearance between the face of the sensors and the lamination stack. As a result, although the position sensor can detect all four rotor teeth, the extended effective airgap of one of the teeth gives rise at a corresponding asymmetry in detection of that particular tooth edge. This is illustrated by the measured Hall sensor signals of figure 7.20 that were measured at a speed of 7300rpm. The control algorithm therefore included provision to account for this asymmetry, hence enabling absolute position to be measured to a reasonable degree of accuracy (approximately $3^{\circ}$ mechanical), albeit with some initial calibration. A schematic of the prototype EATG system including the connections to the ancillary components is shown in figure 7.21, while figure 7.22 shows a photograph of the complete prototype system on the bench. 


\subsubsection{CONTROL SYSTEM IMPLEMENTATION}

The control algorithm was implemented in a dSPACE hardware-in-the-loop system, comprising a DS1005 processor board, and a DS4002 timing and digital I/O [7.1]. The DS4002 timing and digital I/O board has 8 I/O channels that can be individually programmed to operate in either input or output mode and two external triggers. In input mode the signals are monitored for level changes, i.e. rising or falling edges, the events are recorded and the timestamps stored in the local RAM, each with a resolution of 200ns. These signal timestamps coupled with the two external triggers provide a high degree of flexibility for the definition of parameters such as the frequency and duty cycle of the rotor position sensor input signal. This flexibility is particularly advantageous in this case as it allows a degree of signal "correction" and frequency averaging to be applied to the input signals to accommodate the nonuniform duty cycle pulse train from the rotor position sensors. In output mode the level of the channels can be updated by the continual specification of a series of commands which include a time delay (again with a resolution of 200ns) and the signal level to be attained after the delay has passed. The 3 phase output signals can be appropriately timed with an externally created trigger event based on the Hall sensor input and hence the commutation signals can be referenced to the absolute rotor position.

The control software was implemented using a series of macros specific to the real time workshop environment and compiled using a Texas Instruments $C$ program compiler. The code is downloaded to the processor board using the dSPACE real time interface (RTI1005), which is executed within the Matlab/Simulink environment. Finally data acquisition, variable display and manual commutation angle control (for example) is undertaken in the dSPACE ControlDesk environment, an example of which is shown in figure 7.23. 


\subsection{Test Results}

\subsubsection{MEASUREMENT OF PHASE SELF-INDUCTANCE AND RESISTANCE}

The phase self inductance of the prototype was measured at various rotor angles in order to both validate the calculations undertaken in chapter 3 , and to determine the additional inductance contributed by the end-windings (which is unaccounted for in two-dimensional analysis). The winding of one phase was excited with a $50 \mathrm{~Hz}$ sinusoidal current waveform (generated by a power amplifier in constant current mode) with an rms value of $5.0 \mathrm{~A}$ (which is considerably lower than the saturation threshold of the prototype machine). The rotor angular position was measured using a Heidenhain precision optical rotary transducer. The inductive power flow, $Q$ and the rms current $I_{r m s}$, were measured using a high-precision Norma power analyser (D 6100). The self inductance under these operating conditions was determined using equation 7.1.

$$
L_{p h}=\frac{Q}{2 \pi f I_{m s}^{2}}
$$

Figure 7.24 shows the measured inductance as a function of rotor position, together with the corresponding calculated inductance variation derived from the finite element analysis presented in chapter 3 . The curves are in reasonable agreement, but there is a relatively constant offset of a magnitude of some $90 \mu \mathrm{H}$ between the measured and predicted values. This can be attributed in part to the constant endwinding inductance of the prototype device that was not accounted for in the twodimensional finite element model.

The resistance of each phase was measured at room temperature using a Rhopoint 4 terminal milli-ohmeter. There was some spread in the measured results with 
maximum and minimum values of $19 \mathrm{~m} \Omega$ and $20 \mathrm{~m} \Omega$ respectively. These values are in reasonable agreement with the value of $18 \mathrm{~m} \Omega$ predicted using equation 3.18 in section 3.6.1.

\subsubsection{LOW-SPEED, LOW-POWER BENCH TESTING}

A limited number of low-speed, and low-power tests were performed with the EATG system mounted on a test-stand rather than directly on the engine. The purposes of these low-power tests were to check the operation of the DSP, the power electronic converter, and the position sensors and to verify the DSP control code. Further, the measurements taken during these tests provided data for the initial validation of the dynamic simulation technique developed in chapter 3 and the iron loss calculation developed in chapter 4.

Three operating conditions at the three test points are detailed in table 7.1. Tests 1 and 2 were conducted at $32 \mathrm{~V}$ in order to limit the peak current being drawn from the laboratory power supply at low speeds. The Voltage was increased to $50 \mathrm{~V}$ for test 3 , in order to accommodate the VA rating of the power supply to provide the higher levels of power required under these conditions.

\begin{tabular}{|l|c|c|c|}
\hline & Case 1 & Case 2 & Case 3 \\
\hline Speed & 10100 & 14900 & 18400 \\
\hline Voltage & 32 & 32 & 50 \\
\hline$\theta_{\text {on }}$ & -27 & -30 & -31 \\
\hline$\theta_{\text {dwell }}$ & 10 & 19 & 22 \\
\hline
\end{tabular}

Table 7.1. Operating conditions of the three test points. 


\subsubsection{MEASURED DYNAMIC PERFORMANCE}

Phase current and phase voltage waveforms were measured for each of the 3 test points using a Tektronix A6303 current probe and a Chauvin Arnoux DP25 active differential probe respectively, and captured using a LeCroy 140 digital storage oscilloscope. The resulting waveforms are shown in figures 7.25 , from which a number of interesting features can be observed, particularly in the measured voltage waveforms. In each of the three cases, following the extinction of the current, the phase voltage waveform only slowly returns to $0 \mathrm{~V}$. Given that for these particular commutation conditions there are no currents flowing in other phases during this interval, the emf in the winding can only be attributed to a degree of remanence in the stator and/or rotor laminations. Another notable feature is the considerable influence that the various switching device voltage drops have when operating at such low DC link voltages. By means of example, the voltage across the winding during the decay of the current in figure $7.25 \mathrm{a}$ (test case 1 ) is $-34.5 \mathrm{~V}$ as compared to $29 \mathrm{~V}$ during the rise of the current, in turn markedly influencing the rate of rise and fall of current. This is not directly accounted for in the simulations presented in chapter 3, albeit that it will have considerably less effect at the specific DC link voltage of $96 \mathrm{~V}$.

The captured waveforms of figure 7.25 can be used to determine the net electrical power into the machine, providing care is taken to perform the integration of the product of voltage and current over a complete cycle. By means of example, figure 7.26 shows the variation during one electrical cycle of the energy drawn from and subsequently returned to the supply for test case 1 . The current waveforms of figure 7.25 can be used to calculate the average copper loss by employing a value of phase resistance that was measured (at the winding terminations) directly at the end of each test (in order to provide some allowance for any temperature rise in the winding). Table 7.2 shows the measured resistance immediately following each of the three tests 


\begin{tabular}{|l|c|}
\hline Test case & Measured phase resistance $(\mathrm{m} \Omega)$ \\
\hline 1 & 46 \\
\hline 2 & 45 \\
\hline 3 & 47 \\
\hline
\end{tabular}

Table 7.2. Measured phase resistance immediately following each of the three tests

Whereas the electrical input power and the copper losses in the machine can be measured to a reasonable accuracy using the techniques described above, the iron loss cannot be measured directly, but must be inferred from a loss subtraction technique. A key requirement in this regard is a reliable estimate of the mechanical load on the machine, i.e. the work done by the compressor and turbine wheels and any mechanical losses within the system (principally bearing friction and rotor windage). Since an SR machine is a singly excited machine, in its unexcited state there is nominally no iron loss in the machine (although as will be demonstrated remanent magnetism in the laminations will give rise some degree of iron loss). The absence of any significant iron loss in an un-excited machine enables the mechanical load and losses on the system at a given speed to be quantified by means of a socalled 'run-down' test. In such a test, the rotor is accelerated up to specified rotational speed, and having achieved steady state conditions, the excitation is removed. The variation of speed during the subsequent run-down is measured using the rotor position sensors and the DSP system. Given the polar moment of inertia of the rotating components (established from results in chapters 2 and 6) this speed-time curve can be converted to a decelerating torque versus time characteristic, and hence to a torque-speed curve for the total mechanical load on the shaft. Figure 7.27 shows a series of measured load torque versus speed characteristics which were measured on the test stand at various starting speeds up to 19400rpm

Having measured the electrical input power, the copper losses and the mechanical load/losses, an estimation of the iron loss at a particular operating point can be derived. Table 7.3 summarises the values of the various components of measured 
power for the various test operating conditions together with the corresponding values of iron loss inferred from these measurements.

\begin{tabular}{|l|c|c|c|}
\cline { 2 - 4 } \multicolumn{1}{c|}{} & $\begin{array}{c}\text { case1 } \\
32 \mathrm{~V}, 10100 \mathrm{rpm}\end{array}$ & $\begin{array}{c}\text { Case 2 } \\
32 \mathrm{~V}, 14900 \mathrm{rpm}\end{array}$ & $\begin{array}{c}\text { Case 3 } \\
50 \mathrm{~V}, 18400 \mathrm{rpm}\end{array}$ \\
\hline Measured electrical input power & 81 & 197 & 538 \\
\hline Measured copper loss & 5 & 10 & 23 \\
\hline Measured mechanical losses & 25 & 60 & 175 \\
\hline $\begin{array}{l}\text { Iron losses inferred from } \\
\text { measurements }\end{array}$ & 51 & 127 & 340 \\
\hline
\end{tabular}

Table 7.3 Division of measured electrical input power

\subsubsection{SIMULATED PERFORMANCE AT TEST-POINTS}

In order to validate the simulation techniques presented in chapters 3 and 4 , i.e. the dynamic electrical simulation and the iron loss calculation techniques, these simulations were performed under the 3 specific operating conditions detailed in table 7.1.

The dynamic simulation was performed at the three operating points, with the current waveforms, calculated copper loss and machine mechanical power being compared with measured results. Figures $7.28 \mathrm{a}, 7.28 \mathrm{~b}$ and $7.28 \mathrm{c}$ show the measured and predicted current waveforms at each of the three operating points respectively. The form of the measured and predicted current waveforms is in good agreement. However the current decays much more rapidly in the measured waveforms a factor which is attributed to the remanence of the lamination material as discussed in section 7.3.3.

Table 7.4 shows a summary of this measured and predicted power and peak current data. As is evident, there is a good correlation in terms of the current waveform. 
However, whereas the agreement between measured and predicted power appears to be relatively poor, it should be borne in mind that the dynamic simulation does not account for iron losses.

\begin{tabular}{|c|c|c|c|c|c|c|}
\cline { 2 - 7 } \multicolumn{1}{c|}{} & \multicolumn{2}{c|}{ Case 1 } & \multicolumn{2}{c|}{ Case 2 } & \multicolumn{2}{c|}{ Case 3 } \\
\cline { 2 - 7 } \multicolumn{1}{c|}{} & Measured & Predicted & Measured & Predicted & Measured & Predicted \\
\hline Output power & 25 & 40 & 60 & 118 & 175 & 332 \\
\hline Peak current & 21.8 & 21.9 & 23.1 & 21.7 & 30.1 & 33.0 \\
\hline Copper loss & 4.5 & 4.6 & 10 & 11 & 23 & 26 \\
\hline
\end{tabular}

Table 7.4 Comparison of measured and predicted dynamic performance

The iron loss modelling technique discussed in chapter 4 is based on a combination of finite element analysis and a decomposition loss model and is specific to a particular current waveform and operating speed. As a consequence, it was necessary to undertake three further iron loss calculations in order to replicate the operating conditions encountered in the low-power testing. Further, the iron loss predictions in chapter 4 were not directly undertaken using the geometry of the eventual prototype, which includes a number of practically driven modifications, in particular the inclusion of oil-channels in the stator and the increased fillet radius required to accommodate the shaft sleeve. Therefore, the mesh shown in figure 7.29, which consists of 15563 nodes and 30920 elements was employed to calculate the iron loss. The mesh includes the oil-channels in the stator and the revised rotor design, but neglects the array of radial fins since they have negligible effect on the flux density distribution within the stator laminations as demonstrated previously in figure 7.8. The iron loss calculations were performed using the measured current waveforms. A typical series of field distributions, (for case 2) are shown in figure 7.30, while the corresponding loss contours are shown in figure 7.31 The calculated iron loss values together with the results from the loss subtraction technique discussed in section 7.3.3 are shown in table 7.5. 


\begin{tabular}{|c|c|c|c|}
\hline & Case1 & Case 2 & Case 3 \\
\hline Iron losses from measurements & $\overline{51}$ & $\overline{127}$ & $\overline{340}$ \\
\hline Calculated iron loss & 39 & 112 & 233 \\
\hline
\end{tabular}

Table 7.5 Summary of the iron loss calculated by applying the loss subtraction technique to the measured data and the calculated iron loss using technique discussed in chapter 4.

Although there is reasonable agreement, the iron losses inferred from the measurements are in all cases higher than those predicted by the finite element / loss model technique. There are 3 factors that may account for some of the observed discrepancies:

1. Remanence in the Laminations - As was observed previously in the voltage waveforms of figures 7.25 , there is a degree of remanence in the stator and/or rotor laminations in the absence of any current, which is relatively significant when compared to the flux densities achieved at these low-power test points. However, it is difficult to directly determine the impact of this behaviour on the measured iron losses. It is likely that it will increase the measured iron loss since in some respects its effect is analogous to the presence of an additional 'permanent-magnet' source, albeit one whose properties are time-varying and a complex function of excitation. This additional loss is not modelled in the finite element analysis discussed in section 4.4.2 of chapter 4 which is consistent in terms of the underestimates summarised in table 7.5. However it should be borne in mind that some component of iron iron-loss generated by remanence in the laminations may be present during run-down, and is hence accounted for in the measured 'mechanical' loss. Given that the excitation conditions encountered during normal operation and run-down differ considerably and the nature of such remanent losses in soft magnetic materials is extremely complex, there is no sound basis on which the remanent losses can be equated for the two cases. Hence, this possible explanation for the discrepancy between measured and predicted iron losses can only be considered as being qualitative, albeit that it is 
consistent with some degree of underestimation by the finite element analysis and loss model.

2. End-Caps - The windings extend into the solid mild steel end-caps and a degree of flux will be induced in the end caps when the winding is energised. This is evidenced by the measured and predicted inductance variation shown in figure 7.24, where the measured values are consistently greater than those predicted using twodimensional finite element approach. This constant offset can be attributed to the flux passing through the end-caps since it shows little variation with rotor position. It is apparent from the discussion presented in chapter 4 that the presence of time varying flux densities in the end-caps will inevitably give rise to a degree of iron loss in these components, a factor which is not accounted for in the iron loss calculation technique.

3. Loss Subtraction - For the prototype machine it is not possible to directly measure iron losses, and consequently the loss subtraction method was used where at each operating point the input power was measured and the quantifiable rolling resistance, windage and copper loss was subtracted. This method is subject to a high degree of experimental error since it is subject to the accumulation of many experimental errors. This is particularly prevalent for case 3 where the rolling resistance (i.e. the summation of bearing and aerodynamic losses) is seen to rise rapidly at speeds greater than $17,500 \mathrm{rpm}$. Although it is not clear at this stage what causes this rapid rise in rolling resistance it may be attributed to the presence of the first solid body mode critical speed which is predicted to occur at a speed of $18,200 \mathrm{rpm}$ in chapter 6 or aerodynamic effects caused by using an unconnected turbine scroll which results in large a air flow (backwards through the turbine) which is vented to atmospheric pressure. 


\subsection{Engine Testing}

\subsubsection{EXPERIMENTAL APPARATUS}

The engine employed in the testing was a Cummins 4BTA, 3.92 litre, four-cylinder turbo-charged, after-cooled, compression ignition engine used for medium duty industrial automotive applications. In the test-rig, the engine is coupled to a Heenandynamatic dynamometer in which the reaction torque is produced by a circumferentially wound field coil inducing eddy currents in the electrically conducting rotor. The torque is measured using a swinging frame arrangement via a Lebow $2.2 \mathrm{kN}$ load cell. The engine coolant is cooled by means of a $120 \mathrm{~kW}$ heat exchanger supplied with fresh cold water from the laboratory supply. The engine was fitted with a Holset H1C non-wastegated turbocharger and tested with two inlet turbine scrolls of different cross-sectional areas. Firstly, a standard scroll with a nominal inlet area of $16 \mathrm{~cm}^{2}$ was fitted to the engine to provide a reference condition. Secondly a smaller scroll with a nominal inlet area of $12 \mathrm{~cm}^{2}$ was fitted in order to achieve higher turbine powers.

The turbine was closely coupled to the engine using a short, narrow, exhaust manifold in order to maximise the utilisation of the high pressure and temperature. Each scroll features a dual entry design to minimise the detrimental effect of both direct pressure wave interaction and reflection onto the exhaust valves of adjacent cylinders. The centrifugal compressor feeds the inlet air into an engine-mounted after-cooler, which is supplied by cooling water from the engine coolant system.

The fuel system for the engine consists of a rotary type Bosch VE injector pump with an air/fuel ratio control device attached to the governor spring to control emissions. The air mass flow rate into the engine was measured using an Alcock viscous flow meter, where the pressure drop across the element, (which is proportional to the 
volumetric flow rate) was measured with a piezo-resistive differential pressure transducer.

The emissions of hydrocarbons $\left(\mathrm{H}_{\mathrm{x}} \mathrm{C}_{\mathrm{x}}\right)$ and oxides of Nitrogen $\left(\mathrm{NO}\right.$ and $\left.\mathrm{NO}_{2}\right)$ were measured using two Emicont 20 electrochemical exhaust gas analysers. The particulate content in the exhaust was measured using an AVL 415 smoke meter. In this device, a known volume of exhaust gas is drawn through probe mounted in the exhaust system and passed through a filter element where the relative blackening of the filter is measured using a reflectometer head. The volume of gas drawn through the filter is determined automatically whereby an initial $200 \mathrm{ml}$ of gas is sampled to determine the soot content, a more appropriate sample volume of gas is then calculated based upon this estimate and hence very low soot contents can be measured accurately. The results are reported as a filter smoke number (FSN), which vary between 0 and 10 with a resolution of 0.01 FSN and a repeatability of 0.05 FSN. The filter smoke number is subsequently normalised to standard temperature and pressure.

Figure 7.32 shows a schematic arrangement of the engine test facility indicating the various temperature and pressure measuring points, while figure 7.33 shows a photograph of the engine and brake mounted in the sound-proof chamber.

\subsubsection{BASE TESTING RESULTS}

A series of base tests were performed on the engine without the EATG fitted, the purpose of which was to:

1. Verify the preliminary machine specification discussed in chapter 1 , i.e. to confirm the speed and power rating of the EATG.

2. Provide a benchmark on which the performance improvement/degradation of the engine fitted with the EATG can be established. 
3. Establish a comparison between the standard $16 \mathrm{~cm}^{2}$ and the $12 \mathrm{~cm}^{2}$ turbine scrolls in order to determine the most suitable match between the compressor and turbine scroll for use with the EATG.

The engine was tested at speeds between 1000 and $2400 \mathrm{rpm}$ at intervals of $200 \mathrm{rpm}$. The torque applied with the dynamometer varied from a minimum of $50 \mathrm{Nm}$, incremented by intervals of $50 \mathrm{Nm}$ until the limiting value of the engine or dynamometer was reached.

The turbine power can be calculated using the steady flow energy equation. The heat transfer from the turbine to the surrounding ambient is neglected (even though the casing is un-insulated and under some conditions becomes semi-incandescent) since it is assumed to be small in comparison with the total energy flow through the turbine, and hence:

$$
\dot{W}_{t}=\dot{m}_{e} c_{p e}\left(T_{4}-T_{3}\right)
$$

The turbine inlet temperature $T_{3}$ is the numerical average of the temperatures measured in the two entry ports to the turbine (labelled $T_{3 a}$ and $T_{3 b}$ as shown in figure 7.32). It is worth noting that the accurate measurement of exhaust temperature is extremely difficult in practice, due for example, to the radiated heat transfer from the manifold wall to the thermocouples and the temperature fluctuations associated with the pulsating flow from the exhaust valves. The specific heat capacity at constant pressure of the exhaust gas is calculated at the average turbine temperature using

$$
c_{p e}=A+B t+C t^{2}+D t^{3}+E t^{4} \quad[\mathrm{~kJ} / \mathrm{kg} \cdot \mathrm{K}]
$$


Where

$$
\begin{aligned}
& A=1.005+0.787 f \\
& B=2.97 \times 10^{-5}+1.32 \times 10^{-3} f \\
& C=5.98 \times 10^{-7}+1.78 \times 10^{-6} f \\
& D=-6.81 \times 10^{-10}-5.28 \times 10^{-9} f \\
& E=2.32 \times 10^{-13}+3.70 \times 10^{-12} f \\
& t=T(K)-300 \\
& \text { and } f \text { is the fuel/air ratio. }
\end{aligned}
$$

Using equation 7.3, the calculated specific heat capacity of the exhaust gas varied between a minimum of 1.04 and a maximum of 1.23 for the $16 \mathrm{~cm}^{2}$ turbine scroll, while for the $12 \mathrm{~cm}^{2}$ turbine the value varied between 1.04 and 1.22 . The resulting maximum turbine power determined from measured temperatures and flow-rates for the $16 \mathrm{~cm}^{2}$ turbine was $13.2 \mathrm{~kW}$ at $104,280 \mathrm{rpm}$, with the engine developing $84.4 \mathrm{~kW}$ at $2400 \mathrm{rpm}$. When the non-standard $12 \mathrm{~cm}^{2}$ turbine scroll was used further regulation of the engine load was required to prevent over-speed of the turbocharger shaft for safety reasons and hence the engine speed and load were limited so the turbocharger speed did not exceed 100,000rpm. Under these conditions a maximum engine power of $73.3 \mathrm{~kW}$ was achieved at $2000 \mathrm{rpm}$ (the turbine power under these conditions being $11.10 \mathrm{~kW}$ at $96,600 \mathrm{rpm})$. The maximum turbine power recorded using the $12 \mathrm{~cm}^{2}$ turbine was $11.9 \mathrm{~kW}$ at $92,640 \mathrm{rpm}$, with the engine developing $65.4 \mathrm{~kW}$ at $1800 \mathrm{rpm}$

Figures 7.34 show the calculated turbine power for both turbine scrolls. The turbine power increases with increasing engine speed due to the higher mass flow rate of gas through the turbine. As is evident from figure 7.34, the turbine power increases as the load applied to the engine is increased. This behaviour that can be attributed to the increased exhaust gas temperature which occurs as the air/fuel ratio is reduced (as shown in figure 7.35). The average exhaust temperature, (i.e. the numerical average of the temperature measured in the two entries) is shown in figure 7.36. 
As would be expected, for a given mass flow rate, the smaller $\left(12 \mathrm{~cm}^{2}\right)$ turbine scroll exhibits a larger exhaust manifold pressure under given engine operating conditions and hence develops more power over the entire operating range of engine. One notable consequence of the increased turbine power with the $12 \mathrm{~cm}^{2}$ scroll is that under high engine speed and load conditions, the turbocharger will function above its maximum safe operating speed. For the purposes of testing, the engine was operated so the turbocharger speed did not exceed $100,000 \mathrm{rpm}$ in order to provide a reasonable safety margin (the maximum specified operating speed of the standard turbocharger being some $120,000-130,000 \mathrm{rpm})$. The standard engine in its commercial specification employs a $16 \mathrm{~cm}^{2}$ turbine, which is selected on the basis of its maximum speed at the highest engine speed and load conditions, which for the conditions tested was 104,280rpm However, since the EATG allows considerable power to be generated from the turbine, hence reducing the shaft speed, it is permissible for this application to combine the EATG with the $12 \mathrm{~cm}^{2}$ turbine scroll, thus allowing considerable scope for electrical generation over a wider range of engine operating conditions.

The compressor power was calculated for each engine test point using:

$$
-\dot{W}_{c}=\dot{m} c_{p}\left(T_{2}-T_{1}\right)
$$

In the case of the compressor, the specific heat capacity of the inlet air $c_{p}$ is taken as being $1.005 \mathrm{~kJ} / \mathrm{kg}$. Figure 7.37 shows contours of compressor power derived using equation 7.4 and measured temperatures and mass flow rates. As would be expected, the compressor power is higher at a given engine operating point for the case of the smaller turbine, which is consistent with the higher turbine power. The crosssectional area of the turbine scroll only has a minimal effect on the thermal efficiency of the engine, and hence the air/fuel ratio of the engine is slightly higher with the smaller turbine scroll (as was shown previously in figure 7.35). One notable consequence of the slightly greater air/fuel ratios with the smaller turbine is that for a given engine operating condition the level of particulate matter in the exhaust (which 
is heavily influenced by the ai/fuel ratio) will be somewhat lower, as is evidenced by the contours of figure 7.38 .

\subsection{Testing Engine with EATG Fitted}

Having established a series of base tests and determined the most appropriate turbine scroll the EATG was fitted to the engine in place of the standard turbocharger to facilitate a complete system test. The engine fitted with the prototype machine is shown in figure 7.39 (in this case with the flexible inlet duct removed to aid clarity). A Ward-Leonard set with a maximum power capability of some $30 \mathrm{~kW}$ was used to provide the DC link current in preference to a laboratory DC supply.

Preliminary testing identified a problem with the Hall effect position sensors, in that at moderate levels of current (i.e. corresponding to powers greater than some $300 \mathrm{~W}$ ), spurious trigger signals were generated, apparently as a result of magnetic coupling from the machine windings. This conclusion was drawn on the basis that the false triggers were co-incident with switching events, causing erroneous rotor speed and position feedback, in turn causing instability in the operation of the machine. Given that very little could be done with the existing design to minimise this coupling, in order to facilitate further testing an optical sensor was integrated into the compressor inlet. The optical sensor (Honeywell HOA1405 reflective sensor) was located so as to detect a series of dark and bright regions that were painted on the compressor nut, as shown in figure 7.40), giving a 4 pulse-perrevolution signal. Although this allowed position and speed to be detected without interference, the absence of any positive registration with respect to the rotor lamination required a calibration procedure. Further, it will have some detrimental effect on the air-flow in the inlet and is susceptibility to contamination is such that its unlikely to be a long-term solution.

Initially the engine was tested at the lowest speed and power i.e. $1000 \mathrm{rpm}, 50 \mathrm{Nm}$, as this represents the least arduous point for the EATG device both in terms of 
turbocharger speed and exhaust gas temperature. At this engine test point, the original Holset $\mathrm{H} 1 \mathrm{C}$ turbocharger with the $12 \mathrm{~cm}^{2}$ scroll rotates at $25,680 \mathrm{rpm}$ with an exhaust gas temperature of $199^{\circ} \mathrm{C}$, which corresponds to a turbine power of $427 \mathrm{~W}$ (derived using equation 7.2). Given this relatively low turbine power, there is likely to be very little to scope to generate any electrical output power, and indeed initial attempts to extract electrical power using the EATG resulted in an effective "stalling" of the turbocharger. The tests in this case were therefore restricted to the application of motoring power to increase the speed of the turbocharger. For the EATG system the shaft speed with no electrical input was 23,900 rpm (compared with 25,680rpm for the standard turbocharger). The reduced speed can be largely attributed to the use of a different bearing arrangement and the increased aerodynamic loss associated with the SR rotor. Electrical input power was supplied to the system to increase the speed of the shaft, and ultimately a speed of $29,980 \mathrm{rpm}$ was achieved in increments of approximately 2000rpm. Figure 7.41 shows the total electrical power input to the EATG system as a function of shaft speed, with a peak electrical power input of $900 \mathrm{~W}$. The increased boost pressure as a function of shaft speed is shown in figure 7.42, where an overall increase of gauge pressure of $50 \%$ was recorded over the speed range $23,900 \mathrm{rpm}$ to $28,100 \mathrm{rpm}$. Figure 7.43 shows the corresponding increase in mass flow rate of air into the engine, where an increase of $6.5 \%$ is observed over the speed range $23,900 \mathrm{rpm}$ to $29,980 \mathrm{rpm}$. One notable feature of the increased boost pressure and air flow rate is that the efficiency of the compressor, calculated using equation 7.5 , is seen to improve markedly over the range of speeds measured as shown in figure 7.44.

$$
\eta_{c}=\frac{\left(P_{2} / P_{1}\right)^{\frac{\gamma-1}{\gamma}}-1}{T_{2} / T_{1}-1}
$$

Indeed, an overall compressor efficiency increase from $15 \%$ to $22 \%$ is observed for a speed increase from $23,900 \mathrm{rpm}$ to $28,100 \mathrm{rpm}$. This rapid increase in compressor efficiency can be highlighted by the compressor map of figure 7.45 , where the engine 
operating points (with the standard $12 \mathrm{~cm}^{2}$ turbine arrangement fitted) are superimposed onto this compressor map. The engine operating points are arranged as lines of constant speed, where the application of engine load and the corresponding increase in turbocharger speed tend to shift the operating points upward and to the right of the map into regions of increased efficiency. It is apparent that even with the smaller turbine fitted the compressor is operating at efficiency generally well below its optimum value. With the increased speed offered by the use of the EATG in motoring mode it is apparent that the compressor will operate in regions of higher efficiency adding further benefits, in terms of air flow management, to the use of such a system.

Further advantages of the system can be seen when considering the particulate matter emissions, figure 7.46 shows the variation in smoke as a function of the EATG rotor speed. It is apparent from figure 7.46 that even though the smoke emission for these engine operating conditions is already very low (by virtue of the low engine load), considerable gains can be made in terms of reducing smoke emission, indeed an overall reduction of $15 \%$ was recorded over the speed range of $23,900 \mathrm{rpm}$ to $29,980 \mathrm{rpm}$, albeit that the manufacturers specified repeatability is only 0.05 FSN. This reduction is due mainly to the increased oxidation of the particles due to the increased abundance of oxygen in the combustion chamber (section 1.2 of chapter 1) as is evidenced by the increased level of measured air flow rate shown in figure 7.43. Similarly, the concentration of $\mathrm{NO}_{\mathrm{x}}$ emissions is seen to decrease over the speed range by some $13 \%$ as shown in figure 7.47 . However, care must be taken in interpreting this result as the increased mass flow rate of gas into the engine over the speed range (i.e. 6.5\%) will tend to dilute the $\mathrm{NO}_{\mathrm{x}}$ content, and hence the absolute reduction in $\mathrm{NO}_{\mathrm{x}}$ emission is much lower than suggested. Since the formation of $\mathrm{NO}_{\mathrm{x}}$ is a complex function of peak cylinder pressures and temperatures and the timing of the fuel injection, no conclusive findings in relation to $\mathrm{NO}_{\mathrm{x}}$ can reliably be claimed.

In order to establish the benefits of the EATG system in terms of smoke-reduction, a further series of tests was initiated at low engine speeds and high load, specifically $1000 \mathrm{rpm}$ and $150 \mathrm{Nm}$. Unfortunately, while allowing the engine to reach steady-state 
conditions with no electrical input (which typically takes some 45 minutes or so) the turbine-end bearing failed catastrophically at a turbine speed of 31,000 rpm This in turn caused the rotor to ground onto the stator bore, which resulted in a visually discernible bend in the shaft and damaged one stator winding. On disassembly, it became apparent that the phenolic cage of the bearing had disintegrated completely due to the elevated temperature in the end-cap. Whereas features were included to cool the end-cap as discussed in section 7.1.4, it is evident that the measures taken were inadequate to cater for a high load (and hence exhaust temperature condition), particularly given the self loss of the bearings. One possible solution would be to employ direct oil-cooling to the turbine bearing (as opposed to just the end-cap), although this is difficult to achieve in practice given the very limited space available (particularly with regard to axial length) and the need for appropriate sealing. Further, the use of so-called 'cage-less' high-speed bearings could alleviate some of the problems, and indeed, these have been used on larger development turbochargers by Holset Engineering. However, they are highly specialised and expensive bearings, which furthermore were not available in sizes that could be accommodated within the current design nor with the appropriate speed rating.

In view of the degree of failure, and the considerable re-design required to enable testing to be performed with high exhaust gas temperatures, no further testing to establish the benefits of the EATG in reducing smoke at low-engine speed and high load conditions was undertaken. Moreover, given the very severe failure that occurred as a result of bearing over-temperature even at $31,000 \mathrm{rpm}$, it was deemed to be unsafe to test at very high-speeds until extremely reliable bearing operation could be guaranteed, particularly since high turbine speeds necessarily involve high exhaust gas temperatures. Thus, although only very limited results were obtained for the entire system, these nevertheless in part demonstrate the likely advantages of the EATG system 


\section{REFERENCES}

[7.1] "Timing and digital I/O board: DS4002 user guide." dSPACE digital signal processing and control engineering GmbH, Paderborn, Germany. 1997. 


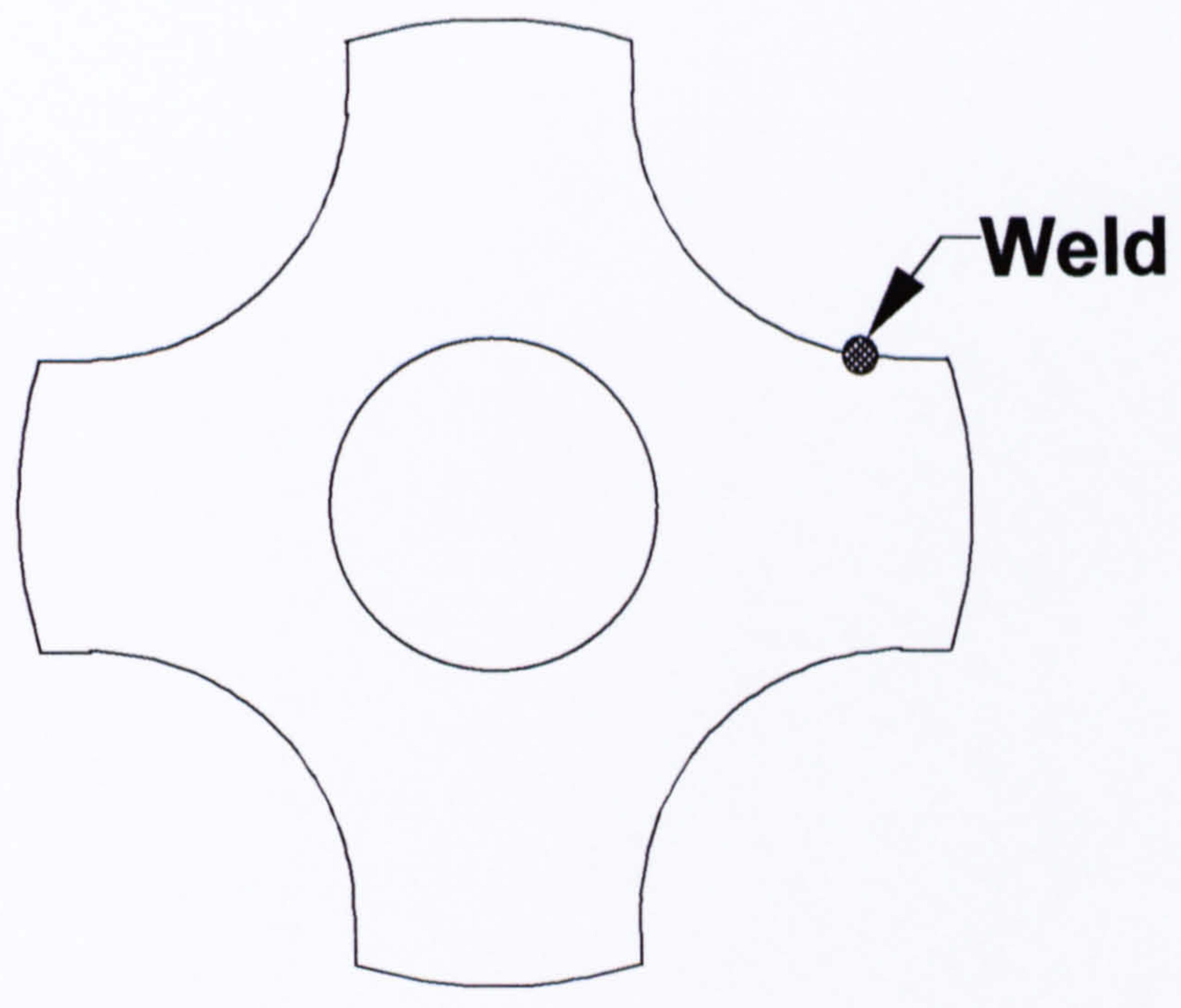

Figure 7.1. Location of rotor lamination welds

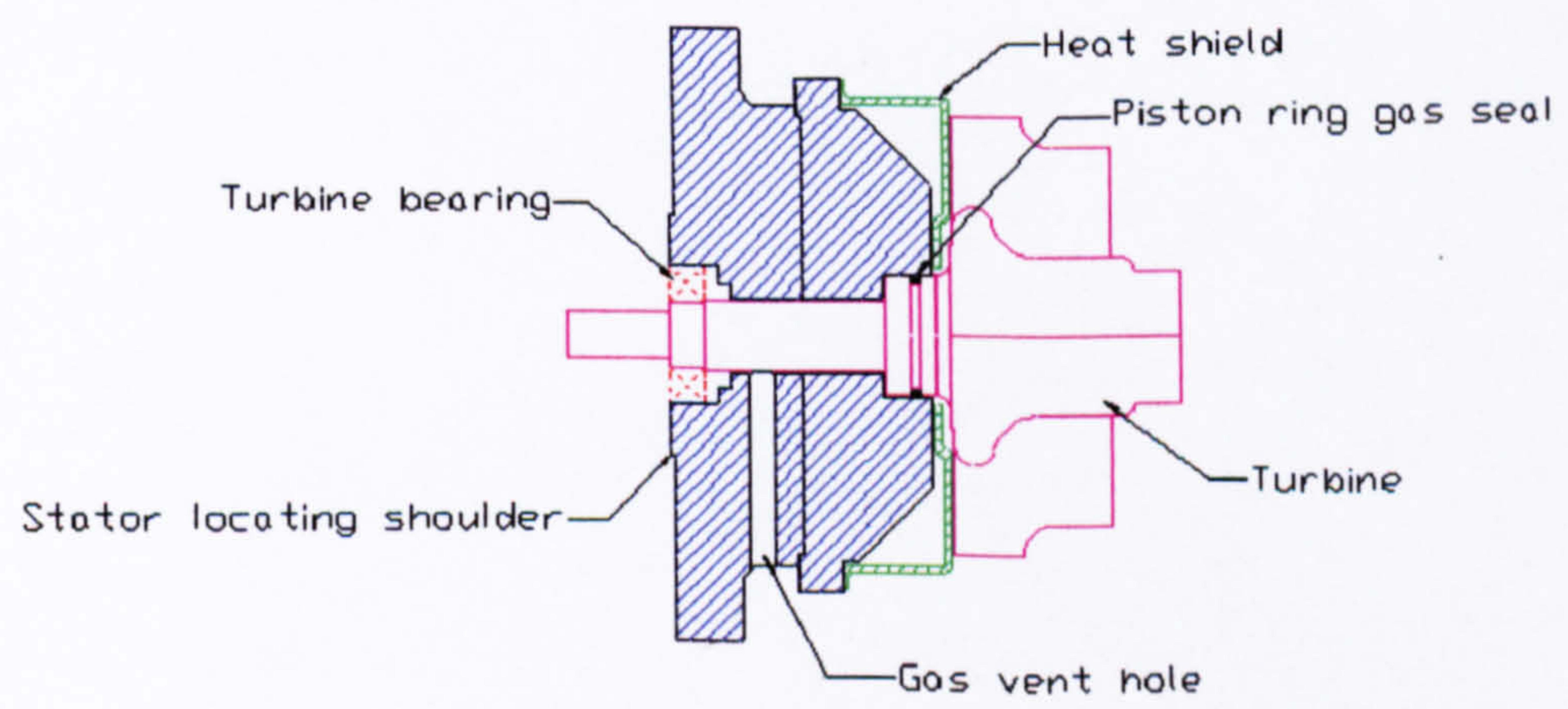

Figure 7.2. Cross-section of turbine end-cap 


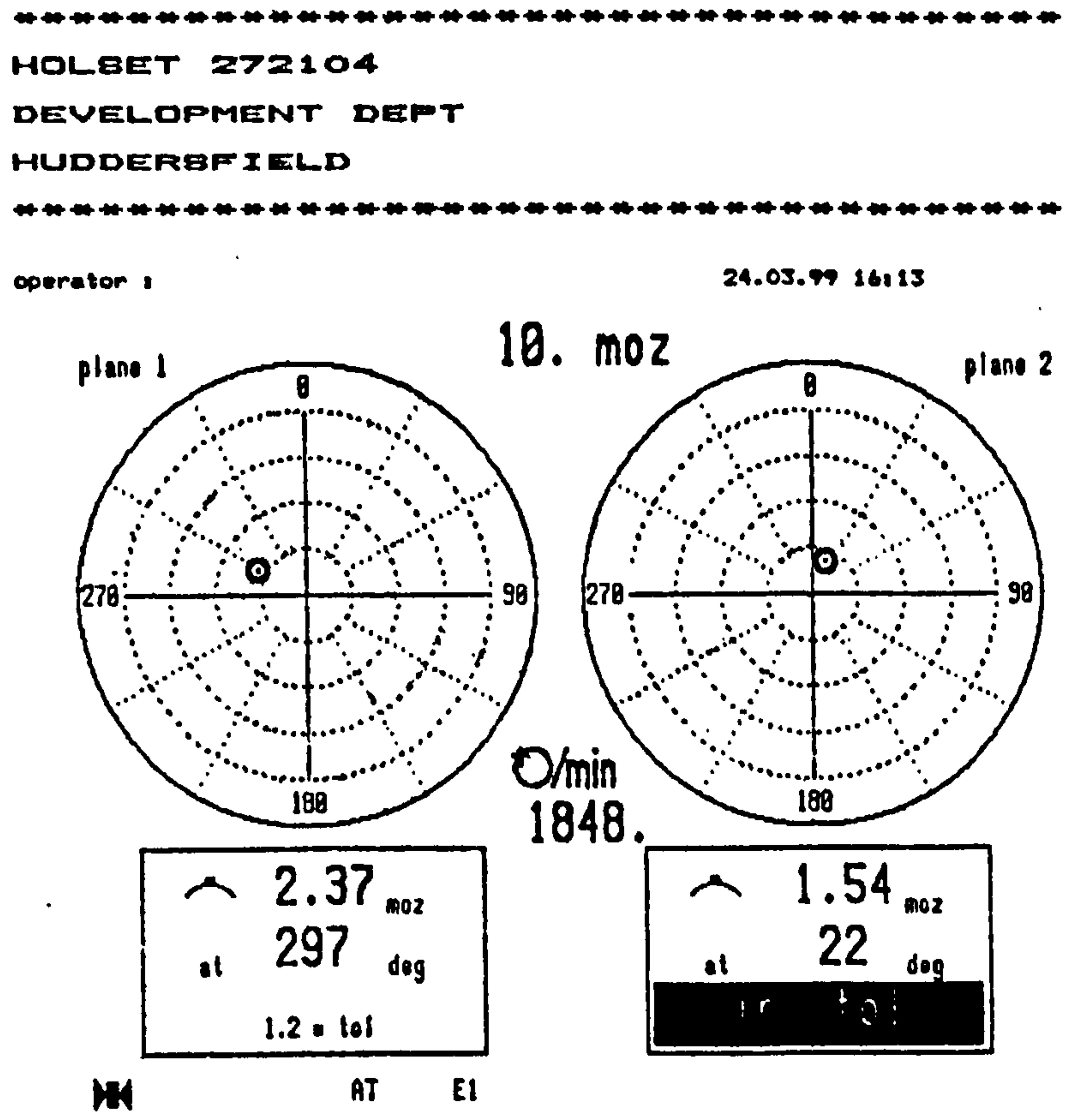

Figure 7.3. Balancing equipment output

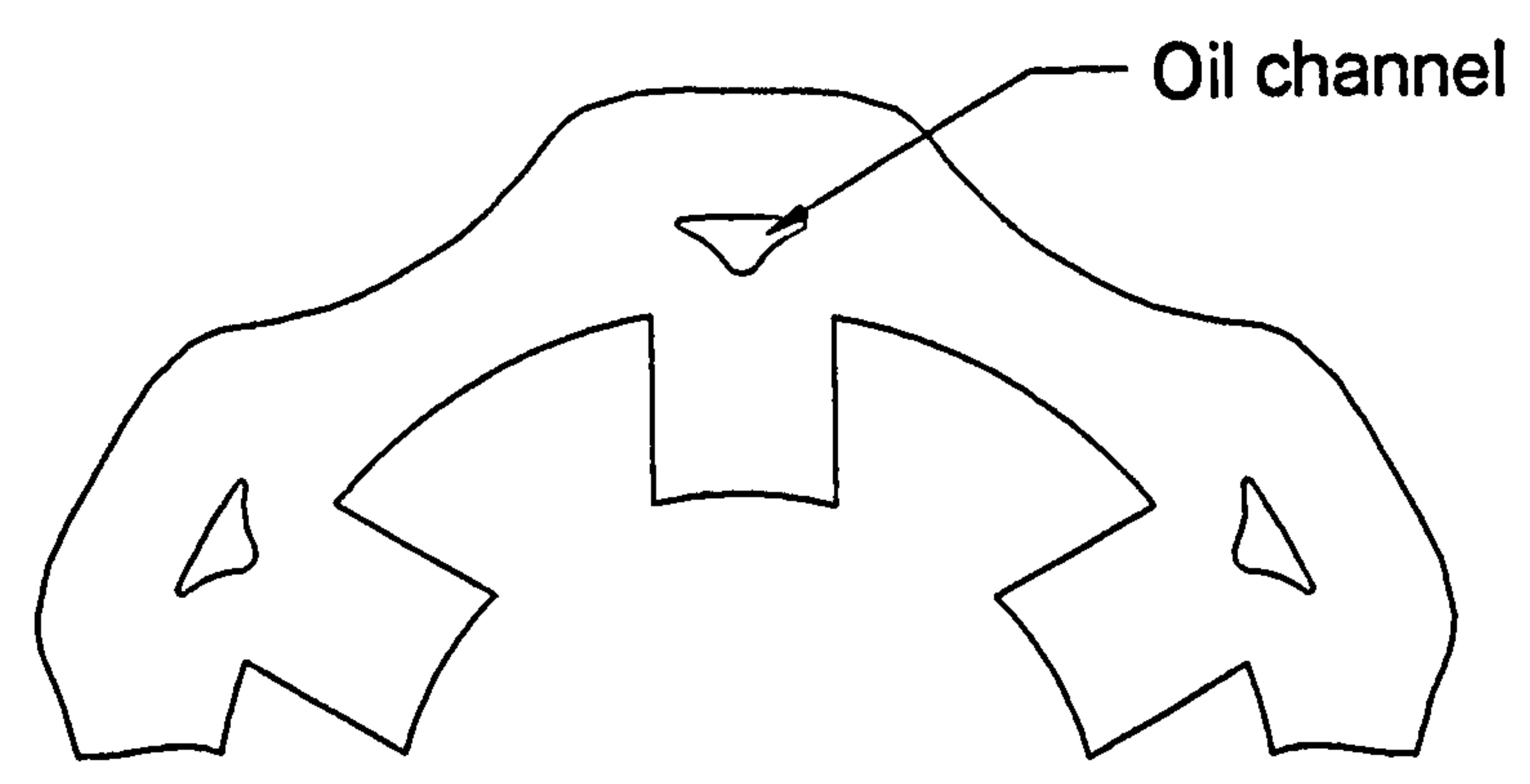

Figure 7.4. Cross-section of stator lamination showing location of oil-channels in the back-iron. 


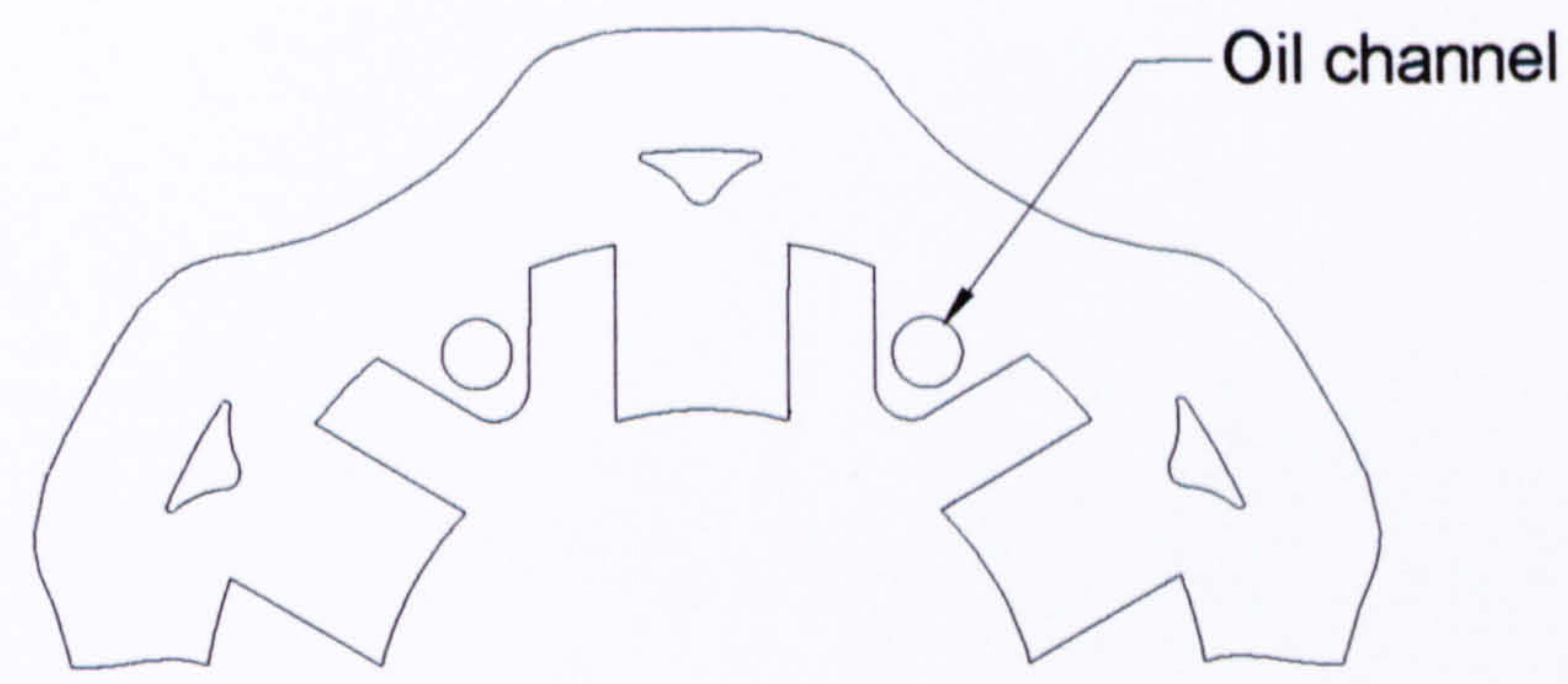

Figure 7.5 Cross-section of stator lamination showing location of oil-channels in the inter-winding region.

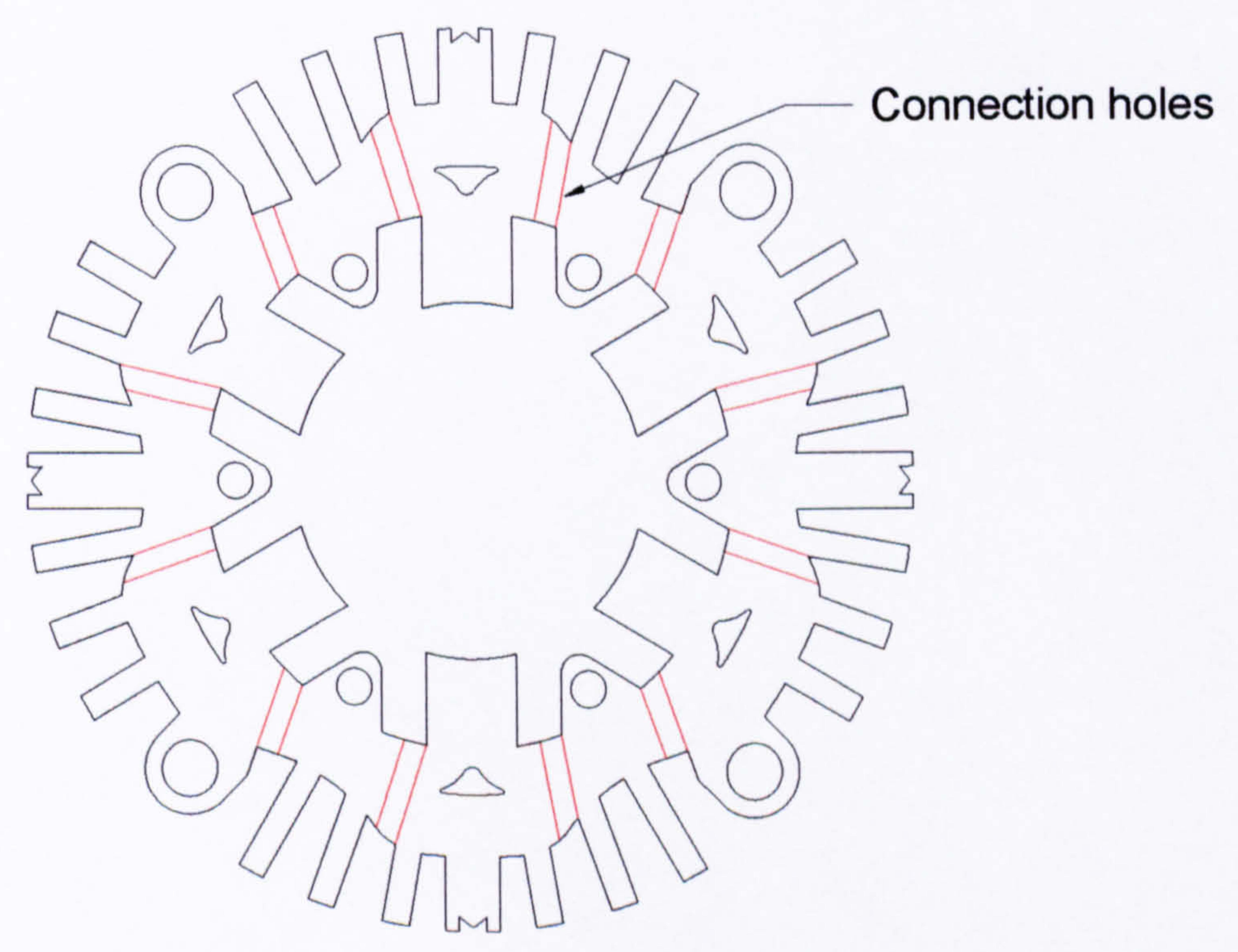

Figure 7.6. Cross section of the final lamination design, including location of winding connection holes 


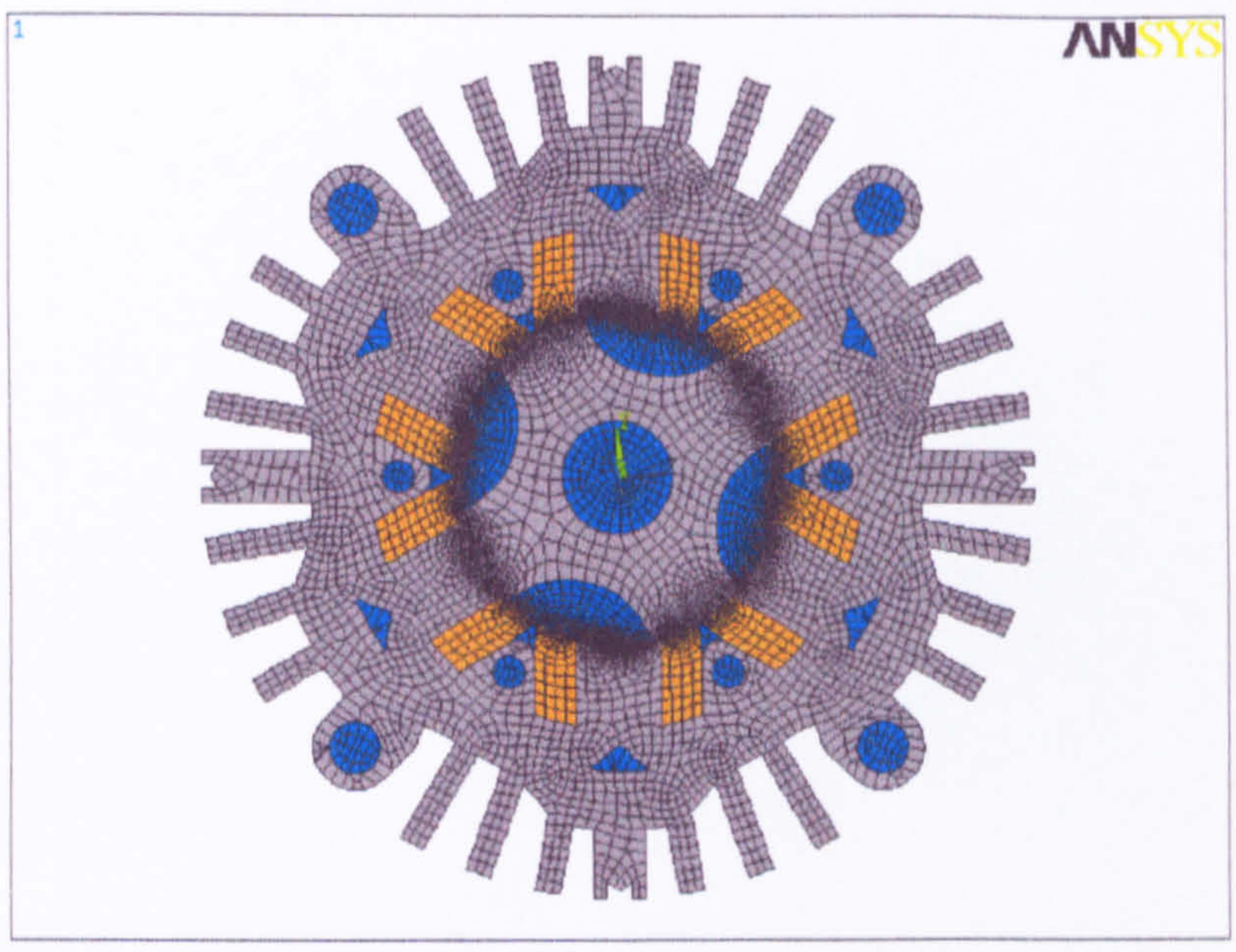

Figure 7.7 Finite element mesh of motor with finned stator consisting of 34,632 nodes and 11,323 second order elements with the rotor $30^{\circ}$ from the aligned position.

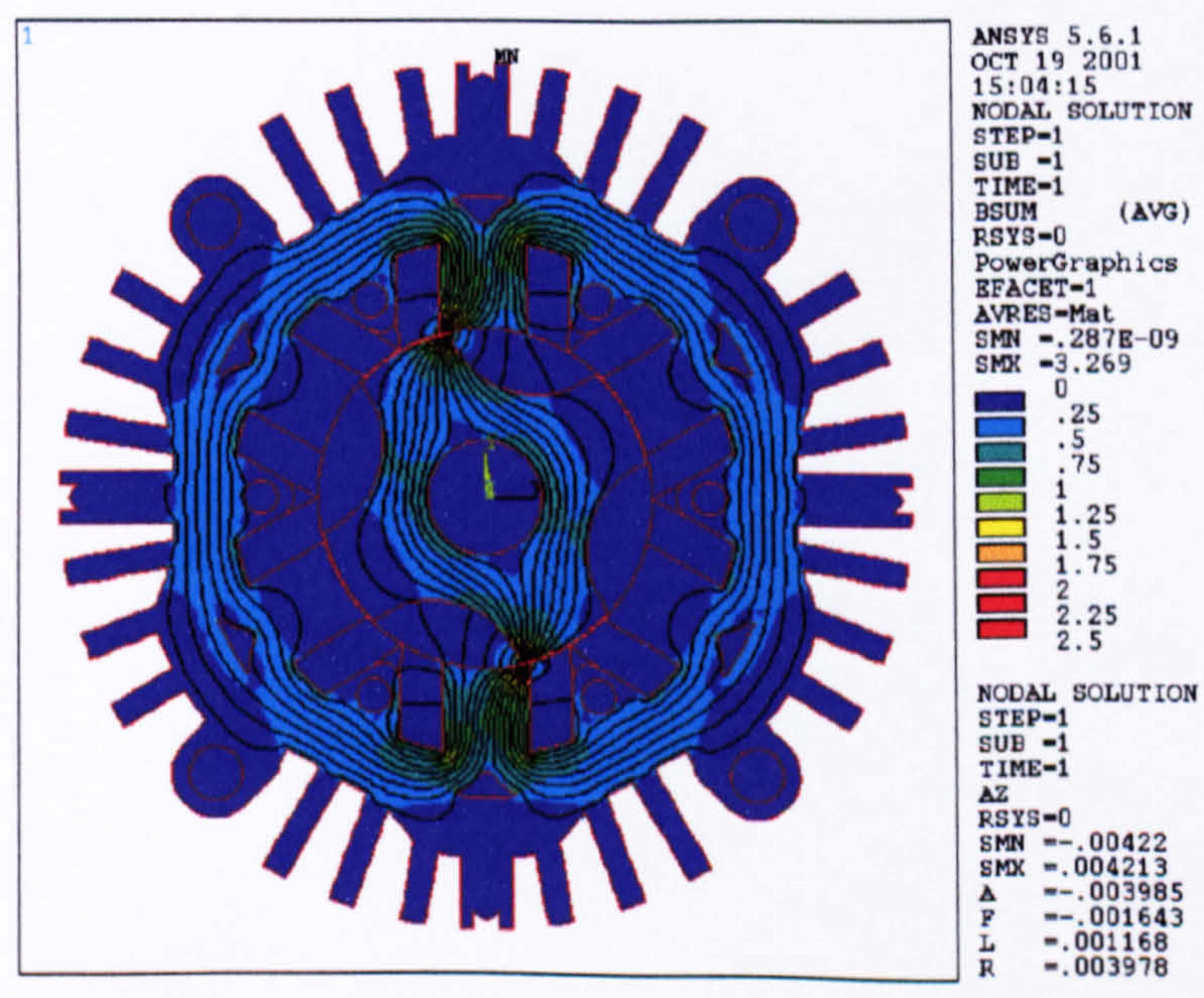

Figure 7.8. Flux density contours and lines of constant vector potential solved using the mesh of figure 7.7 with a current density of $15 \mathrm{~A} / \mathrm{mm}^{2}$ applied to phase A. 


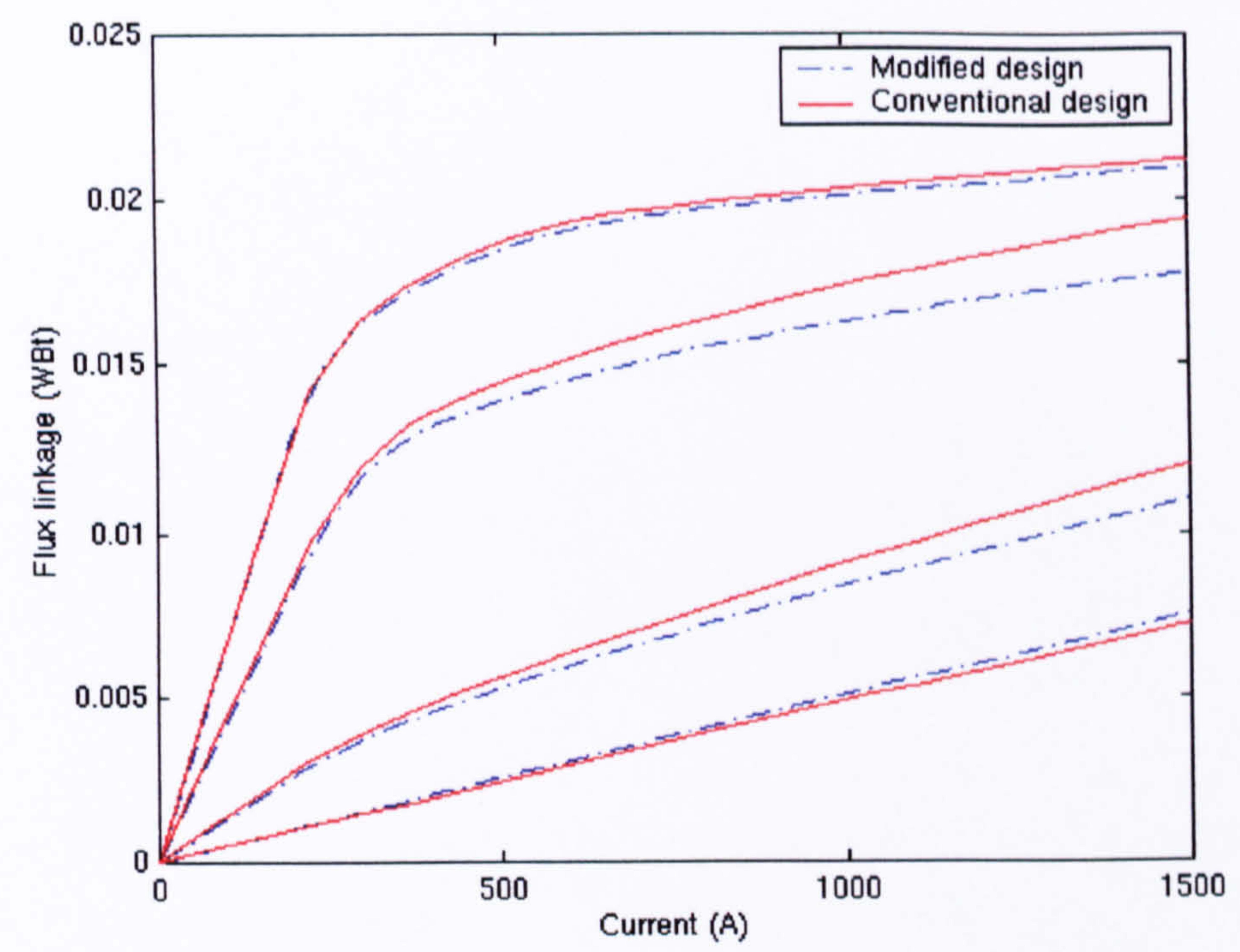

Figure 7.9. $\psi-i$ characteristics for the finned and non-finned (chapter 3 ) stator designs in the aligned and unaligned positions and 15 and $30^{\circ}$ from alignment.

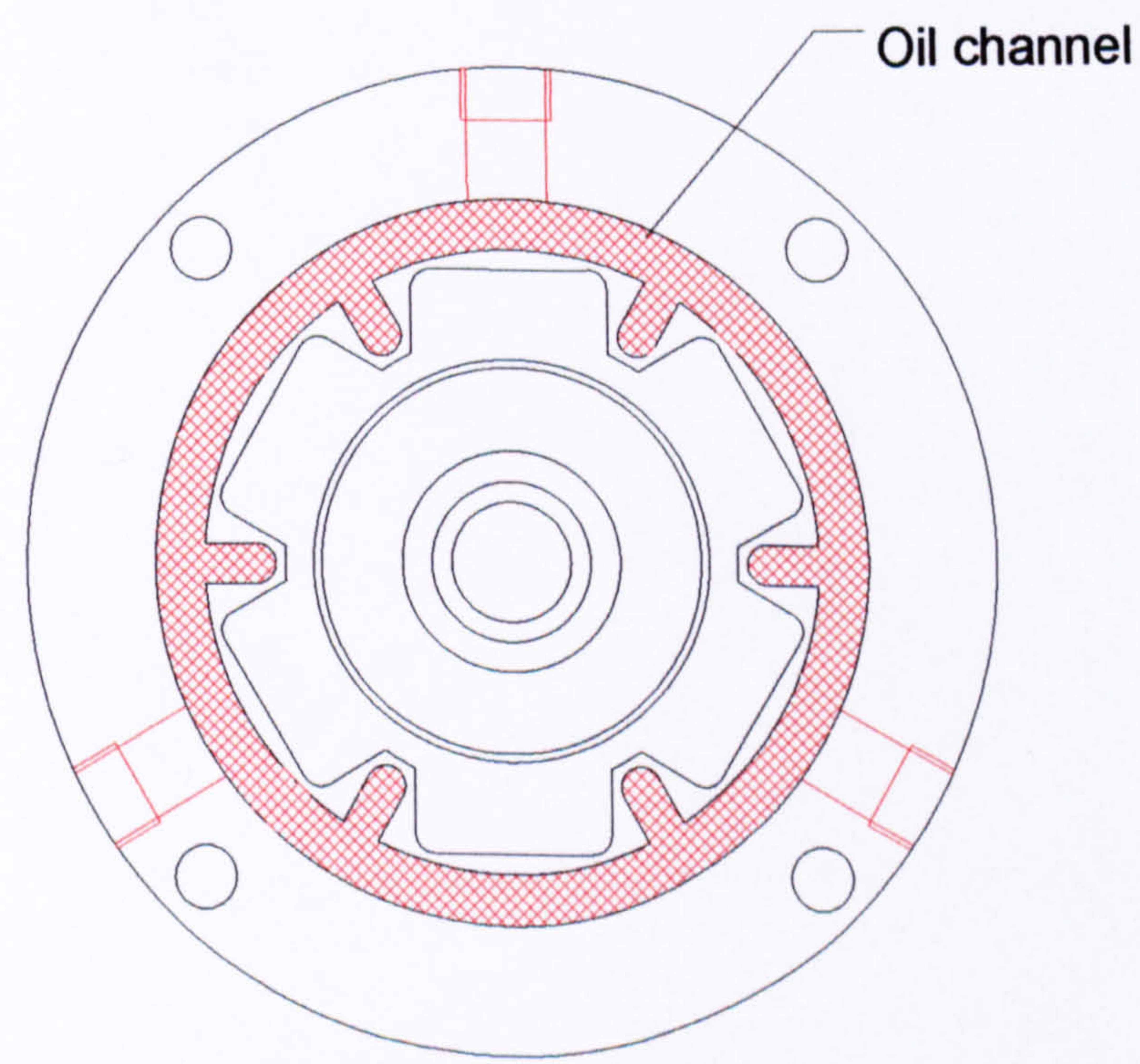

Figure 7.10. Oil-channel milled into the turbine end-cap 

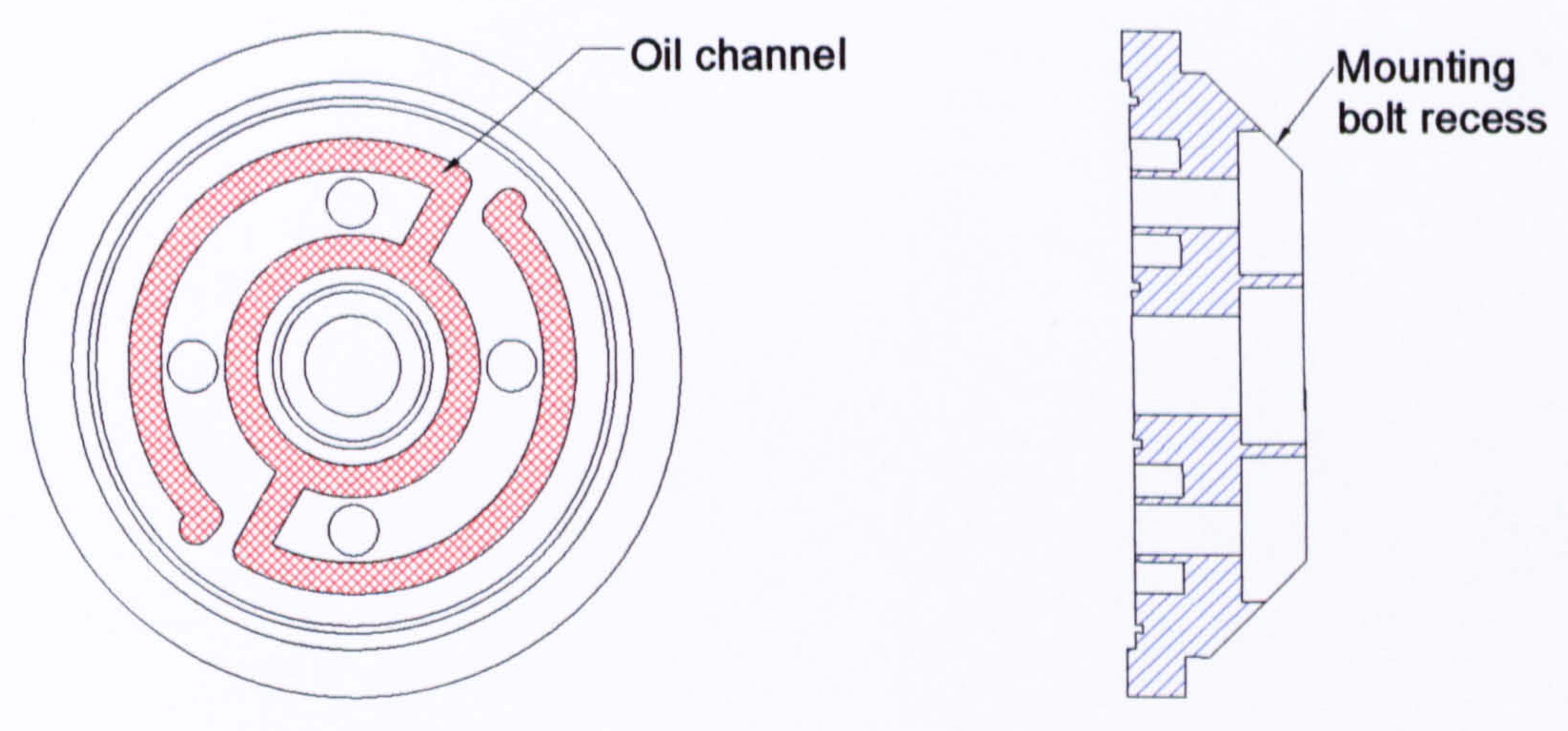

Figure 7.11. Oil channel milled into the interfacing planes of the split turbine endcap.
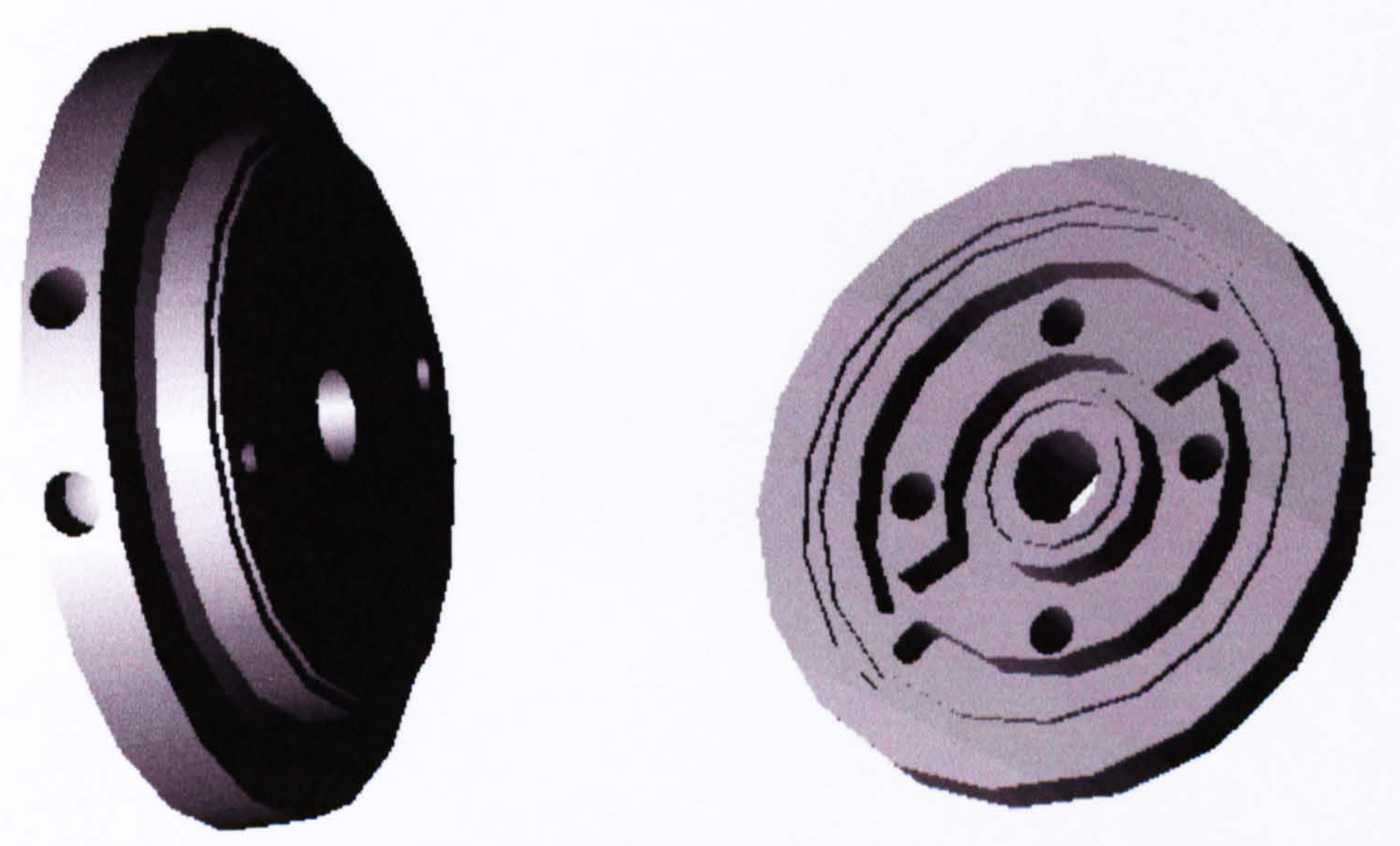

Figure 7.12. Split turbine end-cap assembly showing the oil-circuit arrangement. 


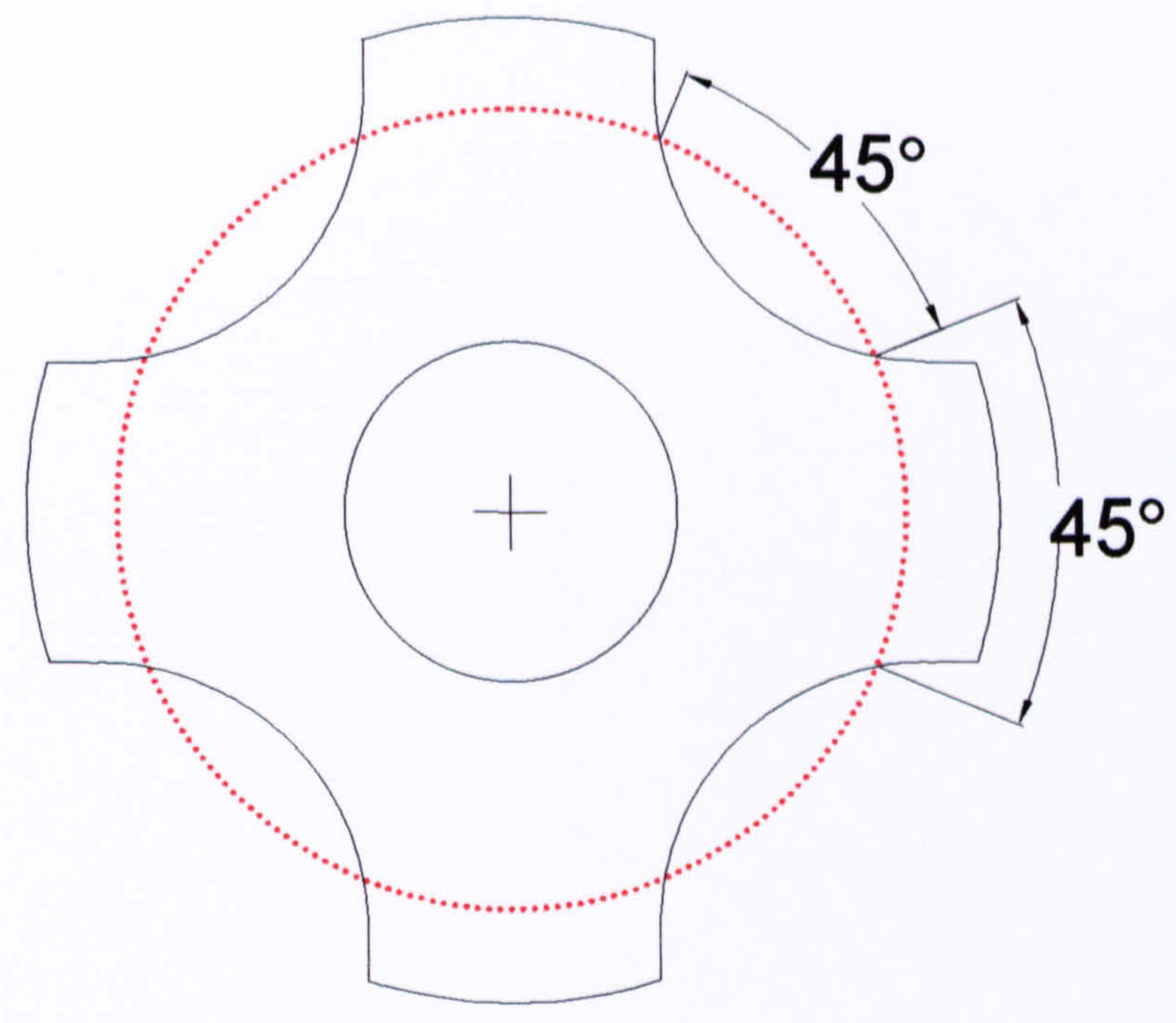

Figure 7.13 . Radius of hall sensors giving a $50 \%$ duty cycle position signal

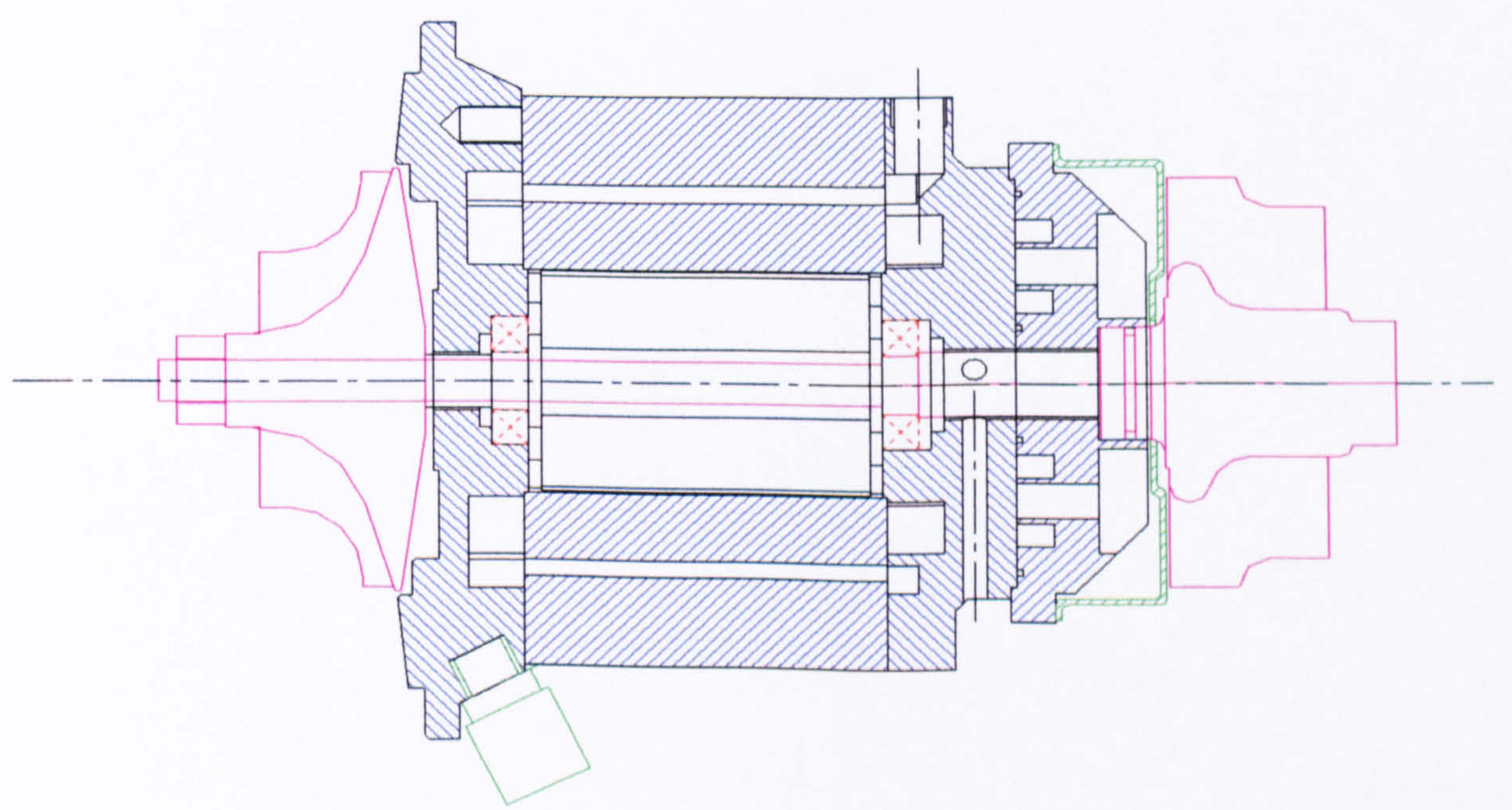

Figure 7.14 Cross section of the prototype EATG 


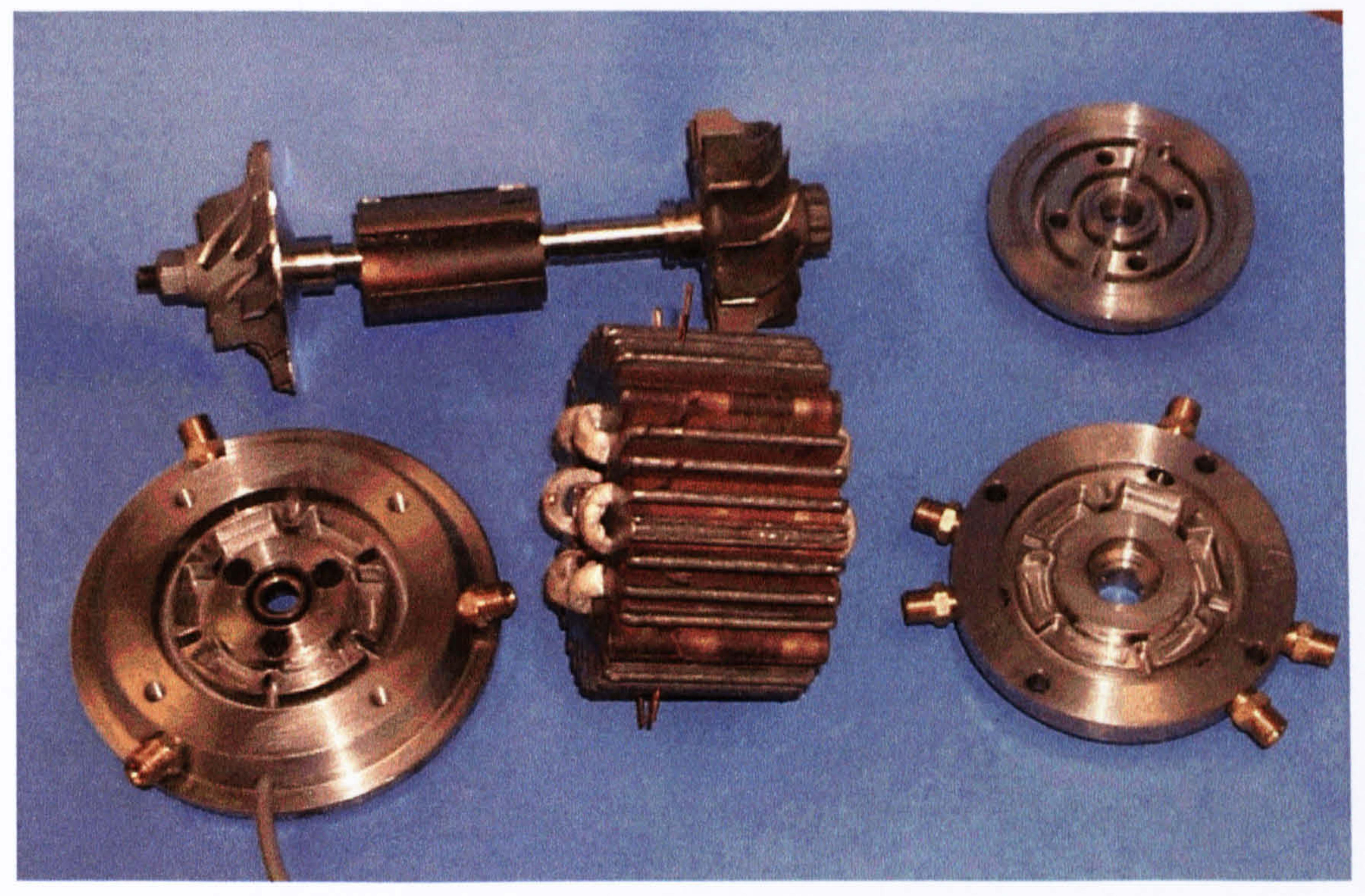

Figure 7.15. Photograph of the components of the prototype EATG.

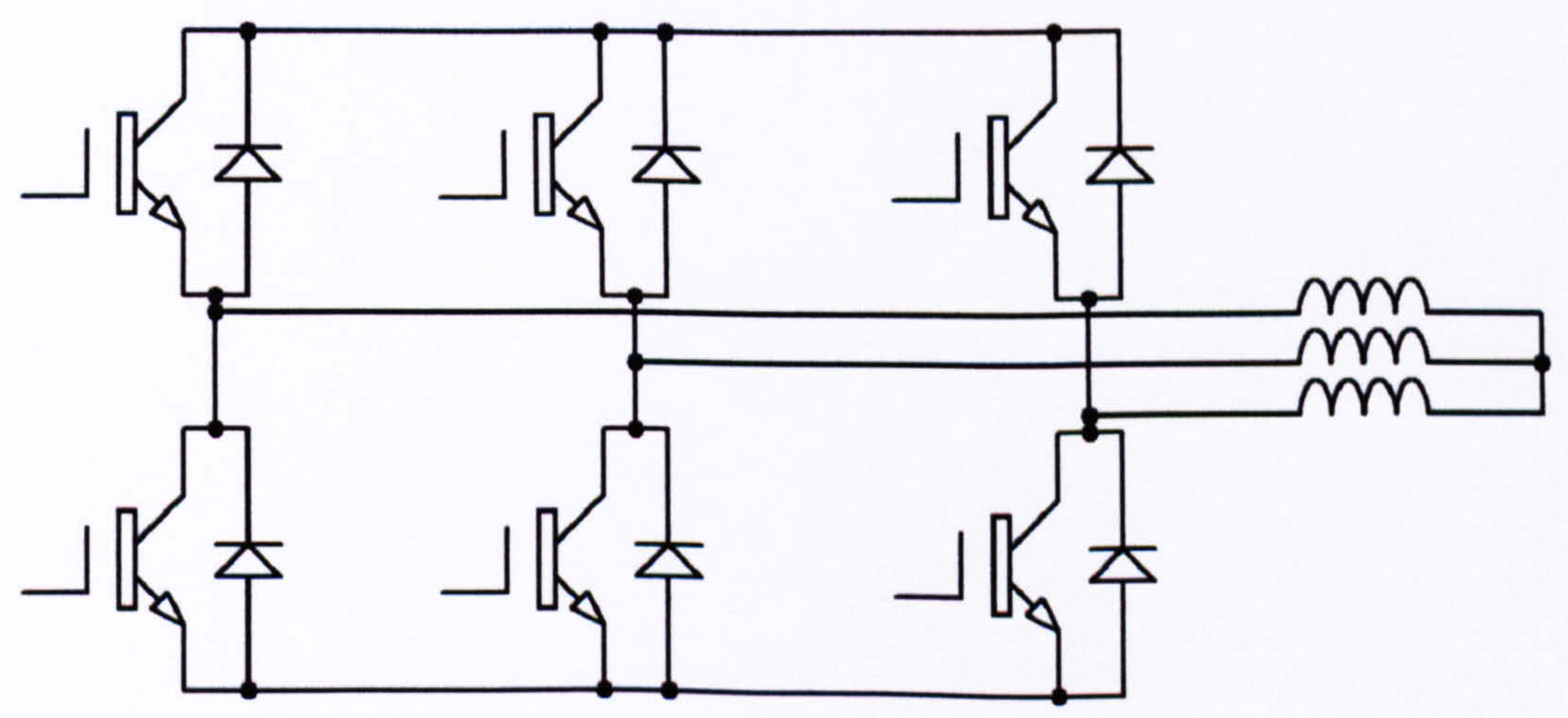

Figure 7.16 Schematic of the 3-phase Fuji IPM showing the internal connections and external connections to a star connected motor 


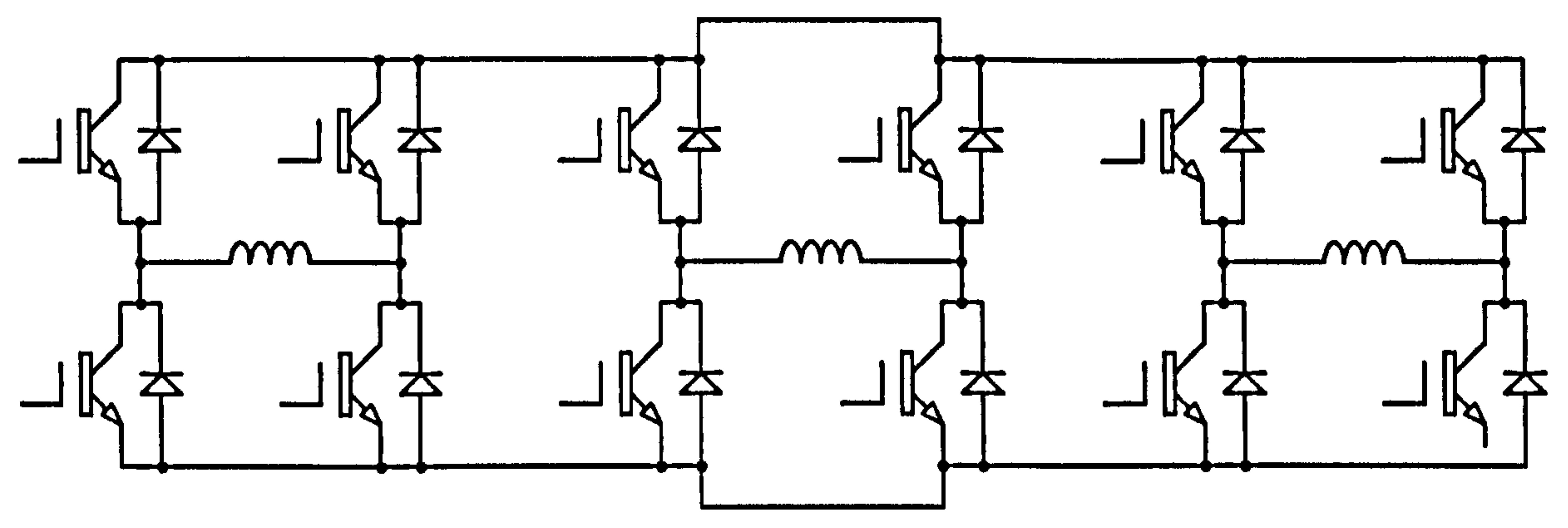

Figure 7.17. Coupling of 2 IPM's to form the basis of the 3-phase SR drive circuit

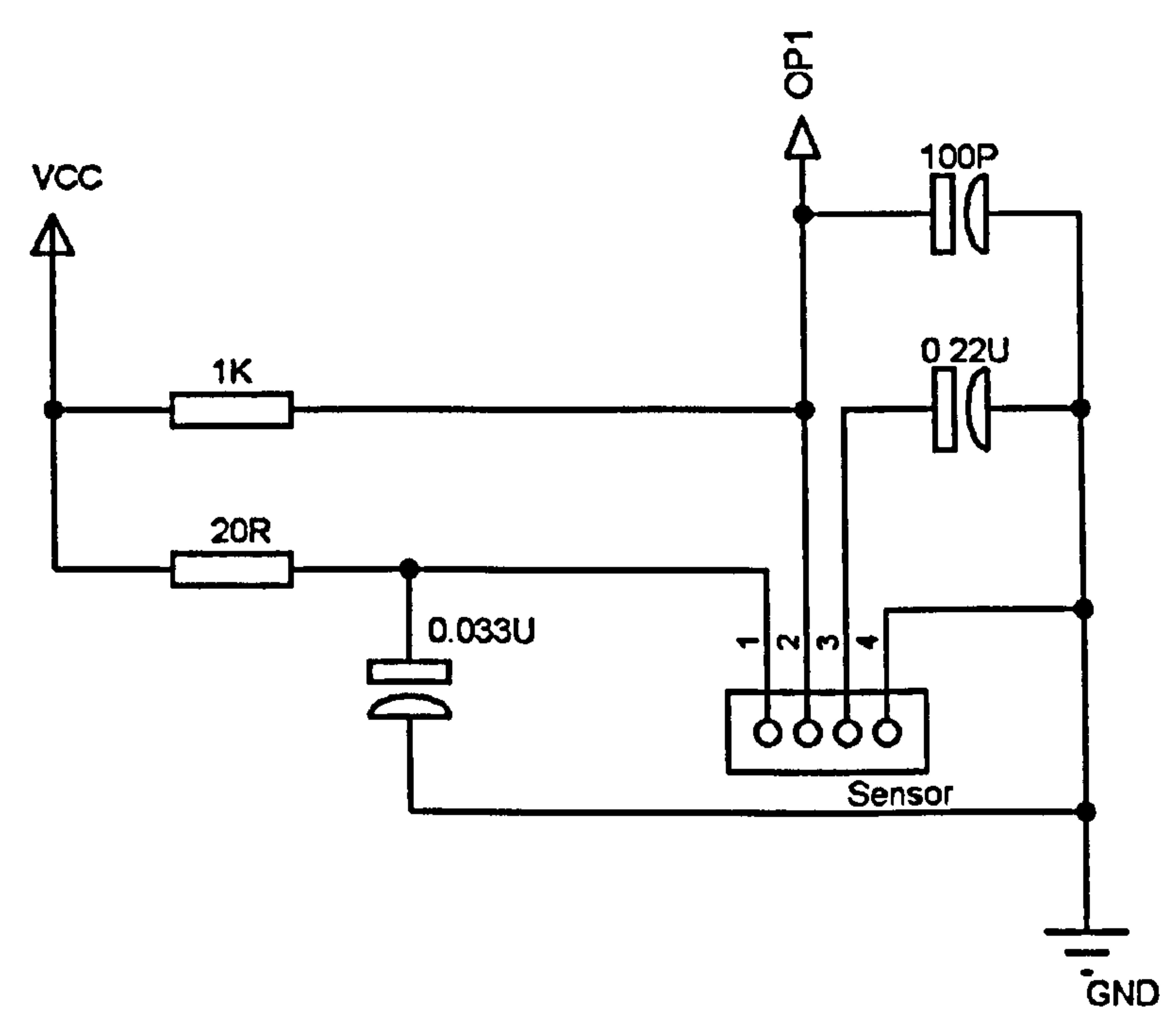

Figure 7.18. Diagram of the low-pass filter constructed using surface mount components and placed on the rear of the hall sensor modules 


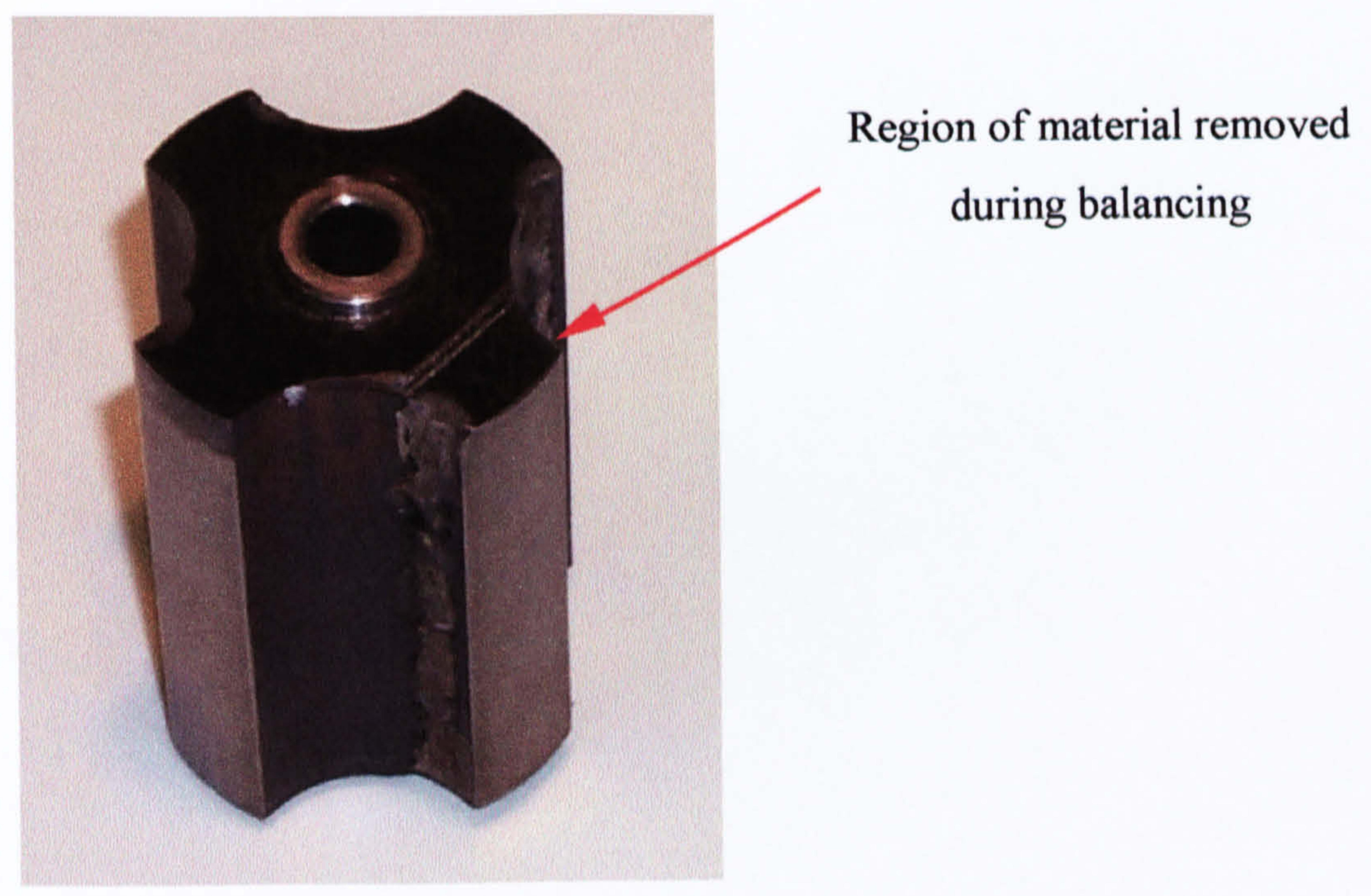

Figure 7.19 Photograph of the rotor after balancing showing the material removed from the face of the structure.

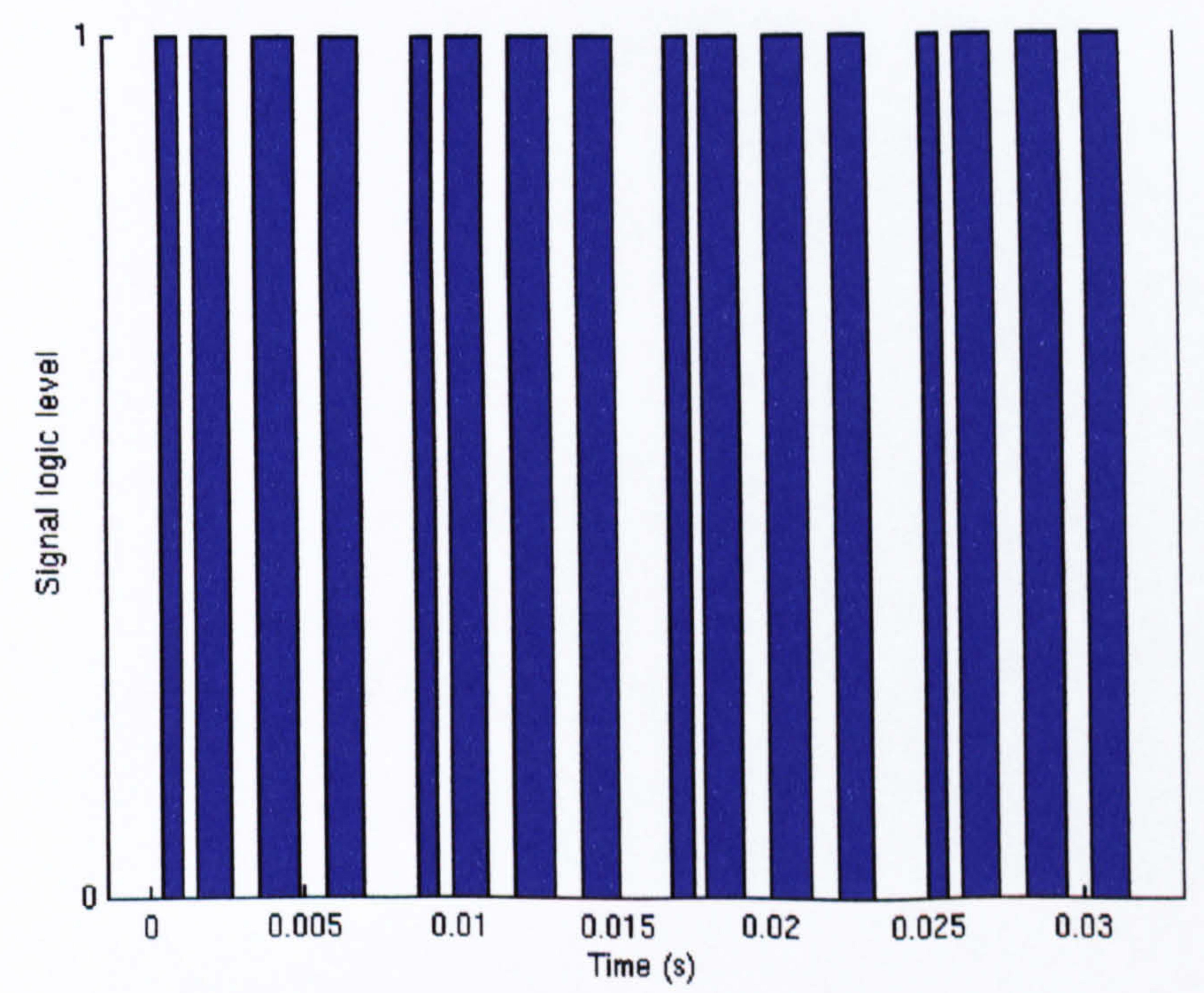

Figure 7.20. Measured pulse pattern from the Hall sensors showing the non-uniform duty cycle. 


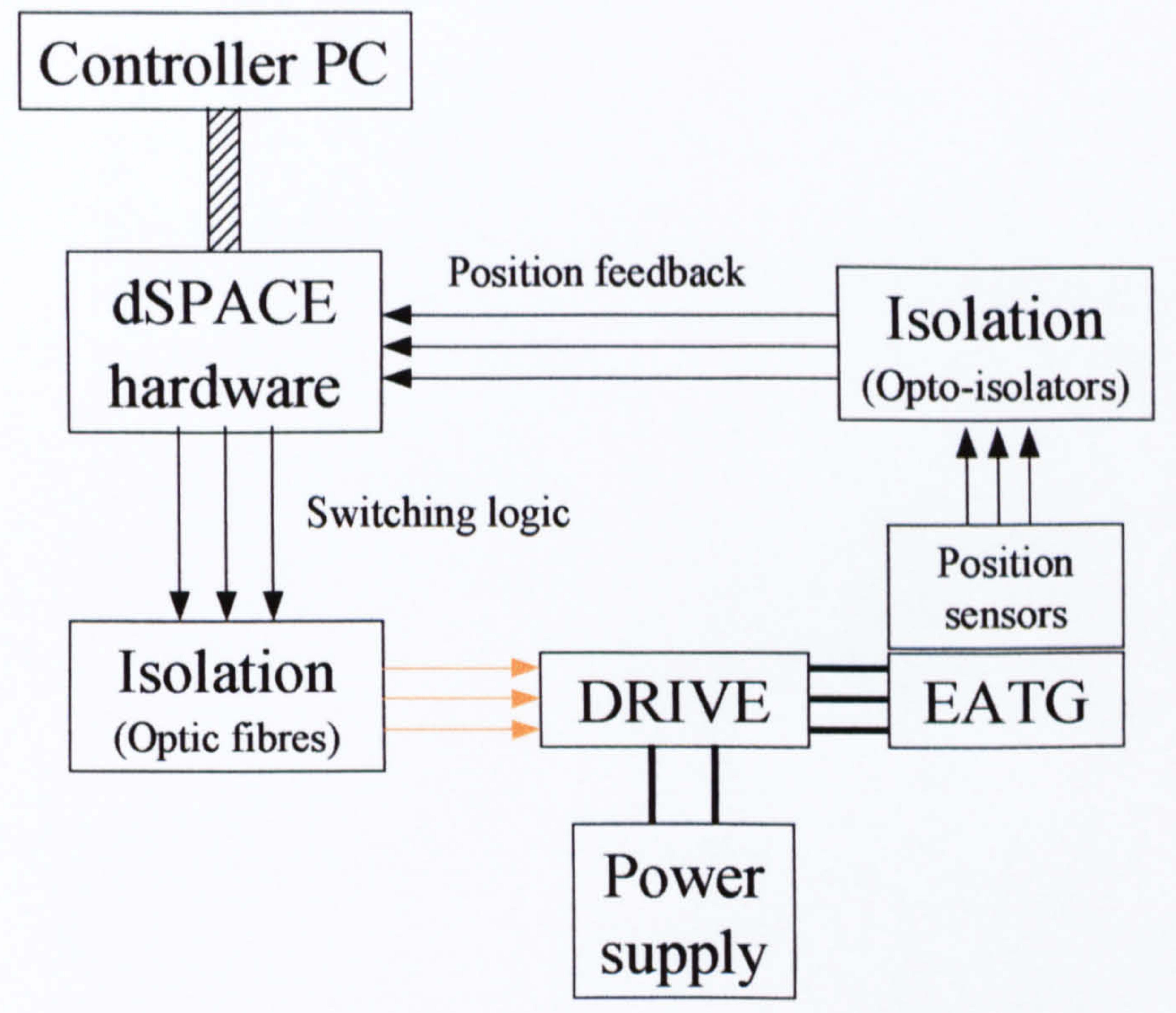

Figure 7.21. Schematic representation of the prototype EATG system.

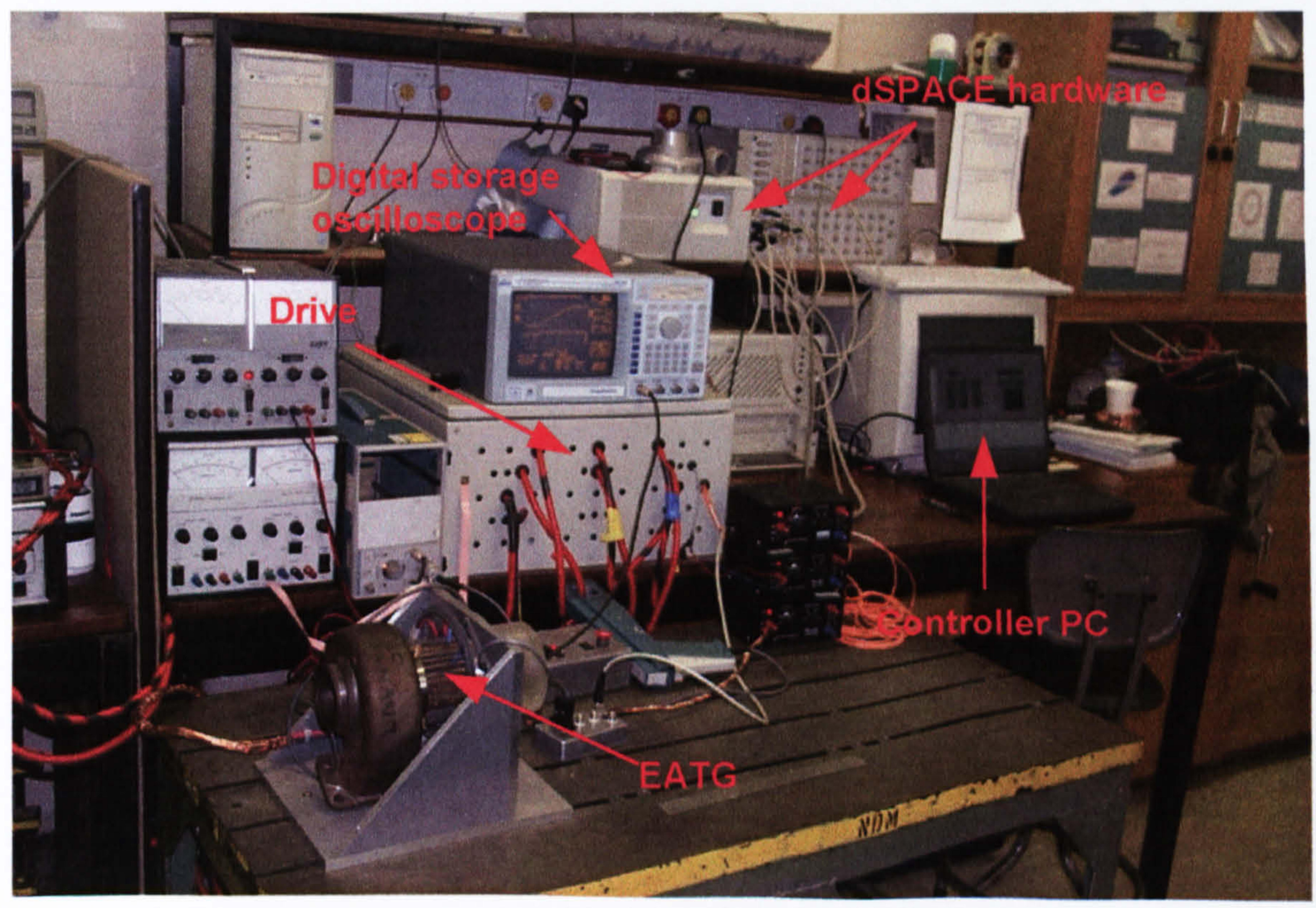

Figure 7.22 Photograph of the prototype system on the bench 


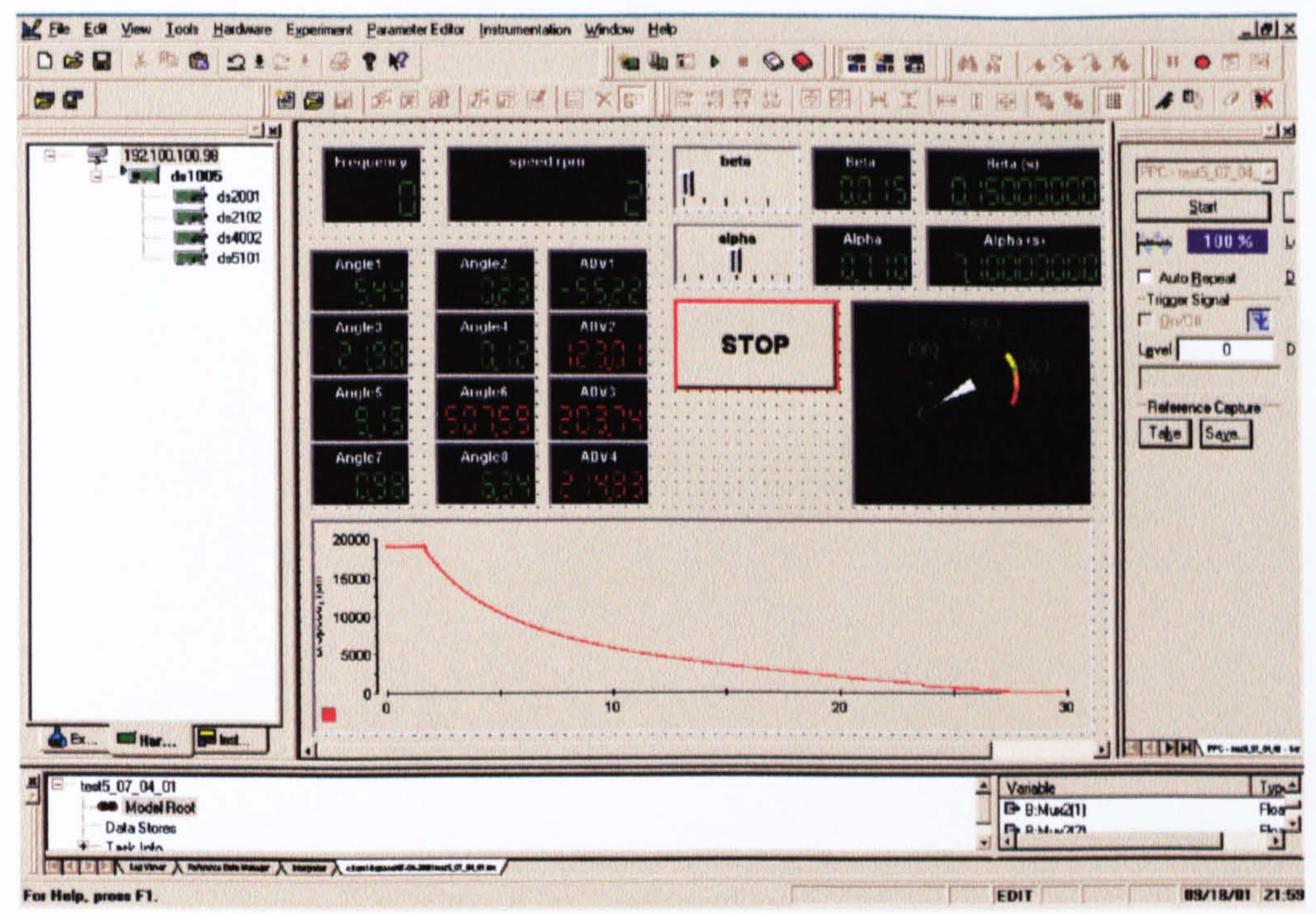

Figure 7.23. Sample screen output from dSPACE control desk.

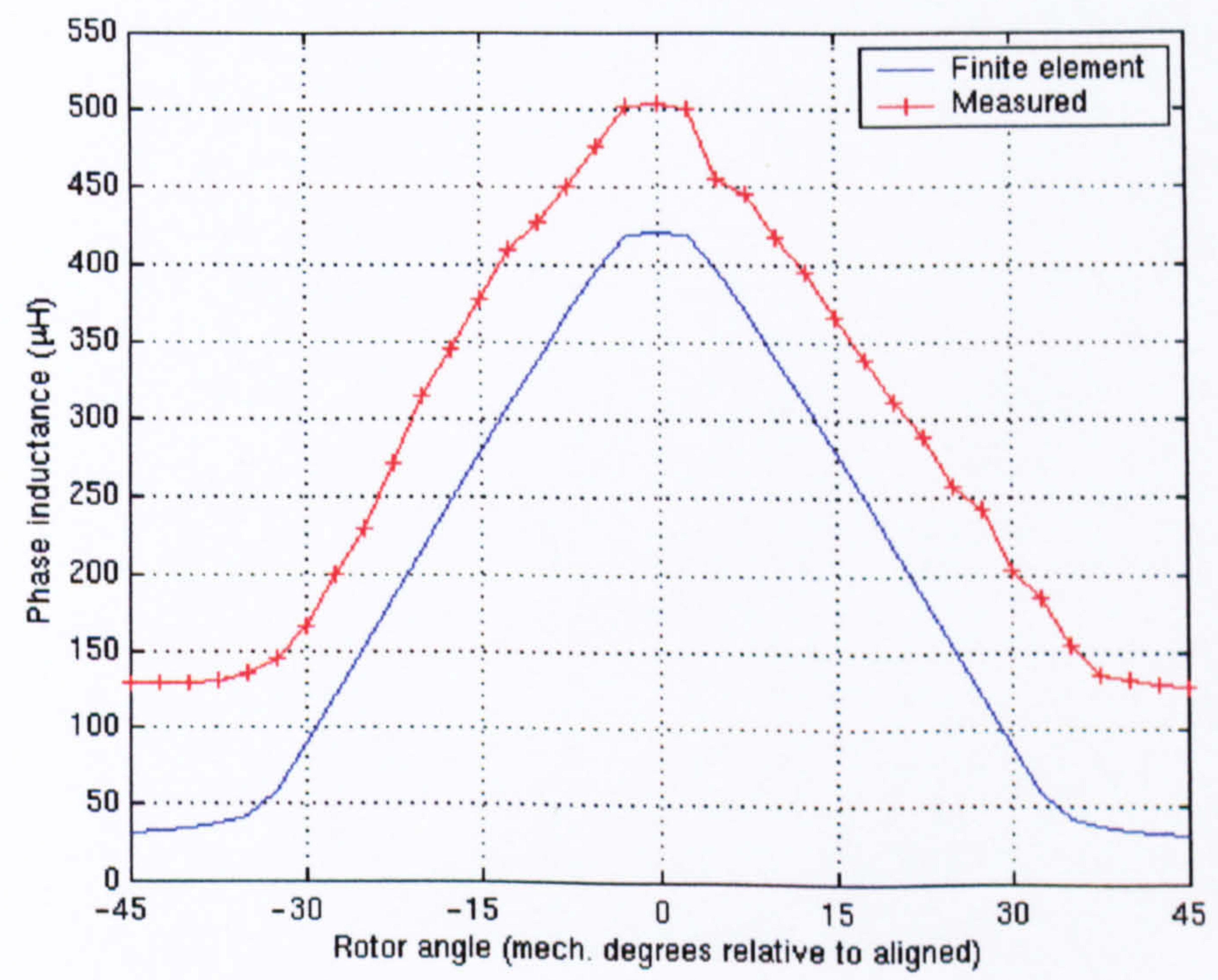

Figure 7.24. Finite element predicted and measured phase inductance as a function of rotor position. 
15-Jun-01 Recalibration Suggested (SYSTEM CONFIG Menu)

$11: 59: 32$

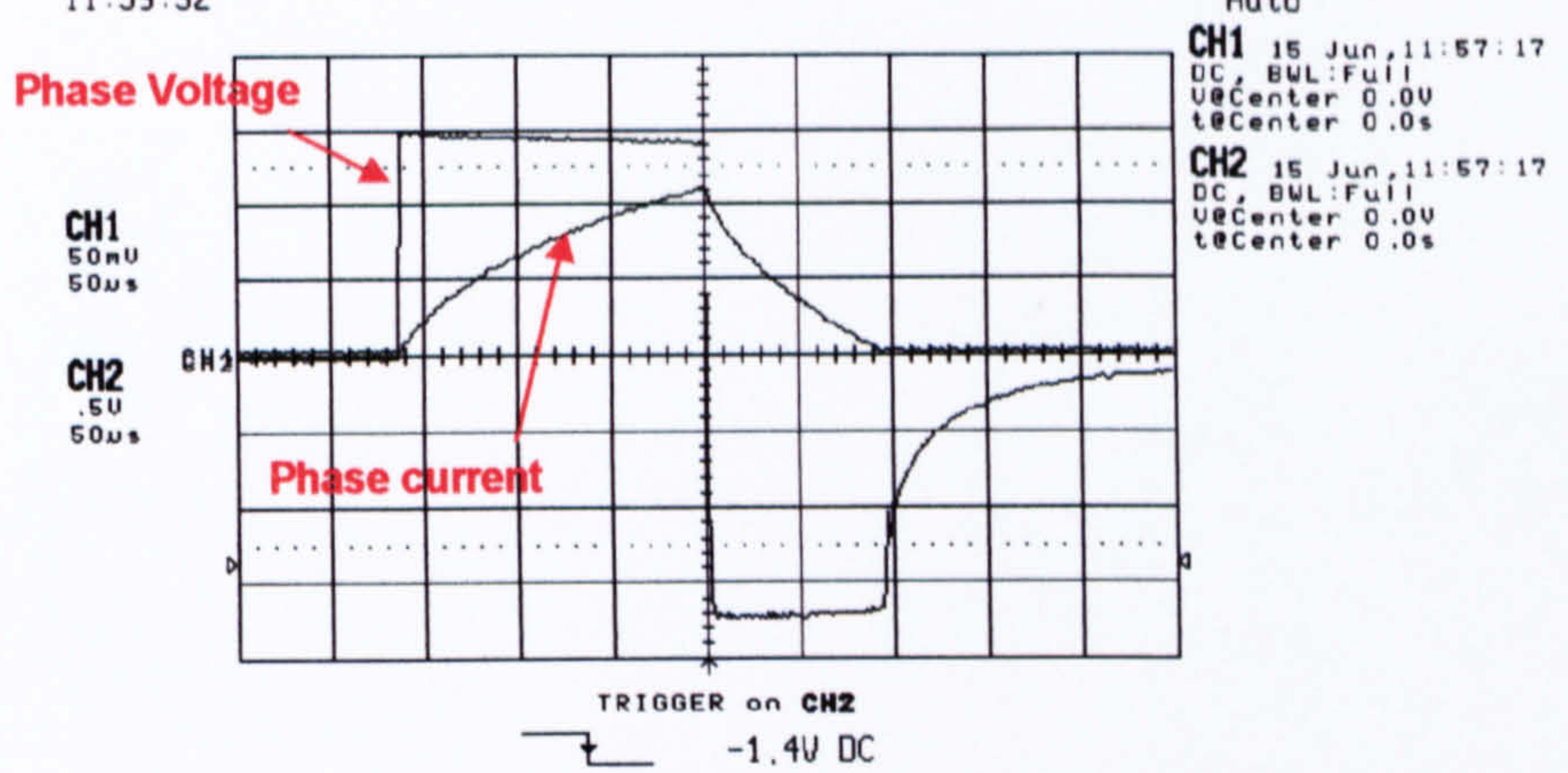

Figure 7.25a. Measured current and voltage waveforms for test case 1.

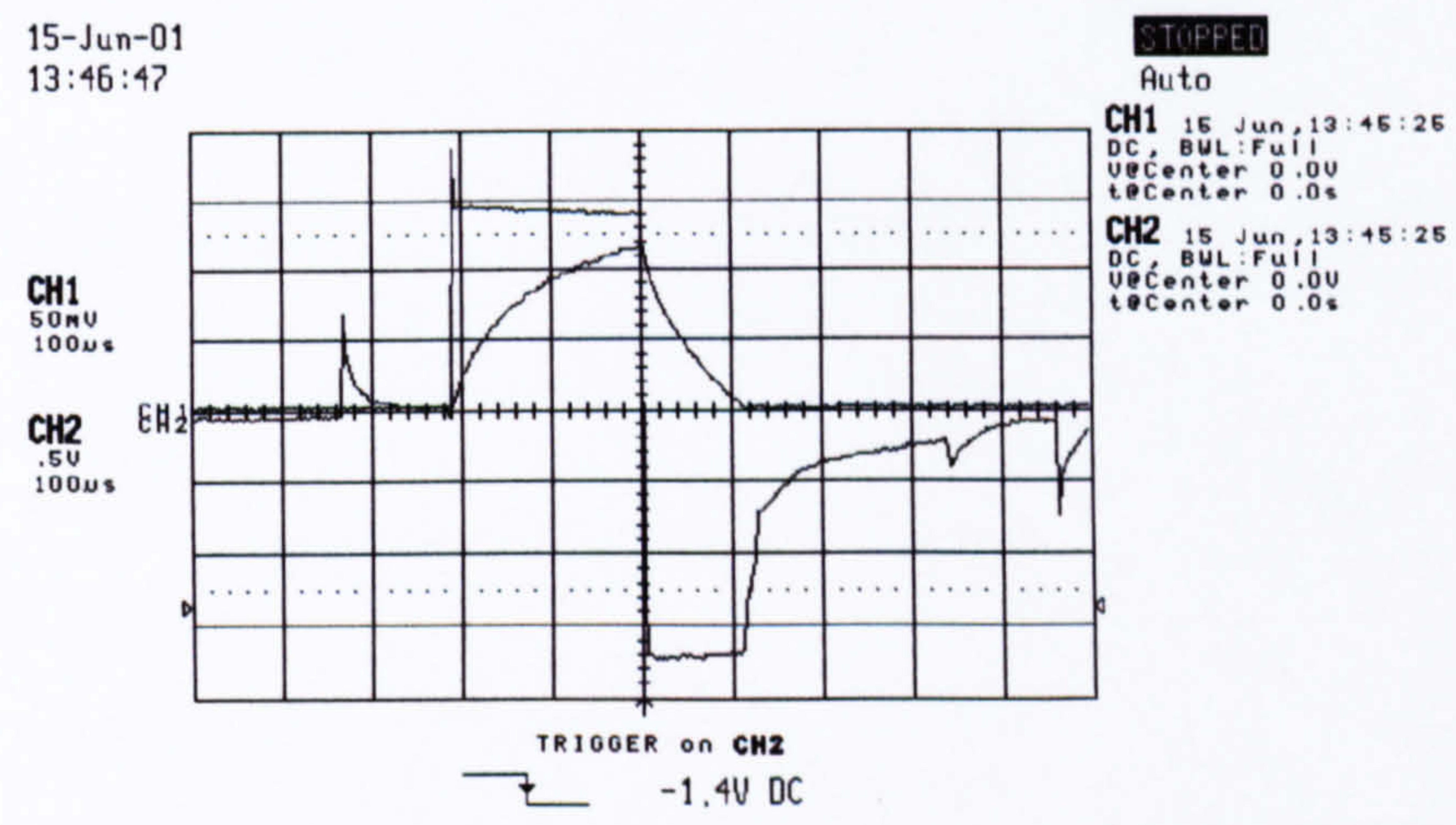

Figure 7.25b. Measured current and voltage waveforms for test case 2.

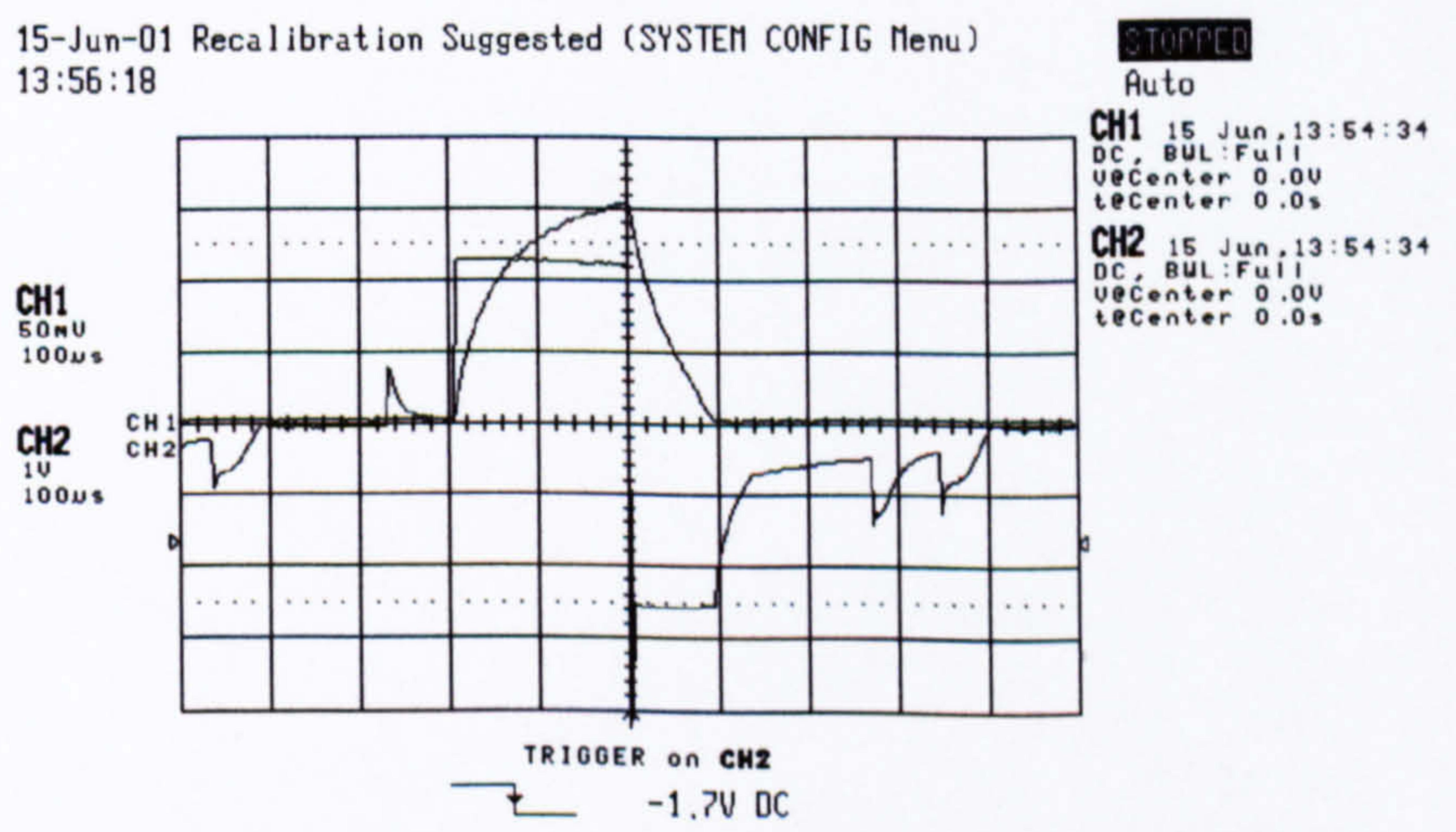

Figure 7.25c. Measured current and voltage waveforms for test case 3. 


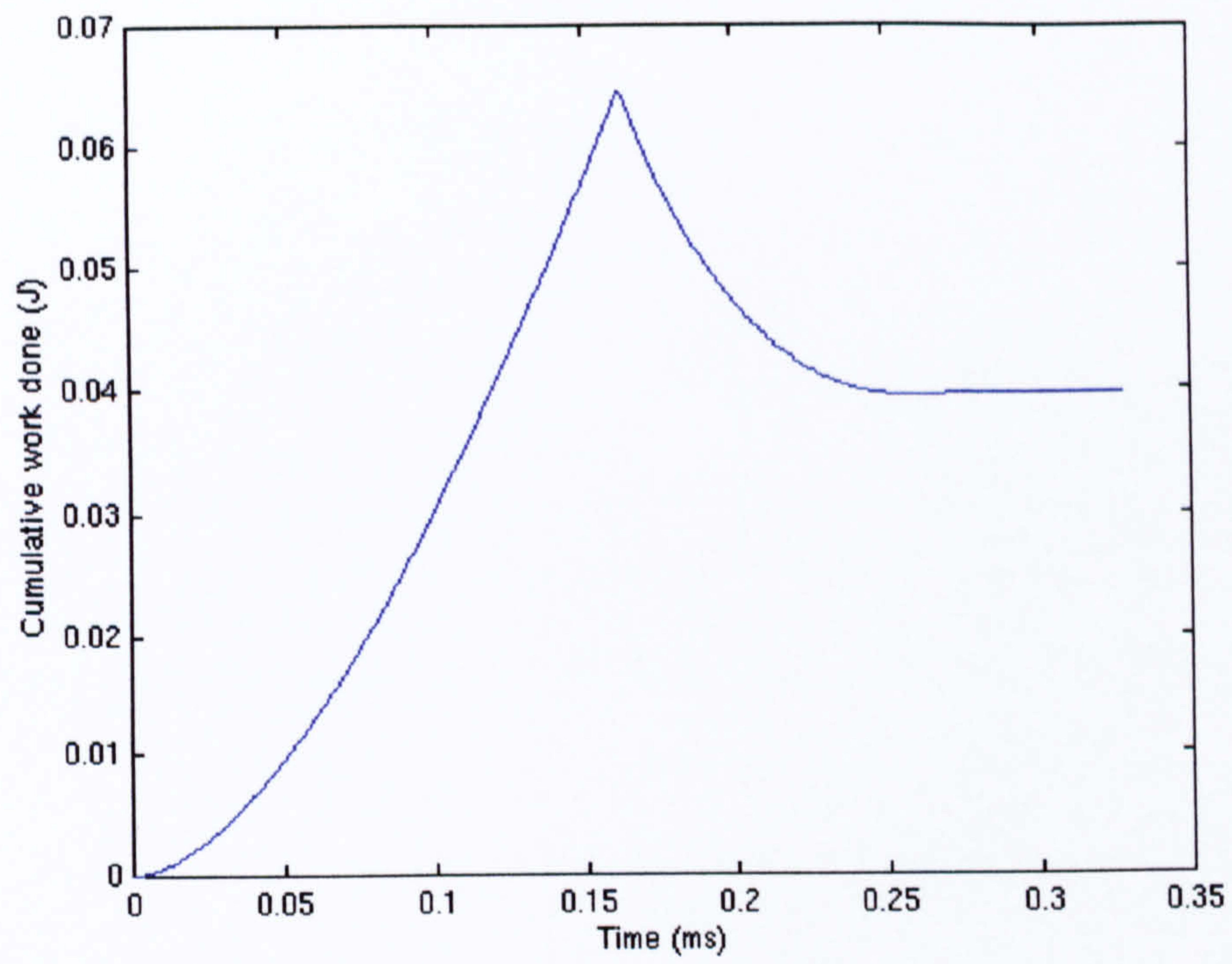

Figure 7.26. Cumulative work done per stroke calculated using measured data from test case 1 .

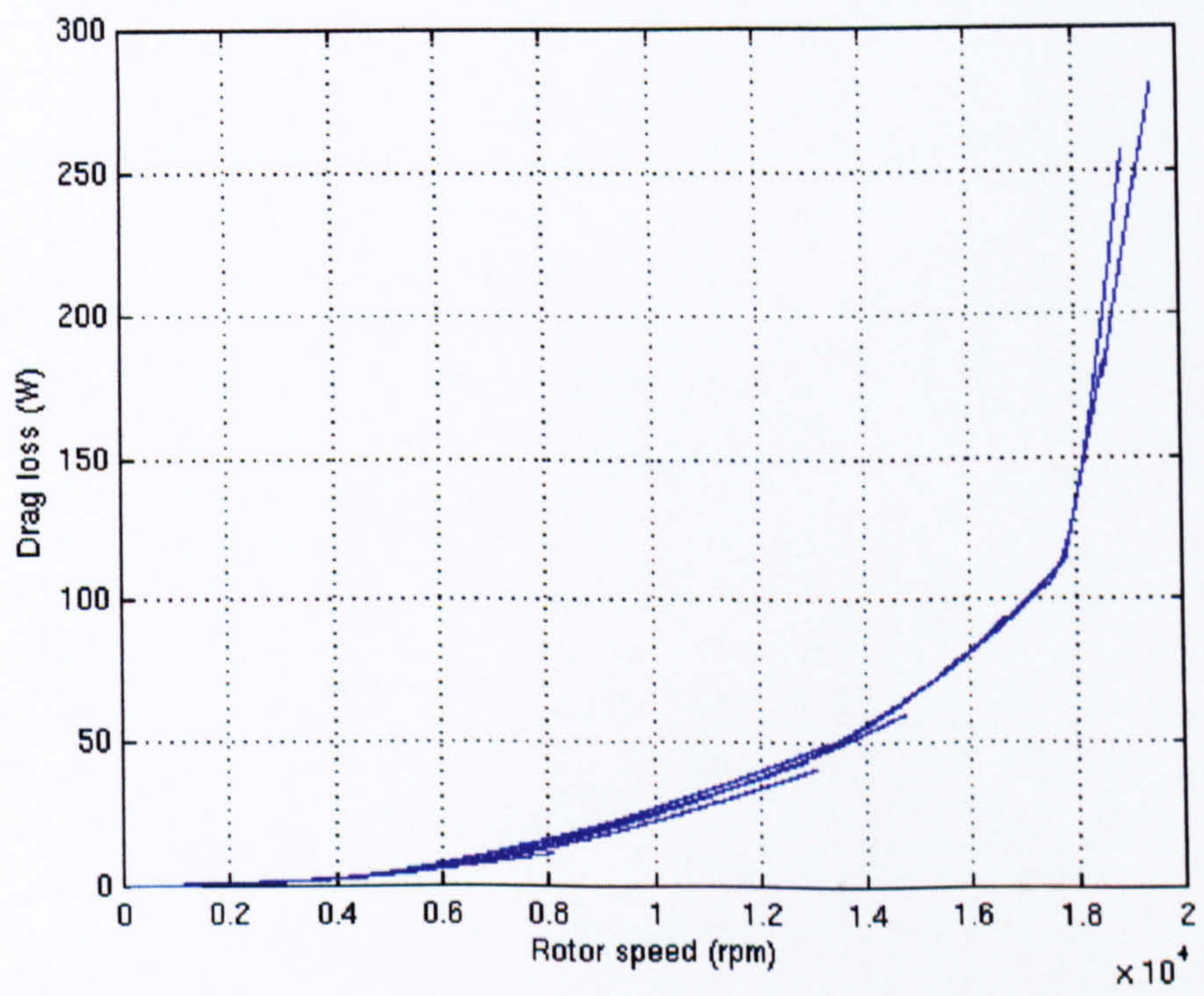

Figure 7.27. Measured variation of rotor drag loss as a function of speed. 


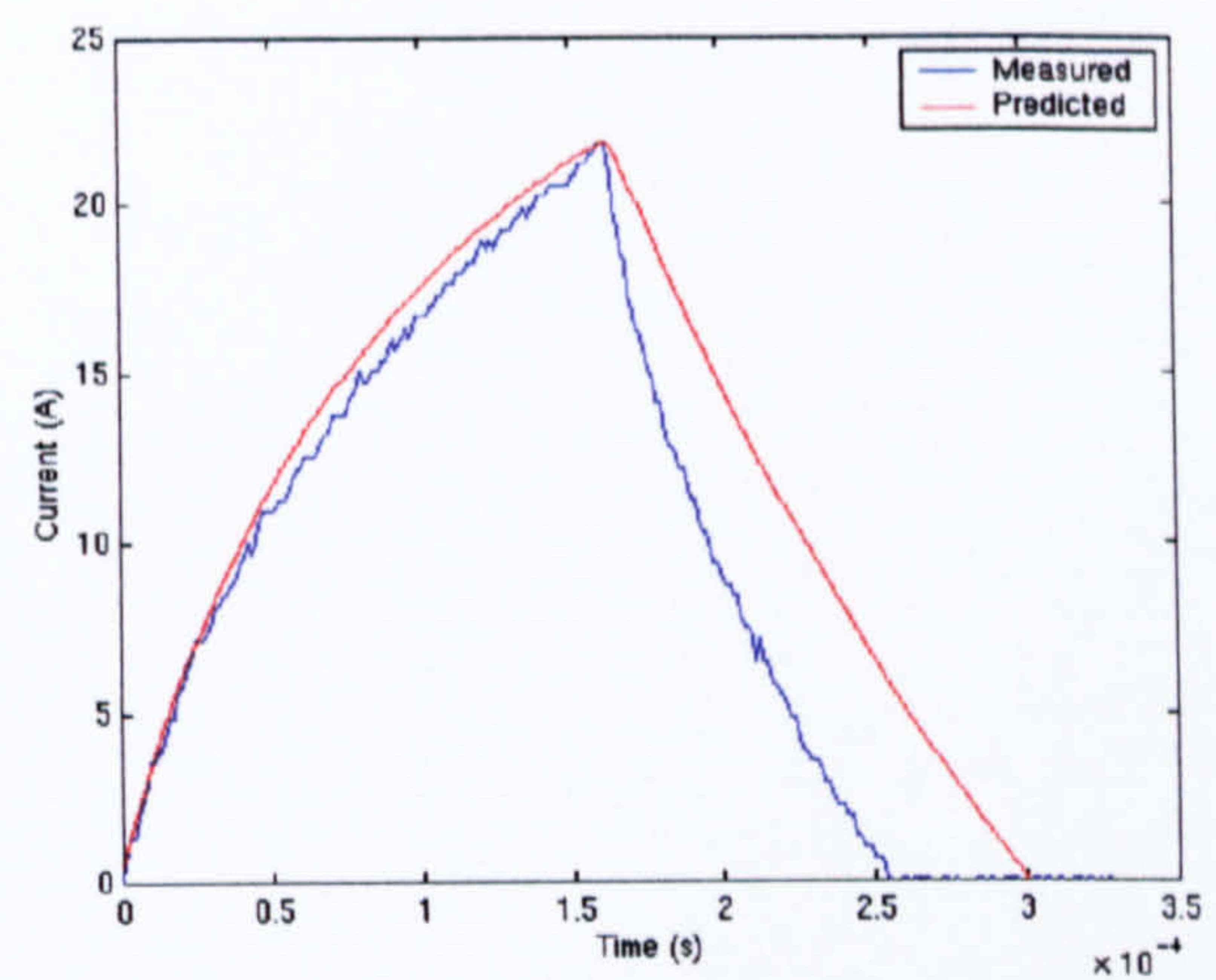

Figure 7.28a. Measured and predicted current waveforms for test case 1

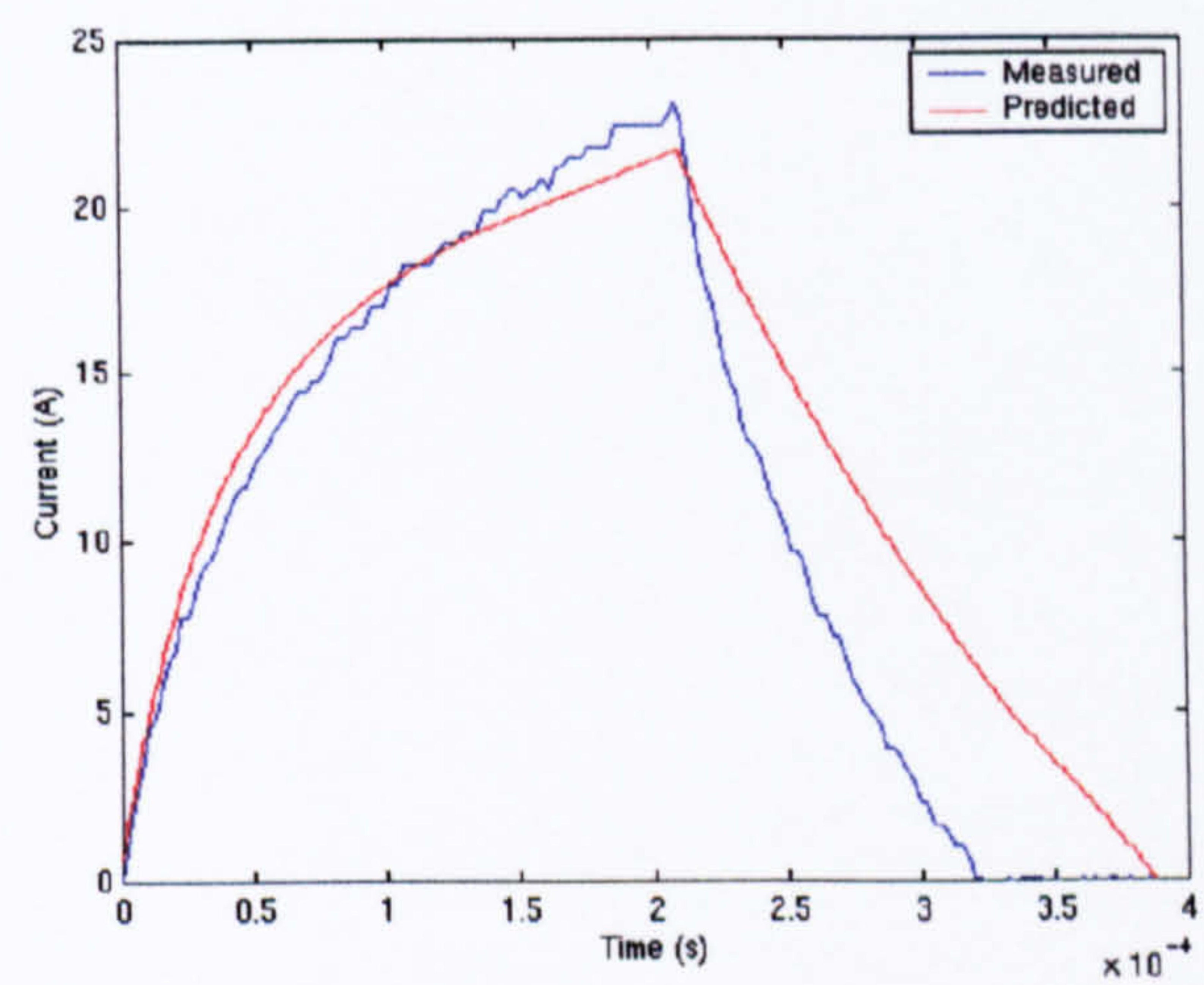

Figure 7.28b. Measured and predicted current waveforms for test case 2

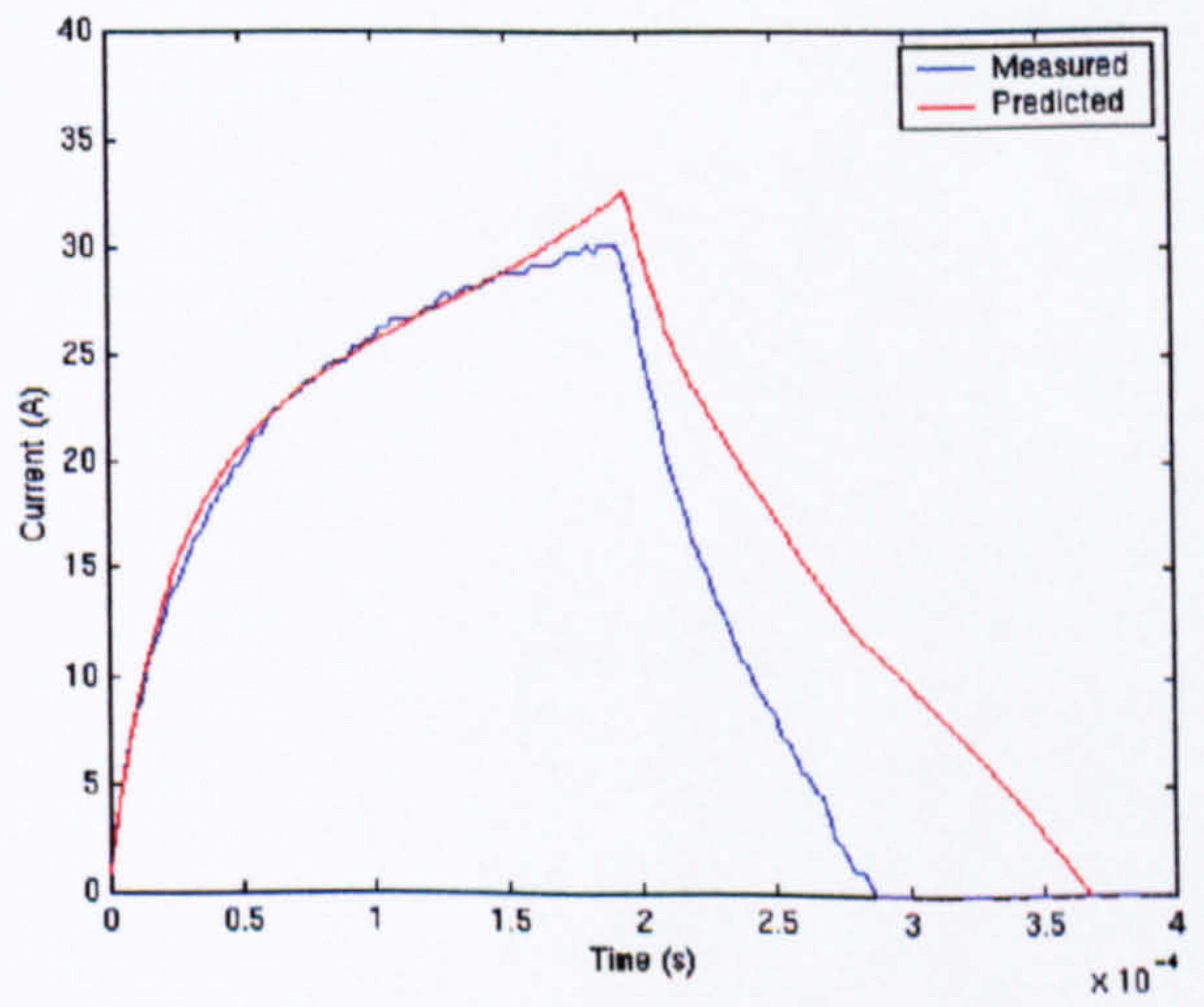

Figure 7.28c. Measured and predicted current waveforms for test case 3 


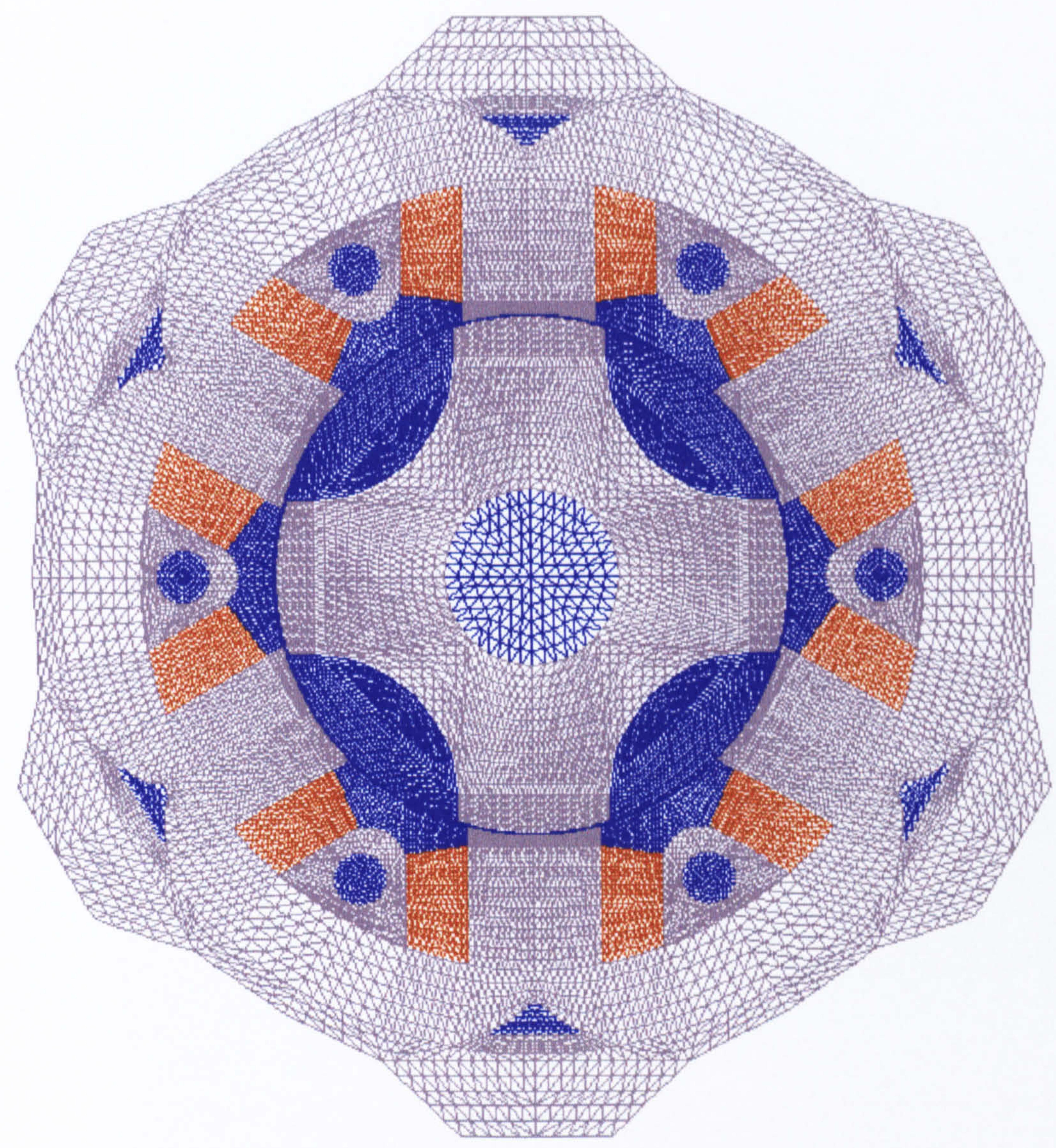

Figure 7.29 Mesh of the prototype machine (without the fins) consisting of 15563 nodes and 30920 first order elements. 


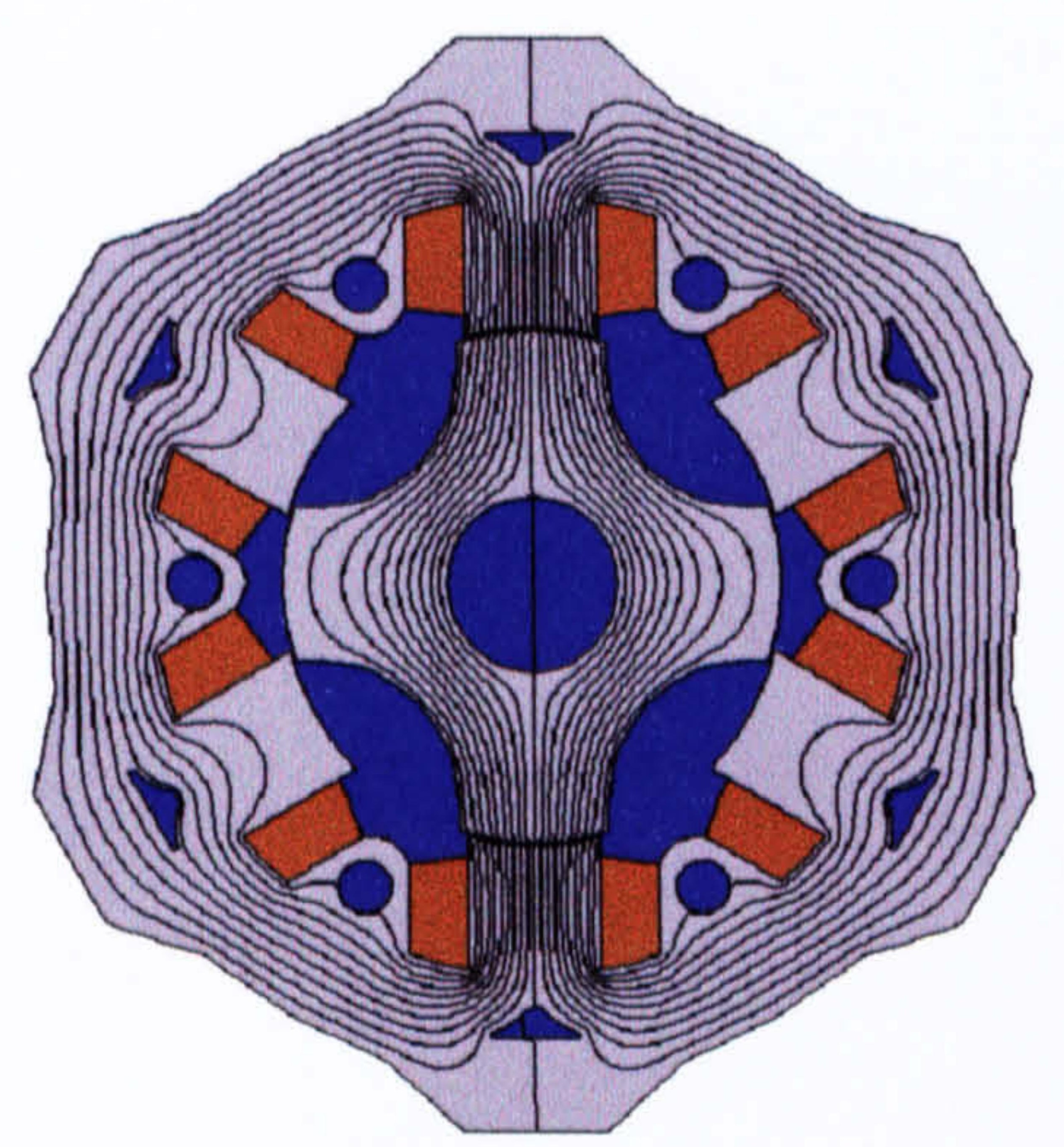

Aligned

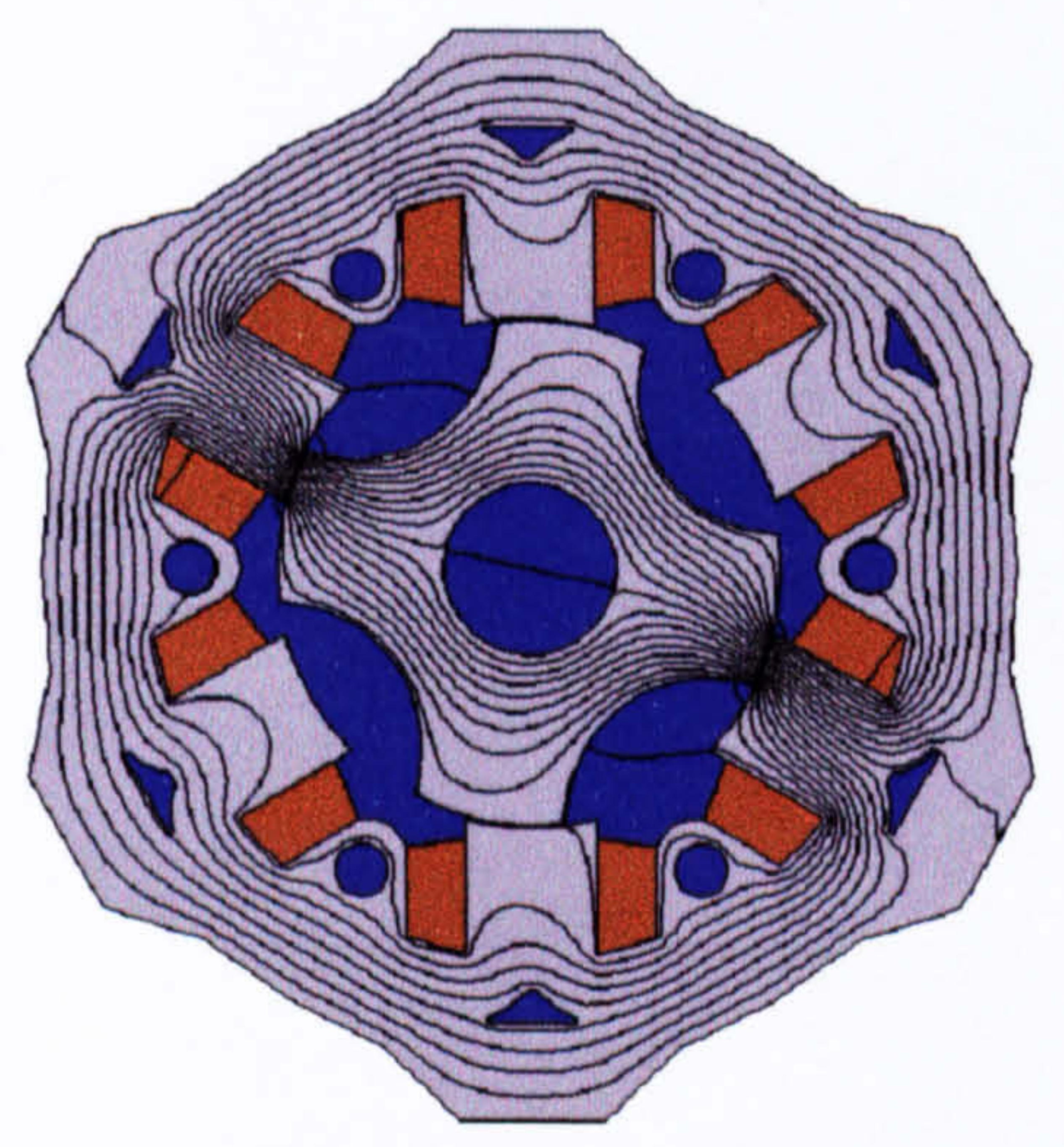

$10^{\circ}$ from alignment

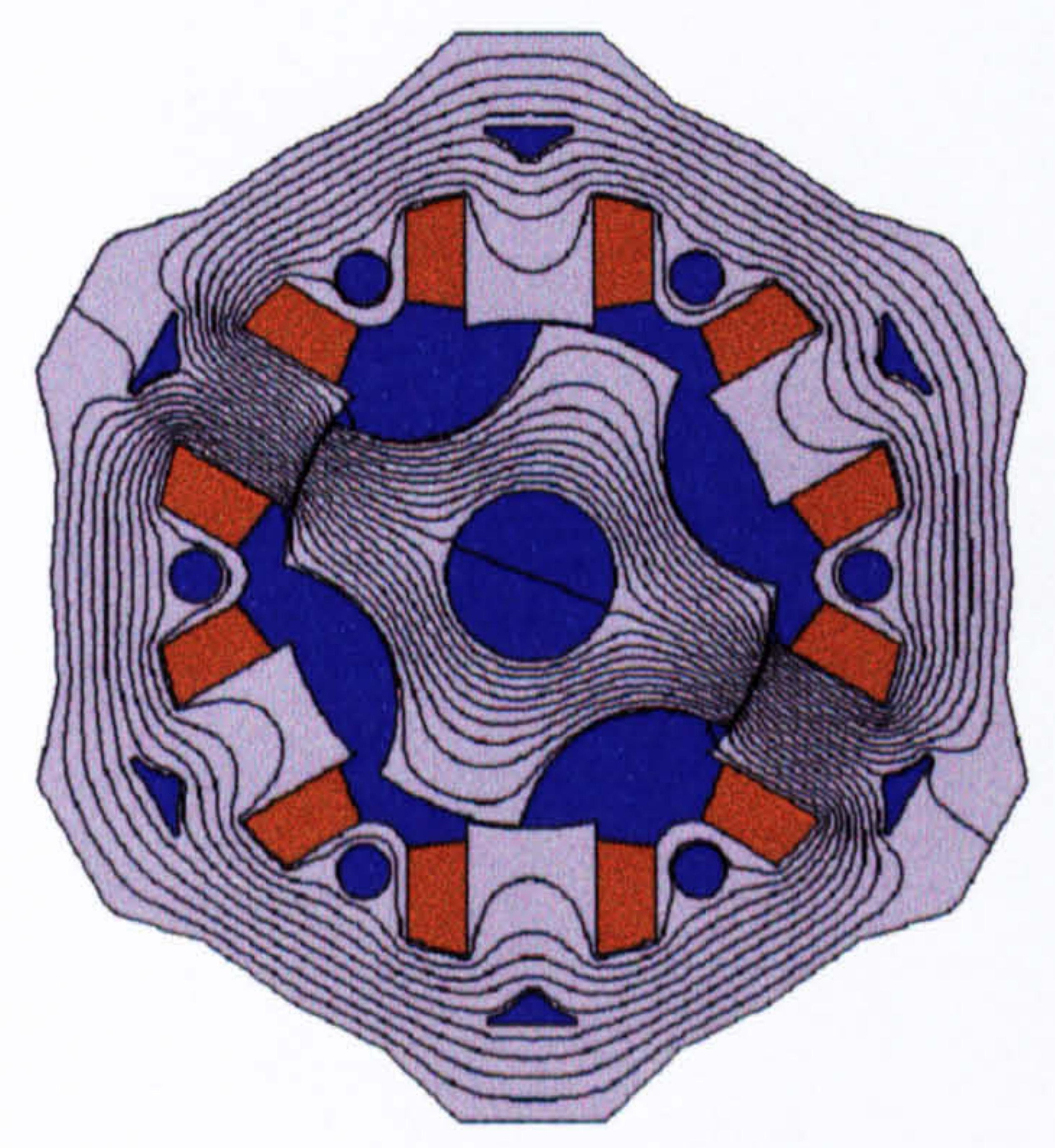

$20^{\circ}$ from alignment

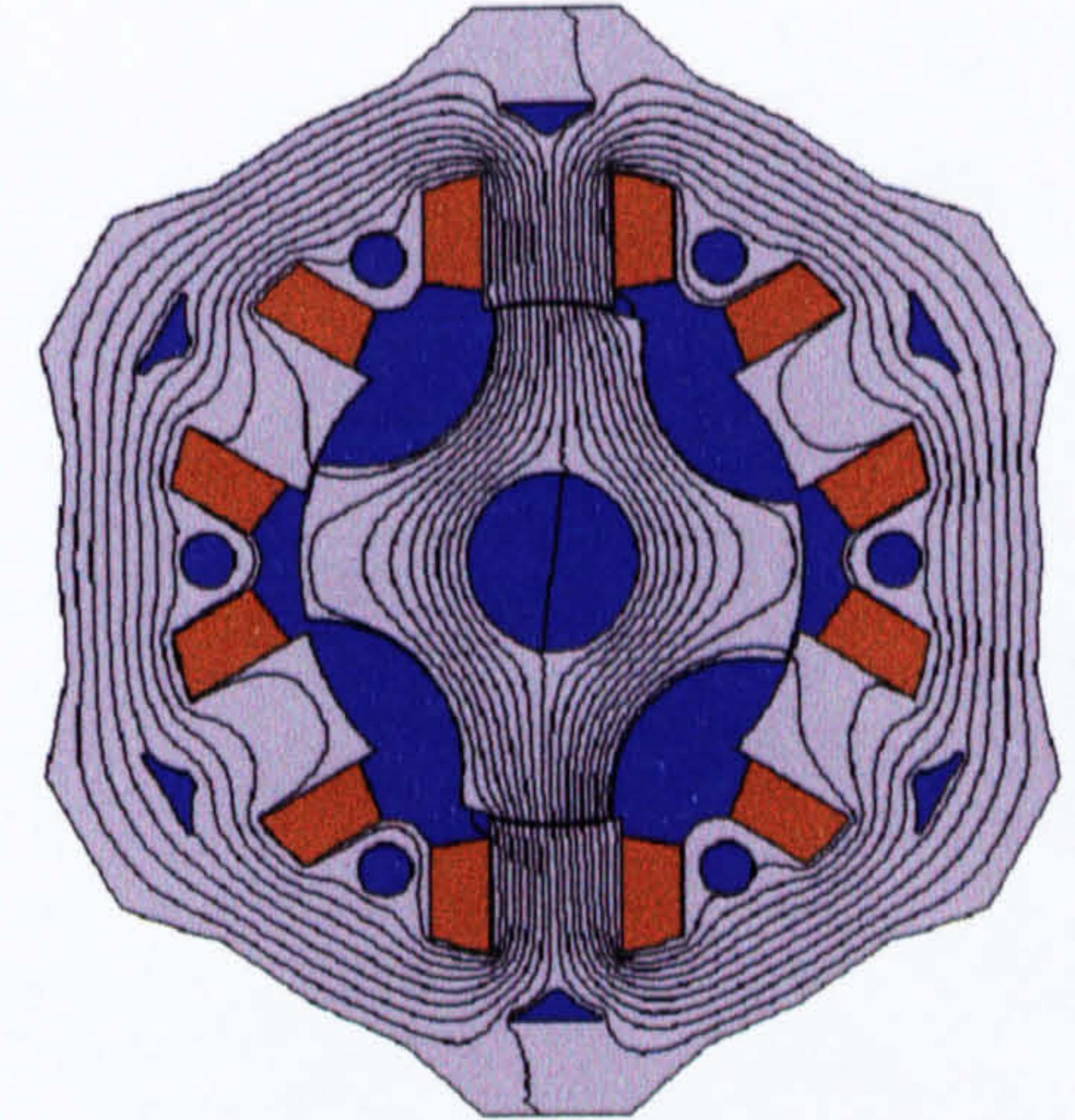

$5^{\circ}$ from alignment

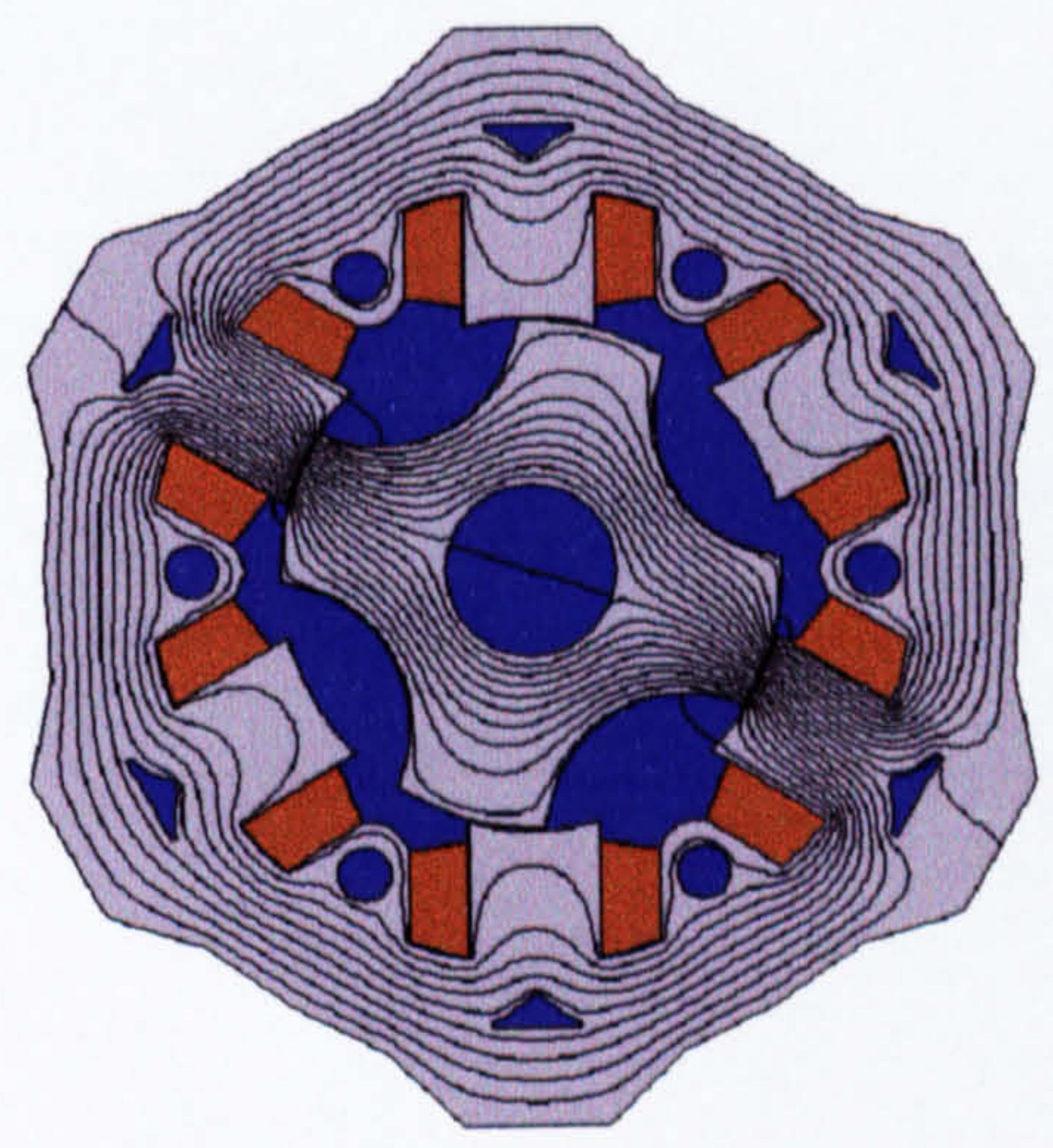

$15^{\circ}$ from alignment

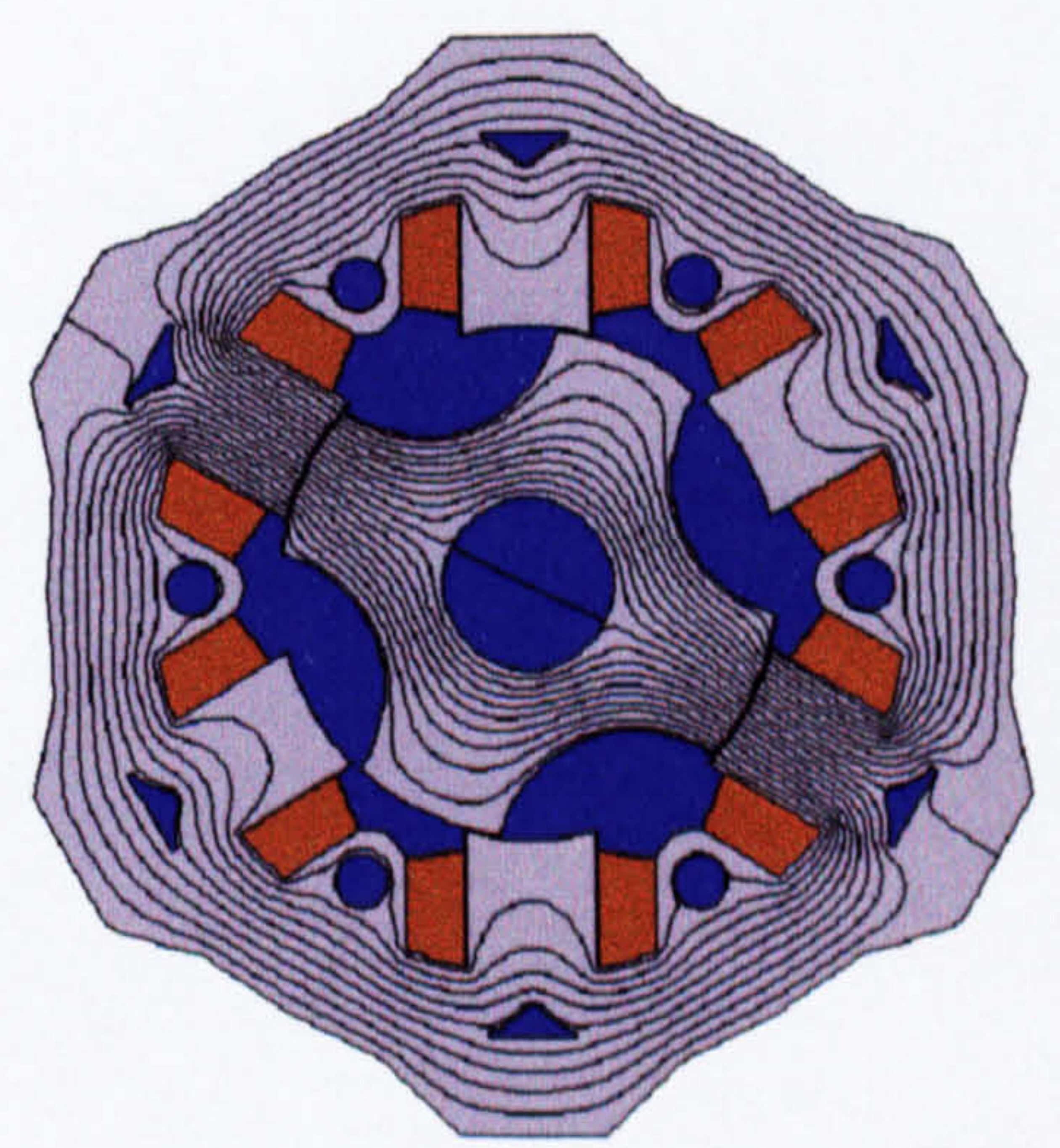

$25^{\circ}$ from alignment

Figure 7.30. Sample field distributions for test case 2 


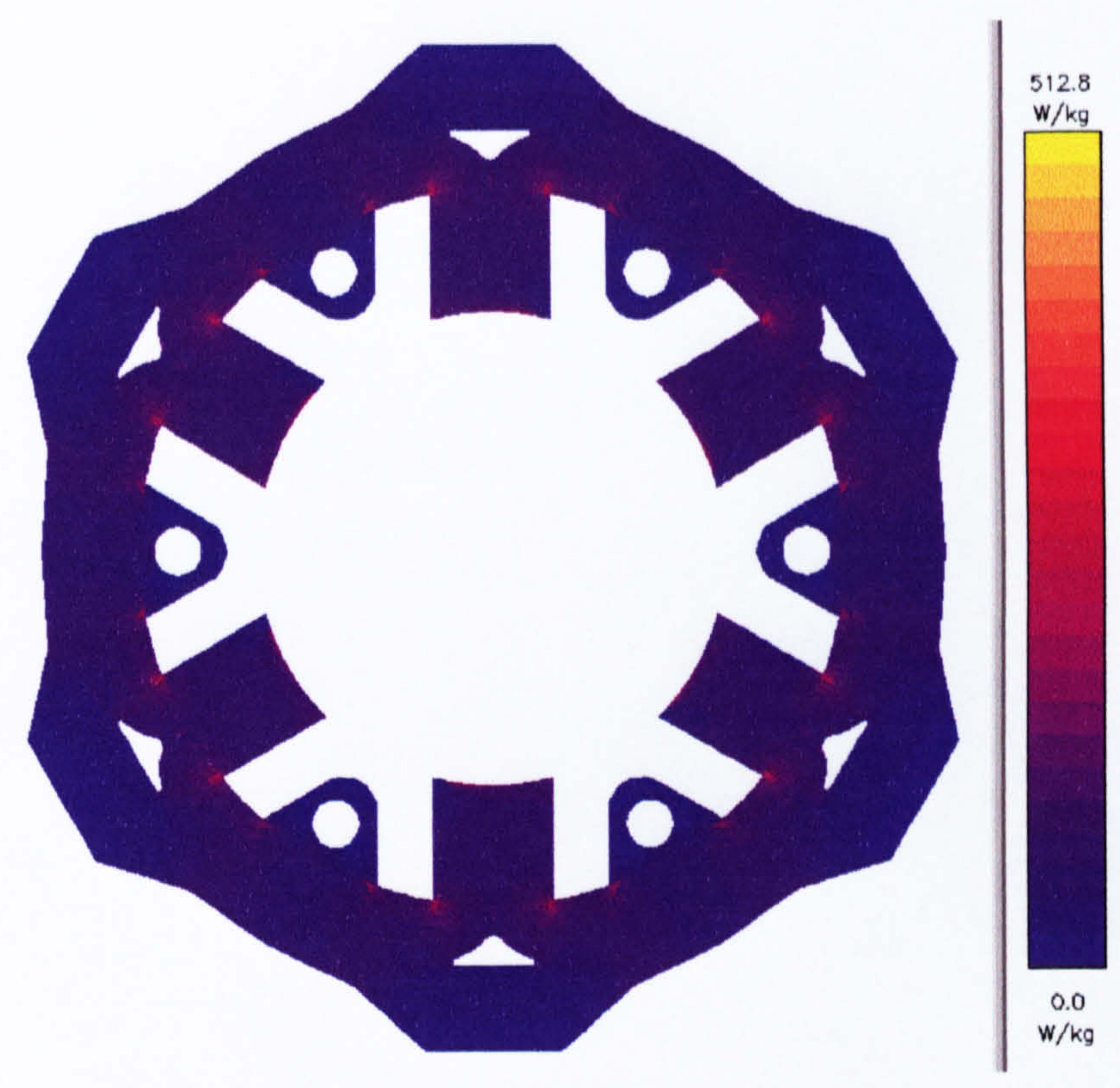

Figure 7.31a. Stator iron loss density contours for test case 2

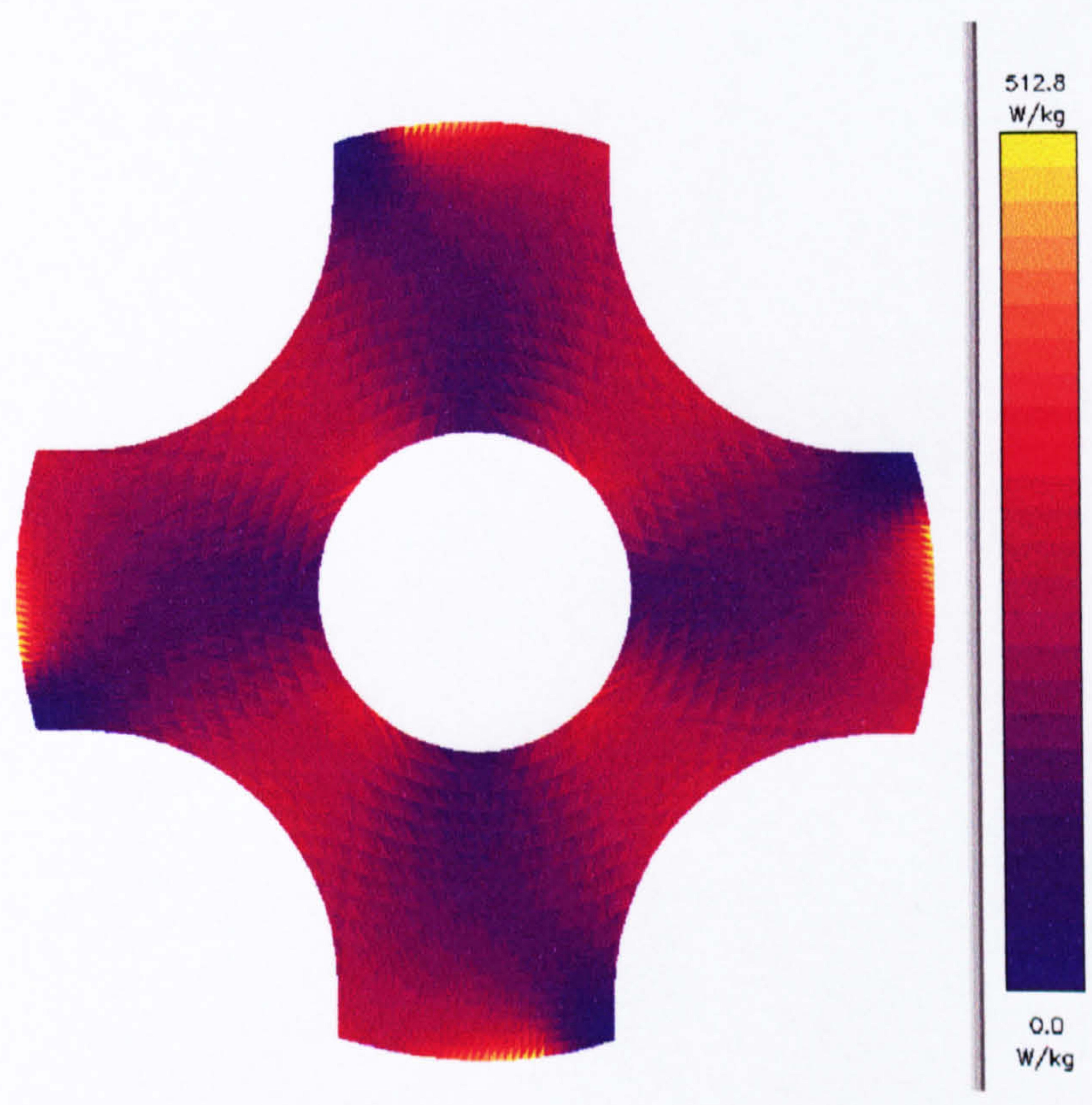

Figure 7.31b. Rotor iron loss contours for test case 2 


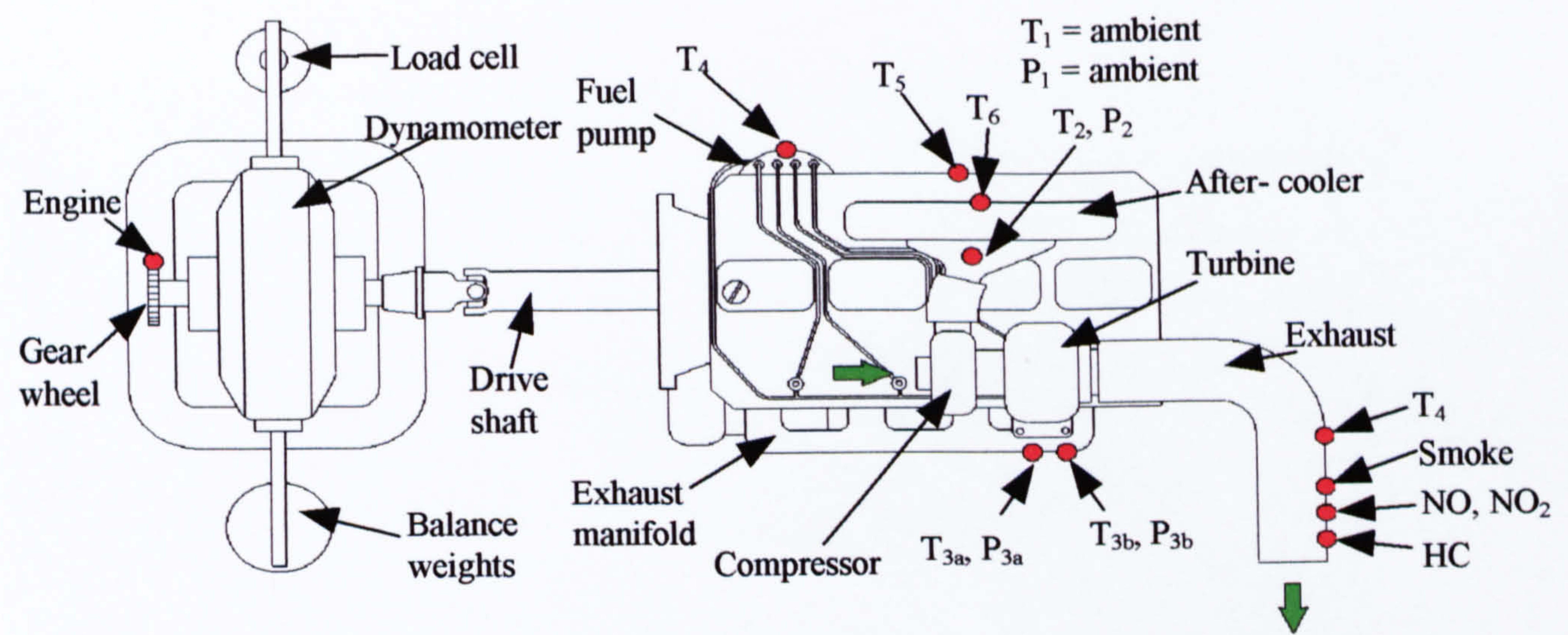

KEY

$\mathrm{P}_{1} \quad$ Ambient pressure

$\mathrm{P}_{2} \quad$ Compressor exit pressure

$\mathrm{P}_{3} \quad$ Turbine inlet pressure

$\mathrm{T}_{1} \quad$ Ambient temperature

$\mathrm{T}_{2} \quad$ Compressor exit temperature

$\mathrm{T}_{3} \quad$ Turbine inlet temperature

$\mathrm{T}_{4} \quad$ Fuel temperature

$\mathrm{T}_{5} \quad$ Oil temperature

$\mathrm{T}_{6} \quad$ Air temperature after after-cooler

Figure 7.32. Schematic of the engine test apparatus. 


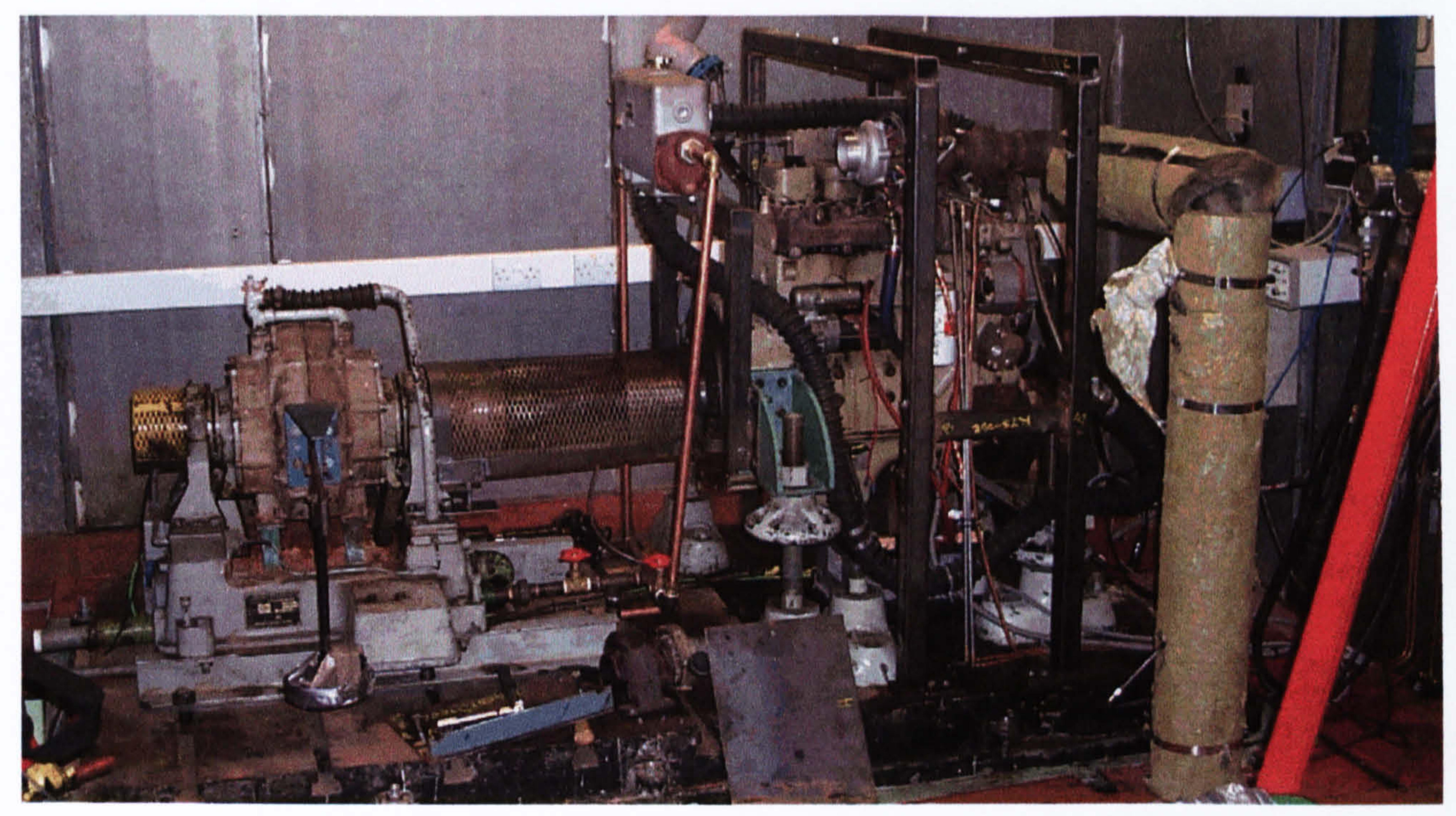

Figure 7.33. Photograph of the engine test facility 


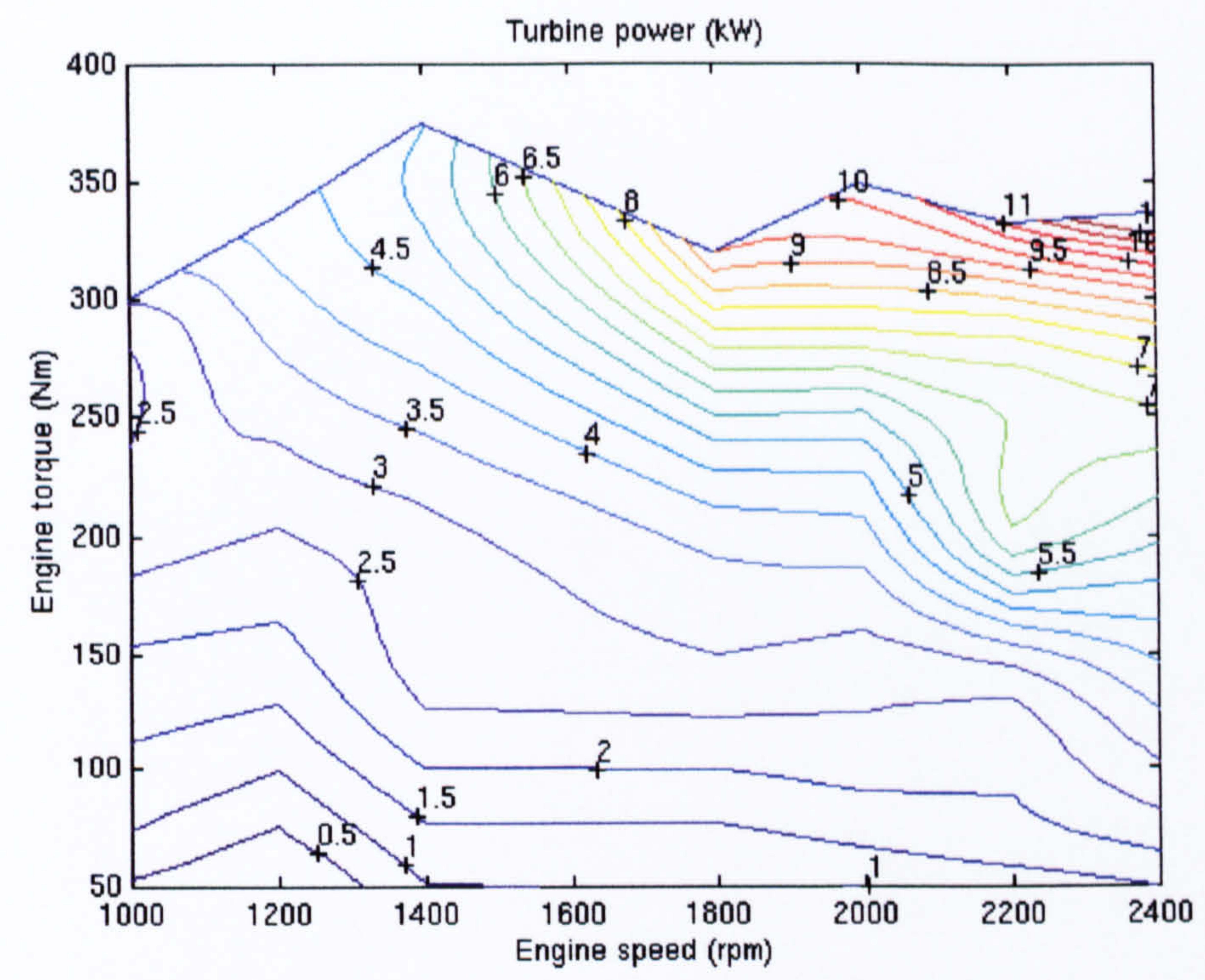

Figure 7.34a. Contours of turbine power for the engine fitted with the $16 \mathrm{~cm}^{2}$ turbine

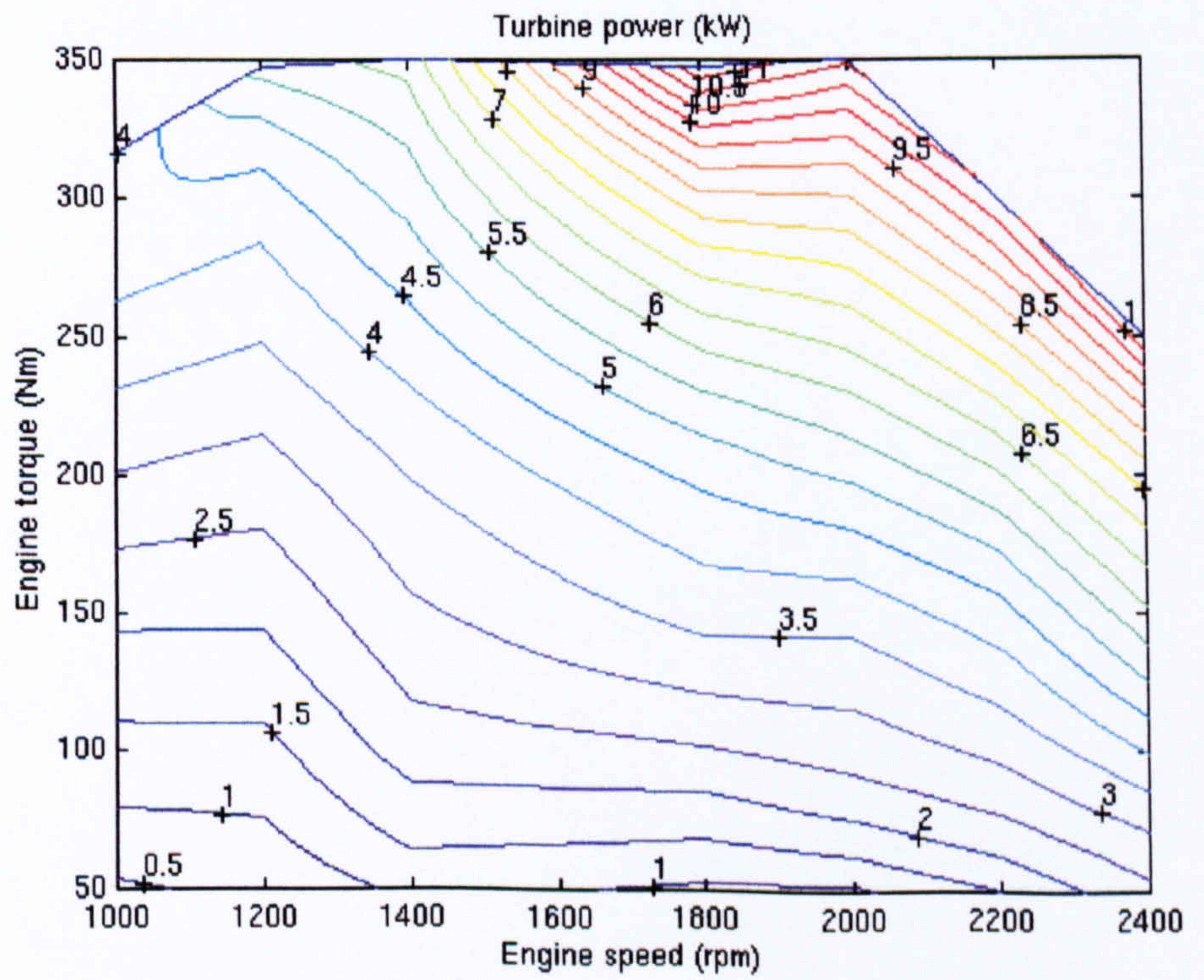

Figure 7.34b. Contours of turbine power for the engine fitted with the $12 \mathrm{~cm}^{2}$ turbine 


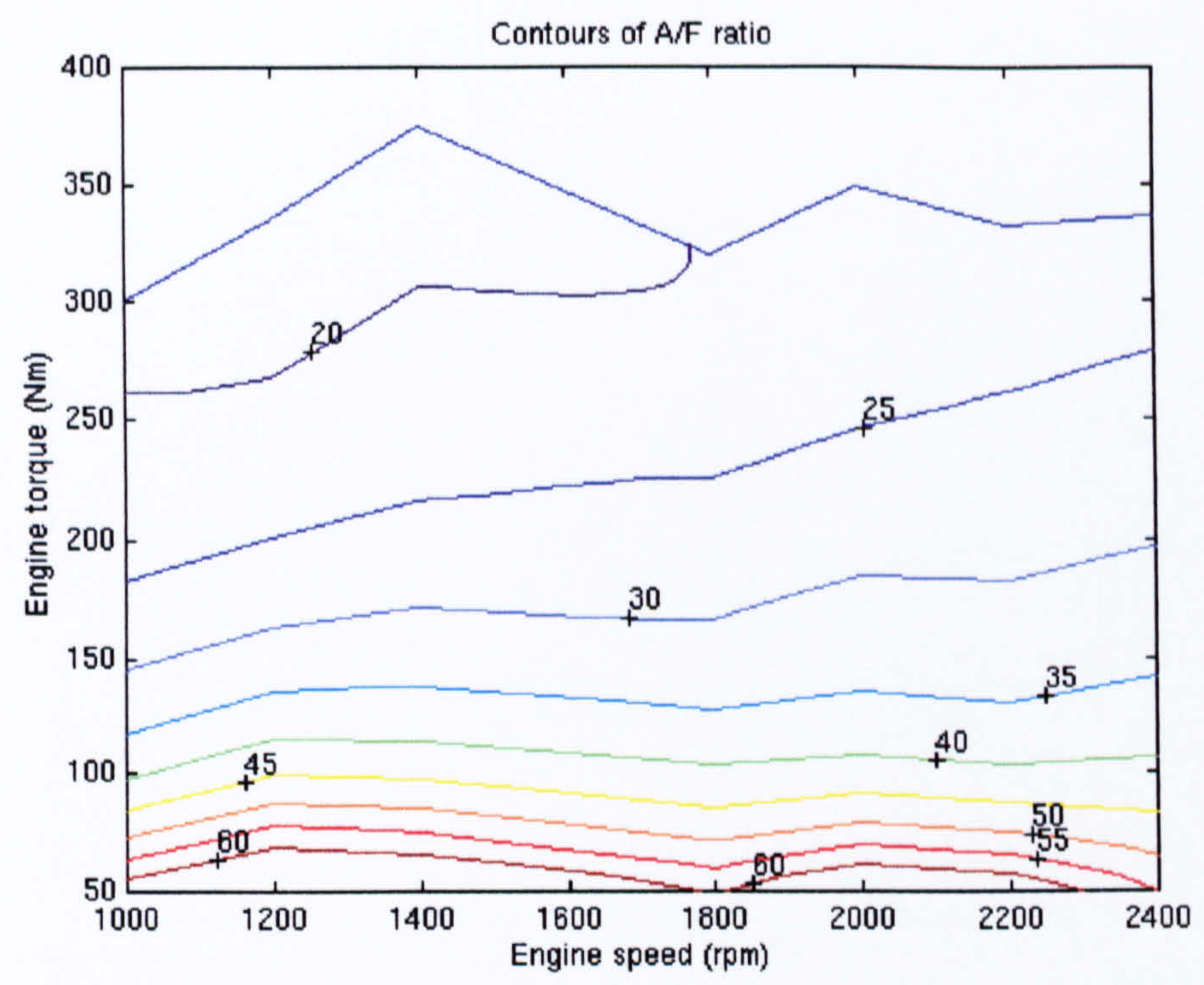

Figure 7.35a. Contours of air/fuel ratio for the engine fitted with the $16 \mathrm{~cm}^{2}$ turbine

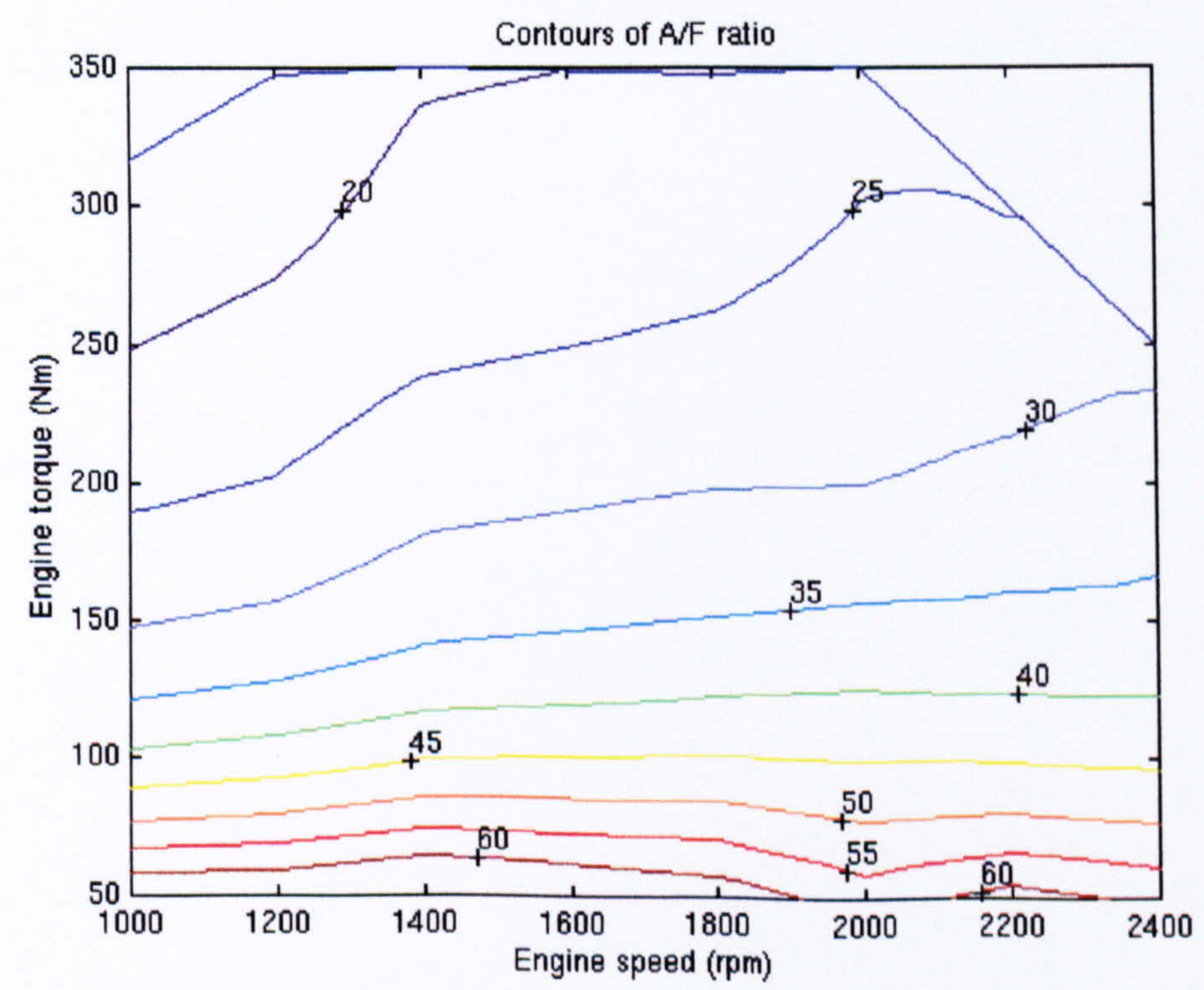

Figure $7.35 \mathrm{~b}$. Contours of air/fuel ratio for the engine fitted with the $12 \mathrm{~cm}^{2}$ turbine 


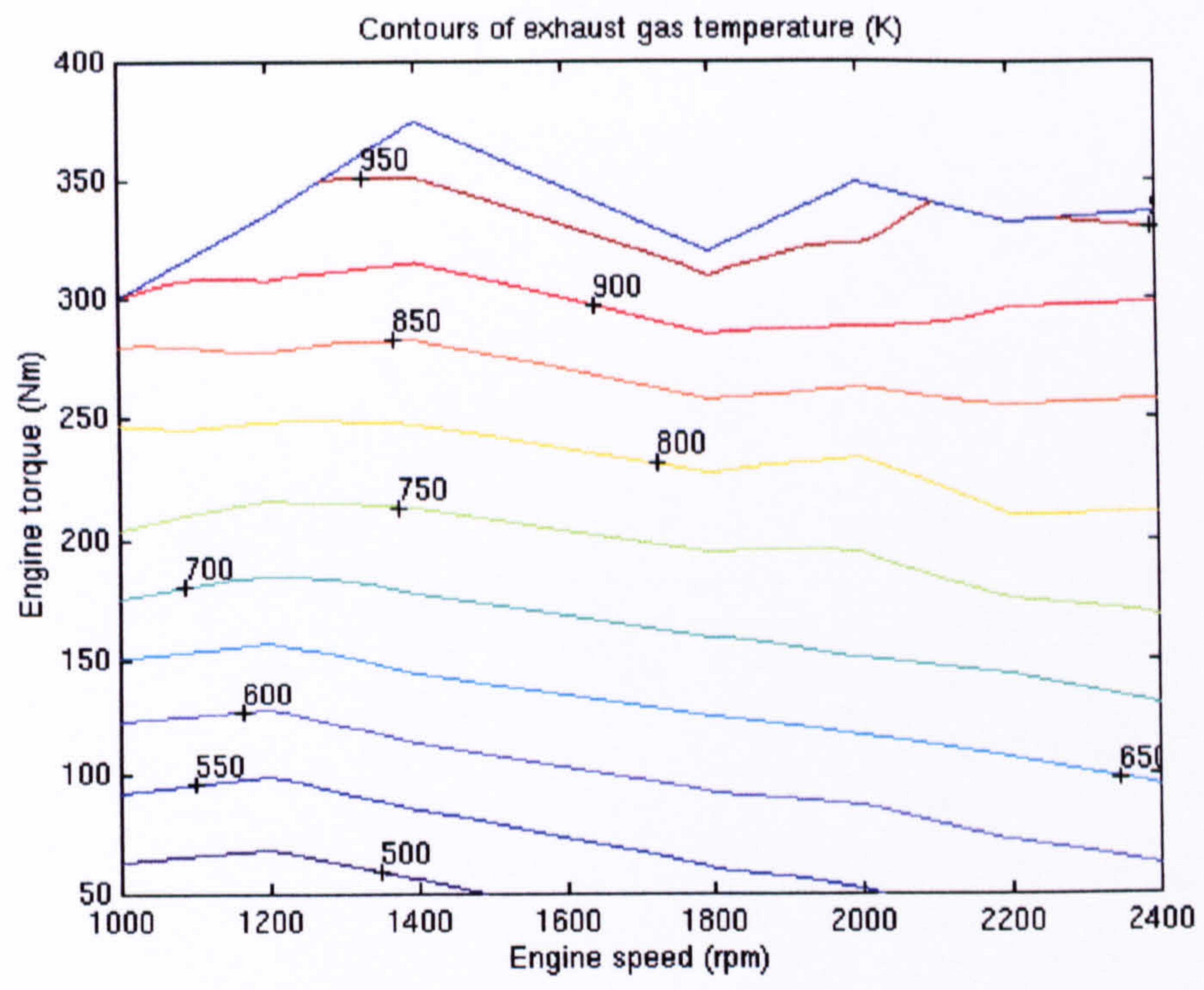

Figure 7.36a. Contours of exhaust gas temperature for the engine fitted with the $16 \mathrm{~cm}^{2}$ turbine

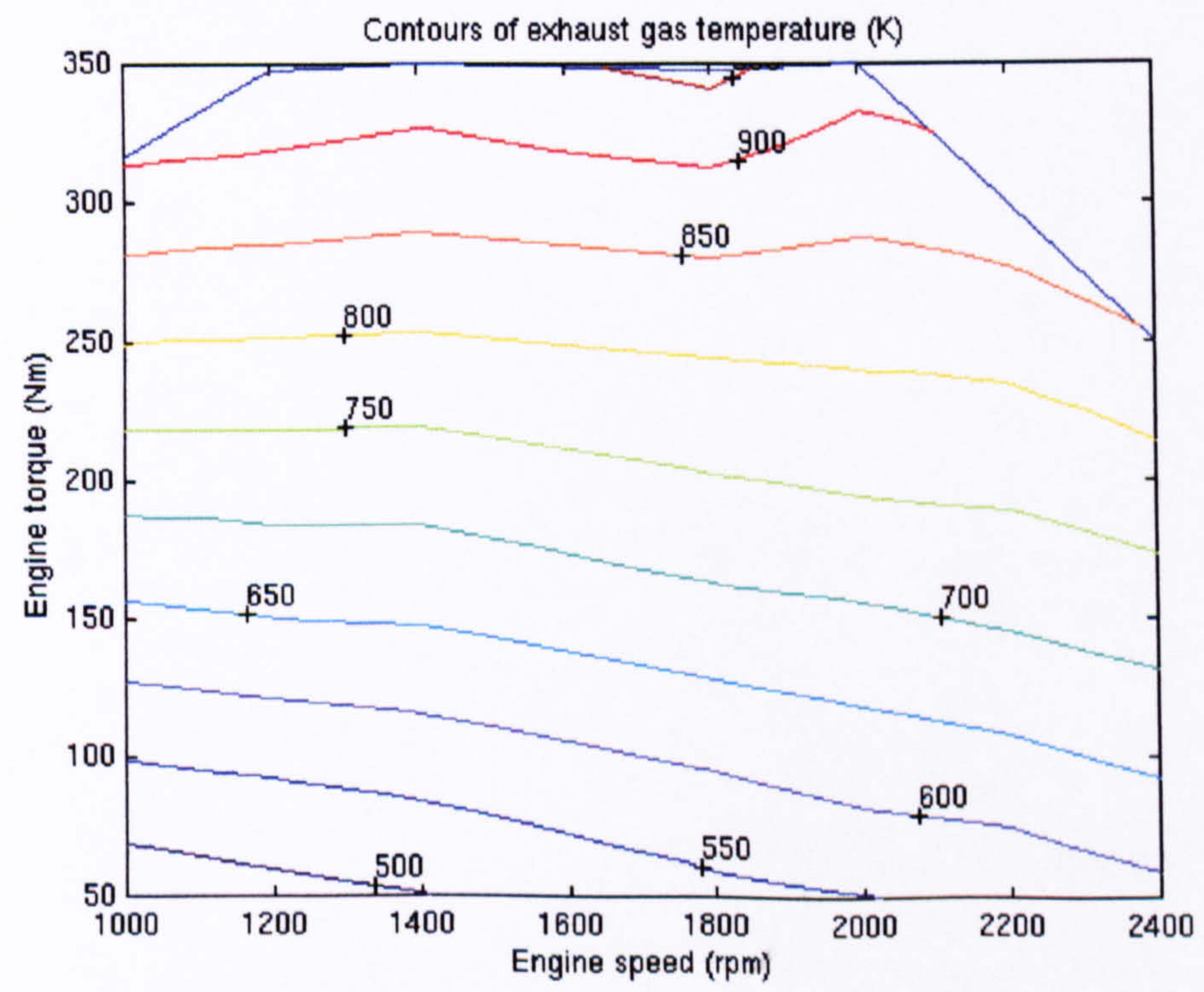

Figure 7.36b. Contours of exhaust gas temperature for the engine fitted with the $12 \mathrm{~cm}^{2}$ turbine 


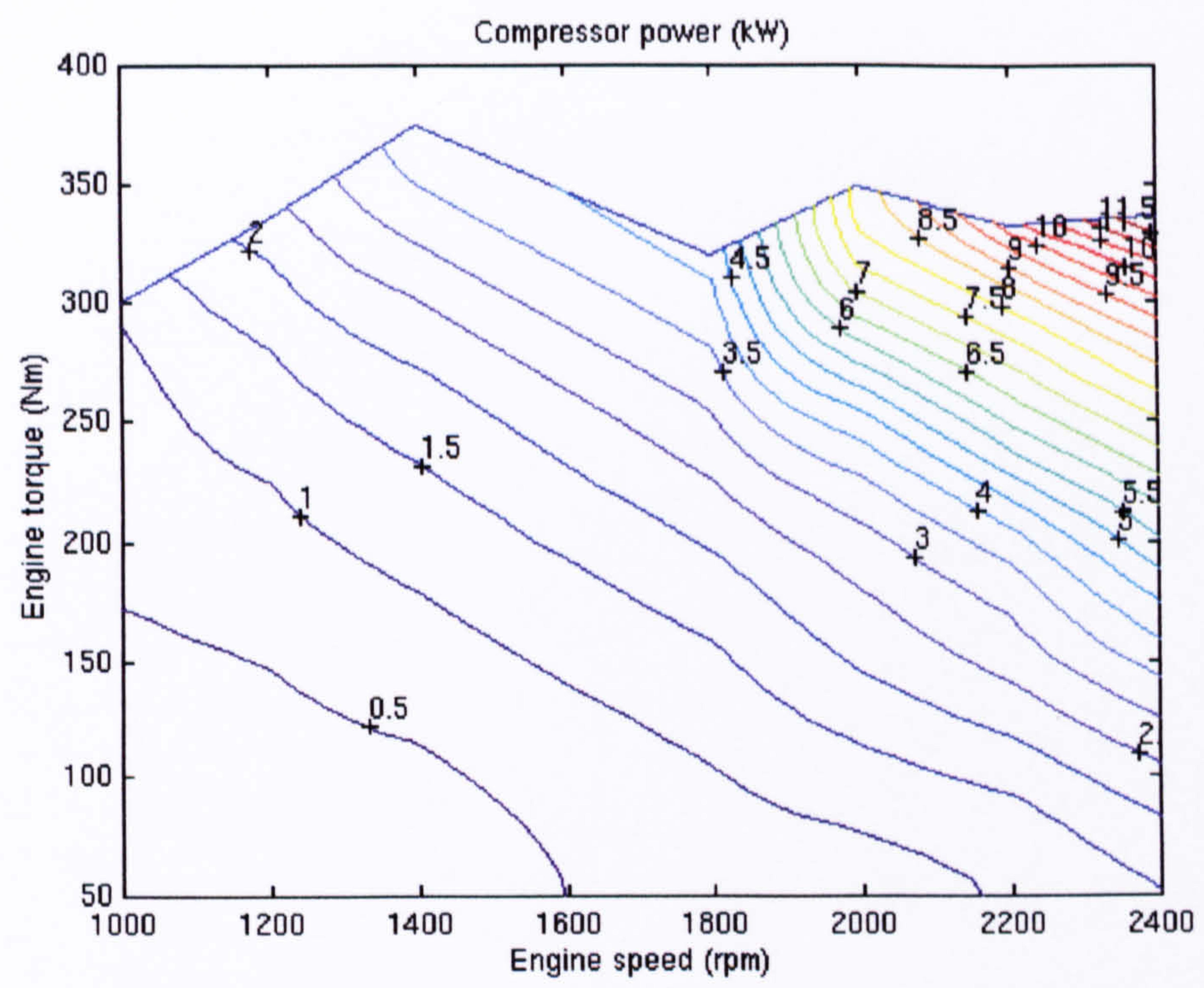

Figure 7.37a. Contours of compressor power for the engine fitted with the $16 \mathrm{~cm}^{2}$ turbine

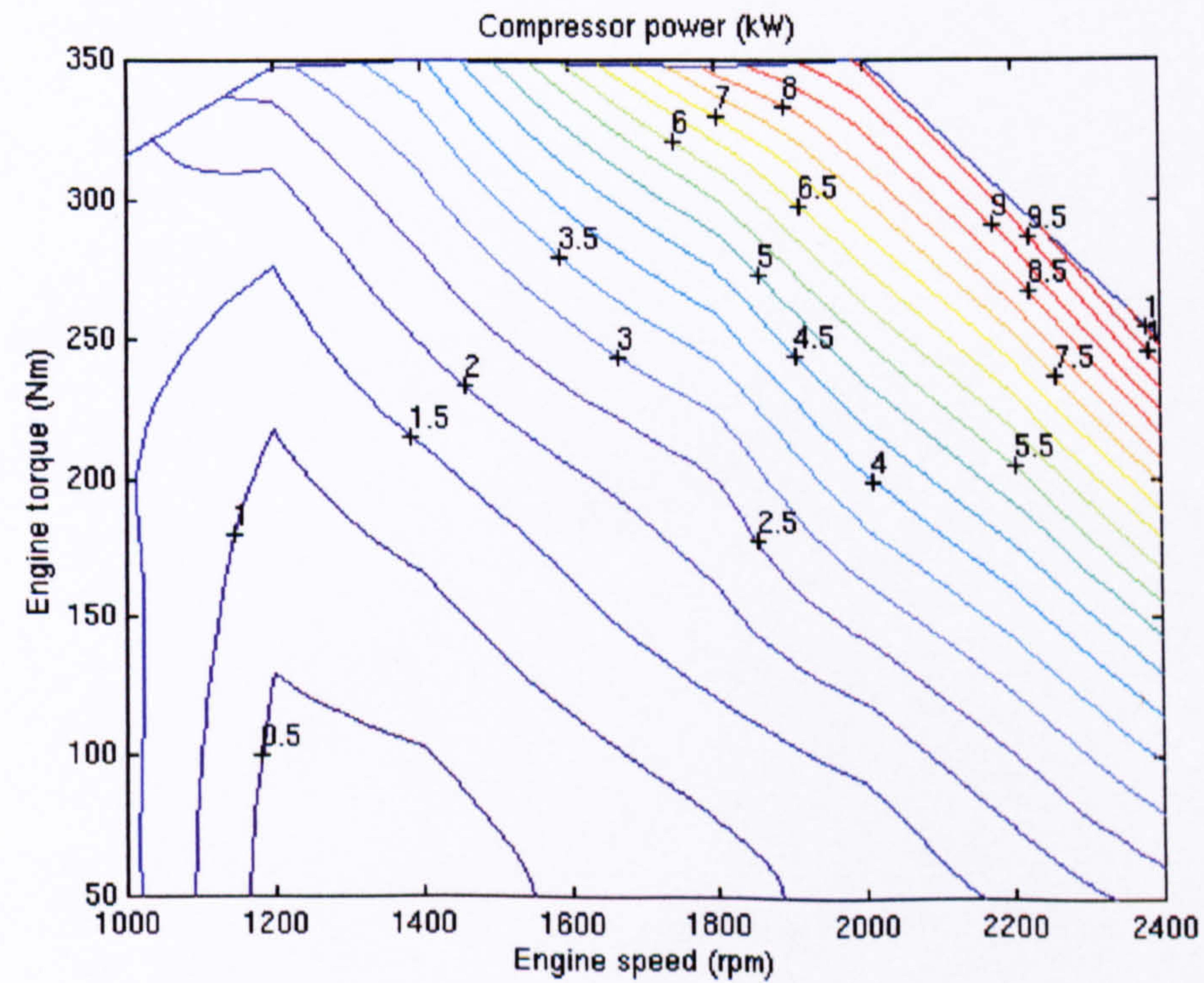

Figure 7.37b. Contours of compressor power for the engine fitted with the $12 \mathrm{~cm}^{2}$ turbine 


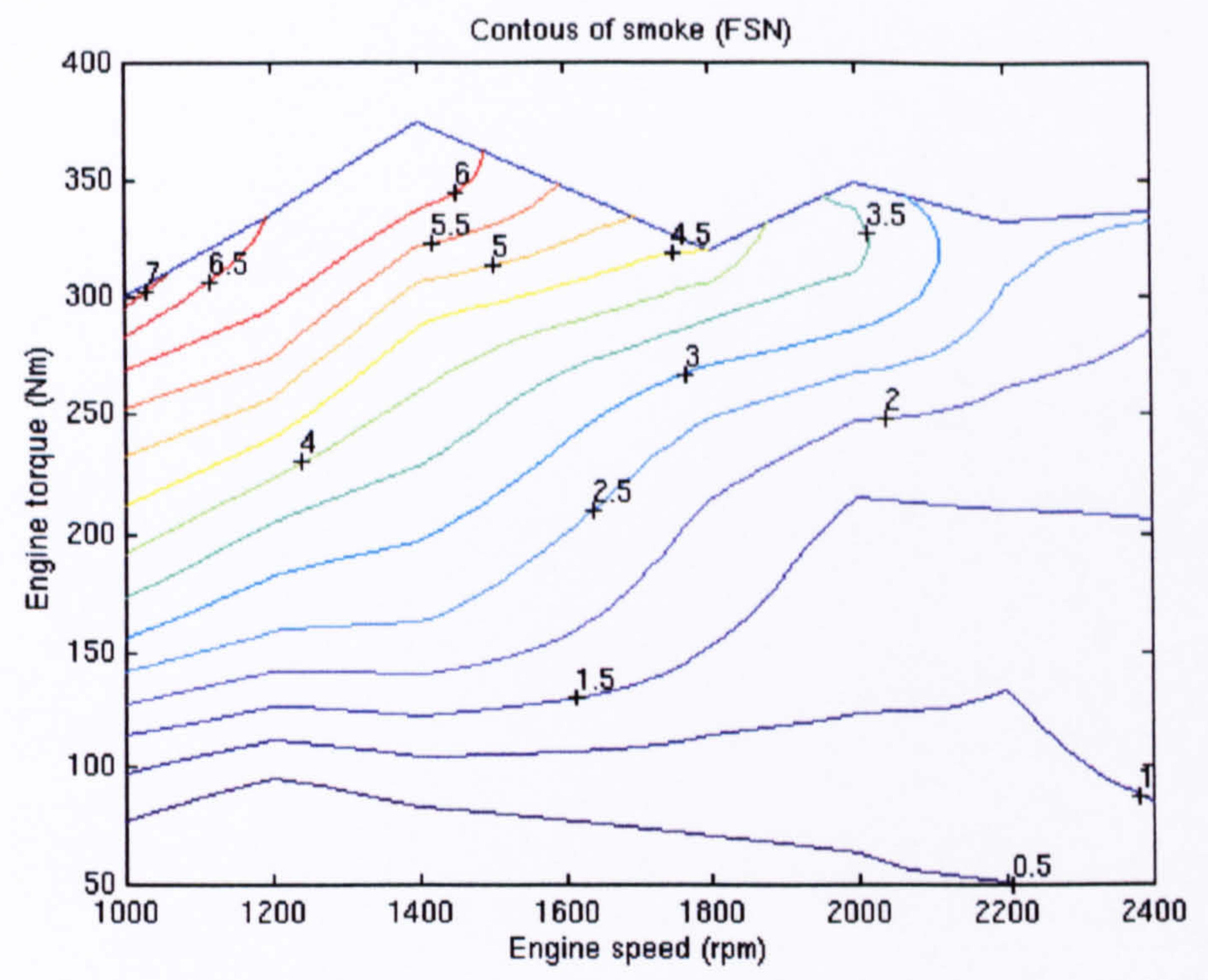

Figure 7.38a. Contours of smoke for the engine fitted with the $16 \mathrm{~cm}^{2}$ turbine

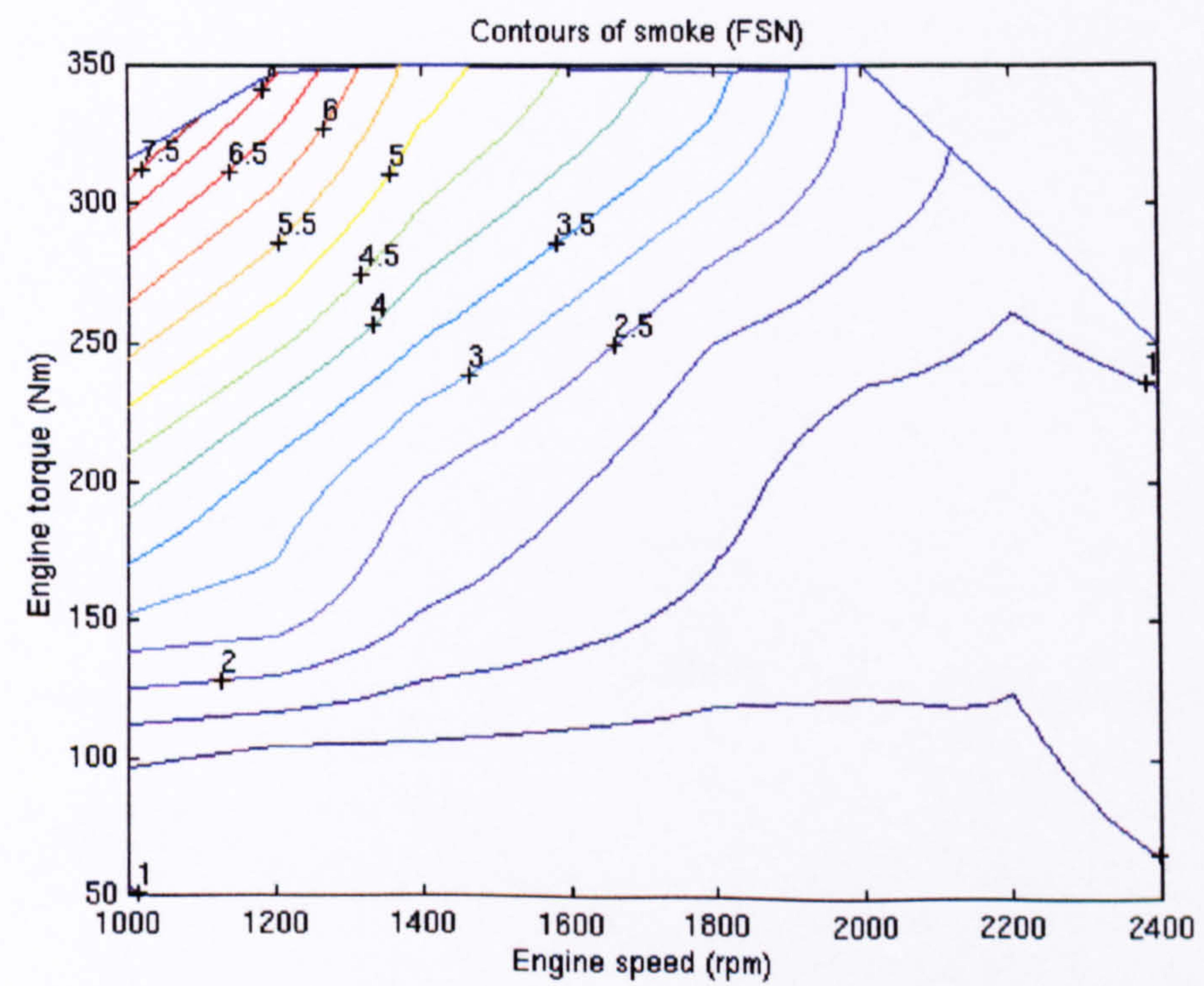

Figure 7.38b. Contours of smoke for the engine fitted with the $12 \mathrm{~cm}^{2}$ turbine 


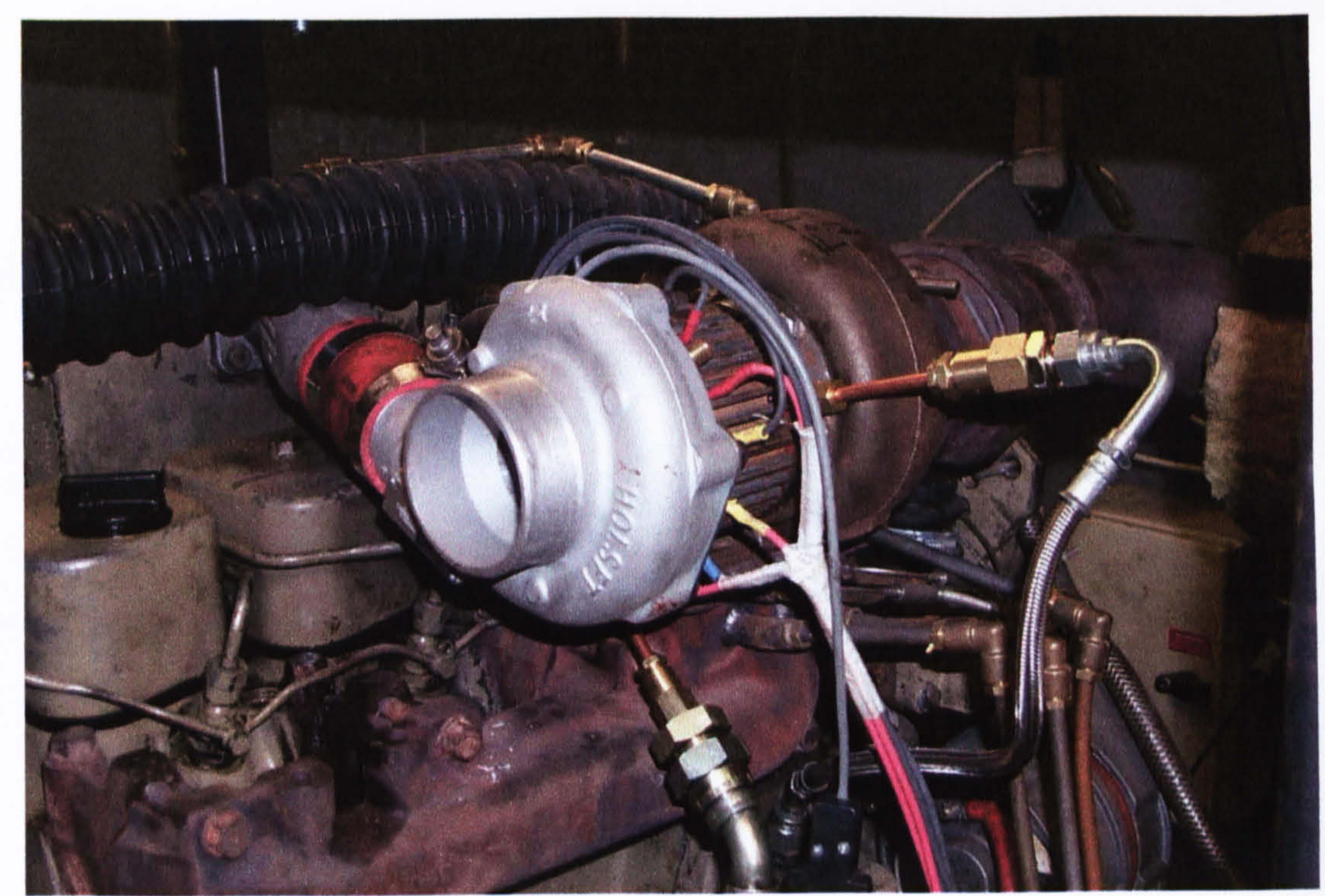

Figure 7.39. Photograph of the prototype EATG fitted to the engine.

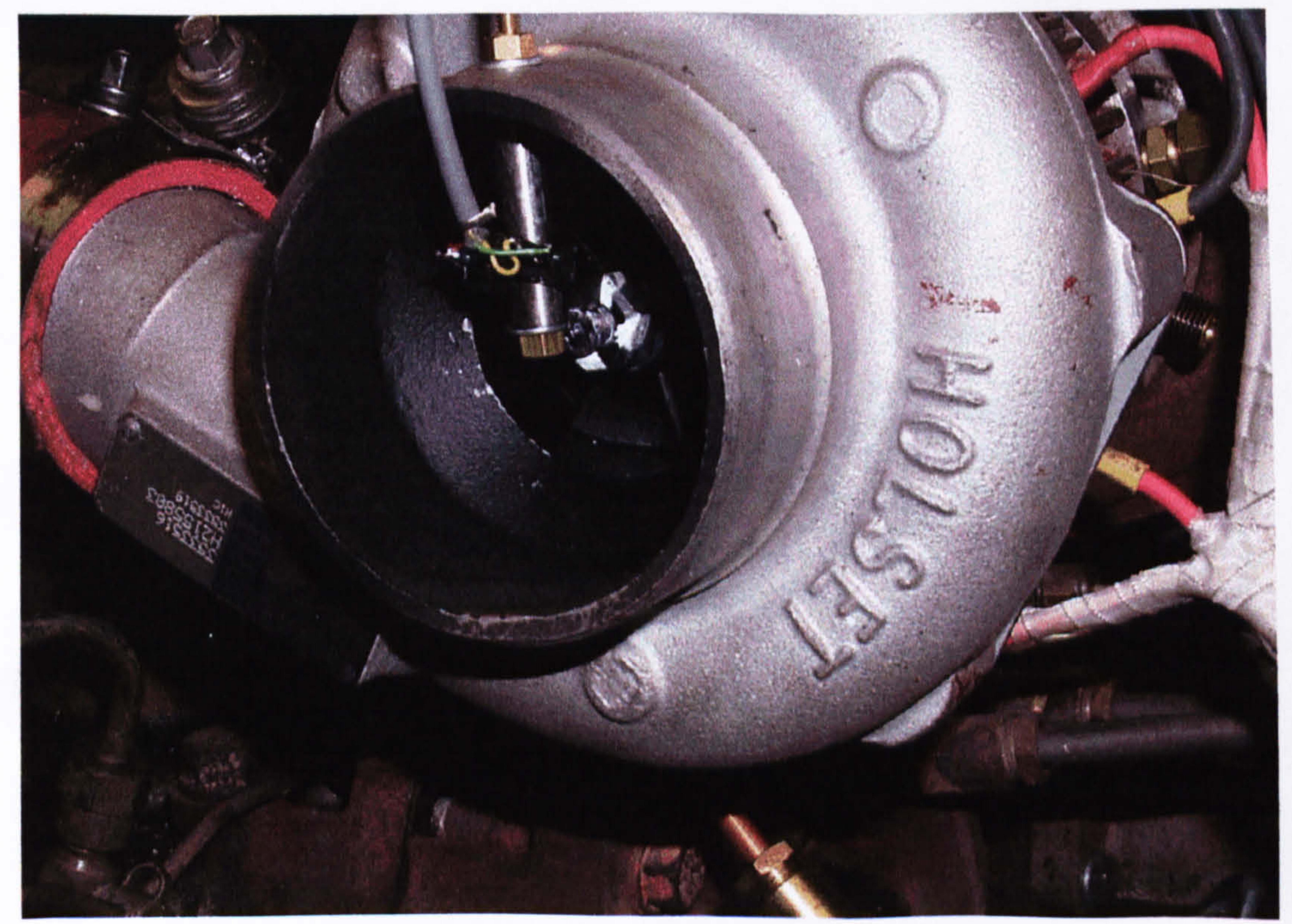

Figure 7.40. Photograph of the optical sensor mounted in the compressor scroll. 


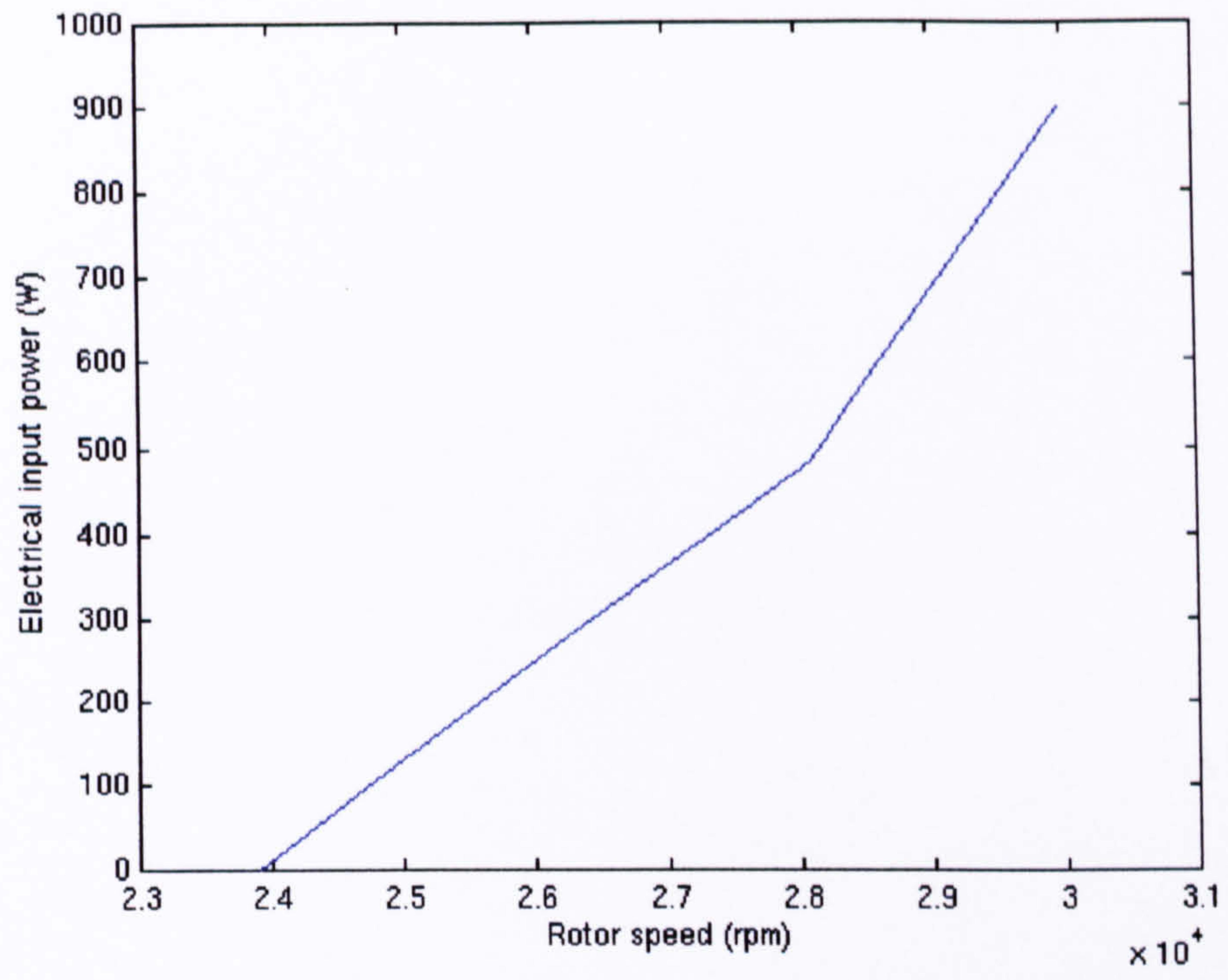

Figure 7.41. Total electrical power input to the EATG as a function of rotor speed.

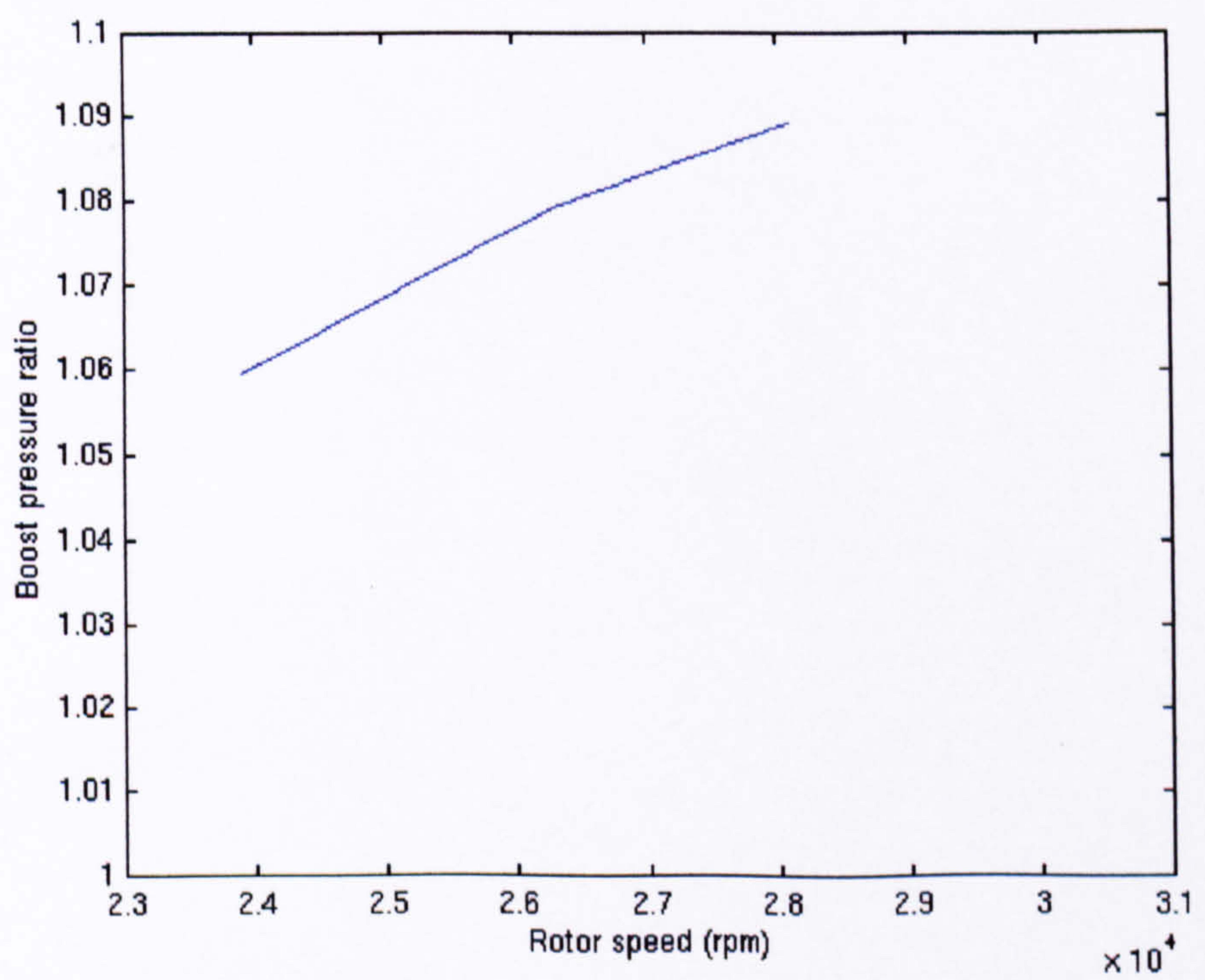

Figure 7.42. Boost pressure ratio as a function of EATG rotor speed. 


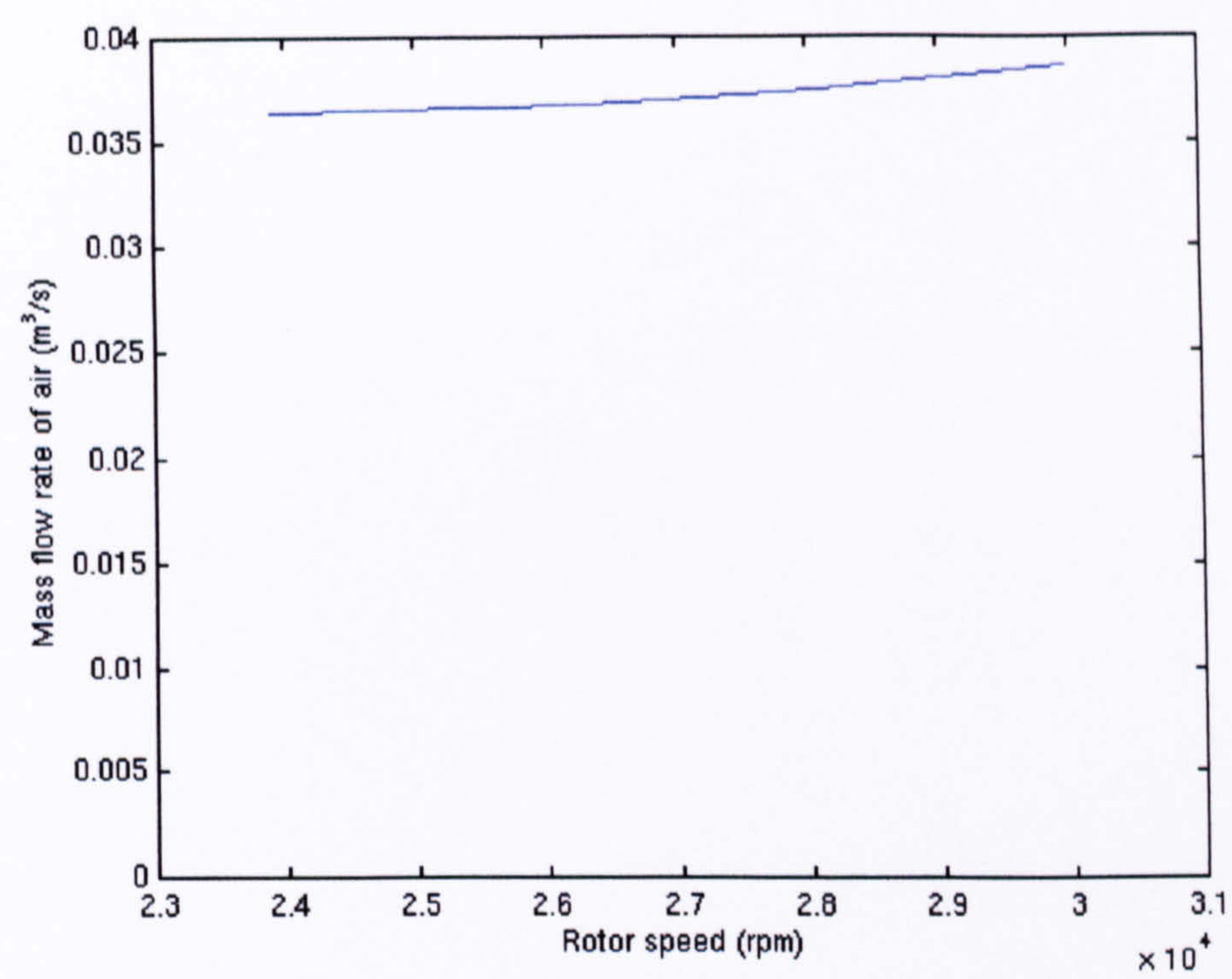

Figure 7.43. Mass flow rate of air into the engine as a function of EATG rotor speed.

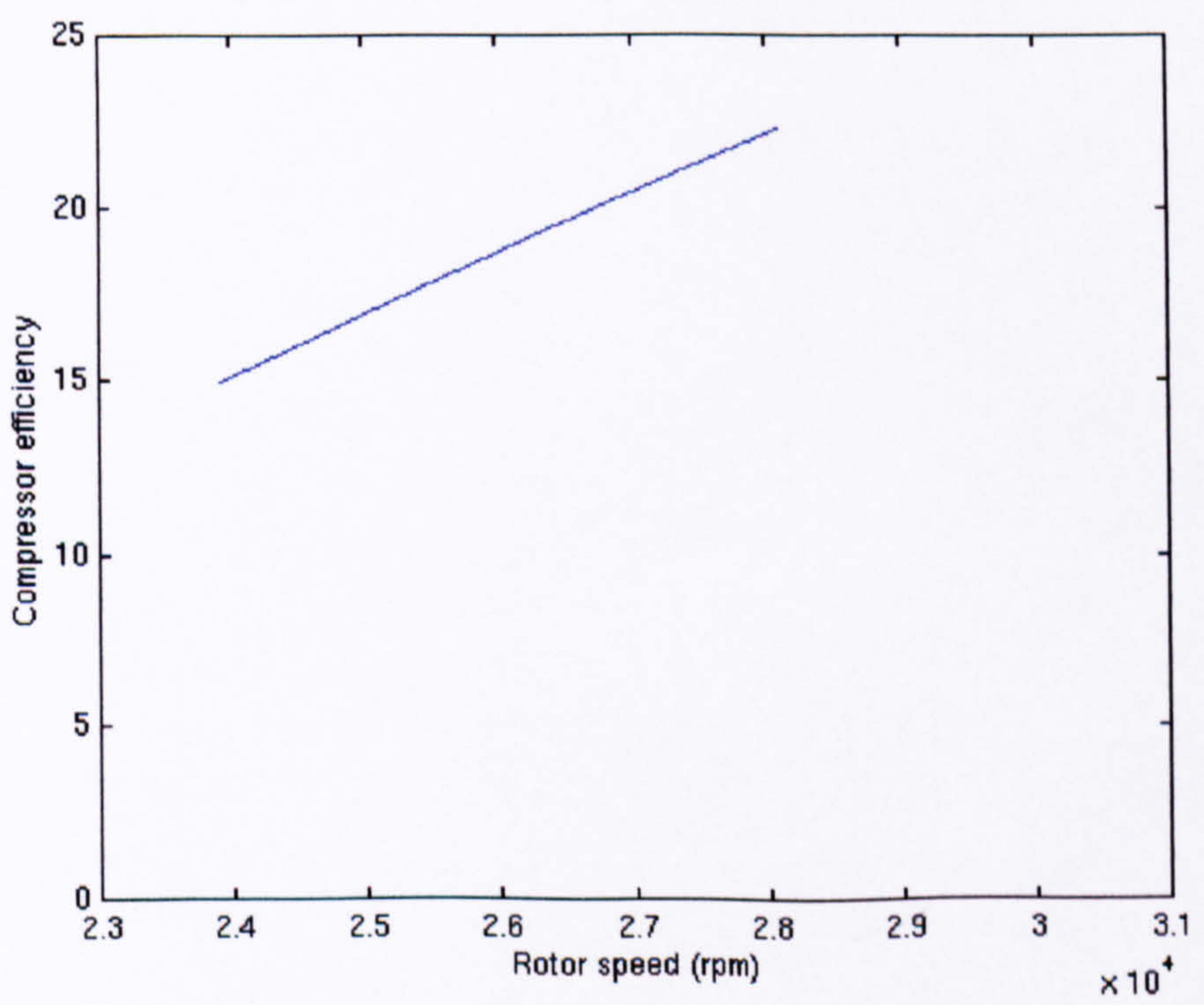

Figure 7.44. Compressor efficiency as a function of EATG rotor speed. 


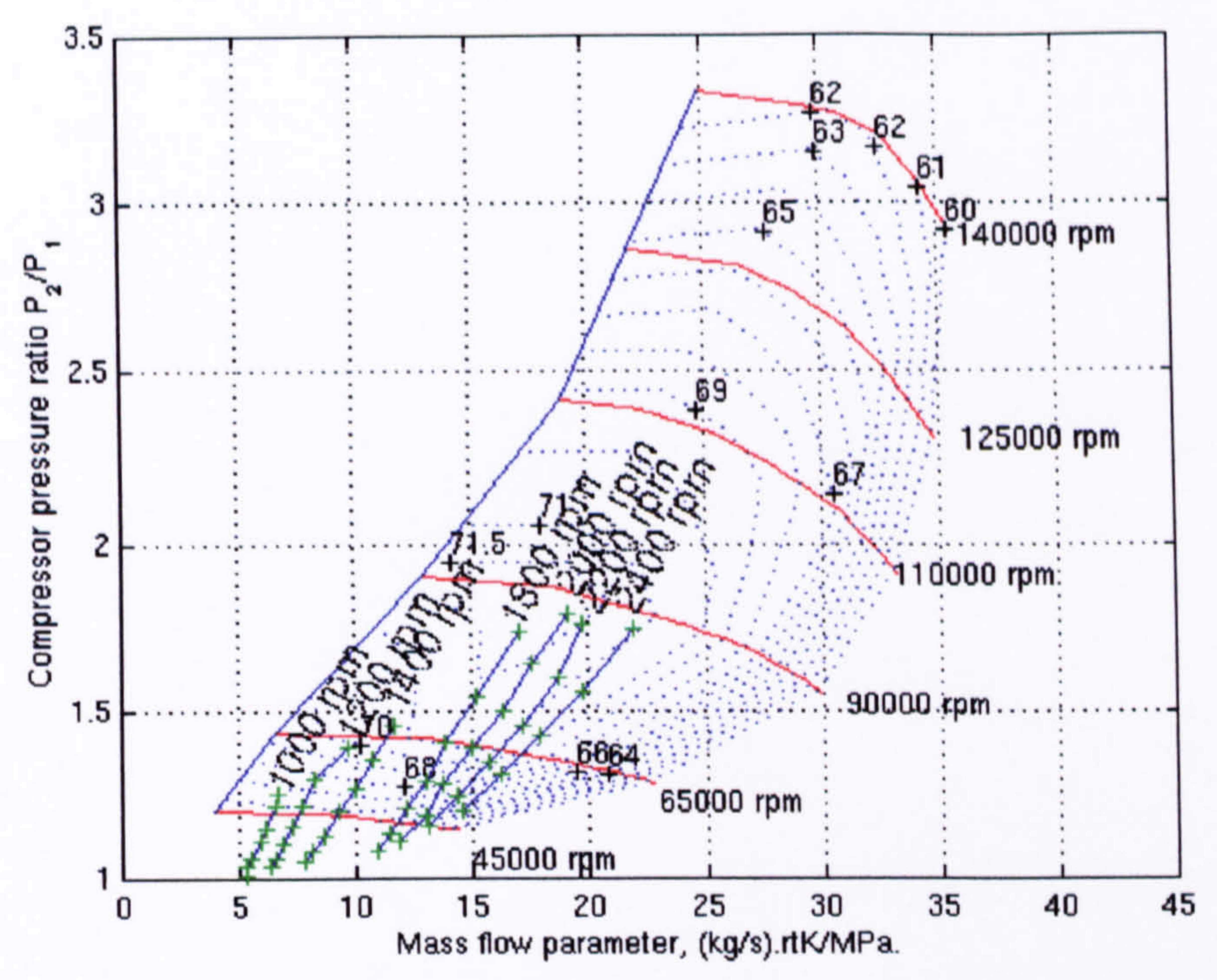

Figure 7.45. Standard compressor map with the engine operating points (with the standard $12 \mathrm{~cm}^{2}$ turbine) superimposed onto the characteristic.

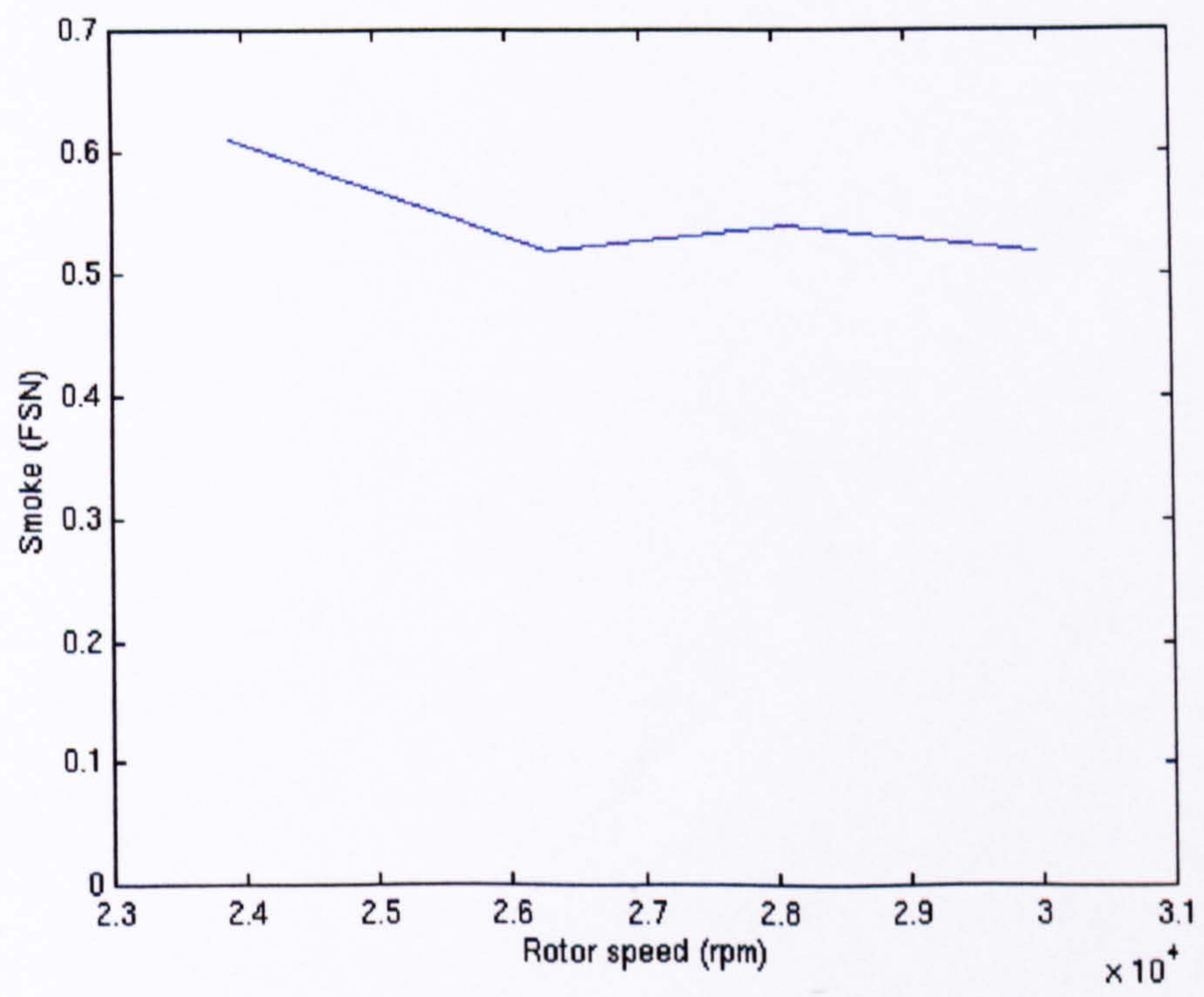

Figure 7.46. Exhaust smoke as a function of function of EATG rotor speed. 


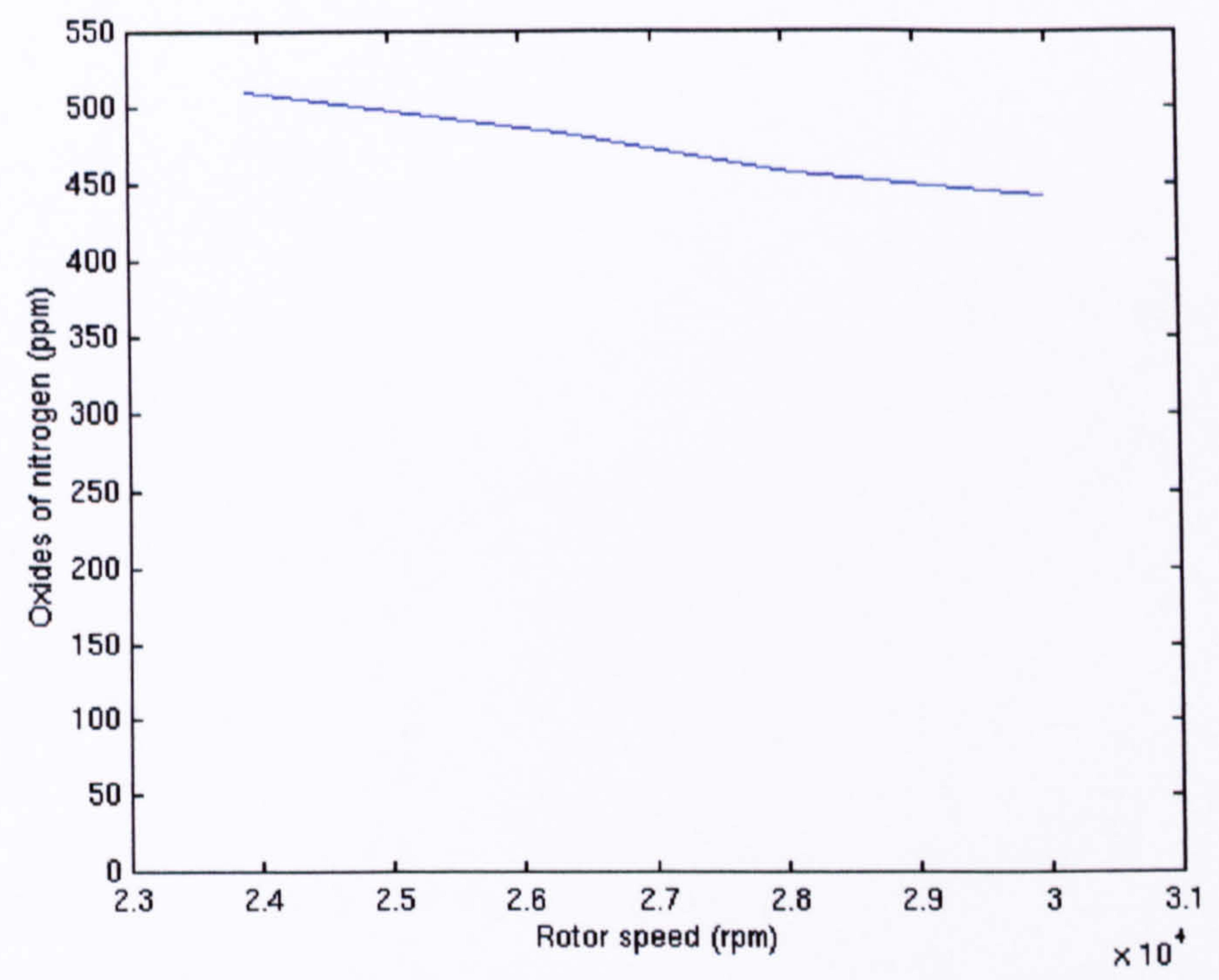

Figure 7.47. Concentration of Oxides of Nitrogen as a function of EATG rotor speed. 


\section{CHAPTER 8}

\section{DISCUSSION AND CONCLUSIONS}

\subsection{Conclusions}

This thesis has presented the findings of a wide-ranging and multi-disciplinary research programme into the feasibility and design issues related to incorporating an electrical machine into a high-speed automotive turbocharger. The research has illustrated the critical importance of a concurrent engineering approach to system design, which encompasses electromagnetics, power electronics, structural analysis, rotor dynamics and computational fluid dynamics. Indeed, although this thesis is primarily concerned with design of an electrical machine and its integration into a system, the key design parameters and constraints are dictated predominantly by mechanical considerations. In addition to describing the design of a specific system, there are a number of generic findings that are likely to be of considerable utility to other applications:

- A generalised technique (albeit one based on extensive finite element analysis) to calculate centrifugal stresses in four-pole SR rotors

- An assessment of the merits of various techniques for calculating aerodynamic losses in SR machines

- A novel technique for estimating the contribution of minor loop excursions to iron losses

The modelling and design aspects of the research have been supported by extensive experimental measurements at component, sub-systcm and system level. In general a 
reasonable degree of correlation was obtained between measured and predicted performance as detailed in the various chapters. A limited testing programme on a complete system was undertaken, with encouraging preliminary findings. However, this was curtailed by severe practical problems associated with over-heating and subsequent failure of the bearings.

In terms of the various specific outcomes of the research programme, the following conclusions can be drawn:

1 Switched reluctance machines are potentially a good fit to the application by virtue of the absence of any no-load iron loss, the robust rotor structure, their ability to operate at high temperatures and their potential for low cost volume manufacture.

2 The maximum rotor diameter in high-speed SR machines is severely constrained by considerations of centrifugal stress and the relatively poor mechanical properties of electrical steels. In the specific case of operation at $100,000 \mathrm{rpm}$ with Silicon steel, the rotor diameter is limited to $38 \mathrm{~mm}$.

3 The maximum stress in any four pole SR rotor can be estimated to a practically useful level of accuracy using a simple equation (i.e. equation 2.12 with a constant of 0.2)

4 Although SR machines tend to be manufactured with small airgaps, (of the order of a few hundred microns) this can more than readily accommodate the likely radial growth of small and medium sized SR machines at high-speeds. By way of example, for the $38 \mathrm{~mm}$ rotor in the prototype, the predicted increase in diameter at $100,000 \mathrm{rpm}$ is only some $8 \mu \mathrm{m}$ as compared to an airgap of $200 \mu \mathrm{m}$.

5 Although linear simulation methods for predicting the dynamic performance of SR machines have numerous drawbacks, e.g. inability to cater for saturation and hence large errors in predicting current waveforms under high levels of excitation, they can nevertheless be extremely useful in terms of winding design (section 3.7.1) and control system design when appropriately applied. 
6 A novel method for approximating the hysteresis loss for waveforms with minor loops has been developed (section 4.5.2.3), and validated by measurements on both material samples, with a typical correlation of $10-12 \%$.

7 Although an analytical approach to iron loss calculation has been proposed, it is apparent that it can result in a considerable underestimate of iron loss even under essentially un-saturated conditions (underestimating by some $50 \%$ the losses predicted by a detailed finite element technique - section 4.6). This is a primarily a consequence of the over simplified representation of the flux distribution near the front regions of the teeth as they come into alignment

8 In a 6-4 SR machine, a NNNSSS winding arrangement results in a reduced iron loss as compared to a NSNSNS, typically by some $8 \%$.

9 A novel test-rig that has been developed based on an iterative open-loop approach to generating flux-density waveforms. Satisfactory performance was obtained for frequencies up to $125 \mathrm{~Hz}$, and the technique offers potentially useful additional features as compared to more conventional analogue feedback systems.

10 In the calculation of aerodynamic losses in SR machines, the vast majority of the loss is generated in the narrow airgap. However, the presence of the inter-polar regions plays a significant role in terms of the magnitude of the overall loss in the narrow airgap region.

11 The aerodynamic losses in an SR rotor are higher than for an equivalent cylindrical rotor of the same diameter, particularly at high speeds.

12 Two-dimensional CFD analysis has a number of limitations in terms of calculating aerodynamic losses, even for rotors with a relatively long axial length, and recourse to three-dimensional analysis is often necessary.

13 Reasonable correlation between measured and predicted losses can be achieved when three-dimensional CFD analysis is appropriately applied (at least in terms of the correlation normally achieved with CFD analysis).

14 Even in high-speed machines, aerodynamic losses are unlikely to constitute the major loss component. By way of example, a 50,000 rpm machine with the same rotor volume as that considered in the experimental measurements, would on the basis of published output coefficients for a typical aerospace specification 
machine, have a torque capability of some $3.5 \mathrm{Nm}$ (based on a torque per unit rotor volume of $30 \mathrm{kNm}^{-2}$ ), and hence a notional power rating of some $18 \mathrm{~kW}$ or so. Thus, the measured aerodynamic losses at $50,000 \mathrm{rpm}$ would constitute a loss of some $0.5 \%$ of rated power.

15 The integration of an SR rotor into a turbocharger shaft introduces a number of additional rotordynamic de-stabilisation mechanisms, which may influence the viability of employing hydrodynamic bearing

16 For the particular rotor configuration selected, two solid body critical speeds lie within the operating speed range of the device, although these can often be driven thorough with no damage to the system. The most destructive vibration mode is likely to be the first bending mode, and again this within the speed range of the system, albeit the top end of the speed range, and is derived with an approximate equivalent stiffness for the bearing.

As discussed above, although a prototype system was manufactured, ultimately its utility could not be demonstrated over its full operating range. In terms of future viability of the concept, the manufacture and testing of the prototype has identified the following key issues:

Rotor position sensing - The harsh environment, in terms of dirt ingress, high temperatures and the electromagnetic interference, dictates that the integration of a robust, repeatable and accurate means of detecting position is extremely challenging. Although in principle, automotive grade variable reluctance sensors can be employed to exploit the salient nature of the SR rotor, in practice the large stray magnetic fields that are present in the vicinity of the end-windings preclude their use. Although some successes was achieved by employing an optical approach, this can only be regarded as a temporary solution for prototype evaluation given its invasive nature in terms of the air-inlet and its likely poor robustness in terms of the build up of contaminants. 
Bearings - Arguably the key enabling technology for any future commercialisation is the bearing system, in particular the turbine-end bearing which operates in a harsh high temperature environment. The testing of the prototype has illustrated that cooling of the turbine-end bearing is critical in terms of realising a viable system However, it is worth noting that ultimately any cost-effective mass-market systems are likely to require the use of hydrodynamic bearings as discussed in chapter 6, rather than high-precision rolling-element bearings. However, as discussed in chapter 6 , this has attendant problems in terms of stability, particularly since SR machines introduce considerable unbalanced magnetic pull and torque pulsations.

In summary, incorporating an SR machine into a turbocharger has very significant potential performance benefits. However, it is an extremely challenging application, in which many of the system components are operating near their limits. Further, there are considerable trade-offs between electrical and mechanical performance, which can only be investigated by detailed analysis. The ultimate commercialisation of such systems is likely to still require major advances in enabling technologies, most notably high-speed bearings that can tolerate the harsh environments and the large loads imposed by SR machines. 